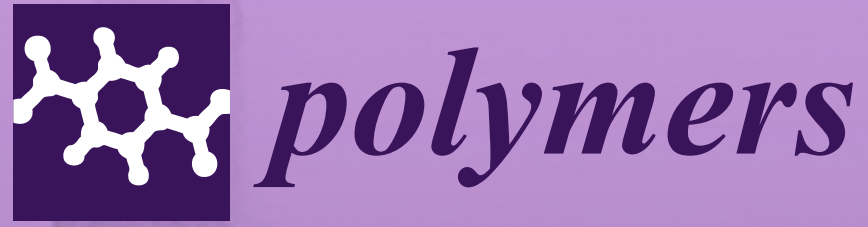

\title{
Polymer Clay \\ Nano-composites
}

Edited by

Stefano Leporatti

Printed Edition of the Special Issue Published in Polymers 


\section{Polymer Clay Nano-composites}





\section{Polymer Clay Nano-composites}

Special Issue Editor

Stefano Leporatti

MDPI • Basel • Beijing • Wuhan • Barcelona • Belgrade

\section{MDPI}


Special Issue Editor

Stefano Leporatti

CNR Nanotec-Istituto di Nanotecnologia

Italy

Editorial Office

MDPI

St. Alban-Anlage 66

4052 Basel, Switzerland

This is a reprint of articles from the Special Issue published online in the open access journal Polymers (ISSN 2073-4360) from 2017 to 2019 (available at: https://www.mdpi.com/journal/polymers/ special_issues/polymer_clay_composites)

For citation purposes, cite each article independently as indicated on the article page online and as indicated below:

LastName, A.A.; LastName, B.B.; LastName, C.C. Article Title. Journal Name Year, Article Number, Page Range.

ISBN 978-3-03921-652-9 (Pbk)

ISBN 978-3-03921-653-6 (PDF)

Cover image courtesy of Stefano Leporatti.

(C) 2019 by the authors. Articles in this book are Open Access and distributed under the Creative Commons Attribution (CC BY) license, which allows users to download, copy and build upon published articles, as long as the author and publisher are properly credited, which ensures maximum dissemination and a wider impact of our publications.

The book as a whole is distributed by MDPI under the terms and conditions of the Creative Commons license CC BY-NC-ND. 


\section{Contents}

About the Special Issue Editor $\ldots \ldots \ldots \ldots \ldots \ldots \ldots$ vii

Stefano Leporatti

Polymer Clay Nano-composites

Reprinted from: Polymers 2019, 11, 1445, doi:10.3390/polym11091445 . . . . . . . . . . . 1

Yang Wu, Yongzhi Zhang, Junping Ju, Hao Yan, Xiaoyu Huang and Yeqiang Tan

Advances in Halloysite Nanotubes-Polysaccharide Nanocomposite Preparation and Applications

Reprinted from: Polymers 2019, 11, 987, doi:10.3390/polym11060987 . . . . . . . . . . 5

Xiandong Zhang and Guangshun Wu

Grafting Halloysite Nanotubes with Amino or Carboxyl Groups onto Carbon Fiber Surface for Excellent Interfacial Properties of Silicone Resin Composites

Reprinted from: Polymers 2018, 10, 1171, doi:10.3390/polym10101171 . . . . . . . . . . . 2

Xiaohu Qiang, Songyi Zhou, Zhuo Zhang, Qiling Quan and Dajian Huang

Synergistic Effect of Halloysite Nanotubes and Glycerol on the Physical Properties of Fish Gelatin Films

Reprinted from: Polymers 2018, 10, 1258, doi:10.3390/polym10111258 . . . . . . . . . . 36

Vladimir Vinokurov, Andrei Novikov, Valentina Rodnova, Boris Anikushin, Mikhail Kotelev, Evgenii Ivanov and Yuri Lvov

Cellulose Nanofibrils and Tubular Halloysite as Enhanced Strength Gelation Agents

Reprinted from: Polymers 2019, 11, 919, doi:10.3390/polym11050919 . . . . . . . . . . . . 50

Angelo Earvin Sy Choi, Cybelle Morales Futalan and Jurng-Jae Yee

Fuzzy Optimization on the Synthesis of Chitosan-Graft-Polyacrylic Acid with Montmorillonite as Filler Material: A Case Study

Reprinted from: Polymers 2019, 11, 738, doi:10.3390/polym11040738 . . . . . . . . . . . . 61

Jinhui Liu, Di Li, Xiangshuai Zhao, Jieting Geng, Jing Hua and Xin Wang

Buildup of Multi-Ionic Supramolecular Network Facilitated by In-Situ Intercalated Organic

Montmorillonite in 1,2-Polybutadiene

Reprinted from: Polymers 2019, 11, 492, doi:10.3390/polym11030492 . . . . . . . . . . . 77

Guiqing Shu, Jing Zhao, Xiu Zheng, Mengdie Xu, Qi Liu and Minfeng Zeng

Modification of Montmorillonite with Polyethylene Oxide and Its Use as Support for $\mathrm{Pd}^{0}$

Nanoparticle Catalysts

Reprinted from: Polymers 2019, 11,755, doi:10.3390/polym11050755 . . . . . . . . . . . 95

Chengcheng Yu, Yangchuan Ke, Xu Hu, Yi Zhao, Qingchun Deng and Shichao Lu

Effect of Bifunctional Montmorillonite on the Thermal and Tribological Properties of Polystyrene/Montmorillonite Nanocomposites

Reprinted from: Polymers 2019, 11, 834, doi:10.3390/polym11050834 . . . . . . . . . . . 106

\section{Ji Zhou, Qiang Cai and Fu Xu}

Nanoscale Mechanical Properties and Indentation Recovery of PI@GO Composites Measured Using AFM

Reprinted from: Polymers 2018, 10, 1020, doi:10.3390/polym10091020 . . . . . . . . . . . 123 
Tingting Jiang, Guangxiang Chen, Xiangyang Shi and Rui Guo

Hyaluronic Acid-Decorated Laponite ${ }^{\circledR}$ Nanocomposites for Targeted Anticancer

Drug Delivery

Reprinted from: Polymers 2019, 11, 137, doi:10.3390/polym11010137 . . . . . . . . . . . . 130

Jorge A. Ramírez-Gómez, Javier Illescas, María del Carmen Díaz-Nava, Claudia Muro-Urista, Sonia Martínez-Gallegos and Ernesto Rivera

Synthesis and Characterization of Clay Polymer Nanocomposites of P(4VP-co-AAm) and Their Application for the Removal of Atrazine

Reprinted from: Polymers 2019, 11, 721, doi:10.3390/polym11040721 . . . . . . . . . . . . . 144

Alexandros K. Nikolaidis, Elisabeth A. Koulaouzidou, Christos Gogos and

Dimitris S. Achilias

Synthesis and Characterization of Dental Nanocomposite Resins Filled with Different Clay Nanoparticles

Reprinted from: Polymers 2019, 11, 730, doi:10.3390/polym11040730 . . . . . . . . . . . . . 164

Cinzia Cristiani, Elena Maria Iannicelli-Zubiani, Giovanni Dotelli, Elisabetta Finocchio, Paola Gallo Stampino and Maurizio Licchelli

Polyamine-Based Organo-Clays for Polluted Water Treatment: Effect of Polyamine Structure and Content

Reprinted from: Polymers 2019, 11, 897, doi:10.3390/polym11050897 . . . . . . . . . . . . . . 185

Yu Liang, Dexin Yang, Tao Yang, Ning Liang and Hao Ding

The Stability of Intercalated Sericite by Cetyl Trimethylammonium Ion under Different Conditions and the Preparation of Sericite/Polymer Nanocomposites

Reprinted from: Polymers 2019, 11, 900, doi:10.3390/polym11050900 . . . . . . . . . . . . . . 201

Yidong Liu, Lingfeng Jian, Tianhua Xiao, Rongtao Liu, Shun Yi, Shiyang Zhang,

Lingzhi Wang, Ruibin Wang and Yonggang Min

High Performance Attapulgite/Polypyrrole Nanocomposite Reinforced Polystyrene (PS) Foam

Based on Supercritical $\mathrm{CO}_{2}$ Foaming

Reprinted from: Polymers 2019, 11, 985, doi:10.3390/polym11060985 . . . . . . . . . . . . . 212

Elodie Bugnicourt, Nicola Brzoska, Esra Kucukpinar, Severine Philippe, Enrico Forlin, Alvise Bianchin and Markus Schmid

Dispersion and Performance of a Nanoclay/Whey Protein Isolate Coating upon its Upscaling as a Novel Ready-to-Use Formulation for Packaging Converters

Reprinted from: Polymers 2019, 11, 1410, doi:10.3390/polym11091410 . . . . . . . . . . . . 222 


\section{About the Special Issue Editor}

Stefano Leporatti, Ph.D., received his Master's degree in Physics at University of Genoa and in 1999 obtained his PhD in Solid State Physics at Max Planck Institute of Colloids and Interface Science with Prof. Dr. Helmuth Mohwald. From 2001 to 2006, he was a Research Scientist at the Institute of Medical Physics \& Biophysics, Universität Leipzig, Leipzig (Germany). From 2006 to 2008 he was CNR Senior Researcher (University Associate Professor Equivalent) at the National Nanotechnology Laboratory (NNL) of CNR-INFM in Lecce, Italy (NanoBioMolecular Division of Prof. R. Rinaldi) and, since 2008, he has been their NanoCarriers and BioMechanics Group Leader. From 2015 he has worked at the newly constituted CNR Institute of Nanotechnology, CNR Nanotec in Lecce (Italy) as Primo Ricercatore CNR (Research Professor). 



\title{
Editorial
}

\section{Polymer Clay Nano-composites}

\author{
Stefano Leporatti \\ CNR Nanotec-Istituto di Nanotecnologia c \o Campus Ekotecne via Monteroni, 73100 Lecce, Italy; \\ stefano.leporatti@nanotec.cnr.it
}

Received: 30 August 2019; Accepted: 3 September 2019; Published: 3 September 2019

Clay-polymer composite materials is an exciting area of research and this Special Issue aims to address the current state-of-the-art of "Polymer Clay Nano-Composites" for several applications, among them antibacterial, environmental, water remediation, dental, drug delivery and others. The original scope of the Special Issue was comprehensively devoted to the synthesis and characterization of polymer clay nano-composites employed for several applications, including nano-clay polymer composites and hybrid nano-assemblies. Furthermore, polymers can be loaded with clay nanoparticles creating novel composite nano-materials enhancing composite strength features. The issue is composed of 16 contributions, fifteen articles and one review. They can be conveniently divided into one group related to Halloysite-composites (four papers including one review), a second group which deals with Montmorillonite-composites (four papers) and a third group, which can be generically referred to Hybrid Clay Nano-Composites (eight contributions).

Relative to the group of Halloysite-composites, in the review [1] Wu et al. summarized the recent progress toward the development of polysaccharide-HNTs composites, paying attention to the main existence forms and wastewater treatment application particularly. The purification of Halloysite Nanotubes (HNTs) and fabrication of the composites were also discussed. Furthermore, they reported the unique characteristics of polysaccharide-HNTs composites and reviewed the recent development of the practical applications. In particular they pointed out that (1) polysaccharide-HNTs composites have improved mechanical, thermal, and swelling properties and good biocompatibility. Therefore they are promising nano-fillers for high-performance polymer composites. (2) HNTs can be combined with polysaccharides by different methods (3) the degree of dispersion of HNTs and the interfacial interactions between polysaccharides and HNTs are key factors affecting the performance of composites. (4) Polysaccharide-HNTs composites has shown promising potential for biomedical applications. Another contribution from Xiandong Zhang and Guangshun Wu [2] dealt with HNTs Carbon Fiber (CFs) composites. The authors achieved for the first time the chemical grafting of halloysite nanotubes (HNTs) with amino or carboxyl groups onto the CFs surface, which was aimed to enhance the composites interfacial strength. Functional groups of HNTs and fiber surface structures were characterized, as well as interfacial properties and anti-hydrothermal aging behaviors. Interfacial reinforcement mechanisms for untreated and modified CF composites were also compared and discussed. Morphology, mechanical properties, water resistance and optical properties of the Fish gelatin (FG)/glycerol (GE)/halloysite (HT) composite films were investigated by Qiang et al. in [3]. Interestingly, they showed that with increasing GE content, the elongation at composite breaks increased significantly, but their tensile strength (TS) and water resistance decreased. Their results indicated that the addition of GE greatly improved film flexibility with a decrease in the TS of the film. Moisture uptake and water solubility were also improved by the addition of GE into the FG matrix, indicating that the water-resistance of the film decreased due to the GE added. Furthermore, the presence of GE enhanced the dispersion of HTs in the FG matrix and thus enhanced the properties of the obtained composite films. In the last paper of this HNTs-composites group, Lvov et al. [4] wanted to answer the following question: What additives could be used to increase the strength of silica gels? To answer this, they prepared colloidal silica gels with various additives and they measured gel strength. It was found 
that cellulose nanofibrils considerably increased the gel strength. Furthermore, cellulose nanofibrils could be produced from cheap industrial-grade cellulose with low-cost industrial chemicals. Therefore, cellulose nanofibrils produced from renewable sources and naturally occurring halloysite nanoclay could be used as complementary reinforcing agents.

In the second group related to Montmorillonite-composites, an interesting article, authored by Choi et al. [5], reported the synthesis of a chitosan-montmorillonite nano-composite material grafted with acrylic acid based on its function in a case study analysis. Fuzzy optimization was used for a multi-criteria decision analysis to determine the best desirable swelling capacity (YQ) of the material synthesis at its lowest possible variable cost. A multi-objective fuzzy optimization showed an innovative approach to determine a solution for the best condition in the material synthesis. Therefore, this approach proved to be a practical method for examining the best possible compromise solution based on the desired function to adequately synthesize a material. Moreover, the incorporation of the criteria of the variable cost in terms of material usage and the cumulative uncertainty of the response successfully ensued essential compromise results in the decision-making process. The development of a sacrificial bond provided unique inspiration for the design of advanced elastomers with excellent mechanical properties, but it was still a big challenge to construct a homogeneous polar sacrificial network in a nonpolar elastomer. In this view, Liu et al. [6] proposed a novel strategy to engineer a multi-ionic network into a covalently cross-linked 1,2-polybutadiene (1,2-PB) facilitated by in situ intercalated organic montmorillonite (OMMT) without phase separation. Overall, their work showed the design of a uniform and strong sacrificial network in the nano-clay/elastomer nano-composite with outstanding mechanical performances under both static and dynamic conditions. Future work will be devoted to further improving the ionic crosslinking density and constructing a stronger sacrificial network to prepare shape memory or self-recovery materials and studying the dynamics of ionic crosslinking. In another article montmorillonites (MMT) were modified by intercalating polyethylene oxide (PEO) macromolecules between the interlayer spaces in an MMT-water suspension system [7]. Shu et al. chose MMT/PEO 80/20 composite as the support platform for immobilization of Pd species in preparing novel heterogeneous catalysts. Their results confirmed that Pd nanoparticles were confined within the interlayer space of MMT and/or dispersed well on the outer surface of MMT. This work offers an alternative approach to the preparation of Pd heterogeneous catalysts with fairly good performances, and heterogeneous catalysts with fairly good performances, and could have broad prospects in both experimental and industrial applications. The effect of doubly functionalized montmorillonite (MMT) on the structure, morphology, thermal, and tribological characteristics of the resulting polystyrene (PS) nano-composites were investigated by Yu et al. [8]. The modification of the MMT was performed using a cationic surfactant and an anionic surfactant or a silane-coupling agent to increase the compatibility with PS matrix. The nanocomposites prepared by a cationic surfactant and a silane-coupling agent exhibited the best thermal stability and tribological performance, providing significant guidance for the future synthesis and application of the PS/OMMT nanocomposites in the oil and gas drilling engineering field to improve drilling fluid lubrication.

The last part of Special Issue is composed of different papers, which can be collected in a common category namely "Hybrid Clay Nano-Composites". Zhu et al. [9] have prepared Polyimide@graphene oxide (PI@GO) composites by way of a simple solution blending method. The nanoscale hardness and Young's modulus of the composites were measured using nano-indentation through atomic force microscopy (AFM). They showed that relatively low GO content could remarkably improve the nanoscale mechanical properties of PI and they demonstrated that 2D nano-materials could improve the self-healing performance of polymer composites. In another paper Jiang et al. [10] conjugated hyaluronic acid (HA) - a natural polysaccharide that can specifically bind to CD44 receptors, onto laponite ${ }^{\circledR}$ (LAP) nano-disks for the encapsulation and targeted delivery of the anti-cancer drug doxorubicin (DOX) to CD44-overexpressed cancer cells. Their results demonstrate that the HA-modified LAP nano-disks with high drug loading efficiency, $\mathrm{pH}$-sensitive drug release properties and CD44 targetability might be an efficient nano-platform for cancer chemotherapy. Another interesting work dealing with the 
adsorption of Atrazine (ATZ) from aqueous solutions using nanocomposite materials, synthesized with two different types of organo-modified clays was written by Jorge A. Ramírez-Gómez et al. [11]. The structural, morphological, and textural characteristics of clays, copolymers, and nano-composites were determined through different analytical and instrumental techniques. They finally demonstrated that the synthesized nano-composites with higher molar fractions of $4 \mathrm{VP}$ obtained the highest removal percentages of ATZ.

The article written by Alexandros K. Nikolaidis et al. [12] covers an interesting area of the application of clay nano-composites: dental materials. It focuses on the reinforcement of dental nano-composite resins with diverse organomodified montmorillonite (OMMT) nanofillers. The aim of this work was to monitor whether the presence of functional groups in the chemical structure of the nanoclay organic modifier may virtually influence the physicochemical and/or the mechanical attitude of the dental resin nano-composites. An enhancement of the flexural modulus was observed, mainly by using clay nanoparticles decorated with methacrylated groups, along with a decrease in the flexural strength at a high filler loading. This work can provide novel information about chemical interaction phenomena between nano-fillers and the organic matrix towards the improvement of dental restorative materials. In the following contribution, Elisabetta Finocchio's group [13] modified a montmorillonite clay with three different aliphatic polyamines and deeply investigated the interaction mechanisms between clay and amines by different experimental techniques among them X-ray powder diffraction (XRD), thermal analysis measurements (DTG), Fourier Transform Infrared Spectroscopy (FT-IR). Their experimental results showed that the amount of amines efficiently immobilized in the solid phase could be enhanced by increasing the initial concentration of polyamines in the clay modification process, envisaging that polyamine-based organo-clays are promising materials for their proposed application in environmental remediation. Layered silicates are suitable for use as fillers in nano-composites based on a large aspect ratio, easy availability, and chemical resistance. Sericite is distinguished for its higher aspect ratio, higher resilience, and ultraviolet shielding and absorption. In this view, Liang et al. [14] studied the stability of the sericite intercalated by CTAB by changing different washing solvents, different temperatures, ultrasonic cleaning, and different solution conditions. Sericite/polymer nano-composites were produced with the stable intercalated sericite, and demonstrated excellent properties compared with pure epoxy resin. Altogether these results have suggested that stable intercalated sericite is a precondition for good adhesion between the sericite and epoxy resin, which gives rise to good nano-composite mechanical properties. In the contribution of Liu et al. [15] an attapulgite (ATP)/polypyrrole (PPy) nano-composite was developed employing the in situ polymerization method to produce the hierarchical cell texture for the PS foam based on the supercritical $\mathrm{CO}_{2}$ foaming. The results showed that the nano-composite could act as an efficient $\mathrm{CO}_{2}$ capturer enabling the random release of it during the foaming process. Therefore, the in situ polymerized ATP/PPy nano-composite makes a supercritical $\mathrm{CO}_{2}$ foaming desired candidate to replace the widely used fluorocarbons and chlorofluorocarbons as PS blowing agents. Finally, Bugnicourt et al. [16] investigated the effect of various preparation methods on different production scales (pilot- and semi-industrial scale) on the barrier performance and morphological properties of the applied nano-composites. A nano-enhanced composition was converted into a so-called "ready-to-use" formulation by means of a solid-state pre-dispersion process using ball-milling. The preparation of a coating formulation using the ready-to-use granules and its up-scaling for roll-to-roll converting of a pilot- and semi-industrial scale was also successfully implemented. Transmission electron microscopy, scanning electron microscopy, as well as oxygen permeability measurements have been employed to characterize the effects of both the production at various scales and ultrasound treatment on the morphology and barrier performance of the nano-composites. Authors concluded that the solid state pre-dispersion of the nano-platelets during the production of the ready-to-use formulation was the predominant process determining the ultimate degree of nanoparticle orientation and dispersion state. 


\section{References}

1. Wu, Y.; Zhang, Y.; Ju, J.; Yan, H.; Huang, X.; Tan, Y. Advances in Halloysite Nanotubes-Polysaccharide Nanocomposite Preparation and Applications. Polymers 2019, 11, 987. [CrossRef] [PubMed]

2. Zhang, X.; Wu, G. Grafting Halloysite Nanotubes with Amino or Carboxyl Groups onto Carbon Fiber Surface for Excellent Interfacial Properties of Silicone Resin Composites. Polymers 2018, 10, 1171. [CrossRef]

3. Qiang, X.; Zhou, S.; Zhang, Z.; Quan, Q.; Huang, D. Synergistic Effect of Halloysite Nanotubes and Glycerol on the Physical Properties of Fish Gelatin Films. Polymers 2018, 10, 1258. [CrossRef] [PubMed]

4. Vinokurov, V.; Novikov, A.; Rodnova, V.; Anikushin, B.; Kotelev, M.; Ivanov, E.; Lvov, Y. Cellulose Nanofibrils and Tubular Halloysite as Enhanced Strength Gelation Agents. Polymers 2019, 11, 919. [CrossRef] [PubMed]

5. Choi, A.E.S.; Futalan, C.M.; Yee, J. Fuzzy Optimization on the Synthesis of Chitosan-Graft-Polyacrylic Acid with Montmorillonite as Filler Material: A Case Study. Polymers 2019, 11, 738. [CrossRef] [PubMed]

6. Liu, J.; Li, D.; Zhao, X.; Geng, J.; Hua, J.; Wang, X. Buildup of Multi-Ionic Supramolecular Network Facilitated by In-Situ Intercalated Organic Montmorillonite in 1,2-Polybutadiene. Polymers 2019, 11, 492. [CrossRef] [PubMed]

7. Shu, G.; Zhao, J.; Zheng, X.; Xu, M.; Liu, Q.; Zeng, M. Modification of Montmorillonite with Polyethylene Oxide and Its Use as Support for Pd0 Nanoparticle Catalysts. Polymers 2019, 11, 755. [CrossRef] [PubMed]

8. Yu, C.; Ke, Y.; Hu, X.; Zhao, Y.; Deng, Q.; Lu, S. Effect of Bifunctional Montmorillonite on the Thermal and Tribological Properties of Polystyrene/Montmorillonite Nanocomposites. Polymers 2019, 11, 834. [CrossRef]

9. Zhou, J.; Cai, Q.; Xu, F. Nanoscale Mechanical Properties and Indentation Recovery of PI@GO Composites Measured Using AFM. Polymers 2018, 10, 1020. [CrossRef]

10. Jiang, T.; Chen, G.; Shi, X.; Guo, R. Hyaluronic Acid-Decorated Laponite ${ }^{\circledR}$ Nanocomposites for Targeted Anticancer Drug Delivery. Polymers 2019, 11, 137. [CrossRef] [PubMed]

11. Ramírez-Gómez, J.A.; Illescas, J.; del Carmen Díaz-Nava, M.; Muro-Urista, C.; Martínez-Gallegos, S.; Rivera, E. Synthesis and Characterization of Clay Polymer Nanocomposites of P(4VP-co-AAm) and Their Application for the Removal of Atrazine. Polymers 2019, 11, 721. [CrossRef]

12. Nikolaidis, A.K.; Koulaouzidou, E.A.; Gogos, C.; Achilias, D.S. Synthesis and Characterization of Dental Nanocomposite Resins Filled with Different Clay Nanoparticles. Polymers 2019, 11, 730. [CrossRef] [PubMed]

13. Cristiani, C.; Iannicelli-Zubiani, E.M.; Dotelli, G.; Finocchio, E.; Stampino, P.G.; Licchelli, M. Polyamine-Based Organo-Clays for Polluted Water Treatment: Effect of Polyamine Structure and Content. Polymers 2019, 11, 897. [CrossRef]

14. Liang, Y.; Yang, D.; Yang, T.; Liang, N.; Ding, H. The Stability of Intercalated Sericite by Cetyl Trimethylammonium Ion under Different Conditions and the Preparation of Sericite/Polymer Nanocomposites. Polymers 2019, 11, 900. [CrossRef] [PubMed]

15. Liu, Y.; Jian, L.; Xiao, T.; Liu, R.; Yi, S.; Zhang, S.; Wang, L.; Wang, R.; Min, Y. High Performance Attapulgite/Polypyrrole Nanocomposite Reinforced Polystyrene (PS) Foam Based on Supercritical $\mathrm{CO}_{2}$ Foaming. Polymers 2019, 11, 985. [CrossRef]

16. Bugnicourt, E.; Brzoska, N.; Kucukpinar, E.; Philippe, S.; Forlin, E.; Bianchin, A.; Schmid, M. Dispersion and Performance of a Nanoclay/Whey Protein Isolate Coating upon its Upscaling as a Novel Ready-to-Use Formulation for Packaging Converters. Polymers 2019, 11, 1410. [CrossRef] [PubMed]

(C) 2019 by the author. Licensee MDPI, Basel, Switzerland. This article is an open access article distributed under the terms and conditions of the Creative Commons Attribution (CC BY) license (http://creativecommons.org/licenses/by/4.0/). 
Review

\title{
Advances in Halloysite Nanotubes-Polysaccharide Nanocomposite Preparation and Applications
}

\author{
Yang $\mathrm{Wu}^{1}$, Yongzhi Zhang ${ }^{1}$, Junping Ju ${ }^{1, *}$, Hao Yan ${ }^{1}$, Xiaoyu Huang ${ }^{2}$ and Yeqiang Tan ${ }^{1, *}$ \\ 1 State Key Laboratory of Bio-fibers and Eco-textiles, Collaborative Innovation Center for Marine Biomass \\ Fibers, Materials and Textiles of Shandong Province, School of Materials Science and Engineering, Qingdao \\ University, Qingdao 266071, China; wy921170920@163.com (Y.W.); zyz18919@163.com (Y.Z.); \\ yanhao1287476167@163.com (H.Y.) \\ 2 Key Laboratory of Synthetic and Self-Assembly Chemistry for organic Functional Molecules, Shanghai \\ Institute of Organic Chemistry, Chinese Academy of Sciences, Shanghai 200032, China; xyhuang@sioc.ac.cn \\ * Correspondence: jujunping@qdu.edu.cn (J.J.); tanyeqiang@qdu.edu.cn (Y.T.); \\ Tel./Fax: +86-571-8595-0961 (Y.T.)
}

Received: 18 April 2019; Accepted: 22 May 2019; Published: 4 June 2019

\begin{abstract}
Halloysite nanotubes (HNTs), novel 1D natural materials with a unique tubular nanostructure, large aspect ratio, biocompatibility, and high mechanical strength, are promising nanofillers to improve the properties of polymers. In this review, we summarize the recent progress toward the development of polysaccharide-HNTs composites, paying attention to the main existence forms and wastewater treatment application particularly. The purification of HNTs and fabrication of the composites are discussed first. Polysaccharides, such as alginate, chitosan, starch, and cellulose, reinforced with HNTs show improved mechanical, thermal, and swelling properties. Finally, we summarize the unique characteristics of polysaccharide-HNTs composites and review the recent development of the practical applications.
\end{abstract}

Keywords: halloysite nanotubes; polysaccharide; interfacial interactions; reinforcing; adsorption

\section{Introduction}

Nanofillers recently have drawn extensive attention from academic and industrial fields due to their unique performance [1]. The traditional materials, such as black carbon, graphite, silica, and silicate, can significantly improve the mechanical properties, thermal stability, and permeability of various polymers [2,3]. Nowadays, clay mineral nanofillers with large aspect ratios, high strength, and relatively low density have attracted intense research interest [4]. Clay minerals, natural materials with proven biocompatibility and abundant storage, exhibit unique properties for various applications [5]. The majority of the research concerning clay minerals is devoted to kaolinite [6], montmorillonite [7], and illite [8]. In recent years, halloysite nanotubes (HNTs), 1D natural materials with a unique tubular nanostructure, large aspect ratio, biocompatibility, and high mechanical strength, have arisen as promising nanofillers to improve the properties of polymers $[9,10]$.

Halloysite was first proposed by Berthier (1826) [11]. Raw halloysite, which is usually white, is exploited from natural sediments and is easily processed into powder. The sizes of halloysite depend on its specific geological deposit, as reported in the literature on the basis of microscopy [12] and scattering techniques [13]. It possesses several typical morphologies, such as spherical, sheet-like, and tubular particles due to the diversity of crystallization conditions and geological occurrence. Among them, the tubular structure is the most common and valuable [14]. The tubular structure is caused by lattice mismatch between adjacent silicone dioxide and aluminum oxide layers [15]. The molecular formula of $\mathrm{HNTs}$ is $\mathrm{Al}_{2} \mathrm{Si}_{2} \mathrm{O}_{5}(\mathrm{OH})_{4} \cdot \mathrm{nH}_{2} \mathrm{O}$, where $\mathrm{n}$ represents hydration or dehydration. HNTs are hydrated when $n$ equals 2 and are dehydrated when $n$ equals 0 [16-19]. Compared with traditional 
nanofillers, such as carbon nanotubes (CNTs) [20] and boron nitride nanotubes (BNNTs) [21], HNTs have a prominent advantage, which is that they are far less expensive [22]. The length of HNTs ranges from 100 to $2000 \mathrm{~nm}$, with the inner diameter from 10 to $30 \mathrm{~nm}$ and the outer diameter from 30 to $50 \mathrm{~nm}$. In terms of functional groups, HNTs contain a large amount of hydroxyl groups situated between layers and on the surface, respectively. Due to the multi-layer structure, most of the hydroxyl groups are inner groups. In addition, the inner surfaces of the HNTs are positively charged, while the outer surfaces are negatively charged [12,23]. The detailed data of HNTs are listed in Table $1[14,24]$.

Table 1. The detailed data of halloysite nanotubes (HNTs) related to combination with polysaccharides.

\begin{tabular}{cc}
\hline Molecular Formula & $\mathrm{Al}_{2} \mathbf{S i}_{2} \mathbf{O}_{5}(\mathbf{O H})_{4} \cdot \mathbf{n H}_{2} \mathbf{O}$ \\
\hline Length & $100-2000 \mathrm{~nm}$ \\
Inner diameter & $10-30 \mathrm{~nm}$ \\
Outer diameter & $30-50 \mathrm{~nm}$ \\
Aspect ratio (L/D) & $10-50$ \\
Eoung's modulus of a single HNTs & $130 \pm 24 \mathrm{GPa}$ \\
Elastic modulus & $460 \mathrm{GPa}$ \\
Interlayer water removal temperature & $400{ }^{\circ} \mathrm{C}$ \\
Water contact angle & $10 \pm 3^{\circ}$ \\
Specific surface area & $22.1-81.6 \mathrm{~m}^{2} / \mathrm{g}$ \\
Total pore volume & $0.06-0.25 \mathrm{~cm}^{3} / \mathrm{g}$ \\
Density & $2.14-2.59 \mathrm{~g} / \mathrm{cm}^{3}$ \\
Mean particle size in aqueous solution & $143 \mathrm{~nm}$ \\
\hline
\end{tabular}

Although the characteristics above generate excellent mechanical, thermal, and regenerable properties, the direct application of HNTs is limited. The drawbacks include difficulty in dissolving, brittleness, and low permeability [25]. With abundantly renewable sources and charming properties, including inherent biocompatibility, polysaccharides have attracted rising attention, and they have been widely applied to the medical [26], textile [27], and food fields, among others [28,29]. By preparing polysaccharide-HNTs composites, we can overcome these shortcomings. Due to the stable tubular morphology, charge distribution, the specific origin, and unique crystal structure, HNTs can be dispersed into single particles easily and the lumen diameter of HNTs fits well to macromolecule and protein diameters, causing the good combination between polysaccharides and HNTs [30-32]. The present research mainly focuses on alginate [33,34], chitosan [35], starch [36], cellulose [37], pectin, and carrageenan [38].

Although general properties of polysaccharide/halloysite nanotube composites and biomedical applications have been reviewed earlier by Liu et al. [39], we review the recent progress toward the development of polysaccharide-HNTs composites, paying attention to the main existence forms, wastewater treatment, and food packaging applications particularly. Through this review, we have a better understanding of unique characteristics of polysaccharide-HNTs composites, which can be helpful to the continuous expansion of their application in the future.

\section{Preparation of Polysaccharide-HNTs Composites}

\subsection{Purification}

Raw halloysite has impurities, such as quartz, illite, and perlite, since it is exploited directly from natural deposits. Therefore, the aggregate nanotubes should be separated to purify the HNTs before use in practical applications [40]. The traditional method of purification is the dispersion-centrifugation-drying technique. Firstly, we slowly added HNTs powder into deionized water under heating and mild stirring conditions. Then, the solution was further processed by lavation with deionized water three times and centrifugation. Finally, the pure HNTs were obtained after desiccation [41]. Figure 1 showed FE-SEM (Left) photos of HNTs and schematic illustration of crystalline structure (Right) of HNTs. 


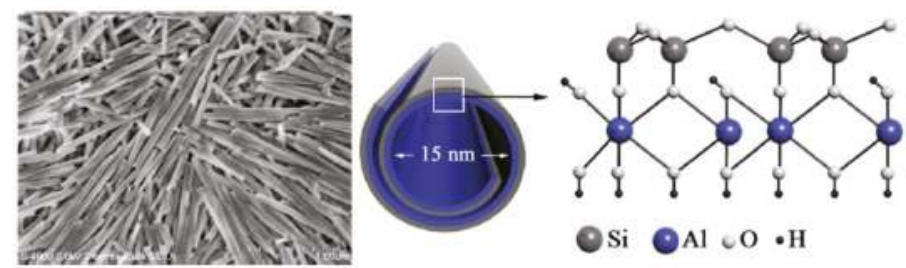

Figure 1. FE-SEM Image of HNTs on Si-Wafer (Left) and Schematic Illustration of Crystalline Structure of HNTs (Right). (Reproduced from [42] with permission from American Chemical Society and Copyright Clearance Center, 2012).

\subsection{HNTs/Polysaccharide Preparations and Formulations}

Using traditional processing techniques, HNTs can be mixed with most polysaccharides, such as alginate, chitosan, starch, cellulose, and carrageenan. The purpose of different fabrication methods is to enhance the interfacial interactions and dispersibility. In this section, we introduce the main existence forms of polysaccharide-HNTs composites.

\subsubsection{Hydrogels}

The hydrophilic structure of hydrogels enables them to hold large amounts of water in the three-dimensional networks. Due to the characteristics of high hygroscopicity and low stiffness, hydrogels are usually described as soft and wet materials $[43,44]$. Chan et al. prepared a HNTs/alginate hydrogel and the effects of HNTs on the physicochemical, thermal, mechanical, and mass transfer properties of alginate hydrogel beads were investigated in detail [45]. It was found that HNTs filled the interspace in the alginate matrix and allowed more efficient load transfer. The HNTs were embedded in the layers of alginate hydrogel networks and they had little effect on the size and on the shape of the alginate beads. The mechanism for enhanced mechanical strength could be attributed to physical interaction between the alginate and HNTs, and the mechanical strength could be improved at lower HNTs loading if chemical interactions were present. Zhou et al. reported alginate/HNTs composite hydrogels via solution mixing and subsequent cross-linking with calcium ions [46]. The static and shear viscosity of composite solutions increases with the increase of HNTs. The rheological behaviors of alginate/HNTs solutions were a shear thinning and fit with the power law model. Due to the good dispersion ability of HNTs, polysaccharides and HNTs are mixed easily via interfacial interactions, such as electrostatic and hydrogen bonding interactions, contributing to the formation of homogeneous composites and enhanced properties. Fourier-transform infrared spectroscopy (FTIR) and X-ray powder diffraction (XRD) are applied to study the interfacial interactions between alginate and HNTs. As shown in Figure 2b, the peaks at $1419 \mathrm{~cm}^{-1}$ shifted to higher wave numbers and no new peaks appeared in the composites, which indicated that hydrogen bond interactions occur between HNTs and alginate but no chemical reaction occurs. The XRD patterns of composites (Figure 2c) were very similar to HNTs no new diffraction peak occurring, which suggested the crystal structure of HNTs was retained in the composites. 
a

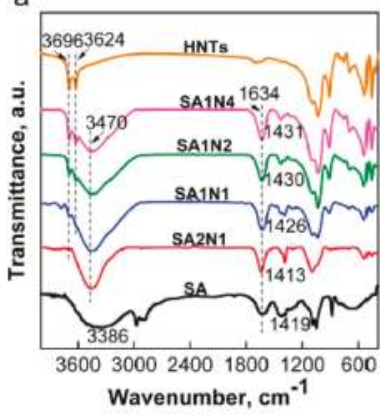

b

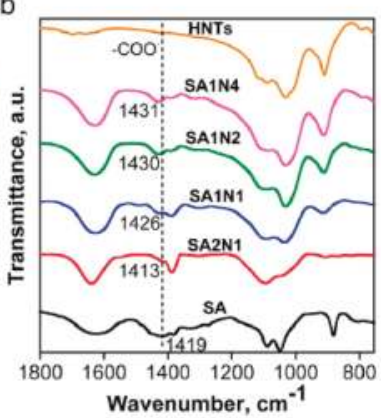

$\mathrm{C}$

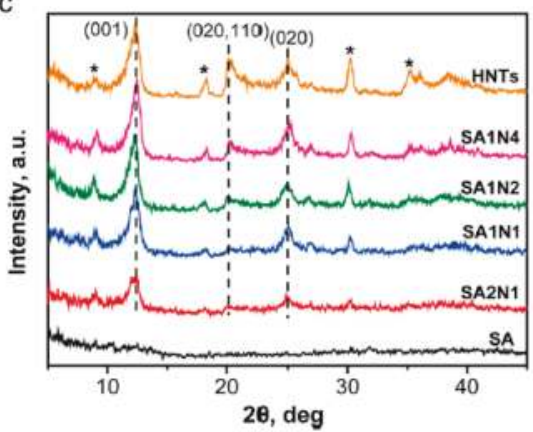

Figure 2. FTIR spectra (a,b) and XRD (c) pattern of HNTs, alginate, and alginate/HNTs composites. (Reproduced from [46] with permission from Elsevier and Copyright Clearance Center, 2017).

The effect of HNTs on the swelling ratios of the polysaccharide/HNTs composites were investigated in $\mathrm{NaCl}$ and water solution. Compared with pure sodium alginate (SA) hydrogel, the SA/HNTs composite hydrogels showed low swelling ratios with the same conditions for soaking time, which gradually decreased with the increasing HNTs loading. This result was attributed to the hydrophilic polymer content in the composite hydrogels decreasing with the addition of HNTs, and the water adsorption of HNTs was lower than SA. In addition, the HNTs used as physical crosslinking points for alginate through the hydrogen bond interactions can greatly improve entanglement of the alginate and lower the mobility of the chains, resulting in water absorption being greatly decreased [47]. Sinem et al. reported a cryogenic technique to modify HNTs. The inner and outer diameters and the surface area of HNTs were evidently increased without disturbing the inherent tubular structure and wall features. Then, modified HNTs were mixed with chitosan to prepared composite hydrogels, showing remarkedly improved mechanical and swelling properties compared with pure chitosan hydrogel [48]. Sharifzadeh et al. synthesized carrageenan/HNTs nanocomposite hydrogels via physical crosslinking. The chemical structure confirmed by FTIR spectroscopy revealed the formation of physical interaction between carrageenan and HNTs in the hydrogels. It was revealed that the thermal stability and swelling of the nanocomposite hydrogels had significantly been improved due to the incorporation of HNTs compared with the pure carrageenan hydrogel [49]. The reasons why HNTs can improve the thermal stability of composites are as follows. The degradation temperature of HNTs is approximately $400{ }^{\circ} \mathrm{C}$, which is higher than most of the polysaccharides. Then, the dispersed HNTs have a blocking effect on mass and heat transfer. Besides, the polysaccharide chains and degraded products enter the inner cavity of HNTs, delaying mass transport and improving the thermal stability. However, the good dispersion of HNTs into the hydrogel is urgently needed for the hydrogel fabrications to broaden their application. The HNTs functionalized via different types of silane coupling agents were used as a way to improve HNTs dispersal in the polymer matrix. Sabbagh et al. prepared novel chitosan/crosslinked 
oxidized starch hydrogels, which were embedded by modified or unmodified HNTs. Incorporation of HNTs significantly affected the swelling behavior and thermal properties of the hydrogel. The increase of the amine groups in HNTs modified with silane reagents made them react with oxidized starch, resulting in good dispersion in the structure of the hydrogel [50]. Figure 3 illustrates the formation of the bio-nanocomposite hydrogel.

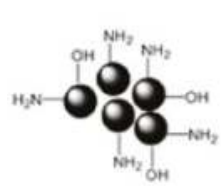

Chitosan (CTS)

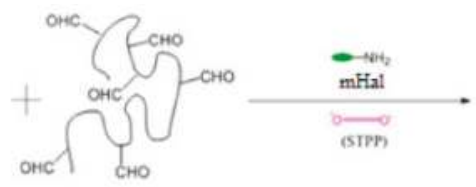

Oxidized-Starch (OSR)

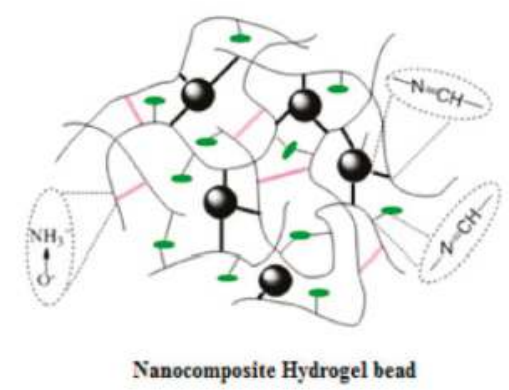

Figure 3. Schematic description of bio-nanocomposite hydrogel formation. (Reproduced from [50] with permission from Elsevier and Copyright Clearance Center, 2017).

The swelling ratio of the chitosan/HNTs hydrogel also decreased compared with the pure chitosan hydrogel, due to the introduction of HNTs content causing the chitosan to contract more [51]. The cellulose/HNTs composite showed a similar variation trend [52].

\subsubsection{Films}

Regenerated cellulose/HNTs nanocomposite films were fabricated in 1-butyl-3-methylimidazolium chloride ionic liquid by solution casting method. Figure 4 showed the cross-sectional FE-SEM images of the cellulose and $6 \mathrm{wt}$ \% HNTs-filled nanocomposite films. The HNTs were well dispersed in cellulose due to good interaction between cellulose and HNTs. Young's modulus and the tensile strength of nanocomposite films were improved by $100 \%$ and $55.3 \%$, respectively, when the loading of HNTs was $6 \mathrm{wt} . \%$, which was owing to tubular geometry and the higher stiffness of the HNTs. The addition of HNTs also improved the thermal stability and char yield of regenerated cellulose, but moisture absorption capacity of the nanocomposites in constant relative humidity was reduced due to the addition of HNTs [53].

Kim et al. reported transparent cellulose-obtained films from cellulose/HNTs solutions. The HNTs could uniformly been dispersed in cellulose because of the repulsive force from its surface charge, and the hydrogen bonding from HNTs and cellulose broke the chain-to-chain interactions of cellulose. The haze of the film was increased due to the introduction of HNTs but the diffuse transmittance could be retained [54]. 

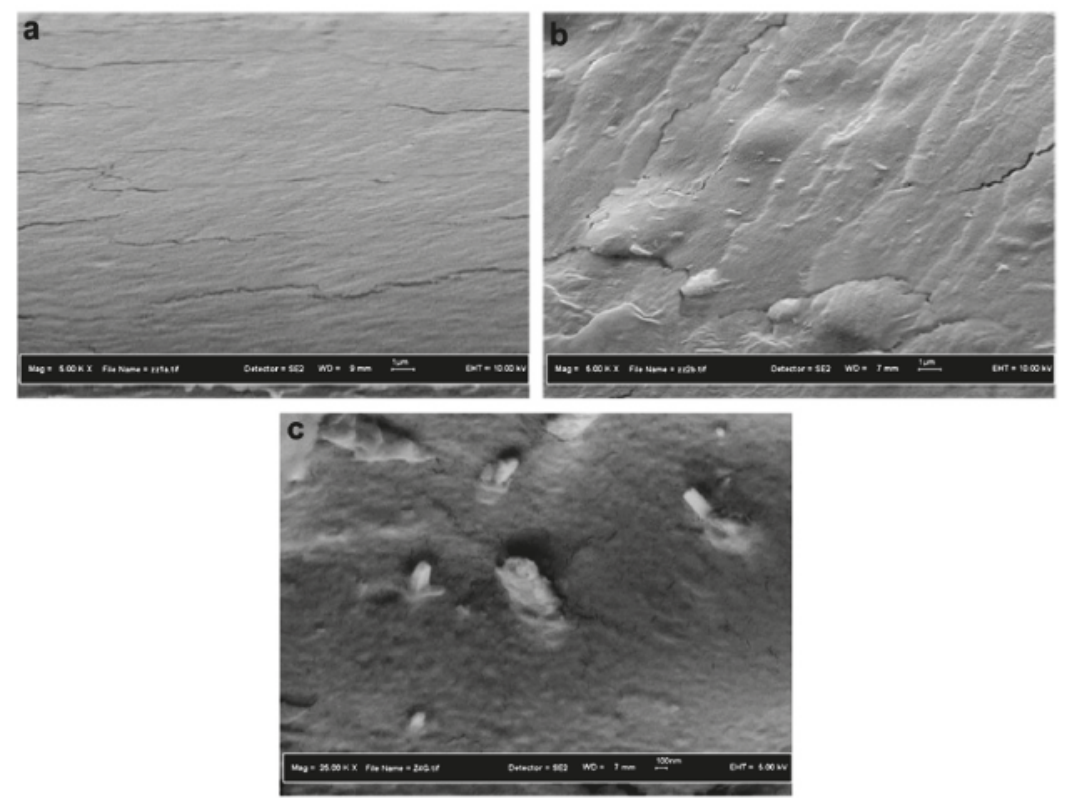

Figure 4. Cross-sectional FE-SEM images of cellulose and $6 \mathrm{wt} \%$ HNTs filled cellulose/HNTs nanocomposite films under (a,b) low magnifications and (c) high magnification. (Reproduced from Reference [53] with permission from Elsevier and Copyright Clearance Center, 2013).

Chang et al. prepared dispersed starch/HNTs composite films by using amylose to wrap the HNTs by ball-milling [55], in order to solve the agglomeration of HNTs. However, the extraction of amylose is expensive and complicated. In one work, polyethylene glycol (PEG) was used as a dispersing agent to mill, modify, and disperse HNTs in different solvents, and certain amounts of glycerin and modified HNTs suspension were added into the slurry. The composite films were obtained after stirring and casting on the stainless-steel plate. SEM of treated HNTs and HNTs/starch films with $3 \mathrm{wt} . \%$ (c and d) and $7 \mathrm{wt} . \%$ HNTs were shown in Figure 5. The HNTs are evenly distributed in the starch matrix. Due to the action of PEG, the treated HNTs were well dispersed in the starch matrix and the tensile strength of the film was effectively improved [56,57]. In another work, chitosan/starch/HNTs ternary nanocomposite films were developed through solution casting method. The interactions between chitosan, starch, and halloysite nanotubes were confirmed by FTIR results. Water absorption capacity, folding strength, and hemocompatibility were remarkedly enhanced owing to the addition of halloysite nanotubes [58]. Then, chitosan-HNTs composites were combined with modified cellulose to produce composite films using a solution casting method [59]; the excellent film formation and increase in surface roughness of the nanocomposite were confirmed by morphological and surface analysis. The kappa carrageenan/HNTs bio-nanocomposite films with enhanced tensile properties were successfully fabricated [60]. 
a

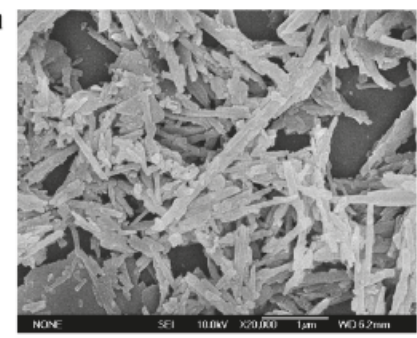

C

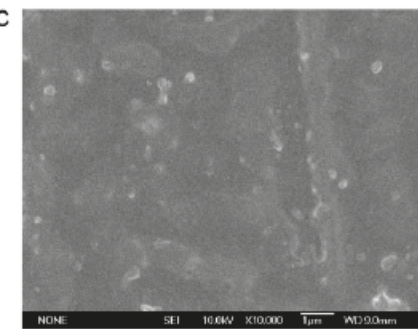

e

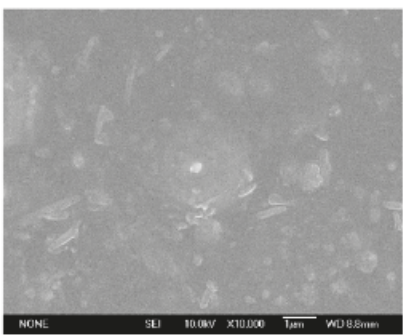

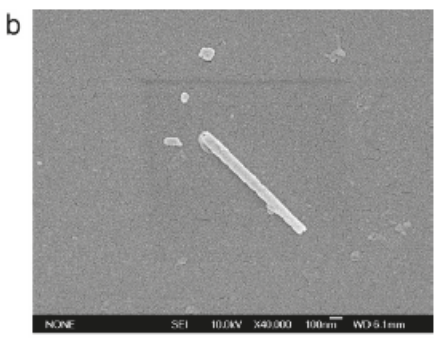
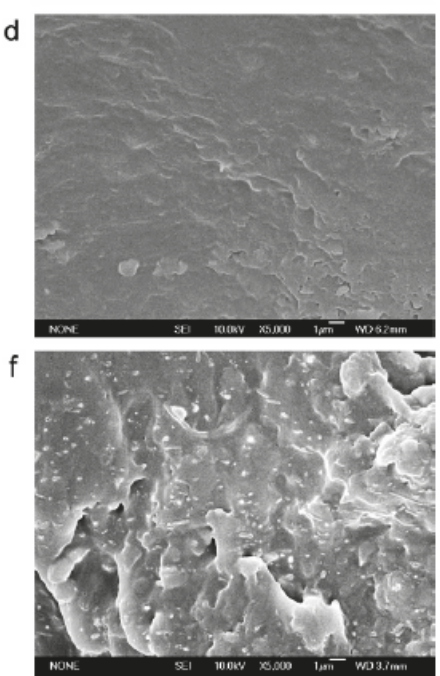

Figure 5. SEM of treated halloysite (a,b) and halloysite/starch films with 3 wt.\% (c,d) and 7 wt.\% halloysite $(\mathbf{e}, \mathbf{f})$. (Reproduced from [56] with permission from Elsevier and Copyright Clearance Center, 2012).

A functional bio-nanocomposite film both with antioxidant and antimicrobial active molecules was successfully prepared by the filling of a pectin matrix with modified HNTs containing peppermint oil. Importantly, the prepared functional film was considered a biocompatible material for packaging applications because of it was composed of eco-compatible molecules [61]. Makaremi et al. developed functional films with antimicrobial properties that can be extended over time by dispersing a HNTs/salicylic acid hybrid into the pectin matrix [62]. Moreover, it was demonstrated that the vacuum pumping in/out procedure can optimize the halloysite loading from the aqueous solution because of the water confinement mechanism [63]. Accordingly, the attained knowledge can offer new routes in the preparation of effective delivery systems based on HNTs.

\subsubsection{Fibers}

Fibers based on polysaccharides with high performance or special functions are becoming an emerging hotspot in both academic and industrial circles, exhibiting great potential in multiple fields.

HNTs can be well-dispersed in some ionic liquids. Song et al. firstly reported fabrication of microcrystalline cellulose/HNTs composite fibers from cellulose/ionic liquid/HNTs solutions by a wet-spinning method. Figure 6 showed schematic apparatus of nanocomposite fibers and SEM images of the morphology of fibers. The uniform dispersion of HNTs in the cellulose matrix was further confirmed by FTIR and wide-angle X-ray diffraction (WAXD) spectra. In addition, the homogeneous dispersion of HNTs and strong interfacial adhesion between HNTs and cellulose chains dramatically enhanced the moisture barrier property of cellulose fibers. Mechanical and thermal properties of HNTs 
nanocomposite fibers were greatly enhanced due to the uniform orientation of the well dispersed HNTs and great interactions from cellulose and HNTs caused by hydrogen bonding, van der Waals, and electrostatic interactions. For example, the tensile strength of the fibers increases from 73.8 to 130.1 MPa with the addition of HNTs from 0 to 7 wt.\% [64].
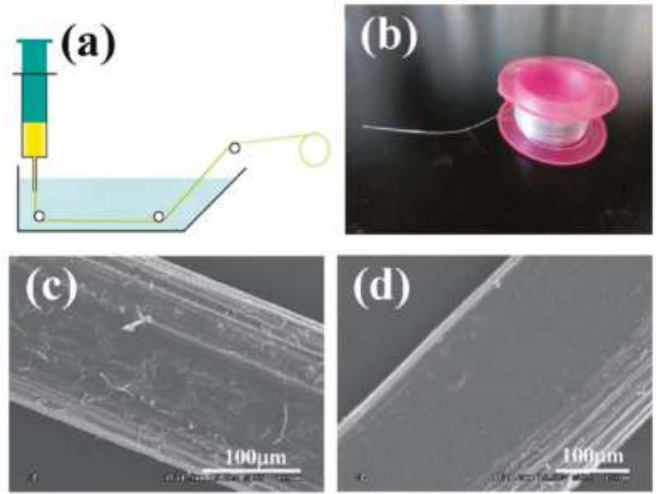

Figure 6. (a) Schematic apparatus for spinning MCC/HNTs nanocomposite fibers. (b) A $3 \mathrm{~m}$ long MCC/HNTs fiber wound on a plastic reel. (c,d) SEM images of the morphology of the side surface sections of regenerated MCC and MCC/HNTs fibers. (Reproduced from [64] with permission from Royal Society of Chemistry and Copyright Clearance Center, 2014).

Silva et al. developed HNTs reinforced alginate nanofibrous scaffolds fabricated by electrospinning. The diameter of alginate-based nanofibers ranged from 40 to $522 \mathrm{~nm}$ with well-aligned HNTs, as shown in Figure 7. The HNTs were well dispersed in the alginate matrix with good uniaxial alignment. Compared to the alginate-based scaffolds without HNTs, the tensile strength and elastic modulus of HNTs-reinforced nanofibrous scaffolds were significantly improved by 3-fold and 2-fold, respectively, when $5 \%(w / w)$ HNTs was added. The incorporation of HNTs also enhanced the thermal stability of the nanofibrous scaffolds [65].
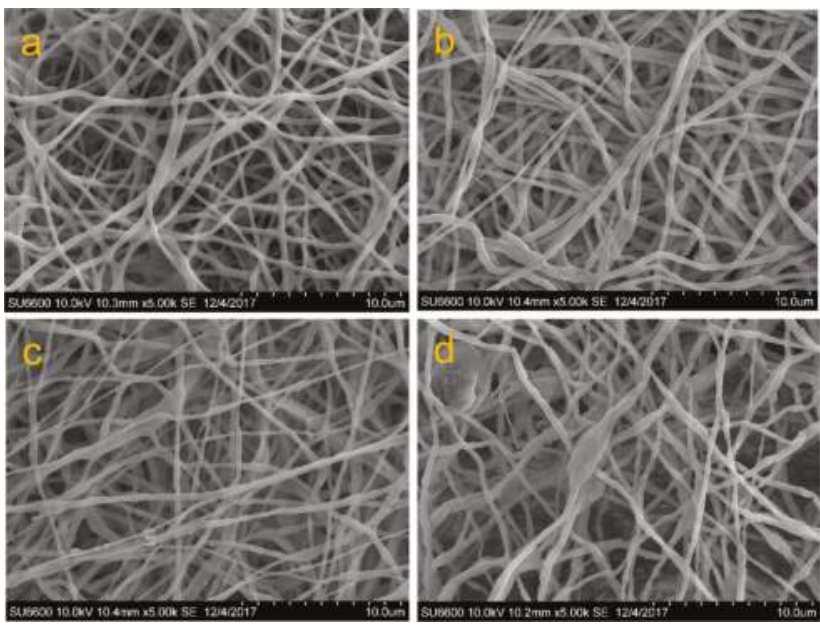

Figure 7. Morphology of electrospun alginate-based scaffolds with (a) 0 , (b,c) 5\%, and (d) $10 \%(w / w)$ of HNTs. (Reproduced from [65] with permission from American Chemical Society and Copyright Clearance Center, 2018). 


\subsubsection{Plasticized Nanocomposites}

Plasticized halloysite nanocomposites are commonly prepared via solvent casting method, but this is limited in the practical processing industries. Thus, the melt mixing technique will be more efficient and productive in a process industry workshop [66].

Schmitt et al. successfully developed plasticized HNTs/wheat starch nanocomposites by melt-extrusion for the first time. A higher loading (up to $6 \mathrm{wt} \%$ ) of HNTs were well-dispersed in a starch matrix. The interactions between external hydroxyl groups of halloysites and C-O-C groups of starch were formed. The thermal stability of the matrix was improved with the addition of HNTs. The tensile strength and Young's modulus of starch were improved up to $29 \%$ and $144 \%$, respectively, without sacrificing ductility [67]. Porous plasticized starch/HNTs nanocomposites were also successfully prepared by melt-extrusion technique. Double benefits could be gained due to the addition of HNTs, which were as a nucleating agent increasing the porosity and as a barrier agent increasing the proportion of small cells [68]. Ren et al. reported plasticized starch/HNTs nanocomposites prepared by melt blending with different polyol plasticizers, such as glycerol, sorbitol, and a mixture of two. Compared to sorbitol or a mixture of two, glycerol offered a more uniform dispersion of HNTs in the starch matrix owing to more stable hydrogen bonds from glycerol and HNTs. The use of mixtures of these polyols was proved a promising way to optimize the mechanical properties of nanocomposites [69].

\section{Applications of Polysaccharide-HNTs Composites}

\subsection{Biomedical Applications}

Liu et al. summarized recent research progress in the biomedical application of polysaccharide-HNTs composites [39]. The interfacial interactions, structure, and properties of the composites were discussed in detail in that review. There were some examples in the biomedical applications, such as tissue engineering, wound healing, and drug carrier systems. Thus, we have listed some examples based on the previous work of Liu et al. in the following.

\subsubsection{Drug Delivery and Release}

HNTs were confirmed as potential drug and gene delivery vehicles [70,71], and the drug release rate slowed down by coating polysaccharides onto the drug-loaded HNTs. Recently, more and more attention has been paid to the drug sustained release based on the polysaccharide-HNTs composites [72,73].

Hydrogels have been researched for the controlled delivery of biomolecules, varying from small molecular weight drugs to biomacromolecules, such as nucleic acids, polysaccharides, and proteins. Moreover, the biocompatible and biodegradable hydrogels were prepared by different natural ingredients. Among them, the chitosan-HNTs composite hydrogels were widely researched due to their low toxicity, good biocompatibility, and degradability by human enzymes [74]. It was found that the chitosan-coated HNTs exhibited reduced release compared with the pure HNTs [24]. For instance, the chitosan-coated HNTs had released only $78 \%$ of the total drug payload, while the uncoated HNTs released $88 \%$ on day 9 . The drug release rate was extremely low, and the residual content was under $10 \%$ of the loaded drug after 20 days. The reason why chitosan-coated HNTs have a lower drug release rate is the additional barrier through which the drug must diffuse provided by chitosan. In practical applications, chitosan-based hydrogels have been used for cancer therapeutics, subcutaneous release, and oral delivery. In recent years, the relevant relationship between plasticizer nature and drug release behavior has been exploited, so the starch/HNTs composite films have been prepared by melt blending technique for drug release applications [75]. In addition to drugs, other active agents, such as antimicrobial agents [76], DNA [77], and proteins, can also be loaded for controlled release. 


\subsubsection{Tissue Engineering Scaffold}

Tissue engineering scaffolds are generally porous structures, biocompatible, and mechanically strong for shaping cell growth [78]. HNTs were added into polysaccharides, such as chitosan [79], alginate [47], and starch [68], to prepare various tissue engineering scaffolds. Compared with pristine chitosan, the chitosan-HNTs nanocomposite scaffolds showed an obvious improvement in mechanical strength, tensile modulus, and thermal stability. Moreover, the addition of HNTs had little influence on the pore structure of chitosan. Thus, the chitosan-HNTs exhibited a highly porous structure. In order to verify the feasibility of the nanocomposite scaffolds, mouse fibroblasts were used for culture. The result showed that mouse fibroblasts could develop on the chitosan-HNTs nanocomposite scaffold surfaces, even at $80 \mathrm{wt}$.\% HNTs loading. In addition, the fibroblast cells cultured on the surfaces exhibited a phenotypic shape, indicating that the cells could penetrate and migrate within the scaffolds, which were similar to the extracellular matrix. Thus, polysaccharide-HNTs composites have promising potential in the tissue engineering scaffold field.

\subsubsection{Wound Dressing}

Wound dressings can be classified as traditional, biomaterial-based, interactive, and bioactive dressings. In recent research studies, biomaterials, such as polysaccharides, which are non-toxic, natural available, non-immunogenic, biocompatible, and biodegradable, have been considered as ideal materials for wound healing [80-82]. Due to the characteristics of biocompatibility, ability to load and release bioactive agents, high water content, and flexibility, hydrogels are most widely adopted for wound dressings [83]. Moreover, HNTs have been added into the polysaccharides, such as alginate $[45,76]$ and chitosan $[84,85]$, to overcome the poor mechanical strength of hydrogels. The alginate-HNTs wound dressing, based on a double barrier with the antibiotic vancomycin as an antimicrobial agent, was prepared. It was concluded that only the antibiotic vancomycin immobilized in HNTs- (3-Aminopropyl)-trimethoxysilane (APTS) and encapsulated in alginate hydrogels can be used as a wound dressing material.

\subsection{Wastewater Treatment Applications}

Among all the causes of wastewater pollutants, heavy metal ions, dyes, and other organic pollutants account for a large proportion [86]. Various methods, such as flocculation [87], precipitation [88], membrane filtration [89], and electrochemical techniques [90] have been restricted in practical usage because of the high cost, secondary pollutants, and poor removal efficiency. Due to its low cost, simple operation, and potential recycling and reuse, adsorption has become one of the most effective alternatives. Traditional absorbents, such as activated carbon [91] and resins [92], have been replaced by HNTs, a novel absorbent which is inexpensive and naturally abundant. In recent research studies, polysaccharide-HNTs composites exhibited promising adsorption capacity and regeneration.

\subsubsection{Applications in Removal of Dyes}

Pristine HNTs with the negative Si-O-Si on the outer surface and the positive Al-OH on the inner surface can adsorb both cationic and anionic dyes, but stable dispersions in water and reusability for its practical application are hard to obtain due to their size. Polysaccharides, such as chitosan, alginate, and starch, have been classified as natural biopolymer adsorbents, as they are non-toxic and biodegradable. However, polysaccharides materials usually have dense layer, low mechanical strength, and rigidity characteristics, which limits their application on a practical scale. Thus, polysaccharide-HNTs composites have been attractive due to combining their benefits for treatment of wastewater.

Cavallaro et al. prepared alginate/HNTs composite hydrogels by encapsulating HNTs into alginate hydrogels using the dropping technique. The adsorption capacity of composite hydrogels was investigated by removing crystal violet (CV) from aqueous media. The results showed that the 
addition of HNTs enhanced the composite hydrogel's ability to capture CV. The weight ratio of alginate and HNTs was 2:1, and the removal rate was increased by $55 \%$ and $45 \%$, respectively, when the stoichiometric adsorbent concentration increased from 0.25 to 0.50 mass $\%$ [93]. It was found that the $\mathrm{pH}$ and temperature had little influence on the adsorption capacity of alginate-HNTs composite beads. The alginate-HNTs composite adsorbents $(10 \mathrm{~g})$ had the ability to treat $29.7 \mathrm{~L}$ of $55 \mathrm{mg} / \mathrm{L}$ methylene blue (MB) solution and the removal efficiency was above $90 \%$. Moreover, the column study verified that the removal efficiency had a slight decline with the increase of bed volumes, but it remained above $90 \%$ after 1500 bed volumes of wastewater was treated [94]. Novel chitosan-HNTs composite hydrogel beads were prepared by the dropping and $\mathrm{pH}$-precipitation technique, exhibiting accelerated adsorption process and improved adsorption capacity $(72.60 \mathrm{mg} / \mathrm{g}$ for $\mathrm{MB}$ and $276.9 \mathrm{mg} / \mathrm{g}$ for malachite green (MG)) compared with the pure chitosan bead. Similar to alginate-HNTs composites, chitosan-HNTs composites also exhibited excellent regeneration properties especially for MB (above 92\%). The removal ratio of dyes increased but the adsorption amount per unit absorbent weight reduced with the addition of the composite hydrogels in solution [95]. The porous starch-HNTs composites were prepared by solvent exchange method using different drying methods. The adsorption performance depended on porosity, which was relative to specific surface area, and the size distribution of the pores and their area changed with different types of drying methods and percentage of ethanol. The adsorption capacity composites were improved due to the incorporation of HNTs [96]. A cryogenic technique to modify HNTs was confirmed to increase the inner and outer diameters and the surface area of HNTs without disturbing the inherent tubular structure and wall features. A small amount of cryo-expanded halloysite was used in chitosan. It was found that the composite hydrogel had a high adsorption capacity for anionic and cationic dyes [48].

Overall, polysaccharide-HNTs composites exhibit reinforced adsorption capacity and regeneration properties. Thus, polysaccharide-HNTs composites can be considered as promising reusable adsorbents for the removal of dyes from wastewater.

\subsubsection{Applications in Removal of Heavy Metal Ions}

HNTs with negatively charged surfaces were regarded as ideal alternatives for removal of heavy metal ions from aqueous media, such as $\mathrm{Cu}^{2+}, \mathrm{Pb}^{2+}$, and $\mathrm{Cr}^{2+}[14,97]$. However, HNTs used as adsorbents face some problems in practical application due to their nanoscale size, such as being easily aggregated, being lost in use, the low permeability of packed adsorption columns, and being difficult to recycle. Thus, HNTs were combined with polysaccharides to improve the affinity and loading capacity for heavy metal ions. Extrusion dripping method was used to produce alginate-HNTs nanocomposite beads with calcium chloride as the curing agent. The results showed that the alginate-HNTs nanocomposite beads had improved adsorption capacity $(325 \mathrm{mg} / \mathrm{g}$ at $0.2 \mathrm{~g}$ HNTs loading). It was also concluded that alginate and HNTs promoted the adsorption of $\mathrm{Pb}^{2+}$ by ion exchange and physisorption, respectively. It was simple to separate the used nanocomposite beads from wastewater by filtration due to the millimetric size, which facilitated the regeneration of the used beads [98]. In other works, the alginate-HNTs hybrid beads with high adsorption capacity for $\mathrm{Cu}^{2+}$ were prepared in the same way. The SEM image showed clearly that the HNTs overlapped loosely together in the interior of the hybrid beads, contributing to the mass transfer of the adsorbents. Furthermore, the regeneration experiment exhibited good adsorption efficiency (approximately 80\%) after three cycles. In addition, the alginate-HNTs hybrid beads also showed good adsorption performance for other heavy metal ions, such as $\mathrm{Ag}^{+}$and $\mathrm{Cd}^{2+}$ [99].

Compared to the traditional chemical methods of removing heavy metal ions from aqueous media, polysaccharide-HNTs composites had advantages, such as low cost, simple operation, regeneration, and being non-secondary pollutants. 


\subsection{Other Applications for Water Treatment}

Currently, the research is focused on the applications of polysaccharide-HNTs composites for the removal of dyes and heavy metal ions in aqueous media. However, polysaccharide-HNTs composites have other practical applications for pollutants removal due to their unique structure and improved properties.

Different types of membranes, such as ultrafiltration, forward osmosis, reverse osmosis, and membrane reactors [100-103], were endowed with special performances by adopting HNTs. Cellulose acetate/L-dopa coated HNTs (LDPHNT) ultrafiltration membranes were prepared by blending in a casting solution. The Energy dispersive spectroscopy (EDS) analysis showed the uniform dispersion of LPDHNTs in the cellulose acetate (CA) membrane matrix. Moreover, the size and number of pores on the membrane surface were increased by the addition of LDPHNTs into CA solution. It was the existence of LDPHNTs that improved the hydrophilicity of the hybrid membranes. In the antifouling test, the composite membranes exhibited higher antifouling performance than pristine CA membranes. Besides, the tensile modulus and elongation at break point of LDPHNTs-CA hybrid membranes were reinforced compared to the pure CA membranes [104]. Some of the phenol-containing pesticides are widely used in agriculture and regarded as endocrine disruptors, exhibiting estrogenic activity and toxicity even at low concentrations. In order to remove phenol-containing pollutants, the special adsorbents based on HNTs were prepared. The chitosan-HNTs hybrid nanotubes were synthesized by simply assembling chitosan onto HNTs. Then, the horseradish peroxidase was immobilized on the hybrid nanotubes for the removal of phenol. The experiment showed that the immobilized HRP exhibited excellent removal efficiency for phenol from wastewater and the activity did not reduce the volume [25].

\subsection{Food Packaging Applications}

Over the years, polysaccharides, owing to their biodegradability, non-toxicity, and good film-forming ability, have raised concerns over their use as food packaging materials for consumer demand and environmental issues. However, these biodegradable polysaccharides have weak stability in processing, poor barrier properties, and high sensitivity to environmental changes. In the previous work, the introduction of HNTs nanoparticles to the starch matrix improved the mechanical properties and decreased permeability to water vapor and oxygen, water adsorption capacity, as well as the water solubility of the films [57], which gave them potential to be used for food packaging purposes. Makaremi et al. prepared biofilms composed of apple pectin and two different types of HNTs-MB with shorter tubes and lower surface area and PT with longer tubes and higher surface area-to obtain a novel functional bio-nanocomposite with enhanced mechanical and thermal properties. Moreover, both HNTs were employed as nanocontainers for salicylic acid, a well-known biocidal agent. On this basis, the HNTs/salicylic acid hybrids were dispersed into the apple pectin matrix to develop functional films with antimicrobial properties that can be extended over time. Thus, the bio-nanocomposite films showed promising potential for food packaging applications [62].

HNTs are able to adsorb active molecules, such as nisin and pediocin. On this basis, Meira et al. added peptides nisin and pediocin into starch films, resulting in active packaging materials with antimicrobial activity against L. monocytogenes and C. perfringens. The addition of HNTs enhanced the mechanical and thermal properties, especially when bacteriocins were adsorbed on the HNTs [105]. The moisture barrier properties of polysaccharide-based films are poor due to their hydrophilic characteristics. Essential oils with antioxidant and antimicrobial activities are commonly incorporated into polysaccharide matrices to overcome these limitations. However, it is rather hard to disperse the essential oils in a hydrophilic polysaccharide matrix. Lee et al. developed chitosan films incorporated with clove essential oil and HNTs. It was confirmed that the essential oils were stabilized by HNTs without any surfactant. The addition of HNTs into the chitosan matrix enhanced the mechanical and water barrier properties of the chitosan films, and the active molecule, clove essential oil, imparted antimicrobial and antioxidant effects to the chitosan-HNTs nanocomposite films. The results showed 
that the films inhibited growth of mold, which was derived from the surrounding environment. Therefore, the nanocomposite films could be used as active food packaging systems because of the antioxidant and antimicrobial properties and enhanced barrier properties against water vapor [106].

\section{Conclusions}

In this review, we summarized the recent research studies regarding polysaccharide-HNT composites. The key points of polysaccharide-HNTs composites are as follows:

(1) HNTs, 1D natural nanoclays, have unique characteristics of tubular structure, high aspect ratio, abundant natural reserves, compatibility, and high mechanical strength. Due to the characteristics of HNTs, polysaccharide-HNTs composites have advantages, such as improved mechanical, thermal, and swelling properties and good biocompatibility. Thus, HNTs are promising nanofillers for high-performance polymer composites.

(2) In addition to the characteristics of HNTs, the degree of dispersion of HNTs and the interfacial interactions between polysaccharides and HNTs (electrostatic and hydrogen bonding interactions) are crucial factors affecting the performance of composites.

(3) HNTs can be combined with polysaccharides by different methods. Polysaccharide-HNTs composite hydrogels can be prepared by solution mixing and freeze-drying method and dropping or PH-precipitation technique; membranes are fabricated by solution casting method and fibers are usually produced by electrospinning technique. The key to this process is to obtain a well-dispersed solution with the HNTs and good interfacial interactions between polysaccharides and HNTs.

(4) Polysaccharide-HNTs composites show promising potential for biomedical applications. The applications in removal of dyes and heavy metal ions are summarized in detail. Polysaccharide-HNTs composites have raised concerns as food packaging materials for consumer demand and environmental issues.

Author Contributions: Writing-original draft preparation, Y.W. and Y.Z.; Writing-review and editing, J.J. and Y.T.; Supervision, H.Y.; Project administration, X.H.; Conceptualization, Y.T.

Funding: This research received no external funding.

Acknowledgments: This work was supported by the National Nature Science Foundation of China (No. 51,403,113 and 51573080), Natural Science Foundation for Distinguished Young Scientists of Shan dong Province (BS2014CL007), Postdoctoral Science Foundation of China and Shandong Province (2016T90610, 2015M571994 and 201501007), Project of Shandong Province Higher Educational Science and Technology Program (J14LA19), and Program for Taishan Scholar of Shandong Province, Postdoctoral Science Foundation of Qingdao City (RZ1900002760), The Open Project Program of key Laboratory of Synthetic and Self-Assembly Chemistry for organic Functional Molecules (K2018-1), Research Project of Innovative Teaching Laboratory of Qingdao University, Shandong Provincial Natural Science Foundation (ZR2019YQ19).

Conflicts of Interest: The authors declare no conflict of interest.

\section{References}

1. Moniruzzaman, M.; Winey, K.I. Polymer nanocomposites containing carbon nanotubes. Macromolecules 2006, 39, 5194-5205. [CrossRef]

2. Tan, Y.Q.; Wang, L.B.; Xiao, J.L.; Zhang, X.; Wang, Y.; Liu, C.; Zhang, H.W.; Liu, C.Z.; Xia, Y.Z.; Sui, K.Y. Synchronous enhancement and stabilization of graphene oxide liquid crystals: Inductive effect of sodium alginates in different concentration zones. Polymer 2019, 160, 107-114. [CrossRef]

3. Lvov, Y.; Abdullayev, E. Functional polymer-clay nanotube composites with sustained release of chemical agents. Prog. Polym. Sci. 2013, 38, 1690-1719. [CrossRef]

4. Fakhrullina, G.I.; Akhatova, F.S.; Lvov, Y.M.; Fakhrullin, R.F. Toxicity of halloysite clay nanotubes in vivo: A Caenorhabditis elegans study. Environ. Sci. Nano 2015, 2, 54-59. [CrossRef] 
5. Lazzara, G.; Cavallaro, G.; Panchal, A.; Fakhrullin, R.; Stavitskaya, A.; Vinokurov, V.; Lvov, Y. An assembly of organic-inorganic composites using halloysite clay nanotubes. Curr. Opin. Colloid Interface Sci. 2018, 35, 42-50. [CrossRef]

6. Mouni, L.; Belkhiri, L.; Bollinger, J.-C.; Bouzaza, A.; Assadi, A.; Tirri, A.; Dahmoune, F.; Madani, K.; Remini, H. Removal of Methylene Blue from aqueous solutions by adsorption on Kaolin: Kinetic and equilibrium studies. Appl. Clay Sci. 2018, 153, 38-45. [CrossRef]

7. Zhu, R.; Chen, Q.; Zhou, Q.; Xi, Y.; Zhu, J.; He, H. Adsorbents based on montmorillonite for contaminant removal from water: A review. Appl. Clay Sci. 2016, 123, 239-258. [CrossRef]

8. Hiranuma, N.; Augustin-Bauditz, S.; Bingemer, H.; Budke, C.; Curtius, J.; Danielczok, A.; Diehl, K.; Dreischmeier, K.; Ebert, M.; Frank, F.; et al. A comprehensive laboratory study on the immersion freezing behavior of illite NX particles: A comparison of 17 ice nucleation measurement techniques. Atmos. Chem. Phys. 2015, 15, 2489-2518. [CrossRef]

9. Tully, J.; Yendluri, R.; Lvov, Y. Halloysite Clay Nanotubes for Enzyme Immobilization. Biomacromolecules 2016, 17, 615-621. [CrossRef]

10. Lvov, Y.; Wang, W.; Zhang, L.; Fakhrullin, R. Halloysite Clay Nanotubes for Loading and Sustained Release of Functional Compounds. Adv. Mater. 2016, 28, 1227-1250. [CrossRef] [PubMed]

11. Joussein, E.; Pettt, S.; Churchman, J.; Theng, B.; Righi, D.; Delvaux, B. Halloysite clay minerals-A review. Clay Min. 2005, 40, 383-426. [CrossRef]

12. Pasbakhsh, P.; Churchman, G.J.; Keeling, J.L. Characterisation of properties of various halloysites relevant to their use as nanotubes and microfibre fillers. Appl. Clay Sci. 2013, 74, 47-57. [CrossRef]

13. Cavallaro, G.; Chiappisi, L.; Pasbakhsh, P.; Gradzielski, M.; Lazzara, G. A structural comparison of halloysite nanotubes of different origin by Small-Angle Neutron Scattering (SANS) and Electric Birefringence. Appl. Clay Sci. 2018, 160, 71-80. [CrossRef]

14. Du, M.; Guo, B.; Jia, D. Newly emerging applications of halloysite nanotubes: A review. Polym. Int. 2010, 25-36. [CrossRef]

15. Vergaro, V.; Abdullayev, E.; Lvov, Y.M.; Zeitoun, A.; Cingolani, R.; Rinaldi, R.; Leporatti, S. Cytocompatibility and Uptake of Halloysite Clay Nanotubes. Biomacromolecules 2010, 11, 820-826. [CrossRef] [PubMed]

16. Zhang, C.-L.; Cui, S.-J.; Wang, Y. Adsorption removal of pefloxacin from water by halloysite nanotubes. J. Ind. Eng. Chem. 2015, 23, 12-15. [CrossRef]

17. Chen, Y.; Zhang, Y.; Zhang, H.; Liu, J.; Song, C. Biofouling control of halloysite nanotubes-decorated polyethersulfone ultrafiltration membrane modified with chitosan-silver nanoparticles. Chem. Eng. J. 2013, 228, 12-20. [CrossRef]

18. Cataldo, S.; Lazzara, G.; Massaro, M.; Muratore, N.; Pettignano, A.; Riela, S. Functionalized halloysite nanotubes for enhanced removal of lead(II) ions from aqueous solutions. Appl. Clay Sci. 2018, 156, 87-95. [CrossRef]

19. Abdullayev, E.; Lvov, Y. Halloysite Clay Nanotubes for Controlled Release of Protective Agents. J. Nanosci. Nanotechnol. 2011, 11, 10007-10026. [CrossRef] [PubMed]

20. Yang, N.; Chen, X.; Ren, T.; Zhang, P.; Yang, D. Carbon nanotube based biosensors. Sens. Actuator B Chem. 2015, 207, 690-715. [CrossRef]

21. Kalay, S.; Yilmaz, Z.; Sen, O.; Emanet, M.; Kazanc, E.; Culha, M. Synthesis of boron nitride nanotubes and their applications. Beilstein J. Nanotechnol. 2015, 6, 84-102. [CrossRef] [PubMed]

22. Liu, R.; Zhang, B.; Mei, D.; Zhang, H.; Liu, J. Adsorption of methyl violet from aqueous solution by halloysite nanotubes. Desalination 2011, 268, 111-116. [CrossRef]

23. Anastopoulos, I.; Mittal, A.; Usman, M.; Mittal, J.; Yu, G.; Núñez-Delgado, A.; Kornaros, M. A review on halloysite-based adsorbents to remove pollutants in water and wastewater. J. Mol. Liq. 2018, 269, 855-868. [CrossRef]

24. Liu, M.; Jia, Z.; Jia, D.; Zhou, C. Recent advance in research on halloysite nanotubes-polymer nanocomposite. Prog. Polym. Sci. 2014, 39, 1498-1525. [CrossRef]

25. Sun, X.; Zhang, Y.; Shen, H.; Jia, N. Direct electrochemistry and electrocatalysis of horseradish peroxidase based on halloysite nanotubes/chitosan nanocomposite film. Electrochim. Acta 2010, 56, 700-705. [CrossRef]

26. Lee, K.Y.; Mooney, D.J. Alginate: Properties and biomedical applications. Prog. Polym. Sci. 2012, 37, $106-126$. [CrossRef] [PubMed] 
27. Aryee, A.N.A.; Agyei, D.; Akanbi, T.O. Recovery and utilization of seaweed pigments in food processing. Curr. Opin. Food Sci. 2018, 19, 113-119. [CrossRef]

28. Galiano, F.; Briceño, K.; Marino, T.; Molino, A.; Christensen, K.V.; Figoli, A. Advances in biopolymer-based membrane preparation and applications. J. Membr. Sci. 2018, 564, 562-586. [CrossRef]

29. Ma, Y.; Qi, P.; Ju, J.; Wang, Q.; Hao, L.; Wang, R.; Sui, K.; Tan, Y. Gelatin/alginate composite nanofiber membranes for effective and even adsorption of cationic dyes. Compos. Pt. B Eng. 2019, 162, 671-677. [CrossRef]

30. Pasbakhsh, P.; De Silva, R.; Vahedi, V.; Jock Churchman, G. Halloysite nanotubes: Prospects and challenges of their use as additives and carriers -A focused review. Clay Min. 2018, 51, 479-487. [CrossRef]

31. Kadam, A.A.; Jang, J.; Jee, S.C.; Sung, J.S.; Lee, D.S. Chitosan-functionalized supermagnetic halloysite nanotubes for covalent laccase immobilization. Carbohydr. Polym. 2018, 194, 208-216. [CrossRef]

32. Lvov, Y.; Price, R.; Gaber, B.; Ichinose, I. Thin film nanofabrication via layer-by-layer adsorption of tubule halloysite, spherical silica, proteins and polycations. Colloid Surf. A Physicochem. Eng. Asp. 2002, 198, 375-382. [CrossRef]

33. Venkatesan, J.; Bhatnagar, I.; Manivasagan, P.; Kang, K.-H.; Kim, S.-K. Alginate composites for bone tissue engineering: A review. Int. J. Biol. Macromol. 2015, 72, 269-281. [CrossRef] [PubMed]

34. Wang, Q.; Ju, J.P.; Tan, Y.Q.; Hao, L.Y.; Ma, Y.L.; Wu, Y.; Zhang, H.W.; Xia, Y.Z.; Sui, K.Y. Controlled synthesis of sodium alginate electrospun nanofiber membranes for multi-occasion adsorption and separation of methylene blue. Carbohydr. Polym. 2019, 205, 125-134. [CrossRef] [PubMed]

35. Ali, A.; Ahmed, S. A review on chitosan and its nanocomposites in drug delivery. Int. J. Biol. Macromol. 2018, 109, 273-286. [CrossRef] [PubMed]

36. Hemamalini, T.; Dev, V.R.G. Comprehensive review on electrospinning of starch polymer for biomedical applications. Int. J. Biol. Macromol. 2018, 106, 712-718. [CrossRef]

37. Nechyporchuk, O.; Belgacem, M.N.; Bras, J. Production of cellulose nanofibrils: A review of recent advances. Ind. Crop. Prod. 2016, 93, 2-25. [CrossRef]

38. Necas, J.; Bartosikova, L. Carrageenan: A review. Vet. Med. 2013, 58, 187-205. [CrossRef]

39. Liu, M.; He, R.; Yang, J.; Long, Z.; Huang, B.; Liu, Y.; Zhou, C. Polysaccharide-halloysite nanotube composites for biomedical applications: A review. Clay Min. 2018, 51, 457-467. [CrossRef]

40. Shchukin, D.G.; Sukhorukov, G.B.; Price, R.R.; Lvov, Y.M. Halloysite nanotubes as biomimetic nanoreactors. Small 2005, 1, 510-513. [CrossRef]

41. Qiu, K.; Netravali, A.N. Halloysite nanotube reinforced biodegradable nanocomposites using noncrosslinked and malonic acid crosslinked polyvinyl alcohol. Polym. Compos. 2013, 34, 799-809. [CrossRef]

42. Yah, W.O.; Xu, H.; Soejima, H.; Ma, W.; Lvov, Y.; Takahara, A. Biomimetic Dopamine Derivative for Selective Polymer Modification of Halloysite Nanotube Lumen. J. Am. Chem. Soc. 2012, 134, 12134-12137. [CrossRef] [PubMed]

43. Nie, J.Y.; Pei, B.Y.; Wang, Z.K.; Hu, Q.L. Construction of ordered structure in polysaccharide hydrogel: A review. Carbohydr. Polym. 2019, 205, 225-235. [CrossRef] [PubMed]

44. Ahmed, E.M. Hydrogel: Preparation, characterization, and applications: A review. J. Adv. Res. 2015, 6, 105-121. [CrossRef] [PubMed]

45. Chiew, C.S.C.; Poh, P.E.; Pasbakhsh, P.; Tey, B.T.; Yeoh, H.K.; Chan, E.S. Physicochemical characterization of halloysite/alginate bionanocomposite hydrogel. Appl. Clay Sci. 2014, 101, 444-454. [CrossRef]

46. Huang, B.; Liu, M.; Long, Z.; Shen, Y.; Zhou, C. Effects of halloysite nanotubes on physical properties and cytocompatibility of alginate composite hydrogels. Mater. Sci. Eng. C Mater. Biol. Appl. 2017, 70, 303-310. [CrossRef]

47. Liu, M.; Dai, L.; Shi, H.; Xiong, S.; Zhou, C. In vitro evaluation of alginate/halloysite nanotube composite scaffolds for tissue engineering. Mater. Sci. Eng. C Mater. Biol. Appl. 2015, 49, 700-712. [CrossRef]

48. Palantoken, S.; Tekay, E.; Sen, S.; Nugay, T.; Nugay, N. A Novel Nonchemical Approach to the Expansion of Halloysite Nanotubes and Their Uses in Chitosan Composite Hydrogels for Broad-Spectrum Dye Adsorption Capacity. Polym. Compos. 2016, 37, 2770-2781. [CrossRef]

49. Sharifzadeh, G.; Wahit, M.U.; Soheilmoghaddam, M.; Whye, W.T.; Pasbakhsh, P. Kappa-carrageenan/ halloysite nanocomposite hydrogels as potential drug delivery systems. J. Taiwan Inst. Chem. Eng. 2016, 67, 426-434. [CrossRef] 
50. Sabbagh, N.; Akbari, A.; Arsalani, N.; Eftekhari-Sis, B.; Hamishekar, H. Halloysite-based hybrid bionanocomposite hydrogels as potential drug delivery systems. Appl. Clay Sci. 2017, 148, 48-55. [CrossRef]

51. Huang, B.; Liu, M.; Zhou, C. Chitosan composite hydrogels reinforced with natural clay nanotubes. Carbohydr. Polym. 2017, 175, 689-698. [CrossRef] [PubMed]

52. Huang, B.; Liu, M.X.; Zhou, C.R. Cellulose-halloysite nanotube composite hydrogels for curcumin delivery. Cellulose 2017, 24, 2861-2875. [CrossRef]

53. Soheilmoghaddam, M.; Wahit, M.U.; Mahmoudian, S.; Hanid, N.A. Regenerated cellulose/halloysite nanotube nanocomposite films prepared with an ionic liquid. Mater. Chem. Phys. 2013, 141, 936-943. [CrossRef]

54. Kim, Y.; Song, Y.; Kim, H. Preparation of transparent cellulose film with controlled haze using halloysite nanotubes. Cellulose 2018, 25, 1239-1248. [CrossRef]

55. Chang, P.R.; Xie, Y.; Wu, D.; Ma, X. Amylose wrapped halloysite nanotubes. Carbohydr. Polym. 2011, 84, 1426-1429. [CrossRef]

56. He, Y.; Kong, W.; Wang, W.; Liu, T.; Liu, Y.; Gong, Q.; Gao, J. Modified natural halloysite/potato starch composite films. Carbohydr. Polym. 2012, 87, 2706-2711. [CrossRef]

57. Sadegh-Hassani, F.; Nafchi, A.M. Preparation and characterization of bionanocomposite films based on potato starch/halloysite nanoclay. Int. J. Biol. Macromol. 2014, 67, 458-462. [CrossRef] [PubMed]

58. Devi, N.; Dutta, J. Development and in vitro characterization of chitosan/starch/halloysite nanotubes ternary nanocomposite films. Int. J. Biol. Macromol. 2019, 127, 222-231. [CrossRef] [PubMed]

59. Cavallaro, G.; Lazzara, G.; Konnova, S.; Fakhrullin, R.; Lvov, Y. Composite films of natural clay nanotubes with cellulose and chitosan. Green Mater. 2014, 2, 232-242. [CrossRef]

60. Wahab, I.F.; Abd Razak, S.I. Bionanocomposite fim of kappa-carrageenan/nanotube clay: Grrowth of hydroxyl apatite and model drug release. Dig. J. Nanomater. Biostruct. 2016, 11, 963-972.

61. Biddeci, G.; Cavallaro, G.; Di Blasi, F.; Lazzara, G.; Massaro, M.; Milioto, S.; Parisi, F.; Riela, S.; Spinelli, G. Halloysite nanotubes loaded with peppermint essential oil as filler for functional biopolymer film. Carbohydr. Polym. 2016, 152, 548-557. [CrossRef] [PubMed]

62. Makaremi, M.; Pasbakhsh, P.; Cavallaro, G.; Lazzara, G.; Aw, Y.K.; Lee, S.M.; Milioto, S. Effect of Morphology and Size of Halloysite Nanotubes on Functional Pectin Bionanocomposites for Food Packaging Applications. ACS Appl. Mater. Interfaces 2017, 9, 17476-17488. [CrossRef] [PubMed]

63. Lisuzzo, L.; Cavallaro, G.; Pasbakhsh, P.; Milioto, S.; Lazzara, G. Why does vacuum drive to the loading of halloysite nanotubes? The key role of water confinement. J. Colloid Interface Sci. 2019, 547, 361-369. [CrossRef] [PubMed]

64. Luo, Z.; Wang, A.; Wang, C.; Qin, W.; Zhao, N.; Song, H.; Gao, J. Liquid crystalline phase behavior and fiber spinning of cellulose/ionic liquid/halloysite nanotubes dispersions. J. Mater. Chem. A 2014, 2, 7327-7336. [CrossRef]

65. De Silva, R.T.; Dissanayake, R.K.; Mantilaka, M.; Wijesinghe, W.; Kaleel, S.S.; Premachandra, T.N.; Weerasinghe, L.; Amaratunga, G.A.J.; de Silva, K.M.N. Drug-Loaded Halloysite Nanotube-Reinforced Electrospun Alginate-Based Nanofibrous Scaffolds with Sustained Antimicrobial Protection. ACS Appl. Mater. Interfaces 2018, 10, 33913-33922. [CrossRef] [PubMed]

66. Kausar, A. Review on Polymer/Halloysite Nanotube Nanocomposite. Polym. Plast. Technol. Eng. 2018, 57, 548-564. [CrossRef]

67. Schmitt, H.; Prashantha, K.; Soulestin, J.; Lacrampe, M.F.; Krawczak, P. Preparation and properties of novel melt-blended halloysite nanotubes/wheat starch nanocomposites. Carbohydr. Polym. 2012, 89, 920-927. [CrossRef] [PubMed]

68. Schmitt, H.; Creton, N.; Prashantha, K.; Soulestin, J.; Lacrampe, M.-F.; Krawczak, P. Preparation and Characterization of Plasticized Starch/Halloysite Porous Nanocomposites Possibly Suitable for Biomedical Applications. J. Appl. Polym. Sci. 2015, 132. [CrossRef]

69. Ren, J.; Khanh Minh, D.; Pollet, E.; Averous, L. Preparation and Characterization of Thermoplastic Potato Starch/Halloysite Nano-Biocomposites: Effect of Plasticizer Nature and Nanoclay Content. Polymers 2018, 10, 808. [CrossRef] [PubMed]

70. Aguzzi, C.; Viseras, C.; Cerezo, P.; Salcedo, I.; Sanchez-Espejo, R.; Valenzuela, C. Release kinetics of 5-aminosalicylic acid from halloysite. Colloid Surf. B Biointerfaces 2013, 105, 75-80. [CrossRef] [PubMed]

71. Forsgren, J.; Jamstorp, E.; Bredenberg, S.; Engqvist, H.; Stromme, M. A Ceramic Drug Delivery Vehicle for Oral Administration of Highly Potent Opioids. J. Pharm. Sci. 2010, 99, 219-226. [CrossRef] [PubMed] 
72. Cavallaro, G.; Lazzara, G.; Milioto, S.; Parisi, F.; Evtugyn, V.; Rozhina, E.; Fakhrullin, R. Nanohydrogel Formation within the Halloysite Lumen for Triggered and Sustained Release. ACS Appl. Mater. Interfaces 2018, 10, 8265-8273. [CrossRef] [PubMed]

73. Karnik, S.; Hines, K.; Mills, D.K. Nanoenhanced hydrogel system with sustained release capabilities. J. Biomed. Mater. Res. Part A 2015, 103, 2416-2426. [CrossRef] [PubMed]

74. Bhattarai, N.; Gunn, J.; Zhang, M.Q. Chitosan-based hydrogels for controlled, localized drug delivery. Adv. Drug Deliv. Rev. 2010, 62, 83-99. [CrossRef] [PubMed]

75. Ren, J.; Han, L.; Cai, H.; Wu, K.; Averous, L.; Guo, W. Functional Biocomposites Based on Plasticized Starch/halloysite Nanotubes for Drug-Release Applications. Starch-Starke 2018, 70, 1700358. [CrossRef]

76. Kurczewska, J.; Pecyna, P.; Ratajczak, M.; Gajecka, M.; Schroeder, G. Halloysite nanotubes as carriers of vancomycin in alginate-based wound dressing. Saudi Pharm. J. 2017, 25, 911-920. [CrossRef]

77. Shi, Y.-F.; Tian, Z.; Zhang, Y.; Shen, H.-B.; Jia, N.-Q. Functionalized halloysite nanotube-based carrier for intracellular delivery of antisense oligonucleotides. Nanoscale Res. Lett. 2011, 6, 1-7. [CrossRef]

78. Del Buffa, S.; Rinaldi, E.; Carretti, E.; Ridi, F.; Bonini, M.; Baglioni, P. Injectable composites via functionalization of $1 \mathrm{D}$ nanoclays and biodegradable coupling with a polysaccharide hydrogel. Colloid Surf. B Biointerfaces 2016, 145, 562-566. [CrossRef]

79. Liu, M.X.; Wu, C.C.; Jiao, Y.P.; Xiong, S.; Zhou, C.R. Chitosan-halloysite nanotubes nanocomposite scaffolds for tissue engineering. J. Mater. Chem. B 2013, 1, 2078-2089. [CrossRef]

80. Dhivya, S.; Padma, V.V.; Santhini, E. Wound dressings-A review. BioMedicine 2015, 5, 24-28. [CrossRef]

81. Ulery, B.D.; Nair, L.S.; Laurencin, C.T. Biomedical Applications of Biodegradable Polymers. J. Polym. Sci. Part B Polym. Phys. 2011, 49, 832-864. [CrossRef] [PubMed]

82. Yadav, P.; Yadav, H.; Shah, V.G.; Shah, G.; Dhaka, G. Biomedical Biopolymers, their Origin and Evolution in Biomedical Sciences: A Systematic Review. J. Clin. Diac. Res. 2015, 9, ZE21-ZE25. [CrossRef] [PubMed]

83. Sood, A.; Granick, M.S.; Tomaselli, N.L. Wound Dressings and Comparative Effectiveness Data. Adv. Wound Care 2014, 3, 511-529. [CrossRef] [PubMed]

84. Liu, M.; Shen, Y.; Ao, P.; Dai, L.; Liu, Z.; Zhou, C. The improvement of hemostatic and wound healing property of chitosan by halloysite nanotubes. RSC Adv. 2014, 4, 23540-23553. [CrossRef]

85. Sandri, G.; Aguzzi, C.; Rossi, S.; Bonferoni, M.C.; Bruni, G.; Boselli, C.; Cornaglia, A.I.; Riva, F.; Viseras, C.; Caramella, C.; et al. Halloysite and chitosan oligosaccharide nanocomposite for wound healing. Acta Biomater. 2017, 57, 216-224. [CrossRef] [PubMed]

86. Yu, L.; Wang, H.; Zhang, Y.; Zhang, B.; Liu, J. Recent advances in halloysite nanotube derived composites for water treatment. Environ. Sci. Nano 2016, 3, 28-44. [CrossRef]

87. Teh, C.Y.; Budiman, P.M.; Shak, K.P.Y.; Wu, T.Y. Recent Advancement of Coagulation-Flocculation and Its Application in Wastewater Treatment. Ind. Eng. Chem. Res. 2016, 55, 4363-4389. [CrossRef]

88. Thorat, A.A.; Dalvi, S.V. Liquid antisolvent precipitation and stabilization of nanoparticles of poorly water soluble drugs in aqueous suspensions: Recent developments and future perspective. Chem. Eng. J. 2012, 181, 1-34. [CrossRef]

89. Leiknes, T. The effect of coupling coagulation and flocculation with membrane filtration in water treatment: A review. J. Environ. Sci. 2009, 21, 8-12. [CrossRef]

90. Natarajan, R.; Al Fazari, F.; Al Saadi, A. Municipal waste water treatment by natural coagulant assisted electrochemical technique-Parametric effects. Environ. Technol. Innov. 2018, 10, 71-77. [CrossRef]

91. Altenor, S.; Carene, B.; Emmanuel, E.; Lambert, J.; Ehrhardt, J.J.; Gaspard, S. Adsorption studies of methylene blue and phenol onto vetiver roots activated carbon prepared by chemical activation. J Hazard Mater. 2009, 165, 1029-1039. [CrossRef] [PubMed]

92. Ozdemir, F.A.; Demirata, B.; Apak, R. Adsorptive removal of methylene blue from simulated dyeing wastewater with melamine-formaldehyde-urea resin. J. Appl. Polym. Sci. 2009, 112, 3442-3448. [CrossRef]

93. Cavallaro, G.; Gianguzza, A.; Lazzara, G.; Milioto, S.; Piazzese, D. Alginate gel beads filled with halloysite nanotubes. Appl. Clay Sci. 2013, 72, 132-137. [CrossRef]

94. Liu, L.; Wan, Y.; Xie, Y.; Zhai, R.; Zhang, B.; Liu, J. The removal of dye from aqueous solution using alginate-halloysite nanotube beads. Chem. Eng. J. 2012, 187, 210-216. [CrossRef]

95. Peng, Q.; Liu, M.; Zheng, J.; Zhou, C. Adsorption of dyes in aqueous solutions by chitosan-halloysite nanotubes composite hydrogel beads. Microporous Mesoporous Mater. 2015, 201, 190-201. [CrossRef] 
96. Oliyaei, N.; Moosavi-Nasab, M.; Tamaddon, A.M.; Fazaeli, M. Preparation and characterization of porous starch reinforced with halloysite nanotube by solvent exchange method. Int. J. Biol. Macromol. 2019, 123, 682-690. [CrossRef]

97. Wang, J.; Zhang, X.; Zhang, B.; Zhao, Y.; Zhai, R.; Liu, J.; Chen, R. Rapid adsorption of Cr (VI) on modified halloysite nanotubes. Desalination 2010, 259, 22-28.

98. Chiew, C.S.C.; Yeoh, H.K.; Pasbakhsh, P.; Krishnaiah, K.; Poh, P.E.; Tey, B.T.; Chan, E.S. Halloysite/alginate nanocomposite beads: Kinetics, equilibrium and mechanism for lead adsorption. Appl. Clay Sci. 2016, 119, 301-310. [CrossRef]

99. Wang, Y.; Zhang, X.; Wang, Q.; Zhang, B.; Liu, J. Continuous fixed bed adsorption of Cu(II) by halloysite nanotube-alginate hybrid beads: An experimental and modelling study. Water Sci Technol. 2014, 70, 192-199. [CrossRef]

100. Yu, H.X.; Zhang, Y.T.; Sun, X.B.; Liu, J.D.; Zhang, H.Q. Improving the antifouling property of polyethersulfone ultrafiltration membrane by incorporation of dextran grafted halloysite nanotubes. Chem. Eng. J. 2014, 237, 322-328. [CrossRef]

101. Ghanbari, M.; Emadzadeh, D.; Lau, W.J.; Lai, S.O.; Matsuura, T.; Ismail, A.F. Synthesis and characterization of novel thin film nanocomposite (TFN) membranes embedded with halloysite nanotubes (HNTs) for water desalination. Desalination 2015, 358,33-41. [CrossRef]

102. Ghanbari, M.; Emadzadeh, D.; Lau, W.J.; Matsuura, T.; Ismail, A.F. Synthesis and characterization of novel thin film nanocomposite reverse osmosis membranes with improved organic fouling properties for water desalination. RSC Adv. 2015, 5, 21268-21276. [CrossRef]

103. Moslehyani, A.; Ismail, A.F.; Othman, M.H.D.; Matsuura, T. Hydrocarbon degradation and separation of bilge water via a novel TiO2-HNTs/PVDF-based photocatalytic membrane reactor (PMR). RSC Adv. 2015, 5, 14147-14155. [CrossRef]

104. Mu, K.G.; Zhang, D.L.; Shao, Z.Q.; Qin, D.J.; Wang, Y.L.; Wang, S. Enhanced permeability and antifouling performance of cellulose acetate ultrafiltration membrane assisted by L-DOPA functionalized halloysite nanotubes. Carbohydr. Polym. 2017, 174, 688-696. [CrossRef] [PubMed]

105. Meister Meira, S.M.; Zehetmeyer, G.; Werner, J.O.; Brandelli, A. A novel active packaging material based on starch-halloysite nanocomposites incorporating antimicrobial peptides. Food Hydrocoll. 2017, 63, 561-570. [CrossRef]

106. Lee, M.H.; Kim, S.Y.; Park, H.J. Effect of halloysite nanoclay on the physical, mechanical, and antioxidant properties of chitosan films incorporated with clove essential oil. Food Hydrocoll. 2018, 84, 58-67. [CrossRef]

(C) 2019 by the authors. Licensee MDPI, Basel, Switzerland. This article is an open access article distributed under the terms and conditions of the Creative Commons Attribution (CC BY) license (http://creativecommons.org/licenses/by/4.0/). 
Article

\title{
Grafting Halloysite Nanotubes with Amino or Carboxyl Groups onto Carbon Fiber Surface for Excellent Interfacial Properties of Silicone Resin Composites
}

\author{
Xiandong Zhang and Guangshun $\mathrm{Wu}$ * \\ School of Chemistry and Materials Science, Ludong University, Yantai 264025, China; zxdldhg@163.com \\ * Correspondence: wugs_hit1@ldu.edu.cn; Tel.: +86-0535-669-7921
}

Received: 5 October 2018; Accepted: 19 October 2018; Published: 22 October 2018

\begin{abstract}
The quality of interphase in carbon fibers (CFs) composites makes a key contribution to overall performance of composites. Here, we achieved for the first time the chemical grafting of halloysite nanotubes (HNTs) with amino or carboxyl groups onto the CFs surface aiming to increase composites interfacial strength. HNTs were grafted using 3-aminopropyltriethoxysilane (APS) followed by succinic anhydride treatment, and HNTs with amino groups $\left(\mathrm{HNT}-\mathrm{NH}_{2}\right)$ or carboxyl groups (HNT-COOH) were separately introduced into the interphase of composites. Functional groups of HNTs and fiber surface structures were characterized, which confirmed the modification success. The wettability between the modified CFs and resin have been enhanced obviously based on the improved fiber polarity and enhanced surface roughness by the introduced two functionalized HNTs with the uniform distributions onto fiber surface. Moreover, interfacial properties and anti-hydrothermal aging behaviors of modified methylphenylsilicone resin (MPSR) composites were improved significantly, especially for $\mathrm{HNT}-\mathrm{COOH}$ grafting. In addition, the interfacial reinforcement mechanisms for untreated and modified CF composites are discussed and compared.
\end{abstract}

Keywords: carbon fibers; surface grafting; halloysite nanotubes; polymer composites; interface

\section{Introduction}

Carbon fibers $(\mathrm{CFs})$ have become one of the ideal reinforcements for matrix resin composites because of their strong specific strength, outstanding chemical resistance and superior environmental stability [1-3]. The quality of interface between the fibers and matrix resin is the main element in the properties of polymer composites, and the weak quality of fiber-matrix interface leads to inferior stress transfer efficiency at the interfacial region and the dissatisfied performance of the resulting composites [4,5]. Unfortunately, untreated CF has a smooth and inert surface, which is difficult to provide desired interfacial interactions, limiting the further application of composites seriously [6,7]. Therefore, various surface treatment methods have been proposed for changing the CFs surface from chemical inert to active with the aim to improve the quality of interface [8-12].

Recently, many researchers have made considerable efforts to graft the surface of CFs with various active nanoparticles to change interfacial microstructure and properties of composites [13-16]. Gao [17] prepared a new hierarchical reinforcement containing octaglycidyldimethylsilyl polyhedral oligomeric silsesquioxane (POSS) and CFs with the remarkable improvement in interfacial strength of composites. Chen [18] effectively introduced polyether imide and graphene oxide into the interface between CFs and poly(ether-ether-ketone) by the sizing process. All the hierarchical CFs/active nanoparticles reinforcements help to change the wettability and surface roughness of the fibers, and thus increase interfacial adhesion and the ultimate properties of the resulting composites. 
Noteworthy, HNTs are a kind of naturally clay silicate minerals with the similar nanostructures of carbon nanotubes (CNTs), which possess an inner gibbsite octahedral sheet groups (Al-OH) and external siloxane groups (Si-O-Si) $[19,20]$. The structure of HNTs can be expressed with the formula $\mathrm{Al}_{2} \mathrm{Si}_{2} \mathrm{O}_{5}(\mathrm{OH})_{4} \cdot \mathrm{nH}_{2} \mathrm{O}$ [21]. As environment-friendly $1 \mathrm{D}$ natural nanofillers, the large surface area, high aspect ratio and the unique hollow nano-tubular structure of HNTs endow high mechanical properties and outstanding thermal stability [22]. Enormous polar groups $(-\mathrm{OH})$ onto the surface of HNTs not only have a good compatibility and effective interactions with polymer resin but also can be used as the bridging sites for further functionalization of HNTs. In addition, HNTs are much cheaper than other active nanoparticles (e.g., CNTs, POSS, and graphene oxide), showing unique advantages for large-scale practical application and industrial production $[23,24]$. Hence, there have been great interests in preparing HNTs/polymer nanocomposites with exceptional interfacial strength, and mechanical and thermal performances of the resulting composites [25-27]. As everyone knows, HNTs are prone to form serious aggregation, and many modified agents have been proposed to enhance the dispersion of HNTs and interfacial properties in the system [24,28]. Yu [29] prepared dextran modified HNTs by the bridging 1,6-hexamethylene diisocyanate (HDI) and incorporated modified HNTs into polyethersulfone ultrafiltration membrane with improved antifouling property. Chao [30] reported surface modification of halloysite nanotubes with dopamine (DA) for the advanced applications. However, the bridging agents HDI or DA are very expensive. More importantly, neither agent is suitable for the preparation of hydrothermal aging resistant interface. The bridging 3-aminopropyltriethoxysilane (APS) can be easily prepared, making it much cheaper than HDI or DA. APS with both ethoxyl and amino groups not only reacts with hydroxyl groups on the surface of HNTs, but also provides massive reactive sites for further introducing active molecules onto HNTs surface. Moreover, based on the same backbone siloxane structures (Si-O) of MPSR matrix, APS molecules with the strong $\mathrm{Si}-\mathrm{O}$ bond can enhance interface compatibility between $\mathrm{HNT}-\mathrm{NH}_{2}$ modified $\mathrm{CFs}$ and MPSR matrix, and fully protect fiber-matrix interface from hydrothermal aging penetration under the harsh environment. In addition, APS is used as the conventional curing agent, which accelerates the curing process of MPSR and reduces defects, and thus increases interfacial properties of composites. Hence, surface grafting of succinic anhydride onto HNTs via the bridging agent APS has been proposed in the study. However, the preparation of modified HNTs/CF hierarchical reinforcements by chemically grafting HNTs onto the CFs surface has rarely been studied.

In this work, $\mathrm{HNT}-\mathrm{NH}_{2}$ and $\mathrm{HNT}-\mathrm{COOH}$ were prepared, and then separately introduced on CFs surface by chemical bonding for the first time with the aim to enhance the quality of interface and properties of composites. Surface element, functional groups and structures of HNTs, HNT-NH $\mathrm{NH}_{2}$ and HNT-COOH were characterized by Fourier transform infrared spectroscopy (FTIR), thermogravimetric analysis (TGA), and X-ray photoelectron spectroscopy (XPS). Surface morphologies and wettability of untreated and modified CFs were examined by scanning electron microscope (SEM) and dynamic contact angle analysis (DCA), respectively. Interfacial properties of MPSR composites reinforced with untreated and grafted CFs were studied systematically via interfacial shear strength (IFSS) and interlaminar shear strength (ILSS), and hydrothermal aging resistance of different composites was also evaluated. In addition, the different interfacial reinforcing mechanisms for untreated and modified CFs composites are discussed and developed. 


\section{Materials and Methods}

\subsection{Materials}

CFs $\left(3 \times 10^{3}\right.$ single filaments per tow, average diameter $7 \mu \mathrm{m}$, tensile strength $3500 \mathrm{MPa}$, and tensile modulus $230 \mathrm{GPa}$ ) were used as the reinforcements, and obtained from Toray Industries, Inc., Tokyo, Japan. MPSR (relative density $1.08 \mathrm{~g} \cdot \mathrm{cm}^{-3}$, molecular weight $2400 \mathrm{~g} \cdot \mathrm{mol}^{-1}$, flexural strength $308 \mathrm{MPa}$ and compressive strength $150 \mathrm{MPa}$ ) and high-purity HNTs (purity > 98\%, diameter 30-70 nm, and length 0.1-2 $\mu \mathrm{m}$ ) were purchased by ShangHai Chemicals Co., Shanghai, China and Guangzhou Runwo Materials Technology Co. Ltd., Guangzhou, China, respectively. APS, succinic anhydride, and triethylamine were received from Aladdin, Shanghai, China. All other drugs and reagents, such as tetrahydrofuran (THF), 4-Dimethylaminopyridine (DMAP), toluene, lithium aluminium hydride $\left(\mathrm{LiAlH}_{4}\right), N, N^{\prime}$-Dicyclohexyl carbodiimide (DCC), concentrated nitric acid $\left(\mathrm{HNO}_{3}\right)$, thionyl chloride $\left(\mathrm{SOCl}_{2}\right)$ and dimethylformamide (DMF) were purchased by Tianjin Bodi Organic Chemicals Co. Ltd., Tianjin, China.

\subsection{Preparation of $\mathrm{HNT}-\mathrm{NH}_{2}$ and $\mathrm{HNT}-\mathrm{COOH}$}

Surface treatment of HNTs by APS and succinic anhydride was carried out using the following procedures. Typically, HNTs (1 g) were added into the solution of $200 \mathrm{~mL}$ toluene by ultrasonic cleaner for $30 \mathrm{~min}$. Then, $5 \mathrm{~mL}$ APS and the catalyst triethylamine were put into HNTs solution, and then reacted at $353 \mathrm{~K}$ for $24 \mathrm{~h}$. After the reaction complete, the grafting of APS onto HNTs was finally centrifuged with deionized water and ethanol each for many times, obtaining $\mathrm{HNT}-\mathrm{NH}_{2}$. To get HNT-COOH, $0.5 \mathrm{~g}$ HNT- $\mathrm{NH}_{2}$ nanoparticles were ultrasonically dispersed into $100 \mathrm{~mL}$ of $0.1 \mathrm{M}$ succinic anhydride in DMF, and then reacted by stirring at room temperature for $24 \mathrm{~h}$. The $\mathrm{HNT}-\mathrm{COOH}$ was obtained after being washed by DMF and deionized water repeatedly and dried. Figure 1a shows the synthesis procedure of $\mathrm{HNT}-\mathrm{NH}_{2}$ and $\mathrm{HNT}-\mathrm{COOH}$.

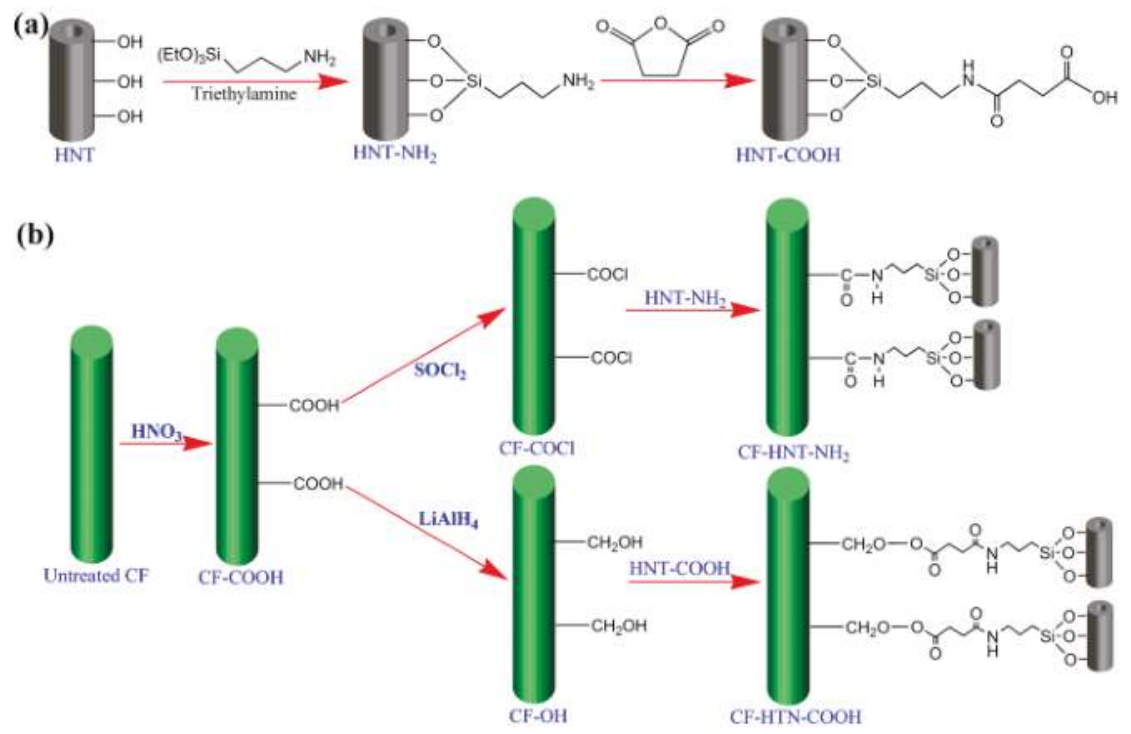

Figure 1. Schematic illustration of the preparation processes of: (a) functionalized HNTs; and (b) $\mathrm{CF}-\mathrm{HNT}-\mathrm{NH}_{2}$ and $\mathrm{CF}-\mathrm{HNT}-\mathrm{COOH}$. 


\subsection{Functionalization of HNTs onto CFs Surface}

\subsubsection{Fiber Desizing, Oxidation, Reduction and Acyl Chloride Modification}

In a typical reaction, the pristine CFs were firstly extracted in supercritical acetone/water at $633 \mathrm{~K}$ for about $30 \mathrm{~min}$ with the aim of removing polymer sizing agents (denoted as untreated CF). Then, untreated $\mathrm{CF}$ was treated by concentrated $\mathrm{HNO}_{3}$ at $353 \mathrm{~K}$ for $4 \mathrm{~h}$ to obtain $\mathrm{CF}-\mathrm{COOH}$, which has many carboxyl functional groups. Subsequently, $\mathrm{CF}-\mathrm{COOH}$ was reacted with $\mathrm{LiAlH}_{4}-\mathrm{THF}$ saturated solution under reflux for $2 \mathrm{~h}$ to introduce hydroxyl groups from the reduction of carboxyl groups (denoted as $\mathrm{CF}-\mathrm{OH}$ ). $\mathrm{CF}-\mathrm{OH}$ could react with $\mathrm{HNT}-\mathrm{COOH}$ easily to prepare $\mathrm{HNT}-\mathrm{COOH}$ modified CFs. To form the covalent bonding with $\mathrm{HNT}-\mathrm{NH}_{2}$, carboxyl groups on the surface of $\mathrm{CF}-\mathrm{COOH}$ are needed to change to acyl chloride groups. Hence, $\mathrm{CF}-\mathrm{COOH}$ was treated with the mixed solution of $\mathrm{SOCl}_{2}(100 \mathrm{~mL})$ and DMF $(5 \mathrm{~mL})$ at $349 \mathrm{~K}$ for $24 \mathrm{~h}$ to get $\mathrm{CF}-\mathrm{COCl}$.

\subsubsection{Separate Grafting of $\mathrm{HNT}-\mathrm{NH}_{2}$ and $\mathrm{HNT}-\mathrm{COOH}$ onto CFs via Chemical Bonds}

HNT- $\mathrm{NH}_{2}$ /CF hierarchical reinforcements were prepared from the chemical reaction between $\mathrm{HNT}-\mathrm{NH}_{2}$ and $\mathrm{CF}-\mathrm{COCl}$. HNT-NH $2(0.1 \mathrm{~g})$ was firstly dispersed by ultrasonic in dried DMF $(50 \mathrm{~mL})$ for forming stable suspensions. Then, $\mathrm{HNT}-\mathrm{NH}_{2}$ suspensions were mixed with $\mathrm{CF}-\mathrm{COCl}$ $(5 \mathrm{~g})$ and reacted at $353 \mathrm{~K}$ for $24 \mathrm{~h}$ under nitrogen atmosphere. After modification, the obtained products (denoted as $\mathrm{CF}-\mathrm{HNT}-\mathrm{NH}_{2}$ ) were washed with $\mathrm{DMF}$ and acetone each many times and dried. To obtain HNT-COOH/CF hierarchical reinforcements via CF-OH bonded with $\mathrm{HNT}-\mathrm{COOH}$, once the suspension of HNT-COOH and DMF was ready, CF-OH (5 g), DMAP (0.01 g) and DCC (0.1 g) as catalysts were quickly added in $\mathrm{HNT}-\mathrm{COOH}$ suspension and then reacted by stirring at $353 \mathrm{~K}$ for $24 \mathrm{~h}$. Finally, CF-HNT-COOH was obtained after being washed by DMF and acetone each for several times and dried. The whole grafting processes are illustrated in Figure $1 \mathrm{~b}$.

\subsection{Preparation of CF/MPSR Composites}

CF/MPSR composite samples used for interfacial properties testing were prepared via the compression molding method. Appropriate untreated CF, CF-HNT-NH $\mathrm{N}_{2}$ and CF-HNT-COOH were separately wrapped around a metal frame tightly. Then, the metal frame was soaked with MPSR solution to make MPSR saturate into the fibers. Subsequently, the unidirectional impregnating samples were obtained by being degassed with a vacuum pump until no bubbles or solvents came out of the samples. Finally, the composites were obtained via using a hot-pressing machine under the controlled curing schedule (atmospheric pressure for $1 \mathrm{~h}$ at 393 and $423 \mathrm{~K}, 20 \mathrm{MPa}$ for $2 \mathrm{~h}$ at $473 \mathrm{~K}$, and $20 \mathrm{MPa}$ for $4 \mathrm{~h}$ at $523 \mathrm{~K}$ in sequence). The fiber contents in composites were about $70 \mathrm{mass} \%$, and the dimensions of the measured cured samples were about $2 \mathrm{~mm} \times 20 \mathrm{~mm} \times 6 \mathrm{~mm}$ adopted for interfacial strength and hydrothermal aging resistance test.

\subsection{Characterization Techniques}

FTIR (Nicolet, Nexus670, Glendale, WI, USA) was used to examine chemical elements of untreated and modified HNTs. The testing specimens were characterized with $2 \mathrm{~cm}^{-1}$ resolution and 64 scans in the wavenumber range of $400-4000 \mathrm{~cm}^{-1}$. Surface composition and functional groups of different HNTs and the fibers before and after modification were also characterized by XPS (ESCALAB 220i-XL, VG, UK) with the monochromatic Al Ka source of $1486.6 \mathrm{eV}$ at a base pressure of $2 \times 10^{-9} \mathrm{mbar}$.

TGA (TA Instruments, Q50, New Castle, DE, USA) was used to examine thermal stability of HNTs, HNT- $\mathrm{NH}_{2}$ and HNT-COOH. The samples of 5-20 mg were added in aluminum pans, and heated from 30 to $800{ }^{\circ} \mathrm{C}$ in nitrogen atmosphere with the heating rate of $5^{\circ} \mathrm{C} / \mathrm{min}$.

Surface characteristics of CFs before and after grafting and the cracked sections of MPSR composites reinforced with different CFs were studied by SEM (Quanta 200FEG, Hitachi Instrument, Inc., Tokyo, Japan). All testing samples were coated with thin gold layers via gold sputtering technique to increase fiber conductivity and obtain stable and clear images. 
The changes of dynamic contact angles and surface energy analysis were carried out using a dynamic contact angle meter (DCAT21, Data Physics Instruments, Filderstadt, Germany), which were used to evaluate the wetting performance of untreated and HNTs modified CFs, using nonpolar diiodomethane and polar deionized water as the testing liquids. The surface free energy, and its dispersive as well as polar components of different $\mathrm{CFs}$ were obtained from the following equations:

$$
\begin{gathered}
\gamma_{1}(1+\cos \theta)=2\left(\gamma_{1}^{\mathrm{p}} \gamma_{\mathrm{f}}^{\mathrm{p}}\right)^{1 / 2}+2\left(\gamma_{1}^{\mathrm{d}} \gamma_{\mathrm{f}}^{\mathrm{d}}\right)^{1 / 2} \\
\gamma_{\mathrm{f}}=\gamma_{\mathrm{f}}^{\mathrm{p}}+\gamma_{\mathrm{f}}^{\mathrm{d}}
\end{gathered}
$$

where $\theta$ and $\gamma_{1}$ represent the dynamic contact angle and surface tension of testing liquids, respectively; $\gamma_{1}^{\mathrm{p}}$ is the polar component; and $\gamma_{1}^{\mathrm{d}}$ represents the dispersive component.

ILSS of untreated and modified composites were examined using a universal testing machine (WD-1, Changchun, China) based on a three-point short-beam bending testing method. Composites ILSS values could be calculated from:

$$
I L S S=\frac{3 P b}{4 b h}
$$

where $b$ and $h$ represent the width $(\mathrm{mm})$ and thickness $(\mathrm{mm})$ of the testing samples, and $P_{b}$ is the maximum breaking load $(\mathrm{N})$.

Anti-hydrothermal aging experiments were carried out by adding CF/MPSR composites to boiling water at $373 \mathrm{~K}$ for $48 \mathrm{~h}$ to examine the effects of different functionalized HNT modifications on the hydrothermal aging resistance of the resulting composites. Afterwards, the hydrothermal aging resistance was characterized by tracing the changes of ILSS results.

\section{Results}

\subsection{Surface Composition and Groups of Functionalized HNTs and CFs}

Figure 2 shows FTIR spectra (Figure 2a), XPS spectra (Figure 2b) and TG curves (Figure 2c) of HNTs, HNT- $\mathrm{NH}_{2}$ and HNT-COOH. As for the FTIR spectrum of HNTs, the strong characteristic bands at about 3622 and $3698 \mathrm{~cm}^{-1}$ correspond to the $\mathrm{Al}-\mathrm{OH}$ bonds stretching vibration, and the band at $907 \mathrm{~cm}^{-1}$ is related to the $\mathrm{Al}-\mathrm{OH}$ bonds bending vibration onto the internal surface of HNTs [31]. The bands at round 1110 and $1033 \mathrm{~cm}^{-1}$ are related to the $\mathrm{Si}-\mathrm{O}-\mathrm{Si}$ bonds stretching vibration and asymmetric stretching vibration, respectively. In contrast to raw HNTs, HNT-NH${ }_{2}$ shows a new characteristic band at round $2934 \mathrm{~cm}^{-1}$ corresponding to the stretching vibration of $\mathrm{C}-\mathrm{H}$ bonds. Besides, the broad peaks in the range of $1600-1200 \mathrm{~cm}^{-1}$ are related to the stretching or bending vibrations of $\mathrm{C}-\mathrm{H}, \mathrm{N}-\mathrm{H}$ and $\mathrm{C}-\mathrm{N}$ bonds. These observed bonds arising from the structure of APS molecules indicate the success grafting of APS onto HNTs surface. For HNT-COOH, a significant band corresponding to the vibrations of carboxylic acids and secondary amides has been observed, and the peak is broad, while the peak intensity is fairly weak owing to few carboxylic acid and secondary amide groups and the existing hydrogen bonding interactions. More importantly, the additional peaks at about 1690 and $1570 \mathrm{~cm}^{-1}$ are due to $\mathrm{C}=\mathrm{O}$ stretching vibrations of the carboxylic acid and amide, respectively [20]. These characteristic bands strongly verify the successful modification of HNTs from hydroxyls to carboxyl groups. As shown in Figure 2b, the XPS spectrum of HNTs is mainly composed of many peaks (O1s, Si2s, Si2p, and Al2p), which are consistent with chemical composition of HNTs mentioned in the literature [31]. Compared to HNTs, a new peak of N1s (401 eV) has been observed on $\mathrm{HNT}-\mathrm{NH}_{2}$ XPS spectra arising from the element of APS. The peak intensity of C1s of HNT-COOH is stronger than that of HNT-NH . The above FTIR and XPS results confirm that APS and succinic anhydride have been successfully grafted onto HNTs surface. Figure 2c presents TG curves of HNTs, HNT-NH $\mathrm{N}_{2}$ and HNT-COOH. HNTs have a weight loss below $200^{\circ} \mathrm{C}$, which can be likely ascribed to the desorption of water. A second weight loss occurred from 450 to $550{ }^{\circ} \mathrm{C}$ owing to the pyrolysis of Al-OH functional groups on HNTs structure [31]. However, compared with TGA curves of HNTs, the 
higher weight loss of $\mathrm{HNT}-\mathrm{NH}_{2}$ and $\mathrm{HNT}-\mathrm{COOH}$ occurred between $200{ }^{\circ} \mathrm{C}$ and $450{ }^{\circ} \mathrm{C}$ because of the pyrolysis of the introduced organic silane onto modified HNTs after modification. A similar conclusion was also drawn by Zhu [32]. In other words, the weight loss of pristine HNTs is $18.3 \%$, whereas the weight losses of HNT- $\mathrm{NH}_{2}$ and $\mathrm{HNT}-\mathrm{COOH}$ reach 21.3 and $24.4 \%$, respectively. TG results are consistent with the analysis of the above FTIR and XPS results, confirming the successful preparation of $\mathrm{HNT}-\mathrm{NH}_{2}$ and HNT-COOH.
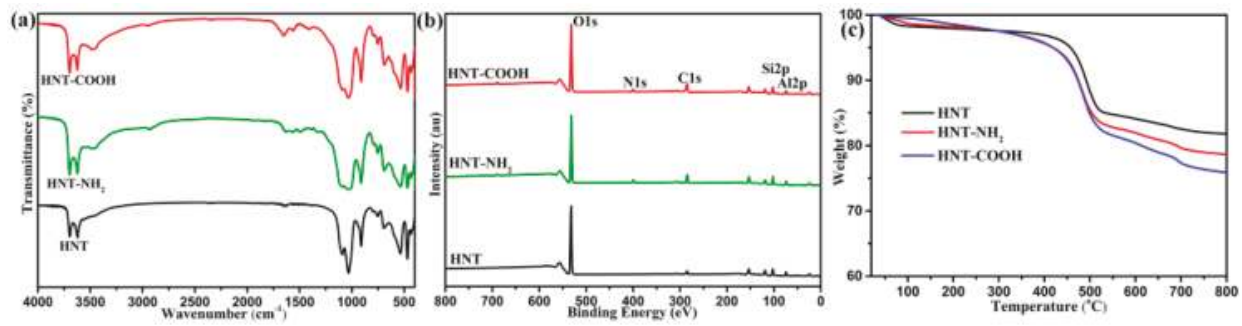

Figure 2. (a) FTIR spectra; (b) XPS spectra; and (c) TGA curves of untreated and modified HNTs.

XPS was also used to characterize chemical composition and groups of different CFs for confirming the success of chemical modification. XPS C1s spectra of untreated CF, CF-HNT-NH${ }_{2}$ and CF-HNT-COOH are presented in Figure 3. For untreated CF (Figure 3a), the XPS spectrum has been decomposed into five characteristic peaks $(\mathrm{C}=\mathrm{C}, 284.5 \mathrm{eV} ; \mathrm{C}-\mathrm{C}, 285.2 \mathrm{eV} ; \mathrm{C}-\mathrm{O}, 286.6 \mathrm{eV} ; \mathrm{C}=\mathrm{O}, 287.8 \mathrm{eV}$; $\mathrm{COOH}$, and $288.9 \mathrm{eV}$ ) [33]. As for the XPS spectrum of CF-HNT-NH 2 (Figure 3b), many significant peaks have been detected. Two new peaks arising from $\mathrm{C}-\mathrm{Si}(283.1 \mathrm{eV})$ and $\mathrm{C}-\mathrm{N}(285.7 \mathrm{eV})$ appear, which may be caused by the introduced $\mathrm{HNT}-\mathrm{NH}_{2}$ structure. Moreover, the presence of $\mathrm{N}-\mathrm{C}=\mathrm{O}$ with the bonding energies of about $287.8 \mathrm{eV}$ further indicates that acyl chloride groups onto fiber surface have already reacted with amino groups of $\mathrm{HNT}-\mathrm{NH}_{2}$. Hence, $\mathrm{HNT}-\mathrm{NH}_{2}$ nanoparticles have been boned with the surface of CFs chemically. As seen from CF-HNT-COOH (Figure 3c), CF-OH was chemically modified with $\mathrm{NHT}-\mathrm{COOH}$, and introduced massive residual carboxyl groups onto CF-HNT-COOH surface. CF-HNT-COOH shows the sharp enhancement in the content of $\mathrm{COOH}$ peak and the obvious decrease of $\mathrm{C}-\mathrm{O}$ content. In addition, the existences of the new peak $\mathrm{O}-\mathrm{C}=\mathrm{O}$ and $\mathrm{C}-\mathrm{Si}$ are responsible for the success of chemical modification for introducing HNT-COOH onto fiber surface. The introduced amino and carboxyl groups help to change fiber inert surface to polar active one, which can improve fiber surface energy and wettability for significantly enhancing interfacial adhesion and mechanical properties of composites.
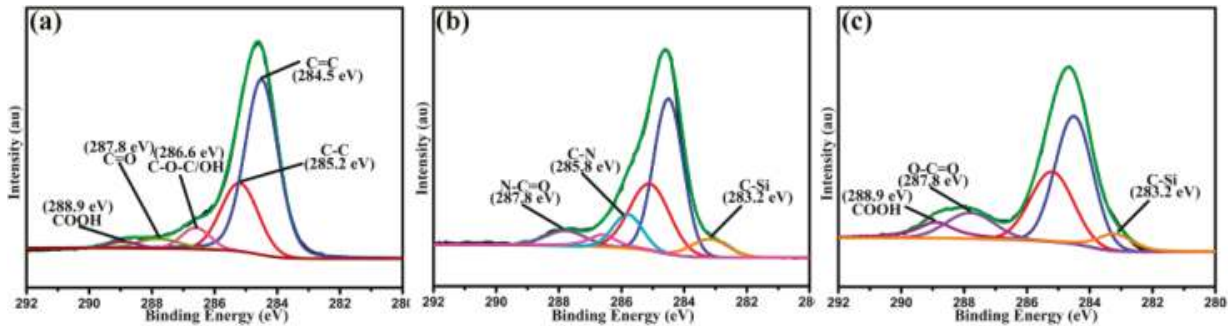

Figure 3. C1s high-resolution XPS peak-fitting curves of: (a) untreated CF; (b) CF-HNT-NH ${ }_{2}$; and (c) $\mathrm{CF}-\mathrm{HNT}-\mathrm{COOH}$. 


\subsection{Surface Microstructures of CFs}

Figure 4 shows surface morphologies for CFs before and after HNTs modification. For untreated CF (Figure 4a,b), the fiber has a smooth and flat surface, and a few narrow parallel grooves are observed. In contrast, surface morphologies of CF-HNT-NH 2 (Figure 4c,d) and CF-HNT-COOH (Figure 4e,f) change dramatically after modification, which increase fiber surface roughness significantly because of the uniform coverage of the fibers surface with grafted HNTs. Both have similar surfaces with uniform distributions of functionalized HNTs at different angles onto the surface and grooves of CFs, which make HNTs modified CFs appear as branched fibers for forming new hierarchical reinforcements. The introduced HNT- $\mathrm{NH}_{2}$ and $\mathrm{HNT}-\mathrm{COOH}$ onto the fiber surface, inserting into the matrix resin, can connect the fibers and matrix resin tightly for increasing interfacial adhesion and mechanical properties of CF composites via increasing surface roughness to provide good mechanical interlocking.

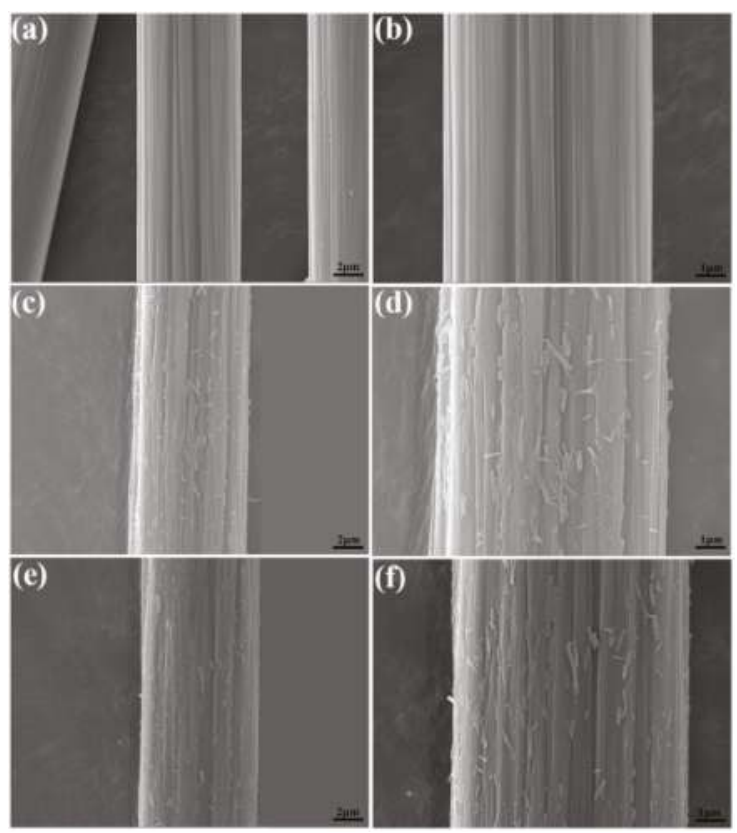

Figure 4. SEM images of different CF surfaces: (a,b) untreated $\mathrm{CF}$, (c,d) CF-HNT-NH $\mathrm{N}_{2}$; and $(\mathbf{e}, \mathbf{f}) \mathrm{CF}-\mathrm{HNT}-\mathrm{COOH}$.

\subsection{Surface Wettability Analysis of CFs}

The changes in chemical activity and surface topography of CFs affect surface energy $(\gamma)$ of CFs. A high $\gamma$ can change the wettability and compatibility between CFs and matrix resin. Hence, to study effects of $\mathrm{HNT}-\mathrm{NH}_{2}$ and $\mathrm{HNT}-\mathrm{COOH}$ modification on fiber surface energy and wettability, advancing contact angle $(\theta)$ and $\gamma$ of untreated CF, CF-HNT- $\mathrm{NH}_{2}$ and CF-HNT-COOH are evaluated and listed in Table 1 . The water contact angle $\left(\theta_{\text {water }}\right)$ and diiodomethane angle $\left(\theta_{\text {diiodomethane }}\right)$ of untreated CF are $78.5^{\circ}$ and $58.9^{\circ}$, respectively. Therefore, $\gamma$ of untreated $C F$ is only $35.87 \mathrm{mN} \cdot \mathrm{m}^{-1}$ (the dispersion component $\left(\gamma^{\mathrm{d}}\right), 31.91 \mathrm{mN} \cdot \mathrm{m}^{-1}$; and the polar component $\left.\left(\gamma^{\mathrm{p}}\right), 12.51 \mathrm{mN} \cdot \mathrm{m}^{-1}\right)$. However, compared with that of untreated $\mathrm{CF}, \mathrm{HNT}-\mathrm{NH}_{2}$ grafting and $\mathrm{HNT}-\mathrm{COOH}$ grafting show remarkably decreased contact angles and sharply enhance fiber surface energy. $\theta_{\text {water }}$ of $\mathrm{CF}-\mathrm{HNT}-\mathrm{NH}_{2}$ and CF-HNT-COOH decreased to $44.28^{\circ}$ and $42.95^{\circ}$. Similarly, $\theta_{\text {diiodomethane }}$ decreased to $40.06^{\circ}$ for $\mathrm{CF}-\mathrm{HNT}-\mathrm{NH}_{2}$ and $38.77^{\circ}$ for $\mathrm{CF}-\mathrm{HNT}-\mathrm{COOH}$. As a result, $\gamma$ of $\mathrm{CF}-\mathrm{HNT}-\mathrm{NH}_{2}$ and $\mathrm{CF}-\mathrm{HNT}-\mathrm{COOH}$ showed remarkable enhancements of $70.17 \%$ and $73.21 \%$ after two different functionalized HNTs grafting. The increased 
$\gamma^{\mathrm{p}}$ and $\gamma^{\mathrm{d}}$ can be related to the introduction of massive amino or carboxyl polar groups and the improvement of surface roughness of CFs grafted with $\mathrm{HNT}-\mathrm{NH}_{2}$ and $\mathrm{HNT}-\mathrm{COOH}$, respectively. Noteworthy, CF-HNT- $\mathrm{NH}_{2}$ and $\mathrm{CF}-\mathrm{HNT}-\mathrm{COOH}$ have similar surface energy and wettability owing to the equal enhancements of chemical polar and surface roughness. As a result, the higher surface energy for the two prepared new hierarchical reinforcements helps to increase the wettability between HNTs modified CFs and MPSR, and then improved composites interfacial properties effectively.

Table 1. Contact angles and surface energy of different CFs.

\begin{tabular}{cccccc}
\hline \multirow{2}{*}{ Samples } & \multicolumn{2}{c}{ Contact Angles $\left(^{\circ}\right)$} & \multicolumn{2}{c}{ Surface Energy $\left(\mathbf{m N} \cdot \mathbf{m}^{-\mathbf{1}}\right)$} \\
\cline { 2 - 6 } & $\boldsymbol{\theta}_{\text {water }}$ & $\boldsymbol{\theta}_{\text {diiodomethane }}$ & $\gamma^{\mathrm{d}}$ & $\gamma^{\mathbf{p}}$ & $\gamma$ \\
\hline Untreated CF & 78.50 & 58.90 & 29.21 & 6.66 & 35.87 \\
CF-HNT-NH & 44.28 & 40.06 & 21.46 & 39.58 & 61.04 \\
CF-HNT-COOH & 42.95 & 38.77 & 21.91 & 40.22 & 62.13 \\
\hline
\end{tabular}

\subsection{Interfacial Property Testing of Composites}

ILSS and IFSS testing results of MPSR composites reinforced with untreated CF, CF-HNT-NH and CF-HNT-COOH are presented in Figure 5a. ILSS and IFSS of untreated CF composites are only 29.47 and $40.37 \mathrm{MPa}$, which is related to fiber smooth and inert surface without providing a good compatibility with MPSR. After grafting, $\mathrm{HNT}-\mathrm{NH}_{2}$ and $\mathrm{HNT}-\mathrm{COOH}$ sharply enhance interfacial properties of composites because HNTs acting as an anchor stick into matrix resin to locally stiffen at the interface region for improving interface quality. For CF-HNT- $\mathrm{NH}_{2}$ composites, the ILSS and IFSS values enhanced to 46.23 and $58.31 \mathrm{MPa}$ compared to those of untreated CF composites, which might be due to the improved interfacial wettability by the introduction of many amino groups as well as the formation of strong mechanical interlocking via the enhanced surface roughness caused by HNT-NH modified onto the fiber surface. After being grafted by $\mathrm{HNT}-\mathrm{COOH}$ and $\mathrm{CF}-\mathrm{HNT}-\mathrm{COOH}$, composites have the highest values of ILSS (51.19 MPa) and ILSS (67.38 MPa), which give rise to $73.70 \%$ and $66.91 \%$ enhancement in comparison with untreated CF composites, and $10.73 \%$ and $15.55 \%$ enhancement compared to $\mathrm{CF}-\mathrm{HNT}-\mathrm{NH}_{2}$ composites. The significant increases in ILSS and IFSS values with respect to $\mathrm{CF}-\mathrm{HNT}-\mathrm{COOH}$ composites can be mainly ascribed to the high compatibility and reactive activity of CFs by introduced massive carboxyl groups onto fiber surface. That is to say, the increased degree of interfacial properties directly correlates with the introduced active groups by functionalized HNTs modification. The formed chemical bonds between $\mathrm{CFs}$ and matrix resin make a critical contribution to the improvement of interfacial properties of composites. Combining the contrast of interfacial strength of $\mathrm{CF}-\mathrm{HNT}-\mathrm{NH}_{2}$ and $\mathrm{CF}-\mathrm{HNT}-\mathrm{COOH}$ composites with a wide range of nanomaterials modification (Supplementary Material, Table S1), the functionalized HNTs grafting strategy is comparable with CFs modified by other nanomaterials, and the enhanced effect on composites interfacial strength is superior to those of many CFs composites. Therefore, HNTs, as environment-friendly and cost-effective natural nanofillers, can be regarded as a commendable alternative to enhance the quality of fiber-matrix interface and mechanical properties of the resulting composites. 

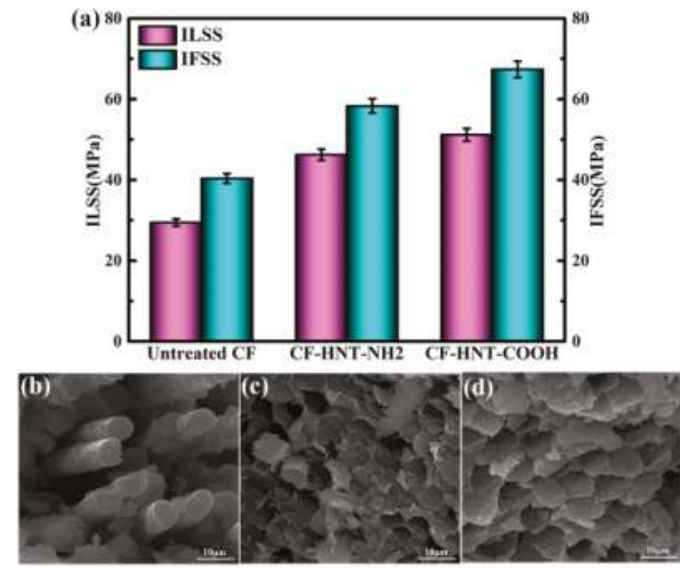

Figure 5. (a) ILSS and IFSS results of composites; and SEM morphologies of the fracture surface of

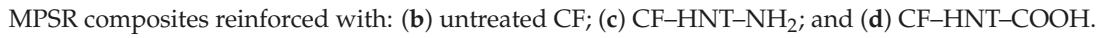

To fully study the interfacial enhancing mechanisms of MPSR composites reinforced with different fibers, composites fracture surfaces after ILSS testing were examined by SEM, as presented in Figure $5 \mathrm{~b}-\mathrm{d}$. Many big holes exist in the cracked sections of untreated CF composites owing to massive the pulled-out fibers (Figure 5b), confirming the weak interfacial adhesion. After being grafted by $\mathrm{HNT}-\mathrm{NH}_{2}$ (Figure $5 \mathrm{c}$ ), the interfacial strength between CF-HNT- $\mathrm{NH}_{2}$ and MPSR is improved greatly. Massive resin fragments are scattered onto composite fracture surfaces with few holes and pulled-out $\mathrm{CFs}$. However, some $\mathrm{CFs}$ remain detached from matrix resin with existing slight breakage of fibers. For CF-HNT-COOH composites (Figure 5d), a favorable and desired fracture surface without pulled-out fibers and fracture steps has been observed, which indicates the sharp improvement of interfacial adhesion and properties of composites via fiber surface grafting of HNT-COOH.

Figure 6 shows schematic illustration of the interfacial reaction of CF-HNT- $-\mathrm{NH}_{2}$ and $\mathrm{CF}-\mathrm{HNT}-\mathrm{COOH}$ composites. For two functionalized HNTs modified CF composites, $\mathrm{HNT}-\mathrm{NH}_{2}$ and HNT-COOH grafting provide similar wettability and mechanical interlocking caused by the obtained fiber surface energy and roughness according to DCA and SEM testing. However, the formation of interfacial reinforcing mechanisms is completely different. For CF-HNT- $\mathrm{NH}_{2}$ composites (Figure 6a), the introduced amino groups of $\mathrm{CFs}$ by $\mathrm{HNT}-\mathrm{NH}_{2}$ grafting using as the basic catalysts for matrix resin cannot react with MPSR resin during the preparation process of composites, but activate the hydroxyl groups of MPSR matrix for accelerating the cross-linking process. This is to say, the introduced HNT- $\mathrm{NH}_{2}$ can only produce sufficient mechanical interlocking for improving interfacial compatibility and adhesion between CF-HNT-NH ${ }_{2}$ and MPSR. For CF-HNT-COOH/MPSR composites (Figure 6b), the presence of carboxyl groups onto the $\mathrm{CF}-\mathrm{HNT}-\mathrm{COOH}$ has the high reaction activity with MPSR. The introduced chemical bonding leads to a significant improvement in the stress transfer from MPSR to HNT-COOH modified hierarchical reinforcing structure, and this is a crucial factor for interfacial improvement. Hence, the chemical bonds combined with sufficient nanosized mechanical interlocking at the interface region via the high strength of the HNT-network modified onto the CF surface inhibit the shear flow via the interface effectively, leading to the best interfacial adhesion of composites. 


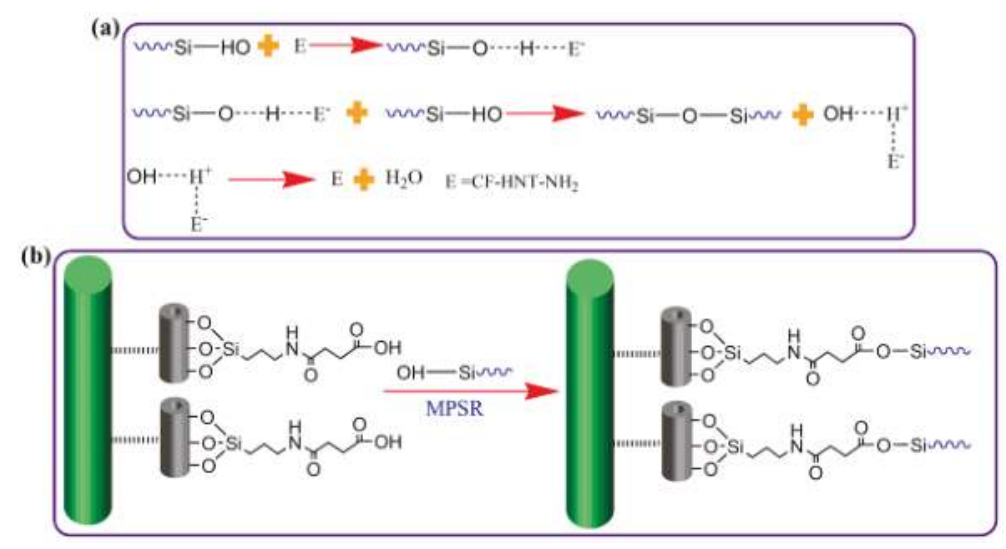

Figure 6. Schematic representation of the interfacial reaction of: (a) CF-HNT-NH $\mathrm{H}_{2}$ composites; and (b) CF-HNT-COOH composites.

\subsection{Hydrothermal Aging Resistance Testing of Composites}

The hydrothermal aging experiments give CF/MPSR composites direct insights to the potential applications in the harsh environment with high humidity. ILSS value retentions of untreated and modified CF composites after aging are shown in Figure 7. After aging, the ILSS values of untreated CF/MPSR composites decline sharply, while ILSS values of MPSR composites reinforced with CF-HNT- $\mathrm{NH}_{2}$ and $\mathrm{CF}-\mathrm{HNT}-\mathrm{COOH}$ declines slower. ILSS values of untreated CF reinforcing MPSR composites decreased from 29.47 MPa without aging to 20.52 MPa, with ILSS retention ratios of $69.63 \%$. However, ILSS retention ratios of MPSR composites reinforced with CF-HNT- $\mathrm{NH}_{2}$ and CF-HNT-COOH are $88.75 \%$ and $93.61 \%$, respectively, confirming that $\mathrm{HNT}-\mathrm{NH}_{2}$ or $\mathrm{HNT}-\mathrm{COOH}$ functionalization can enhance hydrothermal aging resistance of the resulting composites. A poor quality of fiber-matrix interface containing more microcracks and drawbacks is penetrated easily via water molecules because of the obvious difference in coefficient of thermal expansion, which can form the stress concentration at the interfacial region and thus destroy the interface, resulting in a poor hydrothermal aging resistance of untreated CF composites. The introduced $\mathrm{HNT}-\mathrm{NH}_{2}$ or HNT-COOH can strengthen interfacial adhesion between CFs and MPSR and decrease the numbers of microcracks and defects at the interfacial region, which reduce water absorption and protect the interface effectively compared with untreated composites. In addition, compared with CF-HNT- $\mathrm{NH}_{2}$ composites, CF-HNT-COOH composites show higher hydrothermal aging resistance. This might be related to the better quality of $\mathrm{HNT}-\mathrm{COOH}$ interface, which would require stronger acid/base and more energy to destroy. 


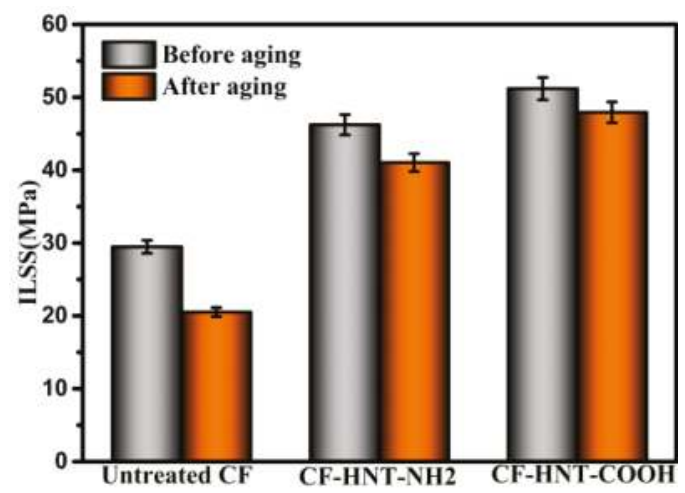

Figure 7. ILSS of composites before and after hydrothermal aging treatment.

\section{Conclusions}

In this study, to improve interfacial properties and anti-hydrothermal aging behaviors of CF/MPSR composites, the chemical grafting of different functionalized HNTs as natural and low-cost nanomaterials onto CFs was reported. Characterization results confirmed the successful modification of functionalized HNTs, and HNT- $\mathrm{NH}_{2}$ or $\mathrm{HNT}-\mathrm{COOH}$ was modified onto the surface of CFs uniformly. HNT- $\mathrm{NH}_{2}$ or $\mathrm{HNT}-\mathrm{COOH}$ modification improved surface wettability significantly through the introduced amino or carboxyl polar groups as well as increased mechanical interlocking between CFs and MPSR obviously via the enhanced surface roughness, leading to the sharp improvement in interfacial strength of composites. Particularly, MPSR composites reinforced with CF-HNT-COOH have the best interfacial properties with ILSS value of $51.19 \mathrm{MPa}$ and IFSS value of $67.38 \mathrm{MPa}$ compared with those of CF-HNT-NH 2 composites (ILSS, 46.23 MPa; and IFSS, 58.31 MPa). The improved mechanical interlocking and the formed chemical bonds between CF-HNT-COOH and MPSR through $\mathrm{HNT}-\mathrm{COOH}$ nanoparticle modification were the main contributors for these highest enhancements. In addition, the introduced $\mathrm{HNT}-\mathrm{NH}_{2}$ or $\mathrm{HNT}-\mathrm{COOH}$ at the interface increased the composites' hydrothermal aging resistance effectively.

Supplementary Materials: The following are available online at http://www.mdpi.com/2073-4360/10/10/ 1171/s1, Table S1: Comparison of interfacial properties among different nanomaterials modified carbon fibers composites.

Author Contributions: X.Z. conducted the experiments, characterization of samples and the analysis of the testing data as well as wrote the manuscript. G.W. contributed to the conception of the study and revised the manuscript. X.Z. and G.W. reviewed the manuscript.

Funding: The work was financially supported by the Natural Science Foundation of Shandong Province (grant number ZR2017PEM008).

Conflicts of Interest: The authors declare no conflict of interest.

\section{References}

1. Chen, S.; Cao, Y.; Feng, J. Polydopamine as an efficient and robust platform to functionalize carbon fiber for high-performance polymer composites. ACS Appl. Mater. Inter. 2014, 6, 349-356. [CrossRef] [PubMed]

2. Xu, F.; Liu, H.-Y.; Du, X. An Analytical Model of Interlaminar Fracture of Polymer Composite Reinforced by Carbon Fibres Grafted with Carbon Nanotubes. Polymers 2018, 10, 683. [CrossRef]

3. Zhu, J.H.; Wei, L.; Guo, G.; Zhu, A. Mechanical and Electrochemical Performance of Carbon Fiber Reinforced Polymer in Oxygen Evolution Environment. Polymers 2016, 8, 393. [CrossRef]

4. Gao, B.; Zhang, J.; Hao, Z.; Huo, L.; Zhang, R.; Shao, L. In-situ modification of carbon fibers with hyperbranched polyglycerol via anionic ring-opening polymerization for use in high-performance composites. Carbon 2017, 123, 548-557. [CrossRef] 
5. Zhang, C.; Wu, G.; Jiang, H. Tuning interfacial strength of silicone resin composites by varying the grafting density of octamaleamic acid-POSS modified onto carbon fiber. Compos. Part A 2018, 109, 555-563. [CrossRef]

6. Jiang, J.; Xu, C.; Su, Y.; Guo, Q.; Liu, F.; Deng, C.; Yao, X.; Zhou, L. Influence of Carbon Nanotube Coatings on Carbon Fiber by Ultrasonically Assisted Electrophoretic Deposition on Its Composite Interfacial Property. Polymers 2016, 8, 302. [CrossRef]

7. Ju, H.; Baschnagel, F.; Rohr, V.; Terrasi, G.P. Fretting Fatigue Behaviour of Pin-Loaded Thermoset Carbon-Fibre-Reinforced Polymer (CFRP) Straps. Polymers 2016, 8, 124.

8. Peng, Q.; Li, Y.; He, X.; Lv, H.; Hu, P.; Shang, Y.; Wang, W.; Wang, R.; Sritharan, T.; Du, S. Interfacial enhancement of carbon fiber composites by poly(amido amine) functionalization. Compos. Sci. Technol. 2013, 74, 37-42. [CrossRef]

9. Ma, L.; Li, N.; Wu, G.; Song, G.; Li, X.; Han, P.; Wang, G.; Huang, Y. Interfacial enhancement of carbon fiber composites by growing $\mathrm{TiO}_{2}$ nanowires onto amine-based functionalized carbon fiber surface in supercritical water. Appl. Surf. Sci. 2018, 433, 560-567. [CrossRef]

10. Shazed, M.A.; Suraya, A.R.; Rahmanian, S.; Mohd Salleh, M.A. Effect of fibre coating and geometry on the tensile properties of hybrid carbon nanotube coated carbon fibre reinforced composite. Mater. Des. 2014, 54, 660-669. [CrossRef]

11. Wu, G.; Ma, L.; Jiang, H.; Liu, L.; Huang, Y. Improving the interfacial strength of silicone resin composites by chemically grafting silica nanoparticles on carbon fiber. Compos. Sci. Technol. 2017, 153, 160-167. [CrossRef]

12. Xu, Z.; Huang, Y.; Zhang, C.; Liu, L.; Zhang, Y.; Wang, L. Effect of $\gamma$-ray irradiation grafting on the carbon fibers and interfacial adhesion of epoxy composites. Compos. Sci. Technol. 2007, 67, 3261-3270. [CrossRef]

13. Li, Y.; Peng, Q.; He, X.; Hu, P.; Wang, C.; Shang, Y.; Wang, R.; Jiao, W.; Lv, H. Synthesis and characterization of a new hierarchical reinforcement by chemically grafting graphene oxide onto carbon fibers. J. Mater. Chem. 2012, 22, 18748-18752. [CrossRef]

14. Xiong, L.; Zhan, F.; Liang, H.; Chen, L.; Lan, D. Chemical grafting of nano- $\mathrm{TiO}_{2}$ onto carbon fiber via thiol-ene click chemistry and its effect on the interfacial and mechanical properties of carbon fiber/epoxy composites. J. Mater. Sci. 2017, 53, 2594-2603. [CrossRef]

15. Zhao, G.; Hu, P.; Zhou, S.; Chen, G.; An, Y.; Cheng, Y.; An, J.; Zhang, X.; Han, W. Ordered Silica Nanoparticles Grown on a Three-Dimensional Carbon Fiber Architecture Substrate with Siliconborocarbonitride Ceramic as a Thermal Barrier Coating. ACS Appl. Mater. Inter. 2016, 8, 4216-4225. [CrossRef] [PubMed]

16. Peng, Q.; He, X.; Li, Y.; Wang, C.; Wang, R.; Hu, P.; Yan, Y.; Srithara, T. Chemically and uniformly grafting carbon nanotubes onto carbon fibers by poly(amidoamine) for enhancing interfacial strength in carbon fiber composites. J. Mater. Chem. 2012, 22, 5928-5931. [CrossRef]

17. Gao, B.; Zhang, R.; He, M.; Wang, C.; Liu, L.; Zhao, L.; Wen, Z.; Ding, Z. Interfacial microstructure and mechanical properties of carbon fiber composites by fiber surface modification with poly(amidoamine)/ polyhedral oligomeric silsesquioxane. Compos. Part A 2016, 90, 653-661. [CrossRef]

18. Chen, J.; Wang, K.; Zhao, Y. Enhanced interfacial interactions of carbon fiber reinforced PEEK composites by regulating PEI and graphene oxide complex sizing at the interface. Compos. Sci. Technol. 2018, 154, 175-186. [CrossRef]

19. Du, M.; Guo, B.; Lei, Y.; Liu, M.; Jia, D. Carboxylated butadiene-styrene rubber/halloysite nanotube nanocomposites: Interfacial interaction and performance. Polymer 2008, 49, 4871-4876. [CrossRef]

20. Joo, Y.; Jeon, Y.; Lee, S.U.; Sim, J.H.; Ryu, J.; Lee, S.; Lee, H.; Sohn, D. Aggregation and Stabilization of Carboxylic Acid Functionalized Halloysite Nanotubes (HNT-COOH). J. Phys. Chem. C 2012, 116, 18230-18235. [CrossRef]

21. Tsoufis, T.; Katsaros, F.; Kooi, B.J.; Bletsa, E.; Papageorgiou, S.; Deligiannakis, Y.; Panagiotopoulos, I. Halloysite nanotube-magnetic iron oxide nanoparticle hybrids for the rapid catalytic decomposition of pentachlorophenol. Chem. Eng. J. 2017, 313, 466-474. [CrossRef]

22. Chiu, F.-C. Halloysite nanotube- and organoclay-filled biodegradable poly(butylene succinate-co-adipate)/ maleated polyethylene blend-based nanocomposites with enhanced rigidity. Compos. Part B 2017, 110, 193-203. [CrossRef]

23. Lin, J.; Zhong, B.; Jia, Z.; Hu, D.; Ding, Y.; Luo, Y.; Jia, M. In-situ fabrication of halloysite nanotubes/silica nano hybrid and its application in unsaturated polyester resin. Appl. Surf. Sci. 2017, 407, 130-136. [CrossRef]

24. Liu, M.; Jia, Z.; Jia, D.; Zhou, C. Recent advance in research on halloysite nanotubes-polymer nanocomposite. Prog. Polym. Sci. 2014, 39, 1498-1525. [CrossRef] 
25. Du, M.; Guo, B.; Jia, D. Thermal stability and flame retardant effects of halloysite nanotubes on poly(propylene). Eur. Polym. J. 2006, 42, 1362-1369. [CrossRef]

26. Albdiry, M.T.; Yousif, B.F. Role of silanized halloysite nanotubes on structural, mechanical properties and fracture toughness of thermoset nanocomposites. Mater. Des. 2014, 57, 279-288. [CrossRef]

27. Jiang, L.; Zhang, C.; Liu, M.; Yang, Z.; Tjiu, W.W.; Liu, T. Simultaneous reinforcement and toughening of polyurethane composites with carbon nanotube/halloysite nanotube hybrids. Compos. Sci. Technol. 2014, 91, 98-103. [CrossRef]

28. Wan, X.; Zhan, Y.; Zeng, G.; He, Y. Nitrile functionalized halloysite nanotubes/poly(arylene ether nitrile) nanocomposites: Interface control, characterization, and improved properties. Appl. Surf. Sci. 2017, 393, 1-10. [CrossRef]

29. Yu, H.; Zhang, Y.; Sun, X.; Liu, J.; Zhang, H. Improving the antifouling property of polyethersulfone ultrafiltration membrane by incorporation of dextran grafted halloysite nanotubes. Chem. Eng. J. 2014, 237, 322-328. [CrossRef]

30. Chao, C.; Liu, J.; Wang, J.; Zhang, Y.; Zhang, B.; Zhang, Y.; Xiang, X.; Chen, R. Surface Modification of Halloysite Nanotubes with Dopamine for Enzyme Immobilization. ACS Appl. Mater. Inter. 2013, 5, 10559-10564. [CrossRef] [PubMed]

31. Zeng, G.; Ye, Z.; He, Y.; Yang, X.; Ma, J.; Shi, H.; Feng, Z. Application of dopamine-modified halloysite nanotubes/PVDF blend membranes for direct dyes removal from wastewater. Chem. Eng. J. 2017, 323, 572-583. [CrossRef]

32. Zhu, J.; Guo, N.; Zhang, Y.; Yu, L.; Liu, J. Preparation and characterization of negatively charged PES nanofiltration membrane by blending with halloysite nanotubes grafted with poly (sodium 4-styrenesulfonate) via surface-initiated ATRP. J. Membrane Sci. 2014, 465, 91-99. [CrossRef]

33. Zhang, G.; Sun, S.; Yang, D.; Dodelet, J.-P.; Sacher, E. The surface analytical characterization of carbon fibers functionalized by $\mathrm{H}_{2} \mathrm{SO}_{4} / \mathrm{HNO}_{3}$ treatment. Carbon 2008, 46, 196-205. [CrossRef]

(C) 2018 by the authors. Licensee MDPI, Basel, Switzerland. This article is an open access article distributed under the terms and conditions of the Creative Commons Attribution (CC BY) license (http:/ / creativecommons.org/licenses/by/4.0/). 
Article

\title{
Synergistic Effect of Halloysite Nanotubes and Glycerol on the Physical Properties of Fish Gelatin Films
}

\author{
Xiaohu Qiang *, Songyi Zhou, Zhuo Zhang, Qiling Quan and Dajian Huang \\ School of Materials Science and Engineering, Lanzhou Jiaotong University, Lanzhou 730070, China; \\ zsy1164826030@126.com (S.Z.); zhang1466rose@163.com (Z.Z.); quanq10329@163.com (Q.Q.); \\ huangdj2015@yeah.net (D.H.) \\ * Correspondence: Qiangxh2018@126.com; Tel.: +86-931-4956-651
}

Received: 3 October 2018; Accepted: 7 November 2018; Published: 13 November 2018

\begin{abstract}
Fish gelatin (FG)/glycerol (GE)/halloysite (HT) composite films were prepared by casting method. The morphology of the composite films was observed by scanning electron microscopy (SEM). The effects of HT and GE addition on the mechanical properties, water resistance and optical properties of the composites were investigated. Results showed that with increasing GE content, the elongation at composite breaks increased significantly, but their tensile strength (TS) and water resistance decreased. SEM results showed that GE can partly promote HT dispersion in composites. TS and water resistance also increased with the addition of HTs. Well-dispersed HTs in the FG matrix decreased the moisture uptake and water solubility of the composites. All films showed a transparency higher than $80 \%$ across the visible light region $(400-800 \mathrm{~nm})$, thereby indicating that light transmittance of the resulting nanocomposites was slightly affected by GE and HTs.
\end{abstract}

Keywords: fish gelatin; halloysite nanotubes; glycerol; mechanical properties; water resistance

\section{Introduction}

Recently, studies using natural polymer materials to replace synthesis plastic for preparing packaging films have attracted wide attention because of the pollution problems caused by synthetic plastic [1-4]. When natural polymer materials are mentioned, gelatin, with low cost, biocompatibility, and biodegradability, is one of the most promising [5-8]. Fish gelatin (FG), a type of gelatin that can provide materials with ultraviolet and oxygen barrier properties, can be extracted from fish bones and skin, and has been used an alternative raw material for a wide range of applications [9-11]. However, FG's inflexible and hydrophilic character, and consequently its poor mechanical properties in the presence of humid environments, limit its application $[10,12,13]$. As a consequence, the flexible properties and water-resistance of FG-based films should be improved further to meet the demand of practical applications.

An effective strategy exploring how to improve the properties of gelatin-based films is adding plasticizer into the gelatin matrix [14-16]. Plasticizers could break the polymer-polymer interactions (such as hydrogen bonds and Van der Waals forces) and form secondary bonds to gelatin molecular chains, thereby increase the flexibility of the films and preventing them from cracking during packing and transportation [16-18]. Among possibilities of additives that can be used as plasticizer in gelatin-based films, glycerol (GE) is the most commonly used in gelatin-based films [18-20]. Compared with other plasticizers such as sorbitol, polyethylene glycol and diethylene glycol, GE has smaller molecular dimensions and higher hydroxyl density, so it is widely used as a plasticizer into a hydrophilic biopolymers matrix. 
Incorporating inorganic nanoparticles such as clay, graphene, zinc oxide and carbon nanotubes into the gelatin matrix has been shown to be an effective method to improve its thermal, mechanical, and barrier properties. Farahnaky et al. found that the mechanical properties and water-resistance of gelatin films were improved by the addition of montmorillonite [21]. Shankar et al. prepared gelatin/ $\mathrm{ZnO}$ nanoparticle nanocomposite films using various types of $\mathrm{ZnO}$ nanoparticle [22]. The results showed that the moisture content, water contact angle, water vapor permeability, and elongation at breaks of $\mathrm{ZnO}$ nanoparticle incorporated films increased, while tensile strength and modulus of elasticity decreased compared with the control gelatin film. Li et al. studied the effect of the concentration of laponite on the physicochemical properties of a gelatin/laponite film [23]. They found that gel strength, mechanical properties, water vapor permeability and water solubility of gelatin/laponite composite films were greatly enhanced with an increase of the concentration of laponite.

Halloysite nanotubes (HTs) are natural aluminosilicates from a clay mineral with a hollow nanotubular structure [24,25]. HTs have been developed to use in controlled release [26], templates [27], and sorption [28] during the past decades. Recently, HTs have been successfully developed as a reinforcing filler to improve the properties of biopolymers. For instance, the mechanical properties and thermal stability of starch films were improved by the addition of HTs [29,30]. Soheilmoghaddam et al. found that the tensile strength of regenerated cellulose film increased from 35.30 to $60.50 \mathrm{MPa}$ when $8 \mathrm{wt} \%$ HTs were added, without loss of ductility [30]. Liu et al. have prepared chitosan/HTs composites via solution casting and the FE-SEM results indicated that HTs are uniformly dispersed in the chitosan matrix [31]. The tensile strength of chitosan was improved by HTs. The low cost and environmentally friendly nature make HTs competitive with other reinforcing fillers for biopolymers. In addition, unlike two-dimensional platelet-like nanoclays [32,33], the rod-like geometry of HTs hardly intertwines, which makes HTs disperse better in a polymer matrix [34].

The objective of this work is to investigate the combined effect of GE and HTs on the properties of FG-based composites. For this purpose, we prepared a series of films containing different amounts of HTs and GE and characterized them using field emission scanning electron microscopy (FE-SEM) and Fourier Transform Infrared Spectroscopy (FT-IR). The mechanical properties, water-resistance, and optical properties of composite films were investigated to establish a structure-property correlation between HTs, GE and the FG matrix.

\section{Materials and Methods}

\subsection{Materials}

FG was purchased from Taosheng Chemical Co., Ltd. (Guangzhou, China). HTs were purchased from Yuanxin Nanotechnology Co., Ltd. (Guangzhou, China). GE is analytical grade, and used without further purification.

\section{Preparation of Composite Films}

Preparation of composite films is on the basis of the method in the literature. FG was added into distilled water at $50{ }^{\circ} \mathrm{C}$ with magnetic stirring for $1 \mathrm{~h}$ to prepare the FG solution $(4 \%, w / w)$. Then, the GE, as a plasticizer, was added into the FG solution at a certain loading $(0,20,30,40 \%$ $(w / w))$ with respect to FG weight. The obtained mixture was stirred for $1 \mathrm{~h}$ to acquire homogeneous solutions. HTs dispersions $(0.5 \%, w / w)$ were prepared by adding appropriate amounts of HTs into distilled water under mechanical agitation at $500 \mathrm{rpm}$ for $1 \mathrm{~h}$ at room temperature. The obtained HTs dispersions were added in the four types of FG/GE composites (pure FG, FG/GE (20\%), FG/GE $(30 \%)$, and FG/GE $(40 \%))$ at $0 \%, 4 \%$ and $8 \%(w / w)$ loadings with respect to FG weight, respectively. Following this, the obtained mixtures were stirred for $1 \mathrm{~h}$ at $50{ }^{\circ} \mathrm{C}$ to acquire homogeneous solutions. These film-forming solutions were placed at room temperature for $1 \mathrm{~h}$ to remove the bubble. After that, the mixtures were poured into $9 \mathrm{~cm}$ diameter polycarbonate petri dishes and evaporated into 
the atmosphere for $72 \mathrm{~h}$; the resulting films were dried in a vacuum oven $\left(\right.$ at $\left.70{ }^{\circ} \mathrm{C}\right)$ for $6 \mathrm{~h}$ to remove traces of the solvents. The named rule of composite films in this work is directly named by the mass fraction of GE and HTs: for example, composite films with $40 \mathrm{wt} \%$ GE loading are named FG/GE40, and composite films with $40 \mathrm{wt} \%$ GE and $10 \mathrm{wt} \% \mathrm{HTs}$ are named FG/GE40/HTs10.

\subsection{Characterization}

The morphology of samples was observed by a field emission scanning electron microscope (FESEM, JEOL JSM-6701F, Japan Electron Optics Limited, Tokyo, Japan), after coating the sample with a layer of gold. Water contact angles (WCA) were measured with a Contact Angle System OCA 20 (Dataphysics, Stuttgart, Germany) at ambient temperature by injecting $5 \mu \mathrm{L}$ of water droplets onto the surface of films. Average WCA values were determined by measuring the same sample at five different positions. Transmission electron microscopy (TEM) analysis of samples was carried out with a TECNAIG2-F30, FEI.

X-ray diffraction (XRD) patterns were collected using an XRD-7000 (XRD, Shimadzu XRD-7000, Shimadzu Co., Ltd., Kyoto, Japan) diffractometer with $\mathrm{Cu} \mathrm{Ka}$ radiation, scanning from $3^{\circ}$ to $40^{\circ}$ at $3^{\circ} / \mathrm{min}$. Attenuated total reflectance Fourier transform infrared spectra (ATR-FTIR) were recorded on an FTIR Spectrophotometer (Thermo Nicolet, iS10, Thermo Fisher, Waltham, MA, USA) in the range of $4000-400 \mathrm{~cm}^{-1}$. Film thickness was studied to the nearest $0.001 \mathrm{~mm}$ with a digital micrometer (Micrometer, Wuxi, China) at 9 random positions around the film.

\subsection{Tensile Tests}

Tensile tests were performed using a an AG-IS material testing machine (Shimadzu Co., Ltd., Kyoto, Japan) equipped with a $200 \mathrm{~N}$ load cell at room temperature, with a gauge length of $40 \mathrm{~mm}$ and crosshead speed of $10 \mathrm{~mm} / \mathrm{min}$. The results were evaluated as an average of at least 5 measurements.

\subsection{Moisture Uptake}

Moisture uptake of the films was measured on samples cut into small pieces $(2 \mathrm{~cm} \times 3 \mathrm{~cm})$. Samples were first dried in an oven at $70{ }^{\circ} \mathrm{C}$ for $8 \mathrm{~h}$. After weighing, the samples were stored over $\mathrm{NaCl}$ saturated salt $(76 \% \mathrm{RH})$ for $72 \mathrm{~h}$ to ensure equilibrium of the moisture before reweighing. The moisture uptake of the samples was calculated as follows:

$$
\text { Moisture uptake }=\left[W_{1}-W_{0}\right] / W_{0} \times 100 \%
$$

where $W_{0}$ and $W_{1}$ are the weight of the samples before exposure to $76 \% \mathrm{RH}$ and at equilibrium, respectively. All tests were carried out with three replicates and an average value for each sample was obtained.

\subsection{Water Solubility}

Water solubility of the films was studied by the following method: Six pieces $(2 \mathrm{~cm} \times 3 \mathrm{~cm})$ of film dried in an air-circulating oven at $70^{\circ} \mathrm{C}$ until reaching constant weight and were weighed $\left(M_{0}\right)$. After this, the films were immersed in $50 \mathrm{~mL}$ of distilled water. After $24 \mathrm{~h}$ of storage in an environmental chamber at $25^{\circ} \mathrm{C}$, samples were recovered and gently rinsed with distilled water. After drying in an air-circulating oven at $70^{\circ} \mathrm{C}$ until reaching constant weight, the films were weighed again $\left(M_{1}\right)$.

The water solubility of films was calculated by the following equation:

$$
\text { Water solubility }=\left[M_{0}-M_{1}\right] / M_{0} \times 100 \%
$$

\subsection{Optical Properties}

The opacity of composite films was studied by a UV-visible spectrophotometer (N4, INESA (Group) Co., Ltd., Shanghai, China). Composite films were cut into rectangular strips and placed 
on the two outer sides of a spectrophotometer cell and light transmission was recorded at room temperature in steps of $1 \mathrm{~nm}$, in the range $200-800 \mathrm{~nm}$.

\subsection{Statistical Analysis}

Averages and standard deviations from at least three measurements of each sample were reported in this study. Student's t-test was used for analysis of test results at the significance level of $p$-value $<0.05$.

\section{Results}

\subsection{Film Thickness}

Film thickness is an important parameter in the calculation of mechanical and other physical properties. As shown in Figure 1, the addition of HT and/or GE generated an increase in the thickness of the composites films. The film thickness increased from $37.0 \mu \mathrm{m}$ for the control FG film to $46.3 \mu \mathrm{m}$ for the FG/GE40/HTs10 composite film. This phenomenon could be due to the increase in dry matter content of composites.

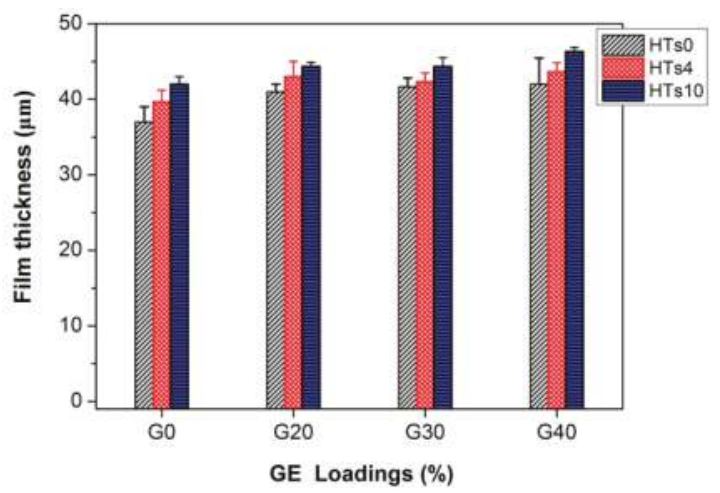

Figure 1. Film thickness of fish gelatin (FG)/glycerol (GE)/halloysite (HT) composites with various amounts of HT and GE loadings.

\subsection{SEM Analysis}

The degree of filler dispersion is a key factor for the final properties of the nanocomposite [35]. SEM micrographs of the fracture surface of selected films are presented in Figure 2. The SEM micrographs of pure FG and FG/GE40 films are shown in Figure 2a,b, respectively. It can be observed that fracture surfaces of the two types of film are smooth and homogeneous, without pores or cracks. Compared with the pure FG film, the fracture surface of FG/GE40 film shows a more dense structure, indicating that there is good compatibility between FG and GE, and that the GE molecular can insert into the FG molecular chains.

The SEM micrographs of FG/HTs4 and FG/GE40/HTs4 films are shown in Figure 2c,d, respectively. These two films showed rougher fractured surface compared with the films without HTs. The bright dots shown in Figure 2c, d are terminals of HTs, which demonstrates that HTs were embedded in the FG matrix successfully. A good dispersion of HTs in the FG or FG/GE40 matrix was observed without obvious agglomeration in the two types of composites, indicating that the HTs, GE and FG were compatible. In order to further observe the dispersion of HTs in the polymers, TEM was used to investigate the FG/GE40/HTs4 sample and the obtained micrographs are displayed in Figure 3. As shown in Figure 3, the HTs possess a hollow cylindrical shape and show a transparent central area along the cylinder with an open end. The HTs were well dispersed in the FG/GE matrix. 
The well-dispersed HTs in the FG or FG/GE40 matrix can be attributed to the following reasons: First, the nanotube morphological character of HTs and the relatively weak nanotube-nanotube interactions allowed to disperse easily in FG by shear force. Second, HTs can interact with FG through hydrogen bonding interactions, which also led to its effective dispersion. As the content of HTs was increased to $10 \%$, some agglomerations were found in the two types of composites (Figure $2 \mathrm{e}, \mathrm{f}$ ), which may be mainly due to the Van der Waals force among HTs. It can be seen that the HTs in the FG/HTs composites exhibit more agglomeration than that of FG/GE40/HTs composites. This phenomenon may be ascribed to the interaction of hydrogen bonds between GE and HTs, and GE could promote the dispersion of HTs in the composites. A similar phenomenon was reported by Lavorgna et al., when they found that the presence of GE improved the chitosan intercalation in the silicate galleries of montmorillonite and prevented the flocculation process, leaving the MMT stacks randomly orientated in the composites [36].
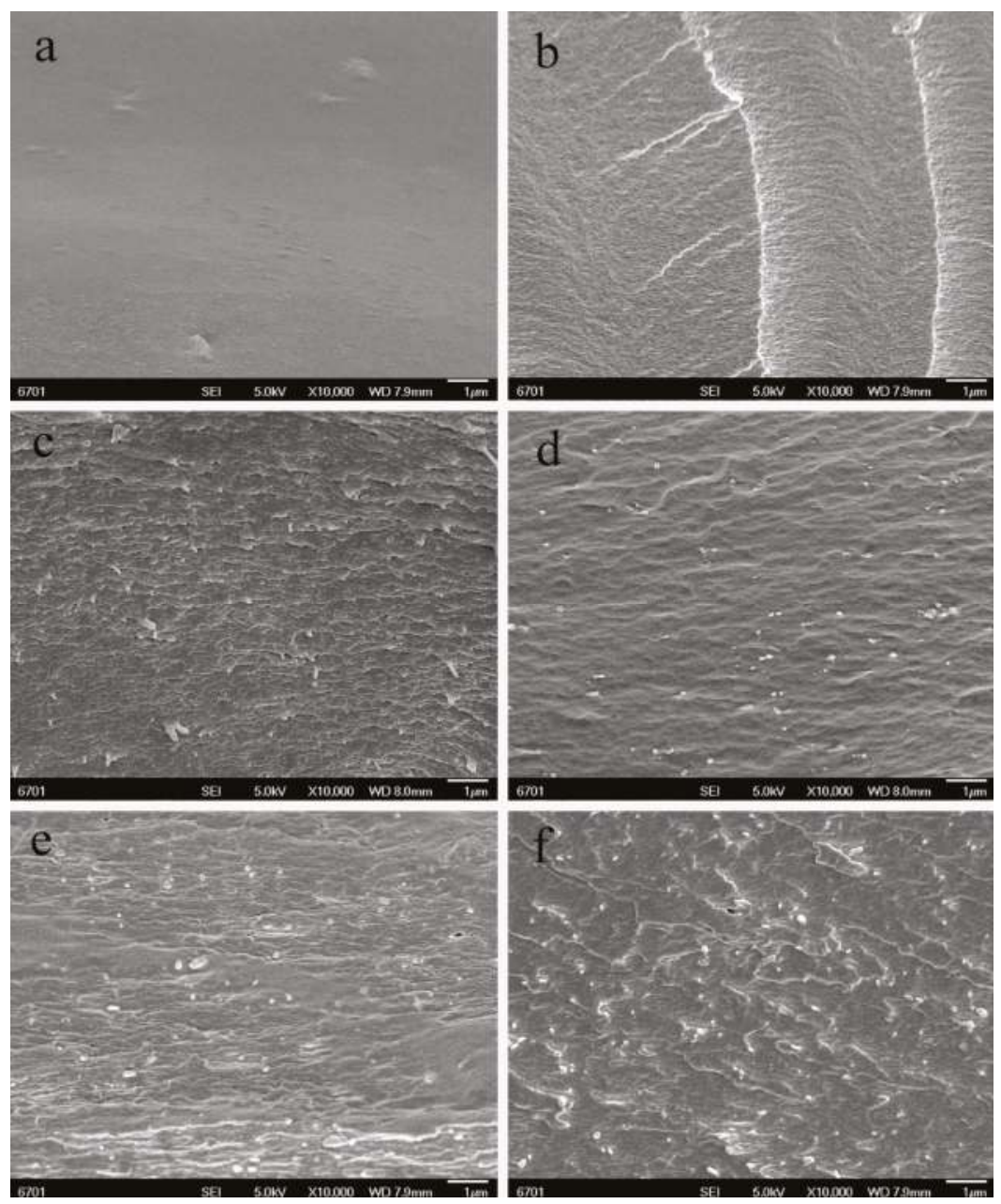

Figure 2. SEM micrographs of (a) pure FG, (b) FG/GE40, (c) FG/HT4, (d) FG/GE40/HTs4, (e) FG/HTs10 and (f) FG/GE40/HTs10. 


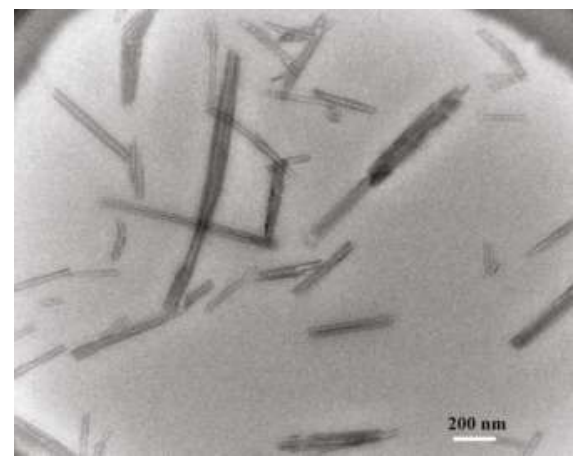

Figure 3. TEM micrographs of FG/GE40/HTs4.

\subsection{XRD Analysis}

Figure 4 shows the XRD patterns of HTs and FG/GE30 composites with HT loadings of $0 \%$, $4 \%$ and $10 \%$. The basal spacing (001) of pristine HTs was approximately $0.72 \mathrm{~nm}$, corresponding to $2 \theta=12.0^{\circ}$ [37], with other peaks appearing at higher angles of $2 \theta=20.22^{\circ}$ and $2 \theta=24.52^{\circ}$; these are assigned to (020), (110) and (002), respectively [31]. Typical XRD peaks for HNT powders appear at $2 \theta$ $=18^{\circ}$, in accordance with reflection planes (002) of gibbsite, which is an impurity in the HTs. Owing to its strong orientation, the peak of gibbsite at $2 \theta=18^{\circ}$ appeared obviously even at a small loading. This XRD peak of gibbsite as an impurity in the HTs also was found in other studies. The FG/GE30 film showed a broad peak in the $2 \theta$ range of $6.2^{\circ}-9.5^{\circ}$, which is a characteristic of amorphous proteins [38]. As for the FG/GE30/HTs composite, the intercalation of FG or GE molecules into the interlayers of HTs' hollow tubular structure does not take place, as the d-spacing values for their weak (001) peaks remain almost unchanged [33]. This peak gradually increased with increasing HT content of the FG/GE30 matrix. In addition, the peaks at $20.22^{\circ}$ and $24.52^{\circ}$ become invisible. This finding is in good agreement with previous work based on polymer/HTs composites [31].

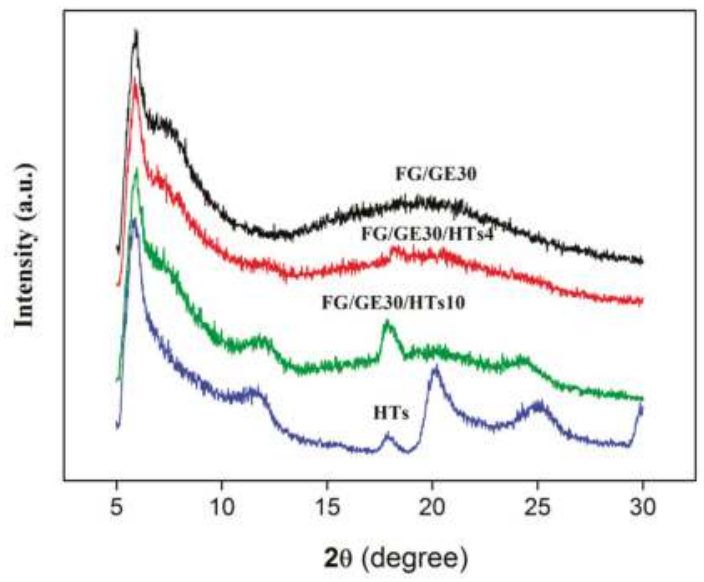

Figure 4. XRD patterns of HTs and FG/GE30 at 0, 4 and 10 wt \%.

\subsection{FTIR Analysis}

ATR-FTIR analysis was performed to study the interactions between HTs and the FG matrix, and the results are presented in Figure 5. FG is an amphoteric polyelectrolyte with both positively and negatively charged amino acids dispersed on the chain backbone [21]. The FT-IR spectrum of pure FG 
film showed the characteristic amide I, amide II and amide III bands. As shown in Figure 1a, the pure FG film showed a major band at $1637 \mathrm{~cm}^{-1}$ (amide I band), which is related to the $\mathrm{C}=\mathrm{O}$ stretching $/ \mathrm{H}$ bonding in combination with COO [21]. The peak at $1540 \mathrm{~cm}^{-1}$ was because of amide-II, produced by bending vibrations of $\mathrm{N}-\mathrm{H}$ groups and stretching vibrations of $\mathrm{C}-\mathrm{N}$ groups [21]. The peak at $1240 \mathrm{~cm}^{-1}$ can be attributed to the vibration of $\mathrm{C}-\mathrm{N}$ and $\mathrm{N}-\mathrm{H}$ groups of bound amide, or vibration of $\mathrm{CH}_{2}$ groups of glycine [39]. The peak at $1033 \mathrm{~cm}^{-1}$ is related to the interaction between GE (OH group of GE) and FG [40]. In the spectrum of FG/GE30 (Figure 1b), the stretching and bending vibrations of the hydrogen bonding -OH group occurred at $3291 \mathrm{~cm}^{-1}$ [20]. The peak $\left(3291 \mathrm{~cm}^{-1}\right)$ is observed to shift to 3286 and $3282 \mathrm{~cm}^{-1}$ (Figure 1c,d) after incorporation of $4 \%$ and 10\% HTs into the FG/GE30 matrix, respectively. These behaviors indicate that HT can interact with FG and GE, and partially destruct the hydrogen bonding between FG and GE [20]. The mechanism of interactions among FG, GE and HTs is illustrated in Scheme 1. A quite similar tendency was found for attapulgite introduction into the PVA/chitosan matrix [31]

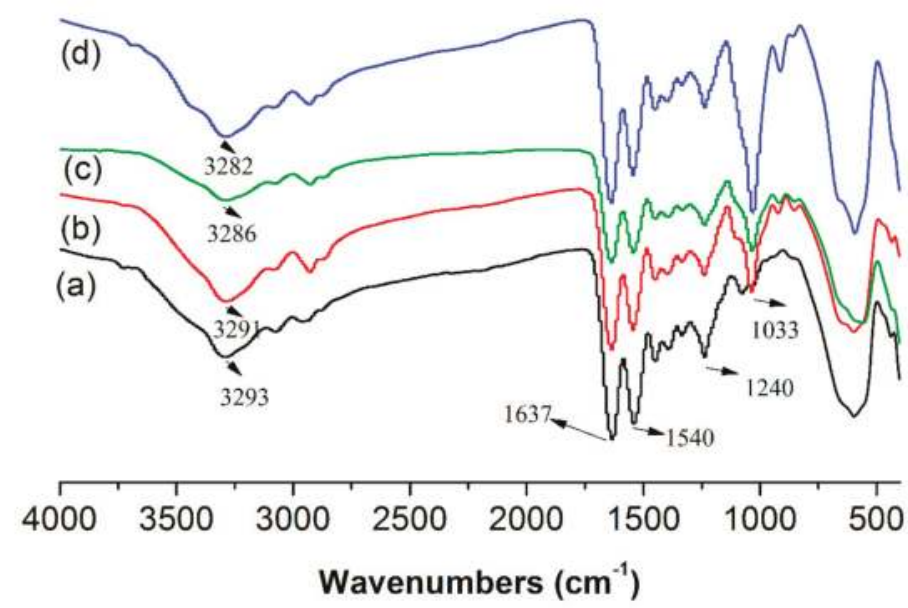

Figure 5. ATR-FTIR spectra of (a) pure FG, (b) FG/GE30, (c) FG/GE30/HTs4 and (d) FG/GE30/HTs10.

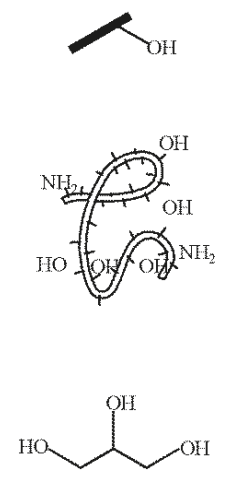

(HT)

(FG)

Scheme 1. Schematic illustration of the mechanism of HTs in the FG/GE composites.

\subsection{Mechanical Properties}

Mechanical properties are one of the most important parameters to packaging materials. Mechanical properties of composite films were characterized by tensile strength (TS) and elongation at break (EB) [41]. The results of TS are shown in Figure 6. As expected, the TS of films decreased 
prominently from 65 to $15 \mathrm{MPa}$ by elevating the proportion of GE from $0 \%$ to $40 \% w / w(p<0.05)$. The effect of GE addition on the EB of films has an inverse behavior (Figure 7) compared with their correspondent TS. Meanwhile, the increase of GE addition from $0 \%$ to $40 \%$ caused EB to greatly increase $(p<0.05)$. This may be due to the role of GE in diminishing the strong intra-molecular attraction between FG chains and improving the formation of hydrogen bonds between GE and FG molecules [14]. Thus, it reduces the TS and increases the EB of composites by subsequently weakening the hydrogen bonds between FG chains. Such disruption and reconstruction of FG molecular chains decreases the rigidity and improves the flexibility of films by endowing them with more chain mobility. Similar results have been reported by Muscat et al.: they found that the elongation of polymeric materials depends on the mobility of their molecular chains [42].

As for the HTs addition on the tensile properties of composites, it is clear that the TS of films was greatly influenced by the addition of HTs. The TS of films was improved continuously by increasing HTs loadings for the FG/GE composites $(p<0.05)$. The improved TS of composites can be explained by the fact that firstly, there is a lot of intermolecular hydrogen bonding between HTs and FG, FG and GE, and GE and HTs, which could form a 3D network (see Scheme 1); and secondly, the large aspect ratio of the HTs is also beneficial to stress transfer [33].

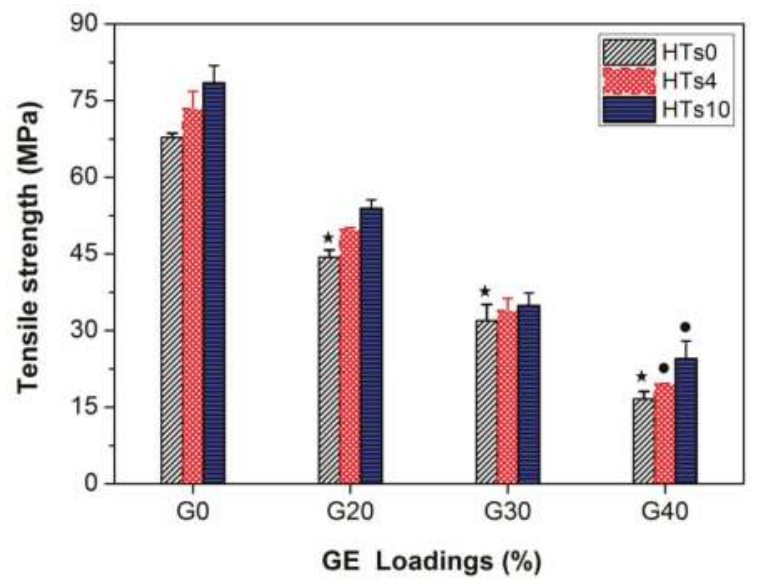

Figure 6. Tensile strength (TS) of FG/GE/HT composites with various HT and GE loadings. * denotes significant difference between FG films containing different percentages of GE $(p<0.05)$. denotes significant difference in FG/GE40 films containing different percentages of HT compared with FG/GE40 only film $(p<0.05)$.

The EB value increased with increasing content of HTs for the FG/GE composites with GE loadings from $0 \%$ to $30 \%$. For FG/GE composites with GE loadings below 30\%, EB values are low $(<10 \%)$, indicating that these composites are still brittle even though plasticized by GE. After incorporation of HTs into FG/GE composites, HTs would act as a bridge to connect the fracture of films at the process of stressing, thus the EB of films was increased. However, when the GE loading was increased to $40 \%$, the EB value reached $20 \%$, which showed flexibility at a certain extent. When incorporating HTs into FG/GE 40\%, the hydrogen bonding between HTs, FG, FG and GE hinders the sliding of the FG chains and consequently the EB values were decreased with the increase of HTs. 


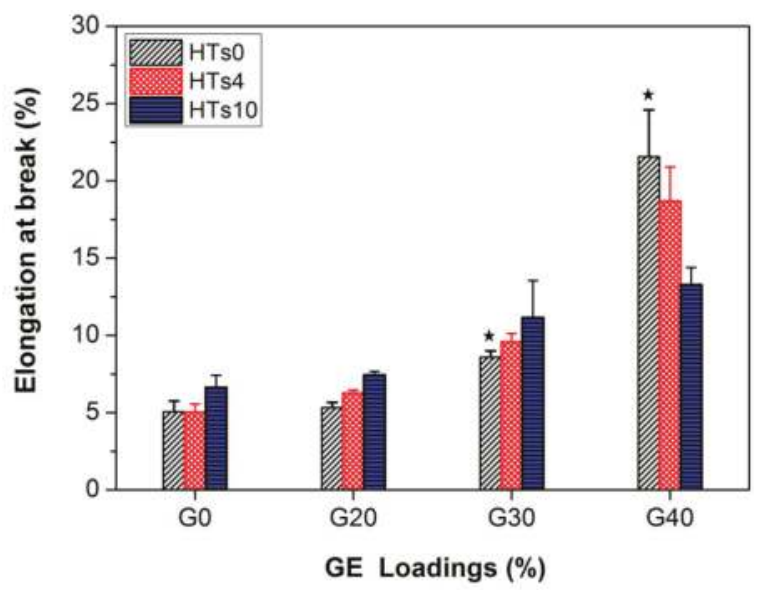

Figure 7. Elongation at break (EB) of FG/GE/HT composites with various HT and GE loadings. * denotes significant difference between FG films containing different percentages of GE $(p<0.05)$.

\subsection{Moisture Uptake and Water Solubility Of Composites}

Moisture uptake and water solubility are common parameters used to characterize the water-resistance of packaging materials [20]. The results for moisture uptake and water solubility of composite films are presented in Figure 8. The moisture uptake of composite films increased continuously with the increase of GE content $(p<0.05)$. This is due to the fact that the GE molecule has high hydroxyl density and is more hydrophilic than FG molecules. However, moisture uptake shows a decreased trend with the addition of HTs for all the FG/GE composites. This reduction in moisture uptake can be ascribed to the following reasons: Firstly, the formation of strong interactions via hydrogen bonds between FG, GE and HTs promotes the cohesiveness of the FG matrix and decrease its water sensitivity [43]. Secondly, moisture uptake of HTs is lower than that of FG and GE, so the addition of HTs reduces the moisture uptake of composites [33].

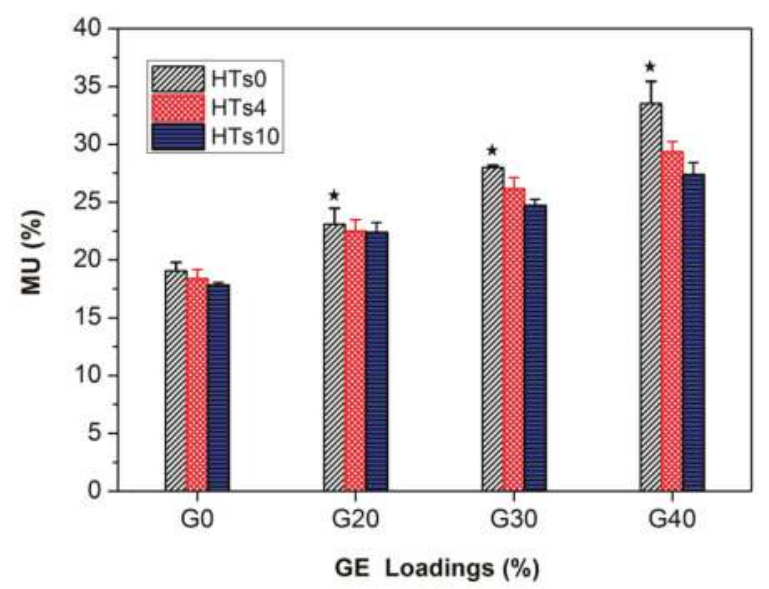

Figure 8. Moisture uptake of FG/GE/HT composites with various HT and GE loadings. * denotes significant difference between FG films containing different percentages of GE $(p<0.05)$.

The results of water solubility of composites are similar with those of moisture uptake, which also increased continuously with the increase of GE content (Figure 9) $(p<0.05)$. This behavior can be 
explained by the fact that GE has higher solubility than FG in the distilled water. In addition, it can be seen that water solubility decreased with the addition of HTs into the FG/GE composites. This is due to the presence of hydrogen bonds between FG, GE and HTs, and the insoluble property of HTs, which reduced the diffusion of water molecules in the composites and thus decreased the water solubility. Based on the above discussion, it was demonstrated that the incorporation of HTs could improve the water resistance properties of the composites.

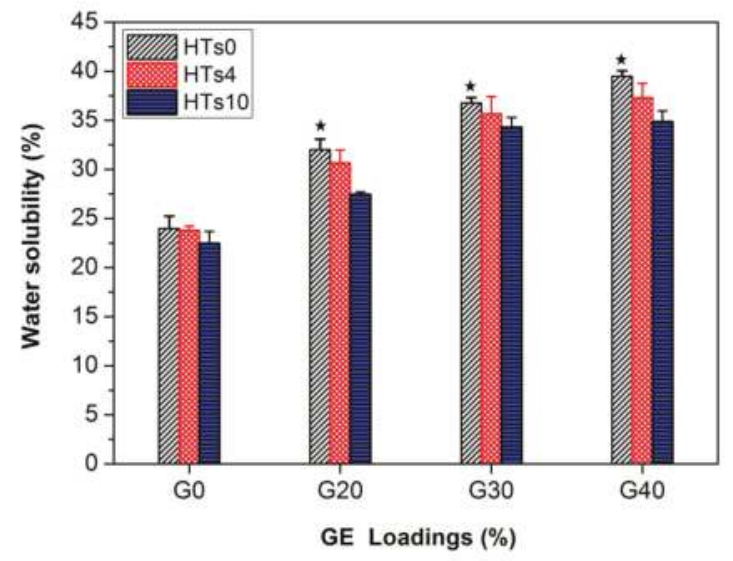

Figure 9. Water solubility of FG/GE/HT composites with various HT and GE loadings. * denotes significant difference between FG films containing different percentages of GE $(p<0.05)$.

Another property of the films is their surface hydrophobicity, which was evaluated through WCA determination. The WCA of the pure FG and composites are shown in Figure 10. The WCA of pure FG film was approximately 87.07, and it increased with the increase of GE content in the composites. GE molecules can be inserted into the FG molecular chains, resulting in the formation of a more compact structure compared with a pure FG film. The compact structure of FG/GE composites blocked the diffusion of large amounts of water molecules into the FG matrix when the water was dropped onto the surface of the films. Thereby, the WCA of FG/GE composites increases with GE addition. When considering a certain GE loading, an increase in HTs content clearly brought an increase in WCA values of the FG/GE composites. This phenomenon can be attributed to the existence of hydrogen bonds among FG, GE and HTs, which also blocked the diffusion of large amounts of water molecules into the FG/GE films when the water was dropped onto the surface of composites.

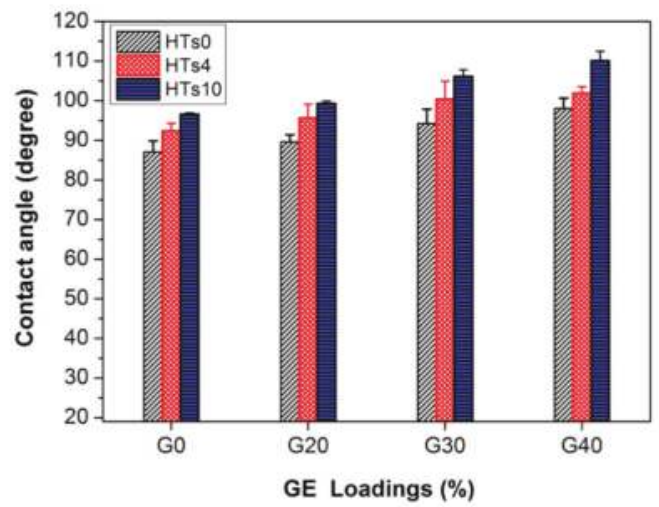

Figure 10. Contact angles of FG/GE/HT composites with various HT and GE loadings. 


\subsection{Optical Properties}

Transparency of packaging film is relevant to their functionality owing to their great impact on the appearance of the products. The light transmittance of the FG/GE films as a function of wavelength is presented in Figure 11a. It is observed that all the FG/GE composites films have high light transmission of above $80 \%$ in the visible region of $400-800 \mathrm{~nm}$, which demonstrates that there is a great consistency between FG and GE, and the FG and GE reach molecule-level dispersion within the composites. Similar phenomena have been reported by researchers in kappa-carrageenan, plasticized with glycerol composite films [44].

On the other hand, light transmittance of FG/GE films showed a general decrease with increasing HTs loading (Figure 11b). Incorporation of HTs induces a decrease in the transparency of films based on FG/GE, owing to changes in light diffraction generated by the dispersed HTs. Film transparency decreases were dependent on the amount of HTs incorporated. It can be seen that the transmittance of FG/GE30 film decreased from $86 \%$ to $80 \%$ when $10 \%$ of HTs was added. Sothornvit et al. reported that transmittance of the PLA film decreased when HTs was added [45]. In spite of decreasing the transparency of films by the HTs, all films containing HTs showed above $75 \%$ transparency across the visible light regions (400-800 nm), indicating that the light transmittance of the resulting nanocomposites films was slightly affected by the HTs owing to their nanometer size and well-dispersed in the composites.
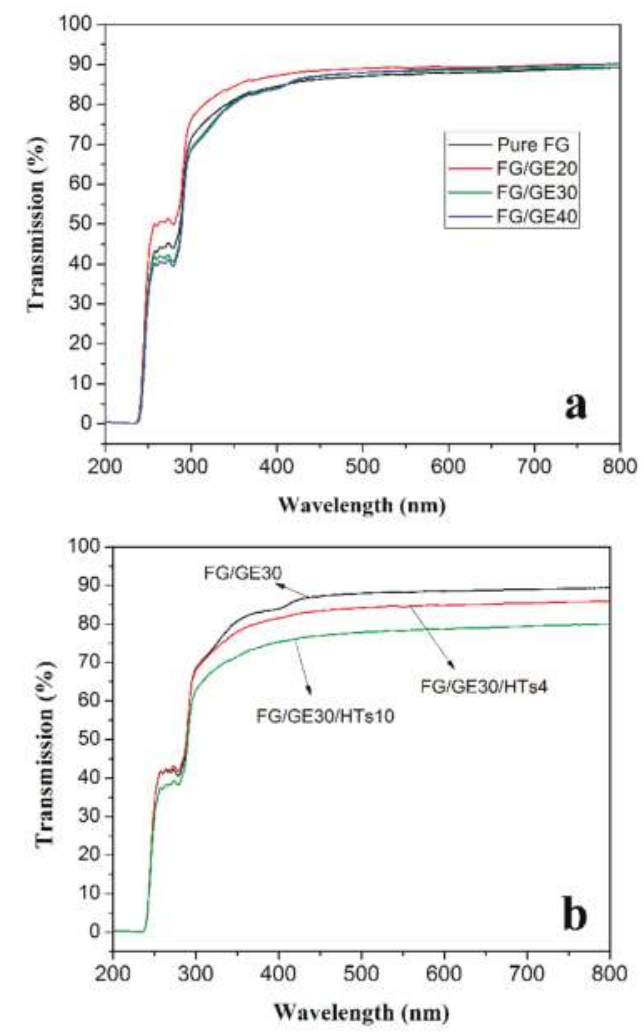

Figure 11. Optical properties of FG/HTs containing 0, 4 and $10 \mathrm{wt} \%$ (a) and FG/FG30/HTs containing 0,4 and 10 wt $\%$ (b). 


\section{Conclusions}

In summary, a series of FG-based composite films at different HTs loadings with and without GE as plasticizer, were prepared using the solution-casting method. Results indicated that the addition of GE greatly improves film flexibility with a decrease in the TS of film. Moisture uptake and water solubility were also improved by the addition of GE into the FG matrix, indicating that the water-resistance of film was decreased by the GE added. The addition of GE into FG matrix had no negative effect on the transparency of films. In addition, the incorporation of HTs into the FG/GE composites could improve the TS and water-resistance of films simultaneously, due to the formation of hydrogen-bonding networks in the composites. Moreover, the presence of GE enhances the dispersion of HTs in the FG matrix and thus enhanced the properties of obtained composite films.

Author Contributions: X.Q. and D.H. conceived and designed the experiments; S.Z. performed the experiments; X.Q. and S.Z. analyzed the data; Z.Z. and Q.Q. contributed reagents/materials/analysis tools; X.Q. wrote the paper.

Funding: This research was funded by [the Science and Technology Support Project of National Natural Science Foundation of Gansu Province] grant number [No. 1506RJZA068].

Conflicts of Interest: The authors declare no conflicts of interest.

\section{References}

1. Vieira, M.G.A.; Da Silva, M.A.; Dos Santos, L.O.; Beppu, M.M. Beppu. Natural-based plasticizers and biopolymer films: A review. Eur. Polym. J. 2011, 47, 254-263. [CrossRef]

2. Nilsuwan, K.; Benjakul, S.; Prodpran, T. Properties and antioxidative activity of fish gelatin-based film incorporated with epigallocatechin gallate. Food. Hydrocoll. 2018, 80, 212-221. [CrossRef]

3. Escárcega-Galaz, A.A.; Sánchez-Machado, D.I.; López-Cervantes, J.; Sanches-Silva, A.; Madera-Santana, T.J.; Paseiro-Losada, P. Paseiro-Losada. Mechanical, structural and physical aspects of chitosan-based films as antimicrobial dressings. Int. J. Biol. Macromol. 2018, 116, 472-481. [CrossRef] [PubMed]

4. Huang, D.; Ma, Z.; Zhang, Z.; Quan, Q. Reducing water sensitivity of chitosan biocomposite films using gliadin particles made by in situ method. Polymers 2017, 9, 583. [CrossRef]

5. Qiao, C.; Ma, X.; Zhang, J.; Yao, J. Molecular interactions in gelatin/chitosan composite films. Food Chem. 2017, 235, 45-50. [CrossRef] [PubMed]

6. Moreno, O.; Cárdenas, J.; Atarés, L.; Chiralt, A. Chiralt. Influence of starch oxidation on the functionality of starch-gelatin based active films. Carbohyd. Polym. 2017, 178, 147-158. [CrossRef] [PubMed]

7. Pérez Córdoba, L.J.; Sobral, P.J.A. Physical and antioxidant properties of films based on gelatin, gelatin-chitosan or gelatin-sodium caseinate blends loaded with nanoemulsified active compounds. J. Food Eng. 2017, 213, 47-53. [CrossRef]

8. Taokaew, S.; Seetabhawang, S.; Siripong, P.; Phisalaphong, M. Biosynthesis and Characterization of Nanocellulose-Gelatin Films. Materials 2013, 6, 782-794. [CrossRef] [PubMed]

9. Park, S.; Seo, S.; Lee, H.; Na, H.; Lee, J.; Woo, H.; Son, T. Preparation of furfuryl-fish gelatin (F-f.gel) cured using visible-light and its application as an anti-adhesion agent. Macromol. Res. 2012, 20, 842-846. [CrossRef]

10. Rouhi, J.; Mahmud, S.; Naderi, N.; Raymond Ooi, C.H.; Mahmood, M.R. Mahmood. Physical properties of fish gelatin-based bio-nanocomposite films incorporated with $\mathrm{ZnO}$ nanorods. Nanoscale Res. Lett. 2013, 8, 1-6. [CrossRef] [PubMed]

11. Chiou, B.; Avena-Bustillos, R.J.; Bechtel, P.J.; Imam, S.H.; Glenn, G.M.; Orts, W.J. Effects of drying temperature on barrier and mechanical properties of cold-water fish gelatin films. J. Food Eng. 2009, 95, 327-331. [CrossRef]

12. Wang, W.; Liu, Y.; Jia, H.; Liu, Y.; Zhang, H.; He, Z.; Ni, Y. Effects of Cellulose Nanofibers Filling and Palmitic Acid Emulsions Coating on the Physical Properties of Fish Gelatin Films. Food Biophys. 2017, 12, $23-32$. [CrossRef]

13. Zadeh, E.M.; Yu, A.; Fu, L.; Dehghan, M.; Sbarski, I.; Harding, I. Harding. Physical and thermal characterization of graphene oxide modified gelatin-based thin films. Polym. Compos. 2014, 35, 2043-2049. [CrossRef]

14. Soradech, S.; Limatvapirat, S.; Luangtana-anan, M. Stability enhancement of shellac by formation of composite film: Effect of gelatin and plasticizers. J. Food Eng. 2013, 116, 572-580. [CrossRef] 
15. Martucci, J.F.; Accareddu, A.E.M.; Ruseckaite, R.A. Preparation and characterization of plasticized gelatin films cross-linked with low concentrations of Glutaraldehyde. J. Mater. Sci. 2012, 47, 3282-3292. [CrossRef]

16. Andreuccetti, C.; Carvalho, R.A.; Grosso, C.R.F. Effect of hydrophobic plasticizers on functional properties of gelatin-based films. Food Res. Int. 2009, 42, 1113-1121. [CrossRef]

17. Hasanzati Rostami, A.; Motamedzadegan, A.; Hosseini, S.E.; Rezaei, M.; Kamali, A. Evaluation of Plasticizing and Antioxidant Properties of Silver Carp Protein Hydrolysates in Fish Gelatin Film. J. Aquat. Food Prod. Technol. 2017, 26, 457-467. [CrossRef]

18. Phetwarotai, W.; Potiyaraj, P.; Aht-Ong, D. Characteristics of biodegradable polylactide/gelatinized starch films: Effects of starch, plasticizer, and compatibilizer. J. Appl. Polym. Sci. 2012, 126, E162-E172. [CrossRef]

19. Staroszczyk, H.; Malinowska-Paczyk, E.; Gottfried, K.; Kolodziejska, I. Fish gelatin-nanoclay films. Part I: Effect of a kind of nanoclays and glycerol concentration on mechanical and water barrier properties of nanocomposites. J. Food Process. Preserv. 2017, 41. [CrossRef]

20. Rivero, S.; Garcia, M.A.; Pinotti, A. Correlations between structural, barrier, thermal and mechanical properties of plasticized gelatin films. Innov. Food Sci. Emerg. Technol. 2010, 11, 369-375. [CrossRef]

21. Farahnaky, A.; Dadfar, S.M.M.; Shahbazi, M. Physical and mechanical properties of gelatin-clay nanocomposite. J. Food Eng. 2014, 122, 78-83. [CrossRef]

22. Shankar, S.; Teng, X.; Li, G.; Rhim, J. Preparation, characterization, and antimicrobial activity of gelatin/ZnO nanocomposite films. Food Hydrocoll. 2015, 45, 264-271. [CrossRef]

23. Li, X.; Liu, A.; Ye, R.; Wang, Y.; Wang, W. Fabrication of gelatin-laponite composite films: Effect of the concentration of laponite on physical properties and the freshness of meat during storage. Food Hydrocoll. 2015, 44, 390-398. [CrossRef]

24. Wu, W.; Cao, X.; Luo, J.; He, G.; Zhang, Y. Morphology, thermal, and mechanical properties of poly(butylene succinate) reinforced with halloysite nanotube. Polym. Compos. 2014, 35, 847-855. [CrossRef]

25. Yuan, P.; Tan, D.; Annabi-Bergaya, F. Properties and applications of halloysite nanotubes: Recent research advances and future prospects. Appl. Clay Sci. 2015, 112-113, 75-93. [CrossRef]

26. Zhong, B.; Wang, S.; Dong, H.; Luo, Y.; Jia, Z.; Zhou, X.; Chen, M.; Xie, D.; Jia, D. Halloysite Tubes as Nanocontainers for Herbicide and Its Controlled Release in Biodegradable Poly(vinyl alcohol)/Starch Film. J. Agric. Food Chem. 2017, 65, 10445-10451. [CrossRef] [PubMed]

27. Liu, Y.; Cai, Q.; Li, H.; Zhang, J. Fabrication and characterization of mesoporous carbon nanosheets using halloysite nanotubes and polypyrrole via a template-like method. J. Appl. Polym. Sci. 2013, 128, 517-522. [CrossRef]

28. Massaro, M.; Colletti, C.G.; Lazzara, G.; Guernelli, S.; Noto, R.; Riela, S. Synthesis and Characterization of Halloysite-Cyclodextrin Nanosponges for Enhanced Dyes Adsorption. ACS Sustain. Chem. Eng. 2017, 5, 3346-3352. [CrossRef]

29. He, Y.; Kong, W.; Wang, W.; Liu, T.; Liu, Y.; Gong, Q.; Gao, J. Modified natural halloysite/potato starch composite films. Carbohyd. Polym. 2012, 87, 2706-2711. [CrossRef]

30. Soheilmoghaddam, M.; Wahit, M.U.; Mahmoudian, S.; Hanid, N.A. Regenerated cellulose/halloysite nanotube nanocomposite films prepared with an ionic liquid. Mater. Chem. Phys. 2013, 141, 936-943. [CrossRef]

31. Liu, M.; Zhang, Y.; Wu, C.; Xiong, S.; Zhou, C. Chitosan/halloysite nanotubes bionanocomposites: Structure, mechanical properties and biocompatibility. Int. J. Biol. Macromol. 2012, 51, 566-575. [CrossRef] [PubMed]

32. Tian, J.; Xu, L.; Wu, H.; Fang, S.; Deng, W.; Peng, T.; Sun, W.; Hu, Y. A novel approach for flotation recovery of spodumene, mica and feldspar from a lithium pegmatite ore. J. Clean. Prod. 2018, 174, 625-633. [CrossRef]

33. Tunç, S.; Duman, O. Duman. Preparation and characterization of biodegradable methyl cellulose/montmorillonite nanocomposite films. Appl. Clay Sci. 2010, 48, 414-424. [CrossRef]

34. Tessema, A.; Zhao, D.; Moll, J.; Xu, S.; Yang, R.; Li, C.; Kumar, S.K.; Kidane, A. Effect of filler loading, geometry, dispersion and temperature on thermal conductivity of polymer nanocomposites. Polym. Test. 2017, 57, 101-106. [CrossRef]

35. Huang, D.; Wang, W.; Xu, J.; Wang, A. Mechanical and water resistance properties of chitosan/poly(vinyl alcohol) films reinforced with attapulgite dispersed by high-pressure homogenization. Chem. Eng. J. 2012, 210, 166-172. [CrossRef]

36. Lavorgna, M.; Piscitelli, F.; Mangiacapra, P.; Buonocore, G.G. Study of the combined effect of both clay and glycerol plasticizer on the properties of chitosan films. Carbohyd. Polym. 2010, 82, 291-298. [CrossRef] 
37. Huang, D.; Wang, W.; Kang, Y.; Wang, A. chitosan/poly(vinyl alcohol) nanocomposite film reinforced with natural halloysite nanotubes. Polym. Compos. 2012, 33, 1693-1699. [CrossRef]

38. Martucci, J.F.; Ruseckaite, R.A. Biodegradable bovine gelatin/Na+-montmorillonite nanocomposite films. structure, barrier and dynamic mechanical properties. Polym. Plast. Technol. Eng. 2010, 49, 581-588. [CrossRef]

39. Muyonga, J.H.; Cole, C.G.B.; Duodu, K.G. Fourier transform infrared (FTIR) spectroscopic study of acid soluble collagen and gelatin from skins and bones of young and adult Nile perch (Lates niloticus). Food Chem. 2004, 86, 325-332. [CrossRef]

40. Hoque, M.S.; Benjakul, S.; Prodpran, T.; Songtipya, P. Properties of blend film based on cuttlefish (Sepia pharaonis) skin gelatin and mungbean protein isolate. Int. J. Biol. Macromol. 2011, 49, 663-673. [CrossRef] [PubMed]

41. Vejdan, A.; Ojagh, S.M.; Adeli, A.; Abdollahi, M. Effect of TiO2 nanoparticles on the physico-mechanical and ultraviolet light barrier properties of fish gelatin/agar bilayer film. LWT-Food Sci. Technol. 2016, 71, 88-95. [CrossRef]

42. Muscat, D.; Adhikari, B.; Adhikari, R.; Chaudhary, D.S. Chaudhary. Comparative study of film forming behaviour of low and high amylose starches using glycerol and xylitol as plasticizers. J. Food Eng. 2012, 109, 189-201. [CrossRef]

43. Li, R.; Liu, C.; Ma, J. Studies on the properties of graphene oxide-reinforced starch biocomposites. Carbohyd. Polym. 2011, 84, 631-637. [CrossRef]

44. Kozlowska, J.; Pauter, K.; Sionkowska, A. Carrageenan-based hydrogels: Effect of sorbitol and glycerin on the stability, swelling and mechanical properties. Polym. Test. 2018, 67, 7-11. [CrossRef]

45. Sothornvit, R.; Rhim, J.; Hong, S. Effect of nano-clay type on the physical and antimicrobial properties of whey protein isolate/clay composite films. J. Food Eng. 2009, 91, 468-473. [CrossRef]

(c) 2018 by the authors. Licensee MDPI, Basel, Switzerland. This article is an open access article distributed under the terms and conditions of the Creative Commons Attribution (CC BY) license (http:/ / creativecommons.org/licenses/by/4.0/). 


\title{
Article \\ Cellulose Nanofibrils and Tubular Halloysite as Enhanced Strength Gelation Agents
}

\author{
Vladimir Vinokurov ${ }^{1}$, Andrei Novikov ${ }^{1, *}$, Valentina Rodnova ${ }^{1,2}$, Boris Anikushin ${ }^{1}$, \\ Mikhail Kotelev ${ }^{1}$, Evgenii Ivanov ${ }^{1}$ and Yuri Lvov ${ }^{1,3, *}$ \\ 1 Functional Aluminosilicate Nanomaterials Lab, Gubkin University, 119991 Moscow, Russia; \\ vinok_ac@mail.ru (V.V.); valentinadinges@rambler.ru (V.R.); anikushin.b@gmail.com (B.A.); \\ kain@inbox.ru (M.K.); ivanov166@list.ru (E.I.) \\ 2 NPK Spetsburmaterialy, Zhukovskiy, 140131 Moscow, Russia \\ 3 Institute for Micromanufacturing, Louisiana Tech University, Ruston, LA 71272, USA \\ * Correspondence: novikov.a@gubkin.ru (A.N.); ylvov@latech.edu (Y.L.); Tel.: +7-499-5078692 (A.N.)
}

Received: 1 May 2019; Accepted: 22 May 2019; Published: 24 May 2019

\begin{abstract}
Silica gels are widely employed in water shutoff services, making them an essential tool in oil well management. Silica nanoparticles may serve as a strengthening additive for polymer hydrogels. In this study, we look at this statement from a different angle: What additives could be used to increase the strength of silica gels? Colloidal silica gels were prepared with various additives, and gel strength was measured by a Veiler-Rebinder apparatus. We found that cellulose nanofibrils considerably increase the gel strength (from $20-25$ to $35-40 \mathrm{kPa}$ ), which is comparable with the industrial anionic polymer Praestol 2540. Cellulose nanofibrils can be produced from cheap industrial-grade cellulose with low-cost industrial chemicals and could be partially replaced by the even less expensive halloysite nanoclay. Cellulose nanofibrils produced from renewable sources and naturally occurring halloysite nanoclay could be used as complementary reinforcing agents.
\end{abstract}

Keywords: gelation kinetics; sol-gel transition; water shutoff; silica sol; cellulose nanofibrils; halloysite nanotubes

\section{Introduction}

Silica gel formation from silica nanoparticles is widely employed in water shutoff services in oil wells $[1,2]$ and was recently proposed to be used in geothermal applications [3].

Silica nanoparticles have been employed as filler in polyacrylamide-based compositions in enhanced oil recovery [4-6]. a converse proposition arises naturally: What can be used to improve the properties of concentrated silica gels formed from silica nanoparticles?

Cellulose nanofibrils (CNFs) are promising materials actively studied and available as water-dispersible powders, films, or hydrogels [7]. They can be produced from a variety of renewable plant sources, including kraft bleached eucalyptus pulp [8], corn stalks [9], and algae [10]. Research into the application of CNFs as reinforcing additives has covered such areas as the paper and cardboard industry, production of polymer nanocomposites [11], development of composites with tunable mechanical properties [12], and preparation of thermo- and $\mathrm{pH}$-sensitive hydrogels [13].

Halloysite nanotubes are widely employed as reinforcing agents in plastic composites [14-17], catalyst carriers [18,19], and biomedical tissue scaffolds [20-22]. The most exciting feature of the halloysite is its double-charged surface, which enables various strategies for the surface modification and loading of halloysite nanotubes for compatibility with hydrophilic or hydrophobic and positively and negatively charged matrices $[23,24]$ and for the encapsulation of functional agents for corrosion protection $[25,26]$, drug delivery $[27,28]$, and other applications. 
The formation of composite materials from cellulose nanofibrils and halloysite nanoclay was recently reported [29], highlighting the possibilities of their co-use as reinforcing agents in composite materials and gels.

The present study was aimed at the possible usage of cellulose nanofibrils and halloysite nanotubes as reinforcing additives for silica sol-based gelation compositions. We found that cellulose nanofibrils provide gel strengthening comparable with that of the synthetic polymer Praestol 2540, and could be partially substituted with halloysite, which would still provide high gel strength.

\section{Materials and Methods}

\subsection{Materials}

Silica sol Polygel ASM K3 was purchased from JSC Polycell (Vladimir, Russia). Praestol 2540 poly(acrylamide) polymer (Ashland Deutschland GmbH, Krefeld, Germany), sodium citrate dihydrate, sulfuric acid, and hydrogen peroxide were purchased from a local dealer Chimmed (Moscow, Russia). Industrial-grade cellulose was kindly provided by Solikamskbumprom (Solikamsk, Perm Krai, Russia). Halloysite nanoclay was purchased from Sigma-Aldrich (product number 685445, St. Louis, MO, USA).

\subsection{Preparation of Cellulose Nanofibrils}

Cellulose nanofibrils were prepared from the industrial-grade cellulose in the following manner: Industrial-grade cellulose was dispersed in distilled water with homogenizer Bosch MSM66110 (Bosch, China), vacuum-filtered, and placed into a tightly closed desiccator overnight to ensure even water content. The cellulose pulp was then sampled for water content determination and subjected to acidic-oxidative treatment as described in [30], with slight modifications. Briefly, concentrated sulfuric acid was added to the $2 \%$ cellulose pulp dispersion in filtered tap water (1.6 kg dry weight of cellulose) to $8 \mathrm{~mol} / \mathrm{kg}$ molality and was held at $55^{\circ} \mathrm{C}$ and $90 \mathrm{rpm}$ for $4 \mathrm{~h}$ in a stirred tank reactor. The treated pulp was then filtered, washed with distilled water until the filtrate $\mathrm{pH}$ was higher than 6.0, and was then redispersed in water to 1.5 mass $\%$ by ultrasound treatment with a flow-through sonifier UIP1000hd (Hielscher Ultrasonics Gmbh, Berlin, Germany) and was then wet milled with a Supermasscolloider MKCA6-5J (Masuko Sangyo Co., Kawaguchi, Japan). The prepared cellulose nanofibrils dispersion was then centrifuged and dried to 20 mass\% cellulose content. Preparative yield of cellulose nanofibrils was $88.7 \%$.

\subsection{Characterization of the Employed Materials}

For the ash content determination, dried cellulose nanofibril samples in porcelain crucibles were calcined in a Nabertherm L 15/13 furnace at $525^{\circ} \mathrm{C}$ according to the TAPPI (Technical Association of the Pulp and Paper Industry) method 211-om 93 [31].

Electron micrographs were acquired with a JEM-2100 transmission electron microscope (Jeol, Japan) and a JIB-4501 scanning electron microscope (Jeol, Japan). The micrographs were analyzed with an ImageJ program suite (not less than 700 particles were measured for each sample). Samples for TEM were prepared by drop-casting the appropriately diluted dispersions onto formvar grids (Ted Pella, Redding, CA, USA). For SEM imaging, the specimens were either drop-cast (as dispersions) onto the aluminum stub or frozen with liquid nitrogen (as solidified gels), broken into pieces, and mounted onto the aluminum stub with carbon tape. All SEM specimens were then sputtered with gold (approx. 5-nm thickness) to ensure their good surface conductivity, using a JFC-1600 magnetron sputtering device (Jeol, Tokyo, Japan).

Zeta potential was measured with an SZ-100 particle size analyzer (Horiba, Kyoto, Japan) by the electrophoretic light scattering method. 


\subsection{Preparation of Reinforced Silica Gels}

Reinforced silica gels were prepared using Polygel ASM K3 industrial-grade silica sol, which has a density of $1.180-1.220 \mathrm{~g} / \mathrm{cm}^{3}$, pH of 8.5-9.5, $\mathrm{SiO}_{2}$ content of $25.0-32.0$ mass $\%$, particle size of 9-11 nm, BET (Brunauer-Emmett-Teller) surface area of $250-300 \mathrm{~m}^{2} / \mathrm{g}$, and viscosity of $10 \mathrm{mPa} \cdot \mathrm{s}$.

Silica sols were mixed with the additives (anionic polymers, halloysite, and cellulose nanofibrils) and the gelation activator (2.4 mass\% sodium citrate) using mechanical stirring and ultrasound treatment in a VBS-3DS ultrasonic bath (Vilitek, Moscow, Russia) for $30 \mathrm{~min}$. Portions of the gelation mixture were then placed in vessels in preparation for the gel strength measurements.

\subsection{Gel Strength Measurements}

After the gel portions had solidified in the vessels, with the special perforated plate immersed, gel strength measurements were performed at certain time intervals. The scheme of the employed apparatus is shown in Appendix A (Figure A1). Gel strength was measured by the Veiler-Rebinder method based on the extraction of the patterned or perforated plate from the gel [32], which is more suitable for the modeling of shear stress of gels in oil well conditions than the routinely employed method of inserting a probe into the gel [33]. Time intervals were counted from the moment of the addition of the gelating activator (sodium citrate).

\section{Results}

\subsection{Characterization of Cellulose Nanofibrils}

The prepared cellulose nanofibrils dispersion was a viscous white liquid with profound thixotropic properties. In several days, the dispersion formed a thick gel. After concentrating up to $20 \%$ cellulose dry weight, the cellulose nanofibrils became a wet white solid, resembling powdered cellulose. This solid mass could be redispersed with sonication into the initial gel-forming liquid.

Electron micrographs of the prepared cellulose nanofibrils are shown in Figure 1. As one can see in Figure 1, cellulose nanofibrils tend to agglomerate into fibers up to $10 \mu \mathrm{m}$ in diameter when dried at the substrate.

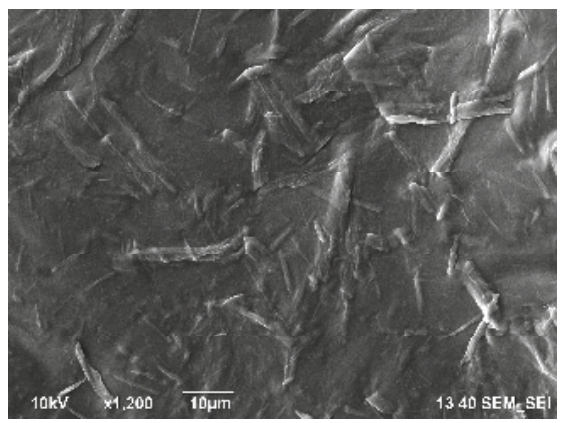

(a)

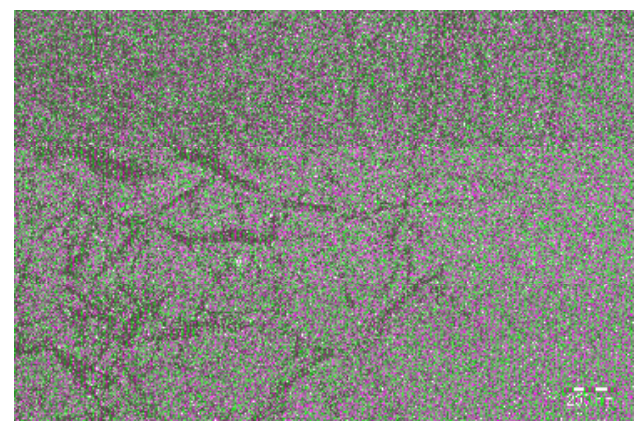

(b)

Figure 1. (a) SEM micrograph of cellulose nanofibrils. Scale bar, $10 \mu \mathrm{m}$; (b) TEM micrograph of cellulose nanofibrils. Scale bar, $200 \mathrm{~nm}$.

Analysis of the TEM micrographs of the cellulose nanofibrils showed that the diameter of cellulose nanofibrils was well described by lognormal distribution with the parameters $\mu=2.7287$ and $\sigma=0.4778$. The histogram and the fitted distribution are shown in Figure 2a. The length of the cellulose nanofibrils was hard to assess, because nanofibrils intertwine easily, even when deposited in a loose layer from the diluted dispersion, as shown in Figure $1 \mathrm{~b}$. While the smallest cellulose nanofibrils were around $200 \mathrm{~nm}$ in length, the bigger nanofibrils were longer than $1 \mu \mathrm{m}$. 
The electrophoretic mobility of the cellulose nanofibrils was $-5.6 \times 10^{-8} \mathrm{~m}^{2} \mathrm{~V}^{-1} \mathrm{~s}^{-1}$, which corresponded to the apparent mean zeta potential of $-69.7 \mathrm{mV}$. The reconstructed zeta potential distribution is shown in Figure 2b. Despite the inherent inaccuracy of the zeta potential reconstruction by the Smoluchowski equation (in the approximation of spherical particles), the estimated zeta potential value reflected the considerable negative charge of the cellulose nanofibrils. Indeed, the cellulose nanofibril dispersion was rather stable and showed only minor sedimentation after several weeks of storage, while gentle shaking restored the homogeneity of the dispersion.

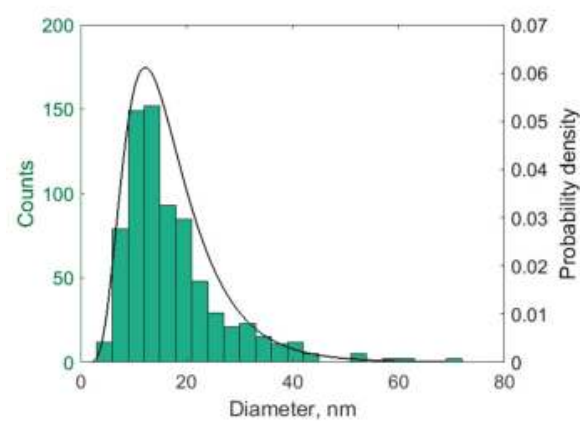

(a)

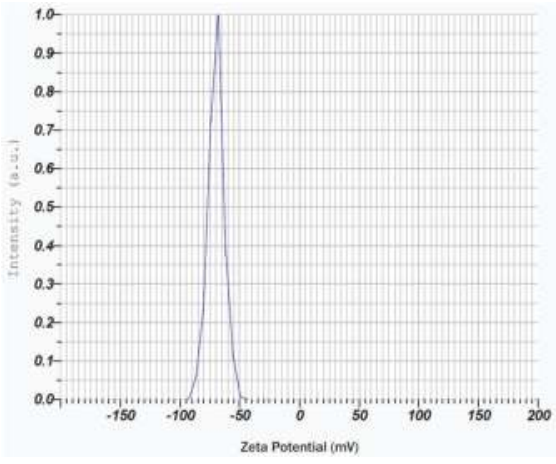

(b)

Figure 2. (a) Diameter distribution of cellulose nanofibrils; (b) Zeta potential distribution of cellulose nanofibrils.

\subsection{Characterization of Halloysite Nanotubes}

Halloysite nanotubes are a white powder, easily dispersed in water. Typically, their aqueous dispersion can be stable for up to $10 \mathrm{~min}$. It is worth noting that, in this study, after some halloysite precipitated, the liquid above the sediment still contained small fractions of halloysite. In the diluted dispersion, the halloysite nanotubes had electrophoretic mobility of $-3.1 \times 10^{-8} \mathrm{~m}^{2} \mathrm{~V}^{-1} \mathrm{~s}^{-1}$, which corresponded to the apparent mean zeta potential of $-40.0 \mathrm{mV}$.

Electron micrographs of the halloysite nanotubes are shown in Figure 3. As one can see in Figure 3, the halloysite nanotubes formed a dense layer when dried on a substrate, while the more dispersible fraction of halloysite aggregated in loose clusters of nanotubes, visible in the TEM micrograph (Figure 3b).

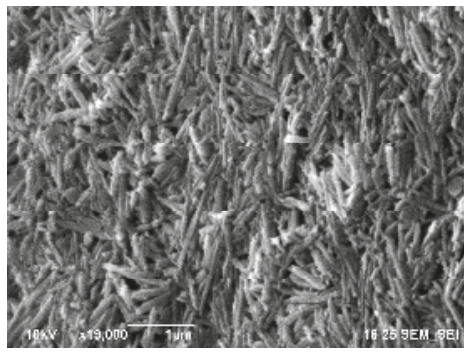

(a)

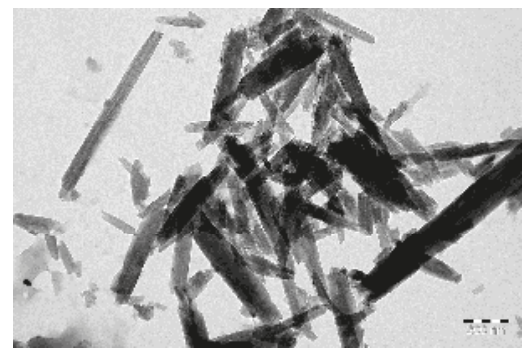

(b)

Figure 3. (a) SEM micrograph of halloysite nanotubes. Scale bar, $1 \mu \mathrm{m}$; (b) TEM micrograph of halloysite nanotubes. Scale bar, $200 \mathrm{~nm}$.

The fraction of halloysite nanotubes visualized by TEM micrographs was characterized by length and diameter distributions. Both length and diameter were well described by lognormal distribution 
with the parameters $\mu=3.8734$ and $\sigma=0.4390$ for diameter, and $\mu=5.3164$ and $\sigma=0.6910$ for length. The histograms and the fitted distributions are shown in Figure 4.

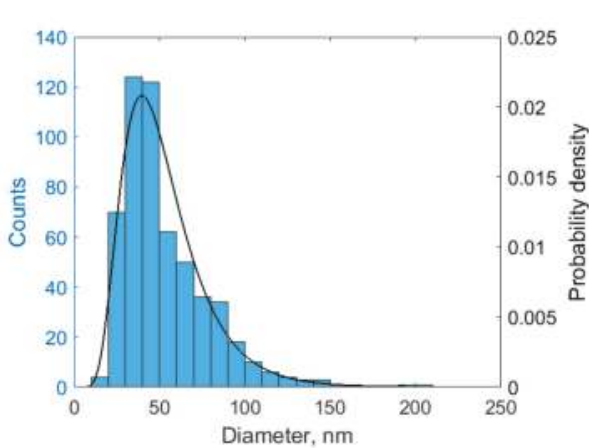

(a)

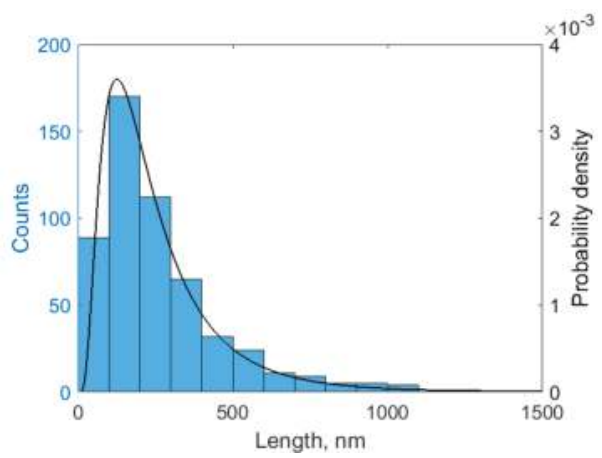

(b)

Figure 4. (a) Diameter distribution of halloysite nanotubes; (b) Length distribution of halloysite nanotubes.

Both the cellulose nanofibrils and halloysite nanotubes were much larger than the silica sol particles (7-9 nm; shown in Figure A2 in Appendix A) employed in the gelation compositions for the isolation squeeze in well servicing and could therefore be useful as reinforcement agents.

\subsection{Gelation Kinetics and Gel Strength}

It is known that the addition of anionic polymers to silica sol leads to the formation of reinforced gel after gelation. One example of a particularly successful reinforcement is the usage of Praestol 2540 poly(acrylamide)-based polymer. In practice, water shutoff compositions must have a low enough viscosity, so the maximum amount of polymer should not exceed 0.05 mass \%. Indeed, silica gel with added 0.05 mass \% of Praestol 2540 is stronger than silica gel without additives, both in the slow $(7.5 \mathrm{~h})$ and fast $(4 \mathrm{~h})$ gelation regimes, as is shown in Figure 5.

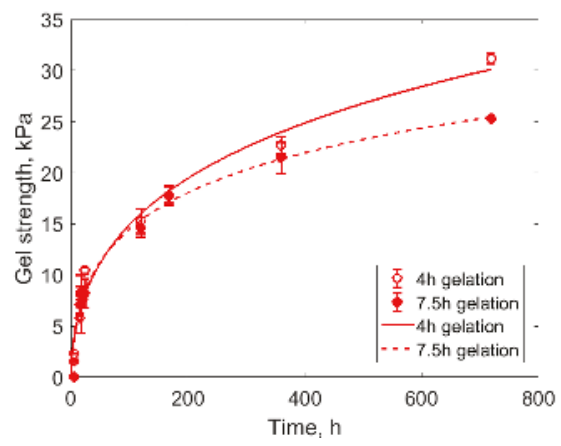

(a)

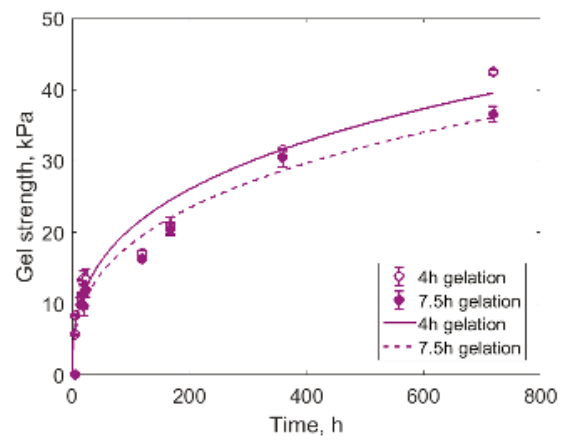

(b)

Figure 5. (a) Silica gelation without additives; (b) Silica gelation with $0.05 \%$ Praestol 2540 added. Gelation times were adjusted by sodium citrate dosage. Lines are Gompertz model fits.

Gelation kinetics are not readily described in terms of the simplest models (such as pseudo-first-order or pseudo-second-order kinetics) but can be approximated with a Gompertz-like model:

$$
s=a_{1} \cdot e^{-a_{2} e^{-a_{3} \log t}}
$$


where $s$ is gel strength, $t$ is time, and $a_{1}-a_{3}$ are fitted parameters. The model reflects the rapid onset of the gel strength after the gelation time and the slow growth of the gel strength during its ripening. In Figure 5, the lines are Equation (1) fits that approximate the experimental points. The fitted parameters for Equation (1) are listed in Table A1 in Appendix A.

The addition of cellulose nanofibrils and halloysite increased the gel strength, as shown in Figure 6a. One would expect a more profound strengthening effect from the halloysite nanotubes at 0.05 mass\% loading, because this value corresponded to the volume fraction of halloysite nanotubes of $0.024 \mathrm{vol} . \%$ or approximately 1.5 nanotubes $/ \mu^{3}$ (assuming the ideal dispersibility of halloysite, density of halloysite of $2.53 \mathrm{~g} / \mathrm{cm}^{3}$, density of silica sol of $1.20 \mathrm{~g} / \mathrm{cm}^{3}$, halloysite length of $126.3 \mathrm{~nm}$, and halloysite diameter of $39.7 \mathrm{~nm}$ ). Apparently, the halloysite was not uniformly distributed throughout the silica sol even after prolonged sonication.

Interestingly, the simultaneous addition of cellulose nanofibrils and halloysite increased the gel strength more than their separate addition, possibly reflecting the difference in the characteristic size of these additives. The influence of both additives was approximated as the quadratic model:

$$
s=a_{1} X+a_{2} X^{2}+a_{3} X Y+a_{4} Y+a_{5} Y^{2}
$$

where $s$ is gel strength at $240 \mathrm{~h}$ after gelation, $X$ is cellulose nanofibrils content (in mass $\%$ ), $Y$ is halloysite content (in mass\%), and $a_{1}-a_{5}$ are fitted parameters. As a result of fitting, the following model was obtained:

$$
s=19.426+833.30 X+9452.6 X^{2}-1985.1 X Y+173.58 Y-976.57 Y^{2},
$$

where the designations are as in Equation (2). The significance of the fitted parameters was checked by Student's statistics. For every parameter, the probabilities derived from the Student's statistics were less than 0.05 , so the model did not need refinement. The fitted model is shown in Figure $6 \mathrm{~b}$.

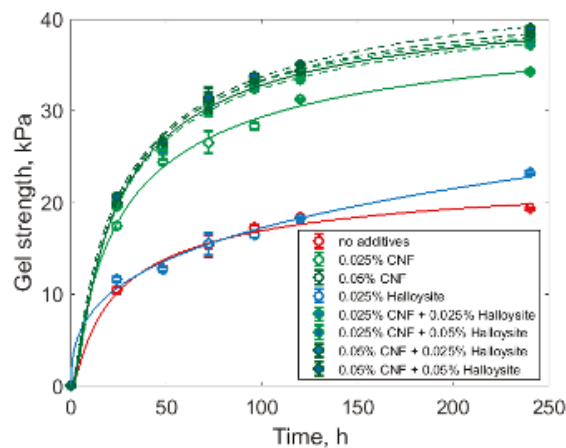

(a)

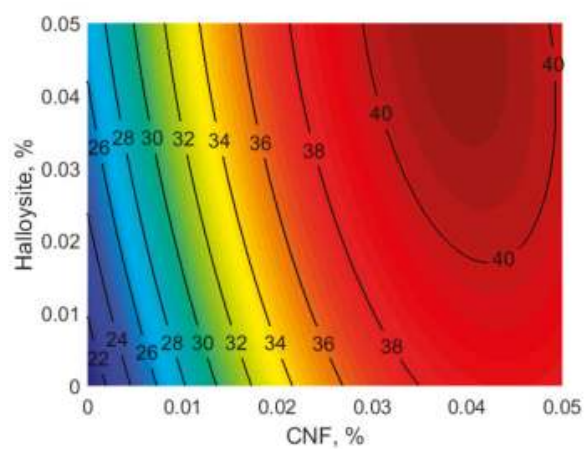

(b)

Figure 6. (a) Silica gelation with various additives. Lines are Gompertz model fits. (b) Gel strength at $240 \mathrm{~h}$ (showed by isolines, $\mathrm{kPa}$ ) as a function of cellulose nanofibrils and halloysite added. CNF: cellulose nanofibrils.

The addition of $\mathrm{CNF}$ and halloysite in various combinations increased the gel strength almost to the same level, around $35-40 \mathrm{kPa}$. Considering the practical limit of $0.05 \mathrm{mass} \%$ of the total additives, we can conclude that about 0.02 mass $\%$ of CNF may be replaced with much cheaper halloysite with the same gel strength (see also Figure A3 in Appendix A). In this regard, the best gelation mixture composition we identified was 0.03 mass\% CNF and 0.02 mass \% halloysite in the silica sol.

The reinforcement of the silica gel by the halloysite nanotubes is confirmed by the SEM images of the rapidly frozen and cleaved gel (see Figures 7 and A4 in Appendix A). 


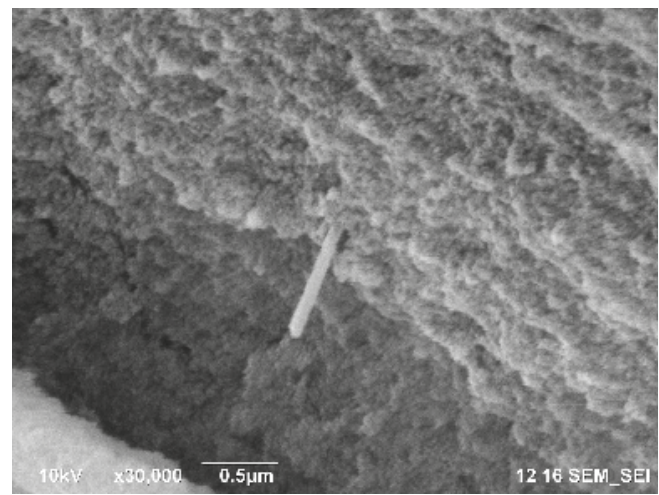

Figure 7. SEM micrograph of the silica gel with $0.05 \%$ halloysite cleaved in liquid nitrogen. The halloysite nanotube is visible in the center of the image. Scale bar, $500 \mathrm{~nm}$.

\section{Discussion}

In agreement with previous studies, sodium citrate induces the sol-gel transition in silica sols with the gelation time being controlled by the sodium citrate concentration [34]. Here, the most durable gels formed when the gelation time was rather short $(4 \mathrm{~h})$, but a further decrease in gelation time was not practical, considering the time necessary for the gelation mixture application in the oil well. Both with slow and short gelation, reinforced gels demonstrated higher strength; this increase in strength was detectable at short gel ripening times (around $240 \mathrm{~h}$ ).

The cellulose nanofibrils produced from the industrial-grade cellulose reinforced the silica gel almost as well as the synthetic polymer Praestol 2540. The halloysite nanotubes also increased the gel strength but not as much as CNF or Praestol 2540. Nevertheless, the combined reinforcement action of CNF and halloysite was comparable to that of the synthetic polymer Praestol 2540. At high CNF loading, halloysite additionally increased the gel strength, thus making possible at least partial replacement of CNF in the reinforcement additive composition.

The reinforcing effect of the studied additives may depend on the characteristic particle size; while Praestol 2540 molecules, which are comparable in size with fine silica sol particles and are known for the efficient removal of fine colloidal particles from water [35], CNF, and especially halloysite, are considerably larger. One would expect high reinforcing ability from the larger fillers, but this effect has been observed only in matrices that are rigid enough, such as in block polymers [36]. Smaller CNF have a higher affinity for the silica particle surface and thus demonstrate a higher reinforcing effect. The different scale of their action may explain the observed synergism of CNF and halloysite in silica gel strengthening. While large halloysite nanotubes provide the rigidity of the gel in the micrometer-size range, CNF bind the silica sol particles, forming intertwined elastic threads in the submicrometer range.

The complementary scales of CNF and halloysite action could be further investigated by the study of the synergism of gel strengthening additives of different sizes, e.g., the study of the combined anionic polymer/CNF/halloysite additive. The observed synergism in the case of a triple additive would confirm the hypothesis of the complementarity of differently sized additives.

Another possible explanation for the different strengthening abilities of the studied additives is their different zeta potential. Weakly charged halloysite nanotubes provide small reinforcing effect, whereas strongly charged CNF and anionic polymers increase the gel strength considerably. The gelation process could involve the formation of joint silica-additive bridge bonds with counter-ions (typically, alkali metal ions). Strongly charged additives could form these bridge bonds more efficiently, thus providing higher gel strength. To test this hypothesis, the study of the reinforcing ability of differently charged halloysite nanotubes is highly desirable. If the gel strengths were found to be dependent on the zeta potential of the halloysite nanotubes, it would confirm this hypothesis. 
The observed reinforcing effect of CNF and halloysite could be employed not only in the gelation compositions for oil well services but also in other industrial gelation products. Additional investigations could open up possibilities for the reinforcement of various materials by additives being hierarchically organized by size.

The economic effect of the observed results is rather promising, because, despite the relatively high cost of commercially available CNF, we can produce it from cheap industrial-grade cellulose with low-cost industrial chemicals (sulfuric acid and hydrogen peroxide). Furthermore, CNF could be partially replaced by even cheaper halloysite-a natural product available in thousands of tons at a reasonable price.

Author Contributions: Conceptualization, A.N. and V.R.; data curation, A.N. and V.R.; funding acquisition, V.V., E.I., and Y.L.; investigation, V.R., B.A., and M.K.; project administration, Y.L.; supervision, V.V. and E.I.; visualization, A.N. and M.K.; writing—original draft, A.N.; writing—review and editing, A.N. and Y.L.

Funding: This research was funded by the Ministry of Science and Higher Education of Russia, unique identifier RFMEFI57717X0265.

Acknowledgments: The authors are grateful to Solikamskbumprom (Solikamsk, Perm Krai, Russia) for the generous gift of the industrial-grade cellulose and also to Dmitry S. Kopitsyn (Gubkin University) for help with the zeta potential measurements.

Conflicts of Interest: The authors declare no conflicts of interest. The funders had no role in the design of the study; in the collection, analyses, or interpretation of the data; in the writing of the manuscript; or in the decision to publish the results.

\section{Appendix A.}

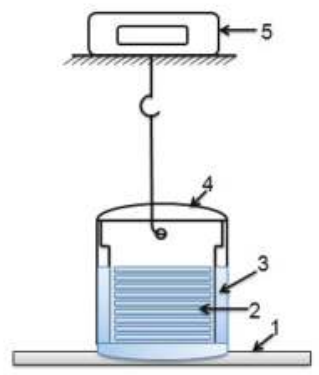

Figure A1. Scheme of the Veiler-Rebinder apparatus for the measurements of gel strength. 1, Z-stage; 2, perforated carbon steel plate; 3, gel; 4, vessel; 5 , analytical balance.

Table A1. The fitted parameters for Equation (1).

\begin{tabular}{|c|c|c|c|}
\hline Experimental conditions & $\mathbf{a}_{1}$ & $\mathbf{a}_{2}$ & $\mathbf{a}_{3}$ \\
\hline \multicolumn{4}{|c|}{ Figure 5} \\
\hline No additives; gelation time $4 \mathrm{~h}$ & $412.89^{1}$ & 5.7940 & 0.12076 \\
\hline No additives; gelation time $7.5 \mathrm{~h}$ & 101.80 & 4.3339 & 0.17328 \\
\hline $0.05 \%$ Praestol 2540 ; gelation time $4 \mathrm{~h}$ & 19395 & 8.6720 & 0.051108 \\
\hline $0.05 \%$ Praestol 2540; gelation time $7.5 \mathrm{~h}$ & 44238 & 9.6360 & 0.046180 \\
\hline \multicolumn{4}{|c|}{ Figure 6} \\
\hline No additives & 24.940 & 6.0196 & 0.59552 \\
\hline $0.025 \% \mathrm{CNF}^{2}$ & 43.437 & 5.7342 & 0.58211 \\
\hline $0.05 \% \mathrm{CNF}$ & 44.446 & 7.6086 & 0.69905 \\
\hline $0.025 \%$ Halloysite & 4322.1 & 7.2512 & 0.059066 \\
\hline $0.025 \% \mathrm{CNF}+0.025 \%$ Halloysite & 44.246 & 7.2674 & 0.68397 \\
\hline $0.025 \% \mathrm{CNF}+0.05 \%$ Halloysite & 43.803 & 8.6246 & 0.74702 \\
\hline $0.05 \%$ CNF $+0.025 \%$ Halloysite & 45.458 & 7.4676 & 0.69053 \\
\hline $0.05 \% \mathrm{CNF}+0.05 \%$ Halloysite & 48.352 & 6.0964 & 0.61393 \\
\hline
\end{tabular}

\footnotetext{
${ }^{1}$ Note that in contrast to the original Gompertz model, the parameter $a_{1}$ in Equation (1) is not necessarily the
} asymptotic value at $t \rightarrow \infty .{ }^{2} \mathrm{CNF}-$ cellulose nanofibrils 


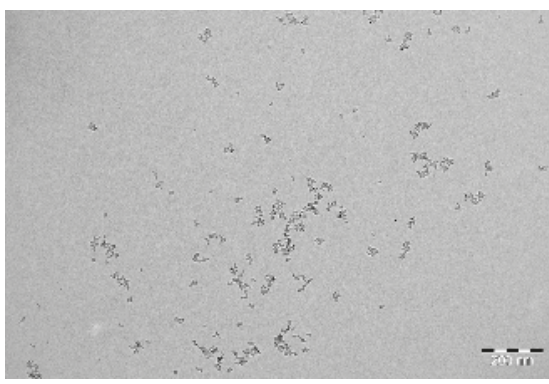

(a)

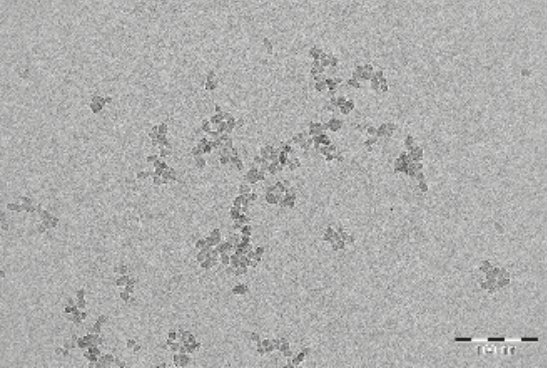

(b)

Figure A2. (a) TEM micrograph of the silica sol before gelation. Scale bar, $200 \mathrm{~nm}$. (b) TEM micrograph of the silica sol before gelation. Scale bar, $100 \mathrm{~nm}$.

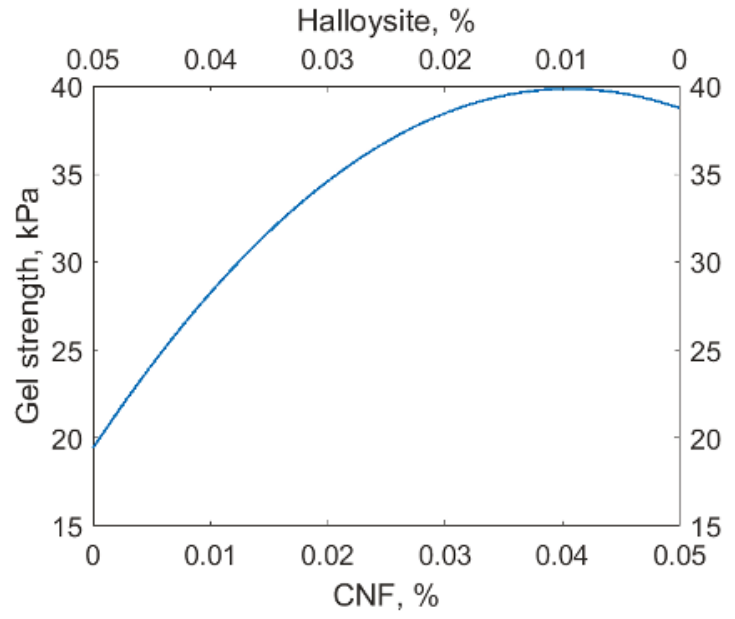

Figure A3. Modeled gel strength at $240 \mathrm{~h}$ for the total added amount of CNF and halloysite of 0.05 mass $\%$.

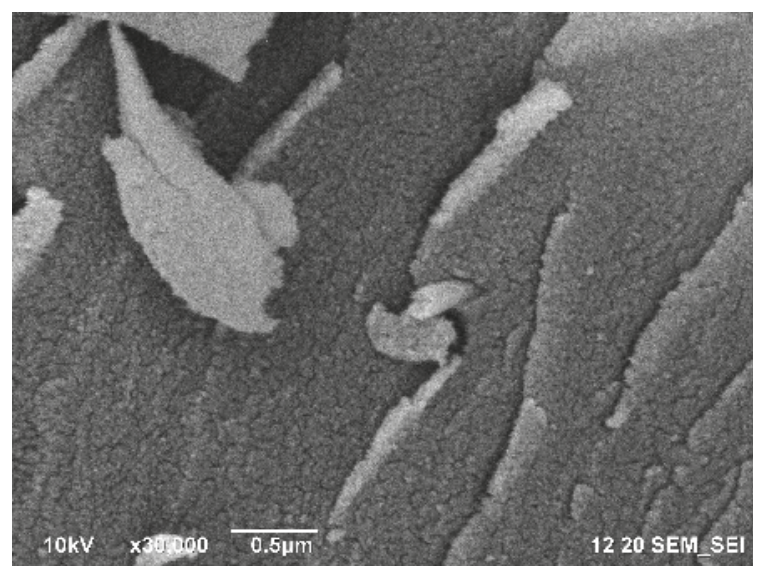

Figure A4. SEM micrograph of the silica gel with $0.05 \%$ halloysite cleaved in liquid nitrogen. The halloysite nanotube is visible in the center of the image. Scale bar, $500 \mathrm{~nm}$. 


\section{References}

1. Almohsin, A.; Huang, J.; Karadkar, P.; Bataweel, M. Nanosilica Based Fluid System for Water Shut-Off. In 22nd World Petroleum Congress, 9-13 July 2017, Istanbul, Turkey; World Petroleum Congress: Houston, TX, USA, 2017.

2. Huang, J.; Al-Mohsin, A.; Bataweel, M.; Karadkar, P.; Li, W.; Shaikh, A. Systematic Approach to Develop a Colloidal Silica Based Gel System for Water Shut-Off. In SPE Middle East Oil \& Gas Show and Conference, 6-9 March 2017, Manama, Kingdom of Bahrain; Society of Petroleum Engineers: Richardson, TX, USA, 2017.

3. Hunt, J.D.; Ezzedine, S.M.; Bourcier, W.; Roberts, S. Kinetics of the Gelation of Colloidal Silica at Geothermal Conditions, and Implications for Reservoir Modification and Management. In Stanford Geothermal Workshop, Palo Alto, California, USA, 11-13 February 2013; Lawrence Livermore National Lab (LLNL): Livermore, CA, USA, 2013.

4. Zhu, D.; Wei, L.; Wang, B.; Feng, Y. Aqueous Hybrids of Silica Nanoparticles and Hydrophobically Associating Hydrolyzed Polyacrylamide Used for EOR in High-Temperature and High-Salinity Reservoirs. Energies 2014, 7, 3858-3871. [CrossRef]

5. Liu, Y.; Dai, C.; Wang, K.; Zou, C.; Gao, M.; Fang, Y.; Zhao, M.; Wu, Y.; You, Q. Study on a Novel Cross-Linked Polymer Gel Strengthened with Silica Nanoparticles. Energy Fuels 2017, 31, 9152-9161. [CrossRef]

6. Asadizadeh, S.; Ayatollahi, S.; ZareNezhad, B. Performance Evaluation of a New Nanocomposite Polymer Gel for Water Shutoff in Petroleum Reservoirs. J. Dispers. Sci. Technol. 2018, 36, 1-9. [CrossRef]

7. Nechyporchuk, O.; Belgacem, M.N.; Bras, J. Production of Cellulose Nanofibrils: a Review of Recent Advances. Ind. Crop. Prod. 2016, 93, 2-25. [CrossRef]

8. Qin, Y.; Qiu, X.; Zhu, J.Y. Understanding Longitudinal Wood Fiber Ultra-Structure for Producing Cellulose Nanofibrils Using Disk Milling with Diluted Acid Prehydrolysis. Sci. Rep. 2016, 6, 35602. [CrossRef] [PubMed]

9. Boufi, S.; Chaker, A. Easy Production of Cellulose Nanofibrils from Corn Stalk by a Conventional High Speed Blender. Ind. Crop. Prod. 2016, 93, 39-47. [CrossRef]

10. Xiang, Z.; Gao, W.; Chen, L.; Lan, W.; Zhu, J.Y.; Runge, T. a Comparison of Cellulose Nanofibrils Produced from Cladophora Glomerata Algae and Bleached Eucalyptus Pulp. Cellulose 2016, 23, 493-503. [CrossRef]

11. Dufresne, A. Cellulose Nanomaterial Reinforced Polymer Nanocomposites. Curr. Opin. Colloid Interface Sci. 2017, 29, 1-8. [CrossRef]

12. Miao, X.; Tian, F.; Lin, J.; Li, H.; Li, X.; Bian, F.; Zhang, X. Tuning the Mechanical Properties of Cellulose Nanofibrils Reinforced Polyvinyl Alcohol Composites: Via Altering the Cellulose Polymorphs. RSC Adv. 2016, 6, 83356-83365. [CrossRef]

13. Lu, J.; Zhu, W.; Dai, L.; Si, C.; Ni, Y. Fabrication of Thermo-And pH-Sensitive Cellulose Nanofibrils-Reinforced Hydrogel with Biomass Nanoparticles. Carbohydr. Polym. 2019, 215, 289-295. [CrossRef] [PubMed]

14. Makaremi, M.; Pasbakhsh, P.; Cavallaro, G.; Lazzara, G.; Aw, Y.K.; Lee, S.M.; Milioto, S. Effect of Morphology and Size of Halloysite Nanotubes on Functional Pectin Bionanocomposites for Food Packaging Applications. ACS Appl. Mater. Interfaces 2017, 9, 17476-17488. [CrossRef]

15. Gaaz, T.S.; Sulong, A.B.; Akhtar, M.N.; Kadhum, A.A.H.; Mohamad, A.B.; Al-Amiery, A.A.; McPhee, D.J. Properties and Applications of Polyvinyl Alcohol, Halloysite Nanotubes and Their Nanocomposites. Molecules 2015, 20, 22833-22847. [CrossRef] [PubMed]

16. Jäger, M.; Zabihi, O.; Ahmadi, M.; Li, Q.; Depalmeanar, A.; Naebe, M. Nano-Enhanced Interface in Carbon Fibre Polymer Composite Using Halloysite Nanotubes. Compos. Part a Appl. Sci. Manuf. 2018, 109, 115-123. [CrossRef]

17. Shuttleworth, P.S.; Díez-Pascual, A.M.; Marco, C.; Ellis, G. Flexible Bionanocomposites from Epoxidized Hemp Seed Oil Thermosetting Resin Reinforced with Halloysite Nanotubes. J. Phys. Chem. B 2017, 121, 2454-2467. [CrossRef] [PubMed]

18. Massaro, M.; Colletti, C.G.; Lazzara, G.; Milioto, S.; Noto, R.; Riela, S. Halloysite Nanotubes as Support for Metal-Based Catalysts. J. Mater. Chem. A 2017, 5, 13276-13293. [CrossRef]

19. Glotov, A.; Levshakov, N.; Stavitskaya, A.; Artemova, M.; Gushchin, P.; Ivanov, E.; Vinokurov, V.; Lvov, Y. Templated Self-Assembly of Ordered Mesoporous Silica on Clay Nanotubes. Chem. Commun. 2019, 55, 5507-5510. [CrossRef]

20. Bonifacio, M.A.; Gentile, P.; Ferreira, A.M.; Cometa, S.; De Giglio, E. Insight into Halloysite Nanotubes-Loaded Gellan Gum Hydrogels for Soft Tissue Engineering Applications. Carbohydr. Polym. 2017, 163, $280-291$. [CrossRef] [PubMed] 
21. Santos, A.C.; Ferreira, C.; Veiga, F.; Ribeiro, A.J.; Panchal, A.; Lvov, Y.; Agarwal, A. Halloysite Clay Nanotubes for Life Sciences Applications: From Drug Encapsulation to Bioscaffold. Adv. Colloid Interface Sci. 2018, 257, 58-70. [CrossRef]

22. Liu, M.; Fakhrullin, R.; Novikov, A.; Panchal, A.; Lvov, Y. Tubule Nanoclay-Organic Heterostructures for Biomedical Applications. Macromol. Biosci. 2019, 19, 1800419. [CrossRef]

23. Cavallaro, G.; Lazzara, G.; Milioto, S.; Parisi, F. Halloysite Nanotubes for Cleaning, Consolidation and Protection. Chem. Rec. 2018, 18, 940-949. [CrossRef]

24. Yang, Y.; Chen, Y.; Leng, F.; Huang, L.; Wang, Z.; Tian, W. Recent Advances on Surface Modification of Halloysite Nanotubes for Multifunctional Applications. Appl. Sci. 2017, 7, 1215. [CrossRef]

25. Shchukina, E.; Grigoriev, D.; Sviridova, T.; Shchukin, D. Comparative Study of the Effect of Halloysite Nanocontainers on Autonomic Corrosion Protection of Polyepoxy Coatings on Steel by Salt-Spray Tests. Prog. Org. Coat. 2017, 108, 84-89. [CrossRef]

26. Shchukina, E.; Shchukin, D.; Grigoriev, D. Effect of Inhibitor-Loaded Halloysites and Mesoporous Silica Nanocontainers on Corrosion Protection of Powder Coatings. Prog. Org. Coat. 2017, 102, 60-65. [CrossRef]

27. Lvov, Y.M.; DeVilliers, M.M.; Fakhrullin, R.F. The Application of Halloysite Tubule Nanoclay in Drug Delivery. Expert Opin. Drug Deliv. 2016, 13, 977-986. [CrossRef]

28. Liu, M.; Chang, Y.; Yang, J.; You, Y.; He, R.; Chen, T.; Zhou, C. Functionalized Halloysite Nanotube by Chitosan Grafting for Drug Delivery of Curcumin to Achieve Enhanced Anticancer Efficacy. J. Mater. Chem. B 2016, 4, 2253-2263. [CrossRef]

29. Yong, C.; Mei, C.; Guan, M.; Wu, Q.; Han, J.; Sun, X. a Comparative Study of Different Nanoclay-Reinforced Cellulose Nanofibril Biocomposites with Enhanced Thermal and Mechanical Properties. Compos. Interfaces 2018, 25, 301-315. [CrossRef]

30. Novikov, A.A.; Anikushin, B.M.; Petrova, D.A.; Konstantinova, S.A.; Mel'nikov, V.B.; Vinokurov, V.A. Acid and Oxidative Treatment of Raw Material for the Production of Nanofibrillar Cellulose. Chem. Technol. Fuels Oils 2018, 54, 564-568. [CrossRef]

31. TAPPI $211 \mathrm{om}-93$. Ash in Wood, Pulp, Paper and Paperboard: Combustion at $525^{\circ} \mathrm{C}$; Committee of the Process and Product Quality Division: TAPPI, Atlanta, GA, USA, 1996.

32. Abubakarova, A.S.; Khadisova, Z.T.; Aleksandrova, E.A.; Krasavtsev, B.E. Study of Structural-Mechanical Properties of Paraffin-Containing Petroleum Products. Chem. Technol. Fuels Oils 2014, 50, 149-155. [CrossRef]

33. Ferrari, F.; Bertoni, M.; Caramella, C.; La Manna, A. Description and Validation of an Apparatus for Gel Strength Measurements. Int. J. Pharm. 1994, 109, 115-124. [CrossRef]

34. Rodnova, V.Y. Gel-Forming Compositions Based on the Alkaline Silica Gel for the Water Shutoff. Ph.D. Thesis, Gubkin University, Moscow, Russia, 20 September 2018.

35. Lee, C.S.; Robinson, J.; Chong, M.F. a Review on Application of Flocculants in Wastewater Treatment. Process Saf. Environ. Prot. 2014, 92, 489-508. [CrossRef]

36. Liu, M.; Jia, Z.; Jia, D.; Zhou, C. Recent Advance in Research on Halloysite Nanotubes-Polymer Nanocomposite. Prog. Polym. Sci. 2014, 39, 1498-1525. [CrossRef]

(C) 2019 by the authors. Licensee MDPI, Basel, Switzerland. This article is an open access article distributed under the terms and conditions of the Creative Commons Attribution (CC BY) license (http://creativecommons.org/licenses/by/4.0/). 
Article

\title{
Fuzzy Optimization on the Synthesis of Chitosan-Graft-Polyacrylic Acid with Montmorillonite as Filler Material: A Case Study
}

\author{
Angelo Earvin Sy Choi ${ }^{1,+}$, Cybelle Morales Futalan ${ }^{1,+}$ and Jurng-Jae Yee ${ }^{2, *}$ \\ 1 National Research Center for Disaster-Free and Safe Ocean City, Busan 49315, Republic of Korea; \\ angelochoi2003@yahoo.com (A.E.S.C.); cmfutalan@gmail.com (C.M.F.) \\ 2 Department of Architectural Engineering, Dong-A University, Busan 49315, Korea \\ * Correspondence: jjyee@dau.ac.kr; Tel.: +82-51-200-7609; Fax: +82-51-294-2256 \\ + Co-first authors.
}

Received: 8 April 2019; Accepted: 21 April 2019; Published: 23 April 2019

\begin{abstract}
In this paper, the synthesis of a chitosan-montmorillonite nanocomposite material grafted with acrylic acid is presented based on its function in a case study analysis. Fuzzy optimization is used for a multi-criteria decision analysis to determine the best desirable swelling capacity $\left(Y_{Q}\right)$ of the material synthesis at its lowest possible variable cost. For $Y_{Q}$, the integrating the result's cumulative uncertainty is an essential element to investigate the feasibility of the developed model equation. The Pareto set analysis is able to set the appropriate boundary limits for $Y_{Q}$ and the variable cost. Two case studies are presented in determining the lowest possible cost: Case 1 for maximum $Y_{Q}$, and Case 2 for minimum $Y_{Q}$. These boundary limits were used in the fuzzy optimization to determine its global optimum results that achieved the overall satisfaction ratings of $67.2 \%$ (Case 1) and $52.3 \%$ (Case 2). The synthesis of the polyacrylic acid/chitosan material for Case 1 resulted in $305 \mathrm{~g} / \mathrm{g} Y_{Q}$ and $10.8 \mathrm{USD} / \mathrm{kg}$, while Case 2 resulted in $97 \mathrm{~g} / \mathrm{g} Y_{Q}$ and $12.3 \mathrm{USD} / \mathrm{kg}$. Thus, the fuzzy optimization approach proves to be a practical method for examining the best possible compromise solution based on the desired function to adequately synthesize a material.
\end{abstract}

Keywords: ammonium persulfate; fuzzy optimization; $N, N^{\prime}$-methylenebisacrylamide; Pareto set; polyacrylic acid; swelling capacity; variable cost

\section{Introduction}

Superabsorbents are smart, functional materials composed of lightly crosslinked hydrophilic polymers with the capacity to hold a large quantity of aqueous and biological fluids even at extreme pressure [1]. It also has the capability to release the collected fluids under a dry environment [2]. Superabsorbents are characterized by their high swelling capacities where saline fluids and water are retained up to 100-1000 times their original dry weight without losing their structural framework and integrity [3]. The preparative methods for superabsorbents involve the attachment of various functional groups such as amide, amine, hydroxyl, sulfonic acid, and carboxylic acid onto the polymer network [4]. Excellent properties of superabsorbents include elasticity, hydrophilicity, high swelling rate, porosity, softness and biodegradability [5-7]. Superabsorbents are classified into three groups: synthetic (derived from petroleum products), natural, and hybrid (vinyl monomers grafted onto natural polymers) [8]. However, petroleum-based polymers have limited utilization due to poor degradability, costly to produce, and toxicity with detrimental impacts to human health and the environment $[9,10]$.

Natural polysaccharides including cashew gum, pectin, chitin, alginate, cellulose, starch, and chitosan $(\mathrm{Ch})$ have gained attention due to their renewability, abundance, biocompatibility, biodegradability, environment-friendliness, and inexpensiveness [11,12]. Ch, which is an amino 
polysaccharide of high molecular weight and crystalline structure, is produced via alkaline deacetylation of chitin that is derived from exoskeletons of krills, lobsters, shrimps, crabs and crawfish. It is composed of binary linear units of $(1 \rightarrow 4)$-2-amino-2-deoxy- $\beta$-D-glucan and $(1 \rightarrow 4)$-2-acetamido-2-deoxy- $\beta$-D-glucan $[1,13,14]$. There are numerous remarkable characteristics of Ch including low-cost, chelating capacity, availability, anti-microbial activity, biodegradability, low immunogenicity and being environmentally benign [15]. However, pure $\mathrm{Ch}$ is known for its low specific gravity and poor mechanical integrity [16]. Graft copolymerization of vinyl monomers, which contain hydrophilic side groups, onto the $\mathrm{Ch}$ backbone has been known to enhance the biodegradability, $\mathrm{pH}$ resistance, thermal stability, water absorptive capability, and salt tolerance as well as minimize the need for oil-based monomers $[1,17,18]$. Acrylic acid (AA) as vinyl monomer has been extensively studied due to its decreased cytotoxicity and improved biocompatibility [19]. However, composite materials derived from natural polymers grafted with vinyl monomers suffer due to their high manufacturing cost and reduced mechanical strength [20].

Recently, incorporation of inorganic fillers such as clay minerals has yielded organic/inorganic composites with improved stiffness and strength, lower cost of production, better handling, enhanced swelling ability, and satisfactory adsorption performance [13,20-23]. Montmorillonite (Mt), which is a member of the smectite group, is comprised of a 2:1 ratio of silica tetrahedron and alumina octahedral sheets [24]. The formula of $\mathrm{Mt}$ is $(\mathrm{Na}, \mathrm{Ca})_{0.33}\left(\mathrm{Al}, \mathrm{Mg}_{2}\left(\mathrm{Si}_{4} \mathrm{O}_{10}\right)(\mathrm{OH})_{2} \cdot n \mathrm{H}_{2} \mathrm{O}\right.$, where it is described as a soft layered silicate with high cation exchange capacity, small particle diameter, and excellent aspect ratio and plane strength $[25,26]$.

In the previous work of Abdel Aziz and Salama [27], Ch-graft-polyacrylic acid (ChPA) with Mt as filler material was prepared as a superabsorbent. The results showed the disappearance of $\mathrm{d}_{001}$ peak at $2 \theta=6.6^{\circ}$ after polymerization. This implies exfoliation of vinyl-modified Mt into individual nanolayers has occurred $[27,28]$. The maximum equilibrium swelling was determined via optimization study using response surface methodology (RSM) with Box-Behnken Design (BBD). The following optimal conditions were determined: $2.98 \mathrm{wt} \%$. initiator, $1.04 \mathrm{wt} \%$ crosslinker and $9.76 \mathrm{AA} / \mathrm{Ch}$ molar ratio [27]. RSM is comprised of statistical and numerical methods utilized in the optimization of multiple factor levels in material preparation and treatment technologies [29,30]. The relationship between process variables and their responses are evaluated where an empirical polynomial model is fitted [31]. RSM also help recognize which of the main variables and their interactions significantly affect the process. However, there are no studies reported on the fuzzy logic technique in the field of material synthesis for superabsorbents. In literature, there is no information that utilized the fuzzy logic system to investigate the cumulative uncertainty error and variable cost on the preparation of superabsorbent.

The fuzzy logic method is a precise modeling tool employed in the evaluation, mapping, modeling, and prediction in areas of environment and ecology [32]. Fuzzy logic allows adaptability of models with a degree of uncertainty near the boundary of unsuitable and suitable areas. The fuzzy system applies sigmoidal adjustment (Gaussian, linear or other method) to adequately define the system's behavior for criteria parameterization [33]. Fuzzy logic system is less likely to be influenced by the analyst's opinion where an acceptable model is defined by the minimum and maximum values of the variables [34].

The present work uses multi-objective optimization with the fuzzy logic concept in the selection of the optimum condition for the synthesis of Ch-Mt composites grafted with PA (ChPA-Mt). The global optimum solution that would yield the best swelling capacity of ChPA-Mt and provide the lowest possible variable cost was determined. The effect of AA/Ch molar ratio, ammonium persulphate as initiator and $N, N^{\prime}$-methylenebisacrylamide as crosslinker on the total variable cost and swelling capacity with respect to the synthesis of ChPA-Mt was investigated.

Using the fuzzy optimization approach, the selection of the ideal swelling capacity was dependent on the end-use (absorbent/adsorbent) of the material. Two cases were considered in the present work where the first case determined the appropriate condition to synthesize an absorbent that would yield 
the maximum swelling capacity for the least variable cost. The second case evaluated the synthesis of an adsorbent where minimal swelling capacity is preferred at the least possible cost. Superabsorbents have been utilized as an adsorbent in the removal of crystal violet dye [35], napthol green dye [35], sunset yellow dye [35], anionic dye [36], $\mathrm{Cu}^{2+}$ [37], $\mathrm{Co}^{2+}$ [37], $\mathrm{Ni}^{2+}$ [37], and $\mathrm{Cd}^{2+}$ [37] from wastewater. Adsorption technology involving industrial fixed-bed reactors requires adsorbent material to have the least swelling capacity to avoid fouling and clogging of the reactor [38].

\section{Methodology}

\subsection{Materials}

Acetic acid, Ch (90\% deacetylation degree), Mt, N,N'-methylenebisacrylamide, ammonium persulphate, and AA were acquired from Sigma-Aldrich (WI, USA). The preparative method utilized for $N$-octyl- $N$-vinyl-2-pyrrolidonium bromide was based on previous work by Naguib et al. [39].

\subsection{Experimental Method}

Approximately $50 \mathrm{~mL}$ of acetic acid $(1 \% \mathrm{v} / \mathrm{v})$ was used to dissolve $2 \mathrm{~g} \mathrm{Ch}$, and the solution was purged for 40 min using $\mathrm{N}_{2}$ gas. The solution was added to a pre-determined amount of ammonium persulphate, after which AA and $N, N^{\prime}$-methylenebisacrylamide were also added to the mixture. Then, mixture was allowed to react for $4 \mathrm{~h}$ at $70{ }^{\circ} \mathrm{C}$. The final solution was allowed to cool, and was then neutralized with $1 \mathrm{M} \mathrm{NaOH}$ until a $\mathrm{pH}$ of 7.0 was attained. The ChPA-Mt material was washed with water to remove any impurities and dried in an oven at $70^{\circ} \mathrm{C}$. Unreacted AA and PA were removed using ethanol in a Soxhlet extractor for $24 \mathrm{~h}$, ensuring that only crosslinked copolymer was attained.

\subsection{Fuzzy Multi-Objective Optimization Method}

The most probable parametric conditions of the variable cost and the total swelling capacity in account of its cumulative uncertainty of results was attained by applying the Pareto set analysis and fuzzy mathematical programming in the optimization studies. For the process parameters (vinylic monomer/biopolymer molar ratio, crosslinker and initiator) of the synthesis of a ChPA-Mt material, the process parameters and the material costs are listed in Table 1. Moreover, Table 2 shows the data derived from the study of Abdel Aziz and Salama [27] with the additional incorporation of the cumulative uncertainty of results. A linguistic value that applies the true value theory, ranging from completely false (0) to completely true (1), was utilized to estimate the reasoning approach in the fuzzy optimization analysis [40]. Figure 1 illustrates the algorithm flowchart of the fuzzy system in the present work. Optimization studies were performed using Lingo 18.0 (Lindo Systems, Chicago, IL, USA) where non-linear programming software uses a global optimizer.

Table 1. (a) Variables and (b) costing parameters for the synthesis of the clay-polymer composite material.

\begin{tabular}{ccc}
\hline (a) Process Parameters & Unit & Range \\
\hline AA/Ch $(A)$ & & $5-10$ \\
$N, N^{\prime}$-methylenebisacrylamide $(B)$ & wt $\%$ & $1-3$ \\
Ammonium persulfate $(C)$ & wt $\%$ & $1-3$ \\
\hline (b) Chemicals & Unit & $*$ Material Cost \\
\hline AA & USD $/ g$ & 0.44 \\
$N, N^{\prime}$-methylenebisacrylamide & USD $/ g$ & 0.41 \\
Ammonium persulfate & USD $/ g$ & 0.39 \\
\hline
\end{tabular}

* Sigma Aldrich (WI, USA); AA: acrylic acid; Ch: chitosan. 


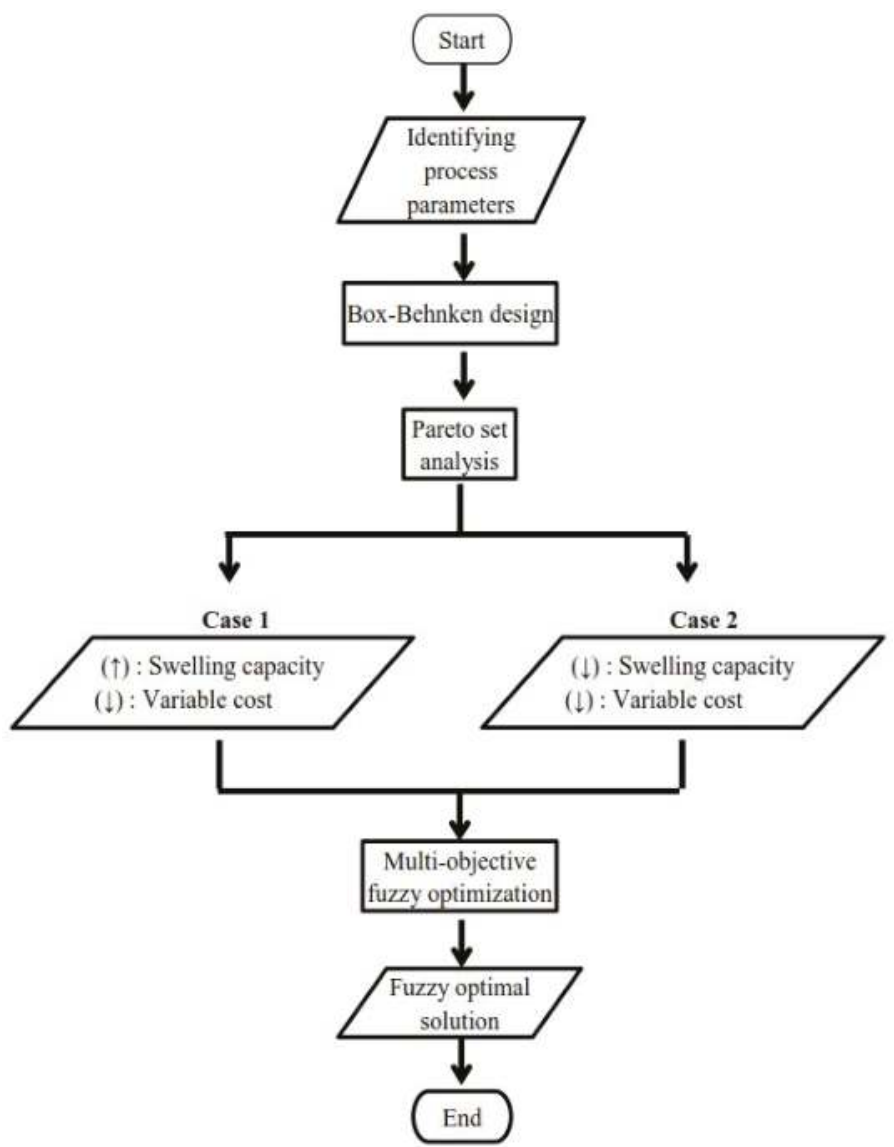

Figure 1. Fuzzy optimization process diagram.

Table 2. Clay-polymer composite material synthesis based on the BBD matrix.

\begin{tabular}{|c|c|c|c|c|c|}
\hline Run & $\begin{array}{l}\mathrm{AA} / \mathrm{Ch} \\
(A)\end{array}$ & $\begin{array}{c}N, N^{\prime} \text {-methylenebisacrylamide } \\
(B: \text { wt \%) }\end{array}$ & $\begin{array}{c}\text { Ammonium } \\
\text { Persulfate }(C: \text { wt \%) }\end{array}$ & $\begin{array}{l}\text { Predicted Swelling } \\
\text { Capacity }(\mathrm{g} / \mathrm{g})\end{array}$ & $\begin{array}{c}{ }^{*} \text { Cumulative } \\
\text { Uncertainty }(\mathrm{g} / \mathrm{g})\end{array}$ \\
\hline 1 & 5 & 2 & 1 & 74 & 19 \\
\hline 3 & 5 & 1 & 2 & 232 & 17 \\
\hline 4 & 5 & 2 & 3 & 164 & 14 \\
\hline 5 & 7.5 & 3 & 3 & 192 & 9 \\
\hline 8 & 7.5 & 3 & 1 & 91 & 17 \\
\hline 9 & 7.5 & 2 & 2 & 220 & 13 \\
\hline 10 & 7.5 & 1 & 3 & 322 & 13 \\
\hline 11 & 7.5 & 1 & 1 & 221 & 19 \\
\hline 12 & 7.5 & 2 & 2 & 220 & 13 \\
\hline 13 & 7.5 & 2 & 2 & 220 & 13 \\
\hline
\end{tabular}

Adapted from Abdel Aziz and Salama [27]; * calculated cumulative uncertainty of the results; BBD: Box-Behnken design. 


\subsubsection{Determination of Boundary Limits through the Pareto Optimality}

The process of choosing an exact number of courses that would generate a substantial overall effect for numerous competing practical solutions was carried out using the Pareto analysis [41]. The initial Pareto optimum solution under several weights of the objective function was obtained using the Pareto set process [42]. Initially, the boundary limits for variable cost and swelling capacity were attained using the method. The multi-objective fuzzy optimization applied the boundary limits. The first objective function in Equation (1) pertains to the swelling capacity in consideration of its uncertainty error $\left(Y_{Q}: \mathrm{g} / \mathrm{g}\right)$. This is either to maximize or minimize depending on desired condition of the case study in the synthesis of the polymer clay nanocomposite material. For $Y_{Q}$ at its maximum value, the upper limits of $Y_{Q}$ and total variable cost $\left(V C_{T}\right.$ : USD $\left./ \mathrm{kg}\right)$ were determined. The lower boundary limits of $Y_{Q}$ and $V C_{T}$ were determined through minimizing the second objective function in Equation (2). In the case of determining the minimum value of $Y_{Q}$, this was able to draw out the lower and upper limits of $Y_{Q}$ and $V C_{T}$, respectively, while its upper and lower boundaries were determined through Equation (2).

Objective functions:

$$
\begin{gathered}
\max / \min Y_{Q} \\
\min V C_{T}
\end{gathered}
$$

In order to satisfy the objective functions, the constraints were subjected to Equations (3)-(7). The sum of the swelling capacity $(Y: \mathrm{g} / \mathrm{g})$ and its corresponding uncertainty error $\left(W_{Y}: \mathrm{g} / \mathrm{g}\right)$ are shown in Equation (3). The response for the model equation in Equation (4) pertains to the swelling capacity of the synthesized polymer clay nanocomposite material. The incorporation of the constraints of the cumulative uncertainty of the response for the swelling capacity and the total variable cost are shown in Equations (5) and (6), respectively. Equation (7) pertains to the feasible regions of the variables tested based on the vinylic monomer/biopolymer molar ratio, crosslinker and initiator.

Constraints:

$$
\begin{gathered}
Y_{Q}=Y+W_{Y} \\
Y=\beta_{0}+\sum_{i=1}^{n} \beta_{i} X_{i}+\sum_{i=1}^{n-1} \sum_{i=1}^{n} \beta_{i j} X_{i} X_{j}+\sum_{i=1}^{n} \beta_{i i} X_{i}^{2} \\
W_{Y}=\left(\sum_{i=n}^{n} \frac{\partial Y}{\partial X_{i}} W_{X_{i}}\right)^{\frac{1}{2}} \\
V C_{T}=\sum_{i=1}^{n} V C_{i} \\
X_{i}^{L} \leq X_{i} \leq X_{i}^{U}
\end{gathered}
$$

where $\beta_{0}$ pertains to the constant; $i$ and $j$ are the single and interacting factor indices, respectively; $n$ denotes the tested number of variables; $\beta_{i}, \beta_{i j}$. and $\beta_{i i}$ are the regression coefficients; $X_{i}$ and $X_{j}$ are the independent variables; $W_{X}$. and $V C$ are the variable uncertainty and variable cost, respectively; while $L$ and $U$ represent the lower and upper boundary limit, respectively.

\subsubsection{Multi-Objective Decision Making through Fuzzy Logic}

The identification of the best solution derived from the recognized set of Pareto optimal conditions would require the utilization of a multi-criteria analysis [42]. The fuzzy mathematical programming system was employed to arrive at a solution from a multi-objective decision-making problem [43-45]. The perception of max-min aggregation that would concurrently enhance the satisfaction degree would be employed to maximize the overall satisfaction degree for the objective function [43-48]. Moreover, 
the degree of satisfaction for overall swelling capacity and the total variable cost of the results must satisfy the overall satisfaction as presented in Equation (8).

Objective function:

$$
\max \mu_{O} \leq \mu_{S C} \text { and } \mu_{V C}
$$

where $\mu_{S C}$ and $\mu_{V C}$ are the individual level of satisfaction of the overall swelling capacity and the total variable cost, respectively.

The constraints used to attain the fuzzy goal are given in Equations (9)-(12). The linear membership function depending on the desired function in either maximizing or minimizing the swelling capacity for the polymer clay nanocomposite material are shown in Equations (9) and (10), respectively. Equation (11) refers to the linear membership function for minimizing the total variable cost involved in the material synthesis. Moreover, the level of satisfaction has a limiting constraint that could only be adjusted within a feasible range as described in Equation (12).

Constraints:

$$
\begin{gathered}
\mu_{S C}=\frac{Y_{Q}-Y_{Q}^{L}}{Y_{Q}^{U}-Y_{Q}^{L}} \\
\mu_{S C}=\frac{Y_{Q}^{U}-Y_{Q}}{Y_{Q}^{U}-Y_{Q}^{L}} \\
\mu_{V C}=\frac{V C_{T}^{U}-V C_{T}}{V C_{T}^{U}-V C_{T}^{L}} \\
\mu_{O}^{L} \leq \mu_{O} \leq \mu_{O}^{U}
\end{gathered}
$$

\section{Results and Discussion}

\subsection{Effect of AA/Ch Molar Ratio on the Swelling Capacity and Its Variable Cost}

In order to investigate the influence of $\mathrm{AA} / \mathrm{Ch}$ molar ratio on the material synthesis, the amount of $N, N^{\prime}$-methylenebisacrylamide and ammonium persulfate were individually kept constant at $2 \mathrm{wt} \%$. The identification of a proper ratio between the vinylic monomer and biopolymer is essential to avoid the competition with AA led to its auto-polymerization aside from the grafting of the $\mathrm{Ch}$ material [49]. Figure 2a illustrates the effect of AA/Ch molar ratio towards the swelling capacity and variable cost.

The trend of the molar ratio from 5 to 10 resulted in a swelling capacity from $141 \pm 15 \mathrm{~g} / \mathrm{g}$ to $264 \pm 11 \mathrm{~g} / \mathrm{g}$, respectively. It was observed that a higher AA concentration leads to a larger swelling capacity upon the synthesis of the polymer clay nanocomposite material. An electrostatic repulsion and ionization in the chain takes place at a higher ratio due to the promotion of available carboxylic groups that is able to expand the originally coiled molecules [50].

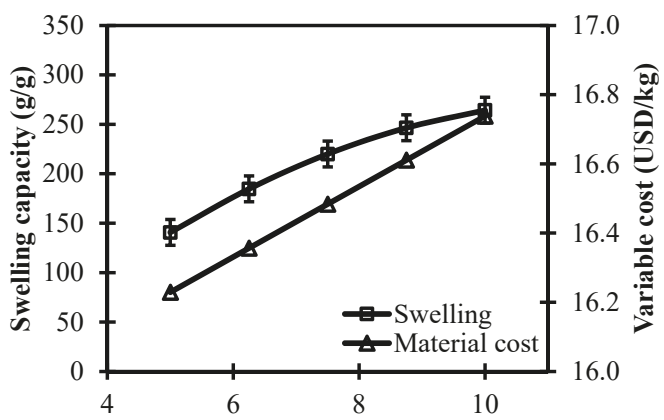

(a) Acrylic acid/Chitosan ( $\mathrm{mol} / \mathrm{mol})$

Figure 2. Cont. 


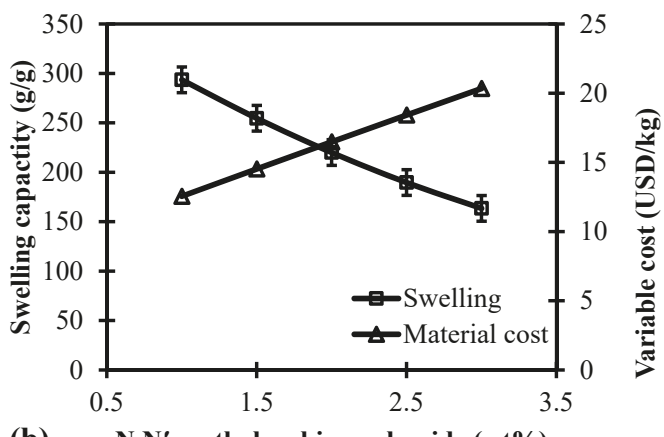

(b) $\quad \mathrm{N}, \mathrm{N}^{\prime}$-methylenebisacrylamide (wt\%)

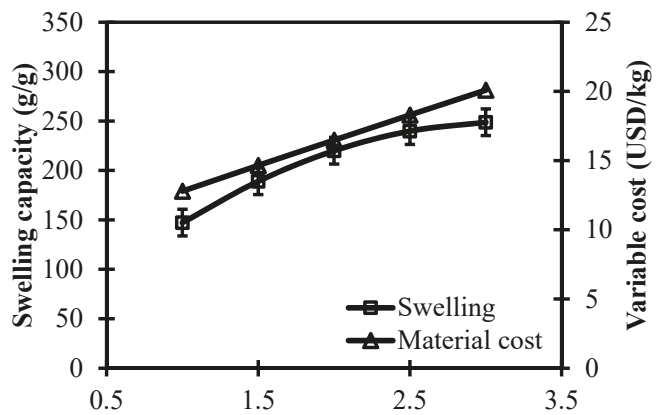

(c)

Ammonium persulfate (wt\%)

Figure 2. Simultaneous analysis of swelling capacity and variable cost at different levels of (a) acrylic acid/chitosan, (b) $N, N^{\prime}$-methylenebisacrylamide, and (c) ammonium persulfate.

In the aspect of the incurred total variable cost, the material synthesis at incremental molar ratio from 5 to 10 showed higher variable cost from $16.2 \mathrm{USD} / \mathrm{kg}$ to $16.7 \mathrm{USD} / \mathrm{kg}$. This is due to a higher AA loading that is utilized to synthesize the polymer clay nanocomposite material. Aside from achieving the desirable swelling capacity, it is also essentially important to minimize the variable cost to achieve an efficient and cheap material according to its function.

\subsection{Effect of $N, N^{\prime}$-methylenebisacrylamide on the Swelling Capacity and Its Variable Cost}

$N, N^{\prime}$-methylenebisacrylamide is used as the crosslinker for the polymer clay nanocomposite material synthesis. Crosslinking is favorable due to its capacity to improve chemical stability and mechanical strength [51]. To determine the effect of the amount of $N, N^{\prime}$-methylenebisacrylamide on the resulting material, the AA/Ch molar ratio of 7.5 and ammonium persulfate content of $2 \mathrm{wt} \%$ were kept constant throughout the variation of the crosslinker dosage. The detailed response of the swelling capacity and variable cost at different $N, N^{\prime}$-methylenebisacrylamide concentration are depicted in Figure 2b.

At 1 wt \% N,N'-methylenebisacrylamide, a swelling capacity of $294 \pm 15 \mathrm{~g} / \mathrm{g}$ was synthesized. Increasing the crosslinker content further to $3 \mathrm{wt} \%$ was able to lower the swelling capacity to $164 \pm 12 \mathrm{~g} / \mathrm{g}$. A higher crosslinker concentration was attributed to the decreasing polymer chain relaxation that led to less swelling [50]. Furthermore, large quantities of crosslinker content increased the crosslink density that tended to restrict additional free volume from being utilized [52].

For the trend from $1 \mathrm{wt} \%$ to $3 \mathrm{wt} \%$ of $N, N^{\prime}$-methylenebisacrylamide, the swelling capacity decreased while variable cost increased. The inverse relationship of the resulting material and its cost proved to be one of the essential criteria for achieving an efficient outcome for its synthesis. Higher 
$N, N^{\prime}$-methylenebisacrylamide implies more material usage that increases the specified variable cost the specified parameter. After selecting the appropriate combination of the crosslinker dosage, the resulting polymer clay nanocomposite material could be utilized in various applications due to its ability to form macromolecular structures [51].

\subsection{Effect of Ammonium Persulfate on the Swelling Capacity and Its Variable Cost}

The use of chemical initiators is essential for its function in generating living free radicals that enable the initiation of polymerization reactions in vinyl monomers [53]. Ammonium persulfate is used as the initiator in the graft polymerization of the AA onto $\mathrm{Ch}$. To determine the effects of ammonium persulfate, the $\mathrm{AA} / \mathrm{Ch}$ molar ratio and $N, N^{\prime}$-methylenebisacrylamide dosage were kept constant at 7.5 and $2 \mathrm{wt} \%$, respectively. Figure 2c shows the results of the variation of ammonium persulfate on the swelling capacity and variable cost.

The results exhibited increasing swelling capacity from $147 \mathrm{~g} / \mathrm{g}$ to $249 \mathrm{~g} / \mathrm{g}$ at the corresponding ammonium persulfate dosages from $1 \mathrm{wt} \%$ to $3 \mathrm{wt} \%$. In terms of the pore distribution, a higher initiator content provides additional free volume that allows higher water mobility leading to a higher swelling degree [52]. Furthermore, an increase in the initiator concentration is able to promote the formation of polymer chain ends in the network leading to higher swelling activities [54].

Aside from the crosslinker, the use of the initiator also contributes to a high production cost in the synthesis of the polymer clay nanocomposite material [49]. The variable cost increased from $12.8 \mathrm{USD} / \mathrm{kg}$ to $20.1 \mathrm{USD} / \mathrm{kg}$ when ammonium persulfate was changed from $1 \mathrm{wt} \%$ to $3 \mathrm{wt} \%$. Higher initiator content increased the material loading that mainly contributed to a larger variable cost. Thus, this is an essential factor for consideration to achieve optimal results upon determining the best compromise solution in a low-cost material synthesis at its ideal swelling capacity.

\subsection{Pareto Set Result}

The Pareto set analysis determined the appropriate upper and lower boundary limits of the swelling capacity with its cumulative uncertainty error and the total variable cost of the polymer clay nanocomposite material. The BBD under the response surface methodology was utilized as the basis to draw out the Pareto efficiency upon the grafting of AA onto $\mathrm{Ch}$. The results of the analysis would then be essentially used towards the preference criterion in the latter part of the decision-making analysis. In the material synthesis of the two case studies, a non-linear model equation is used for the response of the swelling capacity. This type of equation often leads to multiple local optimums and the global optimum are not directly given [55]. The global optimal solution is favorable to carry out the best possible solution in a given set of constraints for the objective function. Thus, the global optimizer option in the Lingo software is utilized to be able to achieve the best resulting solution for synthesis.

The Pareto analysis generates a Ch-graft-PA material through the application of Equations (13)-(27). Equation (13) shows the generated and validated model equation adapted from the study of Abdel Aziz and Salama [27] that describes the swelling capacity of the produced polymer clay nanocomposite material. The cumulative uncertainty of the response in Equation (14) indicates the possible error associated with the synthesis step towards the desired value of the swelling capacity. This is specifically determined through the partial derivatives with respect to AA/Ch molar ratio (Equation (15)), $N, N^{\prime}$-methylenebisacrylamide concentration (Equation (16)), and ammonium persulfate concentration (Equation (17)). The integration of the incurred total variable cost in Equation (18) is a key parameter in the Pareto optimal analysis. Specifically, the individual variable cost of AA, $N, N^{\prime}$-methylenebisacrylamide and ammonium persulfate are shown in Equations (19)-(21), while its calculated material usage are presented in Equations (22)-(24), respectively. Furthermore, Equations (25)-(27) are the boundary limits that denote the respective variables of AA, $N, N^{\prime}$-methylenebisacrylamide and ammonium persulfate.

$$
Y=-7.25+47.9 X_{1}-153 X_{2}+122.25 X_{3}+7.2 X_{1} X_{2}+2.2 X_{1} X_{3}-2.8 X_{1} X_{1}+8.5 X_{2} X_{2}-22 X_{3} X_{3}
$$




$$
\begin{aligned}
W_{Y}=\sqrt{\left(\frac{\partial Y_{Q}}{\partial X_{1}} W_{X_{1}}\right)^{2}+\left(\frac{\partial Y_{Q}}{\partial X_{2}} W_{X_{2}}\right)^{2}+\left(\frac{\partial Y_{Q}}{\partial X_{3}} W_{X_{3}}\right)^{2}} \\
\frac{\partial Y}{\partial X_{1}}=47.9+7.2 X_{2}+2.2 X_{3}-5.6 X_{1} \\
\frac{\partial Y}{\partial X_{2}}=-153+7.2 X_{1}+17 X_{2} \\
\frac{\partial Y}{\partial X_{3}}=122.25+2.2 X_{1}-44 X_{3} \\
V C_{T}=V C_{1}+V C_{2}+V C_{3} \\
V C_{1}=\frac{442.42 A}{(A+I+C+2)} \\
V C_{2}=\frac{421.43 I}{(A+I+C+2)} \\
V C_{3}=\frac{395.96 C}{(A+I+C+2)} \\
A=4.804 x 10^{-4} X_{1} \\
I=\frac{(A+2) X_{2}}{100} \\
C=\frac{(A+2) X_{3}}{100} \\
5 \leq X_{1} \leq 10 \\
1 \leq X_{2} \leq 3 \\
1 \leq X_{3} \leq 3
\end{aligned}
$$

where $X_{1}, X_{2}$ and $X_{3}$ are the variables of AA/Ch molar ratio; $N, N^{\prime}$-methylenebisacrylamide concentration (wt \%) and ammonium persulfate concentration (wt \%), respectively; $W_{X_{1}}, W_{X_{2}}$, and $W_{X_{3}}$ are the uncertainty of the tested variables; $V C_{T}, V C_{1}, V C_{2}$, and $V C_{3}$ are the total variable cost, and the individual variable cost of $\mathrm{AA}, N, N^{\prime}$-methylenebisacrylamide, and ammonium persulfate, respectively, in USD $/ \mathrm{kg}$; $A, I$ and $C$ are the computed material usage for AA, $N, N^{\prime}$-methylenebisacrylamide, and ammonium persulfate in $\mathrm{g}$.

\subsubsection{Case 1: Absorbent Material}

To determine the upper boundary limits of the swelling capacity and total variable cost of the Ch-graft-PA absorbent material, the set objective function for the summation of Equations (13) and (14) were maximized. This is subject to the constraints in Equations (15)-(27) that must be satisfied. Results indicated a swelling capacity at a global maximum of $354 \mathrm{~g} / \mathrm{g}$ and a $10 \mathrm{~g} / \mathrm{g}$ cumulative error of uncertainty. This is under the parametric conditions of $10 \mathrm{AA} / \mathrm{Ch}$ molar ratio, $1.0 \mathrm{wt} \%$ $N, N^{\prime}$-methylenebisacrylamide, and $3.0 \mathrm{wt} \%$ ammonium persulfate with an associated total variable cost of $16.5 \mathrm{USD} / \mathrm{kg}$.

A separate objective function was set to determine the lower boundary limits of the swelling capacity and total variable cost. Equation (18) is minimized under the established conditions of Equations (13)-(17) and (19)-(27). The results of the minimum swelling capacity (165 g/g) and total variable cost $(8.5 \mathrm{USD} / \mathrm{kg}$ ) were obtained in the parameters of $5 \mathrm{AA} / \mathrm{Ch}$ molar ratio, $1.0 \mathrm{wt} \%$ $N, N^{\prime}$-methylenebisacrylamide, and $1.0 \mathrm{wt} \%$ ammonium persulfate. Furthermore, its cumulative error of uncertainty reached $21 \mathrm{~g} / \mathrm{g}$. 
Figure 3a depicts the graphical representation of the Pareto optimality analysis that identified the appropriate boundary limits through determining the maximum swelling capacity at the least possible cost for the material synthesis of Ch-graft-PA absorbent. Results from the Pareto analysis revealed that large swelling capacities incurred higher variable cost. The swelling capacity is characterized by the amount of absorbed water and absorption rate [56]. Synthesizing absorbents with large swelling capacities are favorable to function as a superabsorbent polymer. Typically, the preparation of an AA based superabsorbent utilizes the initiator and crosslinker that contributes to higher production cost [49]. Higher AA/Ch molar ratio and ammonium persulfate concentration facilitate more carboxylic groups that initiate additional sites for crosslinking reaction, while lower $N, N^{\prime}$-methylenebisacrylamide concentration would avoid an extensive crosslinking to favorably increase the swelling characteristic [27]. Thus, a proper compromise between a high swelling capacity and a low variable cost is noteworthy for further investigation.
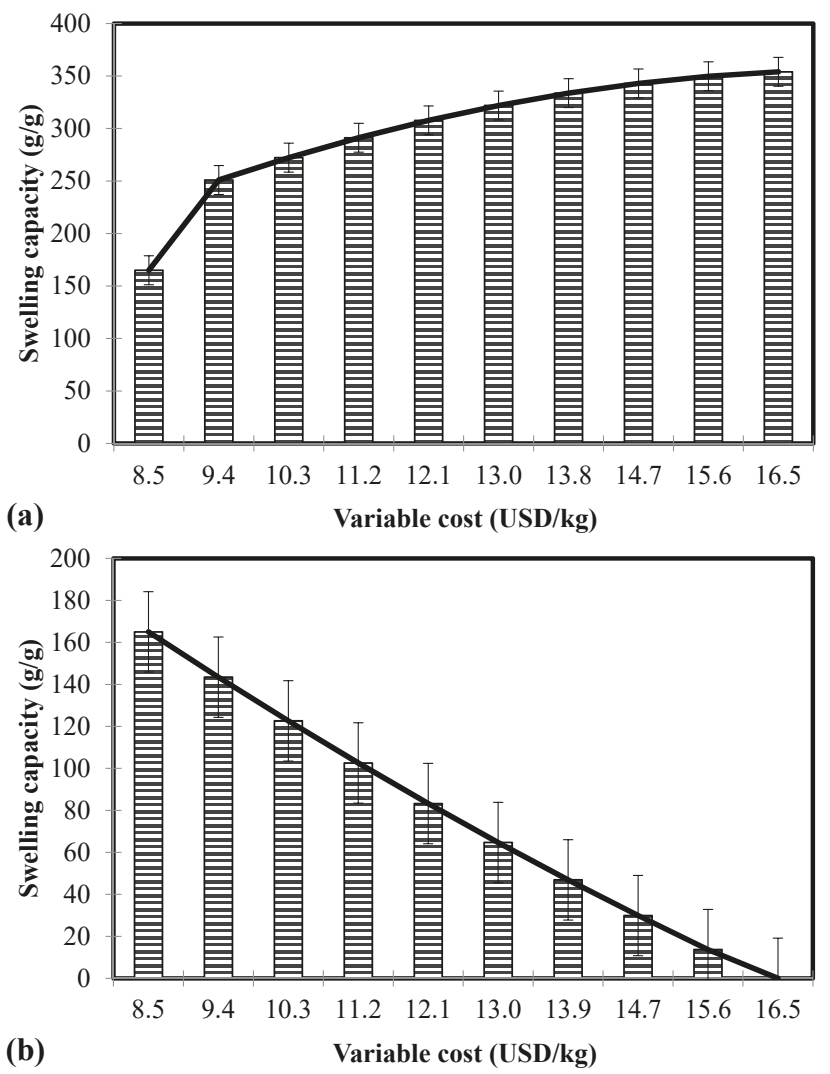

Figure 3. Pareto plot of variable cost with (a) maximum and (b) minimum conditions on the swelling capacity.

\subsubsection{Case 2: Adsorbent Material}

The sum of Equations (13) and (14) are minimized subject to the constraints in Equations (15)-(27) to appropriately establish the lower bound of the swelling capacity according to the desired functionality of the adsorbent material. Results for the global minimum value showed a non-swelling material that has a total variable cost of $16.5 \mathrm{USD} / \mathrm{kg}$ (upper bound for the total variable cost). The parametric conditions ensued five AA/Ch molar ratio, $3.0 \mathrm{wt} \% \mathrm{~N}, \mathrm{~N}^{\prime}$-methylenebisacrylamide and $1.0 \mathrm{wt} \%$ 
ammonium persulfate. Furthermore, the cumulative error of uncertainty in the swelling capacity reached $18 \mathrm{~g} / \mathrm{g}$.

On the other hand, the objective function in Equation (18) is minimized under the conditions of Equations (13)-(17) and (19)-(27). This resulted to an upper boundary limit of the swelling capacity at $165 \mathrm{~g} / \mathrm{g}$ (with a $21 \mathrm{~g} / \mathrm{g}$ cumulative uncertainty of results) and a lower boundary limit of the total variable cost at $8.5 \mathrm{USD} / \mathrm{kg}$ for the synthesis of Ch-graft-PA adsorbent. The variables of acid/Ch molar ratio, $N, N^{\prime}$-methylenebisacrylamide concentration and ammonium persulfate concentration rendered values of 5.0,1.0 $\mathrm{wt} \%$, and $1.0 \mathrm{wt} \%$, respectively.

The results of the Pareto set that comprise the boundary limits to appropriately synthesize the adsorbent material according to its desired minimum swelling capacity and variable cost are depicted in Figure $3 \mathrm{~b}$. In adsorption processes, high swelling materials have the capacity to adsorb large quantities of water molecules that increases the volume of the adsorbent. This would consequently lead to fouling and a sudden stop in the flow of wastewater in the column after a fixed amount of time [38]. It is thus desirable for adsorbents to have a low swelling capacity to be able to capitalize on the material in industrial applications. However, it was revealed in the results that a lower swelling characteristic incurs a higher total variable cost in the Pareto analysis. This is attributed to a higher material usage of the $N, N^{\prime}$-methylenebisacrylamide to lower the swelling capacity due to extensive crosslinking in the adsorbent synthesis [27]. Therefore, an investigation in a multi-objective optimization using the fuzzy logic approach is essential for a decision making analysis.

\subsection{Multi-Objective Fuzzy Optimization Result}

In order to optimize the objective function and the constraints, a decision in a fuzzy environment is required through the analogy of nonfuzzy environments as to select the activities that enables to simultaneously satisfy the objective function and constraints [57]. Based on the functionality of the polymer clay nanocomposite material, the fuzzy optimization was utilized for the decision making strategy of the two case studies presented in this research. The main objective function in Equation (28) is carried out in a global optimization procedure under the interval of 0 (dissatisfied) to 1 (satisfied) of the overall satisfaction rating $\left(\mu_{O}\right)$ in Equation (29). The multi-objective fuzzy optimization process also included the constraints in Equations (13)-(27).

$$
\begin{gathered}
\mu_{O} \leq \mu_{S C} \& \mu_{V C} \\
0 \leq \mu_{O} \leq 1
\end{gathered}
$$

In order to reach the fuzzy goal of the synthesis of an absorbent material (Case 1), the fuzzy constraints are subject to the constraints on the maximum swelling capacity $\left(\mu_{S C}\right)$ and minimum variable cost $\left(\mu_{V C}\right)$ in Equations (30) and (31), respectively.

$$
\begin{aligned}
\mu_{S C} & =\frac{Y_{Q}-185.58}{178.23} \\
\mu_{V C} & =\frac{16.47-V C_{T}}{7.96}
\end{aligned}
$$

For the adsorbent synthesis (Case 2), the fuzzy goal is attained with the minimized fuzzy constraints of the overall swelling capacity (Equation (32)) and total variable cost (Equation (33)).

$$
\begin{aligned}
\mu_{S C} & =\frac{185.58-Y_{Q}}{168.69} \\
\mu_{V C} & =\frac{16.49-V C_{T}}{7.94}
\end{aligned}
$$

The fuzzy objective function and its constraints are characterized by its membership function. Specifically, the linear membership function can facilitate the estimation of non-linear equations 
and is favorable to define the subjective preference of any objective uncertainty [58]. In a graphical representation, the linear membership function can be either maximized (Figure 4a) or minimized (Figure $4 \mathrm{~b}$ ) according to its objective function. Moreover, the boundary limits utilized in the fuzzy optimization for the swelling capacity and variable cost are derived from the Pareto analysis described in the preceding section. The boundary limits for absorbent synthesis (Case 1) are as follows: (1) overall swelling capacity of $364 \mathrm{~g} / \mathrm{g}$ (upper), (2) overall swelling capacity of $186 \mathrm{~g} / \mathrm{g}$ (lower), (3) total variable cost of $16.5 \mathrm{USD} / \mathrm{kg}$ (upper), and (4) total variable cost of $8.5 \mathrm{USD} / \mathrm{kg}$ (lower). For adsorbent synthesis (Case 2), the boundary limits are as follows: (1) overall swelling capacity of $186 \mathrm{~g} / \mathrm{g}$ (upper), (2) overall swelling capacity of $17 \mathrm{~g} / \mathrm{g}$ (lower), (3) total variable cost of $16.5 \mathrm{USD} / \mathrm{kg}$ (upper), and (4) total variable cost of $8.5 \mathrm{USD} / \mathrm{kg}$ (lower).

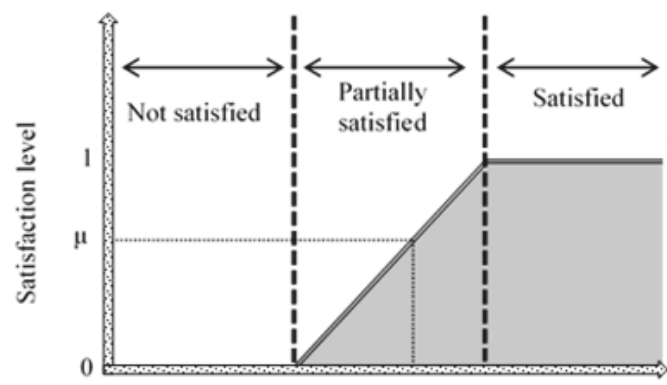

Objective function: Maximization

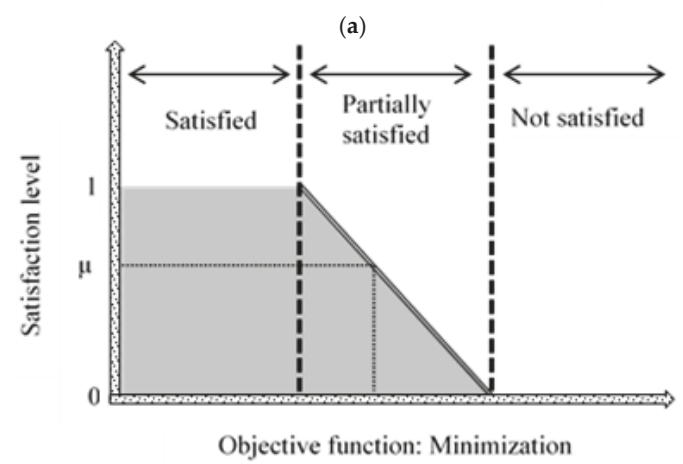

(b)

Figure 4. Linear membership function at goals towards (a) maximization and (b) minimization.

The fuzzy global optimal results from the clay-polymer composite material synthesis are listed in Table 3. Results showed that the maximum $\lambda_{O}$ values for the absorbent and adsorbent material were 0.672 and 0.523 , respectively. This indicates that the material synthesis upon the various matrix of $\mathrm{AA} / \mathrm{Ch}$ molar ratio, $N, N^{\prime}$-methylenebisacrylamide concentration, and ammonium persulfate concentration were able to successfully reach a compromise solution for the absorbent and adsorbent synthesis with an aggregated satisfaction rating of $67.2 \%$ and $52.3 \%$, respectively.

For the absorbent synthesis (Case 1), a proper allocation scheme that achieves the fuzzy goal subjected to its fuzzy constraints reached an overall swelling capacity of $305 \mathrm{~g} / \mathrm{g}$ and a total variable cost of $10.8 \mathrm{USD} / \mathrm{kg}$. The solution that achieved the highest swelling capacity at the least possible cost corresponds to the fuzzy optimum conditions of $10.0 \mathrm{AA} / \mathrm{Ch}$ molar ratio, $1.0 \mathrm{wt} \%$ $N, N^{\prime}$-methylenebisacrylamide, and $1.6 \mathrm{wt} \%$ ammonium persulfate. The resulting solution is desirable for synthesizing a superabsorbent material with a high swelling capacity. Typically, superabsorbents are able to keep large quantity of water that are irremovable under pressure due to its loosely crosslinked 
hydrophilic characteristic as a polymer material. A high-level acid to polymer ratio, low-level crosslinker dose, and mid-level initiator dose are desirable to appropriately initiate a loose crosslinking reaction in the carboxylate groups. In comparison with the study of Abdel Aziz and Salama [27], the optimum results using the RSM model led to a swelling capacity of $351 \mathrm{~g} / \mathrm{g}(9.76 \mathrm{AA} / \mathrm{Ch}$ molar ratio, $1.04 \mathrm{wt}$ $\% \mathrm{~N}^{\prime}$-methylenebisacrylamide, and $2.98 \mathrm{wt} \%$ ammonium persulfate). However, the total variable cost for this synthesis is $16.6 \mathrm{USD} / \mathrm{kg}$ that is $53.7 \%$ more expensive than in the fuzzy optimal solution. Thus, fuzzy optimization was able to reach a compromise solution based on a desirable swelling capacity with a more economical cost for superabsorbent synthesis.

Table 3. Fuzzy optimal solutions based on different objectives for the synthesis of ChPA-Mt material.

\begin{tabular}{|c|c|c|c|}
\hline \multirow[b]{2}{*}{ Parameters } & \multirow[b]{2}{*}{ Unit } & \multicolumn{2}{|c|}{ Goal } \\
\hline & & $\begin{array}{c}\text { Absorbent } \\
(\uparrow): \text { Swelling Capacity } \\
(\downarrow) \text { : Variable Cost }\end{array}$ & $\begin{array}{c}\text { Adsorben } \\
(\downarrow) \text { : Swelling Capacity } \\
(\downarrow) \text { : Variable Cost }\end{array}$ \\
\hline$\mu_{O}$ & $\%$ & 67.2 & 52.3 \\
\hline$\mu_{S C}$ & $\%$ & 67.2 & 52.3 \\
\hline$\mu_{V C}$ & $\%$ & 67.2 & 52.3 \\
\hline Swelling capacity $\left(Y_{Q}\right)$ & $\mathrm{g} / \mathrm{g}$ & 2 & 78 \\
\hline Cumulative uncertainty $\left(W_{Y_{Q}}\right)$ & $\mathrm{g} / \mathrm{g}$ & 15 & 19 \\
\hline $\begin{array}{l}\text { Overall }\left(Y_{Q}+W_{Y_{Q}}\right) \\
\text { Costing: }\end{array}$ & $\mathrm{g} / \mathrm{g}$ & 305 & 97 \\
\hline Arylic acid & $\mathrm{USD} / \mathrm{kg}$ & 1.0 & 0.5 \\
\hline$N, N^{\prime}$-methylenebisacrylamide & $\mathrm{USD} / \mathrm{kg}$ & 4.1 & 8.0 \\
\hline Ammonium persulfate & $\mathrm{USD} / \mathrm{kg}$ & 5.7 & 3.8 \\
\hline Total variable cost & $\mathrm{USD} / \mathrm{kg}$ & 10.8 & 12.3 \\
\hline \multicolumn{4}{|l|}{ Process Parameters: } \\
\hline $\mathrm{AA} / \mathrm{Ch}$ & & 10.0 & 5.0 \\
\hline$N, N^{\prime}$-methylenebisacrylamide & wt $\%$ & 1.0 & 1.9 \\
\hline Ammonium persulfate & wt $\%$ & 1.6 & 1.0 \\
\hline
\end{tabular}

Conversely, materials in adsorption technology that exhibit a high swelling degree are highly unfavorable as adsorbents [38]. The adsorbent synthesis (Case 2) in the fuzzy-based decision making analysis revealed a minimum overall swelling capacity and total variable cost of $97 \mathrm{~g} / \mathrm{g}$ and $12.3 \mathrm{USD} / \mathrm{kg}$, respectively. The fuzzy optimum solution resulted in the parameters of $5.0 \mathrm{AA} / \mathrm{Ch}$ molar ratio, $1.9 \mathrm{wt} \%$ $N, N^{\prime}$-methylenebisacrylamide, and $1.0 \mathrm{wt} \%$ ammonium persulfate. This has been selected to reach a compromise solution by simultaneously minimizing the swelling capacity and its associated costs for synthesis. The acid to polymer ratio (low), crosslinker (mid), and initiator (low) combination are favorable due to promoting an extensive crosslinking reaction leading to smaller swelling capacity in the Ch-graft-PA material. This would ultimately satisfy the functionality of the adsorbent as the swelling characteristic is a crucial factor in adsorption technologies.

\section{Conclusions}

In this study, a strategic decision making analysis for synthesis of ChPA-Mt was investigated depending on its functionality as an absorbent or adsorbent. The tested variables of AA/Ch molar ratio, $N, N^{\prime}$-methylenebisacrylamide concentration, and ammonium persulfate concentration were studied in conjunction with an uncertainty analysis in the response (swelling capacity) and its associated variable cost. The Pareto set analysis indicated the lower to upper boundary limits for the two case studies as follows: (1) absorbent material, overall swelling capacity (186 g/g to $364 \mathrm{~g} / \mathrm{g}$ ) and total variable cost (8.5 USD $/ \mathrm{kg}$ to $16.5 \mathrm{USD} / \mathrm{kg}$ ); and (2) adsorbent material, overall swelling capacity (17 g/g to $186 \mathrm{~g} / \mathrm{g}$ ) and total variable cost (8.5 USD $/ \mathrm{kg}$ to $16.5 \mathrm{USD} / \mathrm{kg})$. A multi-objective fuzzy optimization showed an innovative approach to determine a solution for the best condition in the material synthesis. According to its functionality, the absorbent material ( $305 \mathrm{~g} / \mathrm{g}$ and $10.8 \mathrm{USD} / \mathrm{kg}$ ) and adsorbent material $(97 \mathrm{~g} / \mathrm{g}$ and 
12.3 USD $/ \mathrm{kg}$ ) showed a satisfaction rating in its synthesis of $67.2 \%$ and $52.3 \%$, respectively. Therefore, the application of the global fuzzy optimal solutions and its respective conditions in the case studies proves to be efficient in relation to the material synthesis. Furthermore, the incorporation of the criteria of the variable cost in terms of material usage and the cumulative uncertainty of the response have successfully ensued essential compromise results in the decision making process.

Author Contributions: Conceptualization, methodology, and analysis, A.E.S.C.; writing, reviewing, and editing, A.E.S.C and C.M.F.; supervision and funding acquisition, J.J.Y.

Acknowledgments: The authors would like to acknowledge the National Research Foundation of Korea through Ministry of Education (No. 2016R1A6A1A03012812) for the financial support of this research.

Conflicts of Interest: The authors declare no conflict of interest.

\section{References}

1. Zhang, J.; Wang, Q.; Wang, A. Synthesis and characterization of chitosan-g-poly(acrylic acid)/attapulgite superabsorbent composites. Carbohydr. Polym. 2007, 68, 367-374. [CrossRef]

2. Kabiri, K.; Roshanfekr, S. Converting water absorbent polymer to alcohol absorbent polymer. Polym. Adv. Technol. 2013, 24, 28-33. [CrossRef]

3. Ghazy, M.B.M.; El-Hai, F.A.; Mohamed, M.F.; Essawy, H.A. Potassium fulvate as co-interpenetrating agent during graft polymerization of acrylic acid from cellulose. Int. J. Biol. Macromol. 2016, 91, 1206-1214. [CrossRef] [PubMed]

4. Zheng, Y.; Hua, S.; Wang, A. Adsorption behavior of $\mathrm{Cu} 2+$ from aqueous solutions onto starch-g-poly (acrylic acid)/sodium humate hydrogels. Desalination 2010, 263, 170-175. [CrossRef]

5. Kabiri, K.; Omidian, H.; Hashemi, S.A.; Zohuriaan-Mehr, M.J. Synthesis of fast-swelling superabsorbent hydrogels: Effect of crosslinker type and concentration on porosity and absorption rate. Eur. Polym. J. 2003, 39, 1341-1348. [CrossRef]

6. Singh, J.; Dhaliwal, A.S. Synthesis, characterization and swelling behavior of silver nanoparticles containing superabsorbent based on grafted copolymer of polyacrylic acid/Guar gum. Vacuum 2018, 157, 51-60. [CrossRef]

7. Wang, W.; Wang, J.; Kang, Y.; Wang, A. Synthesis, swelling and responsive properties of a new composite hydrogel based on hydroxyethyl cellulose and medicinal stone. Compos. Part B Eng. 2011, 42, 809-818. [CrossRef]

8. Pourjavadi, A.; Fakoorpoor, S.M.; Hosseini, P.; Khaloo, A. Interactions between superabsorbent polymers and cement-based composites incorporating colloidal silica nanoparticles. Cem. Concr. Compos. 2013, 37, 196-204. [CrossRef]

9. Bao, Y.; Ma, J.; Li, N. Synthesis and swelling behaviors of sodium carboxymethyl cellulose-g-poly (AA-co-AM-co-AMPS)/MMT superabsorbent hydrogel. Carbohydr. Polym. 2011, 84, 76-82. [CrossRef]

10. Bhattacharyya, R.; Ray, S.K. Micro- and nano-sized bentonite filled composite superabsorbents of chitosan and acrylic copolymer for removal of synthetic dyes from water. Appl. Clay Sci. 2014, 101, 510-520. [CrossRef]

11. Fang, S.; Wang, G.; Li, P.; Xing, R.; Liu, S.; Qin, Y.; Yu, H.; Chen, X.; Li, K. Synthesis of chitosan derivative graft acrylic acid superabsorbent polymers and its application as water retaining agent. Int. J. Biol. Macromol. 2018, 115, 754-761. [CrossRef]

12. Gao, J.; Yang, Q.; Ran, F.; Ma, G.; Lei, Z. Preparation and properties of novel eco-friendly superabsorbent composites based on raw wheat bran and clays. Appl. Clay Sci. 2016, 132-133, 739-747. [CrossRef]

13. Da Costa, M.P.M.; De Mello Ferreira, I.L.; De Macedo Cruz, M.T. New polyelectrolyte complex from pectin/chitosan and montmorillonite clay. Carbohydr. Polym. 2016, 146, 123-130. [CrossRef]

14. Jayakumar, R.; Nagahama, H.; Furuike, T.; Tamura, H. Synthesis of phosphorylated chitosan by novel method and its characterization. Int. J. Biol. Macromol. 2008, 42, 335-339. [CrossRef]

15. Fazli, Y.; Shariatinia, Z. Controlled release of cefazolin sodium antibiotic drug from electrospun chitosan-polyethylene oxide nanofibrous Mats. Mater. Sci. Eng. C 2017, 71, 614-652. [CrossRef]

16. Wang, L.; Zhang, J.; Wang, A. Removal of methylene blue from aqueous solution using chitosan- g-poly(acrylic acid)/montmorillonite superadsorbent nanocomposite. Colloids Surf. A Physicochem. Eng. Asp. 2008, 322, 47-53. [CrossRef] 
17. Je, J.Y.; Kim, S.K. Antimicrobial action of novel chitin derivative. Biochim. Biophys. Acta Gen. Subj. 2006, 1760, 104-109. [CrossRef]

18. Pelissari, F.M.; Yamashita, F.; Garcia, M.A.; Martino, M.N.; Zaritzky, N.E.; Grossmann, M.V.E. Constrained mixture design applied to the development of cassava starch-chitosan blown films. J. Food Eng. 2012, 108, 262-267. [CrossRef]

19. Gao, X.; Cao, Y.; Song, X.; Zhang, Z.; Zhuang, X.; He, C.; Chen, X. Biodegradable, pH-responsive carboxymethyl cellulose/poly(acrylic acid) hydrogels for oral insulin delivery. Macromol. Biosci. 2014, 14, 565-575. [CrossRef]

20. Yadav, M.; Rhee, K.Y. Superabsorbent nanocomposite (alginate-g-PAMPS/MMT): Synthesis, characterization and swelling behavior. Carbohydr. Polym. 2012, 90, 165-173. [CrossRef]

21. Feng, E.; Ma, G.; Wu, Y.; Wang, H.; Lei, Z. Preparation and properties of organic-inorganic composite superabsorbent based on xanthan gum and loess. Carbohydr. Polym. 2014, 111, 463-468. [CrossRef] [PubMed]

22. Shi, X.; Wang, W.; Kang, Y.; Wang, A. Enhanced swelling properties of a novel sodium alginate-based superabsorbent composites: NaAlg-g-poly(NaA-co-St)/APT. J. Appl. Polym. Sci. 2012, 125, 1822-1832. [CrossRef]

23. Spagnol, C.; Rodrigues, F.H.A.; Pereira, A.G.B.; Fajardo, A.R.; Rubira, A.F.; Muniz, E.C. Superabsorbent hydrogel composite made of cellulose nanofibrils and chitosan-graft-poly(acrylic acid). Carbohydr. Polym. 2012, 87, 2038-2045. [CrossRef]

24. Murray, H.H. Overview—Clay mineral applications. Appl. Clay Sci. 1991, 5, 379-395. [CrossRef]

25. Kotal, M.; Bhowmick, A.K. Polymer nanocomposites from modified clays: Recent advances and challenges. Prog. Polym. Sci. 2015, 51, 127-187. [CrossRef]

26. Mansoori, Y.; Atghia, S.V.; Zamanloo, M.R.; Imanzadeh, G.; Sirousazar, M. Polymer-clay nanocomposites: Free-radical grafting of polyacrylamide onto organophilic montmorillonite. Eur. Polym. J. 2010, 9, 1844-1853. [CrossRef]

27. Abdel Aziz, M.S.; Salama, H.E. Effect of vinyl montmorillonite on the physical, responsive and antimicrobial properties of the optimized polyacrylic acid/chitosan superabsorbent via Box-Behnken model. Int. J. Biol. Macromol. 2018, 116, 840-848. [CrossRef]

28. Nie, X.; Adalati, A.; Du, J.; Liu, H.; Xu, S.; Wang, J. Preparation of amphoteric nanocomposite hydrogels based on the exfoliation of montmorillonite via in-situ intercalative polymerization of hydrophilic cationic and anionic monomers. Appl. Clay Sci. 2014, 97, 132-137. [CrossRef]

29. Oyekanmi, A.A.; Ahmad, A.; Hossain, K.; Rafatullah, M. Statistical optimization for adsorption of Rhodamine B dye from aqueous solutions. J. Mol. Liq. 2019, 281, 48-58. [CrossRef]

30. Singh, K.P.; Gupta, S.; Singh, A.K.; Sinha, S. Optimizing adsorption of crystal violet dye from water by magnetic nanocomposite using response surface modeling approach. J. Hazard. Mater. 2011, 186, 1462-1473. [CrossRef]

31. Bezerra, M.A.; Santelli, R.E.; Oliveira, E.P.; Villar, L.S.; Escaleira, L.A. Response surface methodology (RSM) as a tool for optimization in analytical chemistry. Talanta 2008, 76, 965-977. [CrossRef] [PubMed]

32. Metternicht, G.; Gonzalez, S. FUERO: Foundations of a fuzzy exploratory model for soil erosion hazard prediction. Environ. Model. Softw. 2005, 20,715-728. [CrossRef]

33. Jiang, H.; Eastman, J.R. Application of fuzzy measures in multi-criteria evaluation in GIS. Int. J. Geogr. Inf. Sci. 2000, 14, 173-184. [CrossRef]

34. Kangas, A.; Kangas, J.; Laukkanen, S. Fuzzy multicriteria approval method and its application to two forest planning problems. For. Sci. 2006, 52, 232-242.

35. Nagarpita, M.V.; Roy, P.; Shruthi, S.B.; Sailaja, R.R.N. Synthesis and swelling characteristics of chitosan and CMC grafted sodium acrylate-co-acrylamide using modified nanoclay and examining its efficacy for removal of dyes. Int. J. Biol. Macromol. 2017, 102, 1226-1240. [CrossRef] [PubMed]

36. Zhao, Y.; Chen, Y.; Zhao, J.; Tong, Z.; Jin, S. Preparation of SA-g-(PAA-co-PDMC) polyampholytic superabsorbent polymer and its application to the anionic dye adsorption removal from effluents. Sep. Purif. Technol. 2017, 188, 329-340. [CrossRef]

37. Shah, L.A.; Khan, M.; Javed, R.; Sayed, M.; Khan, M.S.; Khan, A.; Ullah, M. Superabsorbent polymer hydrogels with good thermal and mechanical properties for removal of selected heavy metal ions. J. Clean. Prod. 2018, 201, 78-87. [CrossRef] 
38. Kyzas, G.Z.; Kostoglou, M. Swelling-Adsorption interactions during mercury and nickel ions removal by chitosan derivatives. Sep. Purif. Technol. 2015, 149, 92-102. [CrossRef]

39. Naguib, H.F.; Abdel Aziz, M.S.; Saad, G.R. Synthesis, Characterization, and Microbial Activity of Nanocomposites of Chitosan-Graft-Poly(4-vinyl pyridine) Copolymer/Organophilic Montmorillonite. Polym. Plast. Technol. Eng. 2015, 54, 1270-1279. [CrossRef]

40. Zhang, F.; Cheng, J. Verification of fuzzy UML models with fuzzy Description Logic. Appl. Soft Comput. J. 2018, 73, 134-152. [CrossRef]

41. Pirouzan, D.; Yahyaei, M.; Banisi, S. Pareto based optimization of flotation cells configuration using an oriented genetic algorithm. Int. J. Miner. Process. 2014, 126, 107-116. [CrossRef]

42. Aviso, K.B.; Tan, R.R.; Culaba, A.B.; Cruz, J.B. Bi-level fuzzy optimization approach for water exchange in eco-industrial parks. Process Saf. Environ. Prot. 2010, 88, 31-40. [CrossRef]

43. Zimmermann, H.J. Fuzzy programming and linear programming with several objective functions. Fuzzy Sets Syst. 1978, 1, 45-55. [CrossRef]

44. Zimmermann, H.J. Fuzzy mathematical programming. Comput. Oper. Res. 1983, 10, 291-298. [CrossRef]

45. Czogala, E.; Zimmermann, H.-J. Decision making in uncertain environments. Eur. J. Oper. Res. 1986, 23, 202-212. [CrossRef]

46. Aviso, K.B.; Tan, R.R.; Culaba, A.B. Designing eco-industrial water exchange networks using fuzzy mathematical programming. Clean Technol. Environ. Policy 2010, 12, 353-363. [CrossRef]

47. Tapia, J.F.D.; Tan, R.R. Fuzzy optimization of multi-period carbon capture and storage systems with parametric uncertainties. Process Saf. Environ. Prot. 2014, 92, 545-554. [CrossRef]

48. Choi, A.E.S.; Roces, S.; Dugos, N.; Wan, M.W. Operating cost study through a Pareto-optimal fuzzy analysis using commercial ferrate (VI) in an ultrasound-assisted oxidative desulfurization of model sulfur compounds. Clean Technol. Environ. Policy 2016, 18, 1433-1441. [CrossRef]

49. Ge, H.; Wang, S. Thermal preparation of chitosan-acrylic acid superabsorbent: Optimization, characteristic and water absorbency. Carbohydr. Polym. 2014, 113, 296-303. [CrossRef]

50. Bukhari, S.M.H.; Khan, S.; Rehanullah, M.; Ranjha, N.M. Synthesis and Characterization of Chemically Cross-Linked Acrylic Acid/Gelatin Hydrogels: Effect of $\mathrm{pH}$ and Composition on Swelling and Drug Release. Int. J. Polym. Sci. 2015, 2015, 187961. [CrossRef]

51. Grisdanurak, N.; Akewaranugulsiri, S.; Futalan, C.M.; Tsai, W.; Kan, C.; Hsu, C.; Wan, M. The Study of Copper Adsorption from Aqueous Solution Using Crosslinked Chitosan Immobilized on Bentonite. J. Appl. Polym. Sci. 2012, 125, E132-E142. [CrossRef]

52. Kumar, P.; Kundu, P.P. Formation of semi-IPN membrane composed of crosslinked SPS-[PVdF-co-HFP/Nafion] for application in DMFC: A fine tuning between crosslinker and initiator. Mater. Chem. Phys. 2015, 164, 188-197. [CrossRef]

53. Wang, W.; Yu, Y.; Wang, P.; Wang, Q.; Li, Y.; Yuan, J.; Fan, X. Controlled graft polymerization on the surface of filter paper via enzyme-initiated RAFT polymerization. Carbohydr. Polym. 2019, 207, 239-245. [CrossRef] [PubMed]

54. Singhal, R.; Tomar, R.S.; Nagpal, A.K. Effect of cross-linker and initiator concentration on the swelling behaviour and network parameters of superabsorbent hydrogels based on acrylamide and acrylic acid. Int. J. Plast. Technol. 2009, 13, 22-37. [CrossRef]

55. Lim, S.; Zhu, J. Integrated data envelopment analysis: Global vs. local optimum. Eur. J. Oper. Res. 2013, 229, 276-278. [CrossRef]

56. Omidian, H.; Hashemi, S.A.; Sammes, P.G.; Meldrum, I. A model for the swelling of superabsorbent polymers. Polymer 1998, 39, 6697-6704. [CrossRef]

57. Zimmermann, H.-J. Individual Decision Making in Fuzzy Environments. In Fuzzy Sets, Decision Making, and Expert Systems; Springer: Dordrecht, The Netherlands, 1987; pp. 15-44. ISBN 978-0-89838-149-8.

58. Wen, B.; Li, H. An approach to formulation of FNLP with complex piecewise linear membership functions. Chin. J. Chem. Eng. 2014, 22, 411-417. [CrossRef]

(C) 2019 by the authors. Licensee MDPI, Basel, Switzerland. This article is an open access article distributed under the terms and conditions of the Creative Commons Attribution (CC BY) license (http://creativecommons.org/licenses/by/4.0/). 
Article

\title{
Buildup of Multi-Ionic Supramolecular Network Facilitated by In-Situ Intercalated Organic Montmorillonite in 1,2-Polybutadiene
}

\author{
Jinhui Liu, Di Li, Xiangshuai Zhao, Jieting Geng, Jing Hua * and Xin Wang * \\ Key Laboratory of Rubber-Plastics Ministry of Education/Shandong Provincial Key Laboratory of \\ Rubber-Plastics, College of Polymer Science and Engineering, Qingdao University of Science and Technology, \\ Qingdao 266042, China; liujinhui7@163.com (J.L.); m13061211032@163.com (D.L.); 15192743117@163.com (X.Z.); \\ 13475862646@163.com (J.G.) \\ * Correspondence: huajing72@qust.edu.cn (J.H.); wangxin@qust.edu.cn (X.W.)
}

Received: 4 February 2019; Accepted: 7 March 2019; Published: 13 March 2019

\begin{abstract}
The development of a sacrificial bond provided unique inspiration for the design of advanced elastomers with excellent mechanical properties, but it is still a huge challenge to construct a homogenous polar sacrificial network in a nonpolar elastomer. In this effort, we proposed a novel strategy to engineer a multi-ionic network into a covalently cross-linked 1,2-polybutadiene (1,2-PB) facilitated by in-situ intercalated organic montmorillonite (OMMT) without phase separation. $\mathrm{XRD}, \mathrm{SEM}$, and TEM analysis were carried out to characterize the microstructure of the resulting polymers. Crosslinking density, dielectric performance, and cyclic tensile tests were used to demonstrate the interaction of zinc methacrylate (ZDMA) and OMMT. The dynamic nature of ionic bonds allowed it to rupture and reform to dissipate energy efficiently. Stretching orientation brought parallelism between polymer chains and OMMT layers which was beneficial for the reconstruction of the ionic network, ultimately resulting in high strength and a low stress relaxation rate. Overall, our work presented the design of a uniform and strong sacrificial network in the nano-clay/elastomer nanocomposite with outstanding mechanical performances under both static and dynamic conditions.
\end{abstract}

Keywords: sacrificial bond; ionic network; organic montmorillonite; 1,2-polybutadiene; in-situ intercalation

\section{Introduction}

The nacreous layer in biological materials—byssus in mollusks and bones in mammals—provide a paradigm for a stiff, strong, and at the same time tough protective engineering [1,2]. The excellent performance of biological materials originates from a brick-and-mortar architecture, in which $95 \mathrm{wt} \%$ hard aragonite tablets (brick) are laminated by $5 \mathrm{wt} \%$ soft biopolymer matrix (mortar). Inspired by biological materials, a tremendous amount of effort has been focused on enhancing the mechanical properties of synthetic polymers by dispersing a small amount of reinforcing nano-clay into a polymer matrix [3-5]. Despite the undoubted success, it has remained extraordinarily challenging to obtain synergetic improvements of strength and toughness through these approaches because the biopolymer is thought to hold the key to the extraordinary toughness in biomaterials to dissipate energy as a kind of sacrificial bond [6-8]. Sacrificial bonds are defined as bonds that rupture before primary bonds under deformation to protect the integrity of the primary network [9]. Sacrificial bonds include irreversible associations, mainly covalent bonds [10] and reversible associations such as hydrogen bonds [11-14], metal-ligand coordinated interactions [15-18], ionic interactions [19,20], electrostatic interactions [21,22], hydrophobic associations [23], and $\pi-\pi$ stacking [24,25]. Such reversible associations can break and reform to dissipate energy repeatedly. To combine the reinforcing effect of 
nano-clay and sacrificial bond, Martikainen et al. [26] prepared biomimetic nanocomposites composed of anionic nano-clay (MMT) and cationic polymer (PDDA), and then modified with dGMP to form multiple hydrogen bonds. Afterward, MMT was as brick moiety and multiple hydrogen bonds as well as electrostatic bonds were as mortar moiety, to synergistically improve the mechanical performance of the biomimetic nanocomposites.

Atactic 1,2-polybutadiene (1,2-PB) rubber with dense vinyl groups and few double bonds on backbone, exhibiting excellent wet-skid resistance, low rolling resistance, low heat build-up, and superior aging resistance, can meet the requirements of high-performance green tire when used in combination with other rubbers such as natural rubber [27-32]. Molybdenum (Mo)-based catalyst system can provide a higher activity for the coordination polymerization of 1,2-PB compared to other catalyst systems such as titanium and chromium-based catalyst system [33]. Nevertheless, the mechanical properties of 1,2-PB are expected to meet various application requirements. If 1,2-PB was reinforced by the combination of nano-clay and sacrificial bonds, it could endow 1,2-PB with unique properties. However, nano-clay and reversible sacrificial bonds are high polar motifs, whereas 1,2-PB is typically nonpolar motifs. The primary challenge to incorporate both sacrificial bonds and nano-clay into elastomers is to achieve effective dispersion. We recently reported an exfoliated nano-clay/rubber nanocomposite by an in-situ polymerization method [34,35]. The in-situ intercalated nano-clay was well dispersed in the rubber matrix with a layer-by-layer structure. OMMT, as an organic modified nano-clay, is composed of a nano-thickness silicate layer with negative charges on its surface. If there are positive charges on the molecular chain, it will interact with OMMT by ionic interactions, thereby enhancing the mechanical properties of 1,2-PB theoretically. Moreover, it has been proven that Zinc dimethacrylate (ZDMA) containing massive - $(\mathrm{COO})_{2} \mathrm{Zn}$ ion pairs can graft onto rubber molecular chains through a peroxide-induced vulcanization system [36,37].

In this work, we incorporated ZDMA-induced ionic sacrificial bond into an in-situ intercalated OMMT /1,2-PB nanocomposite (in-situ-NC) to reinforce the covalently cross-linked elastomer by the interaction of ionic bond and nano-clay. The OMMT layers mostly exfoliated by in-situ polymerization served as bricks and the ionic bonds which could rupture before covalent bond acted as mortar to enhance the strength of the resulting polymer. As expected, such reinforcing engineering brought excellent properties to 1,2-PB.

\section{Experimental Section}

\subsection{Materials}

1,3-Butadiene (Bd) and n-hexane were offered by Qilu Petrochemical Corp., Zibo, China. 1,3-Butadiene was dried over activated aluminum oxide and distilled twice prior to use. n-Hexane was distilled with $67-70^{\circ} \mathrm{C}$ fraction for later use. Na-MMT with the cation exchange capacity (CEC) of 60 70 mmol per $100 \mathrm{~g}$ was supplied by Zhejiang Fenghong Clay Chemical Co., Huzhou, China. Cetyl trimethyl ammonium bromide $(\mathrm{CTAB})$, silver nitrate $\left(\mathrm{AgNO}_{3}\right)$, ethyl alcohol, and hydrochloric acid were purchased from Tianjin Bodi Chemical Co., Ltd., Tianjin, China. The main catalyst (Mo) and co-catalyst (Al) for coordination polymerization were prepared as reported in our previous work [38]. Nitrogen was supplied by Qingdao Heli Chemical Factory, Qingdao, China. Zinc dimethacrylate (ZDMA) was purchased from J\&K China Chemical Ltd., Beijing, China. Dicumyl peroxide (DCP) was used as the initiator and was supplied by Shanghai Shanpu chemical Co., Ltd.,Shanghai, China.

\subsection{Preparation of $O M M T$}

MMT (20 g) was dispersed into $1 \mathrm{~L}$ deionized water, heated to $80^{\circ} \mathrm{C}$ and stirred for $2 \mathrm{~h}$. Thereafter $18 \mathrm{mmol}$ of CTAB in water solution was slowly added to the MMT suspension and stirred for $3 \mathrm{~h}$ with the $\mathrm{PH}$ value being adjusted to 3 to 5 . After the reaction was finished, the suspension was filtered with deionized water, until there was no bromide ion detected by $\mathrm{AgNO}_{3}$ with a concentration of $0.1 \mathrm{M}$. Then the product was dried under vacuum at $80^{\circ} \mathrm{C}$ to constant weight and ground to powder. 


\subsection{Preparation of In-Situ-NC}

Under a nitrogen atmosphere, a certain amount of OMMT and Bd solution in n-hexane (with a concentration of $2.6 \mathrm{M}$ ) were firstly added into a reactor and heated to $60{ }^{\circ} \mathrm{C}$. Then, the main catalyst (Mo/Bd by a molar ratio of $2 \times 10^{-3}$ ) and co-catalyst ( $\mathrm{Al} / \mathrm{Mo}$ by a molar ratio of 30 ) were subsequently injected into the reactor to react for $6 \mathrm{~h}$. Then the polymerization was terminated by ethyl alcohol. After the termination of the polymerization, the resulting nanocomposite was ultimately poured into the boiling water to precipitate and remove most of the main catalyst and co-catalyst. Then the precipitates were dried in vacuum at $80{ }^{\circ} \mathrm{C}$ to a constant weight. The resulting in-situ intercalated OMMT/1,2-PB nanocomposite was named as in-situ-NC.

By contrast, 1,2-PB was fabricated via the polymerization process mentioned above but without OMMT, and then the resulting polymer was physically blended with OMMT (wt \% being of 4 in Bd). The resulting physical blended OMMT/1,2-PB nanocomposite was named as ph-NC.

\subsection{Preparation of In-Situ-NC/ZDMA}

In-situ-NC or ph-NC was compounded with ZDMA in a two-roll mill, respectively. Then the samples were subjected to compression molding at $135^{\circ} \mathrm{C}$. The formulations are listed in Table S1. The resultants were coded as in-situ-NC-x and ph-NC-x, respectively, where $\mathrm{x}$ was the mass fraction of ZDMA to NC.

\subsection{Characterization}

\subsubsection{X-ray Diffraction (XRD)}

XRD patterns were carried out using a Rigaku D/max RB X-ray diffractometer (Tokyo, Japan) with the diffraction angles from $1^{\circ}$ to $10^{\circ} . \mathrm{CuK}_{\alpha}(\lambda=0.154 \mathrm{~nm})$ was used as an $X$-ray source with a generator voltage of $40 \mathrm{kV}$ and a current of $100 \mathrm{~mA}$.

\subsubsection{Gel Permeation Chromatography (GPC)}

The molecular weight and molecular weight distribution of 1,2-PB and the in-situ-NC were characterized with a Waters Co. Maxims 1515 GPC instrument (Milford, MA, USA). The GPC measurements were conducted by injecting $100 \mu \mathrm{L}$ sample solution at a flow rate of $1.0 \mathrm{~mL} / \mathrm{min}$ at room temperature with tetrahydrofuran as the leaching solvent. The specimen concentration was $1 \mathrm{mg} / \mathrm{mL}$.

\subsubsection{Fourier Transform Infrared Spectroscopy (FTIR)}

FTIR was obtained on a Nicolet FTIR-Magna-750 spectrophotometer (Thermo Nicolet Corporation, Madison, WI, USA) in the range of $4000-400 \mathrm{~cm}^{-1}$ by total reflection mode.

\subsection{4. ${ }^{1} \mathrm{H}$ Spectra of Nuclear Magnetic Resonance $\left({ }^{1} \mathrm{H} \mathrm{NMR}\right)$}

${ }^{1} \mathrm{H}$ spectra of NMR were measured on a Bruker Ultra-Shield ${ }^{\mathrm{TM}}$ spectrometer (Karlsruhe, Germany) at $500 \mathrm{MHz}$ with deuterated chloroform as the solvent and tetramethylsilane as an internal chemical shift reference.

\subsubsection{Scanning Electron Microscopy (SEM)}

SEM analysis was performed on a JMS-6700F (JEOL, Mitaka, Japan) scanning microscope equipped with an energy-dispersive X-ray (EDX ISIS 300, Oxford, UK) microanalytical system. The samples were cryo-fractured in liquid nitrogen prior to SEM testing. 


\subsubsection{Energy Dispersive Spectroscopy (EDS)}

The EDS spectra and EDS maps were carried out by energy dispersion spectroscopy (E-max, Hitachi, Tokyo, Japan).

\subsubsection{Transmission Electron Microscopy (TEM)}

For the observation of transmission electron microscopy (TEM), the specimens were ultramicrotomed into thin pieces of $100 \mathrm{~nm}$ in thickness with UC7-532319 (Leica, Solms, Germany) microtome under a liquid nitrogen atmosphere. Then, the observations were carried out on a JEM-3010 (UHR, JEOL, Japan) transmission electron microscope at an acceleration voltage of $100 \mathrm{KV}$.

\subsubsection{Crosslinking Density}

Samples were immersed in toluene at room temperature for $72 \mathrm{~h}$ to achieve their swollen equilibrium. Then, the swollen samples were blotted with tissue paper to remove the excess toluene and immediately weighed. Finally, the samples were dried at $80^{\circ} \mathrm{C}$ to constant weight. The crosslinking density was determined from the Flory-Rehner equation [39]:

$$
-\left[\ln \left(1-\Phi_{r}\right)+\Phi_{r}+\chi \Phi_{r}^{2}\right]=V_{0} n\left(\Phi_{r}^{\frac{1}{3}}-\Phi_{r} / 2\right)
$$

where $\Phi_{r}$ is the volume fraction of the rubber in the swollen mass, $V_{0}$ is the molar volume of the toluene which is $106.2 \mathrm{~cm}^{3}, n$ is the crosslinking density, and $\chi$ is the Flory-Huggins polymer-solvent interaction term, which is 0.393 for toluene [40]. The $\Phi_{r}$ was obtained according to Bala et al. [41]

$$
\Phi_{r}=\frac{m_{2} / \rho_{2}}{m_{2} / \rho_{2}+\left(m_{1}-m_{2}\right) / \rho_{1}}
$$

where $m_{1}$ and $m_{2}$ are the masses of the swollen sample before and after dried, respectively. $\rho_{1}$ and $\rho_{2}$ are the densities of toluene $\left(\rho_{1}=0.865 \mathrm{~g} / \mathrm{cm}^{3}\right)$ and rubber, respectively.

\subsubsection{Differential Scanning Calorimetry (DSC)}

Glass transition temperature $\left(T_{\mathrm{g}}\right)$ was conducted using a TA DSC-Q20 thermal analyzer, Milford, MA, USA, over a temperature range from -80 to $40^{\circ} \mathrm{C}$ at a heating rate of $10^{\circ} \mathrm{C} / \mathrm{min}$.

\subsubsection{Dynamic Mechanical Properties (DMA)}

Dynamic mechanical properties were performed by DMA Q800 (TA, Milford, MA, USA) under temperature sweeping mode from -60 to $80^{\circ} \mathrm{C}$ at a heating rate of $3{ }^{\circ} \mathrm{C} / \mathrm{min}$, an amplitude of $10 \mu \mathrm{m}$ and a frequency of $1 \mathrm{~Hz}$.

\subsubsection{Stress-Relaxation Experiment}

Under different temperatures $\left(40,60,80\right.$, and $\left.100{ }^{\circ} \mathrm{C}\right)$, samples were quickly stretched to $50 \%$ and maintained for $10 \mathrm{~min}$. Then the stress was reduced to zero, and the residual deformation was measured for $10 \mathrm{~min}$.

\subsubsection{Hysteresis Loss}

The loading-unloading cycles were performed on a DMA Q800 (TA, Milford, MA USA) instrument with an extension rate of 50\%/ $\mathrm{min}$ at room temperature. In each cycle, the sample was stretched to $100 \%$ strain. After the second cycle, the sample was heated at $80^{\circ} \mathrm{C}$ for $10 \mathrm{~min}$ and then cooled at $25^{\circ} \mathrm{C}$ for $10 \mathrm{~min}$ to heal the dynamic network, followed by another loading-unloading cycle. 


\subsubsection{Dielectric Performance}

Dielectric performance was performed on an Alpha-A (Novocontrol Technologies, Montabaur, Germany) novocontrol under frequency sweeping mode from $10^{7}$ to $10^{-2} \mathrm{~Hz}$ with a voltage of $1 \mathrm{~V}$ at room temperature. The samples were subjected to gold sputtering for $100 \mathrm{~s}$ before testing.

\subsubsection{Physical and Mechanical Properties}

Tensile and tear strength were tested on a GT-AT-7000M (Taiwan Gotech, Taiwan, China) electronic tension testing machine at a speed of $500 \mathrm{~mm} / \mathrm{min}$. Hardness test was performed on a shore A hardness test instrument according to ISO 7619-1:2010. DIN abrasion test was performed on a GT-7012-D abrasion testing machine according to ISO 4649-2010.

\section{Results and Discussion}

\subsection{In-Situ Intercalation of OMMT}

Organically modified montmorillonite (OMMT) with lower surface energy and larger interplanar spacing exhibits better dispersity in organic materials than pure montmorillonite (Na-MMT). XRD patterns of Na-MMT and OMMT were shown in Figure 1. The diffraction angle of Na-MMT was $6.9^{\circ}$ corresponding to an interlayer spacing of $1.24 \mathrm{~nm}$ according to Bragg's equation $(2 d \sin \theta=n \lambda)$. After the organic modification, the diffraction angle of OMMT was $2.21^{\circ}$, indicating that the interlayer spacing was enlarged to $4.01 \mathrm{~nm}$. Then the OMMT was in-situ intercalated with Bd via Mo-catalyzed coordination polymerization. The relationship between the conversion rate of $\mathrm{Bd}$ monomer and the mass fraction of OMMT was shown in Figure S1 (Supplementary Information). With the increasing mass fraction of OMMT, the conversion rate of Bd decreased and became less than $30 \%$ when the OMMT was more than $6 \mathrm{wt} \%$, because the anions on OMMT would deactivate the Mo-catalyst. To ensure a relative higher conversion rate and more intercalated structure, the mass fraction of OMMT for the following experiments was settled as $4 \%$. Subsequently, the resulting in-situ OMMT/1,2-PB nanocomposite (in-situ-NC) was subjected to XRD analysis. The OMMT in the in-situ-NC showed no peak, which indicated that OMMT was mostly exfoliated as individual OMMT layers in the 1,2-PB matrix. Some small OMMT aggregates still existed in in-situ-NC (as shown by TEM images) but showed no peak. That was attributed to the fact that the amount of the OMMT aggregates and the number of the layers constituting these aggregates were low. Consequently, the small aggregates might be embedded by the rubber matrix and were difficult to detect. By contrast, the physical blended OMMT /1,2-PB nanocomposite (ph-NC) showed a peak at 2.1 $1^{\circ}$, demonstrating that the OMMT was stacked.

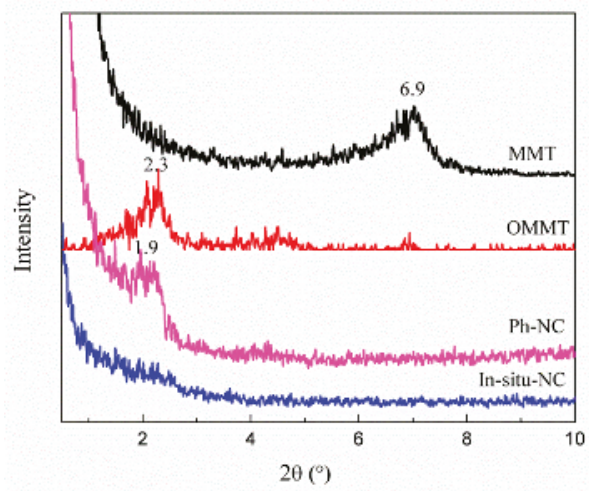

Figure 1. X-ray diffraction patterns of montmorillonite (MMT), organic MMT and 1,2-PB nanocomposite (ph-NC), and in-situ-NC. 
The degree of polymerization and molecular weight distribution were determined by gel permeation chromatography (GPC, Figure S2). The degree of polymerization of 1,2-PB and in situ NC was 12,777 and 16,703 , respectively. For in-situ-NC, the degree of polymerization is larger, because the polymerization is conducted among OMMT layers, in which partial chain transfer reaction was inhibited. Therefore, the life of the active center was prolonged and ultimately resulted in higher molecular weight. The molecular weight distribution of 1,2-PB and in-situ-NC was 2.91 and 2.76, respectively, demonstrating that the OMMT had little effect on it.

The 1,2-unit content of 1,2-PB and in-situ-NC was calculated by NMR spectra (Figure 2). The signals at $\delta=4.9, \delta=5.3$ and $\delta=5.5 \mathrm{ppm}$ were attributed to the olefinic protons of $=\mathrm{CH}_{2}$ of 1,2-unit, $-\mathrm{CH}=$ of 1,4-unit and - $\mathrm{CH}=$ of 1,2-unit, respectively. The 1,2-unit content (C) was calculated according to the following equation:

$$
C=\frac{A_{\delta=4.9}}{A_{\delta=4.9}+\left(A_{\delta=5.3 \sim 5.5}-1 / 2 \times A_{\delta=4.9}\right)} \times 100 \%
$$

where $A$ was the integral area of the corresponding signal peak. After in-situ intercalated with OMMT, the 1,2-unit content of the NC was $83 \%$ which was the same as that of unmodified 1,2-PB. The results of FTIR and ${ }^{1} \mathrm{H}$ NMR showed that, although the negative ions on the surface of OMMT may devitalize the Mo catalyst, it couldn't change the structure of active centers in this coordinative polymerization procedure.

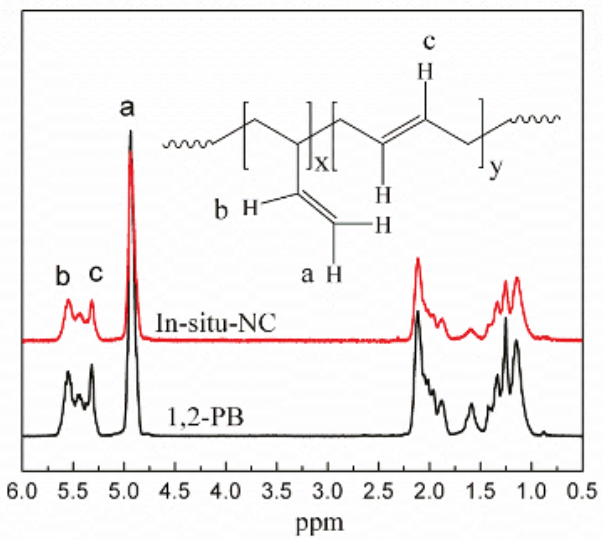

Figure 2. ${ }^{1} \mathrm{H}$ Nuclear Magnetic Resonance (NMR) spectra of 1,2-PB and in-situ-NC.

\subsection{Construction of Multi-Ionic Supramolecular Network}

For chemically cross-linked elastomers, a weak or transient network could be an effective strategy to endow them with enhanced mechanical properties and unexpected functionality. The weak or transient junctions can sustain and sacrifice an initial load and then preferentially break before the rupture of the covalent network of elastomers. In this effort, ZDMA was employed to construct sacrificial bonds anchoring between the rubber skeleton and the OMMT layer. ZDMA was grafted onto the polymer molecular chains by the reaction of the double bond between ZDMA and 1,2-PB (Scheme 1) to incorporate $-(\mathrm{COO})_{2} \mathrm{Zn}$ groups into rubber matrix. Then the Zinc ion $\left(\mathrm{Zn}^{2+}\right)$ was able to joint with the negative ion on OMMT to form OMMT-polymer ionic bonding or with the negative ion on an adjacent polymer to form inter-chain and intra-chain ionic bonding. Additionally, $-(\mathrm{COO})_{2} \mathrm{Zn}$ groups could aggregate together to create a small ion cluster. Therefore, OMMT-polymer ionic bonding, inter-chain, and intra-chain ionic bonding together with ionic cluster constituted a multi-ionic supramolecular 
network (Scheme 2). During stretching, the multi-ionic network would rupture before the covalent network and then was reconstructed after unloading.

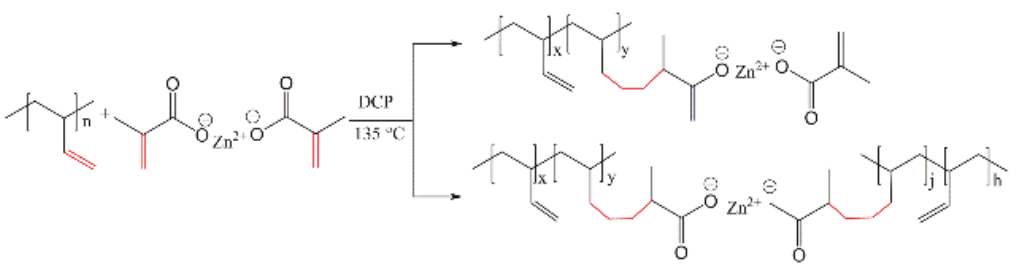

Scheme 1. Reaction between 1,2-PB and zinc methacrylate (ZDMA).

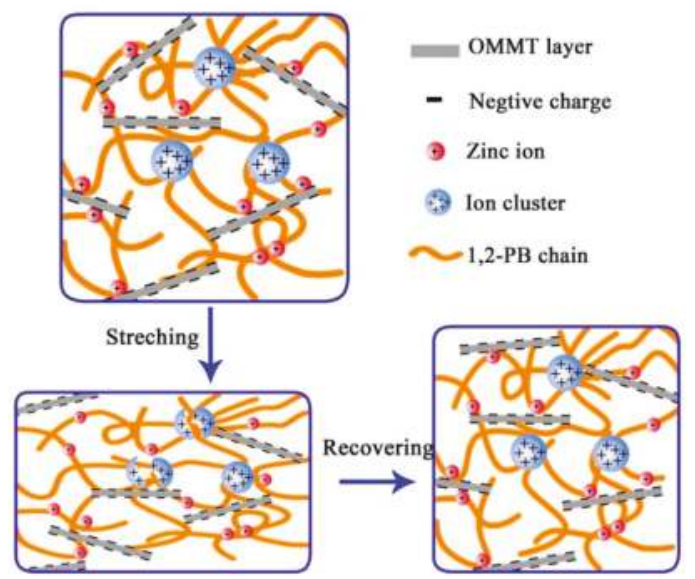

Scheme 2. Proposed mechanism of the rupture and reconstruction of sacrificial bonds in the multi-network system of 1,2-PB.

The organic modification of MMT could be further verified by FTIR analysis. As shown in Figure 3a, after organic modification, new peaks appeared at 2918 and $2848 \mathrm{~cm}^{-1}$, respectively, which were attributed to the asymmetric stretching vibrations of methyl and methylene groups of CTAB. The absorption peaks at 1089 and $1048 \mathrm{~cm}^{-1}$ corresponded to the stretching vibration band of Si-O in MMT. In comparison with ph-NC/ZDMA40, the peak intensity of in-situ-NC/ZDMA40 at 1089 and $1048 \mathrm{~cm}^{-1}$ was stronger, resulting from OMMT tending to aggregate by the physical blending method while it was mostly exfoliated by the in-situ method.
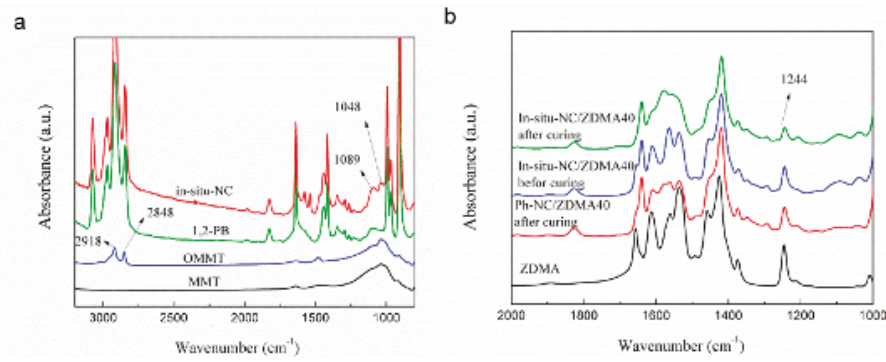

Figure 3. FTIR spectra of (a) MMT, OMMT, 1,2-PB, and in-situ-NC, (b) ZDMA, in-situ-NC/ZDMA40 before and after curing, and ph-NC/ZDMA40. 
Moreover, the exfoliated OMMT layers would leave a more exposed Si-O bond. Additionally, the formation of a covalent linkage between the ZDMA and the double bond on 1,2-PB was illustrated by FTIR analysis (Figure 3b). In the case of ZDMA, the absorption peak at $1244 \mathrm{~cm}^{-1}$ belongs to the vibration band of $\mathrm{C}-(\mathrm{C}=\mathrm{O})-\mathrm{O}$ conjugated with $\mathrm{C}=\mathrm{C}$ double bonds. The relative intensity of in-situ-NC/ZDMA40, after curing, was obviously weak at $1244 \mathrm{~cm}^{-1}$ due to the reaction of $\mathrm{C}=\mathrm{C}$ double bonds. Moreover, more ZDMA was grafted onto 1,2-PB molecular chains for in-situ-NC according to the weaker peak intensity at $1244 \mathrm{~cm}^{-1}$. The main reason was that the negative ions on the surface of OMMT layers led to a better dispersity of ZDMA.

However, the crosslinking of 1,2-PB double bonds and the grafting of ZDMA is a pair of competitive reactions. In this work, we controlled the crosslinking density of covalent and ionic networks by adjusting reaction temperature and time. The curing curves of the in-situ-NC/ZDMA40 under different temperatures were shown in Figure 4. The increased torque value is usually considered to represent the evolution of crosslinking network in rubbers [42]. With the increase in temperature, the torque rate gradually increased because of a shorter half-life period of DCP. However, at a high temperature it would be difficult to control the rate of crosslinking, and at a low temperature it would be difficult to graft ZMDA. Thus, $135{ }^{\circ} \mathrm{C}$ was selected as the curing temperature for the following experiments. Samples were swollen in toluene for $72 \mathrm{~h}$ to calculate the total crosslinking density. To destroy the ionic crosslinks, samples with the same size were swollen in the mixture of toluene/hydrochloric acid/ethyl alcohol for $72 \mathrm{~h}$ to calculate the covalent crosslinking density [36,37]. Then the ionic crosslinking density could be determined by subtraction of the covalent crosslinking density from the total cross-link density. Corresponding digital photographs of in-situ-NC/ZDMA40 cured with different times were shown in Figure S3. It is clear that the swelling volume in the mixture of toluene/hydrochloric acid/ethyl alcohol was larger than that in the toluene. This distinctly evidenced that the supramolecular network was destroyed by the hydrochloric acid, thereby resulting in larger swelling volume. The statistical evidence also demonstrated the existence of the ionic network. As shown in Figure 5a, both covalent and ionic crosslinking density increased with the increasing curing time and the ionic crosslinking density was higher than the covalent crosslinking density when the curing time exceeded $10 \mathrm{~min}$. A high crosslinking density corresponds to a high tensile strength but has a low elongation at break (Figure S4). Consequently, the samples cured at $135^{\circ} \mathrm{C}$ for $10 \mathrm{~min}$ could bring about a better reinforcing effect.
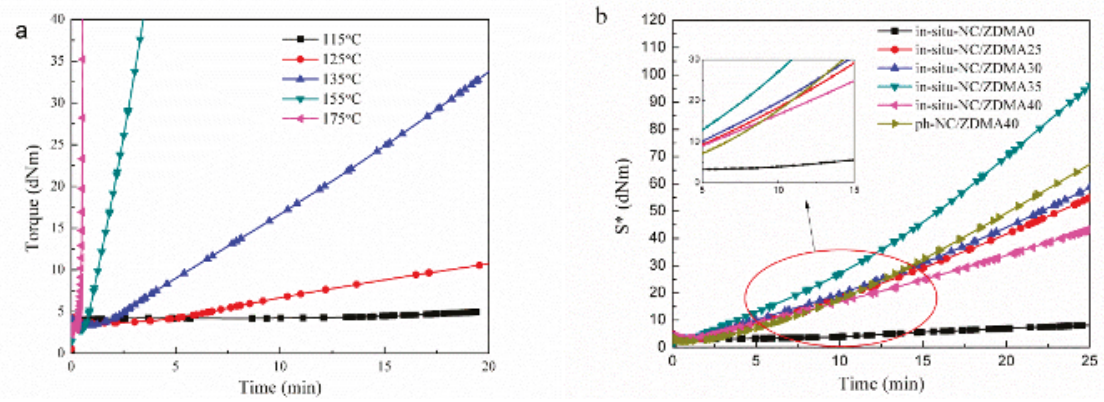

Figure 4. (a) Curing curves of in-situ-NC/OMMT40 at $115,125,135,145$, and $155^{\circ} \mathrm{C}$, (b) curing curves of in-situ-NC/ZMDAs and ph-NC/ZDMA40 at $135^{\circ} \mathrm{C}$.

As shown in Figure $5 \mathrm{~b}$, the ionic crosslinking density of ph-NC/ZDMA40 was $0.07 \times 10^{-4} \mathrm{~mol} / \mathrm{cm}^{3}$ was significantly lower than that of in-situ-NC/ZDMAs due to the non-exfoliated OMMT. Under the same curing condition, the covalent crosslinking density for in-situ-NC was almost zero and for in-situ-NC/ZDMAs was more than $0.8 \times 10^{-4} \mathrm{~mol} / \mathrm{cm}^{3}$. With the increasing mass fraction of ZDMA, both ionic and covalent crosslinking densities for 
in-situ-NC/ZDMAs increased at first and then decreased. The increasing trend illustrated that ZDMA not only determined the formation of the ionic network but also affected the generation of covalent bond for in-situ-NC/ZDMA because the - $(\mathrm{COO})_{2} \mathrm{Zn}$ groups on ZDMA might accelerate the decomposition of DCP. Therefore, the covalent crosslinking density increased significantly for ph-NC/ZDMA40 and in-situ-NC/ZDMAs. However, too much ZDMA in in-situ-NC/ZDMAs is likely to agglomerate into particles which embed DCP and thereby results in the homopolymerization of ZDMA to form poly-ZDMA (PZDMA). Accordingly, the reaction rate for the grafting of ZDMA and the crosslinking of 1,2-PB decreased for in-situ-NC/ZDMA40. Additionally, the total crosslinking density could be further confirmed by the torque value in a curing curve (Figure $4 \mathrm{~b}$ ). In short, the addition of ZMDA into the in-situ-NC could accelerate the formation of the covalent network and construct strong ionic network at the same time.
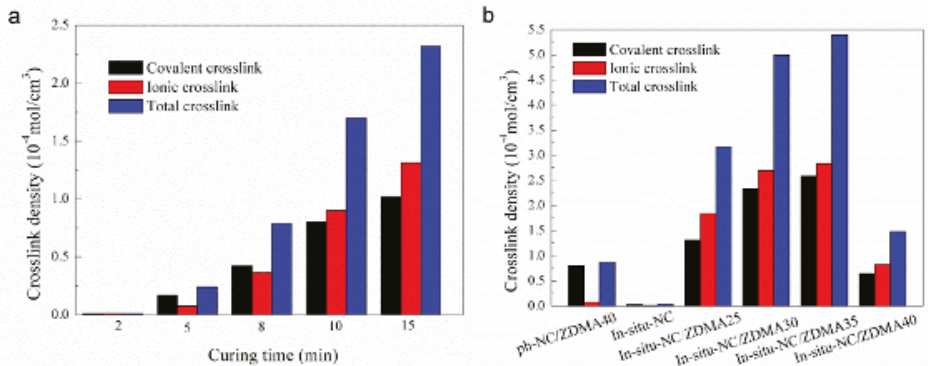

Figure 5. Crosslinking density of (a) in-situ-NC/ZDMA40 under different curing times, (b) Crosslinking density of in-situ-NC, in-situ-NC/ZDMAs, and ph-NC/ZDMA40.

The stress-strain curves of in-situ-NC, in-situ-NC/ZDMAs, and ph-NC/ZDMA40 were presented in Figure 6a. In-situ-NC/ZDMA exhibited high breaking stress resulting from the high total crosslinking density. The reinforcing effect of the ionic network could be verified by the stress-strain curves of in-situ-NC/ZDMA40 and ph-NC/ZDMA40. Considering a similar covalent crosslink, the tensile strength of in-situ-NC/ZDMA40 was significantly higher because of higher ionic crosslinking density (Figure 6b). Nevertheless, the elongation at break of in-situ-NC/ZDMAs was relatively low which was triggered by the low mobility of the polymer chain restricted by covalent as well as ionic crosslinks.
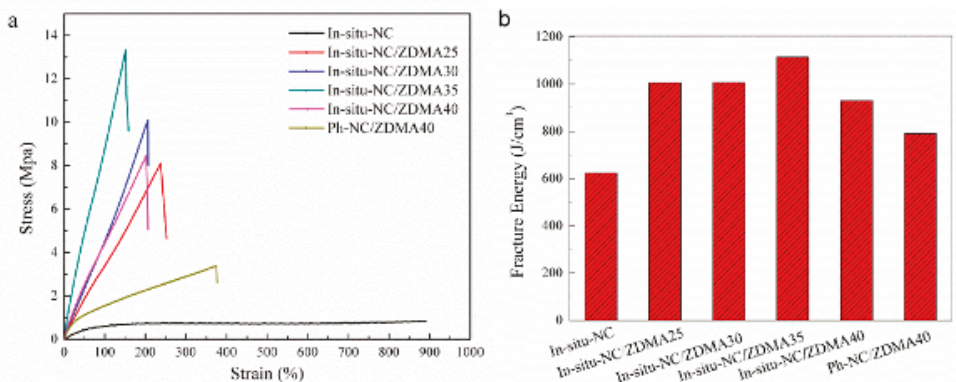

Figure 6. (a) Stress-strain curves of and (b) fracture energy of in-situ-NC, in-situ-NC/ZDMAs, and ph-NC/ZDMA40.

The dispersion morphologies of OMMT and ZDMA in 1,2-PB matrix by in-situ intercalated and physical blending methods were studied by SEM and TEM. As shown in Figure 7a, several aggregates were marked with white ellipses and some fibrils were highlighted with arrows. According to EDS spectra (Figure S5), O, K, Na, Al, and Si elements appeared in these aggregates which indicated 
a composition of OMMT. C, O, Zn elements appeared in those fibrils manifesting that they were composed by ZDMA. Moreover, the composition of these aggregates and fibrils were further verified by EDS maps (Figures S6 and S7), in which the distribution of C, O, Zn, and Si atoms were well consistent with the corresponding SEM image. Therefore, for the in-situ intercalated OMMT, it couldn't be fully exfoliated leaving a few small aggregates in the polymer matrix. Such small OMMT aggregates have little effect on the polymer. For in-situ-NC/ZDMA25 (Figure 7c,d), the ZDMA was well dispersed in the 1,2-PB matrix without any agglomerated fibrils, which was attributed to the fact that the well exfoliated OMMT would facilitate the dispersion of ZDMA via the ionic interaction. However, the ZDMA began to agglomerate with each other when the fraction of ZDMA reached $40 \mathrm{phr}$ (Figure 7e,f). The ZDMA in ph-NC/ZDMA40 (Figure 7g,h), by contrast, formed agglomerates more easily due to the poor dispersity of OMMT by the physical blending method.
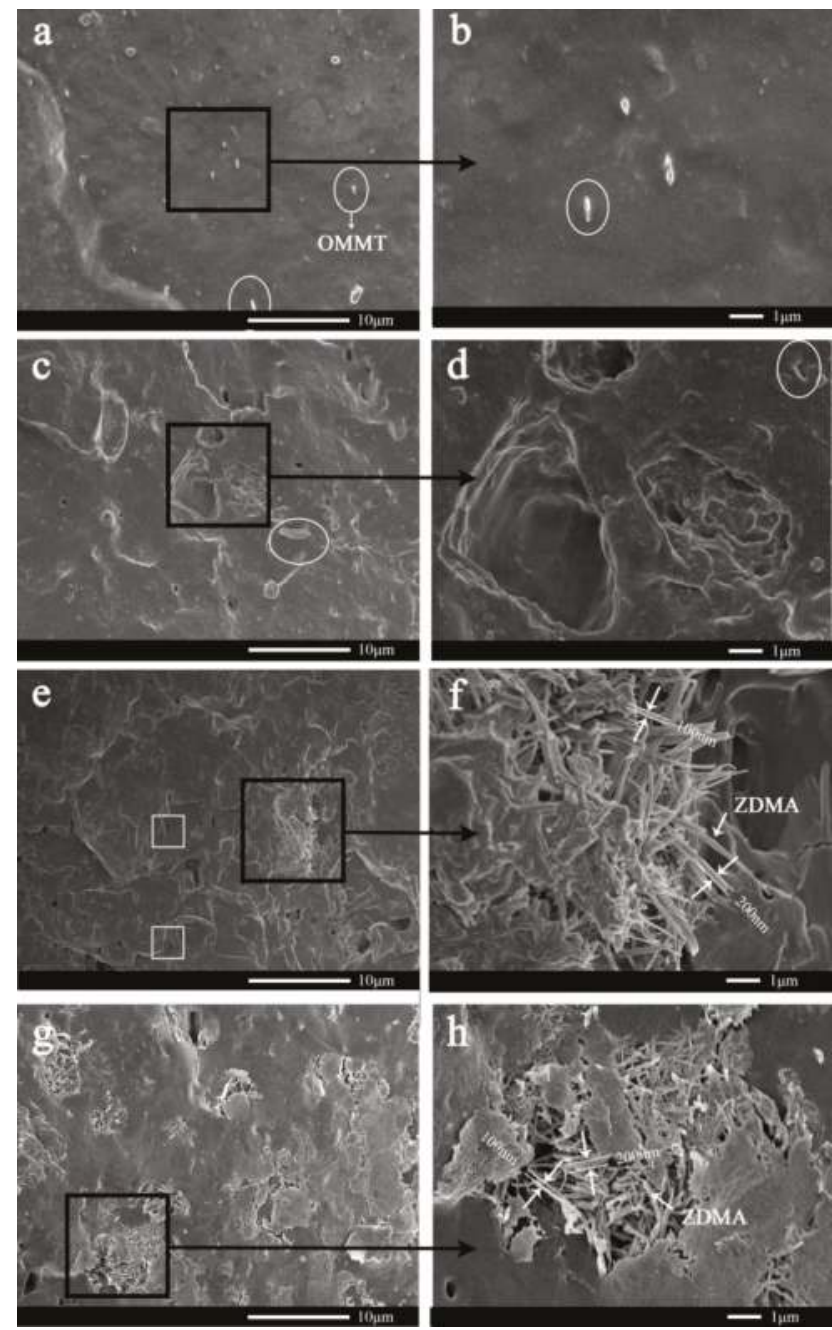

Figure 7. SEM images of (a,b) in-situ-NC, (c,d) in-situ-NC/ZDMA25, (e,f) in-situ-NC/ZDMA40, and $(\mathbf{g}, \mathbf{h})$ ph-NC/ZDMA40. 
The TEM images were shown in Figure 8. In the case of the nanocomposites prepared by the in-situ method, OMMT in the 1,2-PB matrix (Figure 8a) was mostly exfoliated with a few small OMMT aggregates stacked by several layers. However, in the case of the nanocomposite prepared by the physical blending method, OMMT tended to be large aggregates stacked by dozens of layers (Figure 8d). ZDMA was dispersed into in-situ-NC/ZDMA25 (Figure 8b) in nanoscale without agglomerates which was consistent with the result of SEM. ZDMA aggregated to incompact lumps in in-situ-NC/ZDMA40 (Figure 8c) whereas it aggregated to compact chunks in ph-NC/ZDMA40 (Figure 8d), which was in accordance with the analysis of crosslinking density. These large agglomerates would act as impurities under loading and decrease the mechanical properties of the resulting polymer.
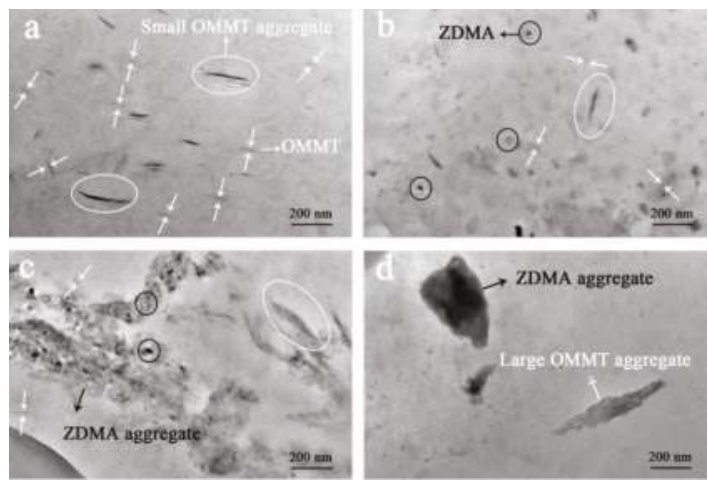

Figure 8. (a) TEM images of in-situ-NC, (b) in-situ-NC/ZDMA25, (c) in-situ-NC/ZDMA40, and (d) ph-NC/ZDMA40.

The polarity of the polymer materials can be discriminated based on the dielectric constant. Generally, a polymer typically exhibits a dielectric constant of less than 10 [43]. As shown in Figure 9, with the same mass fraction of ZDMA, the dielectric constant of ph-NC/ZDMA40 was evidently higher than that of in-situ-NC/ZDMA40, illustrating that the ph-NC displayed a higher polarity. For in-situ-NC/ZDMA40, ZDMA was dispersed in 1,2-PB in nanoscale which was entirely integrated with 1,2-PB/OMMT nanocomposite. Therefore, the polarity of in-situ-NC/ZDMA40 was equivalent to the linear addition of the ZDMA and 1,2-PB/OMMT. However, in the case of ph-NC/ZDMA40, ZDMA dispersed in the 1,2-PB matrix mainly in large aggregates and was easily separated from 1,2-PB and OMMT. Afterward, ZDMA with a higher polarity had a greater effect on the polarity than the linear consideration. Nevertheless, the volume resistivity of in-situ-NC/ZDMA40 was relatively lower because the ion exchange in the ionic network helped charge transport. Consequently, the decrease in volume resistivity indicated that the ionic network could improve the antistatic property of the resulting rubber. The polarity and resistivity results indicated that ZDMA could construct a uniform ionic network in in-situ 1,2-PB/OMMT and it could conduct ion exchange. 

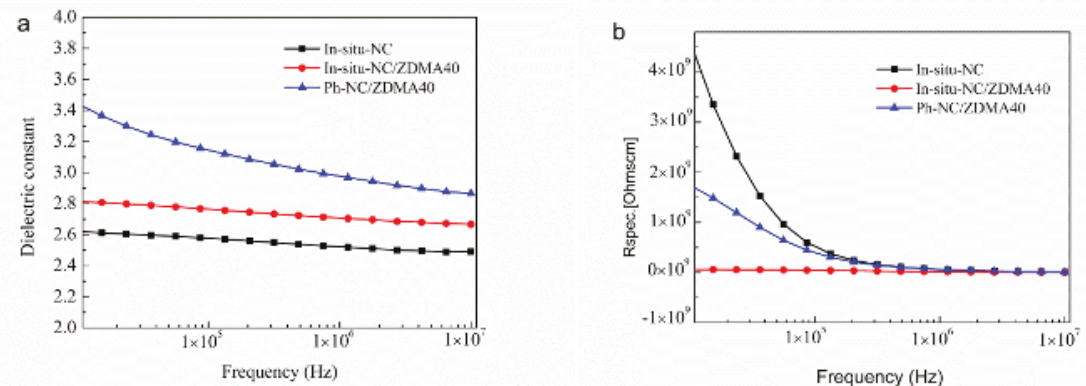

Figure 9. (a) Dielectric constant and (b) volume resistivity of in-situ-NC, in-situ-NC/ZDMA40, and ph-NC/ZDMA40 under the frequency sweeping mode.

DMA testing typically shows a small sample deformation during modulus measurements. In this way, the sample can respond to deformation by changing the chain conformation. When the deformation is accommodated by conformational changes, without bending or breaking of bonds, the relationship between rubbery plateau modulus and crosslinking density (Ve) can be described as follows [44]:

$$
V e=E^{\prime} /(3 \rho \mathrm{RT})
$$

where the modulus $\left(E^{\prime}\right)$ was obtained in the rubbery plateau (usually take the value of peak temperature of loss modulus plus $40^{\circ} \mathrm{C}$ ), $\mathrm{T}$ is the temperature in ${ }^{\circ} \mathrm{K}$ corresponding to the storage modulus value, $\mathrm{R}$ is the gas constant $\left(8.314 \times 10^{7} \mathrm{ergs} / \mathrm{K} \mathrm{mol}\right)$, and $\rho$ is the density of the polymer. Thus the total crosslinking density could be calculated by DMA and the results were shown in Figure S9. The results of the total crosslinking density calculated by DMA were a bit higher than the results calculated by equilibrium swelling method (Figure 5) but the tendency of both methods was similar.

Figure 10a illustrated the temperature dependence of the storage modulus $\left(E^{\prime}\right)$ and loss modulus (E") for NC/ZDMAs. E' of in-situ-NC/ZDMAs was constantly improved with increasing ZDMA fraction because the ionic crosslinks provided a force against deformation. In-situ-NC and ph-NC/ZDMA40 showed a peak of loss modulus at around $-10{ }^{\circ} \mathrm{C}$, while that of in-situ-NC/ZDMAs were at around $0{ }^{\circ} \mathrm{C}$. We suspected that the high crosslinking density restricted the movement of the primary network which would relax at a relatively high temperature. The effect of the ionic network could be proved by the modulus of in-situ-NC/ZDMA40 and ph-NC/ZDMA40. With the almost identical covalent network, the larger ionic network led to a higher storage modulus for in-situ-NC/ZDMA40. The peak value of loss modulus obviously increased for in-situ-NC/ZDMAs since the restricting effect of sacrificial bond on chain segment induced more chain friction during conformational motion. Therefore, the incorporation of ionic network increased the storage and loss modulus at the same time. As shown in Figure 10b, in comparison to in-situ-NC and ph-NC/ZDMA40, the peak value of $\tan \delta$ for in-situ-NC/ZDMAs was significantly suppressed due to the remarkably enhanced $E^{\prime}$ and slightly improved $E^{\prime \prime}$. Moreover, the temperature of $\tan \delta$ peak for in-situ-NC/ZDMA40 was higher than that of ph-NC/ZDMA40 because of the high ionic crosslinking density. For in-situ-NC/ZDMAs, with the increasing mass fraction of ZDMA, the temperature of peak $\tan \delta$ increased at first and then decreased which was consistent with the results of crosslinking density too. In brief, the incorporation of ionic crosslinks led to lower mobility of the polymer chain and resulted in a higher relaxing temperature for polymer chain segment. 

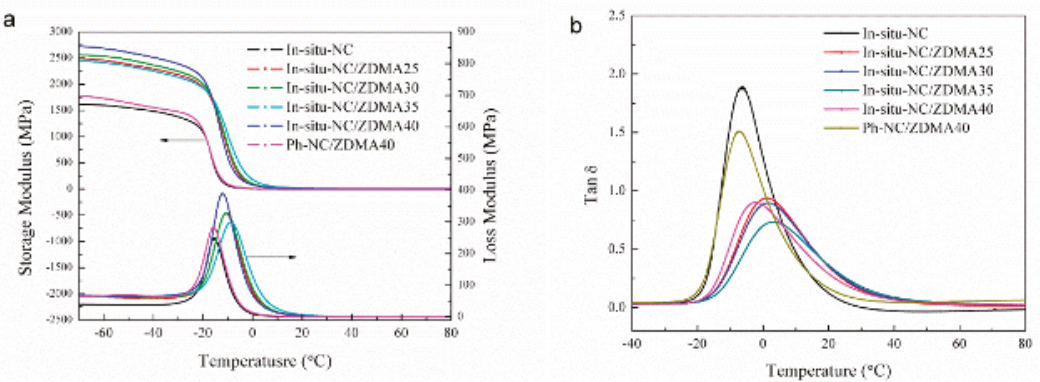

Figure 10. (a) Dependence of $E^{\prime}$ and $E^{\prime \prime}$ and (b) tan $\delta$ versus temperature for in-situ-NC, in-situ-NC/ZDMAs and ph-NC/ZDMA40.

Among the ionic crosslinks, large ionic clusters drastically reduce the dynamics of polymer segments around them and create layers of the trapped polymer with gradually reduced mobility. If the aggregation is significant enough to lead to phase separation, this polymer fraction would show its own glass transition (named ionic transition) [45]. According to the variation of the loss factor with temperature (Figure 10b), there is only one transition of the ionic modified polymers. Additionally, the glass transition temperature $\left(T_{\mathrm{g}}\right.$ ) determined by DSC (Figure 11) also showed no ionic transition in in-situ-NC/ZDMAs. Therefore, the ionic cluster in this system was not strong enough to create phase-separated morphology. Moreover, the $T_{\mathrm{g}}$ of in-situ-NC was about $1.8^{\circ} \mathrm{C}$ higher than that of pure 1,2-PB because of the exfoliated OMMT layers. It could be concluded from the DSC curve of ph-NC/ZDMA40 that ZDMA aggregates would enlarge the inter-chain distance and decrease the $T_{\mathrm{g}}$ of the polymer. For in-situ-NC/ZDMAs, with the increasing mass fraction of ZDMA, the $T_{\mathrm{g}}$ increased first and then decreased slightly. The increase of $T_{\mathrm{g}}$ was attributed to the fact that the ionic crosslinks could enhance the interaction among polymer chains and restrict the movement of the chain segment. Nevertheless, the aggregated ZDMA in in-situ-NC/ZDMA40 reduced the ionic crosslinking density and decreased $T_{\mathrm{g}}$ slightly.

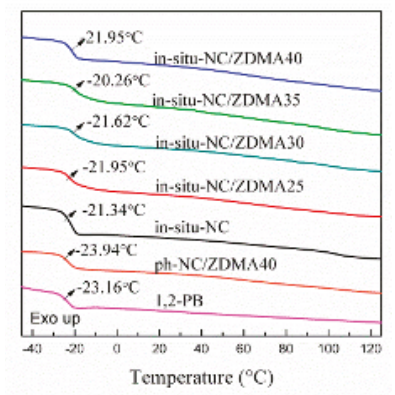

Figure 11. Differential Scanning Calorimetry (DSC) curves of 1,2-PB, in-situ-NC, in-situ-NC/ZDMAs, and ph-NC/ZDMA40.

Sacrificial bond is a general conception of weaker bond in polymer and it is difficult to prove its sacrifice directly. Rief et al. reported that the excellent strength and toughness in natural materials could be characterized by a saw-tooth pattern observed from single-molecule force-extension curves [46]. The saw-tooth pattern was also reported in synthesized polymers with sacrificial bonds and this pattern is a direct evidence of the sacrifice of the sacrificial bonds [17]. Meanwhile, Etienne Ducrot et al. showed that the break of the sacrificial bond could be watched by intensity-colorized images in chemiluminescent cross-linking molecules, which emit light as they break, mapped in real 
time [10]. However, these direct characterization methods could only be carried out by incorporating chemiluminescent molecules or using special modified AFM instrument. Fortunately, some studies reported that the sacrifice of the sacrificial bond could be verified indirectly. Baochun Gou et al. [18,47] demonstrated that the sacrifice of the sacrificial bond could be proved by hysteresis loss and stress relaxation testing. To confirm the sacrifice and reconstruction of the ionic bonds, cyclic tensile tests were performed with stretching to a predefined $100 \%$ strain and the corresponding dissipation of energy was shown in Figure 12. As expected, low hysteresis was observed for in-situ-NC (Figure 12a) and ph-NC/ZDMA40 (Figure 12c) in the first, second, and after heating cycle, which is reasonable for dominance of covalent crosslinks. As for the samples prepared by in-situ polymerization, taking in-situ-NC/ZDMA40 as an example (Figure 12b), significant hysteresis was observed in the first loading-unloading cycle on account of the effect of the ionic network. The dissipation of energy during the loading-unloading test was defined as the area surrounded by the circle and was shown in Figure 12d. The improved dissipation of energy for in-situ-NC/ZDMAs in the first loading-unloading cycle was associated with the rupture of the ionic network. For the second loading, the stress at $100 \%$ strain of in-situ-NC / ZDMA40 was higher than that of the first loading which doesn't exist for in-situ-NC and ph-NC/ZDMA40. We speculated that the stretching orientation of polymer chains and OMMT layers remained after the first unloading due to its slower relaxation rate indicated by $T_{\mathrm{g}}$. The orientation of OMMT layers and polymer chains were in the same direction, which was advantaged for the reconstruction of the ionic network. Meanwhile, the increase in the maximum stress displayed the formation of a denser ionic network induced by the orientation mentioned above. After heating at $80{ }^{\circ} \mathrm{C}$, the orientation of the covalent network of in-situ-NC and ph-NC/ZDMA40 nearly completed self-recovery, and its cyclic tensile curve almost overlapped that of the first cycle. However, in-situ-NC/ZDMA40 showed smaller stress during loading after heating than that of the first cycle before heating. It seems to form a weaker ionic network after heating, which, combining with the denser network by stretching, indicated that the ionic network is sensitive to the conformation of polymer chains. In addition, the samples with other content of ZDMA exhibited similar hysteresis behaviors (Figure S8).
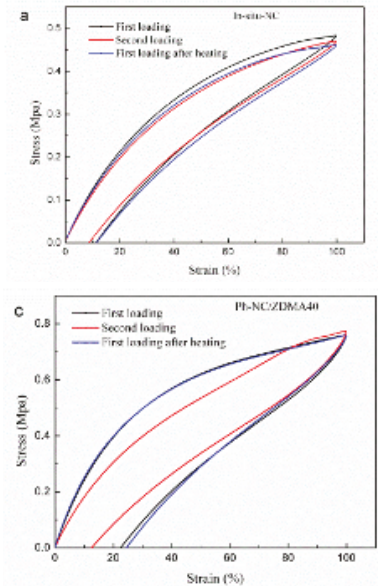

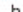

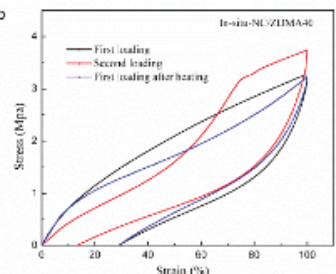

d

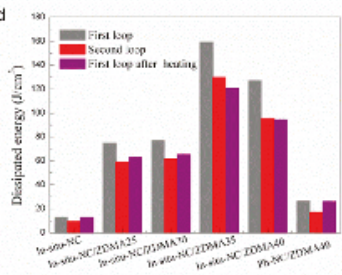

Figure 12. Cyclic tensile curves of (a) in-situ-NC, (b) in-situ-NC/ZDMA40 and (c) ph-NC/ZDMA40 and (d) the corresponding dissipation of energy.

To further illustrate the reinforcing effect of ionic crosslinks, stress relaxation analysis, was performed at $25^{\circ} \mathrm{C}$ (Figure 13a). All the samples were quickly subjected to a strain of $50 \%$, and then the strain was maintained for $600 \mathrm{~s}$. Compared with in-situ-NC and ph-NC/ZDMA40, in-situ-NC/ZDMAs released the applied force much slowly, which implied the protection of ionic 
bonds to the primary network under loading. This relaxing rate was contrary to the result of polymers reinforced by hydrogen bonds and coordination bonds. Baochun Gou et al. [18,47] manifested that the hydrogen bonds and coordination bonds could disassociate under stretching and the relaxing rate was much more rapid than that of the polymer without a sacrificial network. In this effort, the slow stress relaxation rate of in-situ-NC/ZDMAs might result from the reconstruction of the ionic network facilitated by stretching orientation. The stress of in-situ-NC/ZDMA, especially for in-situ-NC/ZDMA35, was a bit higher in the early stage than that of the initial stress, further demonstrating the reconstruction of the ionic network.

a

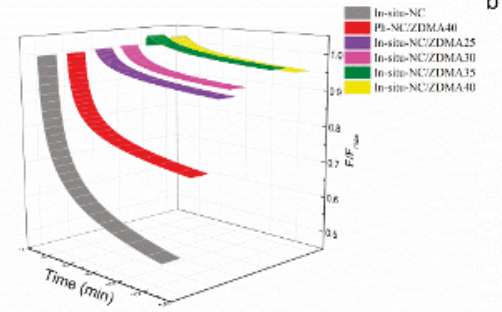

b

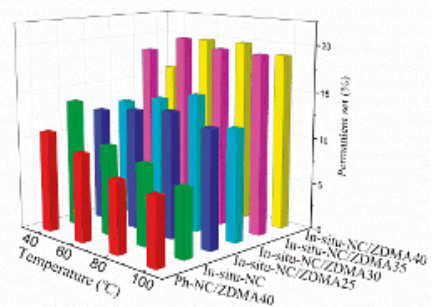

Figure 13. (a) Stress relaxation curves and (b) residual deformation after recovery for 10 min under respective temperatures.

After the relaxation experiment and recovery for $10 \mathrm{~min}$, the residual deformation was measured and was shown in Figure 13b. The residual deformation of in-situ-NC/ZDMA35 was significantly larger than that of the others because of the highest ionic crosslinking density. The ionic crosslinks would break and recombine with the adjacent ions and finally prevent the recovery of the deformed covalent bond. Nevertheless, in-situ-NC/ZDMA40 exhibited higher residual deformation than in-situ-NC/ZDMA25 and 30, which was induced by a relatively low ionic crosslinking density due to PZDMA with low molecular weight. Additionally, as the testing temperature increased, the residual deformation of in-situ-NC and ph-NC/ZDMA40 continually decreased whereas it increased first and then decreased for in-situ-NC/ZDMAs. The deformation of ph-NC/ZDMA40 and in-situ-NC was dominated by the covalent network. At elevated temperatures, the chain segment mobility was improved which resulted in a stronger contractive force, ultimately limiting the residual deformation. However, the deformation of in-situ-NC/ZDMAs was determined by both the covalent and ionic network. Under a quick stretching condition, the partial ionic network was quickly destroyed and was unable to be in-situ reconstructed. Subsequently, the fractured ionic crosslinks reunited with adjacent ions and the reuniting rate increased at elevated temperature, thereby leading to a larger residual deformation. Synchronously, the mobility of the chain segment was improved resulting in a higher contractive force. Therefore, the residual deformation decreased slightly at $100{ }^{\circ} \mathrm{C}$. Overall, the reversible ionic network endowed the polymer with a more plastic property which meant it may be used as a shape memory material.

The mechanical properties of in-situ-NC, in-situ-NC/ZDMAs and ph-NC/ZDMA40 were listed in Table 1. The tensile strength and tear strength of in-situ-NC/ZMDAs were obviously higher than that of ph-NC/ZDMA40 on account of the ionic crosslinks formed between the exfoliated OMMT and the $-(\mathrm{COO})_{2} \mathrm{Zn}$ groups. Nevertheless, the mechanical properties of in-situ-NC/ZDMA40 decreased attributed to the large ZDMA aggregates acting as impurities. Meanwhile, the abrasion resistance of in-situ-NC and in-situ-NC/ZDMAs decreased with the increasing fraction of ZDMA owing to the fact that the ionic contacts decreased the flexibility of the polymer chains. Moreover, the abrasion loss of ph-NC/ZDMA40 was also very high, resulting from the weak interaction of the polymer chain and ZDMA aggregates. 
Table 1. Mechanical properties of in-situ-NC, in-situ-NC/ZDMAs, and ph-NC/ZDMA40.

\begin{tabular}{|c|c|c|c|c|c|c|}
\hline Sample & $\begin{array}{c}\text { ph-NCl } \\
\text { ZDMA40 }\end{array}$ & In-Situ-NC & $\begin{array}{l}\text { In-Situ-NCl } \\
\text { ZDMA25 }\end{array}$ & $\begin{array}{l}\text { In-Situ-NC/ } \\
\text { ZDMA30 }\end{array}$ & $\begin{array}{c}\text { In-Situ-NCl } \\
\text { ZDMA35 }\end{array}$ & $\begin{array}{c}\text { In-Situ-NC/ } \\
\text { ZDMA40 }\end{array}$ \\
\hline Tensile strength (Mpa) & 2.82 & 0.99 & 7.98 & 8.10 & 11.40 & 8.45 \\
\hline Tensile stress at $100 \%$ elongation (MPa) & 1.14 & 0.60 & 4.51 & 3.37 & 5.82 & 4.24 \\
\hline Elongation at break (\%) & 375 & 800 & 205 & 237 & 213 & 202 \\
\hline Tearing strength $(\mathrm{kN} / \mathrm{m})$ & 28.05 & 11.87 & 23.28 & 25.74 & 31.23 & 29.48 \\
\hline Shore A hardness $\left({ }^{\circ}\right)$ & 67 & 45 & 59 & 61 & 66 & 65 \\
\hline Abrasion loss $\left(\mathrm{cm}^{3} \cdot 1.6 \mathrm{~km}^{-1}\right)$ & 0.38 & 0.28 & 0.33 & 0.34 & 0.35 & 0.37 \\
\hline
\end{tabular}

\section{Conclusions}

We developed a nano-clay/elastomer nanocomposite with high tensile strength, tearing strength and low stress relaxation rate which was reinforced by a multi-ionic supramolecular network including OMMT-polymer ionic crosslink, inter-chain, or intra-chain ionic crosslink as well as small ion cluster. The exfoliated OMMT layers prevented the aggregation of ZDMA in nonpolar rubber matrix through ionic interaction between OMMT and ZDMA, and thereby resulted in a uniform ionic network. The ionic network could rupture preferentially during stretching to protect the covalent network and dissipate more energy. Additionally, the ionic crosslinks could be reconstructed and be strengthened through the orientation of OMMT and polymer chain. However, the reversibility of such ionic bonds enlarged the residual deformation of the resulting polymer. In future studies, we will further improve the ionic crosslinking density and construct a stronger sacrificial network to prepare shape memory or self-recovery materials and study the dynamics of ionic crosslinking.

Supplementary Materials: The supplementary materials are available online at http:/ www.mdpi.com/20734360/11/3/492/s1.

Author Contributions: Conceptualization, J.L. and J.H.; methodology, J.L.; software, D.L.; validation, X.Z., D.L. and J.G.; formal analysis, J.L.; investigation, J.L.; resources, J.H.; data curation, X.Z.; writing-original draft preparation, J.L.; writing-review and editing, J.L. and X.W.; visualization, J.L.; supervision, J.H.; project administration, J.L.; funding acquisition, J.H.

Funding: This research was funded by [Natural Science Foundation of Shandong province] grant munber [ZR2016EMM03] and the APC was funded by [Jing Hua].

Conflicts of Interest: The authors declare no conflict of interest.

\section{References}

1. Fratzl, P.; Weinkamer, R. Nature's hierarchical materials. Prog. Mater. Sci. 2007, 52, 1263-1334. [CrossRef]

2. Meyers, M.A.; Chen, P.Y.; Lin, Y.M.; Seki, Y. Biological materials: Structure and mechanical properties. Prog. Mater. Sci. 2008, 53, 1-206. [CrossRef]

3. Satyanarayana, M.S.; Bhowmick, A.K.; Kumar, K.D. Preferentially fixing nanoclays in the phases of incompatible carboxylated nitrile rubber (XNBR)-natural rubber (NR) blend using thermodynamic approach and its effect on physico mechanical properties. Polymer 2016, 99, 21-43. [CrossRef]

4. Mohana, T.P.; Kuriakoseb, J.; Kanny, K. Effect of nanoclay reinforcement on structure, thermal and mechanical properties of natural rubber-styrene butadine rubber (NR-SBR). J. Ind. Eng. Chem. 2011, 17, 264-270. [CrossRef]

5. Carretero-Gonzalez, J.; Retsos, H.; Verdejo, R.; Toki, S.; Hsiao, B.S.; Giannelis, E.P.; Lopez-Manchado, M.A. Effect of Nanoclay on Natural Rubber Microstructure. Macromolecules 2008, 41, 6763-6772. [CrossRef]

6. Espinosa, H.D.; Rim, J.E.; Barthelat, F.; Buehler, M.J. Merger of structure and material in nacre and bone-Perspectives on de novo biomimetic materials. Prog. Mater. Sci. 2009, 54, 1059-1100. [CrossRef]

7. Andreas, W.; Ingela, B.; Jani-Markus, M.; Janne, R.; Lars, B.; Olli, I. Supramolecular control of stiffness and strength in lightweight high-performance nacre-mimetic paper with fire-shielding properties. Angew. Chem. Int. Ed. 2010, 49, 6448-6453.

8. Jackson, A.P.; Vincent, J.F.V.; Turner, R.M. The Mechanical Design of Nacre. Proc. R. Soc. Lond. Ser. 1988, 234, $415-440$. 
9. Li, H.; Oberhauser, A.F.; Fowler, S.B.; Clarke, J.; Fernandez, J.M. Atomic force microscopy reveals the mechanical design of a modular protein. Proc. Natl. Acad. Sci. USA 2000, 97, 6527-6531. [CrossRef]

10. Etienne, D.; Yulan, C.; Markus, B.; Sijbesma, R.P.; Costantino, C. Toughening elastomers with sacrificial bonds and watching them break. Science 2014, 344, 186-189.

11. Damien, M.; Francois, T.; Manuel, H.; Jean-Luc, C.; Ludwik, L. Versatile one-pot synthesis of supramolecular plastics and self-healing rubbers. J. Am. Chem. Soc. 2009, 131, 7966-7967.

12. Philippe, C.; Francois, T.; Corinne, S.Z.; Ludwik, L. Self-healing and thermoreversible rubber from supramolecular assembly. Nature 2008, 451, 977.

13. Chen, Y.; Kushner, A.M.; Williams, G.A.; Guan, Z. Multiphase design of autonomic self-healing thermoplastic elastomers. Nat. Chem. 2012, 4, 467-472. [CrossRef] [PubMed]

14. Kang, J.; Son, D.; Wang, G.I.N.; Liu, Y.; Lopez, J.; Kim, Y.; Jin, Y.O.; Katsumata, T.; Mun, J.; Lee, Y. Tough and Water-Insensitive Self-Healing Elastomer for Robust Electronic Skin. Adv. Sci. 2018, 30, 1706846. [CrossRef] [PubMed]

15. Mark, B.; Liming, T.; Kumpfer, J.R.; Duncan, A.J.; Beyer, F.L.; Fiore, G.L.; Rowan, S.J.; Christoph, W. Optically healable supramolecular polymers. Nature 2011, 472, 334.

16. Kersey, F.R.; Loveless, D.M.; Craig, S.L. A hybrid polymer gel with controlled rates of cross-link rupture and self-repair. J. R. Soc. Interface 2007, 4, 373-380. [CrossRef]

17. Li, C.H.; Wang, C.; Keplinger, C.; Zuo, J.L.; Jin, L.; Sun, Y.; Zheng, P.; Cao, Y.; Lissel, F.; Linder, C. A highly stretchable autonomous self-healing elastomer. Nat. Chem. 2016, 8, 618-624. [CrossRef]

18. Liu, J.; Wang, S.; Tang, Z.; Huang, J.; Guo, B.; Huang, G. Bioinspired engineering of two different types of sacrificial bonds into chemically cross-linked cis-1,4-polyisoprene toward a high-performance elastomer. Macromolecules 2016, 49, 8593-8604. [CrossRef]

19. Kalista, S.J.; Ward, T.C.; Oyetunji, Z. Self-Healing of Poly(Ethylene-co-Methacrylic Acid) Copolymers Following Projectile Puncture. Mech. Adv. Mater. Struc. 2007, 14, 391-397. [CrossRef]

20. Ye, X.; Huang, H.; Peng, X. Synthesis of self-healing waterborne polyurethanes containing sulphonate groups. RSC Adv. 2017, 7, 20093-20100.

21. Bin Ihsan, A.; Sun, T.L.; Kurokawa, T.; Karobi, S.N.; Nakajima, T.; Nonoyama, T.; Roy, C.K.; Luo, F.; Gong, J.P. Self-healing behaviors of tough polyampholyte hydrogels. Macromolecules 2016, 49, 4245-4252. [CrossRef]

22. Lin, S.T.; Takayuki, K.; Shinya, K.; Abu Bin, I.; Taigo, A.; Koshiro, S.; Md Anamul, H.; Tasuku, N.; Ping, G.J. Physical hydrogels composed of polyampholytes demonstrate high toughness and viscoelasticity. Nat. Mater. 2013, 12, 932.

23. Zhang, H.J.; Sun, T.L.; Zhang, A.K.; Ikura, Y.; Nakajima, T.; Nonoyama, T.; Kurokawa, T.; Ito, O.; Ishitobi, H.; Gong, J.P. Tough Physical Double-Network Hydrogels Based on Amphiphilic Triblock Copolymers. Adv. Mater. 2016, 28, 4884-4890. [CrossRef] [PubMed]

24. Burattini, S.; Colquhoun, H.M.; Fox, J.D.; Friedmann, D.; Greenland, B.W.; Harris, P.J.; Hayes, W.M.M.E.; Rowan, S.J. A self-repairing, supramolecular polymer system: Healability as a consequence of donor-acceptor $\pi-\pi$ - stacking interactions. Chem. Commun. 2009, 44, 6717-6719. [CrossRef]

25. Fox, J.; Wie, J.J.; Greenland, B.W.; Burattini, S.; Hayes, W.; Colquhoun, H.M.; Mackay, M.E.; Rowan, S.J. High-strength, healable, supramolecular polymer nanocomposites. J. Am. Chem. Soc. 2012, 134, 5362-5368. [CrossRef] [PubMed]

26. Paramita, D.; Andreas, W. Ionic supramolecular bonds preserve mechanical properties and enable synergetic performance at high humidity in water-borne, self-assembled nacre-mimetics. Nanoscale 2013, 5, 9348-9356.

27. Kai, L.; Lv, Q.; Jing, H. Study on damping properties of HVBR/EVM blends prepared by in situ polymerization. Polym. Test. 2017, 60, 321-325.

28. Jing, H.; Kai, L.; Wang, Z.; Geng, J.; Xin, W. Effect of vinyl and phenyl group content on the physical and dynamic mechanical properties of HVBR and SSBR. J. Appl. Polym. Sci. 2018, 135, 45975.

29. Zhang, W.; Huang, B.; Ai, D.U.; Yao, W.; Yang, S.; Wang, M. Properties of TPI/HVBR/SBR blends. China Rubber Ind. 2002, 49, 69-72.

30. Jinhui, L.; Jieting, G.; Kai, L.; Lingli, Z.; Zhaobo, W.; Jing, H. Effect of electronegativity and steric hindrance of the cocatalyst on the activity and selectivity of butadiene polymerization catalyzed by molybdenum. J. Appl. Polym. Sci. 2019, 136, 46906.

31. Cong, Y.; Ying, L.; Jing, H. Vulcanization formula study on high vinyl polybutadiene rubber synthesized by molybdenum catalyst system. Qilu Petrochem. Technol. 2014, 42, 9-12. 
32. Deng, Z.F.; Guo, L.Y.; Ling, X.U.; Hua, J. Dynamic Property of Molybdenum-catalyzed High 1,2-Polybutadiene Rubber. China Rubber Ind. 2011, 4, 227-230.

33. Wang, L.F.; Wang, W.; Guo, B.S.; Li, M.; Lin, C.Y.; Wang, D.L. Research advance of 1,2-polybutadiene. China Elastom. 2009, 3, 70-75.

34. Hua, J.; Liu, J.; Wang, X.; Yue, Z.; Yang, H.; Geng, J.; Ding, A. Structure and properties of a cis-1,4-polybutadiene/ organic montmorillonite nanocomposite prepared via in-situ polymerization. J. Macromol. Sci. B 2017, 56, 451-461. [CrossRef]

35. Hua, J.; Chen, F.; Tian, Y.; Wang, X.; Cao, K.; Xu, L. Structures and Properties of 1,2-Polybutadiene/Organic Montmorillonite Nanocomposites Prepared by In-situ Polymerization. J. Macromol. Sci. B 2012, 51, 2034-2048. [CrossRef]

36. Xu, C.; Cao, L.; Lin, B.; Liang, X.; Chen, Y. Design of self-Healing supramolecular rubbers by introducing ionic cross-links into natural rubber via a controlled vulcanization. ACS Appl. Mater. Interfaces 2016, 8, 17728-17737. [CrossRef] [PubMed]

37. Xu, C.; Cao, L.; Huang, X.; Chen, Y.; Fu, L. Self-healing natural rubber with tailorable mechanical properties based on ionic supramolecular hybrid network. ACS Appl. Mater. Interfaces 2017, 9, 29363-29373. [CrossRef] [PubMed]

38. Geng, J.; Xin, W.; Jing, H. Grafting of Butadiene-Acrylonitrile Copolymer onto High Vinyl Polybutadiene by Copolymerization. Adv. Polym. Technol. 2016. [CrossRef]

39. Flory, P.J. Statistical Mechanics of Swelling of Network Structures. J. Chem. Phys. 1950, 18, 108-111. [CrossRef]

40. Arroyo, M.; López-Manchado, M.A.; Herrero, B. Organo-montmorillonite as substitute of carbon black in natural rubber compounds. Polymer 2003, 44, 2447-2453. [CrossRef]

41. Bala, P.; Samantaray, B.K.; Srivastava, S.K.; Nando, G.B. Organomodified montmorillonite as filler in natural and synthetic rubber. J. Appl. Polym. Sci. 2004, 92, 3583-3592. [CrossRef]

42. Ping, Z.; Fei, Z.; Yuan, Y.; Shi, X.; Zhao, S. Network evolution based on general-purpose diene rubbers/sulfur/TBBS system during vulcanization (I). Polymer 2010, 51, 257-263.

43. Bai, Y.; Cheng, Z.Y.; Bharti, V.; Xu, H.S.; Zhang, Q.M. High-dielectric-constant ceramic- powder polymer composites. Appl. Phys. Lett. 2000, 76, 3804-3806. [CrossRef]

44. Hill, L.W. Calculation of crosslink density in short chain networks. Prog. Org. Coat. 1997, 31, 235-243. [CrossRef]

45. Basu, D.; Das, A.; Stöckelhuber, K.W.; Jehnichen, D.; Formanek, P.; Sarlin, E.; Vuorinen, J.; Heinrich, G. Evidence for an in situ developed polymer phase in ionic elastomers. Macromolecules 2014, 47, 3436-3450. [CrossRef]

46. Rief, M.; Gautel, M.; Oesterhelt, F.; Fernandez, J.M.; Gaub, H.E. Reversible unfolding of individual titin immunoglobulin domains by AFM. Science 1997, 276, 1109-1112. [CrossRef]

47. Tang, Z.; Jing, H.; Guo, B.; Zhang, L.; Fang, L. Bioinspired engineering of sacrificial metal-ligand bonds into elastomers with supramechanical performance and adaptive recovery. Macromolecules 2016, 49, 1781-1789. [CrossRef]

(C) 2019 by the authors. Licensee MDPI, Basel, Switzerland. This article is an open access article distributed under the terms and conditions of the Creative Commons Attribution (CC BY) license (http:/ / creativecommons.org/licenses/by/4.0/). 
Article

\title{
Modification of Montmorillonite with Polyethylene Oxide and Its Use as Support for $\mathbf{P d}^{0}$ Nanoparticle Catalysts
}

\author{
Guiqing Shu, Jing Zhao, Xiu Zheng, Mengdie Xu, Qi Liu and Minfeng Zeng * \\ Zhejiang Key Laboratory of Alternative Technologies for Fine Chemicals Process, College of Chemistry and \\ Chemical Engineering, Shaoxing University, Shaoxing 312000, China; guiqingshu@163.com (G.S.); \\ jingjingzhao218@sina.com (J.Z.); xiuzheng2019@163.com (X.Z.); joanne7280@163.com (M.X.); \\ lq920819@163.com (Q.L.) \\ * Correspondence: zengmf@usx.edu.cn; Tel.: +86-575-8834-1006
}

Received: 11 March 2019; Accepted: 18 April 2019; Published: 29 April 2019

\begin{abstract}
In this study, montmorillonite (MMT) was modified by intercalating polyethylene oxide (PEO) macromolecules between the interlayer spaces in an MMT-water suspension system. X-ray diffraction results revealed that the galleries of MMT were expanded significantly after intercalation of different loading of PEO. MMT/PEO 80/20 composite was chosen as the support platform for immobilization of Pd species in preparing novel heterogeneous catalysts. After immobilization of Pd species, the interlayer spacing of MMT/PEO (80/20) $(1.52 \mathrm{~nm})$ was further increased to $1.72 \mathrm{~nm}\left(\mathrm{Pd}^{2+} @ \mathrm{MMT} / \mathrm{PEO}\right)$ and $1.73 \mathrm{~nm}\left(\mathrm{Pd}^{0} @ \mathrm{MMT} / \mathrm{PEO}\right)$, confirming the well-immobilization of the Pd species in the interlayer spaces of PEO-modified MMT. High-resolution transmission electron microscopy (HR-TEM) observation results confirmed that Pd nanoparticles were confined inside the interlayer space of MMT and/or dispersed well on the outer surface of MMT. The conversion of $\mathrm{Pd}^{2+}$ to $\mathrm{Pd}^{0}$ species was evidenced by binding energy characterization with $\mathrm{X}$-ray photo electron spectroscopy (XPS). The microstructure variation caused by the Pd immobilization was sensitively detected by positron annihilation lifetime spectroscopy (PALS) studies. The prepared $\mathrm{Pd}^{0} @ \mathrm{MMT} / \mathrm{PEO}(0.2 / 80 / 20)$ catalytic composite exhibits good thermal stability up to around $200{ }^{\circ} \mathrm{C}$, and it showed high activities for Heck reactions between aryl iodides and butyl acrylates and could be recycled for five times. The correlations between the microstructure and properties of the $\mathrm{Pd} @ \mathrm{MMT} / \mathrm{PEO}$ catalytic composites were discussed.
\end{abstract}

Keywords: polyethylene oxide; montmorillonite clays; Pd catalysis; catalytic composite; positron annihilation

\section{Introduction}

The modification of montmorillonite (MMT) with organic polymers has attracted more and more interests for their excellent structural and/or functional performances [1-3]. Many water-soluble polymers, such as polyethylene glycol (PEG) [4], polyethylene oxide (PEO) [5], polyvinyl alcohol (PVA) [6], polyvinyl pyrrolidone (PVP) [7], etc., can be easily intercalated in MMT with the water solution intercalation method. In the MMT/polymer nanocomposites, an obvious increase in the interlayer spacing of MMT is frequently observed, indicating the intercalated polymer chains are well-confined in the narrow galleries between the MMT layers. Meanwhile, the lipophilicity of MMT will be significantly improved after the organic polymer chains intercalated. The prepared MMT/polymer hybrid composites are nowadays frequently applied in many fields, such as preparation of advanced nano-composites, drug delivery, and water treatment, etc. [8-10].

Recently, many researchers shift the attentions towards heterogeneous catalysis topics with use of MMT-based composites [11-14]. In particular, MMT/polymer composites have been recognized 
as good supporting materials for immobilization of transition metal catalysts applied in organic reactions [15-21]. With synergetic processes of ion-exchange with interlayer cations of MMT and complexation with polar polymers, the transition metal cations, like $\mathrm{Pd}^{2+}$ will be easily immobilized on MMT/polymer supports. It has been evidenced that polymers (such as PVA, PVP, chitosan (CS), etc.) modified MMT supported Pd catalytic materials has good comprehensive catalytic performances as applied in Heck and/or Sonogashira coupling reactions. Pd species can be tightly encaged in the narrow galleries between the MMT layers. For same Heck reaction, it is reported $[15,17]$ that Pd@MMT/CS can reuse more times (30 times) than Pd@MMT/PVP catalysts (10 times). It was due to the weaker encaging of Pd species for bigger interlayer space formed in the case of Pd@MMT/PVP and lower chelation ability of PVP than that of CS. This revealed that the catalytic performances can be tailored with different kinds of polymers for the different intercalation structure formed and chelation ability with transition metals.

Polyethylene oxide is another well-known modifier for MMT and the prepared MMT/PEO hybrid composites show potential applications in many fields [22,23]. It was suggested that PEO chains could form single or double-ordered layer arrangements in the interlayer space of MMT. The ending groups of $\mathrm{PEO}$, i.e., - $\mathrm{OH}$ groups, have effective chelation capability with transition metal species. Also, PEO is known as an effective stabilizer for transition metal nanoparticles in reducing of their agglomeration to form big-sized particles [24,25]. PEO itself has been used as a polymer support for Pd catalysts in Heck reactions [26-28]. For example, PEO-supported recyclable NC palladacycle catalysts were developed by Karami and co-workers [28] by blending of dimeric NC palladacycle with PEO melts at $85{ }^{\circ} \mathrm{C}$. However, the size of the prepared Pd nanoparticles is too large (bigger than $50 \mathrm{~nm}$ ) and it just showed moderate catalysis efficiency (up to $80 \%$ yield) for Heck reactions. Therefore, further modification of PEO-supported Pd catalysts is needed. It is expected that PEO-modified MMT should be another good candidate of support for immobilization of transition metals (such as Pd) in heterogeneous catalytic materials preparation.

In this study, PEO-modified MMT supports with different content and Pd-supported on MMT/PEO catalytic composites have been prepared. The microstructure of the PEO chains and Pd species confined in the interlayer space of MMT was characterized by several methods, including X-ray diffraction (XRD), high resolution transmission electron microscopy (HR-TEM), X-ray photo electron spectroscopy (XPS), thermal analysis, differential scanning calorimetry (DSC), and inductively coupled plasma-atomic emission spectroscopy (ICP). The sub-nano level microstructure of Pd@MMT/PEO catalytic composites was further studied by positron annihilation lifetime spectroscopy (PALS). The correlations between the microstructure and catalytic performances of Pd@MMT/PEO catalytic composite in Heck reactions were discussed.

\section{Materials and Methods}

\subsection{Materials}

$\mathrm{Na}^{+}-\mathrm{MMT}$ (G-105 type) of was purchased from Nanocor Co., Arlington Hts, IL, USA and its cationic exchange capacity was $145 \mathrm{mmol} / 100 \mathrm{~g}$. PEO $\left(M_{\mathrm{n}}=2 \times 10^{4} \mathrm{~g} / \mathrm{mol}\right)$ was purchased from Sinopharm Chemical Reagent Co., Ltd. Shanghai, China. $\mathrm{PdCl}_{2}$ (analytical grade) was purchased from Zhejiang Metallurgical Research Institute Co., Ltd. Hangzhou, China. The aromatic halides and acrylate substrates used in Heck reactions were purchased from Energy Chemical, Sun Chemical Technology (Shanghai) Co., Ltd. Shanghai, China. Other used reagents and solvents with no further treatments were purchased from Sinopharm Chemical Reagent Co., Ltd. Shanghai, China.

\subsection{Sample Preparation}

A total of $100 \mathrm{~mL}$ of $2 \mathrm{wt} \%$ of MMT suspension solution was prepared under magnetic stirring. Specific amounts of PEO were dispersed in $100 \mathrm{~mL}$ of deionized water to form homogeneous solution. The PEO solution and the MMT suspension solution were mixed and kept magnetically stirred in 
water bath at $55^{\circ} \mathrm{C}$ for $12 \mathrm{~h}$. The mass ratios of MMT/PEO were set as 100/0, 90/10, 80/20, 70/30, 60/40, and 50/50. $0.3 \mathrm{~g}$ of $\mathrm{PdCl}_{2}$ was dissolved in $100 \mathrm{~mL}$ deionized water with the presence of excessive amounts of $\mathrm{NaCl}$ (about $2 \mathrm{~g}$ ). Then, $2 \mathrm{~mL}$ of the fresh $\mathrm{Pd}^{2+}$ solution was drop-wisely added into the above MMT/PEO (80/20) mixture and kept magnetically stirring for another $6 \mathrm{~h}$. The $\mathrm{Pd}^{2+} @ \mathrm{MMT} / \mathrm{PEO}$ composite was separated by centrifugation and washed with deionized water until neutral $(\mathrm{pH}=7)$. Afterwards, it was dried in an oven at $60^{\circ} \mathrm{C}$. The resultant $\mathrm{Pd}^{2+} @ \mathrm{MMT} / \mathrm{PEO}$ composite was reduced to $\mathrm{Pd}^{0} @ \mathrm{MMT} / \mathrm{PEO}$ with ethylene glycol at $80^{\circ} \mathrm{C}$. According to the ICP-AES determination results, the Pd content within the Pd@MMT/PEO catalytic composite was about $0.2 \mathrm{wt} \%$.

\subsection{Characterizations}

The characterization methods were similar to those in our recent works [19-21]. The XRD analysis of MMT/PEO and Pd@MMT/PEO samples were performed with a PANalytical Empyrean X-ray diffraction system (conditions: $2 \theta$ from $3^{\circ}$ to $70^{\circ}$, scanning rate of $2^{\circ} / \mathrm{min}$ ). The TGA and DSC curves of MMT/PEO and Pd@MMT/PEO samples were recorded with a Mettler Toledo TGA/DSC 2 STAR system (Zurich, Switzerland) (conditions: air atmosphere, from 30 to $700{ }^{\circ} \mathrm{C}$, scanning rate of $20^{\circ} \mathrm{C} / \mathrm{min}$ ). The XPS analysis of Pd@MMT/PEO sample was performed with a Thermo Fisher Scientific ESCALAB 250Xi X-ray photoelectron spectrometer. The Pd@MMT/PEO samples were embedded in epoxy resin and then microtomed for HRTEM observation, which was performed with a JEM-2100F HR-TEM (JEOL Ltd. Tokyo, Japan). The ICP determination of Pd@MMT/PEO samples were performed with a Leemann ICP-AES Prodigy XP inductively coupled plasma atomic emission spectrometry. The positron annihilation lifetime spectroscopy (PALS) analysis of MMT/PEO and Pd@MMT/PEO samples was performed with an EG \& G ORTEC fast-slow system (conditions: time resolution of 198 ps). Before PALS measuments, the MMT/PEO and Pd@MMT/PEO samples were pressed into disks (diameter $\times$ thickness: $1 \mathrm{~cm} \times 2 \mathrm{~mm}$ ) using a 769YP-15A powder tableting machine (Shanghai Xinnuo Instrument Ltd., Shanghai, China). During lifetime spectra measurements, the positron source $\left({ }^{22} \mathrm{NaCl}, 16 \mu \mathrm{Ci}\right.$, deposited between two Kapton foils) was sandwiched between two pre-pressed Pd@MMT/PEO samples disks. The analysis of the positron annihilation spectra was performed with LT-9 (Lifetime-9) and MELT-4 (Maximum Entropy for Lifetime Analysis-4) programs.

\subsection{Catalytic Test}

In a $50 \mathrm{~mL}$ of round bottom flask reactor, a mixture of aromatic halide substrates $(1 \mathrm{mmol})$, acrylates substrates ( $2 \mathrm{mmol}$ ), $\mathrm{Pd}^{0} @ \mathrm{MMT} / \mathrm{PEO}$ catalytic composite $(3 \mu \mathrm{mol}$ of $\mathrm{Pd}), \mathrm{CH}_{3} \mathrm{COOK}$ base ( $3 \mathrm{mmol}$ ), and solvent ( $5 \mathrm{~mL}$ DMSO $+0.2 \mathrm{~mL}$ ethylene glycol) was magnetically stirred at $110{ }^{\circ} \mathrm{C}$ (oil bath heating) for $5 \mathrm{~h}$. The coupling reaction progress was detected with both layer chromatography (TLC) method and gas chromatography-mass spectrometry (GC/MS) analysis (Agilent 6890N/5975 MSD GC/MS, Palo Alto, CA, USA). The coupling reaction yield is according to the GC/MS quantitative analysis results of the reaction mixture. All the coupling products' chemical structure was confirmed by the analysis results of both $\mathrm{H}^{1} \mathrm{NMR}$ and GC/MS, which was consistent with our recent works [21,29]. The recycling experiments of the Pd@MMT/PEO were performed as follows: firstly, filtration out the Pd@MMT/PEO from the reaction system; secondly, repeatedly washing of the filtrated Pd@MMT/PEO with ethanol for 3-5 times and drying; finally, putting the recycled Pd@MMT/PEO into the reaction mixture for use in next reaction run.

\section{Results and Discussions}

Figure 1 shows the XRD patterns of MMT/PEO and Pd@MMT/PEO and the basal spacing $\left(d_{001}\right)$ value for the MMT estimated by Bragg's formula is summarized in Table 1. Meanwhile, when the thickness of the single layer of pure $\mathrm{Na}^{+}$-MMT $(0.96 \mathrm{~nm})$ is taken into account, the interlayer spacing of MMT can be estimated (also shown in Table 1). In the galleries between the MMT layers, the intercalated PEO chains would show different arrangements according to the interlayer spacing values and macromolecular configurations of PEO chains. For pure $\mathrm{Na}^{+}-\mathrm{MMT}$, the $d_{001}$ is $1.25 \mathrm{~nm}$, 
and its corresponding interlayer spacing is $0.29 \mathrm{~nm}$. At the MMT/PEO ratios of 90/10, the $d_{001}$ and interlayer spacing increases to $1.44 \mathrm{~nm}$ and $0.48 \mathrm{~nm}$, respectively. Some of previous works $[23,30]$ showed that the intercalated PEO chains would be in helical conformation. However, it is worth noting that the size of PEO chains with a helical conformation is about $0.8 \mathrm{~nm}(>0.48 \mathrm{~nm})$. It indicates that the helical conformation of PEO chains in the interlayer space of MMT is not to be the case. Similar with recent work by Zhu et al. [31], lateral single layer of PEO chain with random conformation confined inside the galleries between the MMT layers in the case of MMT/PEO (90/10) might be much more possible. At the MMT/PEO ratios of 80/20 and 70/30, the interlayer spacing shows a slow increase to $0.56 \mathrm{~nm}$ (MMT/PEO (80/20)) and $0.62 \mathrm{~nm}$ (MMT/PEO (70/30)), respectively. It should be a wider monolayer of PEG chain confined inside the galleries between the MMT layers. At the MMT/PEO ratios further increase to 60/40 and 50/50, the interlayer spacing shows further increases to $0.85 \mathrm{~nm}$ (MMT/PEO 60/40) and 0.86 nm (MMT/PEO 50/50), indicating bilayers of PEO chains confined inside the galleries between the MMT layers. As we know, PEO is a polymer with high crystalline capability. One more layers highly-ordered arrangements should be beneficial to the formation of PEO crystals. The formation of crystal phase of PEO bulk would lead to a decrease in organic modification efficiency for MMT. Therefore, MMT/PEO (80/20) with wider monolayer arrangement was chosen as the platform for immobilization of $\mathrm{Pd}$ species in preparation of novel heterogeneous catalytic composites. The interlayer spacing of $\mathrm{Pd}^{2+} @ \mathrm{MMT} / \mathrm{PEO}(0.2 / 80 / 20)$ increases to $0.76 \mathrm{~nm}, 0.2 \mathrm{~nm}$ bigger than MMT/PEO (80/20). This confirms that the $\mathrm{Pd}^{2+}$ cations are effectively chelated by the PEO chains and well confined inside the galleries of MMT. Though the calculated mean interlayer spacing of Pd $\mathrm{d}^{0} @ \mathrm{MMT} / \mathrm{PEO}(0.2 / 80 / 20)$ is close to $\mathrm{Pd}^{2+} @ \mathrm{MMT} / \mathrm{PEO}(0.2 / 80 / 20)$, the scattering peak from $\mathrm{Pd}^{0} @ \mathrm{MMT} / \mathrm{PEO}$ becomes a bit narrower. It is presumably caused by the local distortion of the layered structure by in-situ-generated $\mathrm{Pd}^{0}$ nanoparticles with uniform size.

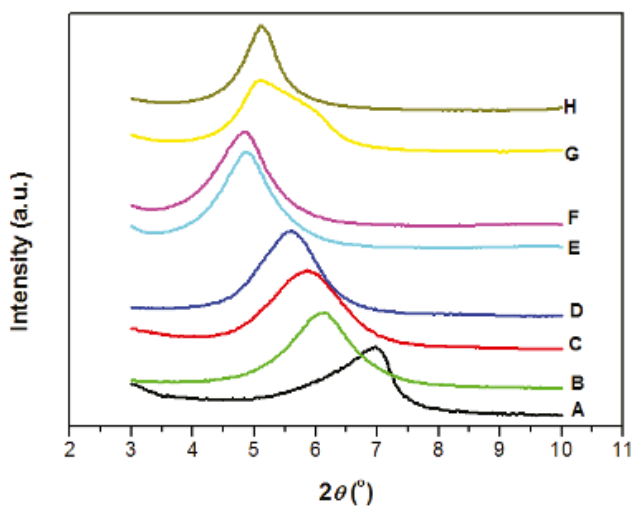

Figure 1. XRD patterns of MMT/PEO hybrids and Pd@MMT/PEO catalytic composites. A. MMT; B. MMT/PEO (90/10); C. MMT/PEO (80/20); D. MMT/PEO (70/30); E. MMT/PEO (60/40); F. MMT/PEO

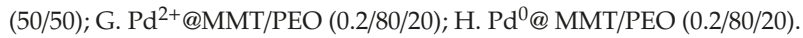


Table 1. Results of $d_{001}$ spacing and corresponding interlayer spacing (subtracting the thickness of MMT layer of $0.96 \mathrm{~nm}$ ) of the investigated MMT from XRD.

\begin{tabular}{|c|c|c|c|}
\hline Sample & $2 \theta\left({ }^{\circ}\right)$ & $d_{001}$ Spacing $(\mathrm{nm})$ & Interlayer Spacing $(\mathrm{nm})$ \\
\hline A. MMT & 7.02 & 1.25 & 0.29 \\
\hline B. MMT/PEO (90/10) & 6.15 & 1.44 & 0.48 \\
\hline C. MMT/PEO (80/20) & 5.78 & 1.52 & 0.56 \\
\hline D. MMT/PEO (70/30) & 5.60 & 1.58 & 0.62 \\
\hline E. MMT/PEO (60/40) & 4.88 & 1.81 & 0.85 \\
\hline F. MMT/PEO (50/50) & 4.84 & 1.82 & 0.86 \\
\hline 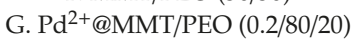 & 5.13 & 1.72 & 0.76 \\
\hline 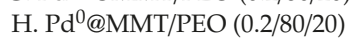 & 5.11 & 1.73 & 0.77 \\
\hline
\end{tabular}

The microstructure of the Pd@MMT/PEO catalytic composite was further characterized with XPS and HR-TEM. As shown in Figure 2A, for $\mathrm{Pd}^{2+} @ \mathrm{MMT} / \mathrm{PEO}$, the binding energy peaks are found at $337.5 \mathrm{eV}\left(\mathrm{Pd}_{3 d_{5 / 2}}\right)$ and $342.9 \mathrm{eV}\left(\mathrm{Pd}_{3} \mathrm{~d}_{3 / 2}\right)$, confirming the presences of $\mathrm{Pd}^{2+}$ species [32]. As shown in Figure 2B, for $\mathrm{Pd}^{0} @ \mathrm{MMT} / \mathrm{PEO}$, the binding energy peaks shift to $335.6 \mathrm{eV}\left(\mathrm{Pd}_{3} \mathrm{~d}_{5 / 2}\right)$ and $340.9 \mathrm{eV}$ $\left(\mathrm{Pd}_{3 / 2}\right)$, respectively, confirming the presences of $\mathrm{Pd}^{0}$ species [32]. Clearly, XPS characterization results supply a powerful evidence for the conversion of $\mathrm{Pd}^{2+}$ to $\mathrm{Pd}^{0}$. Figure 3 shows HR-TEM results of the MMT/PEO (80/20) hybrid and $\mathrm{Pd}^{0} @$ MMT/PEO (0.2/80/20) catalytic composite. MMT/PEO $(80 / 20)$ shows a typical layered structure with well-ordered parallel stacking of silicate MMT layers.

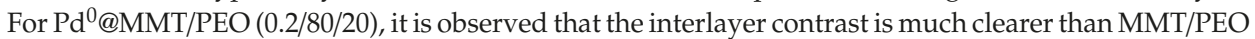
$(80 / 20)$. It should be due to the slight increase in the interlayer spacing after immobilization of Pd species, which is also evidenced by XRD characterization. Meanwhile, it is observed the dispersion states of $\mathrm{Pd}^{0}$ nanoparticles $(2-4 \mathrm{~nm})$ in the MMT/PEO (80/20) matrices are in two forms: (1) being confined inside 1-3 layers of MMT, (2) dispersed on the outer surface of MMT layers.
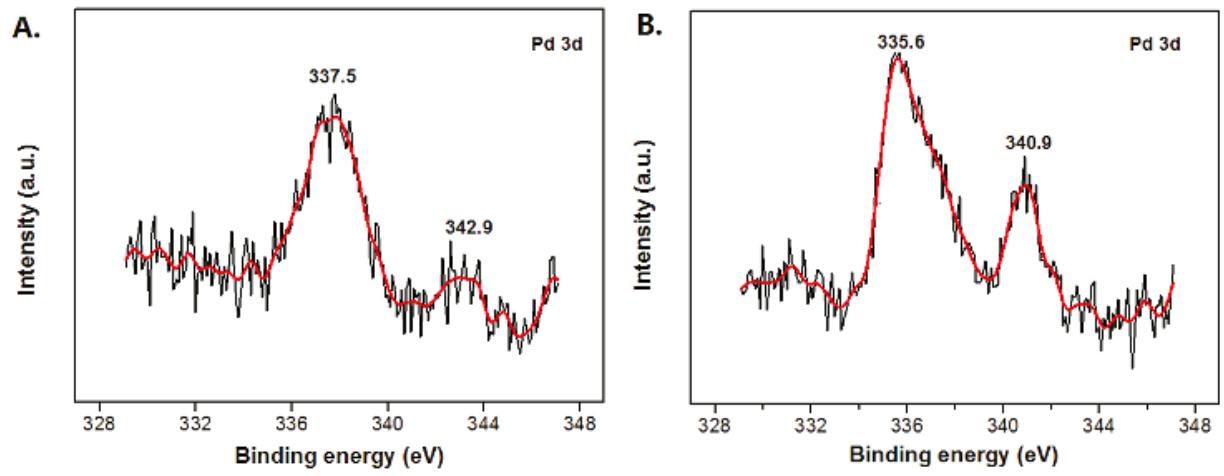

Figure 2. XPS spectra of the $\mathrm{Pd}^{2+} @ \mathrm{MMT} / \mathrm{PEO}(\mathbf{A})$ and $\mathrm{Pd}^{0} @ \mathrm{MMT} / \mathrm{PEO}(\mathbf{B})$ catalytic composites. 

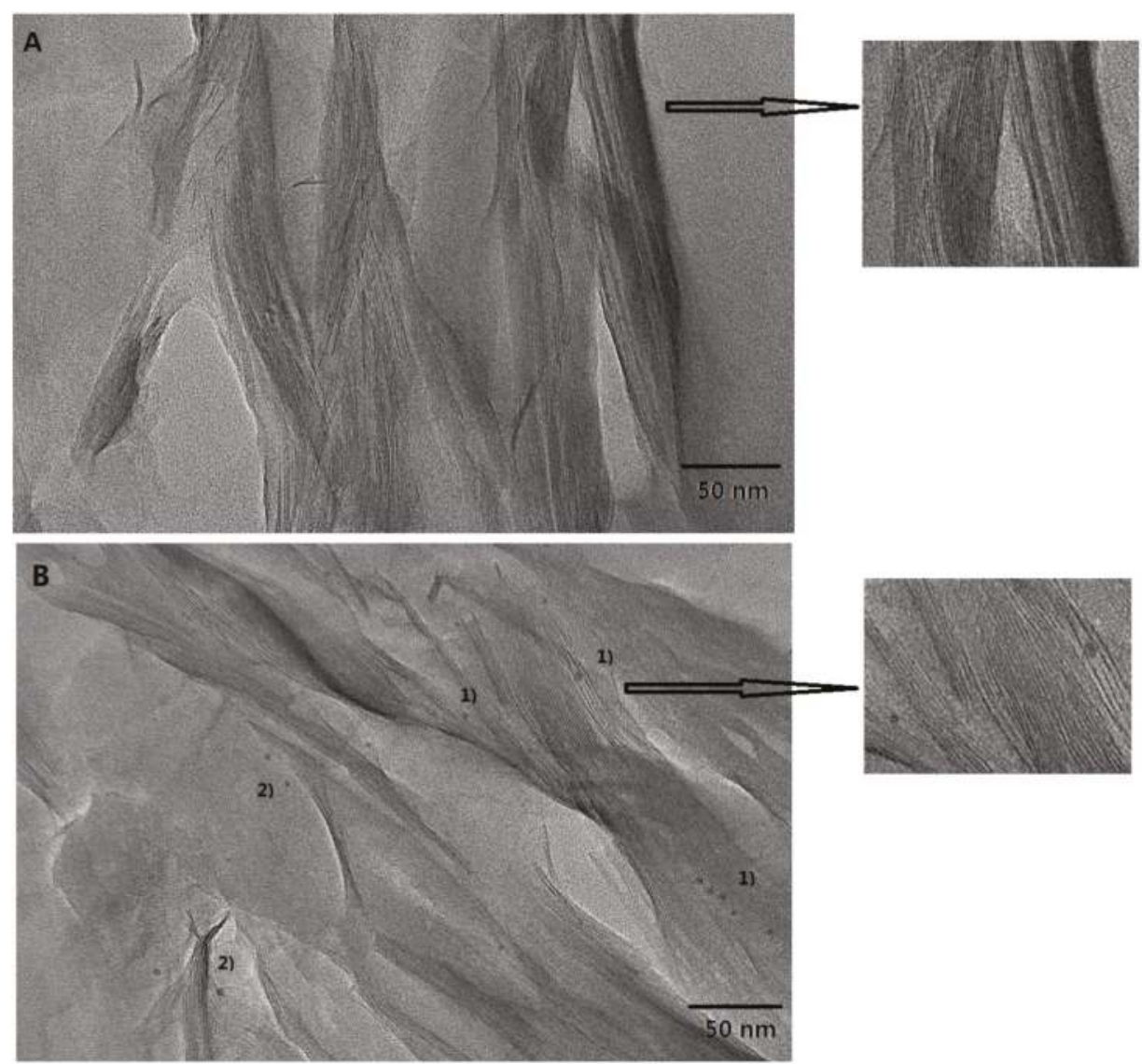

Figure 3. HR-TEM results of the MMT/PEO (80/20) hybrid (A) and $\mathrm{Pd}^{0} @ \mathrm{MMT} / \mathrm{PEO}(0.2 / 80 / 20)$ catalytic composite (B). The insert figures are the enlarged view of the local regions in the HR-TEM photos.

Meanwhile, the effects of the Pd immobilization on the molecular level microstructure of Pd@MMT/PEO catalytic composite have been characterized by PALS. The positron annihilation

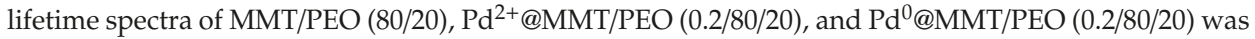
analyzed by three-component fitting using LT-9 and MELT- 4 programs. We mainly take attentions to the longest (third) lifetime component $\left(\tau_{3}\right)$, which is attributed to the ortho-positronium (o-Ps) annihilations inside the interlayer space of MMT [33-35]. The size of the micro defects can be estimated from $\tau_{3}$ with suitable models. It was evidenced that the modified Tao-Eldrup equation for cuboidal voids (Equation (1)) worked well for the MMT-based materials with layered structure [35-38]. Where, $l$ refers to the mean width of cuboidal micro defects, and $\Delta l(0.17 \mathrm{~nm})$ refers to the value of empirical parameter.

$$
\tau_{3}=0.5\left[1-\left(\frac{l}{l+2 \Delta l}+\frac{1}{\pi} \sin \frac{\pi l}{l+2 \Delta l}\right)\right]^{-1}
$$

The sizes of the micro defects estimated with Equation (1) are listed in Table 2. The PALS result of pure MMT sample has been reported in our recent work [20], the o-Ps lifetime is $2.801 \mathrm{~ns}$, and the mean micro defects size $(l)$ of MMT can be calculated as $0.3443 \mathrm{~nm}$. This value is bigger than that of MMT/PEO (80/20), $0.2901 \mathrm{~nm}$. This is due to the fact that the interlayer space of MMT becomes more crowded after the PEO chains intercalate. Meanwhile, the effects of the Pd immobilization and 
reducing treatment on the microstructure have been sensitively detected. Though the interlayer space is expanded obviously after $\mathrm{Pd}^{2+}$ immobilized as determined by XRD, the mean micro defects size (l) show a decrease from $0.2901 \mathrm{~nm}(\mathrm{MMT} / \mathrm{PEO}(80 / 20))$ to $0.2792 \mathrm{~nm}\left(\mathrm{Pd}^{2+} @ \mathrm{MMT} / \mathrm{PEO}(0.2 / 80 / 20)\right)$. By forming effective chelation with $-\mathrm{OH}$ groups, $\mathrm{Pd}^{2+}$ cations play a role similar to cross-linking points for PEO chains, leading to a decrease in micro defects size within the intercalated PEO phase. $\mathrm{Pd}^{0}$ species often has poorer chelation capability with polar groups than $\mathrm{Pd}^{2+}$ species. Therefore, the micro defects size then undergoes an increase to $0.2886 \mathrm{~nm}$ for Pd $\mathrm{d}^{0} @ \mathrm{MMT} / \mathrm{PEO}(0.2 / 80 / 20)$. Figure 4 shows the distribution of the longest lifetime $\left(\tau_{3}\right)$ and corresponding micro-defect's size $(l)$ of the samples as fitted with MELT-4 program. Similar variation trend of the distribution range of the micro-defect's size is found.

Table 2. Variation of longest lifetime, intensity, and mean size of micro-defects of the samples analyzed by LT-9 program.

\begin{tabular}{cccc}
\hline Samples & $\tau_{3}$ (ns) & $\boldsymbol{I}_{3}(\boldsymbol{\%})$ & $\boldsymbol{l}(\mathbf{n m})$ \\
\hline MMT/PEO $(80 / 20)$ & 2.235 & 5.8 & 0.2901 \\
$\mathrm{Pd}^{2+} @ \mathrm{MMT} / \mathrm{PEO}(0.2 / 80 / 20)$ & 2.132 & 3.7 & 0.2792 \\
$\mathrm{Pd}^{0} @ \mathrm{MMT} / \mathrm{PEO}(0.2 / 80 / 20)$ & 2.220 & 4.5 & 0.2886 \\
\hline
\end{tabular}
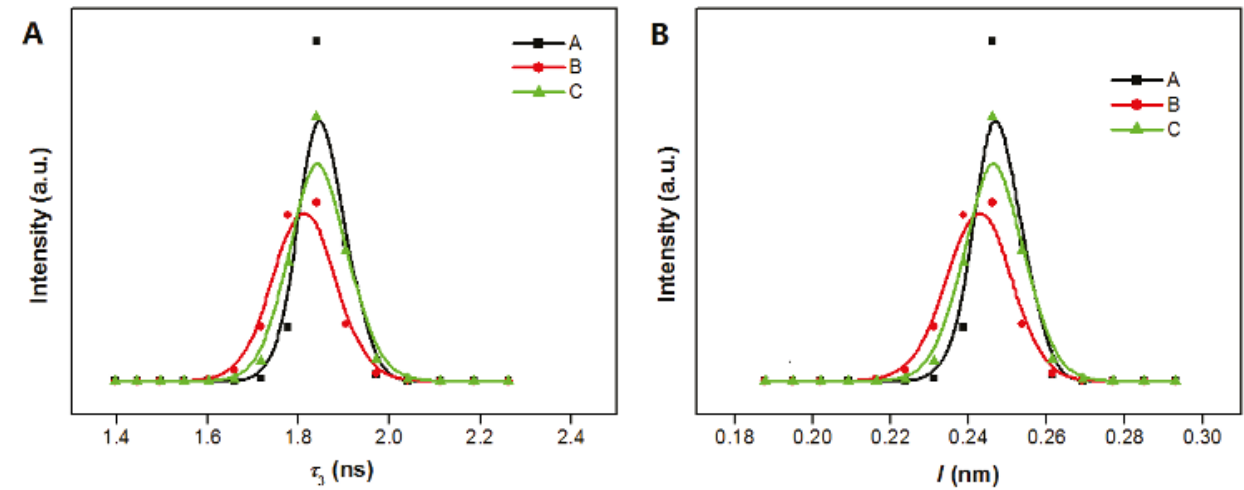

Figure 4. Distribution of the longest lifetime $\left(\tau_{3}\right)(\mathbf{A})$ and corresponding micro-defect's size (l) (B)

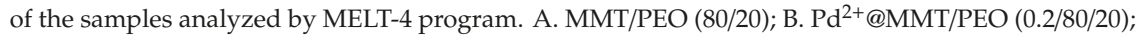

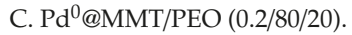

Figure 5A shows the TGA/DTG results of MMT/PEO hybrids and Pd 0 @MT/PEO (0.2/80/20) catalytic composite. As a stable inorganic layered silicate mineral, MMT shows high thermal stability except a weight loss stage caused by the evaporation of absorbed and/or bonded $\mathrm{H}_{2} \mathrm{O}$ before $125{ }^{\circ} \mathrm{C}$. The decomposition temperature (peak temperature of the DTA curve) of PEO is found at about $320^{\circ} \mathrm{C}$. For the MMT/PEO hybrids, decomposition temperature of PEO component shows an obviously decrease to $240{ }^{\circ} \mathrm{C}$ for MMT/PEO (80/20) and $200{ }^{\circ} \mathrm{C}$ for MMT/PEO (60/40), respectively. This decrease should be due to the different aggregation states of PEO chains between pure PEO and MMT/PEO hybrid. Due to the high crystallization ability, most $\mathrm{PEO}$ chains tend to be regularly aggregated to form perfect crystals. As confirmed with DSC curves in Figure 5B, an endothermic peak of PEO crystal at $73{ }^{\circ} \mathrm{C}$ is observed. However, as confined in the narrow galleries of MMT, PEO chains have much lower probabilities in regular aggregation to form crystals. Therefore, no obvious endothermic peak has been detected for the MMT/PEO hybrids and $\mathrm{Pd}^{0} @ \mathrm{MMT} / \mathrm{PEO}(0.2 / 80 / 20)$ catalytic composite. Usually, the formation of perfect crystal of PEO chains is advantageous for higher thermal stability. As a result, PEO component in MMT/PEO hybrids shows lower thermal decomposition temperature in TGA curves. The thermal decomposition temperature of $\mathrm{Pd}^{0} @ \mathrm{MMT} / \mathrm{PEO}(0.2 / 80 / 20)$ catalytic composite is 
close to MMT/PEO (80/20), indicating similar thermal stabilities. Clearly, the prepared $\mathrm{Pd}^{0} @ \mathrm{MMT} / \mathrm{PEO}$ $(0.2 / 80 / 20)$ catalytic composite could be adaptable in organic reactions below $200{ }^{\circ} \mathrm{C}$.
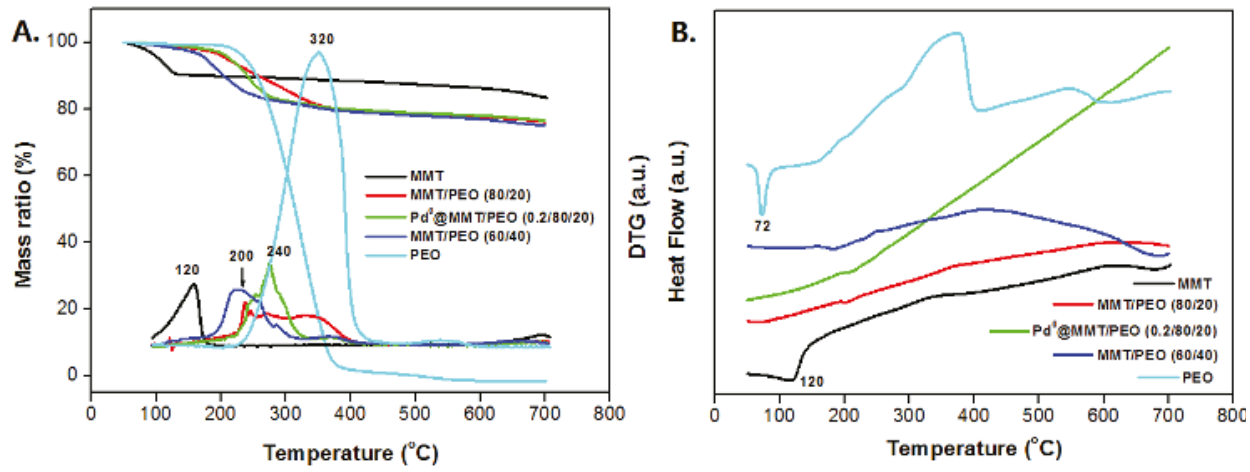

Figure 5. TGA/DTG (A) and DSC (B) curves of the MMT/PEO hybrids and Pd@MMT/PEO catalytic composite.

Heck reactions between aryl halides and butyl acrylate were catalyzed with the prepared $\mathrm{Pd}^{0} @ \mathrm{MMT} / \mathrm{PEO}$ catalytic composite. As shown in Table 3, the Pd0@MMT/PEO catalytic composite shows high catalytic activity for the reaction between iodo benzene and $n$-butyl acrylate (entry $1,91 \%$ yield). It still exhibits high catalytic activity for aryl iodides substituted with either an electron-donating group, such as $p-\mathrm{CH}_{3}$ (entry 2,89\% yield) and $m-\mathrm{CH}_{3} \mathrm{O}$ (entry 3, 84\% yield), or an electron-absorbing group (such as $p$-F (entry $4,88 \%$ yield), $m$-F (entry $5,87 \%$ yield). Heck reaction between aryl iodides and $t$-butyl acrylate can be also effectively catalyzed with the prepared $\mathrm{Pd}^{0} @ \mathrm{MMT} / \mathrm{PEO}$ catalytic composite (entry 6-8). The $\mathrm{Pd}^{0} @ \mathrm{MMT} / \mathrm{PEO}$ catalytic composite show low catalytic activity for the reaction between bromo benzene and $n$-butyl acrylate (entry 9), which is mainly due to the much higher bonding strength of C-Br than C-I to break. Nevertheless, C-Br bond can be activated by substitution of strong electron-absorbing group such as $m-\mathrm{COCH}_{3}$ (entry 10). Clearly, the catalytic activity of $\mathrm{Pd}^{0} @ \mathrm{MMT} / \mathrm{PEO}$ is much higher than PEO-supported recyclable NC palladacycle catalysts [28]. And it is also comparable to recent other reported heterogeneous catalysts for Heck reactions $[39,40]$. After the reaction, the $\mathrm{Pd}^{0} @ \mathrm{MMT} / \mathrm{PEO}$ catalytic composite can be conveniently separated and recycled for the next run. As shown in Figure 6, the catalytic efficiency of the $\mathrm{Pd}^{0} @ \mathrm{MMT} / \mathrm{PEO}$ catalytic composite decrease gradually as the recycling times increase. Similarly, higher

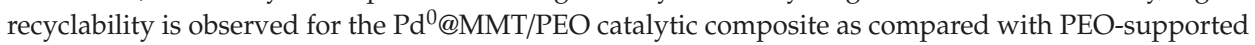
recyclable NC palladacycle catalysts (can recycle 4 times with moderate yield) [28]. However, it is obviously lower than recent prepared $\mathrm{Pd}^{0} @$ MMT/PVA or Pd $\mathrm{Pd}^{0} @ \mathrm{MMT} / \mathrm{PVP}$ catalysts $[19,20]$. For PEO, the polar $-\mathrm{OH}$ groups are distributed in the ending of the chain rather than each repeating unit of the chain like PVA. Reasonably weaker chelation and quicker Pd leaching will occur in the case of $\mathrm{Pd}^{0} @ \mathrm{MMT} / \mathrm{PEO}$. 
Table 3. Catalytic performances of $\mathrm{Pd}^{0} @ \mathrm{MMT} / \mathrm{PEO}$ for Heck reactions between aromatic halides with acrylates.

\begin{tabular}{|c|c|c|c|}
\hline \multicolumn{4}{|c|}{$\mathrm{X}=\mathrm{I}, \mathrm{Br}$} \\
\hline Entry & Aromatic Halides & Acrylates & Coupling Yield ${ }^{a}(\%)$ \\
\hline 1 & $\mathrm{C}_{6} \mathrm{H}_{5} \mathrm{I}$ & $\mathrm{CH}_{2}=\mathrm{CHCOO}\left(n-\mathrm{C}_{4} \mathrm{H}_{9}\right)$ & 91 \\
\hline 2 & $4-\mathrm{CH}_{3} \mathrm{C}_{6} \mathrm{H}_{4} \mathrm{I}$ & $\mathrm{CH}_{2}=\mathrm{CHCOO}\left(n-\mathrm{C}_{4} \mathrm{H}_{9}\right)$ & 89 \\
\hline 3 & $3-\mathrm{CH}_{3} \mathrm{OC}_{6} \mathrm{H}_{4} \mathrm{I}$ & $\mathrm{CH}_{2}=\mathrm{CHCOO}\left(n-\mathrm{C}_{4} \mathrm{H}_{9}\right)$ & 84 \\
\hline 4 & $4-\mathrm{FC}_{6} \mathrm{H}_{4} \mathrm{I}$ & $\mathrm{CH}_{2}=\mathrm{CHCOO}\left(n-\mathrm{C}_{4} \mathrm{H}_{9}\right)$ & 88 \\
\hline 5 & $3-\mathrm{FC}_{6} \mathrm{H}_{4} \mathrm{I}$ & $\mathrm{CH}_{2}=\mathrm{CHCOO}\left(n-\mathrm{C}_{4} \mathrm{H}_{9}\right)$ & 87 \\
\hline 6 & $\mathrm{C}_{6} \mathrm{H}_{5} \mathrm{I}$ & $\mathrm{CH}_{2}=\mathrm{CHCOO}\left(t-\mathrm{C}_{4} \mathrm{H}_{9}\right)$ & 74 \\
\hline 7 & $4-\mathrm{CH}_{3} \mathrm{C}_{6} \mathrm{H}_{4} \mathrm{I}$ & $\mathrm{CH}_{2}=\mathrm{CHCOO}\left(t-\mathrm{C}_{4} \mathrm{H}_{9}\right)$ & 75 \\
\hline 8 & $4-\mathrm{FC}_{6} \mathrm{H}_{4} \mathrm{I}$ & $\mathrm{CH}_{2}=\mathrm{CHCOO}\left(t-\mathrm{C}_{4} \mathrm{H}_{9}\right)$ & 71 \\
\hline 9 & $\mathrm{C}_{6} \mathrm{H}_{5} \mathrm{Br}$ & $\mathrm{CH}_{2}=\mathrm{CHCOO}\left(n-\mathrm{C}_{4} \mathrm{H}_{9}\right)$ & $\operatorname{trace}^{b}$ \\
\hline 10 & $3-\mathrm{COCH}_{3} \mathrm{C}_{6} \mathrm{H}_{4} \mathrm{Br}$ & $\mathrm{CH}_{2}=\mathrm{CHCOO}\left(n-\mathrm{C}_{4} \mathrm{H}_{9}\right)$ & $45^{b}$ \\
\hline
\end{tabular}

${ }^{a}$ GC-MS Yield; ${ }^{\mathrm{b}}$ the reaction time was $10 \mathrm{~h}$.

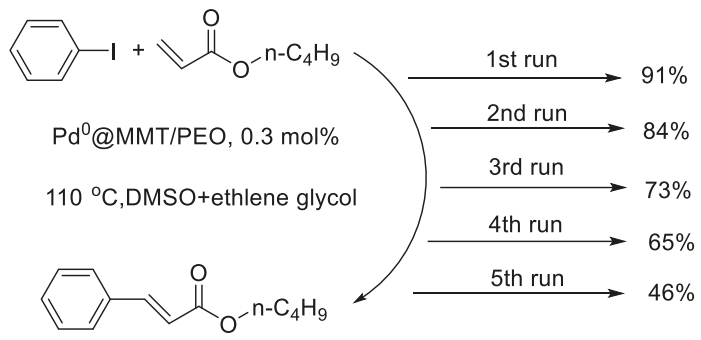

Figure 6. Recycling performances of the $\mathrm{Pd}^{0} @ \mathrm{MMT} / \mathrm{PEO}$ catalytic composites.

\section{Conclusions}

In this study, PEO chains were successfully intercalated into interlayer spaces of $\mathrm{Na}^{+}-\mathrm{MMT}_{\text {, }}$ which can be used as a novel support for $\mathrm{Pd}^{0}$ nanoparticles. PEO chains and Pd species are well confined in the interlayer space of MMT, which is well elucidated by XRD, HR-TEM, XPS, TGA, and PALS. It was demonstrated that $\mathrm{Pd}^{0}$ nanoparticles sized in $2-4 \mathrm{~nm}$ were successfully immobilized on MMT/PEO supports. The sub-nano level micro defects variation of MMT can be sensitively detected by PALS. After PEO intercalation and Pd immobilization, the micro defects undergo a slight decrease in size, which is mainly due to the fact that the interlayer space of MMT becomes more crowded. The prepared $\mathrm{Pd}^{0} @ \mathrm{MMT} / \mathrm{PEO}$ catalytic composite shows high catalytic activities for Heck reactions and can be recycled for five times. The lower recyclability of the $\mathrm{Pd}^{0} @ \mathrm{MMT} / \mathrm{PEO}$ than other reported $\mathrm{Pd}^{0} @ \mathrm{MMT} /$ polar polymers catalytic composites should be mainly due to the weaker chelation of PEO with Pd. Nevertheless, the comprehensive catalytic performances of the $\mathrm{Pd}^{0} @ \mathrm{MMT} / \mathrm{PEO}$ are much improved as compared with Pd heterogeneous catalysts which are prepared by directly supporting of Pd species on PEO chains. This work supplies an alternative approach in the preparation of Pd heterogeneous catalysts with fairly good performances, and might have broad prospects in both experimental and industrial applications.

Author Contributions: M.Z. conceived and designed the experiments; G.S., J.Z., and M.X. performed the materials preparation and characterization experiments; X.Z. and Q.L., performed the catalysis experiments.

Funding: This research was funded by the National Natural Science Foundation of China (Grant No. 11875193 and 11475114), Zhejiang Provincial Fundamental Public Welfare Research Project (Grant No. LGG18E030004), and University Students' Science and Technology Innovation Activity Plan Project of Zhejiang Province (Grant No. 2018R432012). 
Acknowledgments: We are grateful to the Xingzhong Cao and Baoyi Wang (Institute of High Energy Physics, The Chinese Academy of Science, China) for the positron annihilation characterization supports.

Conflicts of Interest: The authors declare no conflict of interest.

\section{References}

1. LeBaron, P.C.; Wang, Z.; Pinnavaia, T.J. Polymer-layered silicate nanocomposites: An overview. Appl. Clay Sci. 1999, 15, 11-29. [CrossRef]

2. Pavlidou, S.; Papaspyrides, C.D. A review on polymer-layered silicate nanocomposites. Prog. Polym. Sci. 2008, 33, 1119-1198. [CrossRef]

3. Bee, S.L.; Abdullah, M.A.A.; Bee, S.T.; Sin, L.T.; Rahmat, A.R. Polymer nanocomposites based on silylated-montmorillonite: A review. Prog. Polym. Sci. 2018, 85, 57-82. [CrossRef]

4. Zampori, L.; Dotelli, G.; Stampino, P.G.; Cristiani, C.; Zorzi, F.; Finocchio, E. Thermal characterization of a montmorillonite, modified with polyethylene-glycols (PEG1500 and PEG4000), by in situ HT-XRD and FT IR: Formation of a high-temperature phase. Appl. Clay Sci. 2012, 59-60, 140-147. [CrossRef]

5. Shen, Z.Q.; Simon, G.P.; Cheng, Y.-B. Comparison of solution intercalation and melt intercalation of polymer-clay nanocomposites. Polymer 2002, 43, 4251-4260. [CrossRef]

6. Strawhecker, K.E.; Manias, E. Structure and properties of poly (vinyl alcohol)/ $\mathrm{Na}^{+}$montmorillonite nanocomposites. Chem. Mater. 2000, 12, 2943-2949. [CrossRef]

7. Papp, S.; Patakfalvi, R.; Dekany, I. Metal nanoparticle formation on layer silicate lamellae. Colloid Polym. Sci. 2008, 286, 3-14. [CrossRef]

8. Paul, D.R.; Robeson, L.M. Polymer nanotechnology: Nanocomposites. Polymer 2008, 49, 3187-3204. [CrossRef]

9. Meirelles, L.M.A.; Raffin, F.N. Clay and polymer-based composites applied to drug release: A scientific and technological prospection. J. Pharm. Pharm. Sci. 2017, 20, 115-134. [CrossRef] [PubMed]

10. Floody, M.C.; Theng, B.K.G.; Reyes, P.; Mora, M.L. Natural nanoclays: Applications and future trends-chilean perspective. Clay Miner. 2009, 44, 161-176. [CrossRef]

11. Zhou, C.H. An overview on strategies towards clay-based designer catalysts for green and sustainable catalysis. Appl. Clay Sci. 2011, 53, 87-96. [CrossRef]

12. Kumar, B.S.; Dhakshinamoorthy, A.; Pitchumani, K. K10 montmorillonite clays as environmentally benign catalysts for organic reactions. Catal. Sci. Technol. 2014, 4, 2378-2396. [CrossRef]

13. Hechelski, M.; Ghinet, A.; Louvel, B.; Dufrenoy, P.; Rigo, B.; Daich, A.; Waterlot, C. From conventional lewis acids to heterogeneous montmorillonite K10: Eco-friendly plant-based catalysts used as green lewis acids. ChemSusChem 2018, 11, 1249-1277. [CrossRef] [PubMed]

14. Nasrollahzadeh, M.; Atarod, M.; Alizadeh, M.; Hatamifard, A.; Sajadi, S.M. Recent advances in the application of heterogeneous nanocaalysts for Sonogashira coupling reactions. Curr. Org. Chem. 2017, 21, 708-749. [CrossRef]

15. Heirati, S.Z.D.; Shirini, F.; Shojaei, A.F. Sulfonated PEG-intercalated montmorillonite [(Mt/PEG)-SO3H] as efficient and ecofriendly nanocatalyst for synthesis of alpha, alpha'-bis(substituted benzylidene) cycloalkanones. Res. Chem. Intermediat. 2017, 43, 6167-6186. [CrossRef]

16. Kar, P.; Nayak, A.; Bhoi, Y.P.; Mishra, B.G. Preparation and catalytic application of sulfonated PVA-Zr-pillared clay nanocomposite materials towards one pot synthesis of hexahydropyrimidines. Microporous Mesoporous Mater. 2016, 223, 176-186. [CrossRef]

17. Senarathna, K.G.C.; Randiligama, H.M.S.P.; Rajapakse, R.M.G. Preparation, characterization and oxygen reduction catalytic activities of nanocomposites of $\mathrm{Co}(\mathrm{II}) /$ montmorillonite containing polypyrrole, polyaniline or poly(ethylenedioxythiophene). RSC Adv. 2016, 6, 112853-112863. [CrossRef]

18. Li, T.; Sun, Y.L.; Zhou, R.; Long, M.D.; Liu, Y.F. Fabrication and application of porous Pd@Mt/PC composite as an efficient green heterogeneous catalyst for Suzuki cross-coupling reaction. Micro Nano Lett. 2018, 13, 969-973.

19. Xu, M.D.; Zhao, J.; Shu, G.Q.; Liu, Q.; Zeng, M.F. Heterogeneous catalytic composites from palladium nanoparticles in montmorillonite intercalated with poly (vinyl pyrrolidone) chains. Polymers 2018, 10, 669. [CrossRef]

20. Zhao, J.; Xu, M.D.; Shu, G.Q.; Yang, Z.; Liu, Q.; Zeng, M.F.; Qi, C.Z.; Cao, X.Z.; Wang, B.Y. Positron annihilation characteristics and catalytic performances of poly (vinyl alcohol) intercalated montmorillonite supported $\mathrm{Pd}^{0}$ nanoparticles composites. Radiat. Phys.Chem. 2018, 153, 164-172. [CrossRef] 
21. Zeng, M.F.; Wang, Y.D.; Liu, Q.; Yuan, X.; Zuo, S.F.; Feng, R.K.; Yang, J.; Wang, B.Y.; Qi, C.Z.; Lin, Y. Encaging palladium nanoparticles in chitosan modified montmorillonite for efficient, recyclable catalysts. ACS Appl. Mater. Interfaces 2016, 8, 33157-33164. [CrossRef]

22. Zampori, L.; Stampino, P.G.; Cristiani, C.; Cazzola, P.; Dotelli, G. Intercalation of poly (ethylene-oxides) in montmorillonite: Tailor-made nanocontainers for drug delivery systems. Appl. Clay Sci. 2010, 50, 266-270. [CrossRef]

23. Aranda, P.; Ruiz-Hitzky, E. Poly (ethylene oxide)-silicate intercalation materials. Chem. Mater. 1992, 4, 1395-1403. [CrossRef]

24. Doherty, S.; Knight, J.G.; Backhouse, T.; Abood, E.; Alshaikh, H.; Clemmet, A.R.; Ellison, J.R.; Bourne, R.A.; Chamberlain, T.W.; Stones, R.; Warren, N.J.; Fairlamb, I.J.S.; Lovelock, K.R.J. Heteroatom donor-decorated polymer-immobilized ionic liquid stabilized palladium nanoparticles: efficient catalysts for room-temperature Suzuki-Miyaura cross-coupling in aqueous media. Adv. Syn. Catal. 2018, 360, 3716-3731. [CrossRef]

25. Zharmagambetova, A.K.; Zamanbekova, A.T.; Darmenbayeva, A.S.; Auyezkhanova, A.S.; Jumekeyeva, A.I.; Talgatov, E.T. Effect of polymers on the formation of nanosized palladium catalysts and their activity and selectivity in the hydrogenation of acetylenic alcohols. Theor. Exp. Chem. 2017, 53, 265-269. [CrossRef]

26. Pires, M.J.D.; Purifição, S.I.; Santos, A.S.; Marques, M.M.B. The role of PEG on Pd- and Cu-catalyzed cross-coupling reactions. Synthesis 2017, 49, 2337-2350.

27. Iranpoor, N.; Firouzabadi, H.; Riazi, A.; Shakerpoor, A. Phosphorylated PEG (PPEG) as a new support for generation of nano-Pd(0): Application to the Heck-Mizoroki and Suzuki-Miyaura coupling reactions. Appl. Organomet. Chem. 2013, 27, 451-458. [CrossRef]

28. Karami, K.; Moghadam, Z.K.; Hosseini-Kharat, M. Polyethylene glycol-supported recyclable NC palladacycle catalyst for Heck cross-coupling reactions. Catal. Commun. 2014, 43, 25-28. [CrossRef]

29. Zeng, M.F.; Qi, C.Z.; Yang, J.; Wang, B.Y.; Zhang, X.-M. A highly efficient and stable palladium catalyst entrapped within the cross-linked chitosan membrane for Heck reactions. Ind. Eng. Chem. Res. 2014, 53, 10041-10050. [CrossRef]

30. Ruiz-Hitzky, E.; Aranda, P. Polymer-salt intercalation complexes in layer silicates. Adv. Mater. 1990, 2, 545-547. [CrossRef]

31. Zhu, S.P.; Chen, J.Y.; Li, H.L.; Cao, Y. Structure and conformation of poly(ethylene glycol) in confined space of montmorillonite. Appl. Surf. Sci. 2013, 264, 500-506. [CrossRef]

32. Wagner, C.D.; Riggs, W.M.; Davis, L.E.; Mullenberg, G.E. Handbook of X-ray Photoelectron Spectroscopy; Perkin-Elmer: Waltham, MN, USA, 1979.

33. Joshi, J.M.; Sodaye, H.S.; Pujari, P.K.; Srisaila, S.; Bajpai, M.B. Positron annihilation spectroscopic investigation of Al-pillared montmorillonites. Catal. Lett. 1998, 51, 109-112. [CrossRef]

34. Sano, M.; Murakami, H.; Ichimura, K. Positronium in a layered-structure material: Montmorillonite. J. Radioanal. Nucl. Chem. 1999, 239, 325-328. [CrossRef]

35. Consolati, G.; Natali-Sora, I.; Pelosato, R.; Quasso, F. Investigation of cation-exchanged montmorillonites by combined X-ray diffraction and positron annihilation lifetime spectroscopy. J. Appl. Phys. 2002, 91, 1928-1932. [CrossRef]

36. Jasińska, B.; Koziol, A.E.; Goworek, T. Voids shape and o-Ps lifetime in molecular crystals. Acta Phys. Pol. A 1999, 95, 557-561. [CrossRef]

37. Tao, S.J. Positronium annihilation in molecular substances. J. Chem. Phys. 1972, 56, 5499-5510. [CrossRef]

38. Eldrup, M.; Lightbody, D.; Sherwood, J.N. The temperature-dependence of positron lifetimes in solid pivalic acid. Chem. Phys. 1981, 63, 51-58. [CrossRef]

39. Bradshaw, M.; Zou, J.L.; Byrne, L.; Iyer, K.S.; Stewart, S.G.; Raston, C.L. Pd(II) conjugated chitosan nanofibre mats for application in Heck cross-coupling reactions. Chem. Commun. 2011, 47, 12292-12294. [CrossRef] [PubMed]

40. Zhan, K.; You, H.H.; Liu, W.Y.; Lu, J.; Lu, P.; Dong, J. Pd nanoparticles encaged in nanoporous interpenetrating polymer networks: A robust recyclable catalyst for Heck reactions. React. Funct. Polym. 2011, 71, 756-765. [CrossRef]

(C) 2019 by the authors. Licensee MDPI, Basel, Switzerland. This article is an open access article distributed under the terms and conditions of the Creative Commons Attribution (CC BY) license (http://creativecommons.org/licenses/by/4.0/). 


\title{
Article \\ Effect of Bifunctional Montmorillonite on the Thermal and Tribological Properties of Polystyrene/Montmorillonite Nanocomposites
}

\author{
Chengcheng Yu, Yangchuan Ke *, Xu Hu, Yi Zhao, Qingchun Deng and Shichao Lu \\ Nanotechnology Center of Energy Resources, CNPC Nanochemistry Key Laboratory, College of Science, \\ China University of Petroleum, Beijing 102249, China; yccycc9999@163.com (C.Y.); xuhu0001@126.com (X.H.); \\ yccycc9999@126.com (Y.Z.); qingchundeng@163.com (Q.D.); shichaolu@yeah.net (S.L.) \\ * Correspondence: kyc010@sohu.com
}

Received: 26 April 2019; Accepted: 7 May 2019; Published: 8 May 2019

\begin{abstract}
In this work, the effect of doubly functionalized montmorillonite (MMT) on the structure, morphology, thermal, and tribological characteristics of the resulting polystyrene (PS) nanocomposites was investigated. The modification of the MMT was performed using a cationic surfactant and an anionic surfactant or a silane coupling agent to increase the compatibility with PS matrix. The polystyrene/organo-montmorillonite (PS/OMMT) nanocomposite particles were prepared by soap-free emulsion polymerization. The OMMT was studied using Fourier-transform infrared (FTIR), $\mathrm{X}$-ray diffraction (XRD), and thermogravimetric analysis (TGA). The structural and morphological changes of PS/OMMT nanocomposites were further characterized by dynamic light scattering (DLS), scanning electron microscopy (SEM), and transmission electron microscopy (TEM). The thermal stability of all the PS/OMMT nanocomposites was higher than that of the pure PS. The anti-wear properties of the polyalphaolefin (PAO) were significantly improved due to the introduction of the $\mathrm{PS} / \mathrm{OMMT}$ nanocomposite particles. The nanocomposites prepared by a cationic surfactant and a silane coupling agent exhibited the best thermal stability and tribological performance. Our results provide the valuable insights needed to guide the design of lubrication and friction reducing materials.
\end{abstract}

Keywords: doubly functionalized montmorillonite; polystyrene; soap-free emulsion polymerization; thermal stability; tribological property

\section{Introduction}

Clay-polymer nanocomposites have received great attention in recent years due to their exceptional properties, such as increased thermal stability, high fire resistance, and enhanced mechanical characteristics, compared with traditional polymer composites [1,2]. The improvement of many properties of clay-polymer nanocomposites could be achieved using a relatively low clay loading (usually $<10 \mathrm{wt} \%$ ) in a polymer matrix [3]. Among the layered silicates, montmorillonite (MMT) was one of the most widely utilized to fabricate clay-polymer nanocomposite, mainly because the MMT was easily available with a high aspect ratio and high expansion capacity [4]. The unit crystal structure of the MMT consisted of two silicon tetrahedral sheets and a central sheet of alumina octahedrons. The negative charge was generated on the surfaces of the MMT layers due to the isomorphic substitutions of lower valance ions $\left(\mathrm{Mg}^{2+}, \mathrm{Fe}^{2+}\right)$ for the central atoms $\left(\mathrm{Al}^{3+}, \mathrm{Si}^{4+}\right)$ in the interior crystal layers of the MMT [5]. To neutralize the negative charges, hydrated cations $\left(\mathrm{Na}^{+}, \mathrm{Ca}^{2+}\right)$ were electrostatically adsorbed inside the interlayer space and on the surface of the MMT. Therefore, the natural state of the MMT was a hydrophilic character, which was inherently incompatible with the vast majority of organic polymers [6]. The poor affinity between the hydrophilic MMT and the hydrophobic polymer will not result in a clay-polymer nanocomposite with improved properties 
compared with the pure polymer matrix. To obtain the highest enhancement of properties, the surface modification of the MMT was desirable to increase the interfacial interactions between the MMT and the polymer matrix [7]. The surface treatment not only enhanced the compatibility of the MMT with the organic polymer, but also enlarged the interlayer space between the layers of MMT.

The most traditional and convenient method for the MMT modification was ion exchange reaction with quaternary alkylammonium salts [8]. However, the ammonium surfactants could not provide an effective thermal stability for high temperature applications because the decomposition of the ammonium surfactants could be initiated above $200{ }^{\circ} \mathrm{C}$ [9]. Thus, the modification of the MMT with phosphonium surfactants has attracted much interest from researchers. Several research papers found that the thermal stability of organoclays modified with phosphonium surfactants was superior to that of organoclays modified with ammonium surfactants $[10,11]$. On the other hand, the anionic surfactant was also used to improve the thermal stability of the organoclays [12]. However, it could be rather difficult to significantly expand the interlayer space of the MMT via the anionic surfactant alone. Therefore, a novel kind of organo-montmorillonite (OMMT) by the synergistic effect between the anionic and cationic surfactants has been prepared in recent years [13]. Another method for the modification of the MMT involved the use of silane coupling agents. These silanes could react with the MMT OH groups to form the chemical bonds with a covalent character between the molecules of the silane and the surface of the MMT [14]. The grafting reaction mainly occurred at the broken edges and at the structural defects situated at the external surfaces of the MMT layers [15]. However, the employment of both a phosphonium surfactant and an anionic surfactant, or both a phosphonium surfactant and a silane coupling agent, to fabricate the OMMT to increase the dispersion degree in the polymer matrix was rarely reported.

Polystyrene (PS) is a synthetic thermoplastic that is widely used in industry due to its excellent performance of high abrasion resistance, strong plasticity and load bearing capacity [16]. However, the relatively weak thermal stability and heat resistance of PS has become a significant challenge, hindering its further applications in a variety of areas. To compensate this demerit, the addition of inorganic clay to the PS matrix can be further helpful to enhance the properties of the PS matrix [17]. Recently, an attractive technique to synthesize the clay-polymer nanocomposite is the emulsion polymerization due to the great advantage of controlled morphology and employment of water as a dispersion medium [18]. However, the surfactants were usually used in emulsion polymerization to provide colloidal stability, which may affect the final properties of the nanocomposite. The soap-free emulsion polymerization is a clean and effective way to prepare clay-polymer particles due to the use of little to no surfactant (below critical micelle concentration) in such a polymerization system $[19,20]$. Though the soap-free emulsion polymerization was extensively studied for the preparation of clay-polymer nanocomposites, a few papers related to the investigation of the effect of doubly organo-modified montmorillonite on the final properties of the resulting PS/OMMT nanocomposites have been published so far.

In this work, two kinds of doubly organo-modified MMT were successfully prepared by using both a cationic surfactant and an anionic surfactant or both a cationic surfactant and a silane coupling agent. Then, the PS/OMMT nanocomposites were produced using styrene monomer and the obtained OMMT samples via the soap-free emulsion polymerization. The obtained PS/OMMT nanocomposites were dispersed into polyalphaolefin (PAO) to create lubricants. The effect arising from the doubly organo-modified MMT on the structure, morphology, thermal, and tribological properties of the PS/OMMT nanocomposites were studied and evaluated.

\section{Materials and Methods}

\subsection{Materials}

The natural sodium montmorillonite (MMT, cationic exchange capacity (CEC) of $100 \mathrm{mmol} / 100 \mathrm{~g}$ ) was provided by Huai An Saibei Technology Co. Ltd., Zhangjiakou, China. The tributyltetradecylphosphonium chloride (TTPC, 97\%), sodium lauryl sulfonate (SLS, 99\%), and $\gamma$-methacryloxypropyltrimethoxysilane 
(KH570, 97\%) were purchased from Aladdin Industrial Corporation, Shanghai, China. Styrene (St, 98\%), divinylbenzene (DVB, 80\%), methacrylic acid (MAA, 98\%), and potassium persulfate (KPS, 99\%) were supplied by the Tianjin Guang fu Fine Chemicals Research Institute, Tianjin, China. The St was washed with $\mathrm{NaOH}$ solution to remove the polymerization inhibitors, and then distilled under reduced pressure before use. Polyalphaolefin (PAO, 99\% purity) was provided by Shanghai Fox Chemical Technology, Shanghai, China. Other reagents were of analytical grade and used as received. Deionized water was applied for all experiments.

\subsection{Preparation of OMMT}

\subsubsection{Preparation of P-MMT}

The modification of MMT with TTPC was carried out by the method reported in a previous paper [21]. Briefly, $2.5 \mathrm{~g}$ of MMT was dispersed into $75 \mathrm{~mL}$ of deionized water with stirring for $30 \mathrm{~min}$ at $75^{\circ} \mathrm{C}$. Then, $25 \mathrm{~mL}$ of aqueous solution containing the TTPC surfactant equivalent to $1.2 \mathrm{CEC}$ of MMT was slowly added into the clay dispersion. The mixture was further stirred at $75^{\circ} \mathrm{C}$ for $11 \mathrm{~h}$ and then filtered by vacuum filtration followed by washing with deionized water several times to remove excess TTPC molecules. The final product was dried at $60^{\circ} \mathrm{C}$ for one day in a vacuum oven then ground into powder. The sample formed was referred to as P-MMT. The preparation process was shown in Figure 1 (Stage A).

\subsubsection{Preparation of P-SLS-MMT}

The P-MMT was further modified using the SLS surfactant to produce the doubly organo-modified MMT (P-SLS-MMT) as shown in Figure 1 (Stage B) [22]. In detail, $2.5 \mathrm{~g}$ of P-MMT and $75 \mathrm{~mL}$ of deionized water were introduced in the reaction flask and stirred for $0.5 \mathrm{~h}$ at $75^{\circ} \mathrm{C}$. Then, the desired amount of SLS surfactant was dissolved in $25 \mathrm{~mL}$ of deionized water and added slowly into the suspension. The amount of SLS used was equivalent to 0.6 CEC of MMT. The mixture was stirred vigorously for $9 \mathrm{~h}$ at $75^{\circ} \mathrm{C}$. Finally, the resultant product was separated by centrifugation and washed four times using a mixture of ethanol/deionized water to remove the residual surfactants. The final product was dried at $60^{\circ} \mathrm{C}$ overnight to obtain the P-SLS-MMT sample.

\subsubsection{Preparation of P-KH570-MMT}

The silane functionalization of P-MMT was carried out following the method described in a previous report [23]. In a typical experiment (Figure 1 (Stage C)), the KH570 (2.483 g) was dissolved into methanol $(90 \mathrm{~mL}) /$ deionized water $(10 \mathrm{~mL})$ solution with stirring, and the $\mathrm{pH}$ of the mixture was adjusted to 4.0 with acetic acid. The mixture was stirred for $1 \mathrm{~h}$ at room temperature to obtain the hydrolyzed KH570. Then, $2.5 \mathrm{~g}$ of P-MMT was introduced into the mixture and the suspension was further mechanically stirred with reflux at $75^{\circ} \mathrm{C}$ for $24 \mathrm{~h}$. Finally, the product was filtered and washed with methanol to remove the undesired siane molecules, and then dried under a vacuum oven at $60^{\circ} \mathrm{C}$ for $24 \mathrm{~h}$. The collected product was named as P-KH570-MMT.

\subsection{Preparation of PS/OMMT Nanocomposite}

The soap-free emulsion polymerization was applied to prepare the PS/OMMT nanocomposites according to previous reports [24,25]. Briefly, a mixture of OMMT $(0.6 \mathrm{~g}, 3.0 \mathrm{wt} \%$ based on St), St (20.0 g), DVB (1.8 g) and an auxiliary monomer MAA (1.0 g) was ultrasonicated for $10 \mathrm{~min}$ in an ice bath and then magnetically stirred at room temperature for $1 \mathrm{~h}$ (Figure 1 (Stage D)). Meanwhile, $0.1 \mathrm{~g}$ of SLS was dissolved in $180 \mathrm{~mL}$ of deionized water with stirring for 30 min under nitrogen atmosphere. Both were later mixed in a 500-mL round-bottomed flask and mechanically stirred at $75^{\circ} \mathrm{C}$ for $30 \mathrm{~min}$ under nitrogen atmosphere. Then, $0.109 \mathrm{~g}$ of KPS initiator was dissolved in $40 \mathrm{~mL}$ of deionized water and slowly introduced into the reactor. The resulting mixture was polymerized at $75^{\circ} \mathrm{C}$ for $24 \mathrm{~h}$ under nitrogen atmosphere and mechanical stirring (Figure 1 (Stage E)). The final products were 
obtained by centrifugation and washing three times using excess hot ethanol to remove the unbound polymers, then dried overnight at $60^{\circ} \mathrm{C}$. The nanocomposite prepared with P-MMT, P-SLS-MMT, and P-KH570-MMT was abbreviated as PS/P-MMT, PS/P-SLS-MMT, and PS/P-KH570-MMT, respectively.
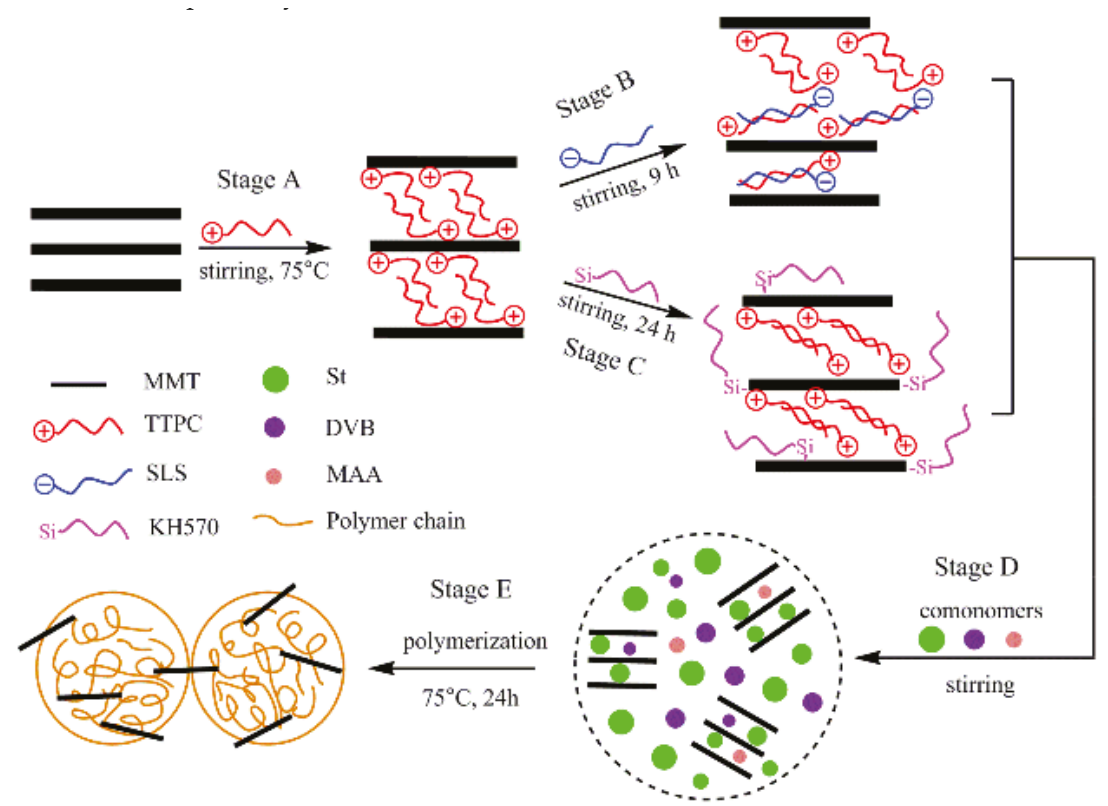

Figure 1. Schematic representation of the processes of preparing the organo-montmorillonite (OMMT) and the polystyrene (PS)/OMMT nanocomposites.

\subsection{Preparation of the PAO-PS/OMMT Lubricant}

The PAO-PS/OMMT lubricant was prepared to investigate the tribological performance of the PS/OMMT nanocomposites as additives. The PS/OMMT nanocomposites (1.0 wt \% based on PAO) were added to the PAO, and the mixture was mechanically stirred at $1000 \mathrm{rpm}$ for $30 \mathrm{~min}$ followed by ultrasonication for $1 \mathrm{~h}$ at room temperature to allow the dispersion and homogenization of the nanocomposites in the PAO.

\subsection{Characterization}

Fourier-transform infrared (FTIR) spectra were conducted over the range of $4000-400 \mathrm{~cm}^{-1}$ using an FTS-3000 spectrophotometer (American Digilab) via a KBr pressed disk technique at room temperature.

X-ray diffraction (XRD) measurements were carried out with a Bruker D8 Advance X-ray Diffractometer (Stadi P, Karlsruhe, Germany) using Cu-Ka radiation $(\lambda=0.1540 \mathrm{~nm}$ ) with a scanning rate of $2^{\circ} / \mathrm{min}, 40 \mathrm{kV}$, and $30 \mathrm{~mA}$ over the $2 \theta$ range of $1.5^{\circ}-20^{\circ}$ by a scanning interval of $0.02^{\circ}$. The corresponding basal space was computed by applying the Bragg's Law.

Dynamic light scattering (DLS) was performed using a Malvern Mastersizer 2000 (Malvern Instruments, Malvern, UK) to determine the particle size and size distribution of the samples at room temperature.

Scanning electron microscopy (SEM, SU8010, Hitachi, Tokyo, Japan) coupled with an energy dispersing spectrometer (EDS) was used to study the surface morphology and elements of the nanocomposites. The samples were coated with a thin layer of gold prior to SEM observation. The EDS analysis was registered three times in different points, and the result was the average of three obtained values. 
Transmission electron microscopy (TEM) analyses were performed on a JEM-2100 TEM operating at an accelerating voltage of $200 \mathrm{kV}$.

Thermogravimetric analysis (TGA) was carried out on a NETZSCH thermogravimetric analyzer (TGA, STA409PC, Bavaria, Germany) at a heating rate of $10{ }^{\circ} \mathrm{C} / \mathrm{min}$ from $25{ }^{\circ} \mathrm{C}$ to $800{ }^{\circ} \mathrm{C}$ under a nitrogen flow. Differential thermogravimetry (DTG) curves were calculated from the TGA results.

The tribological measurements were performed using a four-ball tester (MRS-1J, Jinan, China) under a load of $392 \mathrm{~N}$ at a rotating speed of $1450 \mathrm{r} / \mathrm{min}$ for $1 \mathrm{~h}$ at room temperature. Prior to testing, the steel bearing balls GCr15 (diameter of $12.7 \mathrm{~mm}$, grade 10) were cleaned ultrasonically for $30 \mathrm{~min}$ with ethanol. The average values of coefficient of friction (COF) were reported from three measurements of each sample.

\section{Results}

\subsection{Surface Treatments of Montmorillonite}

\subsubsection{FTIR Analysis}

The FTIR spectra of MMT, PMMT, P-SLS-MMT, and P-KH570-MMT are given in Figure 2. In the spectra of MMT, the broad absorption bands at 3446.4 and $1643.4 \mathrm{~cm}^{-1}$ corresponded to the -OH stretching vibration and $\mathrm{H}-\mathrm{O}-\mathrm{H}$ bending vibration of adsorbed water within MMT interlayer, respectively. The characteristic absorption peak at $3632.5 \mathrm{~cm}^{-1}$ was attributed to the stretching vibration of the $\mathrm{Al}-\mathrm{OH}$ and $\mathrm{Si}-\mathrm{OH}$ in the silicate layers, and this $-\mathrm{OH}$ group was responsible for the reaction with the silane coupling agent [26]. The bands at 1036.1 and $793.4 \mathrm{~cm}^{-1}$ were representative of the stretching vibration of $\mathrm{Si}-\mathrm{O}$ and $\mathrm{Si}-\mathrm{O}-\mathrm{Al}$, respectively. In addition of these characteristic peaks of MMT, novel vibration bands related to surfactants and/or silane coupling agents appeared in the spectra of OMMT (Figure 2B-D). For the P-MMT, the asymmetric and symmetric stretching vibration of $-\mathrm{CH}_{2}$ of phosphonium surfactants was observed at 2928.2 and $2854.0 \mathrm{~cm}^{-1}$, respectively. This is direct evidence for the successful intercalation of TTPC surfactants into the interlayer space of MMT. In comparison with the P-MMT, the intensity of the absorption bands at 2928.2 and $2854.0 \mathrm{~cm}^{-1}$ increased for the P-SLS-MMT, which can be reasonably attributed to the introduction of the SLS surfactants. Besides, the absorption band at $1090.2 \mathrm{~cm}^{-1}$ corresponded to the asymmetric stretching vibration of $-\mathrm{SO}_{3} \mathrm{H}$ group of SLS surfactants. These results indicated that the TTPC and SLS surfactants were synergistically incorporated into the interlayer space of MMT. The spectrum of P-KH570-MMT not only had the characteristic bands of P-MMT, but also exhibited a novel band at $1723.9 \mathrm{~cm}^{-1}$, which was assigned to the stretching vibration of the $\mathrm{C}=\mathrm{O}$ in the $\mathrm{KH} 570$ molecules. In addition, higher intensity of the absorption bands at 2928.2 and $2854.0 \mathrm{~cm}^{-1}$ were also found for the P-KH570-MMT sample, implying that KH570 was successfully grafted on the surfaces and edges of the silicate layers [27]. These observations suggested that the MMT was doubly organo-modified by a phosphonium surfactant and a silane coupling agent. It is worth mentioning that a significant decrease of the relative intensity of the bands at 3632.5-3446.4 $\mathrm{cm}^{-1}$ and $1639.8 \mathrm{~cm}^{-1}$ was observed for the OMMT compared with the MMT, which indicated that the surface of OMMT changed from a hydrophilic to a hydrophobic character and the content of water over OMMT decreased. The above results indicated that the target doubly organo-modified MMT was successfully synthesized. 


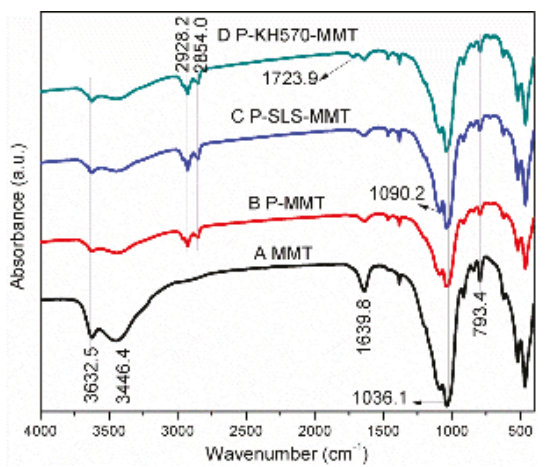

Figure 2. Fourier-transform infrared (FTIR) spectra of (A) sodium montmorillonite (MMT), (B) P-MMT, (C) P-SLS-MMT, and (D) P-KH570-MMT.

\subsubsection{XRD Analysis}

The XRD patterns obtained for the MMT and OMMT are presented in Figure 3. The MMT showed a typical XRD reflection at $2 \theta=7.28^{\circ}$, corresponding to a basal spacing of $1.21 \mathrm{~nm}$ according to Bragg's law. This value is in agreement with similar hydrated clays reported in the literature [28]. The significant increase in the interlayer space of the MMT was observed after the modification with surfactants. In the case of P-MMT, the characteristic peak shifted from $7.28^{\circ}$ to $3.84^{\circ}$, corresponding to an increase in basal space from $1.21 \mathrm{~nm}$ to $2.36 \mathrm{~nm}$. This result confirms that the intercalation of phosphonium surfactant was really achieved, which is consistent with the FTIR results. In the XRD spectrum of P-SLS-MMT, the distribution of interlayer space of platelets was characterized by two prominent reflections that appeared at $2 \theta=2.87^{\circ}$ and $5.79^{\circ}$, corresponding to interlayer spaces of $3.07 \mathrm{~nm}$ and $1.52 \mathrm{~nm}$, respectively. The basal space of P-SLS-MMT was $0.71 \mathrm{~nm}$ higher than that of P-MMT, which indicated that SLS successfully entered the interlayer space of P-MMT and cation-anion type OMMT was prepared. The anionic surfactants can form ion-pairs with the cationic surfactants to intercalate into the interlayer space of MMT. This result was also observed by other researchers $[29,30]$. The appearance of a second peak in the XRD pattern of P-SLS-MMT was attributed to the (002) reflection. The diffraction peak of P-KH570-MMT at $4.21^{\circ}$ corresponded to an interlayer space of $2.09 \mathrm{~nm}$. Compared with P-MMT, the decrease in the interlayer space of P-KH570-MMT was mainly due to partly intercalated cationic surfactants becoming washed out with the use of hydroalcoholic solution as a solvent in the silylation reaction. This result indicated that the KH570 was mainly grafted onto the broken edges and external surfaces or undergoing ion exchange with the pre-existing surfactants in a very low amount, thus merely had a significant impact on the interlayer structure of P-MMT [31].

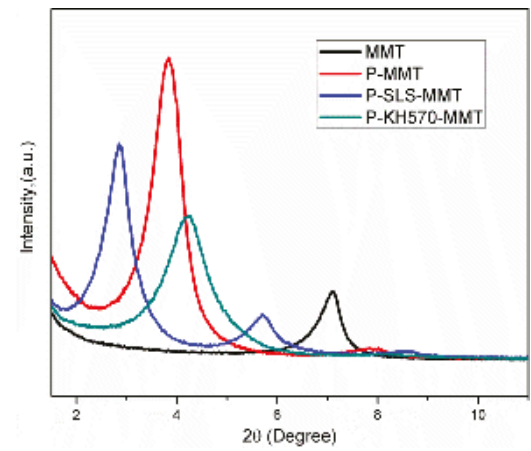

Figure 3. XRD patterns of MMT, P-MMT, P-SLS-MMT, and P-KH570-MMT. 


\subsubsection{TGA and DTG Analysis}

Figure 4 shows the thermogravimetric analysis (TGA) and derivative thermogravimetry (DTG) curves of MMT, PMMT, P-SLS-MMT, and P-KH570-MMT samples. In Table 1, the mass loss associated with the different temperature ranges is listed. Two distinct thermal degradation events were obtained in the DTG curves of the MMT. The first mass loss, occurring at a temperature of $75^{\circ} \mathrm{C}$, was assigned to the elimination of physically adsorbed water, and the second at $660^{\circ} \mathrm{C}$ corresponded to the dihydroxylation of clay layers [32]. The mass loss of OMMT was higher than that of MMT at $200-600^{\circ} \mathrm{C}$, which was evidence of intercalated surfactants and/or grafted silane coupling agents. For the P-MMT, the mass loss below $200{ }^{\circ} \mathrm{C}$ was lower when compared with the MMT, implying less adsorbed water in the P-MMT. Furthermore, the mass loss of the P-MMT between $200^{\circ} \mathrm{C}$ and $600^{\circ} \mathrm{C}$ was obviously higher than that of the MMT, which was attributed to the degradation of adsorbed surfactant molecules on the external surface and of intercalated surfactant molecules in the interlayer space of the MMT. The overall mass loss of P-MMT (20.7 wt \%) was higher than that of MMT (5.6 wt \%). Compared with P-MMT, a greater mass loss in the range of $200^{\circ} \mathrm{C}$ to $600^{\circ} \mathrm{C}$ was found in P-SLS-MMT, which could be attributed to the intercalation of SLS molecules into the interlayer space of P-MMT. In addition, the maximum mass loss rate temperature of P-SLS-MMT occurred at $499^{\circ} \mathrm{C}$, higher than $15^{\circ} \mathrm{C}$ in P-MMT, indicating the thermal properties of P-MMT were enhanced owing to the introduction of the SLS molecules. These results suggested that the SLS molecules were successfully intercalated into the interlayer space of the P-MMT. This result was consistent with the results of XRD analysis. In the case of P-KH570-MMT, the first mass loss occurred at a temperature below $200{ }^{\circ} \mathrm{C}$ which was attributed to the evaporation of adsorbed water and/or of free molecules of solvent in the pores of MMT. The mass loss observed over the range of $200-600{ }^{\circ} \mathrm{C}$ was assigned to the loss of surfactant molecules combined with the decomposition of adsorbed and/or grafted KH570 molecules introduced during the silylation reaction. Compared with the P-MMT, the observed increase in mass loss for the P-KH570-MMT between $200{ }^{\circ} \mathrm{C}$ and $600{ }^{\circ} \mathrm{C}$ was attributed to the intercalated and/or grafted silane molecules. Combining this with the result of XRD, the interlayer space of P-KH570-MMT was smaller than that of P-MMT, the increasing amount of KH570 molecule was mainly grafted on the broken edges and the external surface of MMT, not intercalating in the interlayer space of MMT [33].
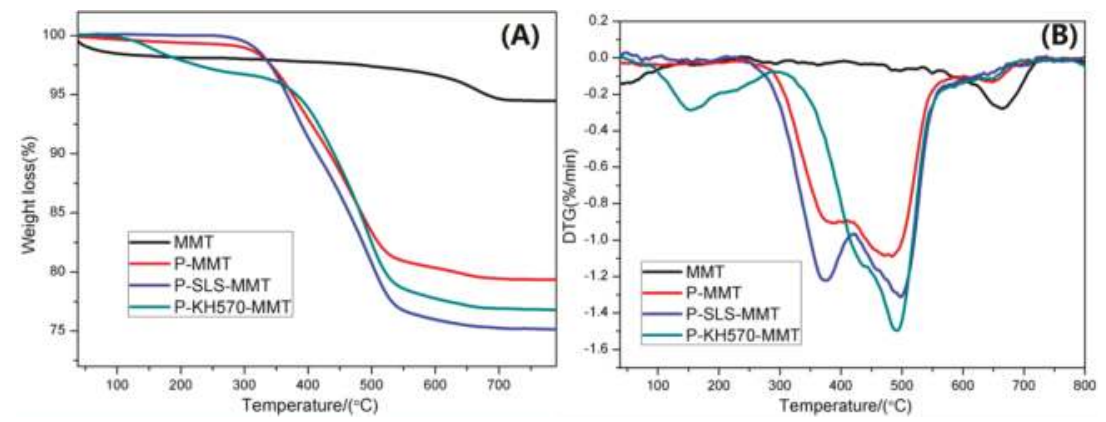

Figure 4. (A) Thermogravimetric analysis (TGA) and (B) Derivative thermogravimetry (DTG) curves of MMT, P-MMT, P-SLS-MMT, and P-KH570-MMT.

Table 1. Relative mass loss during decomposition of investigated samples (wt \%).

\begin{tabular}{cccc}
\hline \multirow{2}{*}{ Sample } & \multicolumn{3}{c}{ Temperature Range } \\
\cline { 2 - 4 } & $<\mathbf{2 0 0} \pm \mathbf{1 . 5}{ }^{\circ} \mathbf{C}$ & $\mathbf{2 0 0 - 6 0 0 \pm \mathbf { 1 . 9 }}{ }^{\circ} \mathbf{C}$ & $\mathbf{3 0 - 8 0 0 \pm \mathbf { 2 . 2 }}{ }^{\circ} \mathbf{C}$ \\
\hline MMT & 1.9 & 1.5 & 5.6 \\
P-MMT & 0.6 & 19.0 & 20.7 \\
P-SLS-MMT & 0.2 & 23.9 & 24.9 \\
P-KH570-MMT & 2.3 & 19.9 & 23.3 \\
\hline
\end{tabular}




\subsection{Characterization of PS/OMMT Nanocomposite}

\subsubsection{FTIR Analysis}

The FTIR spectra of the PS, PS/P-MMT, PS/P-SLS-MMT, and PS/P-KH570-MMT are shown in Figure 5. In the case of PS, the characteristic bands at 3027.6 and $2914.0 \mathrm{~cm}^{-1}$ were attributed to the $\mathrm{CH}$ aromatic stretching vibrations and $\mathrm{CH}_{2}$ asymmetric stretching vibrations, respectively. Two absorption bands at 1602.6 and $1449.1 \mathrm{~cm}^{-1}$ were ascribed to the stretching vibration of the aromatic $\mathrm{C}=\mathrm{C}$. There were two strong absorption bands at 763.0 and $691.1 \mathrm{~cm}^{-1}$ that corresponded to the $\mathrm{CH}$ out-of-plane deformation of benzene rings. In addition, the peak at $1725.7 \mathrm{~cm}^{-1}$ was due to the $\mathrm{C}=\mathrm{O}$ stretching vibration of methacrylic acid. These observations indicate that the polymerization reaction of styrene monomer has been occurred [34]. The FTIR spectra of PS/P-MMT not only had the characteristic bands of PS, but also exhibited new absorption bands at $1039.7 \mathrm{~cm}^{-1}$, which were assigned to Si-O stretching vibrations of the MMT. This result proved the formation of PS/P-MMT nanocomposites. The presence of the characteristic peak of MMT in the polymer matrix was also observed in the samples of PS/P-SLS-MMT and PS/P-KH570-MMT.

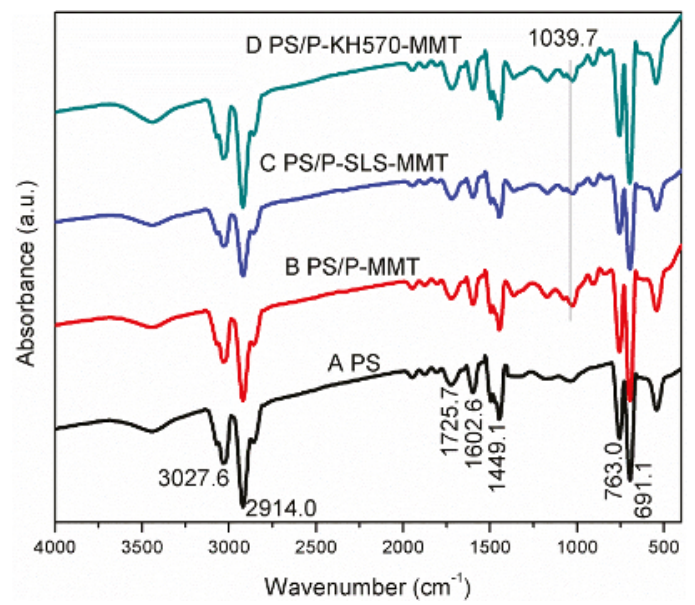

Figure 5. FTIR spectra of (A) PS, (B) PS/P-MMT, (C) PS/P-SLS-MMT, and (D) PS/P-KH570-MMT.

\subsubsection{XRD Analysis}

Figure 6 shows the XRD patterns of the PS, PS/P-MMT, PS/P-SLS-MMT, and PS/P-KH570-MMT nanocomposites. As shown in Figure 6, no characteristic diffraction peak of OMMT was observed for the PS/P-MMT, PS/P-SLS-MMT, or PS/P-KH570-MMT in the range of $1.5^{\circ}-10.0^{\circ}$ of $2 \theta$. This result suggested that the PS chains entered into the interlayer space of OMMT and destroyed the intercalated clay structure, leading to exfoliated structure in the resulting PS/OMMT nanocomposite during the soap-free emulsion polymerization. The clay platelets could be exfoliated in the PS matrix owing to the role of the intercalated phosphonium surfactants, SLS molecules, and/or adsorbed KH570 silanes that obviously enlarged the interlayer space of the MMT, which could provide a bigger space for the ease of PS macromolecular chains penetration. In addition, the complete exfoliation of individual clay platelets in the polymer matrix was also because of the favorable miscibility between the organic clay platelets and polymer matrix [35]. Furthermore, the KH570 molecules that grafted on the broken edges of the MMT could improve the hydrophobicity of the layered silicates, thus increasing their compatibility with the PS matrix [36]. However, the disappearance of XRD peaks does not necessarily suggest complete delamination of the clay platelets. Thus, other analytical tools, such as TEM, were needed to prove the dispersion and degree of exfoliation of the clay platelets. In order to determine the 
deductions from XRD results, the morphology of the nanocomposites was further analyzed by the use of TEM. Figure 7 shows the typical TEM images of the PS and PS/OMMT nanocomposites.

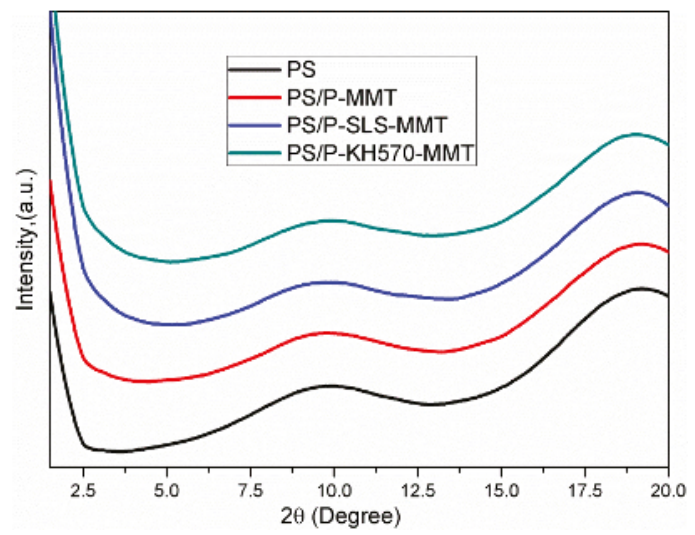

Figure 6. XRD patterns of PS, PS/P-MMT, PS/P-SLS-MMT, and PS/P-KH570-MMT.

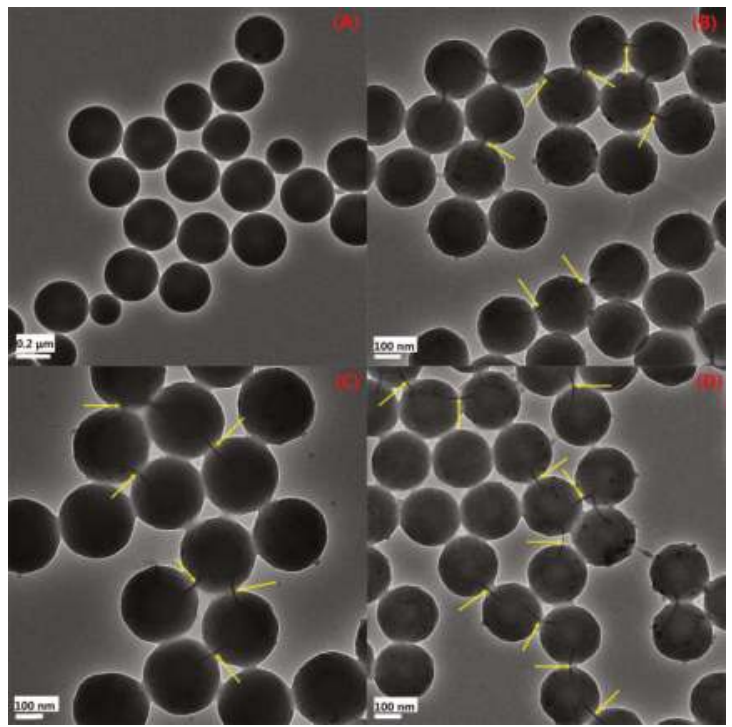

Figure 7. Transmission electron microscopy (TEM) images of (A) PS, (B) PS/P-MMT, (C) PS/P-SLS-MMT, and (D) PS/P-KH570-MMT.

\subsubsection{TEM Analysis}

As shown in Figure 7A, spherical particles could be observed for the pure PS. However, the size distribution of particles was broad, and the particle size was in the range of approximately 200-600 nm. In contrast, when the OMMT was introduced into the polymer matrix, the particle sizes of the PS/OMMT nanocomposites became much smaller, as shown in Figure 7B-D. Moreover, the size distribution of PS/OMMT particles was narrow and monomodal, and the particle size was approximately between $250 \mathrm{~nm}$ and $400 \mathrm{~nm}$. These results indicated that the morphology of the polymer particles was changed by the presence of OMMT. The reason was probably that the movement of the styrene monomers and growth of the PS chains were effectively reduced via the shielding effects of the clay platelets. 
As denoted by the yellow arrows in Figure 7B-D, the existence of exfoliated clay layers encapsulated inside the polymer particles was clearly visible. The TEM images of PS/OMMT nanocomposites showed that the nanoclay layers were completely exfoliated and dispersed homogeneously within the PS matrix. It showed that a highly exfoliated structure of PS/OMMT nanocomposite was obtained, which was in accordance with the corresponding XRD results. This result implied that the clay layers of 3 wt \% OMMT could maintain the polymer colloidal stability, thus resulting resulted in the complete exfoliation of the clay layers and formation of exfoliated structure of PS/OMMT nanocomposites [37].

\subsubsection{DLS Analysis}

Figure 8 shows the size distributions of the pure PS and PS/OMMT nanocomposite samples, and the average particle size data and PDI values are summarized in Table 2. The particle size distributions of all samples showed only one narrow peak, and the average diameters of the polymer particles were in the range of $241.3-378.6 \mathrm{~nm}$. These results were consistent with the results of the TEM analysis. The average particle size of the pure PS was $378.6 \mathrm{~nm}$, while the average particle sizes of the PS/OMMT nanocomposites were $290.4 \mathrm{~nm}, 338.8 \mathrm{~nm}$, and $241.3 \mathrm{~nm}$ for the PS/P-MMT, PS-P-SLS-MMT, and PS/P-KH570-MMT, respectively. The decrease in the average particle size of the nanocomposites was owing to the addition of OMMT would more likely to facilitate the nucleation to form smaller and more uniform particles. On the other hand, the PDI values, which were indicative of the monodispersity of polymer particles, were observed to decrease for the PS/OMMT nanocomposites compared with the pure PS. This observed decrease was owing to the self-arrangement of the modified MMT layers within the PS particles might affect the surface tension and cause fine and stable particles. Moreover, the hydrophobic MMT layers could act as stabilizers to improve the monodispersity of the PS particles [38]. The organic modification of MMT improved its compatibility with the monomer phase and promoted the exfoliation of the MMT, which was beneficial to the encapsulation of clay platelets by the polymer particles. The smallest particle size and PDI value were obtained for the PS/P-KH570-MMT sample, which was due to the ability of the vinyl group of the KH570 molecule to participate in the polymerization reaction.

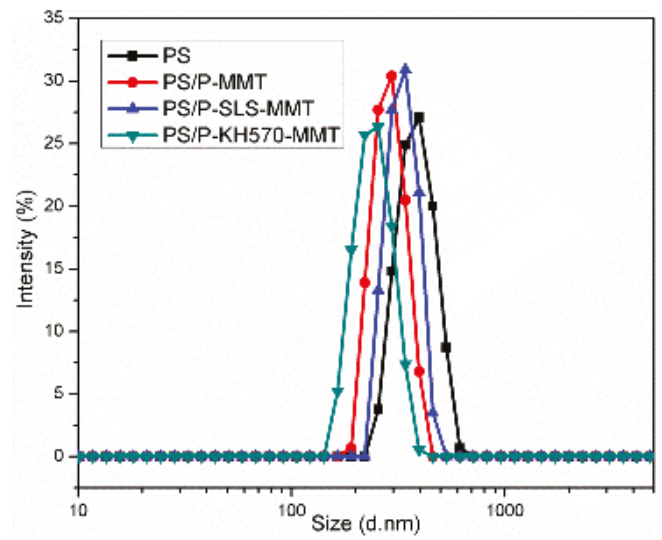

Figure 8. Size distribution curves of PS, PS/P-MMT, PS/P-SLS-MMT, and PS/P-KH570-MMT.

Table 2. Particle size distribution of PS, PS/P-MMT, PS/P-SLS-MMT, and PS/P-KH570-MMT samples.

\begin{tabular}{ccc}
\hline Sample & Z-Average $(\mathbf{n m})$ & PDI \\
\hline PS & $378.6 \pm 8.9$ & 0.232 \\
PS/P-MMT & $290.4 \pm 3.8$ & 0.159 \\
PS/P-SLS-MMT & $338.8 \pm 3.6$ & 0.160 \\
PS/P-KH570-MMT & $241.3 \pm 2.1$ & 0.074 \\
\hline
\end{tabular}




\subsubsection{SEM-EDS Analysis}

Figure 9 shows the SEM images of the PS, PS/P-MMT, PS/P-SLS-MMT, and PS/P-KH570-MMT nanocomposites. As seen in Figure 9A, the particles of pure PS were mostly spherical and the particle size was approximately $380 \mathrm{~nm}$ with a broad size distribution. In particular, a certain degree of coagulation of the particles was observed due to the poor stability. Compared with the pure PS, the particle morphology of the PS/OMMT nanocomposites was changed by the presence of the OMMT. When the OMMT was introduced in Figure 9B-D, the particle size distribution became narrow, and the particle size decreased to $250-350 \mathrm{~nm}$. These results were in good agreement with the results of TEM and DLS. Moreover, each PS/OMMT particle kept good dispersion stability because of the stabilizing effect of OMMT for the polymer particle.

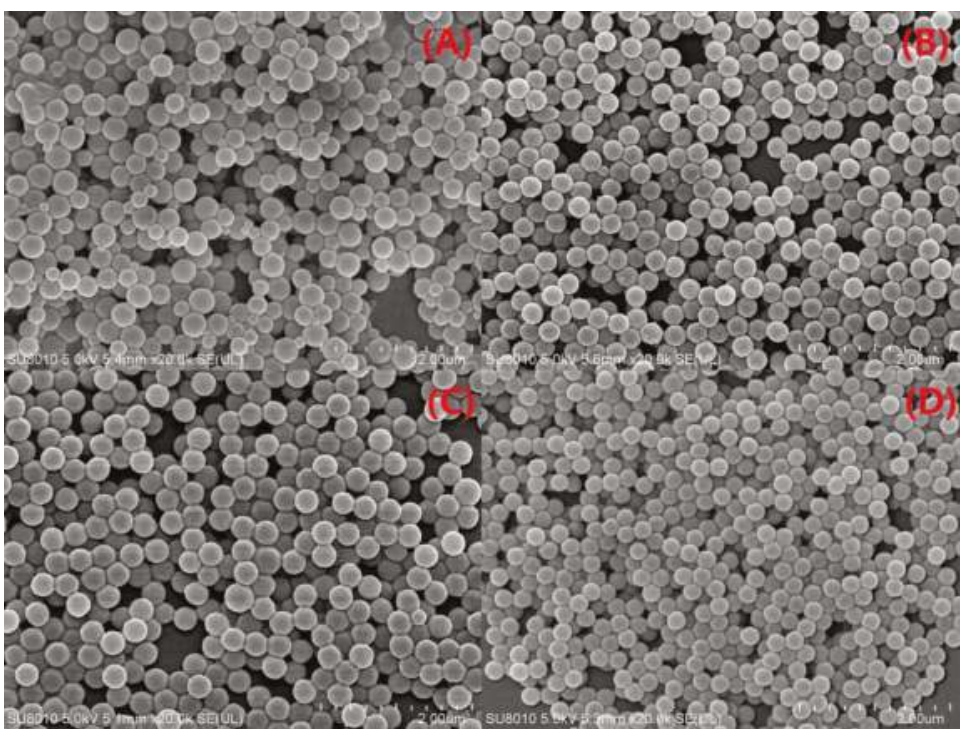

Figure 9. SEM images of (A) PS, (B) PS/P-MMT, (C) PS/P-SLS-MMT, and (D) PS/P-KH570-MMT.

The results of the SEM-EDS analysis are given in Table 3. The most pronounced distinction between the pure PS and the PS/OMMT nanocomposites was the drastic increases in the amount of $\mathrm{Al}$ and $\mathrm{Si}$ content in the PS/OMMT samples. This result indicated that the OMMT was successfully introduced in the polymer matrix.

Table 3. Distribution of the elements in PS, PS/P-MMT, PS/P-SLS-MMT, and PS/P-KH570-MMT samples found by EDS measurement related with SEM images given in Figure 9.

\begin{tabular}{ccccc}
\hline Sample & $\mathbf{C}(\mathbf{w t} \mathbf{\%})$ & O $(\mathbf{w t}$ \%) & Al (wt \%) & Si (wt \%) \\
\hline PS & $68.44 \pm 0.46$ & $30.33 \pm 0.36$ & $0.70 \pm 0.09$ & $0.53 \pm 0.07$ \\
PS/P-MMT & $63.62 \pm 0.24$ & $31.45 \pm 0.40$ & $3.52 \pm 0.17$ & $1.41 \pm 0.12$ \\
PS/P-SLS-MMT & $64.15 \pm 0.30$ & $31.36 \pm 0.38$ & $3.05 \pm 0.19$ & $1.44 \pm 0.13$ \\
PS/P-KH570-MMT & $67.92 \pm 0.33$ & $28.61 \pm 0.29$ & $2.33 \pm 0.11$ & $1.14 \pm 0.12$ \\
\hline
\end{tabular}

\subsubsection{TGA and DTG Analysis}

Figure 10 presents TGA and DTG curves for the pure PS, PS/P-MMT, PS/P-SLS-MMT, and PS/P-KH570-MMT. It can be seen from the TGA curves that the weights of residue left of PS/OMMT nanocomposites at $550{ }^{\circ} \mathrm{C}$ were increased compared with the pure PS, which indicated the presence of 
the MMT in the polymer matrix. Furthermore, in contrast to the pure PS, the PS/OMMT nanocomposites exhibited significantly improved thermal stability, which could be attributed to the addition of OMMT in the polymer matrix [39]. The onset decomposition temperature corresponding to a mass loss of 10 wt $\%\left(T_{10} \%\right)$ of pure PS was $383{ }^{\circ} \mathrm{C}$. As for the PS matrix filled with OMMT, the onset decomposition temperatures of PS/P-MMT, PS/P-SLS-MMT, and PS/P-KH570-MMT were about $399{ }^{\circ} \mathrm{C}, 406{ }^{\circ} \mathrm{C}$, and $416{ }^{\circ} \mathrm{C}$, respectively, that was, $16{ }^{\circ} \mathrm{C}, 23{ }^{\circ} \mathrm{C}$, and $33^{\circ} \mathrm{C}$ higher than that of pure PS. The maximum mass loss temperature $\left(T_{\max }\right.$, observed from DTG) was raised from $419{ }^{\circ} \mathrm{C}$ for the pure PS to $440{ }^{\circ} \mathrm{C}, 443{ }^{\circ} \mathrm{C}$, and $449^{\circ} \mathrm{C}$ for PS/P-MMT, PS/P-SLS-MMT, and PS/P-KH570-MMT, respectively. The enhancement in the thermal stability was due to the MMT layers forming a three-dimension network and a labyrinth pathway in the clay-polymer nanocomposite to prevent out-diffusion of the volatile decomposition products during thermal decomposition [40]. Meanwhile, the exfoliated structure of the MMT worked as a mass transport barrier, or a nucleating agent in the matrix, by limiting the heat transfer and absorbing the heat to decelerate the degradation of the polymers [41]. However, it was observed that the $T_{10 \%}$ and $T_{\max }$ of PS/P-SLS-MMT were higher than those of PS/P-MMT. The reason was perhaps that the maximum mass loss temperature of P-SLS-MMT was higher than that of P-MMT, as shown in Figure 4B, due to the electrostatic interaction between the anionic and cationic surfactants. This improvement in thermal stability was also observed in the PS/P-KH570-MMT nanocomposite. These results revealed that the double-modified MMT-containing PS nanocomposites showed higher thermal stability than that of the single-modified one. Interestingly, the $T_{10 \%}$ and $T_{\max }$ of the PS/P-KH570-MMT were higher than those of the PS/P-SLS-MMT. This result indicated that the double bonds of silanes grafting on the edges and surfaces of the MMT could react with styrene monomer increased chemical interaction between the MMT and the polymers, resulting in the improved interface interactions and enhanced the thermal stability [42]. In this case, the PS chains were covalently bonded on the surface of MMT, and the grafted KH570 molecules worked as the "bridge" connecting the PS chains and silicate layers, leading to an increase in the thermal stability.

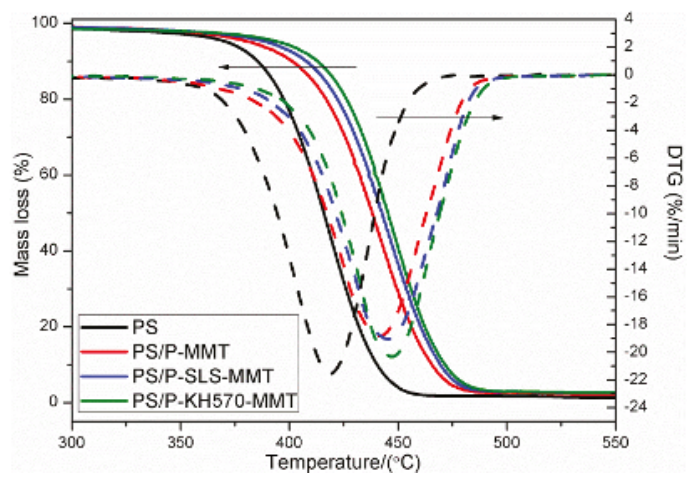

Figure 10. TGA and DTG curves of PS, PS/P-MMT, PS/P-SLS-MMT, and PS/P-KH570-MMT.

\subsubsection{Friction Performance Analysis}

Figure 11 shows the coefficient of friction (COF) versus time of PAO and PAO with PS/OMMT nanocomposite samples. As it can be seen from the figure, the COF of PAO was around 0.095 at the beginning and then increased dramatically to about 0.125 within $1000 \mathrm{~s}$, followed by a stable period of approximately $2700 \mathrm{~s}$. The COF then increased from 0.120 to about 0.145 in the rest of the test. For PAO-PS/P-MMT, the COF rose up sharply from 0.082 to 0.110 , dropped drastically to 0.090 within $250 \mathrm{~s}$, and then experienced a stable period with fluctuation around $1500 \mathrm{~s}$. The COF then increased slowly to 0.115 at the end of the test. The COF of PAO-PS/P-SLS-MMT started at 0.070, increased slowly to about 0.100 around $2200 \mathrm{~s}$, and then remained steady during the rest of the test. The COF of PAO-PS/P-KH570-MMT was 0.070 at the beginning, increased to 0.085 within $250 \mathrm{~s}$, and then rose 
up slowly. At the end of the test, the COF reached 0.090. It can be found from the test results that the adding PS/OMMT nanocomposites to the PAO decreased the COF values and clearly enhanced the friction reduction properties. Furthermore, with the same content of nanocomposite particles, PS/P-KH570-MMT performed the lowest COF value and the best friction reduction properties.

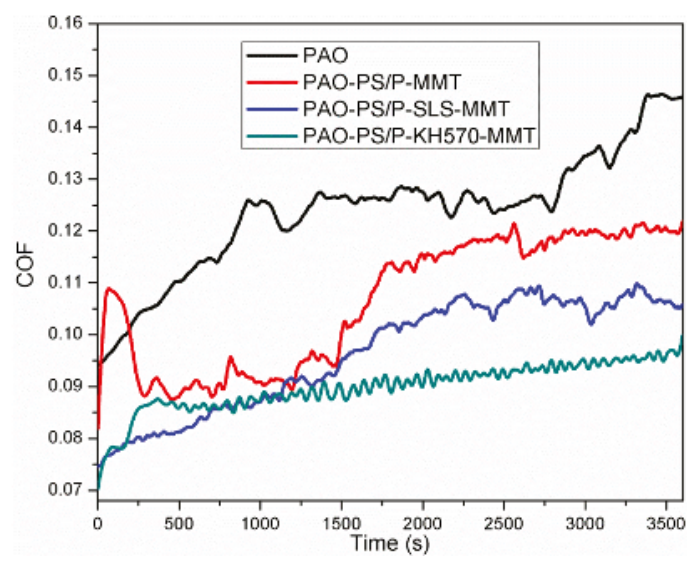

Figure 11. The COF versus time of PAO, PAO-PS/P-MMT, PAO-PS/P-SLS-MMT, and PAO-PS/P-KH570-MMT.

To further characterize the tribological properties for the PS/OMMT nanocomposites, we examined the wear surfaces of the PAO and PAO-PS/OMMT nanocomposites using SEM. Figure 12A-D and Figure 13A-D show the SEM morphology of the wear surfaces of the upper and lower ball of the four-ball machine for PAO, PAO-PS/P-MMT, PAO-PS/P-SLS-MMT, and PAO-PS/P-KH570-MMT, respectively. From Figure 12A, a wide and deep plowing groove was found on the wear surface, indicating serious abrasive wear. This worn morphology was also observed in Figure 13A. However, when the PS/OMMT nanocomposites were added into the PAO, as shown in Figure 12B-D and Figure 13B-D, the grooves on the wear surface became shallower and narrower, which indicated that the surface roughness was improved and the COF was reduced. These results indicated that the PS/OMMT nanocomposites exhibited excellent anti-wear and friction reduction properties in their tribological properties in a lubricant, which were consistent with the results of Figure 11. During the lubrication process, the nanocomposite particles were able to fill in the microgrooves on the wear surfaces and reduce the roughness of the metal surfaces to reduce friction and wear [43]. Furthermore, the nanocomposite particles could support the metal surfaces and prevent them from contacting each other and roll like a bearing ball, thereby reducing the COF and wear [44]. Among the wear surfaces, there was a smoother wear surface with microgrooves and nanogrooves in Figures 12D and 13D. This result suggested that the best anti-wear and friction reduction properties among all the type of nanocomposites were observed with the PS/P-KH570-MMT nanocomposite in this study. This may be due to the smaller PS/P-KH570-MMT nanocomposite particle filling the grooves more effectively than the larger particles. The nanocomposite particles with smaller diameters could interact with the metal surface more effectively and form the transfer film more easily to protect the metal surface and reduce the wear. Meanwhile, with higher thermal stability, the PS/P-KH570-MMT nanocomposite particles might roll better between two wear surfaces and reduce the friction. The above results indicated that the improvements of tribological properties of PS/OMMT nanocomposite particles showed promising applications in the drilling fluid lubrication. 


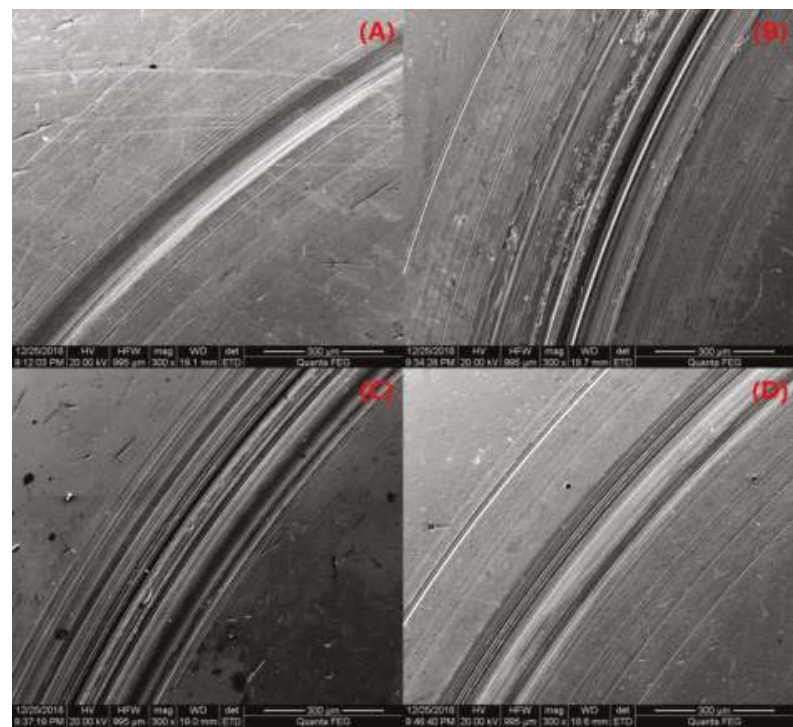

Figure 12. SEM images of the upper ball of (A) PAO, (B) PAO-PS/P-MMT, (C) PAO-PS/P-SLS-MMT, and (D) PAO-PS/P-KH570-MMT.

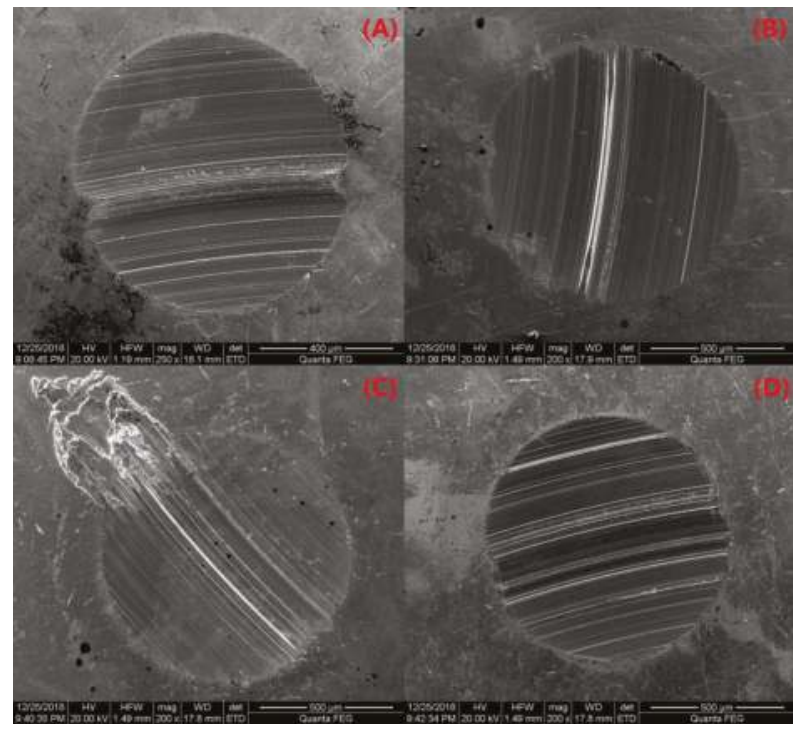

Figure 13. SEM images of the lower ball of (A) PAO, (B) PAO-PS/P-MMT, (C) PAO-PS/P-SLS-MMT, and (D) PAO-PS/P-KH570-MMT.

\section{Conclusions}

Novel PS/OMMT nanocomposite particles were synthesized by soap-free emulsion polymerization in the presence of doubly organo-modified MMTs and investigated. The anionic surfactant, SLS, could be effectively used to further increase the interlayer space of P-MMT by the electrostatic interaction. The silane coupling agent, KH570, could be introduced to the external surfaces and broken edges of the P-MMT due to the silylation reaction. The complete exfoliation of the OMMT within the PS 
matrix was confirmed by XRD and TEM analysis. The introduction of OMMT significantly decreased the average particle size and PDI values of PS, as evidenced by DLS and SEM results, and this became more pronounced in the PS/OMMT nanocomposites filled with P-KH570-MMT. The results of TGA-DTG analysis confirmed that the thermal stability of the PS/OMMT nanocomposites was significantly enhanced compared with pure PS. The $T_{\max }$ increased from $419^{\circ} \mathrm{C}$ of pure PS to $449^{\circ} \mathrm{C}$ of $\mathrm{PS} / \mathrm{P}-\mathrm{KH} 570-\mathrm{MMT}$. Moreover, the COF values of PAO-PS/OMMT nanocomposites were well below than that of pristine PAO, indicating increased friction reduction properties. Available results in this work can provide significant guidance for the future synthesis and application of the PS/OMMT nanocomposites in the oil and gas drilling engineering field to improve drilling fluid lubrication.

Author Contributions: Data curation, S.L. and Q.D.; Investigation, C.Y.; Methodology, Y.K.; Software, Y.Z.; Writing—original draft, C.Y.; Writing—review \& editing, X.H.

Funding: This work was funded by the National Natural Science Foundation of China (Grant No. 51674270), National Major Project (Grant No. 2017ZX05009-003), Major project of the National Natural Science Foundation of China (No.51490650), and the Foundation for Innovative Research Groups of the National Natural Science Foundation of China (Grant No. 51821092 and 51521063).

Conflicts of Interest: The authors declare no conflict of interest.

\section{References}

1. Kawasumi, M. The discovery of polymer-clay hybrids. J. Polym. Sci. A: Polym. Chem. 2004, 42, 819-824. [CrossRef]

2. Chafidz, A.; Ali, I.; Mohsin, M.E.A.; Elleithy, R.; Al-Zahrani, S. Nanoindentation and dynamic mechanical properties of PP/clay nanocomposites. J. Polym. Res. 2012, 19, 1-12. [CrossRef]

3. Li, X.; Tang, S.; Zhou, X.; Gu, S.; Huang, K.; Xu, J.; Wang, X.; Li, Y. Synergistic effect of amino silane functional montmorillonite on intumescent flame-retarded SEBS and its mechanism. J. Appl. Polym. Sci. 2017, 134, 44953. [CrossRef]

4. Silva, A.A.; Dahmouche, K.; Soares, B.G. Nanostructure and dynamic mechanical properties of silane-functionalized montmorillonite/epoxy nanocomposites. Appl. Clay Sci. 2011, 54, 151-158. [CrossRef]

5. Sarier, N.; Onder, E.; Ersoy, S. The modification of Na-montmorillonite by salts of fatty acids: An easy intercalation process. Colloids Surf. A Physicochem. Eng. Asp. 2010, 371, 40-49. [CrossRef]

6. Owusu-Adom, K.; Guymon, C.A. Chemical compatibility and reaction-induced exfoliation in photopolymerizable clay nanocomposites. Macromolecules 2009, 42, 180-187. [CrossRef]

7. Xidas, P.I.; Triantafyllidis, K.S. Effect of the type of alkylammonium ion clay modifier on the structure and thermal/mechanical properties of glassy and rubbery epoxy-clay nanocomposites. Eur. Polym. J. 2010, 46, 404-417. [CrossRef]

8. Giannakas, A.; Spanos, C.G.; Kourkoumelis, N.; Vaimakis, T.; Ladavos, A. Preparation, characterization and water barrier properties of PS/organo-montmorillonite nanocomposites. Eur. Polym. J. 2008, 44, 3915-3921. [CrossRef]

9. Ezquerro, C.S.; Ric, G.I.; Miñana, C.C.; Bermejo, J.S. Characterization of montmorillonites modified with organic divalent phosphonium cations. Appl. Clay Sci. 2015, 111, 1-9. [CrossRef]

10. Mittal, V. Modification of montmorillonites with thermally stable phosphonium cations and comparison with alkylammonium montmorillonites. Appl. Clay Sci. 2012, 56, 103-109. [CrossRef]

11. Abdallah, W.; Yilmazer, U. Novel thermally stable organo-montmorillonites from phosphonium and imidazolium surfactants. Thermochim. Acta 2011, 525, 129-140. [CrossRef]

12. Wang, H.; Wang, H.; Chen, K.; Song, Y.; Wei, Z.; Xue, M. The modification of lanthanum-exchanged montmorillonite with anionic surfactants to enhance the thermal stability of polyvinyl chloride. J. Appl. Polym. Sci. 2015, 132, 41535. [CrossRef]

13. Fu, M.; Zhang, Z.; Wu, L.; Zhuang, G.; Zhang, S.; Yuan, J.; Liao, L. Investigation on the co-modification process of montmorillonite by anionic and cationic surfactants. Appl. Clay Sci. 2016, 132-133, 694-701. [CrossRef]

14. Bertuoli, P.T.; Piazza, D.; Scienza, L.C.; Zattera, A.J. Preparation and characterization of montmorillonite modified with 3-aminopropyltriethoxysilane. Appl. Clay Sci. 2014, 87, 46-51. [CrossRef] 
15. Asgari, M.; Sundararaj, U. Silane functionalization of sodium montmorillonite nanoclay: The effect of dispersing media on intercalation and chemical grafting. Appl. Clay Sci. 2018, 153, 228-238. [CrossRef]

16. Bhanvase, B.A.; Pinjari, D.V.; Gogate, P.R.; Sonawane, S.H.; Pandit, A.B. Synthesis of exfoliated poly(styrene-co-methyl methacrylate)/montmorillonite nanocomposite using ultrasound assisted in situ emulsion copolymerization. Chem. Eng. J. 2012, 181-182, 770-778. [CrossRef]

17. Mirzataheri, M.; Khamisabadi, S.; Salimi, A. Characterization of styrene-co-butyl acrylate/Cloisite $\mathrm{Na}^{+}$ nanocomposite film synthesized via soap free emulsion polymerization. Prog. Org. Coat. 2016, 99, 274-281. [CrossRef]

18. Bourgeat-Lami, E.; Lansalot, M. Organic/inorganic composite latexes: The marriage of emulsion polymerization and inorganic chemistry. Adv. Polym. Sci. 2010, 233, 53-123.

19. Yamamoto, T.; Kawaguchi, K.; Takahashi, Y. Particle size control in the soap-free emulsion polymerization of styrene by an oil-soluble initiator with a weakly acidic water-soluble initiator. Colloids Surf. A Physicochem. Eng. Asp. 2016, 502, 1-5. [CrossRef]

20. Hu, Z.; Yang, X.; Liu, J.; Yan, Y.; Wang, L.; Zeng, Y. An investigation of the effect of sodium dodecyl sulfate on quasi-emulsifier-free emulsion polymerization for highly monodisperse polystyrene nanospheres. Eur. Polym. J. 2011, 47, 24-30.

21. Hojiyev, R.; Ulcay, Y.; Celik, M.S.; Carty, W.M. Effect of CEC coverage of hexadecyltributylphosphonium modified montmorillonite on polymer compatibility. Appl. Clay Sci. 2017, 141, 204-211. [CrossRef]

22. Sun, J.; Zhuang, G.; Wu, S.; Zhang, Z. Structure and performance of anionic-cationic-organo-montmorillonite in different organic solvents. RSC Adv. 2016, 6, 54747-54753. [CrossRef]

23. Alvi, M.U.; Zulfiqar, S.; Yavuz, C.T.; Kweon, H.; Sarwar, M.I. Influence of aminosilane coupling agent on aromatic polyamide/intercalated clay nanocomposites. Ind. Eng. Chem. Res. 2013, 52, 6908-6915. [CrossRef]

24. Cao, X.; Pan, G.; Huang, P.; Guo, D.; Xie, G. Silica-coated core-shell structured polystyrene nanospheres and their size-dependent mechanical properties. Langmuir 2017, 33, 8225-8232. [CrossRef] [PubMed]

25. Chen, J.; Liu, H.; Hong, X.; Wang, M.; Cai, C.; Zhang, Q. Polystyrene/MMT nanocomposites prepared by soap-free emulsion polymerization with high solids content. Colloid Polym. Sci. 2012, 290, 1955-1963. [CrossRef]

26. Angaji, M.T.; Rafiee, R.; Hemmati, M.; Abdollahi, M.; Razavi Aghjeh, M.K. Parametric studies on the grafting of poly (methyl methacrylate) onto organophilic montmorillonite using silylated clay platelets. J. Macromol. Sci. Part B 2014, 53, 957-974. [CrossRef]

27. Park, S.; Kim, B.; Seo, D.; Rhee, K.; Lyu, Y. Effects of a silane treatment on the mechanical interfacial properties of montmorillonite/epoxy nanocomposites. Mater. Sci. Eng. A 2009, 526, 74-78. [CrossRef]

28. Benbayer, C.; Saidi-Besbes, S.; Taffin De Givenchy, E.; Amigoni, S.; Guittard, F.; Derdour, A. Synergistic effect of organoclay fillers based on fluorinated surfmers for preparation of polystyrene nanocomposites. J. Appl. Polym. Sci. 2015, 132, 1-12. [CrossRef]

29. Zhang, Z.; Zhang, J.; Liao, L.; Xia, Z. Synergistic effect of cationic and anionic surfactants for the modification of Ca-montmorillonite. Mater. Res. Bull. 2013, 48, 1811-1816. [CrossRef]

30. Wu, S.; Zhang, Z.; Wang, Y.; Liao, L.; Zhang, J. Influence of montmorillonites exchange capacity on the basal spacing of cation-anion organo-montmorillonites. Mater. Res. Bull. 2014, 59, 59-64.

31. Silva, T.F.; Soares, B.G.; Ferreira, S.C.; Livi, S. Silylated montmorillonite as nanofillers for plasticized PVC nanocomposites: Effect of the plasticizer. Appl. Clay Sci. 2014, 99, 93-99. [CrossRef]

32. Xie, W.; Gao, Z.; Pan, W.P.; Hunter, D.; Singh, A.; Vaia, R. Thermal degradation chemistry of alkyl quaternary ammonium montmorillonite. Chem. Mater. 2001, 13, 2979-2990. [CrossRef]

33. Romanzini, D.; Piroli, V.; Frache, A.; Zattera, A.J.; Amico, S.C. Sodium montmorillonite modified with methacryloxy and vinylsilanes: Influence of silylation on the morphology of clay/unsaturated polyester nanocomposites. Appl. Clay Sci. 2015, 114, 550-557. [CrossRef]

34. Yu, C.; Ke, Y.; Deng, Q.; Lu, S.; Ji, J.; Hu, X.; Zhao, Y. Synthesis and characterization of polystyrene-montmorillonite nanocomposite particles using an anionic-surfactant-modified clay and their friction performance. Appl. Sci. 2018, 8, 964. [CrossRef]

35. Romanzini, D.; Frache, A.; Zattera, A.J.; Amico, S.C. Effect of clay silylation on curing and mechanical and thermal properties of unsaturated polyester/montmorillonite nanocomposites. J. Phys. Chem. Solids 2015, 87, 9-15. [CrossRef] 
36. Zhang, J.; Gupta, R.K.; Wilkie, C.A. Controlled silylation of montmorillonite and its polyethylene nanocomposites. Polymer 2006, 47, 4537-4543. [CrossRef]

37. Bourgeat-Lami, E.; Guimara, T.R.; Pereira, A.M.C.; Alves, G.M.; Moreira, J.C.; Putaux, J.; Santos, A.M. High solids content, soap-free, film-forming latexes stabilized by laponite clay platelets. Macromol. Rapid Comm. 2010, 31, 1874-1880. [CrossRef]

38. Ianchis, R.; Donescu, D.; Petcu, C.; Ghiurea, M.; Anghel, D.F.; Stanga, G.; Marcu, A. Surfactant-free emulsion polymerization of styrene in the presence of silylated montmorillonite. Appl. Clay Sci. 2009, 45, 164-170. [CrossRef]

39. Ianchis, R.; Corobea, M.C.; Donescu, D.; Rosca, I.D.; Cinteza, L.O.; Nistor, L.C.; Vasile, E.; Marin, A.; Preda, S. Advanced functionalization of organoclay nanoparticles by silylation and their polystyrene nanocomposites obtained by miniemulsion polymerization. J. Nanopart. Res. 2012, 14, 1233. [CrossRef]

40. Bee, S.L.; Abdullah, M.A.A.; Mamat, M.; Bee, S.T.; Sin, L.T.; Hui, D.; Rahmat, A.R. Characterization of silylated modified clay nanoparticles and its functionality in PMMA. Compos. Part B: Eng. 2017, 110, 83-95. [CrossRef]

41. Wang, L.; Wang, X.; Chen, Z.; Ma, P. Effect of doubly organo-modified vermiculite on the properties of vermiculite/polystyrene nanocomposites. Appl. Clay Sci. 2013, 75-76, 74-81. [CrossRef]

42. Chen, S.; Lu, X.; Zhang, Z.; Wang, T.; Pan, F. Preparation and Characterization of Poly(methyl methacrylate)/Reactive Montmorillonite Nanocomposites. Polym. Composite 2016, 37, 2396-2403. [CrossRef]

43. Sui, T.; Song, B.; Zhang, F.; Yang, Q. Effects of functional groups on the tribological properties of hairy silica nanoparticles as an additive to polyalphaolefin. RSC Adv. 2016, 6, 393-402. [CrossRef]

44. Sui, T.; Song, B.; Wen, Y.; Zhang, F. Bifunctional hairy silica nanoparticles as high-performance additives for lubricant. Sci. Rep. 2016, 6, 22696. [CrossRef] [PubMed]

(C) 2019 by the authors. Licensee MDPI, Basel, Switzerland. This article is an open access article distributed under the terms and conditions of the Creative Commons Attribution (CC BY) license (http://creativecommons.org/licenses/by/4.0/). 
Communication

\title{
Nanoscale Mechanical Properties and Indentation Recovery of PI@GO Composites Measured Using AFM
}

\author{
Ji Zhou ${ }^{1,2}$, Qiang Cai ${ }^{2}$ and Fu Xu ${ }^{2, *}$ \\ 1 College of Civil Engineering, Hunan University of Science and Engineering, Yongzhou 425006, China; \\ hnkjxy_zhouji@163.com \\ 2 College of Civil Engineering and Mechanics, Xiangtan University, Xiangtan 411105, China; \\ caiqiangvip@foxmail.com \\ * Correspondence: xufu@xtu.edu.cn; Tel.: +86-731-58293084
}

Received: 24 August 2018; Accepted: 13 September 2018; Published: 13 September 2018

\begin{abstract}
Polyimide@graphene oxide (PI@GO) composites were prepared by way of a simple solution blending method. The nanoscale hardness and Young's modulus of the composites were measured using nanoindentation based on atomic force microscopy (AFM). A nanoscale hardness of $\sim 0.65 \mathrm{GPa}$ and an elastic modulus of $\sim 6.5 \mathrm{GPa}$ were reached with a load of $\sim 55 \mu \mathrm{N}$. The indentation recovery on the surface of PI@GO was evaluated. The results show that relatively low GO content can remarkably improve the nanoscale mechanical properties of PI.
\end{abstract}

Keywords: polyimide; graphene oxide; composite; mechanical properties; indentation recovery; AFM

\section{Introduction}

Polyimide (PI) is a well-known high-performance polymer that has excellent thermal, mechanical, and electrical properties, as well as outstanding chemical resistance [1,2]. PI products are widely used in defense and aerospace applications, as well as in the electronics industry, for a variety of interconnect and packaging applications. Nowadays, electronic products require the PI to possess a high glass transition temperature and better thermal mechanical strength. To address this concern, PI-based composites with various fillers including carbon nanotubes [3], $\mathrm{SiC}$, graphene [4], graphene oxide [5], $\mathrm{SiO}_{2}$ [6], and aramid fibers [7] have been explored. The composites are highly affected by the reinforcements, in which the dimension, dispersion state, and the interaction of the reinforcements play significant roles. Actually, the aggregation of nanosize fillers easily results in performance deterioration of the composites. Thus, the preparation of uniformly dispersed PI-based composites is critical to the applications. Among the nanosize fillers, graphene oxide (GO) nanosheets have attracted much attention due to their high dispersibility, easy preparation, and low cost.

Recently, PI@GO composites have been prepared by various methods such as in situ polymerization [8], chemical cross-linking [9], and thermal imidization [10]. Dynamic mechanical analysis indicates that the storage modulus and the glass transition temperature of PI are improved by addition of GO. Besides this, the thermal stability of PI is enhanced with increasing GO content [10]. However, most of the previous studies focused on the macroscopic properties of PI@GO composites [11,12] while, actually, the nanoscale properties of PI-based composites are crucial to their applications in aerospace power and propulsion components. Unfortunately, only limited methods are available to probe the nanomechanical performance of polymer composites [13-15]. Indentation analysis is used to determine the mechanical properties of an indented material by plotting the indentation force versus depth, and has been well established for homogeneous materials on Hysitron or MTS systems. However, the load resolution and size limitations of the indenter restrict its 
application [16]. Relative to indentation analysis, atomic force microscopy (AFM) is considered more accurate at nanoscale due to the smaller tip radius and indentation depth, which eliminate the effects of adhesion and plastic deformation on the measurement. Herein, PI@GO composites were prepared by way of a simple solution blending method. The nanoscale mechanical properties and indentation recovery of the composites were measured using nanoindentation based on AFM.

\section{Materials and Methods}

Soluble PI powders (Matrimid@ 5218, $M_{\mathrm{W}}=80,000$ ) were purchased from Zhuzhou Time New-Materials Tech. Co, Ltd. (Zhuzhou, Hunan, China). Dimethylacetamide (DMAc) was purchased from Tianjin Hengxing Chemical Reagent Co, Ltd. (Tianjin, China). GO used in our research was purchased from Suzhou Tanfeng Tech. Co, Ltd. (Suzhou, Jiangsu, China). All materials are commercially available and were used without further purification. A certain amount of PI was dissolved in the DMAc, then an amount of GO (0.1 wt \% based on the weight of PI) was ultrasonically dispersed into the PI solution. Then, the as-prepared solution was transferred into a metal mold, followed by drying in vacuum at $100{ }^{\circ} \mathrm{C}$ for $48 \mathrm{~h}$ under a pressure of 4 mbar. After the solvent was evaporated, PI@GO composites were obtained. For comparison, a pure PI sample was prepared analogously to the PI@GO. Before being submitted to AFM testing, the PI and PI@GO samples were cut into $1 \times 1 \mathrm{~cm}$ pieces.

The mechanical properties of the PI@GO composites at nanoscale were characterized using AFM (Bruker, Santa Barbara, CA, USA). Nanoindentation was performed with AFM at three different areas by using a hand-crafted natural diamond tip with a spring constant of $\sim 225 \mathrm{~N} / \mathrm{m}$ (Bruker, Santa Barbara, CA, USA). The height of the tripyramidal tip is $50 \mu \mathrm{m}$, and the front, back, and side angles of the tripyramid are $55^{\circ}, 35^{\circ}$, and $51^{\circ}$, respectively. The tip radius is $50 \mathrm{~nm}$. The morphology of the samples was examined by using a common silicon nitride tip (Bruker, Santa Barbara, CA, USA).

In a typical AFM indentation test, a controlled diamond tip is driven into the specimen surface, and the displacement of the indenter tip is continuously monitored by high-resolution sensors. Various mechanical properties (most typically the elastic modulus and hardness) of the indented material can be measured by analyzing the indentation data. The Oliver-Pharr model was employed to evaluate the hardness and Young's modulus in this paper. A schematic diagram of a typical indentation is shown in Figure 1. The indentation hardness proposed by Olive and Pharr is defined as [17]

$$
H=\frac{P_{\max }}{A}
$$

where $P_{\max }$ is the maximum load and $A$ is the projected area of the indentation. The projected contact area is linked to the contact depth $h_{c}$ by the tip geometry. In case of a nonideal probe, the deviations of the contact area can be expressed in the form of a fitting function as shown below [18]:

$$
\begin{gathered}
A=24.5 h_{c}^{2}+793 h_{c}+4238 h_{c}^{1 / 2}+332 h_{c}^{1 / 4}+0.059 h_{c}^{1 / 8} \\
+0.069 h_{c}^{1 / 16}+8.68 h_{c}^{1 / 32}+35.4 h_{c}^{1 / 64}+36.9 h_{c}^{1 / 128} \\
\text { with } h_{c}=h-\varepsilon \frac{P_{\max }}{S}
\end{gathered}
$$

where $h$ and $s$ are the indent depth and contact stiffness, respectively. $\varepsilon$ is a constant that depends on the indenter geometry ( $\varepsilon=0.75$ for a Berkovich indenter).

Contact stiffness $S$ and Young's modulus $E$ of the material can be expressed as

$$
\begin{aligned}
& \frac{1}{E_{r}}=\frac{1-v^{2}}{E}+\frac{1-v_{i}^{2}}{E_{i}} \\
& S=\frac{\mathrm{d} P}{\mathrm{~d} h}=\frac{2}{\sqrt{\pi}} E_{r} \sqrt{A}
\end{aligned}
$$


where $E_{r}$ is the reduced elastic modulus, which accounts for the fact that elastic deformation occurs in both the sample and the indenter. $E$ and $v$ are the elastic modulus and Poisson's ratio for the sample, respectively. $E_{i}$ and $v_{i}$ are the same quantities for the indenter. For diamond, $E_{i}=1141 \mathrm{GPa}$ and $v_{i}=0.07[19,20]$.
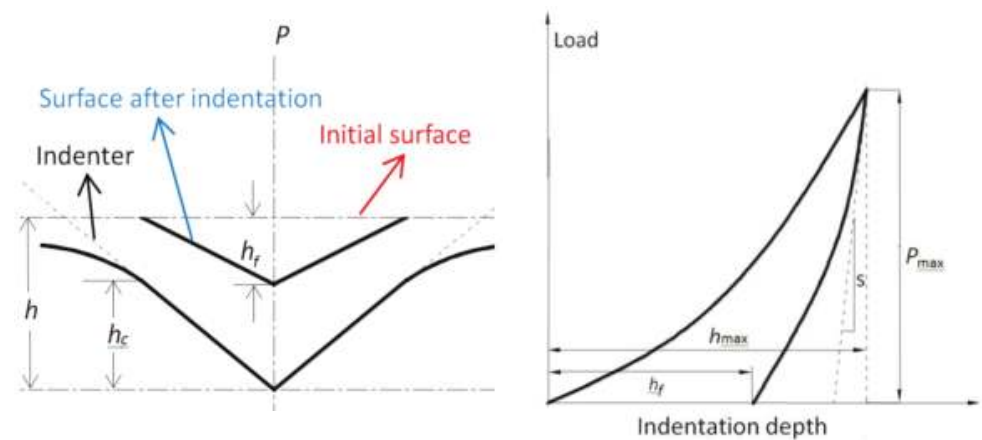

Figure 1. Schematic diagram of a typical indentation.

\section{Results and Discussion}

A diamond cone tip was used as the indenter, and its sensitivity was calibrated using a sapphire standard specimen. Typical topographical features of the PI@GO sample before and after indentation tests are shown in Figure 2. A relatively flat surface $\left(R_{\mathrm{q}}=2.24 \mathrm{~nm}\right)$ can be observed before AFM nanoindentation (Figure 2a). After capturing the morphology, the ramp mode was employed to perform the nanoindentation. A $3 \times 3$ array of indentations was conducted at different indent forces. Each residual indent was imaged by the indenter in tapping mode immediately after the indentation and later by a new and sharp silicon tip in tapping mode. As shown in Figure 2b, all AFM images of the three indents scanned with the indenter show the same triangular shape that is similar to the expected cross section of a three-sided pyramidal tip. The shapes and sizes of the three indentations under the same loading level are highly consistent with each other. No detectable pile-up or significant crack along the edges is observed, indicating that the applied load is not high enough to drive cracks. Due to the homogenous dispersion of GO in the matrix, no nanosheets can be observed in the AFM image. Figure 2c shows the morphology of the GO nanosheets. The lateral size of the GO sheets is in the range of $200 \mathrm{~nm} \sim 3 \mu \mathrm{m}$. The thickness of a single-layer GO nanosheet is about $1.2 \mathrm{~nm}$.
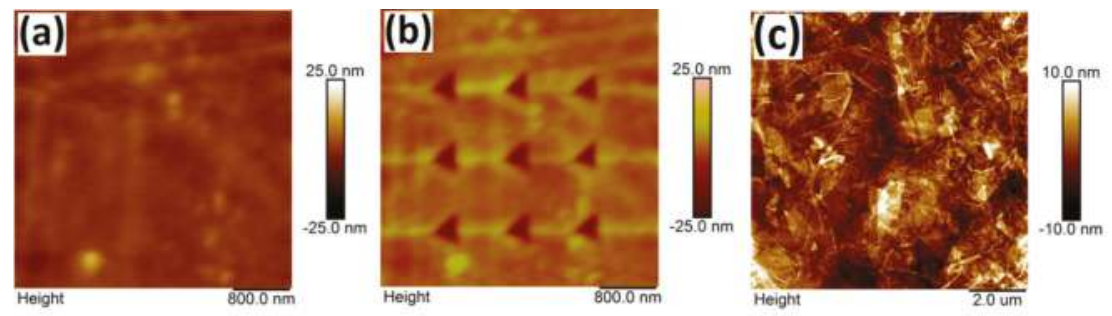

Figure 2. Typical topographical features of the polyimide@graphene oxide (PI@GO) sample before (a) and after (b) indentation tests. (c) AFM image of GO nanosheets.

Figure 3 shows typical deflection-distance curves of the PI@GO composite under a load of up to $\sim 91.9 \mu \mathrm{N}$. A deflection-distance curve recorded by AFM can be converted to a force-indentation plot. The force can be calculated by multiplying the cantilever deflection with calibration factors that have been experimentally determined [21]. Here, the voltage setpoints 1.5, 2.0, and $2.5 \mathrm{~V}$ correspond to loading forces 55.1, 73.5, and $91.9 \mu \mathrm{N}$, respectively. As shown in the loading curve, 
the deflection increased linearly with increasing indenting force. The unloading and loading curves are not overlapped. Resident depth can be measured from the deflection-distance curve. Based on these measurements, it was assumed that the indenter is a rigid body.

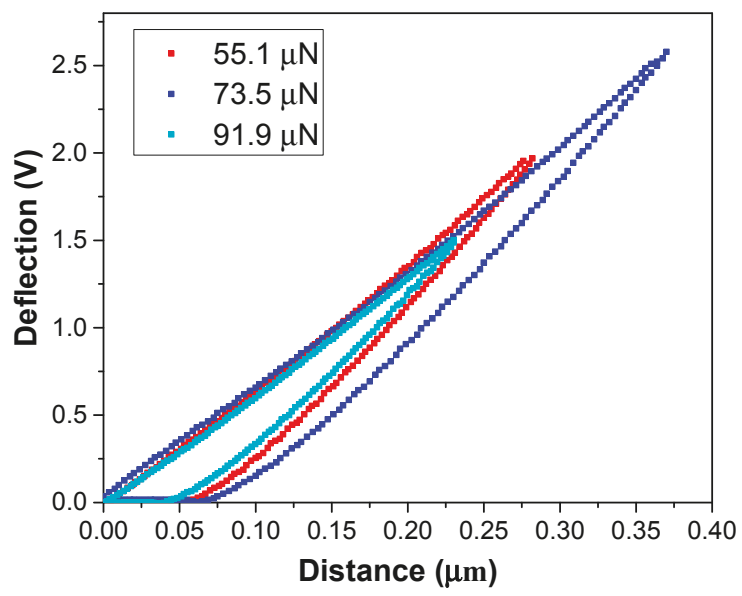

Figure 3. Typical deflection-distance curves of the PI@GO composite.

The hardness and elastic modulus of PI and PI@GO composite were calculated as shown in Figure 4. The hardness of the PI under different loading forces is in the range of 0.5 0.56 GPa (Figure 4a). The hardness of PI does not show an obvious decrease with the increase in indent force, indicating that no size effect is presented in the hardness. The reason for the lack of noticeable size effect might be that the three load forces are relatively close to each other. It was reported that monolayer graphene oxide has an effective Young's modulus of $200 \mathrm{GPa}$ [22]. With the addition of GO nanosheets, the hardness of PI@GO was boosted remarkably. The homogeneous dispersion of GO nanosheets is critical to the enhancement of the mechanical properties of PI. Compared with graphene, the presence of oxygen functional groups makes GO compatible with a polymer matrix. As we expected, the PI@GO composite exhibits a higher elastic modulus compared with pure PI (Figure 4b). The GO nanosheets in the PI matrix effectively resist the penetration of the indenter, resulting in the higher elastic modulus. Robust GO sheets can prevent crack initiation and propagation very well.
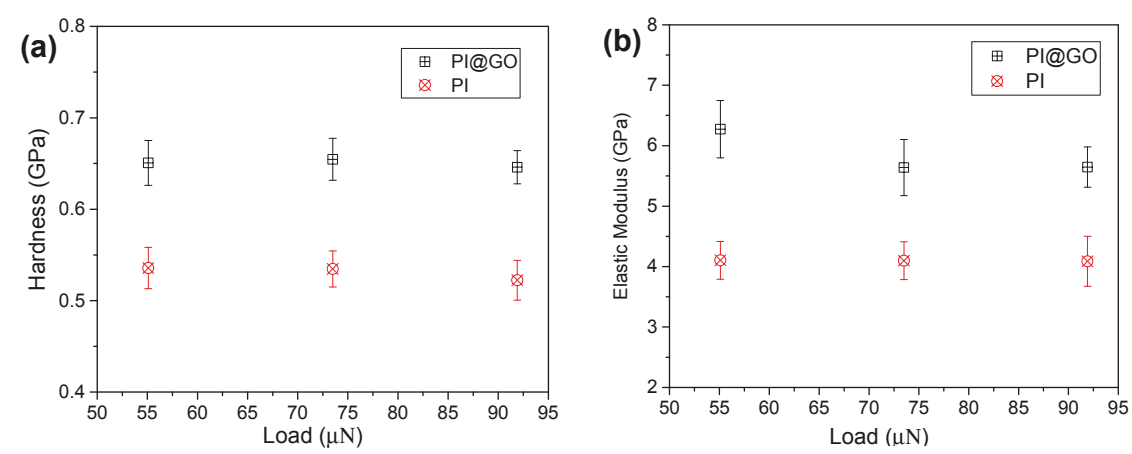

Figure 4. Hardness (a) and elastic modulus (b) of PI and PI@GO composite under different loads. 
The indentation recovery of polymer surfaces has been widely studied in the past decades [23]. The evaluation of indentation topography enables a definitive evaluation of the indentation hardness characterization of polymers and a quantitative determination of the viscoelastic recovery at deformed surfaces. Yanhuai Ding et al. discussed the time-dependent viscoelastic recovery of indentations on a polymethyl methacrylate surface [24]. A simple Kelvin model was employed to predict the indentation recovery. As shown in Figure 5a, the inverse AFM images clearly demonstrate the morphology changes of indentations on the PI@GO surface with increasing recovery time. The residual indentation depth was measured using a sharp AFM tip and is summarized in Figure 5b. A nonlinear dependence between the residual indentation depth and time can be observed. The recovery rate decreased dramatically over time. After several hours, the residual depth remained virtually unchanged, which is well known as the plastic deformation. By comparison, PI@GO composites show a higher recovery rate than does PI, which can be ascribed to the excellent mechanical properties of the additives. The imbedding of GO sheets in the polymer matrix accelerated the indentation recovery by releasing the deformation energy [25]. The results indicate that two-dimensional nanomaterials can boost the indentation recovery due to their unique morphological characteristics.

(a)

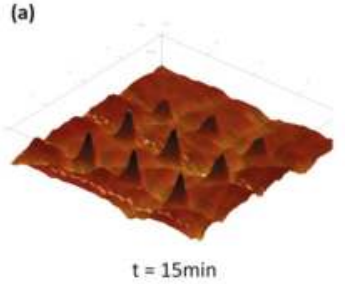

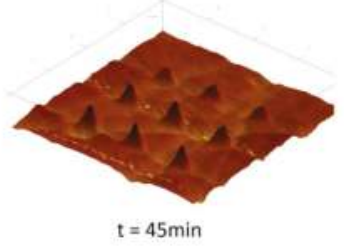

$\mathrm{t}=45 \mathrm{~min}$

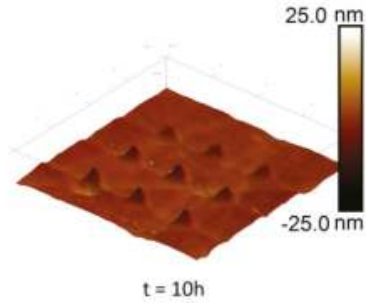

$\mathrm{t}=10 \mathrm{~h}$

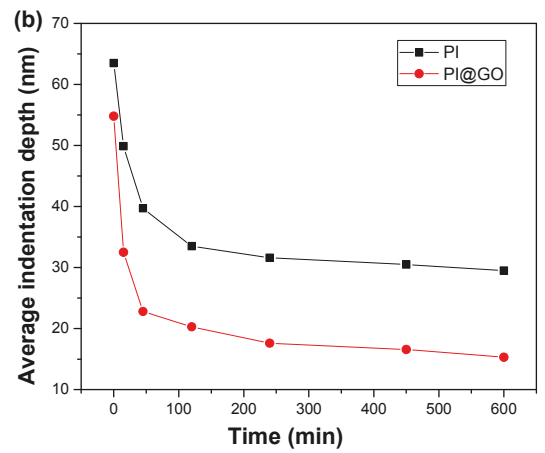

Figure 5. (a) AFM images of the indentation recovery of PI@GO. (b) Indentation recovery data of PI and PI@GO under the load of $91.9 \mu \mathrm{N}$.

\section{Conclusions}

In summary, the nanoscale hardness and elastic modulus of PI@GO composites were characterized by way of AFM nanoindentation. The indentation recovery of the PI@GO surface was measured compared with that of raw PI. The results show that the hardness and elastic modulus of the PI are enhanced by the addition of GO. The imbedded GO sheets in the polymer matrix accelerate the indentation recovery by releasing the deformation energy. This work demonstrates that 2D nanomaterials can improve the self-healing performance of polymer composites.

Author Contributions: Conceptualization, J.Z. and F.X.; methodology, Q.C.; investigation, J.Z.; writing-original draft preparation, Q.C.; writing—review and editing, J.Z. and F.X.; supervision, F.X.; project administration, F.X.; funding acquisition, F.X. 
Funding: This research was funded by National Natural Science Foundation of China (No. 51401176) and Natural Science Foundation of Hunan Province (No. 2018JJ2394).

Acknowledgments: We appreciate the helpful comments from Yanhuai Ding.

Conflicts of Interest: The authors declare no conflict of interest.

\section{References}

1. Qu, C.; Hu, J.; Liu, X.; Li, Z.; Ding, Y. Morphology and mechanical properties of polyimide films: The effects of UV irradiation on microscale surface. Materials 2017, 10, 1329. [CrossRef] [PubMed]

2. Wu, G.; Cheng, Y.; Wang, Z.; Wang, K.; Feng, A. In situ polymerization of modified graphene/polyimide composite with improved mechanical and thermal properties. J. Mater. Sci. 2017, 28, 576-581. [CrossRef]

3. Nayak, L.; Rahaman, M.; Aldalbahi, A.; Kumar Chaki, T.; Khastgir, D. Polyimide-carbon nanotubes nanocomposites: Electrical conduction behavior under cryogenic condition. Polym. Eng. Sci. 2017, 57, 291-298. [CrossRef]

4. Liu, Y.; Liu, J.; Ding, Q.; Tan, J.; Chen, Z.; Chen, J.; Zuo, X.; Tang, A.; Zeng, K. Polyimide/Graphene nanocomposites with improved gas barrier and thermal properties due to a "dual-plane" structure effect. Macromol. Mater. Eng. 2018, 303, 1800053. [CrossRef]

5. Lu, Y.; Hao, J.; Xiao, G.; Zhao, H.; Hu, Z.; Wang, T. In situ polymerization and performance of alicyclic polyimide/graphene oxide nanocomposites derived from 6FAPB and CBDA. Appl. Surf. Sci. 2017, 394, 78-86. [CrossRef]

6. Chen, M.; Qi, M.; Yao, L.; Su, B.; Yin, J. Effect of surface charged SiO2 nanoparticles on the microstructure and properties of polyimide $/ \mathrm{SiO}_{2}$ nanocomposite films. Surf. Coat. Technol. 2017, 320, 59-64. [CrossRef]

7. Yu, J.; Zhang, T.; Xu, L.; Huang, P. Synthesis and characterization of aramid fiber-reinforced polyimide/carbon black composites and their use in a supercapacitor. Chin. J. Chem. 2017, 35, 1586-1594. [CrossRef]

8. Liu, H.; Li, Y.; Wang, T.; Wang, Q. In situ synthesis and thermal, tribological properties of thermosetting polyimide/graphene oxide nanocomposites. J. Mater. Sci. 2012, 47, 1867-1874. [CrossRef]

9. Kong, J.-Y.; Choi, M.-C.; Kim, G.Y.; Park, J.J.; Selvaraj, M.; Han, M.; Ha, C.-S. Preparation and properties of polyimide/graphene oxide nanocomposite films with Mg ion crosslinker. Eur. Polym. J. 2012, 48, 1394-1405. [CrossRef]

10. Ramakrishnan, S.; Dhakshnamoorthy, M.; Jelmy, E.J.; Vasanthakumari, R.; Kothurkar, N.K. Synthesis and characterization of graphene oxide-polyimide nanofiber composites. RSC Adv. 2014, 4, 9743-9749. [CrossRef]

11. Díez-Pascual, A.M.; Gómez-Fatou, M.A.; Ania, F.; Flores, A. Nanoindentation in polymer nanocomposites. Prog. Mater. Sci. 2015, 67, 1-94. [CrossRef]

12. Yongqiang, L.; Chunzheng, P. Improved interfacial properties of PI composites through graphene oxide and carbon nanotubes on carbon fiber surface. Surf. Interface Anal. 2018, 50, 634-639. [CrossRef]

13. Li, L.; Encarnacao, L.M.; Brown, K.A. Polymer nanomechanics: Separating the size effect from the substrate effect in nanoindentation. Appl. Phys. Lett. 2017, 110, 043105. [CrossRef]

14. Qu, Z.; Bu, J.; Pan, X.; Hu, X. Probing the nanomechanical properties of PLA/PC blends compatibilized with compatibilizer and nucleation agent by AFM. J. Polym. Res. 2018, 25, 138. [CrossRef]

15. Zheng, L.; Jiang, X.; Deng, X.H.; Yin, J.R.; Jiang, Y.; Zhang, P.; Ding, Y.H. Mechanical characterization of PMMA by AFM nanoindentation and finite element simulation. Mater. Res. Express 2016, 3, 115302. [CrossRef]

16. Moeller, G. AFM nanoindentation of viscoelastic materials with large end-radius probes. J. Polym. Sci. Part B 2009, 47, 1573-1587. [CrossRef]

17. Oliver, W.C.; Pharr, G.M. An improved technique for determining hardness and elastic modulus using load and displacement sensing indentation experiments. J. Mater. Res. 1992, 7, 1564-1583. [CrossRef]

18. Nili, H.; Walia, S.; Bhaskaran, M.; Sriram, S. Nanoscale electro-mechanical dynamics of nano-crystalline platinum thin films: An in situ electrical nanoindentation study. J. Appl. Phys. 2014, 116, 163504. [CrossRef]

19. Savvides, N.; Bell, T. Microhardness and Young's modulus of diamond and diamondlike carbon films. J. Appl. Phys. 1992, 72, 2791-2796. [CrossRef]

20. Doerner, M.F.; Nix, W.D. A method for interpreting the data from depth-sensing indentation instruments. J. Mater. Res. 2011, 1, 601-609. [CrossRef] 
21. Clifford, C.A.; Seah, M.P. Quantification issues in the identification of nanoscale regions of homopolymers using modulus measurement via AFM nanoindentation. Appl. Surf. Sci. 2005, 252, 1915-1933. [CrossRef]

22. Suk, J.W.; Piner, R.D.; An, J.; Ruoff, R.S. Mechanical properties of monolayer graphene oxide. ACS Nano 2010, 4, 6557-6564. [CrossRef] [PubMed]

23. Tweedie, C.A.; Van Vliet, K.J. On the indentation recovery and fleeting hardness of polymers. J. Mater. Res. 2011, 21, 3029-3036. [CrossRef]

24. Ding, Y.H.; Deng, X.H.; Jiang, X.; Zhang, P.; Yin, J.R.; Jiang, Y. Nanoscale mechanical characterization of PMMA by AFM nanoindentation: A theoretical study on the time-dependent viscoelastic recovery. J. Mater. Sci. 2013, 48, 3479-3485. [CrossRef]

25. Ghosh, P.; Rameshbabu, A.P.; Dhara, S. Citrate cross-linked gels with strain reversibility and viscoelastic behavior accelerate healing of osteochondral defects in a rabbit model. Langmuir 2014, 30, 8442-8451. [CrossRef] [PubMed]

(C) 2018 by the authors. Licensee MDPI, Basel, Switzerland. This article is an open access article distributed under the terms and conditions of the Creative Commons Attribution (CC BY) license (http:/ / creativecommons.org/licenses/by/4.0/). 
Article

\title{
Hyaluronic Acid-Decorated Laponite ${ }^{\circledR}$ Nanocomposites for Targeted Anticancer Drug Delivery
}

\author{
Tingting Jiang, Guangxiang Chen, Xiangyang Shi * and Rui Guo * \\ Key Laboratory of Science \& Technology of Eco-Textile, Ministry of Education, College of Chemistry, Chemical \\ Engineering and Biotechnology, Donghua University, Shanghai 201620, China; \\ tingting_jiang1993@foxmail.com (T.J.); gxchen0429@163.com (G.C.) \\ * Correspondence: xshi@dhu.edu.cn (X.S.); ruiguo@dhu.edu.cn (R.G.); \\ Tel.: +86-21-67792750 (X.S.); +86-21-67792750 (R.G.)
}

Received: 5 December 2018; Accepted: 7 January 2019; Published: 14 January 2019

\begin{abstract}
In this study, hyaluronic acid (HA), a natural polysaccharide that can specifically bind to $\mathrm{CD} 44$ receptors, was conjugated onto laponite ${ }^{\circledR}$ (LAP) nanodisks for the encapsulation and specific delivery of the anti-cancer drug doxorubicin (DOX) to CD44-overexpressed cancer cells. The prepared LM-HA could encapsulate DOX efficiently and release drug in a continuous manner with $\mathrm{pH}$-responsiveness. In vitro cell viability assay proved that LM-HA had good biocompatibility, and drug-loaded LM-HA/DOX exhibited targeted anti-tumor effects against HeLa cells with CD44 receptors overexpressed. In addition, the flow cytometric detection and confocal laser scanning microscope results confirmed that LM-HA/DOX could be specifically internalized by HeLa cells via CD44-mediated endocytosis. Therefore, the HA-modified LAP nanodisks with high drug loading efficiency, $\mathrm{pH}$-sensitive drug release properties and $\mathrm{CD} 44$ targetability might be an efficient nanoplatform for cancer chemotherapy.
\end{abstract}

Keywords: LAP; hyaluronic acid; doxorubicin; CD44 receptor targeted

\section{Introduction}

Chemotherapy is one of the most widely used and indispensable tools in cancer treatments. Small molecular anticancer drugs could interfere with cell mitosis and effectively inhibit cell proliferation, but the lack of specific selectivity to tumor cells and fast body metabolism may cause severe side effects and low drug bioavailability, limiting their clinical applications [1]. To address these problems, nanosized drug delivery systems have been developed to prolong the circulation time in the blood, and enhance the drug accumulation in the tumor via the enhanced permeability and retention (EPR) effect [2]. In addition, a series of tumor-targeting moieties were functionalized on the surface of nanocarriers to improve the specific delivery and uptake of drugs by cancer cells, including RGD (Arg-Gly-Asp) [3], folic acid (FA) [4], phenylboronic acid (PBA) [5], hyaluronic acid (HA) [6], etc. [7-9]. However, it remains a challenge to construct a desired vehicle with a high drug loading efficiency, a controlled drug release profile, and specific targeting to cancer cells [10].

Until now, various kinds of inorganic and organic materials have been used in constructing nano-drug delivery systems, such as liposomes [11], dendrimers [12], polymer micelles [13], nanogels [14], mesoporous silica nanoparticles (MSN) [15], carbon nanotubes (CNTS) [16] and nanoclays [17]. Among them, laponite (LAP) nanodisks were considered as one of the most promising delivery vehicles due to their unique structure, good stability, excellent biocompatibility and ease of surface functionalization [18]. As a type of artificial nanoclay, LAP has a similar composition and layered structure to native hectorite, and can be degraded into nontoxic products under physiological 
conditions [19]. Moreover, LAP can be dispersed in aqueous solution as nanodisks with about $25 \mathrm{~nm}$ in diameter and $1 \mathrm{~nm}$ in thickness, which means that LAP has a highly-specific surface area and strong cationic exchange capability for the effective encapsulation of various drug molecules [20], such as doxorubicin (DOX) [21], amoxicillin [22], tetracycline [23], and dexamethasone [24]. In our previous work, LAP has been applied to encapsulate the positively-charged anticancer drug DOX with $\mathrm{pH}$-sensitive drug-release properties and an effective drug encapsulation efficiency of $98 \%$. The prepared LAP/DOX complexes showed a more efficient therapeutic efficacy compared to free DOX in vitro, by the virtue of their nano-size [20]. Moreover, tumor targeting agents could be further decorated on LAP to improve specific targeting to cancer cells. For example, DOX-loaded, FA-modified LAP nanocomposites displayed significantly enhanced therapeutic efficacy in treating cancer cells overexpressing FA receptors [25]. PEG-lactobionic acid modified LAP possessed great colloidal stability and exhibited selective cytotoxic properties to targeted hepatocarcinoma cells overexpressing asialoglycoprotein receptors [26].

A cluster of differentiation-44 (CD44) is a transmembrane glycoprotein over-expressed in a variety of solid tumors, such as breast cancer, gastric carcinoma, hepatocarcinoma, and melanoma [27]. Hence, many studies have focused on targeting the overexpression of CD44 receptors to improve the specific deliver and endocytotic uptake of drugs in cancer cells [28,29]. Hyaluronic acid is recognized as the primary CD44 binding molecule because of the existence of the binding site, which connects HA and CD44 receptors on the surface of cancer cells [30]. For instance, Zhang et al. synthesized HA-modified, single-walled carbon nanotubes to load DOX. In vitro experiments demonstrated that the drug delivery system could induce significantly higher cytotoxicity against a human cervical cancer cell line (HeLa cells) overexpressing CD44 receptors than normal fibroblasts, and histological examinations proved their lower toxicity to vital organs in comparison with DOX [31]. He et al. used $\mathrm{HA}$ to functionalize PS/ $\mathrm{CaCO}_{3} / \mathrm{DNA}$ nanoparticles (HNP) for targeted gene delivery. After $4 \mathrm{~h}$ of incubation with HeLa cells, HNP displayed a significant higher mean fluorescence intensity than non-targeted NPs, indicating that the enhanced cell uptake of HNP is caused by CD44-mediated cellular uptake [32]. In Cheng's work, cisplatin-incorporated Cy5.5-PEG-g-HA nanoparticles could selectively inhibit the proliferation of HeLa cells, and exhibited an efficacious accumulation in tumors [33]. Therefore, HA modification could increase cell uptake and preferential accumulation in tumors overexpressing CD44 receptors, resulting in reduced residual toxicity and an improved therapeutic effect [34].

In this study, HA-modified LAP nanocomposites were synthesized to load the antitumor drug DOX for targeted delivery to cancer cells overexpressing CD44 receptors. The structure of the prepared LM-HA was characterized by ${ }^{1} \mathrm{H}$ NMR, FT-IR spectrometry, thermal gravimetric analyses (TGA), dynamic light scattering (DLS) and UV-vis spectrometry. The release profile of LM-HA/DOX nanocomposites was investigated under both acidic and physiological conditions. Furthermore, CD44 overexpressed HeLa cells were utilized as model cells to estimate the specific targeting and antitumor efficacy of LM-HA/DOX nanocomposites by 3-(4,5-Dimethylthiazol-2-yl)-2,5-diphenyltetrazolium bromide (MTT) assay, flow cytometric analysis, cell morphology and confocal laser scanning microscopic (CLSM) observation.

\section{Experiment}

\subsection{Preparation and Characterization of DOX-Loaded Nanocomposites}

According to the previous literature [35], $50 \mathrm{mg}$ of laponite ${ }^{\circledR}$ (LAP) powder was dissolved in $5 \mathrm{~mL}$ of ultrapure water and magnetically stirred at $50{ }^{\circ} \mathrm{C}$ overnight to obtain completely LAP aqueous solution. Then $1 \mathrm{~mL}$ APMES aqueous solution $(20 \mathrm{mg} / \mathrm{mL})$ was slowly injected into the LAP aqueous solution and stirred at $50{ }^{\circ} \mathrm{C}$ for $16 \mathrm{~h}$. The resulting solution was then dialyzed against a dialysis bag with a molecular weight cutoff of $8000-14,000$ for 3 days (2 L, 3 times per day). After dialysis was 
completed, the product in the dialysis bag was transferred to a $50 \mathrm{~mL}$ EP tube and stored at $4{ }^{\circ} \mathrm{C}$ to obtain $\mathrm{LM}-\mathrm{NH}_{2}$.

Then, HA ( $84.35 \mathrm{mg})$ was dissolved in ultrapure water $(5 \mathrm{~mL})$ and activated by EDC (104.1 mg) and NHS (62.51 mg) at room temperature for $3 \mathrm{~h}$. Next, the activated HA aqueous solution was added to $\mathrm{LM}_{-\mathrm{NH}_{2}}(6.276 \mathrm{~mL}, 52.33 \mathrm{mg})$ and stirred for 3 days. Finally, the reaction mixture was extensively dialyzed against water (2 L, 3 times per day) for 3 days using a dialysis bag with a molecular weight cutoff of 8000-14,000 to obtain targeted LM-HA.

The DOX $/ \mathrm{HCl}$ powder was dissolved in ultrapure water to obtain DOX aqueous solution ( $2 \mathrm{mg} / \mathrm{mL})$. Then, LM-HA aqueous solution $(6 \mathrm{mg} / \mathrm{mL})$ was mixed with DOX aqueous solution in a mass ratio of 3:1. The drug loaded LM-HA/DOX can be obtained by magnetically stirring for $24 \mathrm{~h}$ in darkness. Subsequently, the solution was purified by centrifugation (15 min, $8500 \mathrm{rpm})$ and washed 3 times with ultrapure water to achieve drug-loaded LM-HA/DOX nanocomposites. The drug loading efficiency and loading capacity can be calculated using Equations (1) and (2), respectively:

$$
\begin{gathered}
\text { Loading efficiency }=\left(\mathrm{M}_{\mathrm{t}} / \mathrm{M}_{0}\right) \times 100 \% \\
\text { Loading capacity }=\mathrm{M}_{\mathrm{t}} /\left(\mathrm{M}_{\mathrm{t}}+\mathrm{M}_{\mathrm{L}}\right) \times 100 \%
\end{gathered}
$$

where $M_{t}, M_{0}$ and $M_{L}$ stand for the mass of the encapsulated DOX, the initial DOX, and the LM-HA/DOX nanocomplexes, respectively.

Details of these methods can be found in the Supplementary Materials, including materials and characterization techniques, and in vitro drug release.

\subsection{Cell Culture}

HeLa cells were continuously cultured in a $75 \mathrm{~cm}^{2}$ tissue culture flask with $15 \mathrm{~mL}$ Dulbecco's modified Eagle's medium (DMEM) containing 10\% FBS, $100 \mathrm{U} / \mathrm{mL}$ penicillin, and $100 \mu \mathrm{g} / \mathrm{mL}$ streptomycin in a humidified incubator with $5 \% \mathrm{CO}_{2}$ at $37{ }^{\circ} \mathrm{C}$. HA pre-treated HeLa cells were obtained by pre-treating HeLa cells with HA-containing DMEM medium ( $3 \mathrm{mM})$ for $1 \mathrm{~h}$ before the addition of LM-HA or LM-HA/DOX [36,37].

\subsection{In Vitro Cytotoxicity Assay and Cell Morphology Observation}

HeLa cells were collected and seeded in the 96-well plate at the density of $1 \times 10^{4}$ cells/well, with $100 \mu \mathrm{L}$ DMEM medium for $24 \mathrm{~h}$. The next day, the medium was discarded and replaced with $100 \mu \mathrm{L}$ of fresh medium, containing $10 \mu \mathrm{L}$ phosphate buffered saline (PBS) of LM-HA nanodisks at different final concentrations $(0,1.5,3.0,6.0,12,24,48$ and $96 \mu \mathrm{g} / \mathrm{mL}$, respectively) $(\mathrm{n}=5)$. PBS was used as control. After a $24 \mathrm{~h}$ culture to bring the cells to confluence, the medium was discarded and cells were rinsed with PBS 3 times, followed by the addition of $90 \mu \mathrm{L}$ medium and $10 \mu \mathrm{L}$ MTT solution, and continued to incubate for $4 \mathrm{~h}$. Then the original medium was washed out and $100 \mu \mathrm{L}$ DMSO solution was added to each hole in shaking table for $20 \mathrm{~min}$. A Thermo Scientific Multiskan MK3 ELISA reader (Waltham, MA) was used to record the absorbance of each well at $570 \mathrm{~nm}$.

To check the therapeutic efficacy of LM-HA/DOX nanocomposites, HeLa cells and HA pre-treated HeLa cells were incubated with DOX and LM-HA/DOX at different DOX concentrations (0.5, 1.0, 2.0, 4.0 and $8.0 \mu \mathrm{g} / \mathrm{mL})(\mathrm{n}=5)$ for $24 \mathrm{~h}$. The morphology of cells was observed by using a Leica DM IL LED inverted phase contrast microscope (Wetzlar, Germany) with a magnification of $100 \times$ for each sample. MTT assay was also applied to evaluate the cell viabilities against a standard protocol.

\subsection{Cellular Uptake}

Cover slips with a diameter of $14 \mathrm{~mm}$ were pretreated with $5 \% \mathrm{HCl}, 30 \% \mathrm{HNO}_{3}$, and $75 \%$ alcohol, placed in 24-well tissue culture plates, and then soaked by medium for $24 \mathrm{~h}$. HeLa cells were seeded into each well with a density of $1 \times 10^{5}$ cells/well. After culturing for $24 \mathrm{~h}$ to bring the cells to attach onto the cover slips, the cells were treated with $0.3 \mathrm{~mL}$ fresh medium, which contained free DOX and 
LM-HA/DOX at a final DOX concentration of $0.5 \mu \mathrm{g} / \mathrm{mL}$, and then were cultured for another $4 \mathrm{~h}$. After that, the medium was discarded, and the cells were rinsed with PBS 3 times. Then the cells were fixed with glutaraldehyde $(2.5 \%)$ for $15 \mathrm{~min}$ at $4{ }^{\circ} \mathrm{C}$ and counterstained with DAPI $(1 \mu \mathrm{g} / \mathrm{mL})$ for $15 \mathrm{~min}$ at $37^{\circ} \mathrm{C}$, using a standard procedure. Finally, the mean fluorescence intensity was observed by a confocal laser scanning microscope using a $63 \times$ oil-immersion objective lens (CLSM, Carl Zeiss LSM 700, Jena, Germany).

The targeting specificity of LM-HA/DOX nanocomposites was quantitatively analyzed via flow cytometry. The HeLa cells were collected and seeded in the 6-well plate at the density of $4 \times 10^{5}$ cells $/$ well for $24 \mathrm{~h}$. Then, the medium was replaced with $1 \mathrm{~mL}$ fresh medium containing free DOX and LM-HA/DOX at a final DOX concentration of $0.5 \mu \mathrm{g} / \mathrm{mL}$, then cultured for a further $4 \mathrm{~h}$. After that, the medium was discarded and the cells were rinsed with PBS 3 times, trypsinated, centrifuged, and resuspended in $1 \mathrm{~mL}$ PBS. The intensity of DOX fluorescence was measured using a Becton Dickinson FACScan flow cytometer (FCM, BD Biosciences, Franklin Lake, NJ, USA).

\section{Results and Discussion}

\subsection{Synthesis and Characterization of LM-HA/DOX}

In this study, LAP was first silanized using 3-aminopropyldimethylethoxysilane (APMES) to introduce amine groups on the surface, and then hyaluronic acid was conjugated by the reaction between their carboxyl groups and amine groups on LM via 1-ethyl-3-(3-dimethylaminopropyl) carbodiimide hydrochloride (EDC) chemistry. Finally, the formed LM-HA nanodisks were utilized to load the chemotherapeutic agent doxorubicin by physical absorption (Scheme 1).

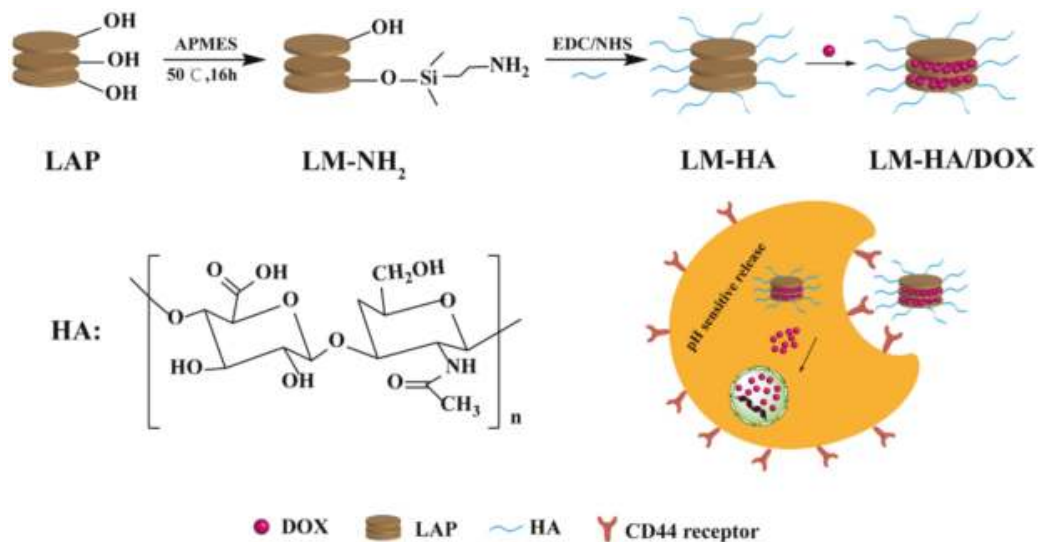

Scheme 1. Schematic illustration of the preparation of LM-HA/DOX.

The structure of LM-NH$H_{2}$ and LM-HA were evaluated by ${ }^{1} \mathrm{H}$ NMR, as shown in Figure 1. Compared to pristine LAP, the emergence of peaks at 1.2, 2.8, and $3.6 \mathrm{ppm}$ in the spectrum of $\mathrm{LM}_{-} \mathrm{NH}_{2}$ were associated to the silane coupling agent APMES, indicating the successful conjugation of APMES on LAP [38]. After the modification of HA, LM-HA displayed a prominent peak at 1.9 ppm in the spectrum, which was attributed to $-\mathrm{CH}_{3}$ protons of HA moieties [39]. This result demonstrated that HA was successfully conjugated on LAP via EDC reaction between the carboxyl group of HA and the amine group on $\mathrm{LM}-\mathrm{NH}_{2}$. 


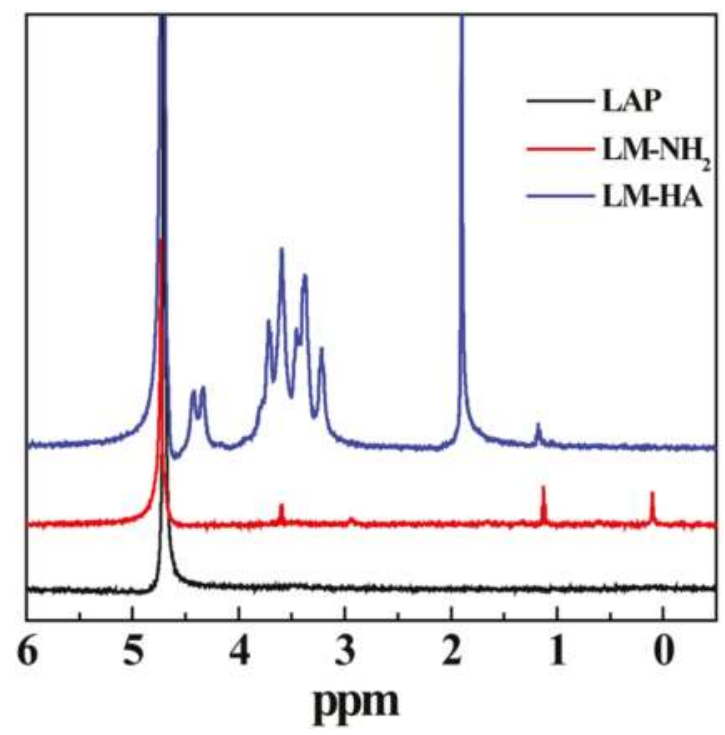

Figure 1. ${ }^{1} \mathrm{H}$ NMR spectra of laponite nanodisks (LAP), $\mathrm{LM}^{-\mathrm{NH}_{2}}$ and $\mathrm{LM}-\mathrm{HA}$.

The successful synthesis of LM-HA was also verified by FT-IR, as seen in Figure 2. Compared with LAP, $\mathrm{LM}-\mathrm{NH}_{2}$ displayed a typical peak at $1245 \mathrm{~cm}^{-1}$ triggered by the asymmetric stretching vibration of the Si-O-Si bond, indicating that APMES were successfully decorated onto LAP [21]. After the modification of HA, there were three kinds of prominent peaks at $1692 \mathrm{~cm}^{-1}, 2895 \mathrm{~cm}^{-1}$ and $1152 \mathrm{~cm}^{-1}$. They were assigned to the $\mathrm{C}=\mathrm{O}$ stretching vibration of the carboxyl group and the amide $\mathrm{I}$ band, the symmetric stretching vibration of $\mathrm{C}-\mathrm{H}$, and the $\mathrm{C}-\mathrm{O}$ stretching of the proteoglycan sugar ring of HA, respectively [40]. Hence, the FT-IR result confirmed the successful conjugation of the targeting agent HA on LAP.

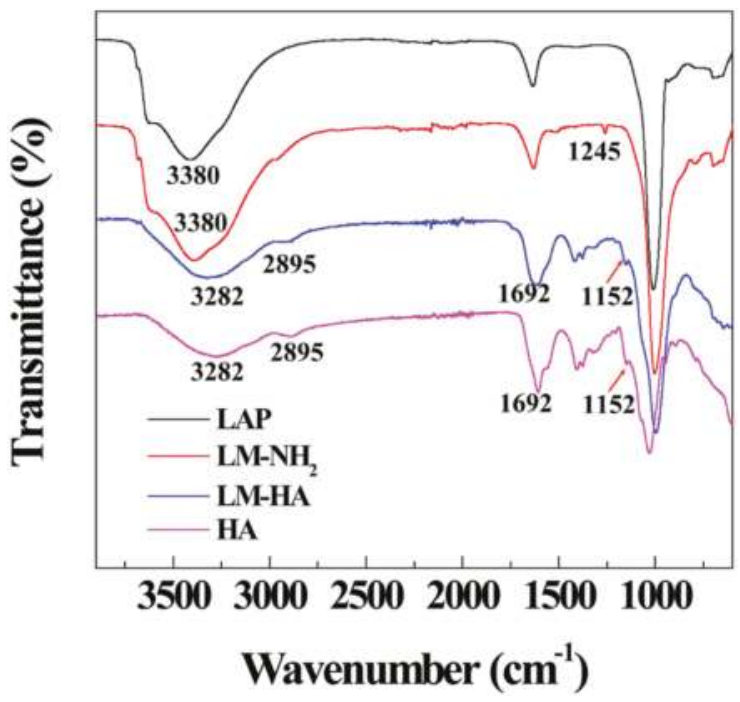

Figure 2. FT-IR spectra of LAP, LM-NH $\mathrm{N}_{2}$ LM-HA, and HA. 
TGA was then used to quantify the component of LM-HA by measuring the weight loss of LAP, $\mathrm{LM}-\mathrm{NH}_{2}$, and LM-HA from $100{ }^{\circ} \mathrm{C}$ to $700{ }^{\circ} \mathrm{C}$ (Figure 3). Compared with the pristine LAP, $\mathrm{LM}^{-\mathrm{NH}_{2}}$ showed a slight weight loss of $5.79 \%$ during the heating process, which was attributed to the thermal decomposition of the APMES [21]. This result demonstrated the conjugation of APMES on LAP. LM-HA exhibited a higher weight loss of $22.70 \%$, and by deducting the residue weight of APMES, the amount of HA modified on LAP was about $13.80 \%$ of the final LM-HA product. Therefore, the TGA result further verified the successful synthesis of LM-HA.

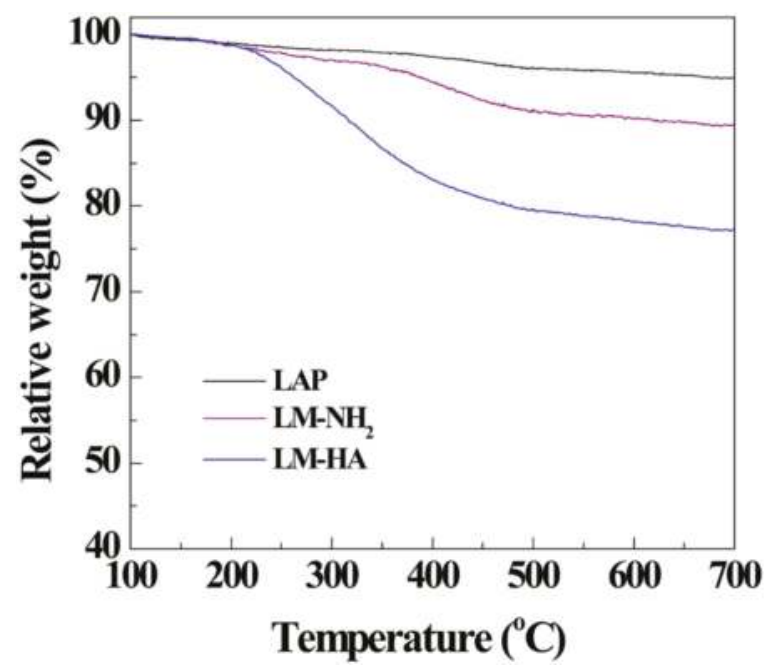

Figure 3. TGA curves of LAP, $\mathrm{LM}-\mathrm{NH}_{2}$, and LM-HA, respectively.

Furthermore, zeta potential measurement and DLS were used to evaluate the change of hydrodynamic and surface potential after modification (Table 1). After silanization, the hydrodynamic diameter increased from $86.5 \mathrm{~nm}$ of pristine LAP to $184.0 \mathrm{~nm}$ of $\mathrm{LM}-\mathrm{NH}_{2}$, and the zeta potential surged from $-39.6 \mathrm{mV}$ to $-12.0 \mathrm{mV}$, due to the introduction of amine groups on the surface. After the modification of HA, LM-HA showed a lower surface potential of $-14.1 \mathrm{mV}$, which was attributed to the inherent negative charge of HA moieties on surface. Meanwhile, the hydrodynamic size of LM-HA expanded to $282.6 \mathrm{~nm}$ because of the conjugation of hydrophilic HA chains, which may provide additional colloidal stability for nanocomposites.

Table 1. $\zeta$-potential and hydrodynamic diameter of LAP, LM-NH $\mathrm{N}_{2}, \mathrm{LM}-\mathrm{HA}$, and LM-HA/DOX, respectively. All the samples were dispersed in water at the concentration of $0.5 \mathrm{mg} / \mathrm{mL}$.

\begin{tabular}{|c|c|c|}
\hline Materials & $\zeta$-Potential (mV) & Hydrodynamic Size (nm) \\
\hline LAP & $-39.6 \pm 0.7$ & $86.5 \pm 5.1$ \\
\hline $\mathrm{LM}-\mathrm{NH}_{2}$ & $-12.0 \pm 2.0$ & $184.0 \pm 1.9$ \\
\hline LM-HA & $-14.1 \pm 1.4$ & $282.6 \pm 12.7$ \\
\hline LM-HA/DOX & $-10.8 \pm 0.7$ & $412.7 \pm 16.1$ \\
\hline
\end{tabular}

\subsection{DOX Encapsulation and Release}

In this study, LM-HA was used to load anticancer drug DOX by mixing the aqueous solution of LM-HA and DOX for $24 \mathrm{~h}$. UV-vis spectroscopy test was conducted to confirm the encapsulation of DOX into LM-HA nanodisks (Figure 4). Obviously, the solutions of LM-HA did not show any absorbance over $400 \mathrm{~nm}$, while drug-loaded materials displayed an obvious DOX-related absorbance peak at $480 \mathrm{~nm}$, demonstrating that LM-HA could encapsulate DOX effectively [41]. Although the 
size of LM-HA/DOX expanded to $412.7 \mathrm{~nm}$ after drug loading, they still exhibited good stability in PBS, culture medium (DMEM) and fatal bovine serum (FBS) for two weeks (Table S1 and Figure S1). The drug loading efficiency and loading capacity was calculated to be $85.1 \pm 1.5 \%$ and $22.1 \pm 0.22 \%$ respectively, using a standard calibration curve. The reason for slightly reduced drug loading efficiency compared to our prior study may be that the increase of surface potential after modification caused a decrease of adsorbed drugs onto LAP via electrostatic interactions [20].

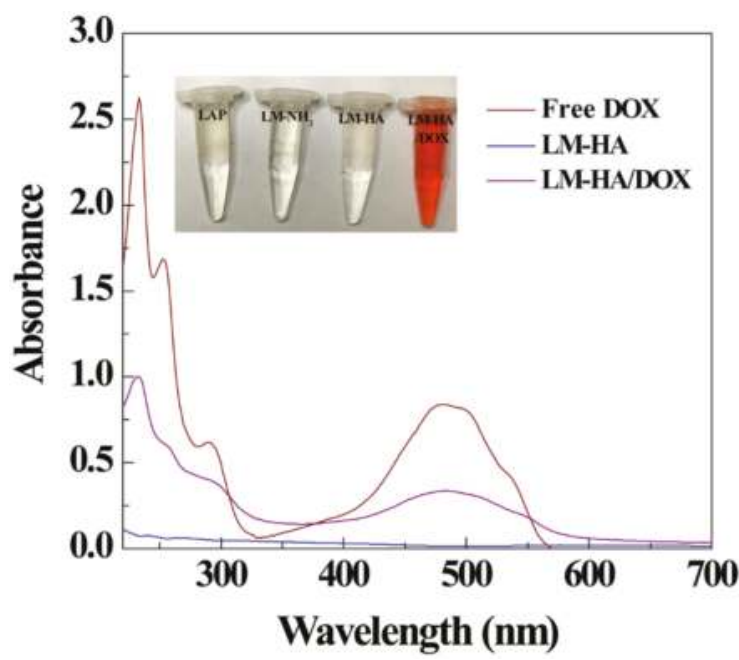

Figure 4. UV-vis spectra of free doxorubicin (DOX), LM-HA, and LM-HA/DOX, respectively.

As seen in Figure 5, the release profiles of LM-HA/DOX at pH 5.4 or 7.4 were investigated. Obviously, at a weak acid condition, LM-HA/DOX initially exhibited a fast release of DOX in the first $48 \mathrm{~h}$, and then a sustained release for a long duration. After $168 \mathrm{~h}$, LM-HA/DOX nanocomposites released about $44 \%$ of DOX at $\mathrm{pH}$ 5.4. The two-phase release profile of LM-HA/DOX may help to restrain the proliferation of cancer cells immediately after administration and keep a relatively high drug concentration for continuous chemotherapy. In contrast, at $\mathrm{pH} 7.4$ condition, the drug release rate was very low, and around 13\% of DOX was desorbed after $168 \mathrm{~h}$. The reason for the $\mathrm{pH}$-sensitive release property of LM-HA/DOX may be the good solubility of the salt form (DOX.HCl) of DOX under acidic $\mathrm{pH}$ conditions, and its hydrophobic neutral structure under physiological $\mathrm{pH}$ conditions [42]. Considering the slightly acidic microenvironment in tumors, a drug released in a sustained manner with $\mathrm{pH}$-responsiveness may be beneficial for chemotherapy, and the decreased leakage of the drug during circulation in physiological conditions may decrease the side effect of DOX. 


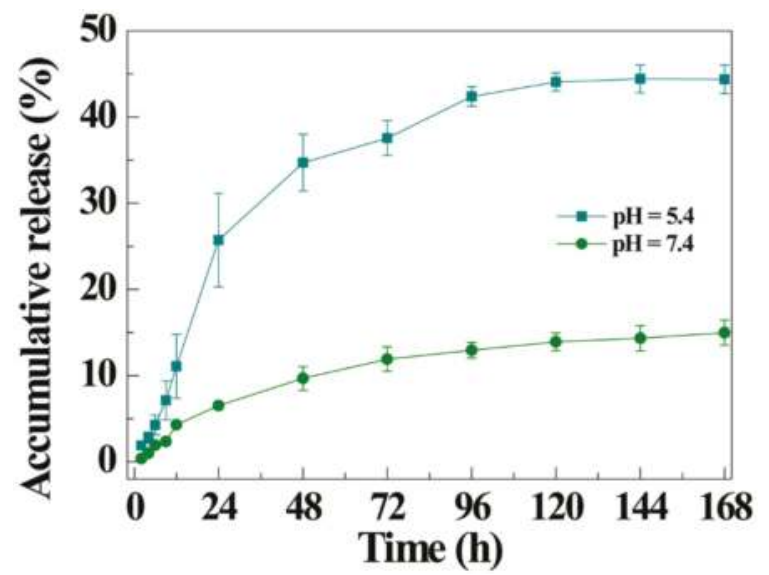

Figure 5. In vitro DOX release profile of LM-HA/DOX at $37^{\circ} \mathrm{C}$ under $\mathrm{pH} 5.4$ and 7.4 conditions.

\subsection{Targeted Therapeutic Efficacy of LM-HA/DOX}

To evaluate the targeted therapeutic efficacy of LM-HA/DOX, HeLa cells with overexpressing CD44 receptors were used as model cells, and free HA-pretreated HeLa cells were also prepared as a block control by culturing cells in HA-containing DMEM for $1 \mathrm{~h}$ before the experiment. Firstly, an MTT assay was conducted to evaluate the cytotoxicity of LM-HA on HeLa cells and HA-pretreated HeLa cells (Figure 6). It was obvious that the viability of both cells could remain over $90 \%$ after being treated with LM-HA within the concentration range of 1.5 to $96 \mu \mathrm{g} / \mathrm{mL}$, demonstrating the good biocompatibility of the LM-HA nanocomposites.

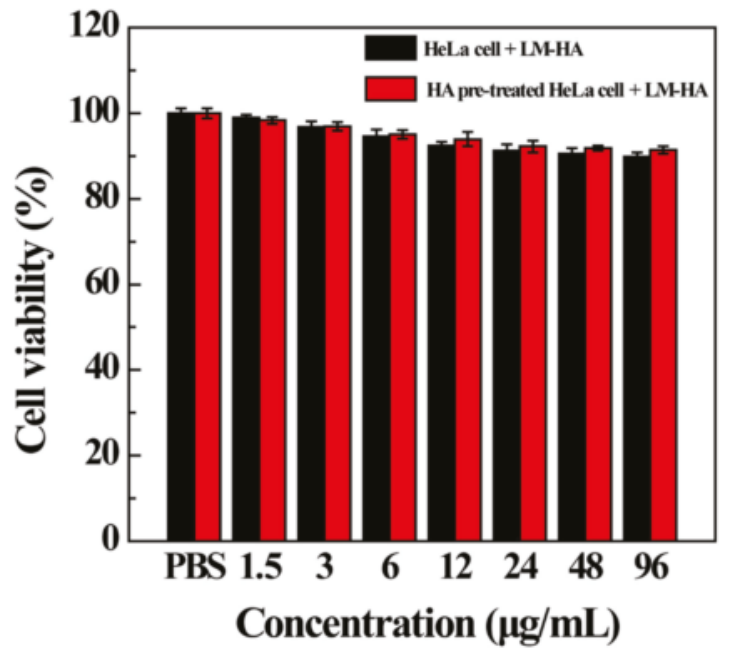

Figure 6. MTT viability assay of HeLa cells and HA pre-treated HeLa cells treated with LM-HA for $24 \mathrm{~h}$, respectively.

To evaluate the therapeutic efficacy of LM-HA/DOX nanocomposites, the cell viability of HeLa cells was assessed by MTT assay after being treated with free DOX and LM-HA/DOX with different DOX concentrations for $24 \mathrm{~h}$, and the viability of HA-pretreated HeLa cells treated with LM-HA/DOX was also measured (Figure 7). Both free DOX and LM-HA/DOX could restrain the proliferation of 
HeLa cells in a dose-dependent manner, demonstrating the prominent chemotherapeutic effect of DOX. Moreover, LM-HA/DOX displayed a significantly enhanced inhibition of HeLa cells in comparison with free DOX at the same DOX concentration, demonstrating the superior therapeutic effect of LM-HA/DOX. Importantly, the cell viability of HA-pretreated HeLa cells was much higher than that of HeLa cells when treating with the same dose of LM-HA/DOX. The half-maximal inhibitory concentration $\left(\mathrm{IC}_{50}\right)$ of LM-HA/DOX in treating HA-pretreated HeLa cells $(1.62 \mu \mathrm{g} / \mathrm{mL})$ was found to be about 3.38 folds higher than that of HeLa cells $(0.48 \mu \mathrm{g} / \mathrm{mL})$, demonstrating that LM-HA/DOX could specifically inhibit HeLa cells via the CD44-mediated targeting. In summary, LM-HA/DOX nanocomposites can exert enhanced antitumor efficacy on cancer cells overexpressing CD44 receptors.

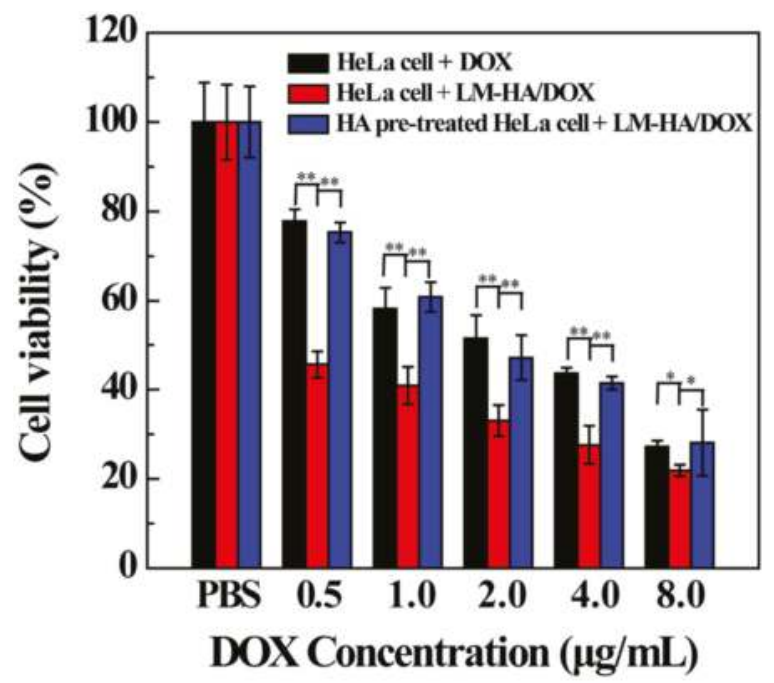

Figure 7. MTT viability assay of HeLa cells and HA pre-treated HeLa cells treated with free DOX and LM-HA/DOX nanocomposites for $24 \mathrm{~h}$ with different DOX concentrations.

Finally, the targeted antitumor efficacy of the LM-HA/DOX nanocomposites was confirmed by morphology observation (Figure S2). Obviously, with the increase of DOX concentration, an increasing portion of HeLa cells and HA-pretreated HeLa cells became rounded and non-adherent, confirming the inhibition efficiency of LM-HA/DOX. In addition, LM-HA/DOX exhibited a higher inhibition rate in treating HeLa cells in comparison with HA-pretreated HeLa cells. This result is consistent with the MTT assay results, demonstrating the targeted inhibition of CD44-overexpressed cancer cells by LM-HA/DOX.

\subsection{CD44-Mediated Cellular Uptake}

HeLa cells and HA-pretreated HeLa cells were incubated with LM-HA/DOX nanocomposites for $4 \mathrm{~h}$ to assess the intracellular uptake of LM-HA/DOX by CLSM observation (Figure 8). The cell nuclei displayed blue fluorescence after being stained by DAPI, and the uptake and distribution of LM-HA/DOX could be illustrated by the self-fluorescence of DOX. Compared with the PBS control group, HeLa cells displayed DOX-related fluorescence in cell nuclei and cytoplasma after treated with free DOX and LM-HA/DOX. And the red fluorescence of LM-HA/DOX group was brighter than that of free DOX group, indicating the effective uptake of LM-HA/DOX by cells. Additionally, the red fluorescence signal accumulated in HA pre-treated HeLa cells was lower than that in HeLa cells, which was possibly due to the blockage of CD44 receptors on the cell surface by free HA. This outcome verified that the modification of HA could enhance the cellular uptake of LM-HA/DOX through CD44-mediated specific targeting. 


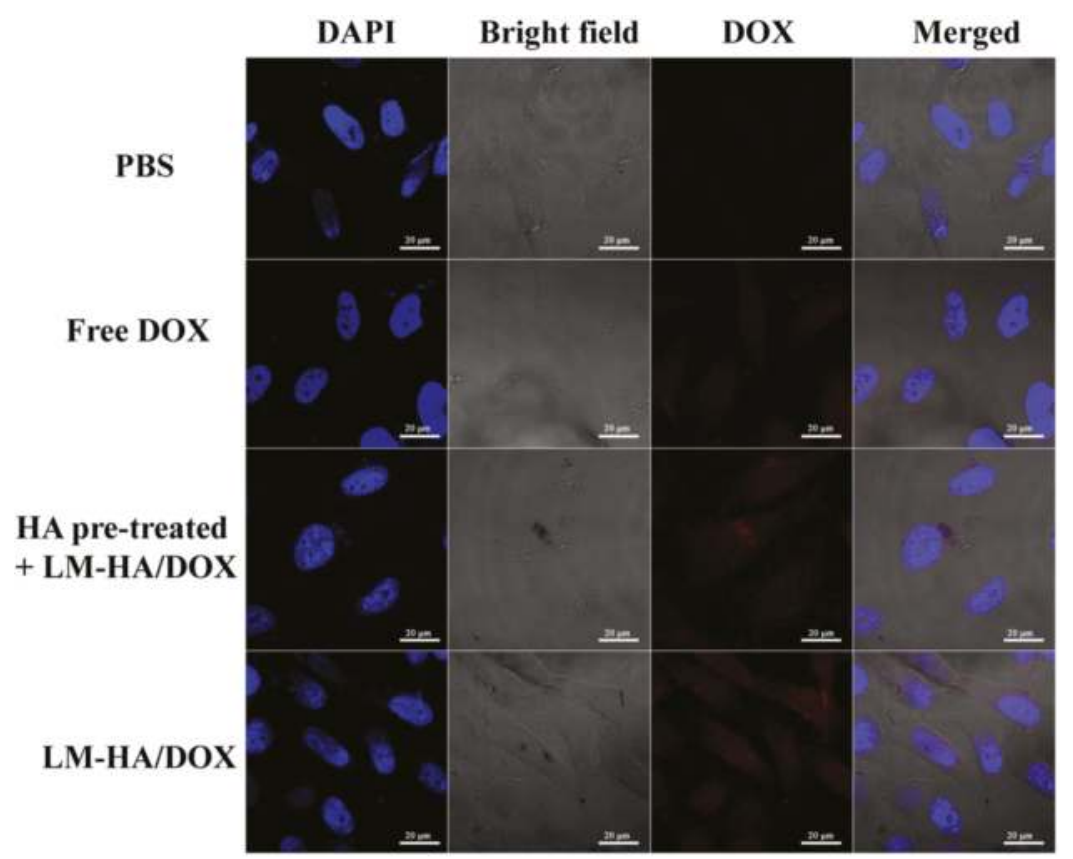

Figure 8. CLSM images of HeLa cells and HA pre-treated HeLa cells treated with PBS, free DOX and LM-HA/DOX nanocomposites for $4 \mathrm{~h}$, at same DOX concentrations of $0.5 \mu \mathrm{g} / \mathrm{mL}$.

Finally, flow cytometry measurement was performed to further demonstrate the targeting specificity of LM-HA/DOX in HeLa cells (Figure 9). When compared to PBS control, both DOX and LM-HA/DOX displayed an obvious mean fluorescence enhancement, indicating the uptake of DOX and LM-HA/DOX by HeLa cells. Moreover, HeLa cells exhibited a significant enhanced mean fluorescence after being treated with LM-HA/DOX in comparison to HA pre-treated HeLa cells, in consistent with CLSM result. This phenomenon clearly demonstrated that HA modification could increase the cellular uptake by CD44-mediated endocytosis. Overall, LM-HA/DOX could selectively bind and internalize into HeLa cells due to the specific interaction between HA and CD44 overexpressed on cell surface, resulting in enhanced therapeutic efficacy in treating CD44-overexpressed cancer cells. 


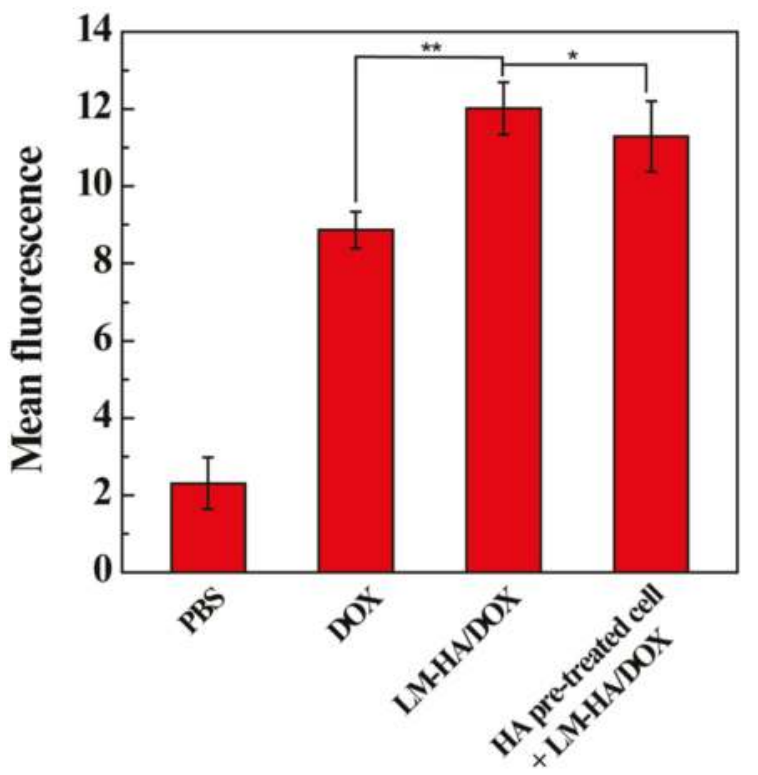

Figure 9. Mean fluorescence intensity of HeLa cells and HA pre-treated HeLa cells treated with PBS, free DOX and LM-HA/DOX nanocomposites for $4 \mathrm{~h}$, at the same DOX concentrations of $0.5 \mu \mathrm{g} / \mathrm{mL}$.

\section{Conclusions}

To conclude, hyaluronic acid-decorated LAP nanocomposites were successfully synthesized for targeted delivery of anticancer drugs to tumor cells overexpressing CD44 receptors. Obviously, the prepared LM-HA could load DOX effectively $(85.1 \pm 1.5 \%)$, and release drug in a pH-sensitive profile. In vitro cell viability assay, FCM, and CLSM results demonstrated that LM-HA/DOX nanocomposites exhibited a significantly enhanced antitumor efficacy than free DOX in treating CD44-overexpressed HeLa cells, due to their specific delivery and enhanced uptake via CD44-mediated endocytosis. Therefore, the designed LM-HA/DOX nanocomposites can be considered as a promising targeted anticancer drug delivery system for cancer treatment.

Supplementary Materials: The following are available online at http://www.mdpi.com/2073-4360/11/1/137/s1. Figure S1. Photographs of LM-HA/DOX dispersed in different solvents, Figure S2. Micrographs of HeLa cells and HA pre-treated HeLa cells treated with PBS, LM-HA/DOX for $24 \mathrm{~h}$ with different concentrations of DOX $(1.0,2.0$, and $4.0 \mu \mathrm{g} / \mathrm{mL})$, Table S1. $\zeta$-potential and hydrodynamic diameter of LM-HA/DOX dispersed in water, PBS, DMEM, and FBS, respectively.

Author Contributions: R.G. proposed the idea and writing thought of the work. T.J. carried out some experiments, analyzed the data and wrote the manuscript. G.C. performed some of the experimental work. X.S. proposed the idea of the experimental part of the whole work.

Funding: This research was funded by Shanghai Natural Science Foundation (17ZR1401200), the National Natural Science Foundation of China (21785031 and 81761148028).

Conflicts of Interest: The authors declare no conflict of interest.

\section{References}

1. Liu, D.; Yang, F.; Xiong, F.; Gu, N. The Smart Drug Delivery System and Its Clinical Potential. Theranostics 2016, 6, 1306-1323. [CrossRef] [PubMed]

2. Oku, N. Innovations in Liposomal DDS Technology and Its Application for the Treatment of Various Diseases. Biol. Pharm. Bull. 2017, 40, 119-127. [CrossRef] [PubMed] 
3. Xu, F.; Liu, M.; Li, X.; Xiong, Z.; Cao, X.; Shi, X.; Guo, R. Loading of indocyanine green within polydopamine-coated laponite nanodisks for targeted cancer photothermal and photodynamic therapy. Nanomaterials 2018, 8, 347. [CrossRef] [PubMed]

4. Zhu, J.; Zheng, L.; Wen, S.; Tang, Y.; Shen, M.; Zhang, G.; Shi, X. Targeted cancer theranostics using alpha-tocopheryl succinate-conjugated multifunctional dendrimer-entrapped gold nanoparticles. Biomaterials 2014, 35, 7635-7646. [CrossRef] [PubMed]

5. Wang, D.; Yan, J.; Jiang, J.; Liu, X.; Tian, C.; Xu, J.; Yuan, M.; Han, X.; Wang, J. Polydiacetylene liposomes with phenylboronic acid tags: A fluorescence turn-on sensor for sialic acid detection and cell-surface glycan imaging. Nanoscale 2018, 10, 4570-4578. [CrossRef] [PubMed]

6. Xu, W.; Qian, J.; Hou, G.; Wang, Y.; Wang, J.; Sun, T.; Ji, L.; Suo, A.; Yao, Y. A dual-targeted hyaluronic acid-gold nanorod platform with triple-stimuli responsiveness for photodynamic/photothermal therapy of breast cancer. Acta Biomater. 2018, 1742-1756. [CrossRef] [PubMed]

7. Alonso, S. Exploiting the bioengineering versatility of lactobionic acid in targeted nanosystems and biomaterials. J. Controlled Release 2018, 287, 216-234. [CrossRef]

8. Zhao, L.; Zhu, J.; Cheng, Y.; Xiong, Z.; Tang, Y.; Guo, L.; Shi, X.; Zhao, J. Chlorotoxin-Conjugated Multifunctional Dendrimers Labeled with Radionuclide I-131 for Single Photon Emission Computed Tomography Imaging and Radiotherapy of Gliomas. ACS Appl. Mater. Interfaces 2015, 7, 19798-19808. [CrossRef]

9. Zhang, Y.; Wu, X.; Hou, C.; Shang, K.; Yang, K.; Tian, Z.; Pei, Z.; Qu, Y.; Pei, Y. Dual-responsive dithio-polydopamine coated porous $\mathrm{CeO} 2$ nanorods for targeted and synergistic drug delivery. Int. J. Nanomed. 2018, 13, 2161-2173. [CrossRef] [PubMed]

10. Zhang, Y.; Guo, Z.; Cao, Z.; Zhou, W.; Zhang, Y.; Chen, Q.; Lu, Y.; Chen, X.; Guo, Q.; Li, C.; et al. Endogenous albumin-mediated delivery of redox-responsive paclitaxel-loaded micelles for targeted cancer therapy. Biomaterials 2018, 183, 243-257. [CrossRef]

11. Hatakeyama, H. Development of a Novel Liposomal DDS by Manipulating Pharmacokinetics and Intracellular Trafficking for Drug Therapy and Nucleic Acid Medicine. Yakugaku Zasshi 2018, 138, 591-598. [CrossRef] [PubMed]

12. Wang, Y.; Guo, R.; Cao, X.; Shen, M.; Shi, X. Encapsulation of 2-methoxyestradiol within multifunctional poly (amidoamine) dendrimers for targeted cancer therapy. Biomaterials 2011, 32, 3322-3329. [CrossRef] [PubMed]

13. Wang, F.; Chen, Y.; Zhang, D.; Zhang, Q.; Zheng, D.; Hao, L.; Liu, Y.; Duan, C.; Jia, L.; Liu, G. Folate-mediated targeted and intracellular delivery of paclitaxel using a novel deoxycholic acid-o-carboxymethylated chitosan-folic acid micelles. Int. J. Nanomed. 2012, 7, 325-337.

14. Zhou, T.; Xiao, C.; Fan, J.; Chen, S.; Shen, J.; Wu, W.; Zhou, S. A nanogel of on-site tunable pH-response for efficient anticancer drug delivery. Acta Biomater. 2013, 9, 4546-4557. [CrossRef] [PubMed]

15. Rahoui, N.; Jiang, B.; Hegazy, M.; Taloub, N.; Wang, Y.; Yu, M.; Huang, Y.D. Gold modified polydopamine coated mesoporous silica nano-structures for synergetic chemo-photothermal effect. Colloids Surf. B 2018, 171, 176-185. [CrossRef] [PubMed]

16. Yu, S.; Li, Q.; Wang, J.; Du, J.; Gao, Y.; Zhang, L.; Chen, L.; Yang, Y.; Liu, X. A targeted drug delivery system based on carbon nanotubes loaded with lobaplatin toward liver cancer cells. J. Mater. Res. 2018, 33, 2565-2575. [CrossRef]

17. Ingram, S.E.; Liggat, J.J.; Pethrick, R.A. Properties of epoxy nanoclay system based on diaminodiphenyl sulfone and diglycidyl ether of bisphenol F: Influence of post cure and structure of amine and epoxy. Polym. Int. 2007, 56, 1029-1034. [CrossRef]

18. Ding, L.; Hu, Y.; Luo, Y.; Zhu, J.; Wu, Y.; Cao, X.; Peng, C.; Shi, X.; Guo, R. LAPONITEreg-stabilized iron oxide nanoparticles for in vivo MR imaging of tumors. Biomater. Sci. 2016, 4, 474-482. [CrossRef]

19. Li, Y.; Maciel, D.; Tomás, H.; Rodrigues, J.; Ma, H.; Shi, X. pH sensitive laponite/alginate hybrid hydrogels: Swelling behaviour and release mechanism. Soft Matter 2011, 7, 6231-6238. [CrossRef]

20. Wang, S.; Wu, Y.; Guo, R.; Huang, Y.; Wen, S.; Shen, M.; Wang, J.; Shi, X. Laponite nanodisks as an efficient platform for doxorubicin delivery to cancer cells. Langmuir 2013, 29, 5030-5036. [CrossRef]

21. Zhuang, Y.; Zhou, L.; Zheng, L.; Hu, Y.; Ding, L.; Li, X.; Liu, C.; Zhao, J.; Shi, X.; Guo, R. Laponite-polyethylenimine based theranostic nanoplatform for tumor-targeting CT imaging and chemotherapy. ACS Biomater. Sci. Eng. 2017, 3, 431-442. [CrossRef] 
22. Wang, S.; Zheng, F.; Huang, Y.; Fang, Y.; Shen, M.; Zhu, M.; Shi, X. Encapsulation of amoxicillin within laponite-doped poly (lactic-co-glycolic acid) nanofibers: Preparation, characterization, and antibacterial activity. ACS Appl. Mater. Interfaces 2012, 4, 6393-6401. [CrossRef] [PubMed]

23. Ghadiri, M.; Hau, H.; Chrzanowski, W.; Agus, H.; Rohanizadeh, R. Laponite clay as a carrier for in situ delivery of tetracycline. RSC Adv. 2013, 3, 20193-20201. [CrossRef]

24. Fraile, J.M.; Garcia-Martin, E.; Gil, C.; Mayoral, J.A.; Pablo, L.E.; Polo, V.; Prieto, E.; Vispe, E. Laponite as carrier for controlled in vitro delivery of dexamethasone in vitreous humor models. Eur. J. Pharm. Biopharm. 2016, 108, 83-90. [CrossRef] [PubMed]

25. Wu, Y.; Guo, R.; Wen, S.; Shen, M.; Zhu, M.; Wang, J.; Shi, X. Folic acid-modified laponite nanodisks for targeted anticancer drug delivery. J. Mater. Chem. B 2014, 2, 7410-7418. [CrossRef]

26. Chen, G.; Li, D.; Li, J.; Cao, X.; Wang, J.; Shi, X.; Guo, R. Targeted doxorubicin delivery to hepatocarcinoma cells by lactobionic acid-modified laponite nanodisks. New J. Chem. 2015, 39, 2847-2855. [CrossRef]

27. Lim, E.K.; Kim, H.O.; Jang, E.; Park, J.; Lee, K.; Suh, J.S.; Huh, Y.M.; Haam, S. Hyaluronan-modified magnetic nanoclusters for detection of CD44-overexpressing breast cancer by MR imaging. Biomaterials 2011, 32, 7941-7950. [CrossRef]

28. Lee, H.; Park, H.; Noh, G.J.; Lee, E.S. pH-responsive hyaluronate-anchored extracellular vesicles to promote tumor-targeted drug delivery. Carbohydr. Polym. 2018, 202, 323-333. [CrossRef]

29. Jang, E.; Lim, E.K.; Choi, Y.; Kim, E.; Kim, H.O.; Kim, D.J.; Suh, J.S.; Huh, Y.M.; Haam, S. pi-Hyaluronan nanocarriers for CD44-targeted and pH-boosted aromatic drug delivery. J. Mater. Chem. B 2013, 1, 5686-5693. [CrossRef]

30. Mandaliti, W.; Nepravishta, R.; Pica, F.; Vallebona, P.S.; Garaci, E.; Paci, M. Thymosin 1 interacts with hyaluronic acid electrostatically by its terminal sequence lkekk. Molecules 2017, 22, 1843. [CrossRef]

31. Mo, Y.; Wang, H.; Liu, J.; Lan, Y.; Guo, R.; Zhang, Y.; Xue, W.; Zhang, Y. Controlled release and targeted delivery to cancer cells of doxorubicin from polysaccharide-functionalised single-walled carbon nanotubes. J. Mater. Chem. B 2015, 3, 1846-1855. [CrossRef]

32. He, X.; Liu, B.; Xu, C.; Zhuo, R.; Cheng, S. A multi-functional macrophage and tumor targeting gene delivery system for the regulation of macrophage polarity and reversal of cancer immunoresistance. Nanoscale 2018, 10, 15578-15587. [CrossRef] [PubMed]

33. Cheng, C.; Meng, Y.; Zhang, Z.; Li, Y.; Zhang, Q. Tumoral acidic pH-responsive cis-diaminodichloroplatinum-incorporated Cy5.5-PEG-g-A-HA nanoparticles for targeting delivery of cddp against cervical cancer. ACS Appl. Mater. Interfaces 2018, 10, 26882-26892. [CrossRef] [PubMed]

34. Mattheolabakis, G.; Milane, L.; Singh, A.; Amiji, M.M. Hyaluronic acid targeting of CD44 for cancer therapy: From receptor biology to nanomedicine. J. Drug Targeting 2015, 23, 605-618. [CrossRef] [PubMed]

35. Parafiniuk, K.; Monnereau, C.; Sznitko, L.; Mettra, B.; Zelechowska, M.; Andraud, C.; Miniewicz, A.; Mysliwiec, J. Distributed feedback lasing in amorphous. Polymers with covalently bonded fluorescent dyes: The influence of photoisomerization process. Macromolecules 2017, 50, 6164-6173. [CrossRef]

36. Mayol, L.; de Stefano, D.; de Falco, F.; Carnuccio, R.; Maiuri, M.C.; de Rosa, G. Effect of hyaluronic acid on the thermogelation and biocompatibility of its blends with methyl cellulose. Carbohydr. Polym. 2014, 112, 480-485. [CrossRef]

37. Hang, C.; Zou, Y.; Zhong, Y.; Zhong, Z.; Meng, F. NIR and UV-responsive degradable hyaluronic acid nanogels for CD44-targeted and remotely triggered intracellular doxorubicin delivery. Colloids Surf. B 2017, 158, 547-555. [CrossRef]

38. Daehler, A.; Boskovic, S.; Gee, M.L.; Separovic, F.; Stevens, G.W.; O'Connor, A.J. Postsynthesis vapor-phase functionalization of MCM-48 with hexamethyldisilazane and 3-aminopropyldimethylethoxylsilane for bioseparation applications. J. Phys. Chem. B 2005, 109, 16263-16271. [CrossRef]

39. Sun, F.; Yu, Y.; Yang, Z.; Wang, Z.; Li, Y.; Wang, F.; Tan, H. Hyaluronic acid-endostatin2-alft1 (HA-ES2-AF) nanoparticle-like conjugate for the target treatment of diseases. J. Controlled Release 2018, 288, 1-13. [CrossRef]

40. Chahuki, F.F.; Aminzadeh, S.; Jafarian, V.; Tabandeh, F.; Khodabandeh, M. Hyaluronic acid production enhancement via genetically modification and culture medium optimization in Lactobacillus acidophilus. Int. J. Biol. Macromol. 2019, 121, 870-881. [CrossRef] 
41. Zhang, B.; Chang, B.; Sun, T. Synthesis and Study of Hypoxia-Responsive Micelles Based on Hyaluronic Acid. Acta Chim. Sin. 2018, 76, 35-42. [CrossRef]

42. Luo, Z.; Li, Y.; Wang, B.; Jiang, J. pH-Sensitive Vesicles Formed by Amphiphilic Grafted Copolymers with Tunable Membrane Permeability for Drug Loading/Release: A Multiscale Simulation Study. Macromolecules 2016, 49, 6084-6094. [CrossRef]

(C) 2019 by the authors. Licensee MDPI, Basel, Switzerland. This article is an open access article distributed under the terms and conditions of the Creative Commons Attribution (CC BY) license (http://creativecommons.org/licenses/by/4.0/). 
Article

\title{
Synthesis and Characterization of Clay Polymer Nanocomposites of P(4VP-co-AAm) and Their Application for the Removal of Atrazine
}

\author{
Jorge A. Ramírez-Gómez ${ }^{1}$, Javier Illescas ${ }^{1, *, \dagger}$, María del Carmen Díaz-Nava ${ }^{1}$, \\ Claudia Muro-Urista ${ }^{1}$, Sonia Martínez-Gallegos ${ }^{1}$ and Ernesto Rivera ${ }^{2}$ \\ 1 Tecnológico Nacional de Mexico/Instituto Tecnológico de Toluca, División de Estudios de Posgrado e \\ Investigación, Av. Tecnológico S/N, Col. Agrícola Bellavista, Metepec 52149, Mexico; \\ j.ramirez4499@gmail.com (J.A.R.-G.); cdiazn@toluca.tecnm.mx (M.d.C.D.-N.); \\ cmurou@toluca.tecnm.mx (C.M.-U.); soniakorn@yahoo.com (S.M.-G.) \\ 2 Instituto de Investigaciones en Materiales, Universidad Nacional Autónoma de Mexico, Circuito Exterior \\ S/N, Ciudad Universitaria, Mexico City 04510, Mexico; riverage@unam.mx \\ * Correspondence: fillescasm@toluca.tecnm.mx; Tel.: +52-722-208-7224 \\ † Consejo Nacional de Ciencia y Tecnología (CONACYT) Research Fellow.
}

Received: 30 March 2019; Accepted: 15 April 2019; Published: 19 April 2019

\begin{abstract}
Atrazine (ATZ) is an herbicide which is applied to the soil, and its mechanism of action involves the inhibition of photosynthesis. One of its main functions is to control the appearance of weeds in crops, primarily in corn, sorghum, sugar cane, and wheat; however, it is very toxic for numerous species, including humans. Therefore, this work deals with the adsorption of ATZ from aqueous solutions using nanocomposite materials, synthesized with two different types of organo-modified clays. Those were obtained by the free radical polymerization of 4-vinylpyridine $(4 \mathrm{VP})$ and acrylamide (AAm) in different stoichiometric ratios, using tetrabutylphosphonium persulfate (TBPPS) as a radical initiator and $N, N^{\prime}$-methylenebisacrylamide (BIS) as cross-linking agent. The structural, morphological, and textural characteristics of clays, copolymers, and nanocomposites were determined through different analytical and instrumental techniques, i.e., X-ray diffraction (XRD), Fourier-transform infrared spectroscopy (FTIR), and thermogravimetric analysis (TGA). Adsorption kinetics experiments of ATZ were determined with the modified and synthesized materials, and the effect of the ratio between $4 \mathrm{VP}$ and AAm moieties on the removal capacities of the obtained nanocomposites was evaluated. Finally, from these sets of experiments, it was demonstrated that the synthesized nanocomposites with higher molar fractions of $4 \mathrm{VP}$ obtained the highest removal percentages of ATZ.
\end{abstract}

Keywords: clay-polymer nanocomposites; atrazine; radical polymerization; hexadecyltrimethylammonium bromide; phenyltrimethylammonium chloride; FTIR; TGA; adsorption

\section{Introduction}

Atrazine (ATZ) is an herbicide that belongs to the family of triazines; its International Union of Pure and Applied Chemistry (IUPAC) name is 6-chloro- $N$-ethyl- $N$-(1-methylethyl)-1,3,5-triazine-2,4-diamine (Figure 1). It is a selective herbicide that is applied to the soil; mainly, its action mechanism involves the inhibition of photosynthesis. Moreover, in plants, it is absorbed through the roots or leaves and it is applied before or after the germination of seeds. One of the main applications of ATZ is for controlling the occurrence of broadleaf and grassy weeds [1,2]. Additionally, ATZ is also known as an endocrine disruptor; this term defines a diverse and heterogeneous set of chemical compounds capable of altering the hormonal balance. 


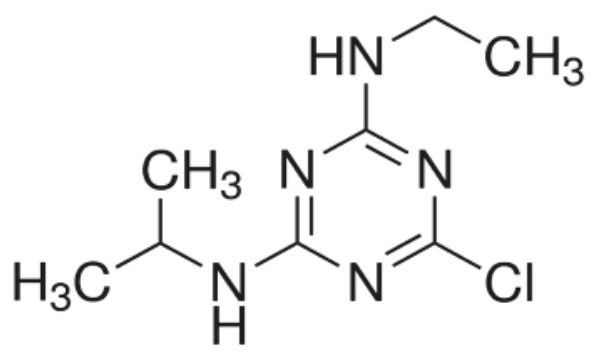

Figure 1. Structure of the atrazine (ATZ) herbicide.

There are many factors that contribute to the pollution of water sources destined to human consumption, through runoff with ATZ. Some of the properties that determine the mobility of ATZ and its metabolites in the environment are their low affinity to the soil components and their high persistence. Moreover, the relative "high" solubility of ATZ in water is the most frequent cause of finding it in surface and underground water bodies [3,4]. The Environmental Protection Agency of the United States (EPA) reported that this herbicide is toxic to numerous aquatic and reptile species, at levels of up to two parts per billion (ppb). Moreover, the EPA recommendation for the treatment of drinking water, polluted with triazines, is filtration with granular activated carbon (GAC) [5].

The degradation of ATZ can be carried out by both biological and chemical reactions. Biological degradation occurs through the activity of microorganisms and it is considered as the main process by which this herbicide is transformed [6-9]. However, the degradation of ATZ by means of microorganisms is not the most appropriate technology due to the formation of its metabolites, which are more toxic than the herbicide itself. Meanwhile, the chemical degradation of ATZ is carried out mainly by two processes: hydrolysis and photolysis. Hydrolysis commonly leads to the production of hydroxylated compounds such as hydroxyatrazine, desethylhydroxyatrazine, and deisopropylhydroxyatrazine, each one with variable persistence and toxicity [10].

One alternative method employed for the removal of ATZ is the adsorption process through the development of novel adsorbent materials, which is widely used. Some advantages of this method are (i) effectiveness, since it can reduce ATZ concentration to prevent the formation of a metabolite of the herbicide; (ii) simplicity, because its application is the use of columns through which water is passed; (iii) environmentally friendly, thanks to the use of small amounts of adsorbents that can be regenerated; and (iv) economical, because materials are cheaper than those used in other methods. One example of these kinds of materials involves clays modified with a cationic surfactant, also called organo-modified clays, because the nature of the hydrophilic clay is modified by organic cations to form a richer organophilic surface, which confers them a great affinity for organic compounds, being able to remove them from water bodies [11-14].

These organo-modified clays are incorporated into polymer matrices to obtain clay polymer nanocomposites that attracted attention as adsorbents, since they can be reusable and have a high retention capacity [15-17]. It is noteworthy that a nanocomposite consists of at least two main components that are chemically distinct and insoluble. The first of them is the matrix that serves as the continuous phase and could be a polymeric, a metallic, or a ceramic material. The second one is the filler, in the nanoscale range, i.e., from 1 to $100 \mathrm{~nm}$, whose main function is to reinforce the matrix; some examples of fillers are graphene, dichalcogenide materials like $\mathrm{MoS}_{2} \mathrm{MoSe}_{2}, \mathrm{WS}_{2}$, or $\mathrm{MoTe}_{2}$, or natural materials such as clays or zeolites. Specifically, in our research group, we synthesized different types of clay polymer nanocomposites for different purposes, i.e., for the removal from aqueous solutions of azo dyes [18,19], a triarylmethane dye [20], or even phenolic compounds [21].

The compound 4-vinylpyridine (4VP) is a weak base that, when protonated, can increase its volume due to the incorporation of solvent and electrostatic repulsion between the charged sites. Moreover, the presence of the pyridine ring offers the possibility of anchoring different species, making 
it a good adsorbent [22]. Acrylamide (AAm) has two reactive centers: the amide group carries out the characteristic reactions of an aliphatic amide and also has weakly acidic and basic properties [23,24]. The double bond of AAm is deficient in electrons and produces Michael-type addition reactions, many of which are reversible. AAm is used in the treatment of water to flocculate solids. On the other side, sepiolite is a raw clay mineral that is used for its organo-modification. This is because of the enhancement in the mechanical properties that it offers to any polymer matrix and because it is very easy to modify with a cationic surfactant [25-27].

In this work the organo-modification of a raw clay mineral, consisting mainly of sepiolite, and its nanocomposites, comprising monomers $4 \mathrm{VP}$ and AAm in different percentages, were synthesized and characterized. For this purpose, a raw clay mineral, a sepiolite, from Puebla State, in Mexico, was organo-modified with two different cationic surfactants, namely, hexadecyltrimethylammonium bromide (HDTMA-Br) or phenyltrimethylammonium chloride (PTMA-Cl). Afterwards, these organo-modified clays were incorporated into the copolymer structures, $\mathrm{p}(4 \mathrm{VP}-\mathrm{co}-\mathrm{AAm})$, to obtain two different series of nanocomposites. At last, their morphology, their thermal properties, their spectroscopic characteristics, and their ATZ kinetics adsorption were gauged.

\section{Materials and Methods}

\subsection{Materials}

All the chemicals and materials used in the synthesis and characterization of nanocomposites, as well as those for the ATZ adsorption tests, were used as received without any further purification, unless otherwise stated. Firstly, 4-vinylpyridine $\left(4 \mathrm{VP} ;(\mathrm{FW})=105.14 \mathrm{~g} \cdot \mathrm{mol}^{-1}, \mathrm{~b} . \mathrm{p} .=62-65^{\circ} \mathrm{C}\right)$ and acrylamide (AAm; FW $=71.08 \mathrm{~g} \cdot \mathrm{mol}^{-1}, \mathrm{~m} \cdot \mathrm{p},=82-86^{\circ} \mathrm{C}$ ) monomers, $N, N^{\prime}$-methylenebis(acrylamide) (BIS; FW $=154.17 \mathrm{~g} \cdot \mathrm{mol}^{-1}, \mathrm{~m} . \mathrm{p} . \geq 300^{\circ} \mathrm{C}$ ) cross-linking agent, hexadecyltrimethylammonium bromide (HDTMA-Br; FW $=364.45 \mathrm{~g} \cdot \mathrm{mol}^{-1}, \mathrm{~m} \cdot \mathrm{p} .=212{ }^{\circ} \mathrm{C}$ ) and phenyltrimethylammonium chloride (PTMA-Cl; $\left.\mathrm{FW}=171.67 \mathrm{~g} \cdot \mathrm{mol}^{-1}, \mathrm{~m} . \mathrm{p} .=246-248^{\circ} \mathrm{C}\right)$ cationic surfactants, and formamide $\left(\mathrm{FW}=45.04 \mathrm{~g} \cdot \mathrm{mol}^{-1}\right.$, b.p. $\left.=210^{\circ} \mathrm{C}\right)$ solvent were obtained from Sigma-Aldrich. Sodium chloride $(\mathrm{NaCl}, \mathrm{FW}=74.55$ $\mathrm{g} \cdot \mathrm{mol}^{-1}, T_{\mathrm{m}}=776^{\circ} \mathrm{C}$ ) was obtained from JT Baker (Mexico City, Mexico). The ionic-liquid radical initiator of the polymerization, tetrabutylphosphonium persulfate (TBPPS; FW $=710 \mathrm{~g} \cdot \mathrm{mol}^{-1}$ ), was synthesized according to the procedure previously reported elsewhere [28]. Atrazine (ATZ, 6-chloro- $N$-2-ethyl- $N$-4-isopropyl-1,3,5-triazine-2,4-diamine $\geq 90 \%$ ) was kindly supplied by Servicios Tecnológicos para la Agrigultura S.A. de C.V. (Irapuato, Mexico) in its commercial form "Calibre 90 DF". Finally, a raw clay mineral, with sepiolite, supplied by Zeolitech S.A. de C.V. (Puebla, Mexico) and identified by the supplier as A1, was employed.

\subsection{Raw Clay Mineral Organo-Modification and Cationic Exchange Capacity (CEC)}

Firstly, the raw clay mineral (sepiolite) was milled and sieved until it was obtained with a particle size lesser than $44 \mu \mathrm{m}$ (325 mesh); subsequently, it was stored in plastic bottles free of moisture until it was used. Afterward, $50 \mathrm{~g}$ of this clay was put in contact with $500 \mathrm{~mL}$ of a $\mathrm{NaCl}$ solution $(0.1 \mathrm{M})$ for $3 \mathrm{~h}$ with reflux. Next, phases were separated and the clay was decanted; then, another $500 \mathrm{~mL}$ of $\mathrm{NaCl}$ solution was added, repeating the same procedure until it completed $6 \mathrm{~h}$ of reflux. At the end of the total reflux time, the clay was allowed to cool to room temperature and the solution was decanted. After its homoionization, the clay material was washed with deionized water for the elimination of chloride ions, which was verified using silver nitrate $\left(\mathrm{AgNO}_{3}\right)$. In addition, $\mathrm{CEC}$ of the clay material was performed as reported by the American Petroleum Institute, API [29,30]. Briefly, $1 \mathrm{~g}$ of the raw clay material, $10 \mathrm{~mL}$ of distilled water, $15 \mathrm{~mL}$ of hydrogen peroxide $\left(\mathrm{H}_{2} \mathrm{O}_{2} ; 3 \% \mathrm{v} / \mathrm{v}\right)$, and $0.5 \mathrm{~mL}$ of a sulfuric acid $\left(\mathrm{H}_{2} \mathrm{SO}_{4}\right)$ solution $(0.5 \mathrm{~N})$ were mixed. Subsequently, the mixture was heated and allowed to boil for $10 \mathrm{~min}$. After this time, distilled water was added until a volume of $50 \mathrm{~mL}$ was obtained; finally, it was titrated with a methylene blue solution of $0.01 \mathrm{mEq}$. 


\subsection{Modification of the Homoionized Clay Mineral with HDTMA-Br and PTMA-Cl}

The modification of the homoionized clay mineral was carried out with two different cationic surfactants, namely, HDTMA-Br and PTMA-Cl. In the case of the modification of the clay mineral with HDTMA-Br, it was carried out as reported by Hernández-Hernández et al. [21]. Specifically, $10 \mathrm{~g}$ of clay material was put in contact with $100 \mathrm{~mL}$ of a solution of HDTMA-Br $\left(0.03 \mathrm{M}, 30 \mathrm{mEq} \cdot \mathrm{L}^{-1}\right)$ for $48 \mathrm{~h}$ at $30^{\circ} \mathrm{C}$ and $100 \mathrm{rpm}$. In the case of the modification with PTMA-Cl, $10 \mathrm{~g}$ of clay material were put in contact with $100 \mathrm{~mL}$ of a $0.03 \mathrm{M}$ PTMA-Cl solution $\left(30 \mathrm{mEq} \cdot \mathrm{L}^{-1}\right)$, applying the same conditions of temperature and contact time. The concentration of the used surfactant was in concordance with the CEC that was calculated for the raw clay mineral. At the end of this time, the two organo-modified clay minerals, with HDTMA-Br (OMH) or with PTMA-Cl (OMP), were washed with deionized water until the complete removal of bromide or chloride ions, respectively.

\subsection{Synthesis of the Organo-Modified Clay Nanocomposites (CC)}

Organo-modified clay nanocomposites (CC) were obtained according to the following procedure: appropriate amounts of $4 \mathrm{VP}$ and AAm comonomers, OMH or OMP organo-modified clays, BIS cross-linking agent, TBPPS polymerization initiator, and a solvent mixture consisting of formamide and deionized water $(50 \% v / v)$ were added to a polymerization reactor (Table 1$)$. In all cases, the total amount of the two comonomers $(0.1 \mathrm{~mol})$, initiator (TBPPS, $0.25 \mathrm{~mol}$ \% referred to the total molar amount of the two comonomers), cross-linking agent (BIS, $3 \mathrm{~mol}$. \% referred to the total molar amount of the two comonomers), and the solvent mixture $(2 \mathrm{~mL})$ were kept constant. Then, mixtures without TBPPS to avoid spontaneous polymerization were placed in an ultrasonic bath for $3 \mathrm{~h}$ at $100 \mathrm{rpm}$ to exfoliate and disperse the clay [17]. After, TBPPS initiator was added and the polymerization reactors were again ultrasonicated until TBPPS was totally dissolved. Finally, nitrogen was bubbled for $30 \mathrm{~min}$ and reactors were sealed and placed in an oil mineral bath at $55{ }^{\circ} \mathrm{C}$ for $24 \mathrm{~h}$. After polymerization, all samples were extracted and washed with deionized water for several days to remove the solvent mixture and all reagents that did not react.

Table 1. Experimental data of the synthesized samples. 4VP-4-vinylpyridine; AAm-acrylamide; $\mathrm{OMH}$ —organo-modified with hexadecyltrimethylammonium bromide; OMP—organo-modified with phenyltrimethylammonium chloride; BIS $N, N^{\prime}$-methylenebis(acrylamide).

\begin{tabular}{|c|c|c|c|c|c|}
\hline Sample Codes & 4VP (mol. \%) & AAm (mol. \%) & OMH/OMP (wt. \%) & BIS (mol. \%) & TBPPS (mol. \%) \\
\hline CC01 & 100 & 0 & - & 3 & 0.25 \\
\hline $\mathrm{CCO} 2$ & 85 & 15 & - & 3 & 0.25 \\
\hline $\mathrm{CCO3}$ & 65 & 35 & - & 3 & 0.25 \\
\hline $\mathrm{CCO} 4$ & 50 & 50 & - & 3 & 0.25 \\
\hline $\mathrm{CCO5}$ & 30 & 70 & - & 3 & 0.25 \\
\hline $\mathrm{CC} 06$ & 10 & 90 & - & 3 & 0.25 \\
\hline $\mathrm{CCO}$ & 0 & 100 & - & 3 & 0.25 \\
\hline СCH01 & 100 & 0 & 5 & 3 & 0.25 \\
\hline $\mathrm{CCH} 02$ & 85 & 15 & 5 & 3 & 0.25 \\
\hline $\mathrm{CCH} 03$ & 65 & 35 & 5 & 3 & 0.25 \\
\hline $\mathrm{CCH} 04$ & 50 & 50 & 5 & 3 & 0.25 \\
\hline $\mathrm{CCH} 05$ & 30 & 70 & 5 & 3 & 0.25 \\
\hline $\mathrm{CCH} 06$ & 10 & 90 & 5 & 3 & 0.25 \\
\hline $\mathrm{CCH} 07$ & 0 & 100 & 5 & 3 & 0.25 \\
\hline ССР01 & 100 & 0 & 5 & 3 & 0.25 \\
\hline ССР02 & 85 & 15 & 5 & 3 & 0.25 \\
\hline ССР03 & 65 & 35 & 5 & 3 & 0.25 \\
\hline ССР04 & 50 & 50 & 5 & 3 & 0.25 \\
\hline ССР05 & 30 & 70 & 5 & 3 & 0.25 \\
\hline ССР06 & 10 & 90 & 5 & 3 & 0.25 \\
\hline ССР07 & 0 & 100 & 5 & 3 & 0.25 \\
\hline
\end{tabular}




\subsection{Swelling Behavior}

Equilibrium swelling behaviors were recorded for the synthesized dry materials, one for each stoichiometric ratio, and their initial weight (Po) was recorded. Dry disc samples were immersed in 50 $\mathrm{mL}$ of deionized water at room temperature. Weights of swollen discs were measured at different time intervals after excess surface water was removed. This procedure was repeated until there was no weight change. The swelling percentage was calculated according to the following equation:

$$
\mathrm{Sw}(\%)=\left[\left(W_{\mathrm{s}}-W_{\mathrm{d}}\right) / W_{\mathrm{d}}\right] \times 100,
$$

where $W_{\mathrm{d}}$ and $W_{\mathrm{s}}$ are the initial dried disc sample and the final swollen disc sample weights after a certain period of time, respectively.

On the other side, the critical $\mathrm{pH}$ points of the samples were studied while they were immersed in distilled water for $8 \mathrm{~h}$, at room temperature in solutions of $\mathrm{pH}$ ranging from 2.2 to 11 . In all cases, the inflexion point of the swelling as a function of $\mathrm{pH}$ gives us the critical $\mathrm{pH}$ point.

Finally, the $\mathrm{pH}$ sensitivity was defined from the following equation:

$$
(\mathrm{pH})_{\mathrm{s}}=W_{3} / W_{7},
$$

where $W_{3}$ and $W_{7}$ are the swollen weights of the samples at $\mathrm{pH}=3$ and 7 , obtained after $8 \mathrm{~h}$ of swelling.

\subsection{Characterization}

\subsubsection{Raw Clay Mineral, Organo-Modified Clay Materials, and CC}

X-ray diffraction (XRD) of both the raw clay mineral and the organo-modified clay materials was obtained on a Rigaku X-ray diffractometer to identify the main crystalline phases that compose them. The analyses were performed by the powder method, running the samples from $2^{\circ}$ to $85^{\circ}$ in $2 \theta$ and with a step size of $0.02^{\circ}$.

FTIR spectra for all samples were recorded on an infrared absorption spectrophotometer with Fourier transform (FTIR Varian 640-IR) equipped with a diamond ATR (attenuated total reflectance) accessory. The absorption was measured in a wavenumber range between 4000 and $600 \mathrm{~cm}^{-1}$, with a resolution of $16 \mathrm{~cm}^{-1}$ and 16 scans.

The morphology of the clay minerals and CC was studied by means of scanning electron microscopy (SEM) using a scanning electron microscope SEM JEOL model JSM 5900LV (Mexico City, Mexico).

In the case of the thermal analysis, the thermal stability of the samples was studied through the thermogravimetric analysis (TGA) in a thermogravimetric analyzer, TA Instruments, model Q5000 TGA (Mexico City, Mexico) under $\mathrm{N}_{2}$ atmosphere, from 25 to $850{ }^{\circ} \mathrm{C}$, at a heating rate of $10{ }^{\circ} \mathrm{C} \cdot \mathrm{min}^{-1}$. Moreover, differential scanning calorimetry (DSC), using a differential scanning calorimeter, TA Instruments model 2910 (Mexico City, Mexico), was employed in order to determine the thermal transitions of the polymeric materials. For each sample, two consecutive scans were performed in a temperature range from -80 to $200{ }^{\circ} \mathrm{C}$ under $\mathrm{N}_{2}$ atmosphere, with a heating rate of $20^{\circ} \mathrm{C} \cdot \mathrm{min}^{-1}$.

\subsubsection{ATZ Quantification and Adsorption Kinetics Evaluation}

Firstly, absorption spectra were obtained at different ATZ concentrations, from 1 to $11 \mathrm{mg} \cdot \mathrm{L}^{-1}$, in a Perkin Elmer Lambda 35 ultraviolet-visible light (UV-Vis, Metepec, Mexico) spectrophotometer, at a wavelength value of $222 \mathrm{~nm}$. Subsequently, mixtures of $35 \mathrm{mg}$ of raw clay mineral, OMH, OMP, or CCC samples, and $10 \mathrm{~mL}$ of ATZ solutions $\left(5 \mathrm{mg} \cdot \mathrm{L}^{-1}\right)$ were shaken in an orbital bath for different periods of time, from 4 to $24 \mathrm{~h}$, at $25^{\circ} \mathrm{C}$ and $100 \mathrm{rpm}$. Lastly, samples were decanted, and the supernatants were centrifuged at $1500 \mathrm{rpm}$ for $20 \mathrm{~min}$, and the concentration of ATZ in the supernatant was determined 
by means of the UV-Vis technique, following the procedure reported previously [16]. It is noteworthy that all adsorption kinetics experiments were performed at least in triplicate.

The $q_{\mathrm{e}}$ values (equilibrium adsorption capacity) were determined from the adsorption kinetics curve: once the $q_{\mathrm{t}}$ values did not change, we could assume that the equilibrium was reached; therefore, $q_{\mathrm{e}}=q_{\mathrm{t}}$.

In addition, $q_{\mathrm{t}}$ was determined from the following equation:

$$
q_{\mathrm{t}}=\left[\left(C_{\mathrm{i}}-C_{\mathrm{t}}\right) / w\right] \mathrm{V}
$$

where $C_{\mathrm{i}}$ is the initial concentration of the ATZ solution in $\mathrm{mg} \cdot \mathrm{L}^{-1}, C_{\mathrm{t}}$ is the ATZ concentration in a determined time $\mathrm{t}\left(\mathrm{mg} \cdot \mathrm{L}^{-1}\right), \mathrm{w}$ is the mass of the dry adsorbent material $(\mathrm{g})$, and $\mathrm{V}$ is the volume of the solution (L).

\section{Results}

\subsection{Organo-Modification of the Raw Clay Mineral}

\subsubsection{CEC}

Table 2 summarizes the obtained CEC results for the raw clay mineral (sepiolite), as reported by the API [29]; this procedure was carried out in triplicate. Furthermore, to be more accurate, 0.1 and $0.5 \mathrm{~g}$ samples were weighed to compare the obtained results. The mineral presented a CEC of $29.6 \mathrm{mEq} / 100 \mathrm{~g}$, which is in concordance with what is reported in the literature for a sepiolite [31-33].

Table 2. Cationic exchange capacity (CEC) results for the raw clay mineral.

\begin{tabular}{ccc}
\hline Raw Clay Mineral (g) & Volume of Methylene Blue (mL) & CEC (meq/100 g) \\
\hline 0.5006 & 15 & 29.96 \\
0.5003 & 15 & 29.86 \\
0.4996 & 14 & 28.02 \\
0.1006 & 3 & 29.82 \\
0.1004 & 3 & 29.88 \\
0.1002 & 3 & 29.94 \\
\hline
\end{tabular}

\subsubsection{XRD Analysis}

Figure 2a shows the XRD patterns of the raw clay mineral, the OMH, and the HDTMA-Br cationic surfactant. In the case of the first pattern, reflections corresponding to the main clay minerals present in the structure were identified and corresponded to sepiolite (JCPDS 00-023-0330), albite (JCPDS 00-001-0739), and quartz (JCPDS 00-005-0490). Moreover, the pattern corresponding to the HDTMA-Br surfactant was also observed, and it was compared to the JCPDS 00-030-1746 card. In general terms, it can be seen that the obtained XRD pattern maintained the same crystallographic structure of the HDTMA cationic surfactant. Finally, the pattern of the OMH presented a displacement to the right, in angles $2 \theta$, compared to the raw clay mineral, which was a result of the decrease of the interlaminar space and is proof that a change in the crystalline structure of the material was carried out mainly in the interlaminar zone. 


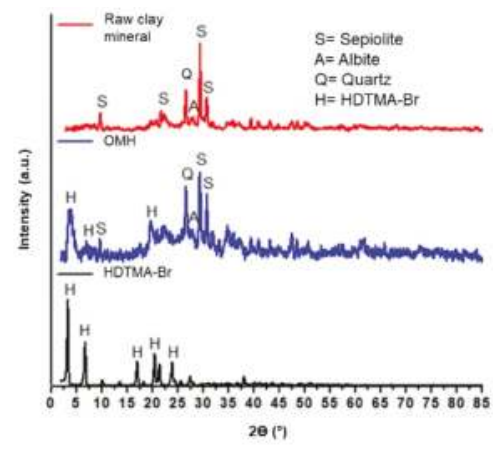

(a)

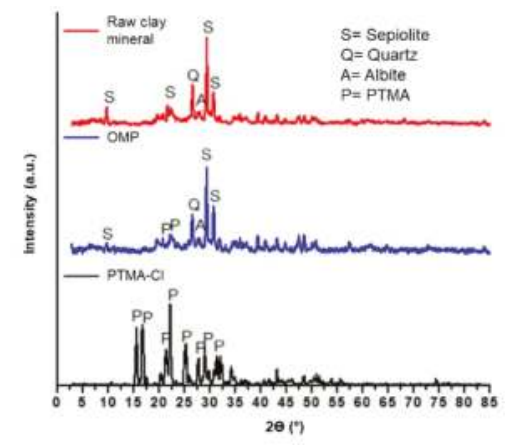

(b)

Figure 2. X-ray diffraction (XRD) patterns of the (a) raw clay mineral, the organo-modified with hexadecyltrimethylammonium bromide $(\mathrm{OMH})$, and the hexadecyltrimethylammonium bromide (HDTMA-Br) cationic surfactant. (b) The raw clay mineral, the organo-modified with phenyltrimethylammonium chloride (OMP) and the phenyltrimethylammonium chloride (PTMA-Cl) cationic surfactant.

On the other side, the XRD patterns of the raw clay mineral, the OMP, and the PTMA-Cl cationic surfactant are shown in Figure 2b; however, a different behavior was observed. In this case, peaks did not present any shift and maintained the same pattern. Therefore, it is suggested that the exchange process of the PTMA cations within the clay structure was achieved mainly with the external cations present in the surface of the raw clay mineral [21].

\subsection{Organo-Modified Clay Nanocomposite (CC) Characterization}

\subsubsection{Swelling Behavior}

Firstly, we studied the swelling properties of the synthesized materials. Figure 3a shows the swelling percentage of a synthesized $\mathrm{p}(4 \mathrm{VP}-\mathrm{co}-\mathrm{AAm})$ copolymer, sample CC04, and the homopolymers $\mathrm{p}(4 \mathrm{VP})$ and $\mathrm{p}(\mathrm{AAm})$, which correspond to samples CC01 and CC07, respectively. Sample CC07 reached its equilibrium after $8 \mathrm{~h}$ with a maximum swelling percentage of $400 \%$. However, it showed poor mechanical properties when it was swollen, since its structure collapsed. Meanwhile, the sample CC01 reached its maximum swelling capacity at $12 \mathrm{~h}$ with a maximum percentage of $140 \%$. Finally, the copolymer CC04 had its maximum swelling capacity at $12 \mathrm{~h}$ with a percentage of $190 \%$; this decrease in the swelling capacity, compared to the homopolymer CC07, was mainly due to the presence of the $4 \mathrm{VP}$ moiety in the copolymer matrix, which is more hydrophobic than the AAm moiety.

Figure $3 b$ shows the equilibrium swelling of nanocomposites with $\mathrm{OMH}$, which occurred very quickly during the first $4 \mathrm{~h}$ for all the materials. In the case of nanocomposite $\mathrm{CCH} 07$, a maximum swelling percentage of $400 \%$ was found. On the other side, the nanocomposite $\mathrm{CCH} 01$ had a maximum swelling percentage of $140 \%$. Finally, the nanocomposite CCH04 presented a maximum swelling percentage of $190 \%$. It is important to mention that the swelling capacity between polymers and their nanocomposites with $\mathrm{OMH}$ did not vary significantly; however, the time in which the equilibrium was reached changed significantly, going from an equilibrium swelling time of 12 to $4 \mathrm{~h}$.

At last, Figure $3 \mathrm{c}$ shows the swelling behavior for nanocomposites synthesized with OMP. For all synthesized materials, the equilibrium time was found after $8 \mathrm{~h}$; in the case of nanocomposite CCP07, it reached a maximum percentage of $290 \%$. Meanwhile, the nanocomposite CCP01 exhibited a maximum swelling percentage of $190 \%$, and the nanocomposite CCP04 showed a swelling capacity of $200 \%$. 


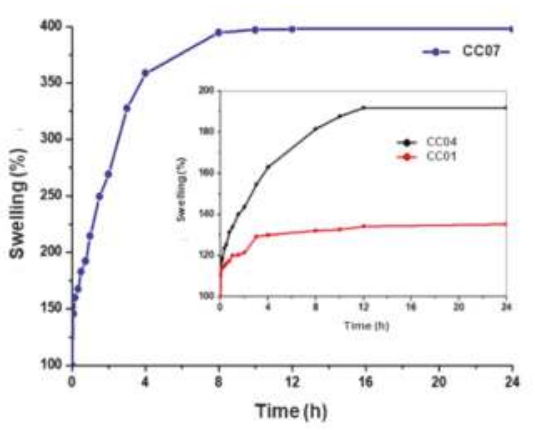

(a)

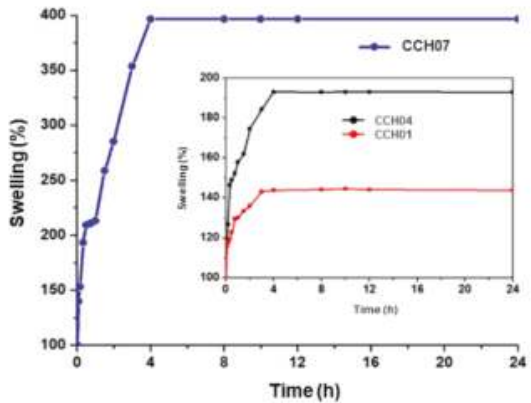

(b)

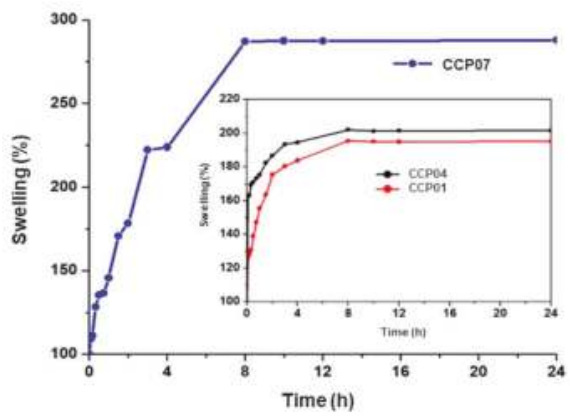

(c)

Figure 3. Equilibrium swelling time of different systems: (a) CC01, CC04, and CC07; (b) CCH01, CCH04, and $\mathrm{CCH} 07$ nanocomposites modified with HDTMA; (c) CCP01, CCP04, and CCP07 nanocomposites modified with PTMA.

Figure 4a displays the critical $\mathrm{pH}$ value of the synthesized homopolymers and copolymer. For the homopolymer $\mathrm{CC} 07$, it was confirmed that the swelling capacity did not depend on the $\mathrm{pH}$ of the aqueous solution; therefore, it is a polymer that does not respond to the $\mathrm{pH}$ stimulus. In contrast, the homopolymer CC01 showed variable swelling capacities due to the basic nature of the pyridine nitrogen ring [34]. The critical $\mathrm{pH}$ value determined was found to be 4.9 , which was very similar to that reported by Clara-Rahola et al. [35]. This homopolymer presented a significant swelling capacity, because, at these values, the pyridine groups of $\mathrm{p}(4 \mathrm{VP})$ are protonated; thus, the repulsive electrostatic contribution induces the swelling of the polymer structure forming hydrogen bonds with the water molecules in the medium. Finally, the copolymer CC04 showed a very similar behavior to that of homopolymer $\mathrm{CC} 01$, with a critical $\mathrm{pH}$ value of 5.0; thus, it is understood that the behavior of this copolymer was significantly influenced by the $\mathrm{p}(4 \mathrm{VP})$ moiety in its structure.

Figure $4 \mathrm{~b}$ illustrates the critical $\mathrm{pH}$ value of nanocomposites synthesized with the organo-modified clay with HDTMA. From this figure, it can be seen that, for nanocomposite $\mathrm{CCH} 01$, the critical $\mathrm{pH}$ value did not show any significant difference compared to the homopolymer $\mathrm{CC} 01$ and it was practically the same, i.e., 4.9. Similarly, it was observed that the nanocomposite $\mathrm{CCH} 04$ did not show any significant difference with the copolymer CC04, with a critical $\mathrm{pH}$ value of 5 .

Finally, Figure $4 \mathrm{c}$ represents the critical $\mathrm{pH}$ value of nanocomposites synthesized with the organo-modified clay with PTMA. From this figure, it can be observed that the critical $\mathrm{pH}$ values for nanocomposites with this organo-modified clay had a similar behavior to the synthesized nanocomposites with HDTMA, i.e., $\mathrm{pH}=4.9$. 


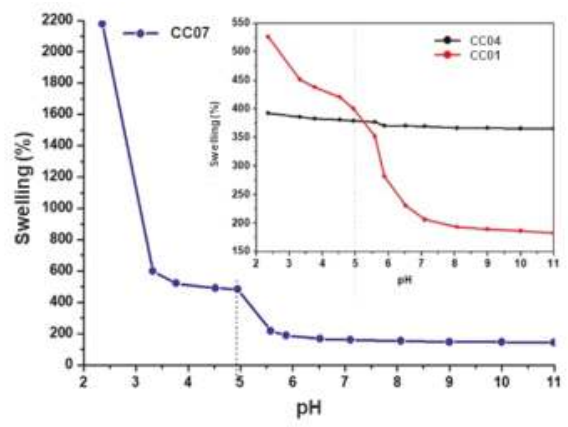

(a)

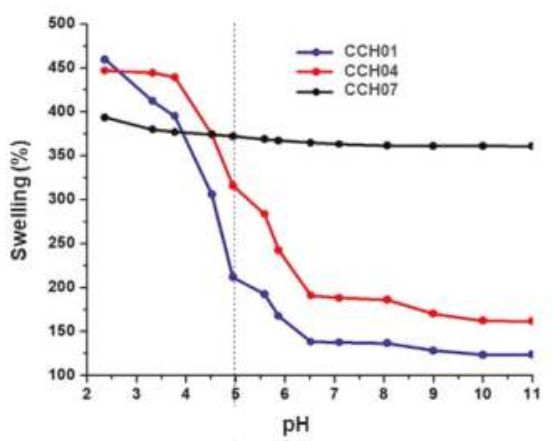

(b)

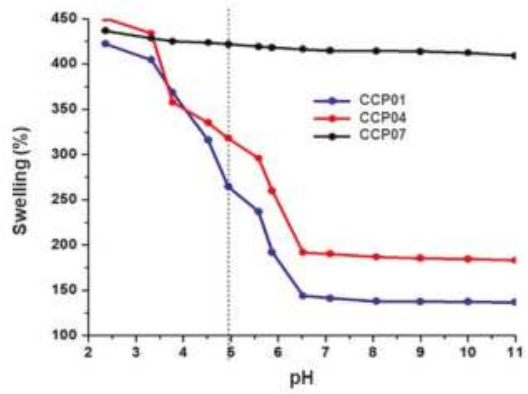

(c)

Figure 4. Critical pH value of different systems: (a) CC01, CC04, and CC07 copolymers; (b) $\mathrm{CCH} 01, \mathrm{CCH} 04$, and CCH07 nanocomposites modified with HDTMA; (c) CCP01, CCP04, and CCP07 nanocomposites modified with PTMA.

Figure 5a,b show the reversibility of the extended and collapsed states for the synthesized $\mathrm{p}(4 \mathrm{VP}-\mathrm{co}-\mathrm{AAm})$ nanocomposites with the organo-modified clays HDTMA and PTMA, respectively. Measurements were performed below and above the critical $\mathrm{pH}$ value, that is, at $\mathrm{pH}=3.3$ and $\mathrm{pH}$ $=9$, after swelling for 4 or $8 \mathrm{~h}$, depending on the nanocomposite sample. In the case of Figure 5a, nanocomposites $\mathrm{CCH} 01$ and $\mathrm{CCH} 04$ showed very good reversibility response with pH sensitivity, $\mathrm{S}_{\mathrm{w} 9} / \mathrm{S}_{\mathrm{w} 3.3}=2.56$. In the case of nanocomposite $\mathrm{CCH} 07$, since AAm did not have a response to $\mathrm{pH}$, it did not demonstrate any response to this external stimulus. Figure $5 b$ corresponds to nanocomposites CCP01, CCP04, and CCP07. Similarly, these materials displayed a $\mathrm{pH}$ sensitivity value, $\mathrm{S}_{\mathrm{w} 9} / \mathrm{S}_{\mathrm{w} 3.3}=2.54$, which is in concordance with that obtained for nanocomposites modified with HDTMA. 


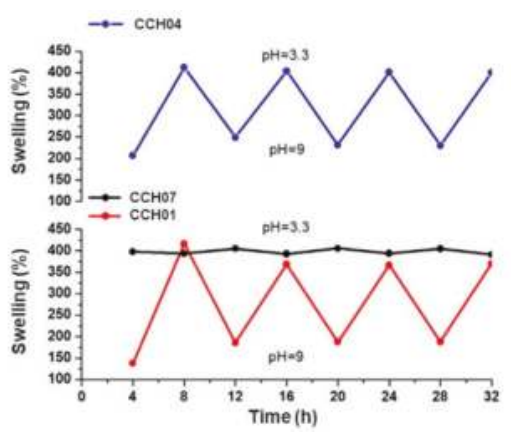

(a)

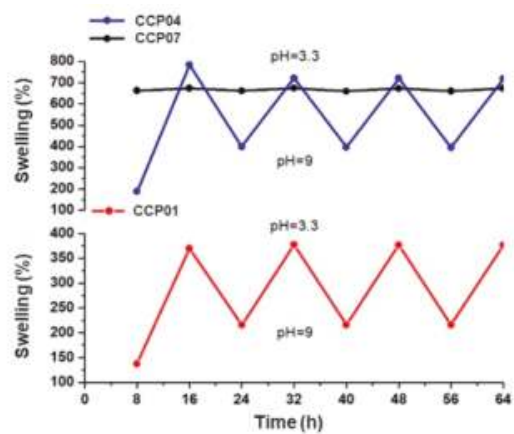

(b)

Figure 5. pH reversibility for different systems: (a) $\mathrm{CCH} 01, \mathrm{CCH} 04$, and $\mathrm{CCH} 07$ nanocomposites modified with HDTMA; (b) CCP01, CCP04, and CCP07 nanocomposites modified with PTMA.

\subsubsection{FTIR}

Figure 6a shows FTIR spectra corresponding to raw clay mineral, OMH, and HDTMA cationic surfactant. In this case, three important signals were observed that confirmed the $\mathrm{OMH}$ organo-modification with HDTMA; namely, the first signals between 2918 and $2843 \mathrm{~cm}^{-1}$ were due to the stretching vibrations of the $-\mathrm{CH}_{2}$ groups present in the hydrocarbon chain, and the third signal at $1470 \mathrm{~cm}^{-1}$ corresponded to the bending vibration of this same bond. In addition, the increase in the intensity of all these signals was related to the amount of ammonium salt used to obtain this organo-modified clay. In the case of Figure $6 b$, the most important signal representative of the organo-modification with PTMA was found at $1502 \mathrm{~cm}^{-1}$, which could be attributed to the bending vibrations of the $\mathrm{C}-\mathrm{N}$ bond of the quaternary ammonium salt.

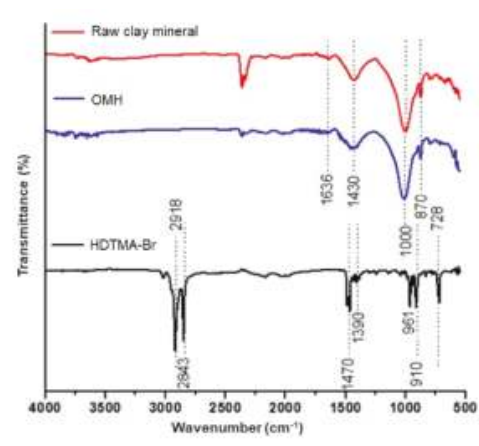

(a)

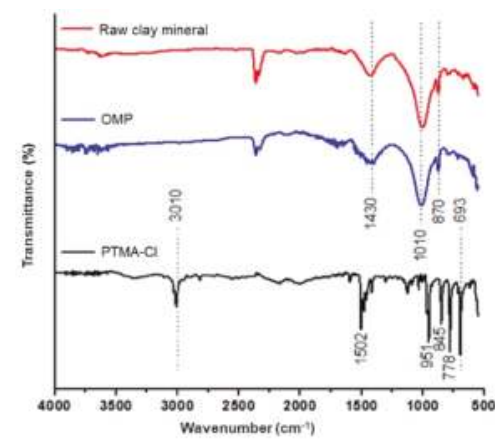

(b)

Figure 6. Fourier-transform infrared (FTIR) spectra of (a) raw clay mineral, OMH, and HDTMA-Br cationic surfactant; (b) raw clay mineral, OMP, and PTMA-Cl cationic surfactant.

Figure 7 shows the FTIR spectra of samples CC01, CC04, and CC07. In the case of homopolymer CC01, the absorption bands located between 1600 and $1419 \mathrm{~cm}^{-1}$ corresponded to the stretching vibrations of the pyridine ring, while the band at $821 \mathrm{~cm}^{-1}$ was associated with the symmetric vibrations of the monosubstituted ring of pyridine. Moreover, bands at 1492 and $1451 \mathrm{~cm}^{-1}$ were characteristic of the $\mathrm{C}-\mathrm{C}$ stretching vibrations of the benzyl ring and the stretching vibrations of the $\mathrm{C}-\mathrm{H}$ bond of this same ring, respectively. Finally, other bands in this spectrum were associated with the stretching vibration of the aliphatic $\mathrm{CH}_{2}$, at $2914 \mathrm{~cm}^{-1}$, and aromatic $\mathrm{CH}$, at $3026 \mathrm{~cm}^{-1}$, and the medium intensity bands, located in the range between 1250 and $1000 \mathrm{~cm}^{-1}$, with $\mathrm{C}=\mathrm{N}$ bonds. 


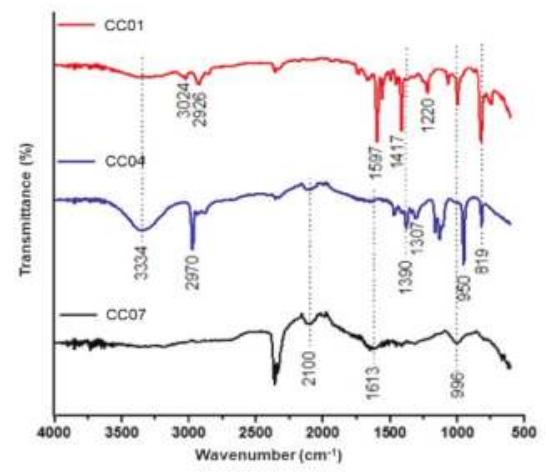

Figure 7. FTIR spectra of CC01, CC04, and CC07 copolymer matrices.

Meanwhile, in the spectrum corresponding to the copolymer CC04, the characteristic bands of $\mathrm{p}(4 \mathrm{VP})$ could be observed at $2914 \mathrm{~cm}^{-1}$, associated with the stretching vibration of the aliphatic $\mathrm{CH}_{2}$ bond, and at $821 \mathrm{~cm}^{-1}$, corresponding to the symmetric vibrations of the monosubstituted pyridine ring. In the same way, the bands corresponding to acrylamide were found at $2100 \mathrm{~cm}^{-1}$ which corresponds to the $\mathrm{N}-\mathrm{H}$ combination of stretching and torsional vibrations, and at $1613 \mathrm{~cm}^{-1}$, which corresponds to the stretching of the $\mathrm{C}=\mathrm{O}$ bond of the acrylamide.

Lastly, in the case of the homopolymer $\mathrm{CC} 07$, two characteristic bands corresponding to the $\mathrm{N}-\mathrm{H}$ bond were observed; the first one was located at $3359 \mathrm{~cm}^{-1}$, which was due to its asymmetric stretching vibrations, and the second one was at $3203 \mathrm{~cm}^{-1}$, due to its symmetric stretching vibrations. In addition, between 2914 and $2800 \mathrm{~cm}^{-1}$, vibrations of the $\mathrm{CH}_{2}$ bonds of the aliphatic chain were observed. Finally, the absorption band at $1684 \mathrm{~cm}^{-1}$ indicated the stretching vibrations of the carbonyl group, $\mathrm{C}=\mathrm{O}$, corresponding to the primary amides of acrylamide.

\subsubsection{SEM}

Figure 8a-c show micrographs of the raw clay mineral (sepiolite), $\mathrm{OMH}$, and OMP organo-modified clays, respectively. In Figure 8b, the OMH surface showed agglomerates of some particles, which were not identified on the surface of sepiolite, depicted in Figure 8a. The same behavior was observed in Figure $8 c$, which corresponds to the OMP organo-modified clay, where a more homogeneous surface could be observed, with respect to that of sepiolite. These changes could be attributed to the presence of the cationic surfactants that were not present in the structure of the sepiolite, which is in concordance with a previous report concerning the organo-modification of different types of clays [36,37].

In the case of Figure 9a-c, SEM micrographs of copolymer CC04, and nanocomposites CCH04 and CCP04, respectively, are shown. As it can be seen, copolymer CC04 had a more regular surface; in comparison, nanocomposites $\mathrm{CCH} 04$ or CCP04 exhibited a more heterogeneous surface with some little aggregates randomly distributed onto their surface. This change could be attributed to the incorporation of the $\mathrm{OMH}$ or $\mathrm{OMP}$ into the structure of the material that was not present in the sample CC04. 


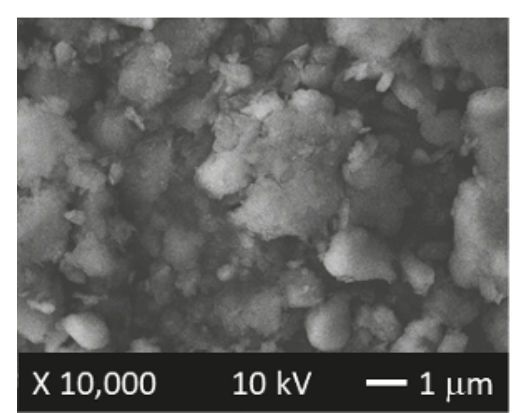

(a)

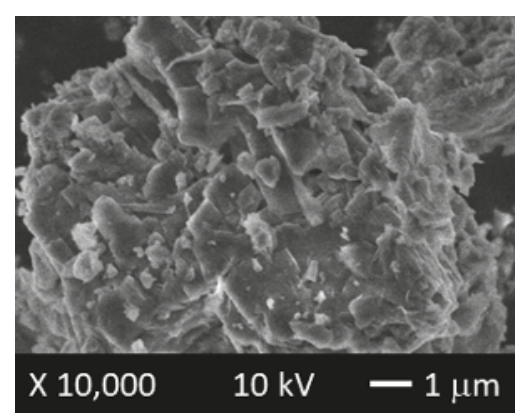

(b)

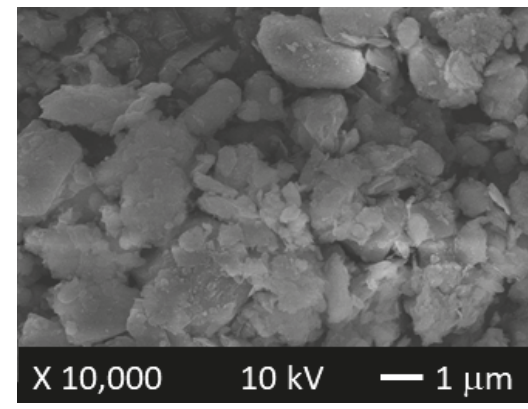

(c)

Figure 8. SEM micrographs of (a) raw clay mineral (sepiolite), and (b) $\mathrm{OMH}$ - and (c) OMP-modified clays.

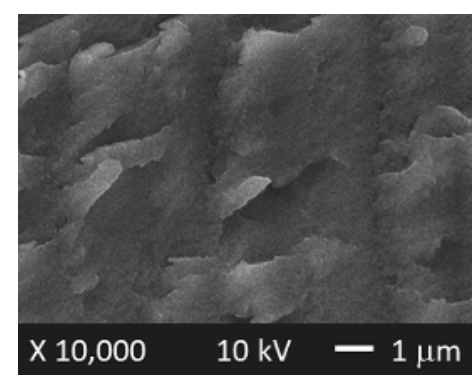

(a)

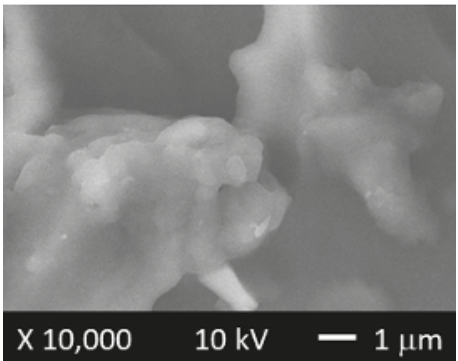

(b)

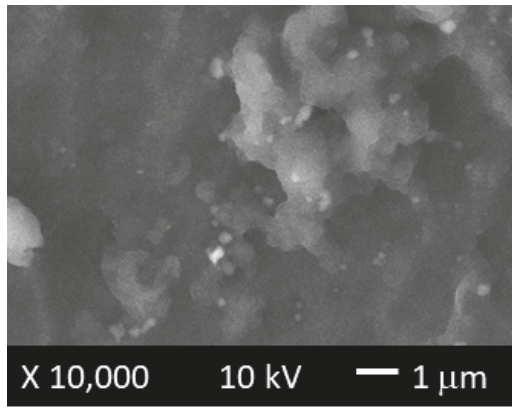

(c)

Figure 9. SEM micrographs of (a) CC04 copolymer, and (b) CCH04 and (c) CCP04 nanocomposites. 


\subsubsection{Thermal Analysis}

TGA and DSC analyses (not shown) of the raw clay mineral (sepiolite), the organo-modified clays (OMH and $\mathrm{OMP}$ ), and $\mathrm{CC} 4, \mathrm{CCH} 4$, and $\mathrm{CCP} 04$ polymer and nanocomposite samples were performed and are summarized in Table 3.

Table 3. Thermogravimetric analysis (TGA) results of the employed materials.

\begin{tabular}{ccc}
\hline Sample Code & $T_{5}\left({ }^{\circ} \mathrm{C}\right)$ & $T_{\mathrm{g}}\left({ }^{\circ} \mathrm{C}\right)$ \\
\hline Raw clay mineral & 500 & $\mathrm{ND}^{1}$ \\
OMH & 480 & $\mathrm{ND}^{1}$ \\
OMP & 520 & $\mathrm{ND}^{1}$ \\
CC04 & 150 & 165 \\
CCH04 & 140 & 167 \\
CCP04 & 140 & 168 \\
\hline \multicolumn{2}{c}{${ }^{1} \mathrm{ND}=$ not determined. }
\end{tabular}

In the case of the TGA analyses, the thermal stability of clay materials was very good, and, for all materials, the obtained $T_{5}$ values (i.e., the temperature at which the sample loses $5 \%$ mass) were found between 480 to $520^{\circ} \mathrm{C}$ depending on the nature of the raw clay mineral or the employed cationic surfactant for its organo-modification. As for the copolymer or nanocomposite samples, these values were found from 140 to $150{ }^{\circ} \mathrm{C}$; however, this stability decreased when the organo-modified clay was incorporated into the copolymer matrix structure, which is in concordance with some previous reports $[18,21]$.

Meanwhile, from the DSC analyses, it was found that $T_{\mathrm{g}}$ values for copolymer and nanocomposite samples were around 165 to $168^{\circ} \mathrm{C}$, depending on the incorporated organo-modified clay into its structure. This slight change could be attributed to the plasticizer effect that clay minerals could provide to the polymer matrix, since they have an organophilic nature due to their organo-modification with the HDTMA or PTMA cationic surfactants.

\subsection{Adsorption Experiments}

\subsubsection{Adsorption Kinetics of the Synthesized Nanocomposites with the CC04 Copolymer}

Adsorption kinetics allows evaluating the potentiality of a material to be used as an adsorbent. Therefore, it is important to determine the time for which the adsorption process reaches its equilibrium, as well as the kinetics parameters, to understand the behavior of the materials in the atrazine adsorption process.

Figure 10 represents the adsorption kinetics for the $\mathrm{CC} 04$ and respective nanocomposites, i.e., $\mathrm{CCHO4}$ and CCP04. From this figure, it can be observed that all the synthesized materials presented a maximum adsorption capacity at $12 \mathrm{~h}$, with ATZ removal being slightly higher in the CCP04 nanocomposite. A similar behavior was obtained for the OMH and OMP (not shown). 


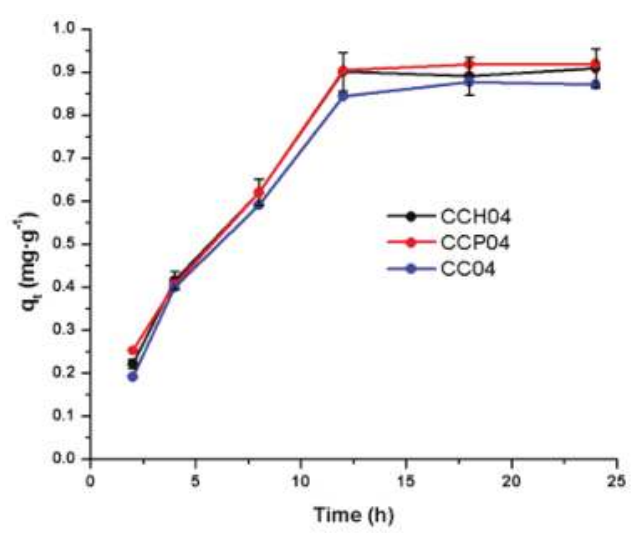

Figure 10. Adsorption kinetics for the synthesized materials: CCO4 copolymer, and CCH04 and CCP04 nanocomposites.

It is noteworthy that, when all materials reached the equilibrium time $(12 \mathrm{~h})$, they maintained a constant adsorption capacity; this indicates that the copolymer matrix contributed to keeping the ATZ molecule stable on the surface of the material during the adsorption process. On the other hand, from this same figure, it can be seen that nanocomposites CCP04 and CCH04 presented $q_{\mathrm{t}}$ values slightly higher with respect to the CC04 copolymer, since the incorporation of the organo-modified clays into the copolymer matrix conferred synergic properties to the nanocomposite materials.

Moreover, in order to know the nature of the system, the experimental data were associated with the following kinetics models: pseudo-first-order and pseudo-second-order, as depicted in Figure 11a-c. Comparing the two proposed models for the ATZ adsorption, it is observed in Table 4 that all the synthesized materials presented a better fit with the pseudo-first-order model, obtaining higher values of the correlation coefficient $\left(R^{2}\right)$ very close to 1 , and lower values of chi-square $\left(\chi^{2}\right)$, indicating that the limiting step for the ATZ adsorption process was the mass transfer of the herbicide from the solution to the surface of the adsorbent [38]. In the case of the value of the constant $\mathrm{K}$, it was observed that nanocomposites $\mathrm{CCH} 04$ and $\mathrm{CCP} 4$ presented higher values than the CC04 copolymer, which was an indication that the adsorption speed of ATZ was higher; consequently, the value of constant $\mathrm{K}$ depended on the content of the quaternary ammonium salt present in the nanocomposite.

Therefore, from these results, it can be observed that the pseudo-first-order model is the one that was best fitted to the experimental data, which is an indication that the mechanism of physisorption was predominant in the adsorption process.

Table 4. Parameters for the kinetics models of the synthesized materials.

\begin{tabular}{|c|c|c|c|c|c|c|c|}
\hline \multirow{2}{*}{ Material } & \multirow{2}{*}{$q_{\mathrm{e}}(\exp )\left(\mathrm{mg} \cdot \mathrm{g}^{-1}\right)$} & \multicolumn{3}{|c|}{ Pseudo-First-Order Model } & \multicolumn{3}{|c|}{ Pseudo-Second-Order Model } \\
\hline & & $K_{\mathrm{L}}\left(\mathrm{h}^{-1}\right)$ & $q_{\mathrm{e}}\left(\mathrm{mg} \cdot \mathrm{g}^{-1}\right)$ & $R^{2}$ & $K_{2}\left(\mathrm{~g} \cdot \mathrm{mg}^{-1} \cdot \mathrm{h}^{-1}\right)$ & $q_{\mathrm{e}}\left(\mathrm{mg} \cdot \mathrm{g}^{-1}\right)$ & $R^{2}$ \\
\hline CC04 & 0.845 & 0.137 & 0.945 & 0.986 & 0.095 & 1.258 & 0.978 \\
\hline $\mathrm{CCH} 04$ & 0.900 & 144 & 0.972 & 0.983 & 0.100 & 1.278 & 0.974 \\
\hline ССР04 & 0.903 & 143 & 0.988 & 0.984 & 0.098 & 1.298 & 0.976 \\
\hline
\end{tabular}




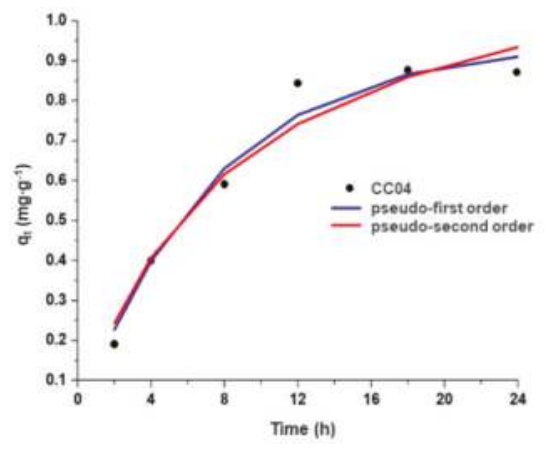

(a)

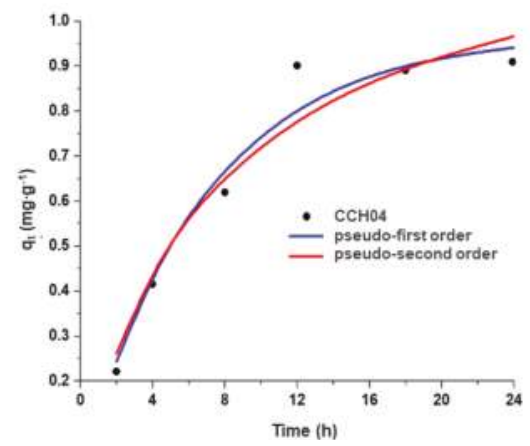

(b)

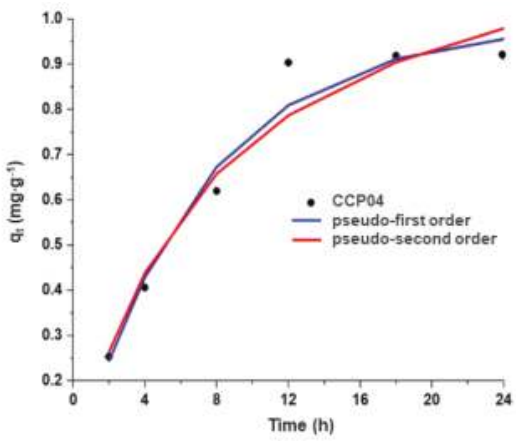

(c)

Figure 11. Kinetics models of the different synthesized materials: (a) CC04 copolymer, and (b) CCH04 and (c) CCP04 nanocomposites.

\subsubsection{Effect of the $4 \mathrm{VP}$ and AAm Molar Fractions in the Obtained Nanocomposites}

Figure 12 presents the contact tests that were carried out for the polymeric matrix, and the nanocomposites with HDTMA or PTMA in all the stoichiometric ratios considered for the synthesis. Briefly, $0.0367 \mathrm{~g}$ of each material was put in contact with $10 \mathrm{~mL}$ of a commercial ATZ solution with an initial concentration of $5 \mathrm{mg} \cdot \mathrm{L}^{-1}$; all tests were carried out at $25^{\circ} \mathrm{C}, 100 \mathrm{rpm}$, with an equilibrium time of $12 \mathrm{~h}$. After this period of time, samples were decanted to separate the aqueous phase from the adsorbent material. From this figure, it can be observed that from the synthesized materials, the p(4VP) homopolymer, and its nanocomposites, with HDTMA and PTMA organo-modified clays, showed the highest adsorption capacities, with $q_{\mathrm{t}}$ values of $1.192 \mathrm{mg} \cdot \mathrm{g}^{-1}$ for the homopolymer, and between 1.286 and $1.293 \mathrm{mg} \cdot \mathrm{g}^{-1}$ for the nanocomposites with HDTMA of PTMA, respectively. 


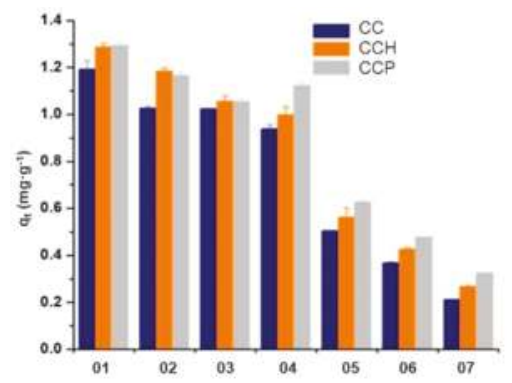

Figure 12. Removal capacities of the synthesized copolymers, with different of 4-vinylpyridine (4VP)/ acrylamide (AAm) co-monomer ratios in their structures.

Moreover, it was also observed that, when the ratio of acrylamide in the copolymer and nanocomposite structures increased, the adsorption capacity of ATZ with these materials decreased. Nevertheless, in the case of copolymers or nanocomposites with 85:15, 65:35, and 50:50 ratios between $4 \mathrm{VP}$ and AAm, the ATZ removal capacity was very similar, i.e., around $15 \%$, which is in agreement with the data previously reported by Gardi et al. [5]. Finally, nanocomposites with PTMA organo-modified clay into their structure were more efficient than HDTMA nanocomposites, which can probably be attributed to the cationic surfactant structure that has an aromatic ring in its chemical structure. Table 5 shows the percentages of the synthesized copolymers, with different molar fractions of $4 \mathrm{VP}$ and AAm in their structures.

Table 5. Removal percentages and q average value for the different synthesized materials.

\begin{tabular}{|c|c|c|c|c|c|c|}
\hline \multirow{2}{*}{ Material } & \multicolumn{2}{|c|}{ Copolymer (CC) } & \multicolumn{2}{|c|}{ HDTMA Nanocomposites (CCH) } & \multicolumn{2}{|c|}{ PTMA Nanocomposites (CCP) } \\
\hline & Removal (\%) & $q_{\mathrm{t}}$ Average $\left(\mathrm{mg} \cdot \mathrm{g}^{-1}\right)$ & Removal (\%) & $q_{\mathrm{t}}$ Average $\left(\mathrm{mg} \cdot \mathrm{g}^{-1}\right)$ & Removal (\%) & $q_{\mathrm{t}}$ Average $\left(\mathrm{mg} \cdot \mathrm{g}^{-1}\right)$ \\
\hline 01 & 92 & $1.192 \pm 0.036$ & 94 & $1.286 \pm 0.015$ & 95 & $1.293 \pm 0.014$ \\
\hline \multirow{2}{*}{02} & 81 & \multirow{2}{*}{$1.026 \pm 0.007$} & 82 & \multirow{2}{*}{$1.185 \pm 0.011$} & 83 & \multirow{2}{*}{$1.165 \pm 0.015$} \\
\hline & 81 & & 82 & & 83 & \\
\hline 03 & 76 & $1.024 \pm 0.002$ & 79 & $1.056 \pm 0.023$ & 80 & $1.054 \pm 0.009$ \\
\hline \multirow{2}{*}{04} & 71 & \multirow{2}{*}{$0.938 \pm 0.019$} & 73 & \multirow{2}{*}{$0.998 \pm 0.033$} & 78 & \multirow{2}{*}{$1.122 \pm 0.015$} \\
\hline & 69 & & 73 & & 78 & \\
\hline \multirow{2}{*}{05} & 37 & \multirow{2}{*}{$0.505 \pm 0.002$} & 39 & \multirow{2}{*}{$0.562 \pm 0.038$} & 44 & \multirow{2}{*}{$0.625 \pm 0.007$} \\
\hline & 36 & & 41 & & 46 & \\
\hline 06 & 28 & $0.366 \pm 0.005$ & 31 & $0.425 \pm 0.007$ & 35 & $0.476 \pm 0.001$ \\
\hline
\end{tabular}

3.3.3. Effect of the Mass-Volume Ratio on the Capacity to Remove ATZ with the Modified and Synthesized Materials

Finally, the relationship between the mass of the adsorbent and the initial concentration of ATZ in the solution was evaluated in this work, and the results are presented in Figure 13. In the first case, an unexpected behavior, i.e., a decrement of the mass of the adsorbent material, from 0.0367 to $0.01 \mathrm{~g}$, favored the ATZ adsorption process with both copolymers and nanocomposites, with an increase in the adsorption capacity of $400 \%$. As for the effect of the initial concentration of ATZ on its adsorption process, when the concentration increased from 5 to $20 \mathrm{mg} \cdot \mathrm{L}^{-1}$ in the aqueous solutions, it was observed that the adsorbed amount of the herbicide with materials was favored, until it reached six times its initial removal capacity. It is worth noticing that this was only observed in the polymeric materials; on the contrary, the organo-modified clays did not show this behavior, since the active sites were occupied quickly by the herbicide, causing saturation of the material. Lastly, the effect of the volume of solutions on the ATZ adsorption capacity was evaluated, and it was observed that this 
parameter was strongly associated with the initial concentration of the herbicide, since, at low ATZ concentrations $\left(5 \mathrm{mg} \cdot \mathrm{L}^{-1}\right.$ ), the adsorption capacity was $200 \%$; however, when the concentration of the analyte was increased to $25 \mathrm{mg} \cdot \mathrm{L}^{-1}$, the adsorption capacity diminished to only $50 \%$. Therefore, the effect of the volume on the adsorption capacity was lesser compared to the effects of variations of the adsorbent mass and initial ATZ concentration.

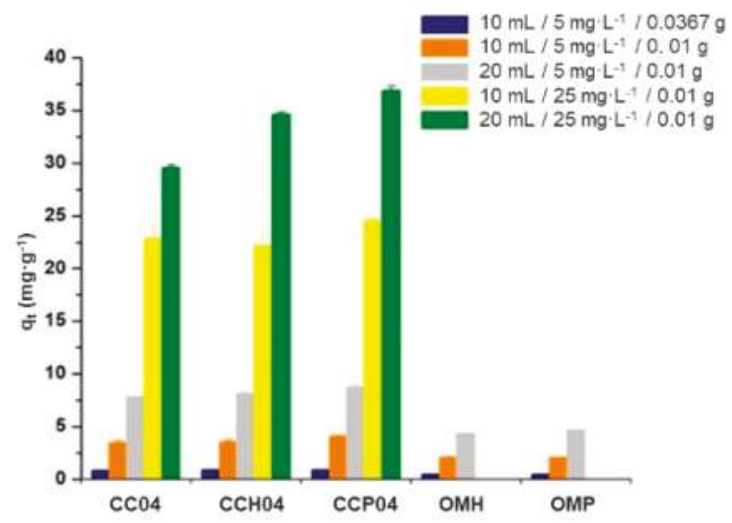

Figure 13. Adsorption experiments varying the mass-volume ratio and the initial concentration of atrazine in the prepared solutions.

\section{Discussion}

From these results, it was demonstrated that $\mathrm{p}(4 \mathrm{VP}-\mathrm{co}-\mathrm{AAm})$ copolymers and their nanocomposites with an organo-modified clay were obtained through the radical polymerization technique. Firstly, the nature of the hydrophilic raw clay mineral was effectively changed to a hydrophobic one, with the incorporation of two different types of organo cationic surfactants, HDTMA of PTMA. From the obtained XRD patterns, it is important to remark that the expansion of the interlaminar spaces could be an indication of the presence of the surfactant molecules in the organo-modified clay and this became evident because of the shift of the peaks toward lower angles in $2 \theta$, as well as the appearance of new ones.

When the percentage of hydrophilic monomer (AAm) in the synthesis of the copolymers was increased, the maximum swelling percentage at the equilibrium was augmented, and the absorption time of water decreased by action of the ionic forces between the functional groups of the copolymer chain and the surrounding medium. This decrement in the equilibrium time was attributed to the incorporation of the organo-modified clay within the polymer matrices, and the swelling capacity was similar between nanocomposites and these matrices, due to the hydrophobic nature of these organo-modified clays. Furthermore, it was observed that, at $\mathrm{pH}$ values below the critical $\mathrm{pH}$ value, the swelling capacity decreased in comparison with the polymer matrices; however, it was also lower compared to the nanocomposite with the HDTMA-modified clay, because the organo-modification of the clay with the PTMA surfactant and the type of structure that it presented prevented the penetration of water inside the matrices; therefore, a decrement in the swelling capacity was observed. Furthermore, all of the synthesized materials confirmed an excellent response to cyclical changes in $\mathrm{pH}$, demonstrated by their stable sensitivity to $\mathrm{pH}$ value.

In the case of FTIR spectra, the successful incorporation of the organo-modified clays into the polymer matrices was confirmed by the appearance of bands related to silicates and the alkyl chains of both cationic surfactants. In addition, according to the TGA, the amount of absorbed water decreased when the organo-modified clays with HDTMA or PTMA were incorporated into the matrix structures. Moreover, the thermal stability of nanocomposites was higher than that of the polymer matrices due to the incorporation of these organo-modified clays in their structures. Specifically, the value of $T_{\mathrm{g}}$ for the 
$\mathrm{p}(4 \mathrm{VP})$ differed from that reported in the literature probably due to the amount of cross-linking agent used in the synthesis of the polymers, which gave them a higher plasticizer effect.

The kinetics results indicated that nanocomposites reached their maximum adsorption capacity from 12 up to $24 \mathrm{~h}$. This indicates that the copolymer matrix contribution was the stability of the ATZ molecule on the surface of the material during the adsorption process. The kinetics model that best fitted the experimental data was the pseudo-first-order model, which pointed out that the mechanism of physisorption was predominant during the adsorption process. Finally, the relationship between mass and volume with the initial concentration of ATZ played an important part in the removal capacity of the obtained materials, and it is an important consideration for future applications.

\section{Conclusions}

In this work, two different series of copolymers and their nanocomposites, obtained from 4VP and AAm with the incorporation of two diverse organo-modified clays, were successfully synthesized. It was confirmed that the swelling capacity of the synthesized materials was influenced by the percentage of the hydrophilic monomer (AAm) into their structure; i.e., the higher the hydrophobic monomer $(4-\mathrm{VP})$ moiety, the lower the swelling capacity of the materials. The critical $\mathrm{pH}$ value $(\mathrm{pH}=4.9)$ did not vary between the polymer matrix and the synthesized nanocomposites because of the amount of the organo-modified clay that was dispersed within the polymer matrix. The $\mathrm{pH}$ sensitivity test showed that the physical properties of the copolymers were not affected by the incorporation of the organo-modified clay within its structure, since this dispersion was very low. As it was previously confirmed, the thermal stability of the obtained nanocomposites was greater than that of the copolymers due to the incorporation of the organo-modified clay into their structure. Lastly, from the adsorption kinetics experiments, it was demonstrated that the modification of the raw clay mineral with the HDTMA-Br and PTMA-Cl surfactants improved its performance for the ATZ removal; and the kinetics model of pseudo first order was the one that best described the ATZ sorption from aqueous solutions.

Author Contributions: Conceptualization, J.I. and M.d.C.D.-N.; data curation, J.I., M.d.C.D.-N., C.M.-U., S.M.-G., and E.R.; investigation, M.d.C.D.-N., C.M.-U., S.M.-G., and E.R.; methodology, J.A.R.-G., J.I., and M.d.C.D.-N.; writing-original draft, J.A.R.-G. and J.I.; writing—review and editing, J.I.

Funding: This research was funded by the Consejo Nacional de Ciencia y Tecnología (CONACyT) through the project "Cátedras CONACyT 3056" and by Tecnológico Nacional de México (TecNM) through the project 6421.19-P.

Acknowledgments: We thank Omar Novelo and Josué Romero from the Instituto de Investigaciones en Materiales UNAM for their assistance and kind discussions of SEM micrographs; Adriana Tejeda and Eriseth Reyes, from Instituto de Investigaciones en Materiales UNAM, for their assistance with XRD determination, and thermal analysis (TGA and DSC), respectively; and Enrique Aguirre and Martha Manjarrez from the Instituto Tecnológico de Toluca for their helpfulness with FTIR measurements. Jorge A. Ramírez-Gómez is grateful to CONACyT for the scholarship.

Conflicts of Interest: The authors declare no conflicts of interest. The funders had no role in the design of the study; in the collection, analyses, or interpretation of data; in the writing of the manuscript, or in the decision to publish the results.

\section{References}

1. Rinsky, J.L.; Hopenhayn, C.; Golla, V.; Browning, S.; Bush, H.M. Atrazine exposure in public drinking water and preterm birth. Public Health Rep. 2012, 127, 72-80. [CrossRef] [PubMed]

2. Golla, V.; Nelms, J.; Taylor, R.; Mishra, S. Pesticide concentrations in drinking water from farm homes: Variation between community water supply and well-water. J. Environ. Sci. Eng. 2011, 5, 955-961.

3. Nödler, K.; Licha, T.; Voutsa, D. Twenty years later-Atrazine concentrations in selected coastal waters of the Mediterranean and the Baltic Sea. Mar. Pollut. Bull. 2013, 70, 112-118. [CrossRef]

4. Graymore, M.; Stagnitti, F.; Allinson, G. Impacts of atrazine in aquatic ecosystems. Environ. Int. 2001, 26, 483-495. [CrossRef] 
5. Gardi, I.; Nir, S.; Mishael, Y. Filtration of triazine herbicides by polymer-clay sorbents: Coupling an experimental mechanistic approach with empirical modeling. Water Res. 2015, 70, 64-73. [CrossRef] [PubMed]

6. Zhu, J.; Fu, L.; Jin, C.; Meng, Z.; Yang, N. Study on the Isolation of Two Atrazine-Degrading Bacteria and the Development of a Microbial Agent. Microorganisms 2019, 7, 80. [CrossRef] [PubMed]

7. Katoon, H.; Nai, J.P.N. Augmentation of Atrazine biodegradation by two Bacilli immobilized on $\alpha$-Fe2O3 magnetic nanoparticles. Sci. Rep. UK 2018, 8, 1-12. [CrossRef] [PubMed]

8. Monard, C.; Vandekoornhuyse, P.; Le Bot, B.; Binet, F. Relationship between bacterial diversity and function under biotic control: The soil pesticide degraders as a case study. ISME J. 2011, 5, 1048-1056. [CrossRef]

9. Meakins, N.C.; Bubb, J.M.; Lester, J.N. The mobility, partitioning and degradation of atrazine and simazine in the salt marsh environment. Mar. Pollut. Bull. 1995, 30, 812-819. [CrossRef]

10. Youssef, L.; Younes, G.; Al-Oweini, R. Photocatalytic degradation of atrazine by heteropolyoxotungstates. J. Taibah Univ. Sci. 2019, 13, 274-279. [CrossRef]

11. Díaz-Nava, M.C.; Solache-Ríos, M.; Contreras-Olivares, N. Enhanced decolorization of dyes by an iron modified clay and thermodynamic parameters. Water Sci. Technol. 2016, 73, 2007-2016.

12. Hernández-Hernández, K.A.; Illescas, J.; Díaz-Nava, M.C.; Muro-Urista, C.; Martínez-Gallegos, S.; Ortega-Aguilar, R.E. Polymer-Clay Nanocomposites and Composites: Structures, Characteristics, and their Applications in the Removal of Organic Compounds of Environmental Interest. Med. Chem. 2016, 6, 201-210. [CrossRef]

13. Hernández-Hernández, K.A.; Solache-Ríos, M.; Díaz-Nava, M.C. Removal of brilliant blue FCF from aqueous solutions using an unmodified and iron modified bentonite and the thermodynamic parameters of the process. Water Air Soil Poll. 2013, 24,1-11. [CrossRef]

14. Díaz-Nava, M.C.; Olguín, M.T.; Solache-Ríos, M. Adsorption of phenol onto surfactants modified bentonite. J. Incl. Phenom. Macrocycl. 2012, 74, 67-75. [CrossRef]

15. Zhang, Q.; Zhang, T.; He, T.; Chen, L. Removal of crystal violet by clay/PNIPAm nanocomposite hydrogels with various clay contents. Appl. Clay Sci. 2014, 90,1-5. [CrossRef]

16. Zadaka, D.; Nir, S.; Radian, A.; Mishael, A.G. Atrazine removal from water by polycation-clay composites: Effect of dissolved organic matter and comparison to activated carbon. Water Res. 2009, 43, 677-683. [CrossRef]

17. Alexandre, M.; Dubois, P. Polymer-layerd silicate nanocomposites preparation, properties and uses of a new class of materials. Mater. Sci. Eng. 2000, 28, 1-63. [CrossRef]

18. Saavedra-Labastida, E.; Díaz-Nava, M.C.; Illescas, J.; Muro, C. Comparison of the removal of an anionic dye from aqueous solutions by adsorption with organically modified clays and their composites. Water Air Soil Pollut. 2019, 230, 1-26. [CrossRef]

19. Ortega-Aguirre, S.; Díaz-Nava, M.C.; Solache-Ríos, M.J.; Illescas, J. Alginate-iron modified zeolite beads biocomposite for removal of azo dye from water medium. MRS Adv. 2018, 3, 3769-3773. [CrossRef]

20. Remigio-Reyes, G.D.; Illescas, J.; Díaz-Nava, M.C. Removal of bromophenol blue dye from aqueous solutions through the synthesis and characterization of a polymer-clay composite. MRS Adv. 2018, 3, 3751-3755. [CrossRef]

21. Hernández-Hernández, K.A.; Illescas, J.; Díaz-Nava, M.C.; Martínez-Gallegos, S.; Muro-Urista, C.; Ortega-Aguilar, R.E.; Rodríguez-Alba, E.; Rivera, E. Preparation of nanocomposites for the removal of phenolic compounds from aqueous solutions. Appl. Clay Sci. 2018, 157, 212-217. [CrossRef]

22. Yanovska, E.S.; Vretik, L.O.; Nikolaeva, O.A.; Polonska, Y.; Sternik, D.; Kichkiruk, O.Y. Synthesis and Adsorption Properties of 4-Vinylpyridine and Styrene Copolymer in Situ Immobilized on Silica Surface. Nanoscale Res. Lett. 2017, 12,1-6. [CrossRef]

23. Rassu, M.; Alzari, V.; Nuvoli, D.; Nuvoli, L.; Sanna, D.; Sanna, V.; Malucelli, G.; Mariani, A. Semi-interpenetrating polymer networks of methyl cellulose and polyacrylamide prepared by frontal polymerization. J. Polym. Sci. Pol. Chem. 2017, 55, 1268-1274. [CrossRef]

24. Scognamillo, S.; Alzari, V.; Nuvoli, D.; Mariani, A. Thermoresponsive super water absorbent hydrogels prepared by frontal polymerization. J. Polym. Sci. Pol. Chem. 2010, 48, 2486-2490. [CrossRef]

25. Martino, L.; Guigo, N.; Van Berkel, J.G.; Sbirrazzuoli, N. Influence of organically modified montmorillonite and sepiolite clays on the physical properties of bio-based poly(ethylene 2,5-furandicarboxylate). Compos. Part B-Eng. 2017, 110, 96-105. [CrossRef] 
26. Chen, H.; Lu, H.; Zhou, Y.; Zheng, M.; Ke, C.; Zeng, D. Study on thermal properties of polyurethane nanocomposites based on organo-sepiolite. Polym. Degrad. Stabil. 2012, 97, 242-247. [CrossRef]

27. Lowe, D.J.; Chapman, A.V.; Cook, S.; Busfield, J.J.C. Natural Rubber Nanocomposites by In Situ Modification of Clay. Macromol. Mater. Eng. 2011, 296, 693-702. [CrossRef]

28. Mariani, A.; Nuvoli, D.; Alzari, V.; Pini, M. Phosphonium-Based ionic liquids as a new class of radical initiators and their use in gas-free frontal polymerization. Macromolecules 2008, 41, 5191-5196. [CrossRef]

29. ANSI/API 13B-1. Recommended Practice for Field Testing-Water-Based Drilling Fluids, 5th ed.; API Publishing Services: Washington, DC, USA, 2017.

30. ASTM C837-09. Standard Test Method for Methylene Blue Index of Clay; ASTM International: West Conshohocken, PA, USA, 2014.

31. Zhuang, G.; Gao, J.; Chen, H.; Zhang, Z. A new one-step method for physical purification and organic modification of sepiolite. Appl. Clay Sci. 2018, 153, 1-8. [CrossRef]

32. Moreira, M.A.; Ciuffi, K.J.; Rives, V.; Vicente, M.A.; Trujillano, R.; Gil, A.; Korili, S.A.; de Faria, E.H. Effect of chemical modification of palygorskite and sepiolite by 3-aminopropyltriethoxisilane on adsorption of cationic and anionic dyes. Appl. Clay Sci. 2017, 135, 394-404. [CrossRef]

33. Lemić, J.; Tomašević-Čanović, M.; Djuričić, M.; Stanić, T. Surface modification of sepiolite with quaternary amines. J. Colloid Interface Sci. 2005, 292, 11-19. [CrossRef]

34. Ortega, A.; Alarcón, D.; Muñoz-Muñoz, F.; Garzón-Fontecha, A.; Burillo, G. Radiation grafting of pH-sensitive acrylic acid and 4-vinylpyridine onto nylon-6 using one- and two-step methods. Radiat. Phys. Chem. 2015, 109, 6-12. [CrossRef]

35. Clara-Rahola, J.; Moscoso, A.; Ruiz-Muelle, A.B.; Laurenti, M.; Formanek, P.; Lopez-Romero, J.M.; Fernández, I.; Diaz, J.F.; Jorge Rubio-Retama, J.; Fery, A.; et al. Au@p4VP core@shell pH-sensitive nanocomposites suitable for drug entrapment. J. Colloid Interface Sci. 2018, 514, 704-714. [CrossRef] [PubMed]

36. Lazo, J.C.; Navarro, A.E.; Sun-Kou, M.R.; Llanos, B.P. Síntesis y caracterización de arcillas organofílicas y su aplicación como adsorbentes del fenol. Rev. Soc. Quím. Perú 2008, 74, 3-19.

37. Kleinübing, S.J.; Gaia, F.; Bertagnolli, C.; Carlos da Silva, M.G. Extraction of alginate biopolymer present in marine alga sargassum filipendula and bioadsorption of metallic ions. Mater. Res. 2013, 16, 481-488. [CrossRef]

38. Ho, Y.S.; McKay, G. Pseudo-second order model for sorption processes. Process Biochem. 1999, 34, 451-465. [CrossRef]

(C) 2019 by the authors. Licensee MDPI, Basel, Switzerland. This article is an open access article distributed under the terms and conditions of the Creative Commons Attribution (CC BY) license (http://creativecommons.org/licenses/by/4.0/). 
Article

\title{
Synthesis and Characterization of Dental Nanocomposite Resins Filled with Different Clay Nanoparticles
}

\author{
Alexandros K. Nikolaidis ${ }^{1, *}$, Elisabeth A. Koulaouzidou ${ }^{1}$, Christos Gogos $^{1}$ and \\ Dimitris S. Achilias ${ }^{2}$ \\ 1 Division of Dental Tissues' Pathology and Therapeutics (Basic Dental Sciences, Endodontology and \\ Operative Dentistry), School of Dentistry, Aristotle University Thessaloniki, 54124 Thessaloniki, Greece; \\ koulaouz@dent.auth.gr (E.A.K.); gogos@dent.auth.gr (C.G.) \\ 2 Laboratory of Polymer and Color Chemistry and Technology, Department of Chemistry, Aristotle University \\ Thessaloniki, 54124 Thessaloniki, Greece; axilias@chem.auth.gr \\ * Correspondence: nikolchem@dent.auth.gr; Tel.: +30-2310-999-616
}

Received: 13 March 2019; Accepted: 19 April 2019; Published: 22 April 2019

\begin{abstract}
Nanotechnology comprises a promising approach towards the update of dental materials.The present study focuses on the reinforcement ofdental nanocomposite resins with diverse organomodified montmorillonite (OMMT) nanofillers. The aim is to investigate whether the presence of functional groups in the chemical structure of the nanoclay organic modifier may virtually influence the physicochemical and/or the mechanical attitude of the dental resin nanocomposites. The structure and morphology of the prepared materials were investigated by means of wide angle X-ray diffraction and scanning electron microscopy analysis. Fourier transform infrared spectroscopy was used to determine the variation of the degree of conversion over time. Measurements of polymerization shrinkage and mechanical properties were conducted with a linear variable displacement transducer apparatus and a dynamometer, respectively. All the obtained nanocomposites revealed intercalated structures and most of them had an extensive filler distribution into the polymer matrix. Polymerization kinetics werefound to be influenced by the variance of the clay organomodifier, whilenanoclays with vinyl groups considerably increased the degree of conversion. Polymerization shrinkage was almost limited up to $50 \%$ by incorporating nanoclays. The absence of reactive groups in the OMMT structure may retain setting contraction atlow levels. An enhancement of the flexural modulus was observed, mainly by using clay nanoparticles decorated with methacrylated groups, along with a decrease in the flexural strength at a high filler loading. The overall best performance was found for the nanocomposites with OMMTs containing double bonds. The significance of the current work relies on providing novel information about chemical interactions phenomena between nanofillers and the organic matrix towards the improvement of dental restorative materials.
\end{abstract}

Keywords: dental resins; nanocomposite materials; organically modified clays; montmorillonite; intercalation; nanotechnology

\section{Introduction}

Composite restorative materials were initially developed to overcome the drawbacks of silicate cements and unfilled resins based on methyl methacrylate monomer and its polymer [1]. Furthermore, aesthetic reasons and concerns associated with amalgam's toxicity [2] established them as modern biomaterials in the dental industry. These materials consist of the following major components: (a) an organic resin matrix, usually containing 2,2-Bis[p-(2'-hydroxy-3'- 
methacryloxypropoxy)phenylene]propane (Bis-GMA) or 1,6-bis(methacryloxy-2-ethoxycarbonylamino)-2,4,4-trimethylhexane (UDMA) and the co-monomer triethylene glycol dimethacrylate (TEGDMA) as viscosity controller, (b) an inorganic reinforcing filler, such as glass, quartz or fused silica, and (c) a coupling agent, such as $\gamma$-methacryloxy propyltrimethoxysilane, to enhance bonding between the filler and resin matrix. The latter contains an activator/initiator system to promote light-activated polymerization of the organic matrix and form cross-linked polymer networks [3-5]. In clinical practice, restorations based on dental composite resins are usually challenged by requirements, such as excellent mechanical properties, low wear, and water solubility or sorption, low polymerization shrinkage and marginal leakage, good biocompatibility, caries-inhibition ability and low toxicity, color matching, and stability, etc. [6,7].

Nanotechnology constitutes a promising approach towards the improvement of biomedical $[8,9]$ and dental applications [10,11], utilizing particles as fillers in nanometer scale with a high surface area that can markedly change resins' macroscopic properties. In recent years, polymer-clay nanocomposites have attracted the strong interest of many materials researchers, as it is possible to achieve impressive enhancements of nanocomposite properties compared to the pure polymers [12-18]. Particularly, when these properties depend on the surface area of the filler particles, only small amounts (typical less than $5 \mathrm{wt} \%$ ) of nanoclay may improve mechanical properties and thermal stability, give better resistant to solvents, and decrease gas and liquid permeability [3,19-22]. Montmorillonite (MMT) is a 2:1 layered silicate, commonly used in polymer nanocomposite formulations. Due to its hydrophilic nature, pristine MMT containing $\mathrm{Na}^{+}$or $\mathrm{Ca}^{2+}$ ions is usually modified with quaternary ammonium ions through an ion exchange reaction, and the resulting organomodifiednanoclay (OMMT) is then compatible to the polymer matrix [23-32]. In general, four types of polymer-clay nanocomposite structures are mainly produced: (1) Exfoliated nanocomposites where the individual nanoclay layers are absolutely delaminated and dispersed in the polymer matrix, while their ordered structure collapses; (2) intercalated nanocomposites formed by the insertion of polymer chains between the intact silicate layers, retaining their regular alternation of galleries and laminas; (3) intermediate nanocomposites which are partially intercalated and partially exfoliated, and (4) conventional composites where layered silicate acts as a conventional micro-sized filler due to the presence of tactoids $[3,14,27,33]$. Several studies have proven that compared to the intercalated nanocomposites, the exfoliated analogs have a higher Young's modulus, larger increase in elongation at break, and better thermal stability, and the extent of exfoliation strongly affects the improvement of the final properties [3,34-36].

Green composites for environmental purposes [37], drug delivery systems [38], and DNA acid nucleic bases adsorption studies [39] constitute some of the numerous modern MMT applications. There are many reports associated with the incorporation of OMMTs in dental composites and their efficacy in terms of the nanocomposite morphology and final properties. It has been shown that at low nanoclay regimes (Cloisite $93 \mathrm{~A}$ and $30 \mathrm{~B}$ up to $10 \mathrm{wt} \%$ ), the polymerization features, mechanical and thermal properties of the final nanocomposites mainly depend on the degree of exfoliation or intercalation of the clay layers [40]. Light-cured methacrylated/MMT nanocomposites with intercalated or exfoliated structures have also been studied by using the commercial Claytone APA. It was found that materials containing $3 \mathrm{wt} \%$ OMMT were extensively cured with increased water uptake, while the presence of the clay had no significant effect on the mechanical properties of nanocomposites [41]. Furthermore, storage modulus and thermal stability increment accompanied with slower polymerization rates and lower degrees of conversion were observed by incorporating up to $15 \mathrm{wt} \%$ OMMT with hexyltrimethylammonium bromide [42]. Intercalated nanocomposites containing 50,60, and $70 \mathrm{wt} \%$ Cloisite $10 \mathrm{~A}$ with lower polymerization shrinkage, high degree of conversion, and lower flexural strength compared to composites analogs filled with silanized silica were also reported [43]. The usage of 50, 60, and $65 \mathrm{wt} \%$ Cloisite 30B was found to induce lower polymerization shrinkage than silanized silica [44], while a similar degree of conversion and higher elastic modulus values in comparison to barium glass fillers were indicated at lower concentrations [45]. Dental nanocomposites exhibited high thermal stability when pre-irradiated 
Cloisite 20A (50 wt \%) was used [46]. Moreover, MMT was successfully functionalized with 2-(methacryloyloxy)ethyltrimethylammonium chloride and further incorporated into experimental dental composites. Physical and biological properties results showed a potential interest inthe application of such nanoclays into dental resin composites [47]. The two commercial OMMTs Viscogel B8 and Dellite 67G were better dispersed into dental resin matrix at $2.5 \mathrm{wt} \%$ improving the final mechanical properties, whereas the increase of filler concentration reduced the crosslinking ability of the system [48].

In the present study, an effort was made to synthesize and characterize dental nanocomposite resins containing OMMTs with a noticeable diversity of the organic clay modification. For this purpose, a commercially available OMMT with ammonium intercalant having hydroxy-polar groups, an OMMT with C16 alkyl chain hydrophobic ammonium intercalating agent, and two OMMTs containing quaternary ammonium functional methacrylates with C8 and C18 alkyl chains, respectively, were incorporated into dental resin formulations. Moreover, the corresponding surface modified OMMTs with silane coupling agent were also utilized. This work aims to investigate whether the presence of functional groups in the chemical structure of the nanoclay organic modifier may virtually influence the physicochemical and mechanical attitude of dental resin nanocomposites.In a few words, it can be concluded that methacrylated OMMTs are generally responsible for the overall improvement of the produced nanocomposites. The obtained findings could play a key role inthe designing of novel dental nanocomposites suitable to meet the requirements of clinical practice.

\section{Materials and Methods}

\subsection{Materials}

The monomers triethylene glycol dimethacrylate (TEGDMA), 95\%, and 2,2-Bis[p-(2'-hydroxy-3'methacryloxypropoxy)phenylene]propane (Bis-GMA) were both provided by Aldrich, Taufkirchen, Germany. Co-initiator 2-(Dimethylamino)ethylmethacrylate (DMAEMA), 99\%, and initiator camphorquinone, $98 \%$, were purchased from J\&K Scientific GmbH, (Lommel, Belgium). Commercially available OMMT, Nanomer ${ }^{\circledR}$ I.34MN, produced by Nanocor Company (Hoffman Estates, IL, USA) and supplied by Aldrich (Taufkirchen, Germany), is an -onium ion modified clay containing 25 to $30 \mathrm{wt}$ $\%$ methyl dixydroxyethyl hydrogenated tallow ammonium ion. OMMTs with different intercalating organomodifiers, such as cetyltrimethylammonium chloride (MMT-CTAC), dimethylaminooctadecyl methacrylate (MMT-DMAODM), dimethylaminohexadecyl methacrylate (MMT-DMAHDM), as well as two surface modified analogs, with 3-(trimethoxysilyl)propyl methacrylate, S.MMT-CTAC, and S.MMT-DMAHDM were all prepared in our previous works $[49,50]$. The specific chemical structures of all MMT organomofidiers are represented in Figure 1. All other chemicals used were of reagent grade.

\subsection{Sample Preparation}

Six groups of experimental composites were prepared by initially mixing a Bis-GMA/TEGDMA base (50:50 wt/wt) which containedcamphorquinone (0.2 wt \%) and DMAEMA (0.8 wt \%) as a photo-initiating system. Afterward, the different organomodified clays were mixed with the resin by manual mixing until the powder was completely wetted with organic matrix, and the obtained mixture was ultrasonicated for $10 \mathrm{~min}$. The nanofiller loading was $50 \mathrm{wt} \%$ to ensure paste handling properties almost similar to a commercial dental composite resin. Bis-GMA/TEGDMA pure matrix was also prepared to the same composition to be used as control material. 


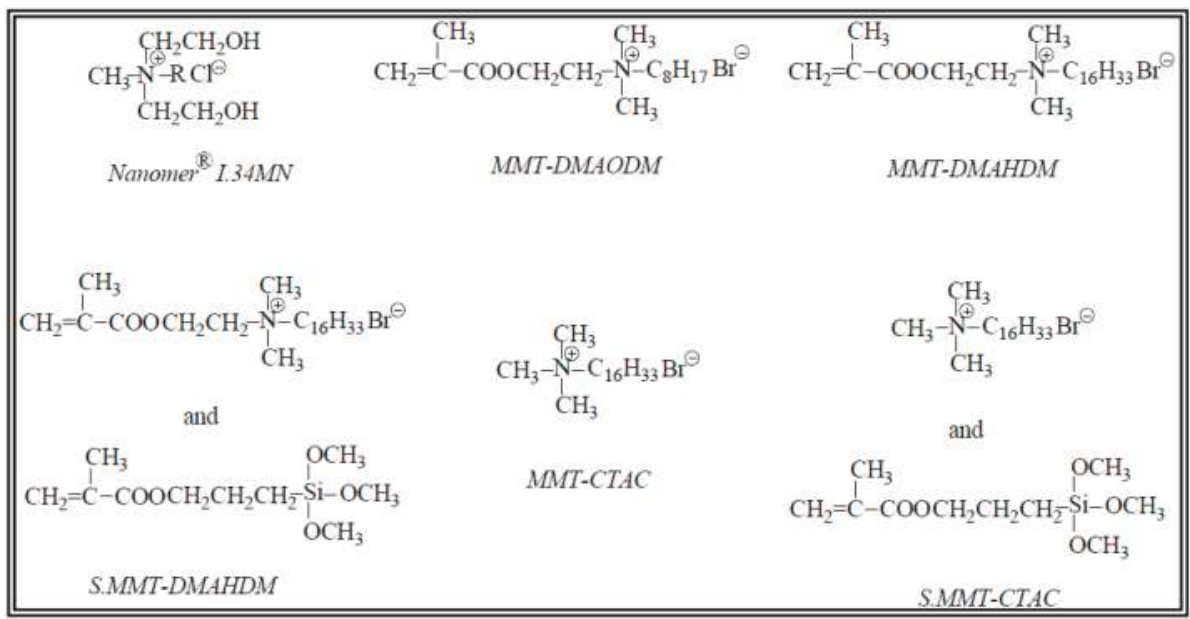

Figure 1. Different types of clay organomodifiers used for nanocomposite synthesis ( $\mathrm{R}$ stands for hydrogenated tallow).

\subsection{Measurements}

X-ray diffraction (XRD) analysis of cured materials and nanopowders (MMT-CTAC and S.MMT-CTAC) were performed over the $2 \theta$ range of $2^{\circ}$ to $10^{\circ}$, at steps of $0.05^{\circ}$, and counting time of 5 s per step, using a Miniflex II XRD system from Rigaku Co. (Tokyo, Japan) with Cu Ka radiation $(\lambda=0.154 \mathrm{~nm})$.

Scanning electron microscopy (SEM) was carried out using a JEOL JSM-6390LV (JEOL USA, Inc., Peabody, MA, USA) scanning microscope equipped with an energy-dispersive X-ray (EDX) INCA PentaFETx3 (Oxford Instruments, Abingdon, England) microanalytical system. All the studied surfaces were coated with carbon black to avoid charging under the electron beam.

Polymerization shrinkage kinetics were conducted according to the "bonded-disk" method which was initially published and further refined by Watts and co-workers [51-53]. Briefly, a disk-shaped un-set specimen with dimensions of $1.0 \mathrm{~mm} \times 8.0 \mathrm{~mm}$ was formed and centrally positioned upon a $3 \mathrm{~mm}$ thick rigid glass plate. A flexible cover-slip diaphragm, supported by an outer peripheral brass ring with internal diameter circa $15 \mathrm{~mm}$, was rested on the upper surface of the specimen disk so as to be adherent. A uniaxial LVDT (linear variable displacement transducer) measuring system was positioned centrally onto the cover slip. The signal from the LVDT was transmitted to a computer by a transducer indicator (E 309, RDP Electronics Ltd., Wolverhampton, UK), and a high-resolution analog to digital converter (ADAM-4016 acquisition module) supported by datalogger software (Advantech Adam/Apax.NET Utility, version 2.05.11). Measurements records were taken by continuous irradiation of specimens with a quartz-tungsten-halogen lamp (Astralis 3, Ivoclar-VivadentSchaan, Liechtenstein) at $650 \mathrm{~mW} \cdot \mathrm{cm}^{-2}$ for $5 \mathrm{~min}$ directly from beneath the glass plate at room temperature. A radiometer (Hilux, Benlioglu Dental Inc., Ankara, Turkey) was used to verify the output irradiance of the light-curing device. Four repetitions $(n=4)$ were made at each specimen. Strain was calculated as:

$$
\varepsilon(\%)=100 \times \frac{\Delta \mathrm{L}}{\mathrm{L}_{0}}
$$

where $\varepsilon(\%)$ represents the strain $(\%), \Delta \mathrm{L}$ and $\mathrm{L}_{0}$ are the shrinkage displacement and the initial specimen thickness, respectively.

Polymerization kinetics wereperformed by placing a small amount of each composite between two translucent Mylar strips, which were pressed to produce a very thin film. The films of unpolymerized composites were exposed to visible light as previously described, and immediately scanned by a 
Spectrum One Perkin-Elmer FTIR spectrometer (PerkinElmer Inc., Waltham, MA, USA) at different curing time intervals $(0,5,10,15,20,25,30,40,60,80,120,180 \mathrm{~s})$. Spectra were obtained over $4000-600 \mathrm{~cm}^{-1}$ region and acquired with a resolution of $4 \mathrm{~cm}^{-1}$ and a total of $32 \mathrm{scans}$ per spectrum. The area of aliphatic $C=C$ peak absorption at $1637 \mathrm{~cm}^{-1}$, and the aromatic $C=C$ peak absorption at $1580 \mathrm{~cm}^{-1}$ were determined, utilizing a base line technique which proved the best fit to the Beer-Lambert law [54]. The aromatic $\mathrm{C}=\mathrm{C}$ vibration was used as an internal standard. The percent degree of monomer conversion (DC \%) of the cured specimen, which expresses the percent amount of double carbon bond reacted at each time period, was determined according to the equation:

$$
\mathrm{DC}(\%)=\left[1-\frac{\left(\frac{\mathrm{A}_{1637}}{\mathrm{~A}_{1580}}\right)_{\text {polymer }}}{\left(\frac{\mathrm{A}_{1637}}{\mathrm{~A}_{1580}}\right)_{\text {monomer }}}\right] \times 100
$$

For flexural tests, bar-specimens were prepared by filling a Teflon mold ( $2 \mathrm{~mm} \times 2 \mathrm{~mm} \times 25 \mathrm{~mm}$ ) with unpolymerized paste in accordance withISO 4049. The mold surfaces were overlaid with glass slides covered with a Mylar sheet to avoid air entrapping and adhesion of the final set material. The assembly was held together with spring clips and irradiated by overlapping on both sides, as previously described. Each overlap irradiation lasted for $40 \mathrm{~s}$. Five specimen bars $(n=5)$ were prepared for each nanocomposite. The specimens were stored at $37 \pm 1{ }^{\circ} \mathrm{C}$ in dark conditions for $24 \mathrm{~h}$ immediately after curing. Afterward, they were bent in a three-point transverse testing rig with 20 $\mathrm{mm}$ between the two supports (3-point bending). The rig was fitted to a universal testing machine (Testometric AX, M350-10kN, Testometric Co. Ltd., Rochdale, England). All bend tests were carried out at a cross-head speed of $0.5 \mathrm{~mm} \cdot \mathrm{min}^{-1}$ until fracture occurred. The load and the corresponding deflection were recorded. The flexural modulus (E) in GPa and flexural strength $(\sigma)$ in MPa were calculated according to the following equations:

$$
\mathrm{E}=\frac{\mathrm{F}_{1} \mathrm{l}^{3}}{4 \mathrm{bd}_{1} \mathrm{~h}^{3}} 10^{-3} \text { and } \sigma=\frac{3 \mathrm{~F}_{\max } \mathrm{l}}{2 \mathrm{bh}^{2}}
$$

where: $F_{1}$ is the load recorded in $N, F_{\max }$ is the maximum load recorded before fracture in $\mathrm{N}, \mathrm{l}$ is the span between the supports $(20 \mathrm{~mm}), \mathrm{b}$ is the width of the specimen in $\mathrm{mm}, \mathrm{h}$ is the height of the specimen in $\mathrm{mm}$, and $\mathrm{d}_{1}$ is the deflection (in $\mathrm{mm}$ ) corresponding to the load $\mathrm{F}_{1}$.

\subsection{Statistical Analysis}

The values of the measured mechanical properties represent mean values \pm standard deviation of replicates. Kruskal-Wallis statistic test, followed by a Dunn's test, for multiple comparisons between means to determine significant differences $(\mathrm{p}<0.001)$, for analysis of the experimental results. This was performed separately for both flexural modulus and strength parameters.

\section{Results and Discussion}

\subsection{Structure and Morphology Characterization}

Diffractogramsfor the synthesized dental nanocomposite resins and their corresponding pure nanoclays appear in Figure 2. The presence of two distinct diffraction peaks is observed for most nanocomposites. Furthermore, the diffraction peaks of the pristine OMMTs are mainly shifted to lower $2 \theta$ angles (first peaks), denoting a possible obtained intercalated structure due to the insertion of macromolecules within clay galleries. Jlassi et al. have proven the co-existence of exfoliated with intercalated clay layers for epoxy nanocomposites with $3 \mathrm{wt} \%$ bentonite acting as intercalated chain transfer agent [55], as well asthe high degree of exfoliation for epoxy resins containing $0.5 \mathrm{wt} \%$ bentonite/4-diphenylamine diazonium/polyaniline nanocomposite filler [56]. However, our findings were also enhanced by predominantly intercalation phenomena previously reported by other researchers 
regarding dental composites filled with MMT nanoparticles at higher clay loadings around $50 \mathrm{wt}$ $\%[45,46,57]$. The secondary peaks of nanocomposites remained in the same angle regions as the initial peaks of the pure OMMTs, implying the portion of nanoclay that did not intercalated by macromolecular chains. Hence, agglomerated MMT nanofillers might be formed usually called as "tactoids". In such relatively high nanofiller loadings, similar structural characteristics were also reported by other researchers [43-45].
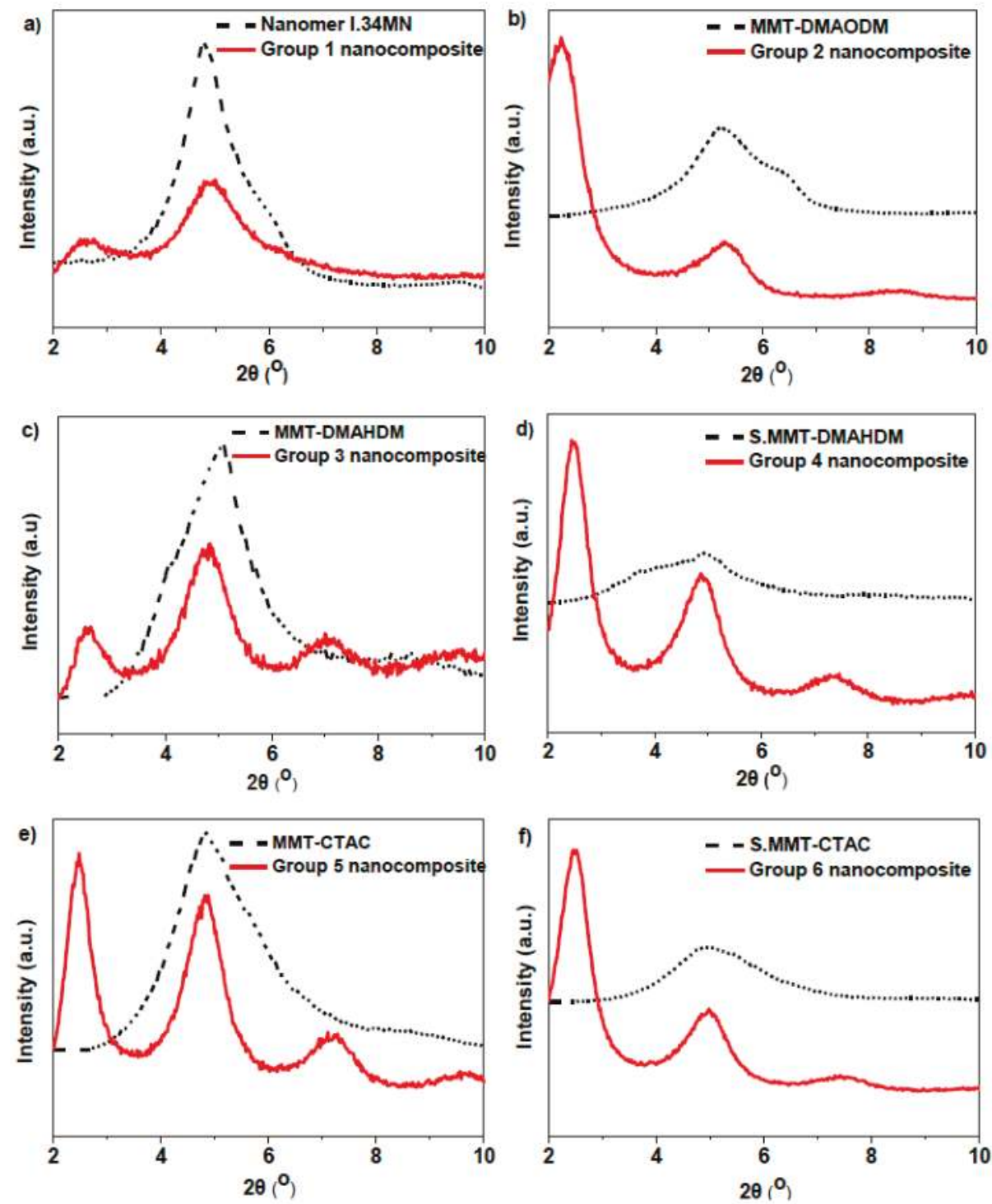

Figure 2. X-ray diffraction (XRD) patterns of the dental nanocomposite resins and the different incorporated organo modified montmorillonite (OMMT) nanoclays. 
The $d_{001}$-basal spacing values for all nanocomposite resin groups were calculated according to the Bragg's law $(\mathrm{n} \lambda=2 \mathrm{~d} \sin \theta)$ and are listed in Table 1 . The $d_{001}$-values for the majority of the used OMMTs were determined in our previous work [50] and are also available in Table 1 for comparative purpose.

Table 1. $d_{001}$-spacing values calculated on the basis of X-ray diffraction (XRD) spectra for different species of nanoclays incorporated in nanocomposite resins.

\begin{tabular}{ccccc}
\hline $\begin{array}{c}\text { Nanocomposite } \\
\text { Resin }\end{array}$ & $\begin{array}{c}\text { OMMT Nanofiller } \\
\text { (50 wt } \mathbf{~})\end{array}$ & \multicolumn{2}{c}{$\begin{array}{c}d_{\mathbf{0 0 1}} \\
(\mathbf{n m})\end{array}$} & $\begin{array}{c}\Delta d_{\mathbf{0 1}} \\
(\mathbf{n m})\end{array}$ \\
\hline & & $\begin{array}{c}\text { Pure OMMT } \\
{[49,50]}\end{array}$ & $\begin{array}{c}\text { OMMT into } \\
\text { Nanocomposite }\end{array}$ & \\
\hline Group 1 & Nanomer ${ }^{\circledR}$ I.34MN & 1.86 & 3.39 & 1.53 \\
Group 2 & MMT-DMAODM & 1.77 & 3.95 & 2.18 \\
Group 3 & MMT-DMAHDM & 1.73 & 3.45 & 1.72 \\
Group 4 & S.MMT-DMAHDM & 1.78 & 3.62 & 1.84 \\
Group 5 & MMT-CTAC & 1.83 & 3.56 & 1.73 \\
Group 6 & S.MMT-CTAC & 1.79 & 3.60 & 1.81 \\
\hline
\end{tabular}

Particularly, the incorporation of MMT-DMAODM nanofillers into dental nanocomposites imposed a considerable change of interlamelar spacing, implying a possible structure composed of highlyintercalated regions along with a small portion of agglomerates. The vinyl groups of DMAODM are capable ofstrongly interacting with methacrylated groups of monomers, and, thus, favor the formation of such structures. Similar performance is also observed for surface modified OMMTs, S.MMT-DMAHDM, and S.MMT-CTAC, as silane coupling agent could also chemically interact with monomers' functional groups (Figure 3). On the other hand, MMT-DMAHDM nanoparticles showed a satisfactory alternation of $d_{001}$-basal spacing values, due to functional end groups, denoting anintercalated structure of nanocomposite. However, this nanocomposite structure seems to be enriched with clay agglomerates as the first diffraction peak is shorter than the secondary. In the same manner, Nanomer ${ }^{\circledR}$ I.34MN and MMT-CTAC exhibited a well-established agglomeration of nanofillers despite their remarkable extent of monomer intercalation.

Figure 4 shows SEM microphotographs taken for all the nanocomposite resins' groups. It can be seen that voids were presented on the observed surfaces, while clay nanofillers (white dots) were extensively distributed into the polymer matrix for the majority of composites, despite the formation of some agglomerates distinguished as larger white dots. These clusters formed by OMMTs' aggregation could be attributed to some restrictions associated with the widely used manual mixing [58-62]. Previous studies based on this technique have proven that the relatively high clay filler loadings (varying from $16 \mathrm{up}$ to $70 \mathrm{wt} \%$ ) might result in the occurrence of a great number of tactoids $[43,45,46,63]$. Nanomer ${ }^{\circledR}$ I.34MN (Figure 4a), MMT-DMAHDM (Figure 4c), and MMT-CTAC (Figure 4e) clays seem to form the most and largest agglomerates. The lower extent of clustering is observed for OMMTs, such as MMT-DMAODM (Figure 4b), S.MMT-DMAHDM (Figure 4d), and S.MMT-CTAC (Figure 4f), with organomodifiers containing methacrylated groups, which maybe due to their chemical affinity with monomers which can promote better dispersion of nanofillers into the organic matrix. The above observations describe a surface nanoparticles' distribution which is almost in agreement with the aforementioned XRD results. 


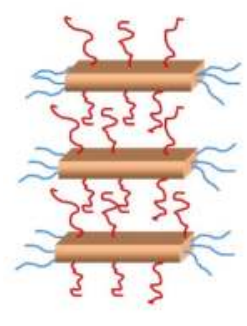

Camphorquinone<smiles>C=C(C)C(=O)OCC(O)COc1ccc(C(C)(C)C2CCC(OCC(O)COC(=O)C(=C)C)CC2)cc1</smiles>

Bis-GMA<smiles>C=C(C)C(=O)OCCOCCOCCOC(=O)C(=C)C</smiles>

TEGDMA

DMAEMA

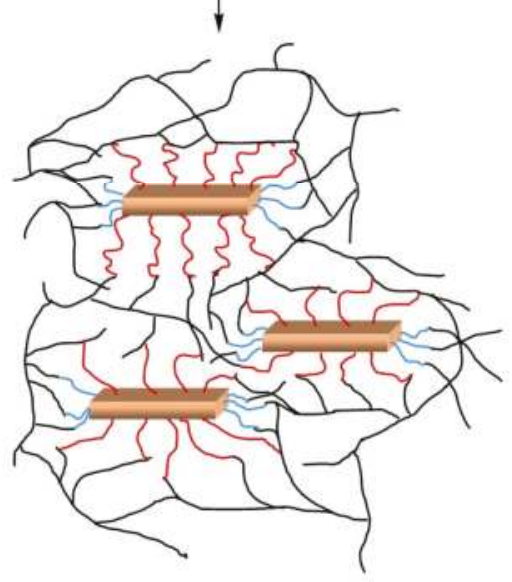

Clay platelet

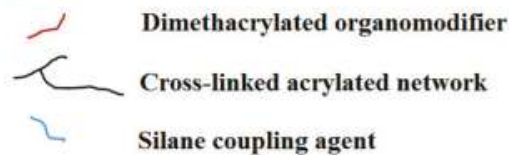

Figure 3. Schematic representation of nanocomposite formation indicating the possible chemical interactions between nanoclayorganomodifier and dental resin monomers. 

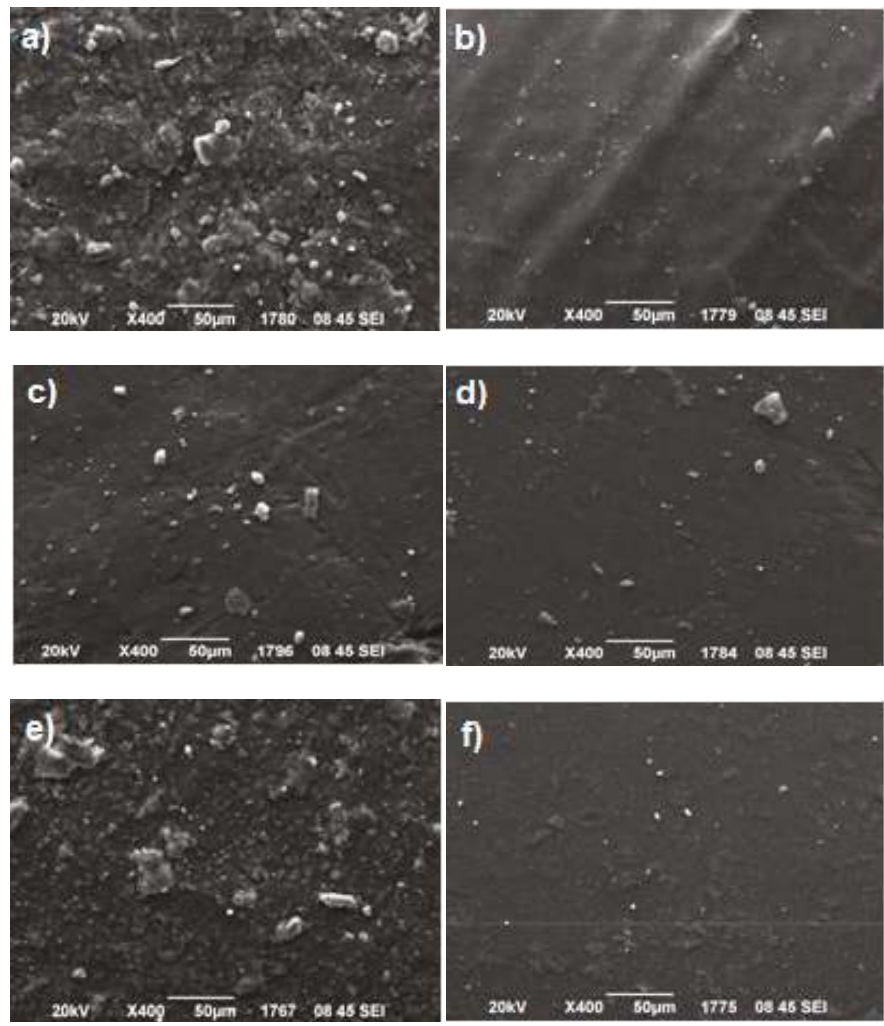

Figure 4. Scanning electron microscopy (SEM) images of a series of experimental dental nanocomposite resins containing (a) Nanomer ${ }^{\circledR}$ I.34MN; (b) Montmorillonite-dimethylaminooctadecyl methacrylate (MMT-DMAODM); (c) Montmorillonite-dimethylaminohexadecyl methacrylate (MMT-DMAHDM); (d) S.MMT-DMAHDM; (e) Montmorillonite-cetyltrimethylammonium chloride (MMT-CTAC); (f) S.MMT-CTAC at concentration $50 \mathrm{wt} \%$.

\subsection{Polymerization Kinetics}

Figure 5a represents the results on the degree of conversion versus time for Bis-GMA/TEGDMA matrix and the nanocomposite resins containing different types of nanoclayorganomodifiers. Typical FTIR absorbance peaks (1635 and $1582 \mathrm{~cm}^{-1}$ ) recorded for Group 1, 2, and 4 nanocomposites, under cured and uncured conditions, which were used to calculate the degree of conversion are given in Figure 5a. The experimental values of the final DC (\%) are listed in Table 2. Representative data for some commercial dental resins $[64,65]$ are also given in Table 2. 
Table 2. Experimental values concerning curing kinetics, polymerization shrinkage and mechanical properties of pure 2,2-Bis[p-(2'-hydroxy-3'- methacryloxypropoxy)phenylene]propane/triethylene glycol dimethacrylate(Bis-GMA/TEGDMA) resin and dental nanocomposite resins.For flexural modulus and strength mean values, the groups with the same superscript letters exhibit statistically significant differences $(\mathrm{p}<0.001)$. Literature data for typical commercially available dental resins are also included $[64,65]$.

\begin{tabular}{|c|c|c|c|c|}
\hline Sample & Final DC (\%) & Total Strain $(\%)$ & $\begin{array}{c}\text { Flexural } \\
\text { Modulus (GPa) }\end{array}$ & $\begin{array}{c}\text { Flexural } \\
\text { Strength (MPa) }\end{array}$ \\
\hline Bis-GMA/TEGDMA & 61.60 & $6.47 \pm 0.19$ & $1.53 \pm 0.40^{a, b, c}$ & $92.59 \pm 13.94^{\mathrm{d}, e, f}$ \\
\hline Nanomer ${ }^{\circledR}$ I.34MN nanocomposite & 56.80 & $3.46 \pm 0.37$ & $2.40 \pm 0.64$ & $29.45 \pm 11.45$ \\
\hline MMT-DMAODM nanocomposite & 70.60 & $2.80 \pm 0.23$ & $3.32 \pm 0.36^{\mathrm{a}}$ & $36.60 \pm 7.28$ \\
\hline MMT-DMAHDM nanocomposite & 60.80 & $2.71 \pm 0.13$ & $2.30 \pm 0.15$ & $26.82 \pm 6.33^{d}$ \\
\hline S.MMT-DMAHDM nanocomposite & 61.70 & $2.86 \pm 0.37$ & $3.14 \pm 0.47^{b}$ & $32.39 \pm 3.52$ \\
\hline MMT-CTAC nanocomposite & 41.00 & $2.51 \pm 0.29$ & $2.50 \pm 0.84$ & $21.14 \pm 6.79^{\mathrm{e}}$ \\
\hline S.MMT-CTAC nanocomposite & 45.80 & $2.54 \pm 0.22$ & $3.48 \pm 0.34^{c}$ & $23.09 \pm 10.23^{f}$ \\
\hline $\begin{array}{c}\text { Filtek }^{\mathrm{TM}} \text { Z350 XT (3M ESPE, St. Paul, MN, } \\
\text { USA) [64] }\end{array}$ & 50.96 & $1.66 \pm 0.15$ & $9.13 \pm 0.66$ & $80.52 \pm 15.88$ \\
\hline $\begin{array}{c}\text { Tetric }{ }^{\circledR} \text { N-Ceram Bulk Fill (Ivoclar-Vivadent, } \\
\text { Schaan, Liechtenstein) [64] }\end{array}$ & 49.50 & $1.36 \pm 0.08$ & $7.05 \pm 0.60$ & $60.37 \pm 11.05$ \\
\hline $\begin{array}{l}\text { Tetric }{ }^{\circledR} \text { Evo Ceram Bulk Fill } \\
\text { (Ivoclar-Vivadent, Schaan, Liechtenstein) [65] }\end{array}$ & 56.70 & - & 6.10 & 94.50 \\
\hline Grandio (Voco, Cuxhaven, Germany) [65] & 62.80 & - & 15.30 & 125.00 \\
\hline
\end{tabular}

According to Figure 5a, for pure Bis-GMA/TEGDMA the reaction conversion eventually reaches the relatively low ultimate value of approximately $62 \%$. This performance is attributed to the glass-effect procedure due to the influence of diffusion-controlled phenomena on the propagation reaction and the constrained mobility of monomer molecules to find and react with a macro-radical [66-69]. Despite available monomers still remaining unreacted, the reaction rate is almost zero. Furthermore, an abrupt increment in the degree of conversion observed at the first $5 \mathrm{~min}$ for the majority of the materials, implies the appearance of the well-known auto-acceleration or gel-effect phenomenon due to the effect of diffusion-controlled phenomena on the termination reaction and the reduced mobility of live macro-radicals to find one another and react [66]. As a result, their concentration increases, leading toelevated reaction rates.

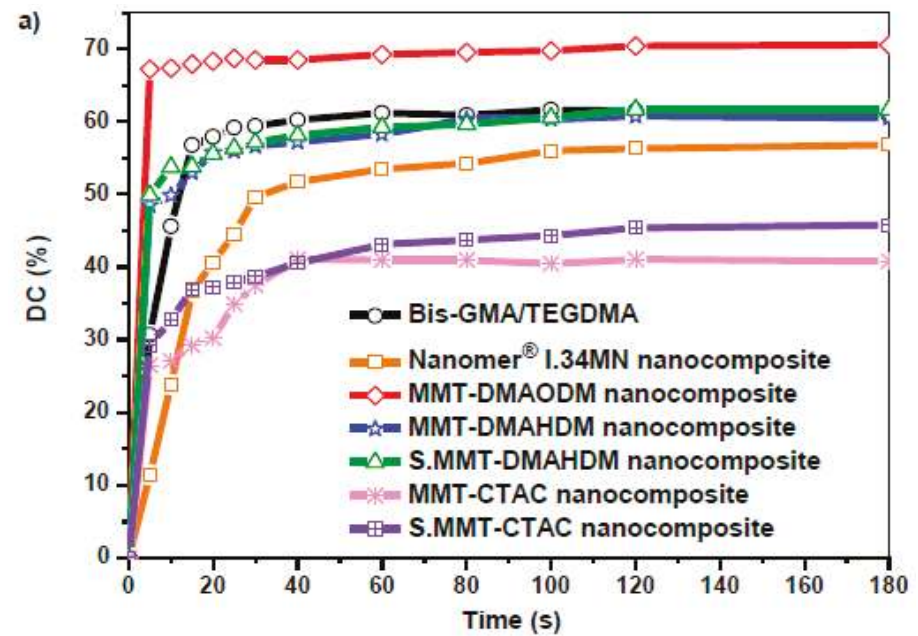

Figure 5. Cont. 

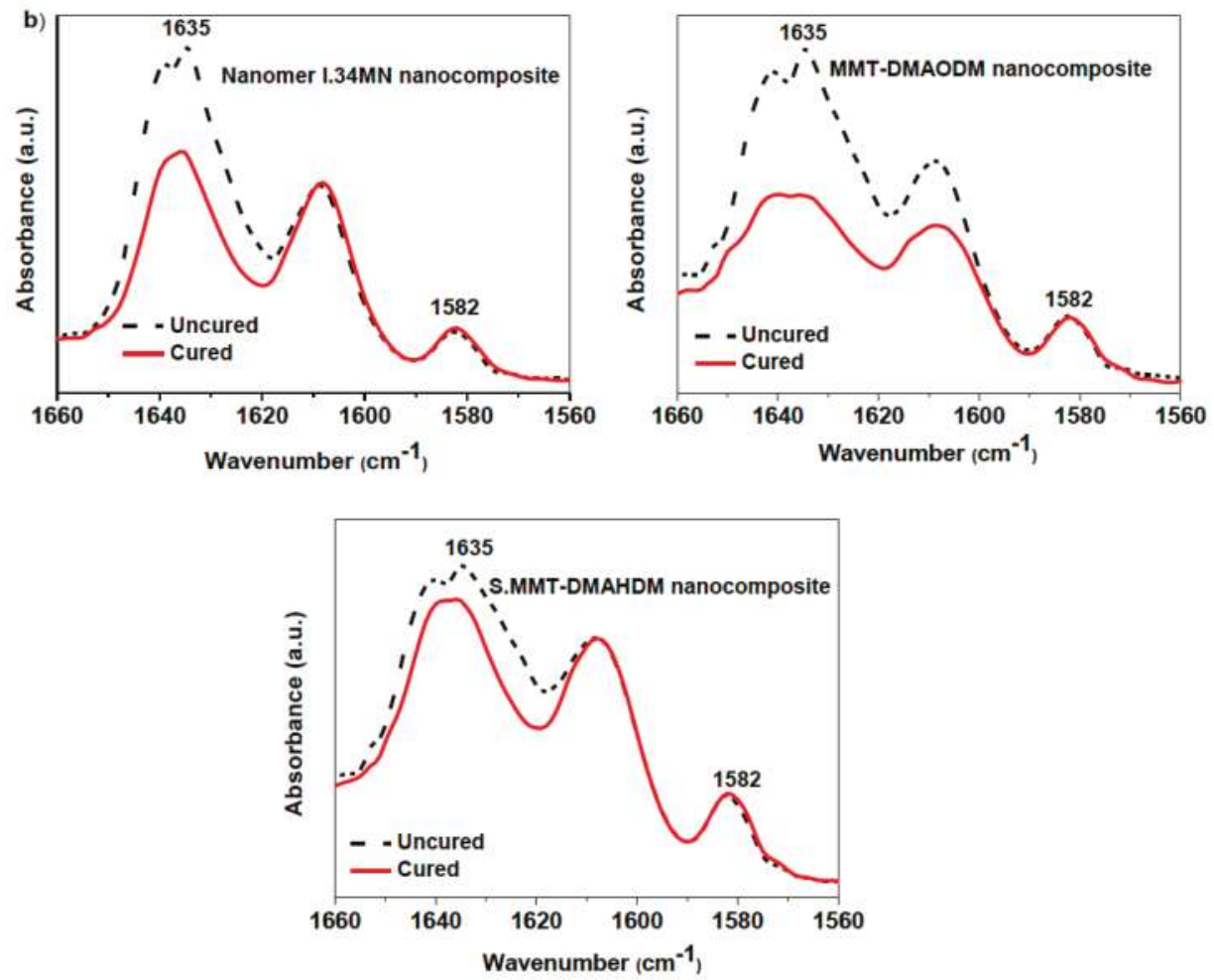

Figure 5. (a) Degree of conversion versus time of 2,2-Bis[p-(2'-hydroxy-3'- methacryloxypropoxy) phenylene]propane/triethylene glycol dimethacrylate(Bis-GMA/TEGDMA) matrix and dental nanocomposite resins filled with different OMMT nanoparticles; (b) FTIR spectra with measured peak areas (1635 and $1582 \mathrm{~cm}^{-1}$ ) used to calculate the percent degree of conversion (DC (\%)) for uncured and cured nanocomposites.

In particular, the gel-effect phenomenon seems to be more effective for MMT-DMAODM, MMT-DMAHDM, and S.MMT-DMAHDM, as the presence of OMMT nanofiller might act as a barrier to the diffusion of macroradicals to find each other and terminate. Thus, the local concentration of radicals increases leading toincreased reaction rates and higher conversion values at the gel-effect period. It is obvious that the high reactivity of MMT-DMAODM nanoparticles arisingfrom the presence of vinyl functional groups in a short C8 alkyl chain of the organomodifier can significantly enhance the auto-acceleration effect up to $68 \%$ degree of conversion, while the final reaction degree reaches almost $70 \%$. The lower conversion value of 50\% observed for MMT-DMAHDM and S.MMT-DMAHDM at the gel-effect period maybe denotes a lower reactivity of vinyl groups in a larger C16 alkyl chain, although the ultimate conversion value is almost comparative to the Bis-GMA/TEGDMA matrix. From the results it is also apparent that for hydrophobic MMT-CTAC and S.MMT-CTAC, as well as for Nanomer ${ }^{\circledR}$ I.34MN containing hydroxy-groups, the reaction rates decrease and the gel-effect phenomenon becomes weaker, as the corresponding degree of conversion values vary from $30 \%$ to $37 \%$, maybe due to a possible occurrence of aggregates as shown from XRD spectra and SEM images, resulting in a lower capability of clay to act as radical scavenger. Moreover, the ultimate reaction degrees deteriorate as the movement of the small monomer molecules decreases, caused by a lower intercalation extent of the OMMTs, so that their diffusion becomes restricted and they do not easily react with macroradicals. The aspect that clay aggregates can act as microfillers affecting absorption 
and scattering of light and, thus, attenuating the light photo-initiation process, has been suggested by other researchers [70]. In particular, hydroxy-groups of Nanomer ${ }^{\circledR}$ I.34MN nanoclay, are not expected to participate in the addition polymerization reaction and, thus, affect the auto-acceleration and glass effect, even if they could interact with ether oxygen atoms of monomers via hydrogen bond formation. An alternative explanation could be that primary ketyl radicals formed from the degradation of the camphorquinone photo-initiator [71] may react with the quaternary ammonium hydroxyls on the MMT surface by abstracting a hydrogen atom. The alkoxy radicals of OMMT may then scavenge a further primary radical, leading to the decrease of the effective number of primary radicals which can find a monomer molecule and initiate polymerization [72].

On the other hand, degree of conversion curves for S.MMT-CTAC and S.MMT-DMAHDM denote that additional double bonds due to the silane coupling agent on the surface of OMMT nanoclay rather favor the occurrence of both gel and glass effect.

A theoretical model to optimize experimental data used for DC (\%) calculations was previously suggested by Ilie and Durner [73]. According to that, the increase of DC can be described by the superposition of two exponential functions, and the correlation function is asfollows:

$$
\mathrm{y}=\mathrm{y}_{0}+\alpha\left(1-\mathrm{e}^{-\mathrm{bx}}\right)+\mathrm{c}\left(1-\mathrm{e}^{-\mathrm{dx}}\right)
$$

where $y$ is the DC $(\%), x$ is the polymerization time, the term $y_{0}$ represents the $y$-intercept, while parameters $a, b, c$, and $d$ are modulation factors of the exponential function to optimize the double exponential function on the measured curve. Typical measuring points recorded for nanocomposites filled with Nanomer ${ }^{\circledR}$ I.34MN, MMT-DMAHDM, and S.MMT-DMAHDM were plotted again (Figure 6), and a line of best fit was inserted through all points.

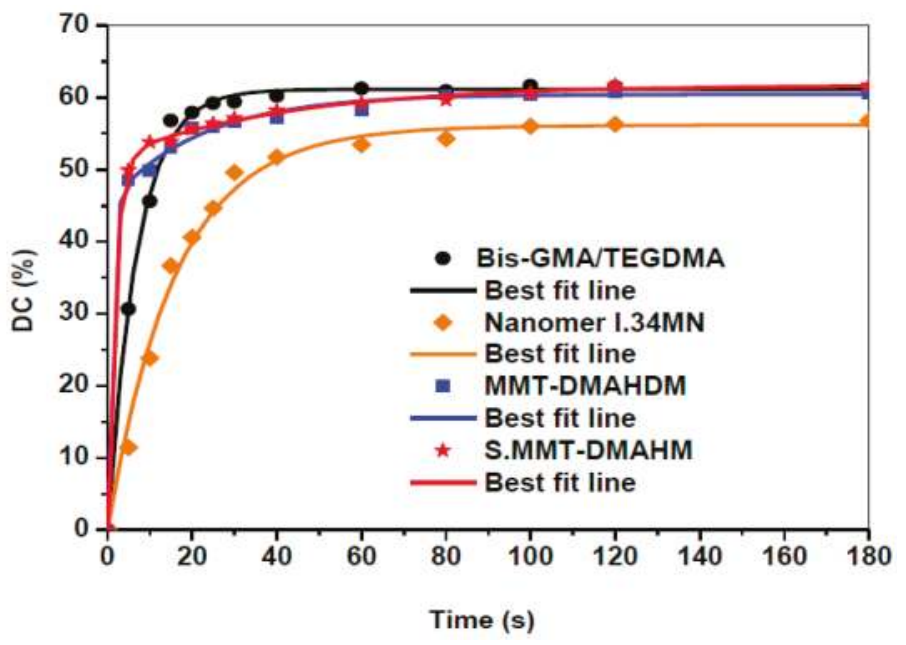

Figure 6. Representative DC-time experimental data and the two exponential approximate functions, for pure Bis-GMA/TEGDMA matrix and nanocomposites filled with Nanomer ${ }^{\circledR}$ I.34MN, MMT-DMAHDM and S.MMT-DMAHDM. Lines of best fit are drawn through all experimental points of the approximate function.

The calculated parameters $a, b, c$, and $d$ for each nanocomposite are given in Table 3. Particularly, the $\mathrm{R}^{2}$ values denote a satisfactory correlation between measured and theoretical data. For a long polymerization time $(x \rightarrow \infty)$ Equation (4) is converted to:

$$
\mathrm{y}=\mathrm{y}_{0}+\alpha+\mathrm{c}
$$


The theoretical final DC (\%) can now be calculated by combining the specific values $y_{0}, a, c$ of Table 3 and Equation (5). By comparing the DC (\%) values of Tables 2 and 3, it can be postulated that the theoretical approach is very close to the obtained experimental data.

Table 3. Parmeters describing the exponential sum function accompanied by the coefficient of deterimination, R2, and the calculated values of the final percent degree of conversion (DC (\%)).

\begin{tabular}{cccccccc}
\hline Resin & $y_{0}$ & $\alpha$ & $b$ & $c$ & $d$ & $R^{2}$ & $\begin{array}{c}\text { Final DC } \\
(\%)\end{array}$ \\
\hline Bis-GMA/TEGDMA & -0.2870 & 6.4892 & 0.1446 & 54.9137 & 0.1447 & 0.9972 & 61.69 \\
\hline $\begin{array}{c}\text { Nanomer }{ }^{\circledR} \text { I.34MN } \\
\text { nanocomposite }\end{array}$ & -1.8527 & 41.3735 & 0.0652 & 16.4640 & 0.0652 & 0.9929 & 55.98 \\
\hline $\begin{array}{c}\text { MMT-DMAHDM } \\
\text { nanocomposite }\end{array}$ & 0.0000 & 15.1918 & 0.0462 & 45.2200 & 1.0484 & 0.9982 & 60.41 \\
\hline $\begin{array}{c}\text { S.MMT-DMAHDM } \\
\text { nanocomposite }\end{array}$ & 0.0000 & 10.2364 & 0.0243 & 51.4829 & 0.5863 & 0.9995 & 61.72 \\
\hline
\end{tabular}

\subsection{Polymerization Shrinkage Kinetics}

Setting contraction of dental composite resins, widely known as polymerization shrinkage, should be ideally limited as much as possible because this achievement favors marginal adaptation, reduces the possibility of a breakdown of the bond to the tooth tissues and prevents the occurrence of secondary caries [5]. Figure 7a illustrates the polymerization shrinkage strain plots versus time for Bis-GMA/TEGDMA matrix and nanocomposite resins containing several types of OMMT nanoclays. Figure $7 \mathrm{~b}$ shows the apparatus used for shrinkage measurements, as was discussed in Section 2.3. The total strain (\%) values are listed in Table 2, while corresponding values are also included for several commercially available dental resins $[64,65]$. As it was expected, the setting contraction proceeds faster for pure matrix than nanocomposites, and reaches an ultimate strain value almost 3-fold higher compared to the majority of nanocomposites. Nanofillers do not participate in the polymerization reaction, and concurrently decrease the concentration of reactive methacrylate groups [2]. The ultimate strain values recorded for the synthesized nanocomposite resins vary from $2.5 \%$ up to $3.5 \%$. In terms of traditional composite resins, values of around $1.5 \%$ to $3.0 \%$ volumetric contraction are typical as opposed to $6 \%$ for acrylics [2]. According to XRD results, OMMT nanoclays are subjected to swelling as their platelets are spread apart via polymerization process. As a result, this expansion mechanism increases the free volume inside the clay and might allow for a reduction of polymerization shrinkage as well as residual stresses [44]. A similar attitude for Bis-GMA/nanoclay systems hasalso been reported by other researchers $[74,75]$. Campos et al. found that dental nanocomposites' shrinkage isreduced by increasing MMT filler content from 20 to $50 \mathrm{wt} \%$, indicating the influence of clay nanoparticles on polymerization shrinkage [57]. 

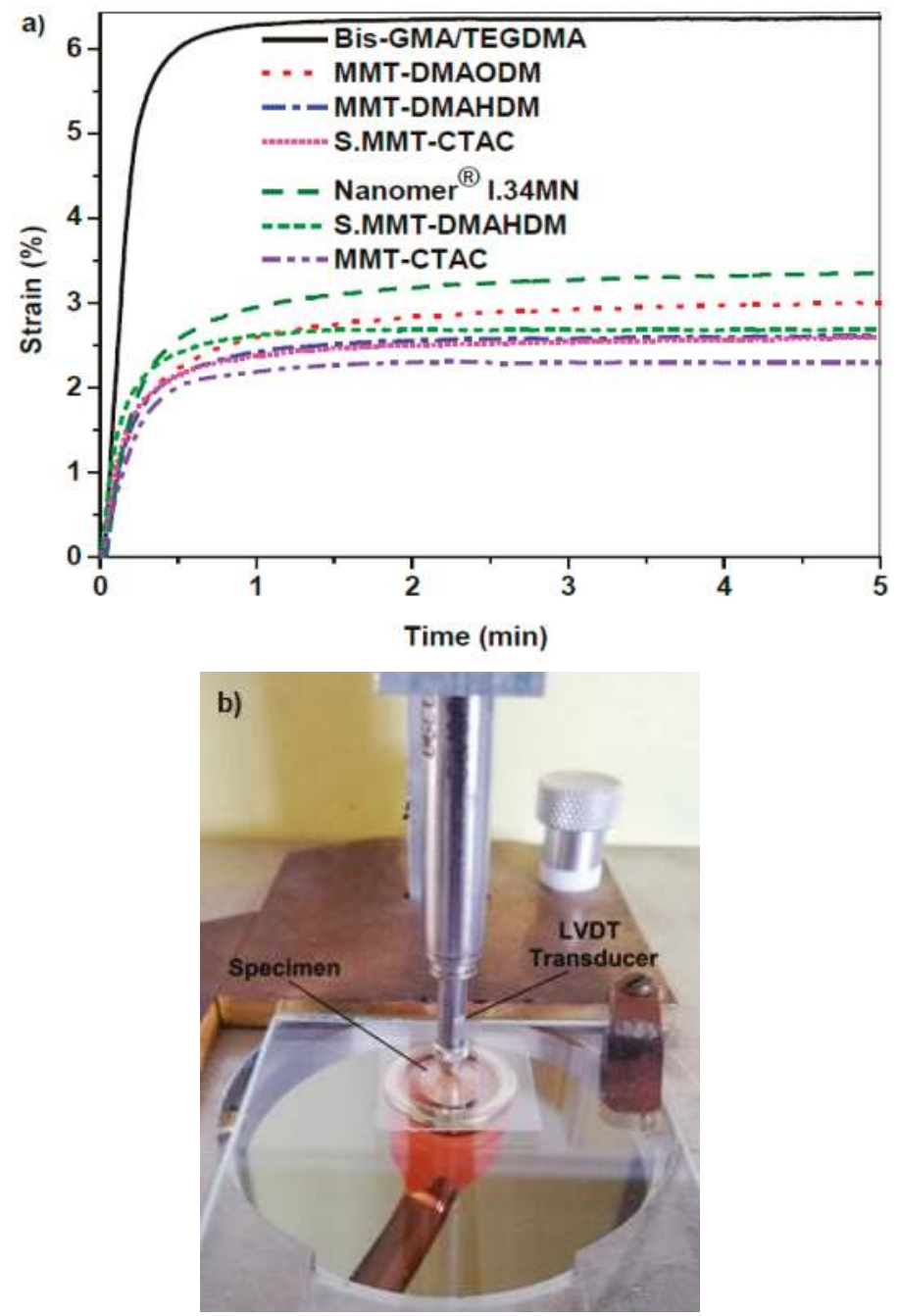

Figure 7. (a) Time dependence of polymerization shrinkage strain of Bis-GMA/TEGDMA matrix and dental nanocomposite resins with diverse types of OMMT nanoclays; (b) Apparatus utilized for polymerization shrinkage measurements.

Figure 7a reveals that nanocomposites filled with OMMTs containing quaternary ammonium methacrylates, such as MMT-DMAODM and MMT-DMAHDM, exhibit higher setting contraction than the corresponding MMT-CTAC with hydrophobic ammonium intercalant throughout the photo-polymerization process. Mahmoodian et al. suggested that not only the degree of conversion but also the volume shrinkage are dependent on the type of clay organomodifier [40]. The presence of functional groups in clay nanoparticles accounts for the observed tendency, as more available double bonds can take part in an addition polymerization reaction between nanoclayorganomodifiers and monomers, leading to the formation of more covalent bonds with a smaller length. Thus, the free volume of the nanocomposite resin is reduced, and the polymerization shrinkageincreases. Moreover, the silane coupling agent on the surface of S.MMT-CTAC and S.MMT-DMAHDM contributes additional double bonds throughout the setting reaction in a similar way, enhancing somewhat the polymerization 
shrinkage of the final nanocomposite resin when compared to MMT-CTAC and MMT-DMAHDM. According to literature data, OMMTs containing $\mathrm{OH}$ polar groups performed as effective setting contraction controllers [40] even at high mass fractions [44]. However, nanocomposite filled with Nanomer ${ }^{\circledR}$ I.34MN yielded the highest strain curve, although hydroxy-groups of its organomodifier are not expected to participate in the polymerization reaction. As it was indicated from XRD and SEM results, possible agglomeration of clay lamellae presented in the intercalated structure of resin could readily reduce the expansion and free volume of Nanomer ${ }^{\circledR}$ I.34MN clay, augmenting the shrinkage strain of the set nanocomposite.

\subsection{Mechanical Properties}

The flexural properties of the dental nanocomposite resins produced were studied in relation to the effect of the different nanoclayorganomodifier, at a constant concentration of $50 \mathrm{wt} \%$. Their flexural modulus and strength dependence on the various OMMT type are shown in Figure $8 \mathrm{a}, \mathrm{b}$, respectively, while the corresponding mean values accompanied by standard deviations are listed in Table 2. Typical data are also included for some commercially available resins based on previous studies [64,65]. Concerning the flexural modulus, significant statistic differences were found to exist between Bis-GMA/TEGDMA and nanocomposites with MMT-DMAHDM, S.MMT-DMAHDM, and S.MMT-CTAC. Force versus displacement representative plots of flexural behavior for Bis-GMA/TEGDMA matrix and nanoclay filled dental resins are given in Figure 8c. It is obvious that all nanocomposite resins exhibited higher flexural values compared to the pristine Bis-GMA/TEGDMA matrix. This specific trend was supposed to be expected, as MMT nanoparticles may give rise to high stiffness and modulus [76], and, thus, exert the high resistance against the plastic deformation, as well as the stretching resistance of the oriented macromolecular networks into the clay galleries [77,78]. In each nanocomposite case, the quaternary ammonium intercalating agent of the organoclay can perform as the 'bridge' connecting the OMMT platelets and inserted macromolecules. The ammonium head groups of the intercalant molecules reside at the silicate layer, and the organic ligands stretch around the silicate surface and target towards the polymer chains [25].

In particular, nanocomposites filled with S.MMT-CTAC, MMT-DMAODM, and S.MMTDMAHDM, yielded the highest stiffness among the rest of the nanocomposites produced, corresponding to a $105 \%$ to $127 \%$ increment of flexural modulus when compared to virgin Bis-GMA/TEGDMA. It seems that vinyl groups of the specific organomodifier contributed to a noticeable stiffening of nanocomposite resins, through a copolymerization process between MMT quaternary ammonium intercalants and methacrylated monomers. A lower improvement percentage of flexural modulus was shown for nanocomposites with Nanomer ${ }^{\circledR}$ I.34MN (57\%), MMT-DMAHDM (50\%), and MMT-CTAC $(63 \%)$.Despite the potential of hydroxy-groups of Nanomer ${ }^{\circledR}$ I.34MN to interact with monomers through hydrogen bonding, and vinyl groups of MMT-DMAHDM to copolymerize with matrix, the relatively smaller number and size of the clay clusters found for the nanocomposites reinforced with MMT-DMAODM, S.MMT-DMAHDM, and S.MMT-CTAC nanoparticles may account for the most intensive stiffening effect rendered by the presence of MMT. It could be stated that the longer chain length (C16) of quaternary ammonium intercalating agent DMAHDM onto MMT sheets might restrict the nanoparticle mobility of the nanoclay through the organic phase of monomers, rather than the MMT-DMAODM intercalant chain (C8), resulting in a probable additional chemical reaction between the separate nanofillers with reactive vinyl groups, and, thus, to a higher extent of clustering. Discacciati et al. also suggested the formation of clay larger agglomerates at high clay concentration, when they used the stereochemically heavy and reactive vinylbenzyltrimethyl ammonium cation as MMT intercalant, due to the strong covalent bonding between clay sheets [63]. 

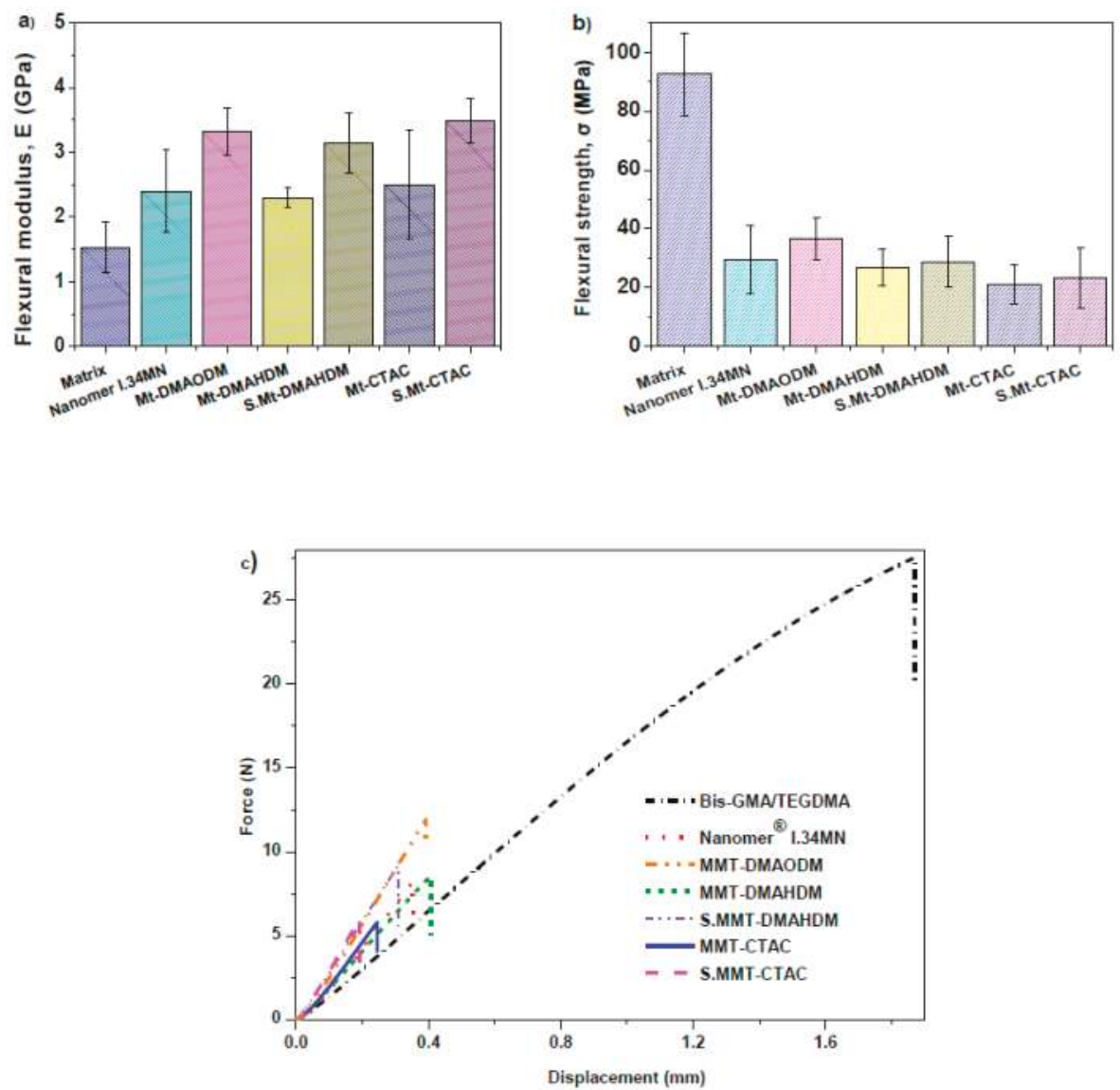

Figure 8. (a) Flexural modulus; (b) Flexural resistance; (c) Graphic representation of force against the displacement of flexural properties, for pure Bis-GMA/TEGMA matrix and dental nanocomposite resins filled with different OMMT nanofillers.

Although MMT nanoparticles were capable of increasing the stiffness for the total of the tested nanocomposite resins compared to the pure Bis-GMA/TEGDMA resin, a considerable weakness of flexural strength was observed (Figure 8b). The corresponding strength values are also listed in Table 2. The flexural strength decrease recorded at $50 \mathrm{wt} \%$ clay concentration contrasts with epoxy nanocomposites employing other types of cation-exchanged clays, such asbentonite/4-diphenylamine diazonium/polyaniline nanocomposite, where a filler loading up to $0.5 \mathrm{wt} \%$ can significantly improve flexural strength [56]. At high nanofiller clay levels as much as $50 \mathrm{wt} \%$, the co-existence of agglomerates along with clay intercalates into the cross-linked matrix, according to the combination of XRD and SEM results may be responsible for the low resistance of nanocomposites against flexural loadings. It is widely known that agglomeration can give rise to stress formation [79], while it moderates the intercalation phenomena which favors the improvement of mechanical properties [80]. As a result, the dispersion of tactoids in the polymer matrix can act as a conventional filler, and the interfacial adhesion between the organoclay and polymer matrix is not strong enough to withstand large deformations (Figure 8c). A similar trend of flexural strength decrease at high nanofiller fractions was also observed by other researchers [45]. In terms of the type of MMT organomodifier, according to Figure $8 \mathrm{~b}$ and Table 2 values, it can be seen that the enrichment of clay functionalization with as 
many as vinyl groups, including silane modifier, might yield somewhat better flexural strength of the nanocomposite when compared to the other tested experimental organoclays. In particular, the better dispersion and a possible co-polymerization of MMT-DMAODM nanoparticles with organic matrix could account for a slight capability of the nanocomposite to resist bending loadings.

\section{Conclusions}

Dental nanocomposite resins were successfully synthesized by inserting different OMMT nanoparticles. The specific type of clay organomodifier was found to affect not only their morphological characteristics but also their physicochemical and mechanical properties. The combination of XRD and SEM results confirmed the intercalation of macromolecular chains between clay platelets, while some agglomerates of clay still remained. Vinyl groups of intercalant and/or silane coupling agent promoted the better dispersion of nanofillers into the resin matrix. Monitoring of polymerization kinetics revealed that the affinity of methacrylatednanoclays with monomers can contribute to the acceleration of the photo-polymerization reaction, improving the final degree of conversion. Particularly, the high reactivity of methacrylated MMT-DMAODM enhanced the gel-effect up to a $68 \%$ degree of conversion, while the final reaction degree reached almost $70 \%$. Experimental data obtained for nanocomposites with Nanomer ${ }^{\circledR}$ I.34MN, MMT-DMAHDM, and S.MMT-DMAHDM were found to be very close to those derived from theoretical calculations. Polymerization shrinkage was lowered by incorporating any kind of the tested nanoclay, especially in the absence of organomodifier reactive groups. Nanocomposite containing the non-reactive hydrophobic MMT-CTAC exhibited the lowest total strain (2.51\%), whereas Nanomer ${ }^{\circledR}$ I.34MN yielded the highest strain curve $(3.46 \%)$ due to a possible agglomeration of clay lamellae.Regarding the mechanical properties, it was verified that the incorporation of OMMT nanofiller improves the stiffness of the dental composite resin. A 105\% to $127 \%$ increment of flexural modulus values was achieved for nanocomposites reinforced with thepolymerizable S.MMT-CTAC (3.48 GPa), MMT-DMAODM (3.32 GPa), and S.MMT-DMAHDM (3.14 GPa) nanoclays, when compared to Bis-GMA/TEGDMA resin matrix (1.53 GPa). However, the flexural strength was decreased due to the aggregation of clay nanoparticles at high concentrations. The significance of the current work relies on providing novel information about chemical interactions phenomena between nanofillers and organic matrix towards the improvement of dental restorative materials.

Author Contributions: A.K.N. and E.A.K. contributed to the project conceptualization; A.K.N., E.A.K., C.G., and D.S.A. conceived the methodology and designed the experiments; A.K.N. performed the experiments and analyzed the data; C.G. conducted the statistical analysis of the experimental data. A.K.N. carried out the writing-original draft preparation. E.A.K., C.G. and D.S.A. contributed to the writing-review of the paper.

Funding: This research was funded by GREEK STATE SCHOLARSHIPS FOUNDATION (IKY), grant number MIS: 5001552. The APC was funded by MIS: 5001552.

Acknowledgments: The experimental procedures were performed at the Department of Basic Dental Sciences, Division of Dental Tissues Pathology and Therapeutics, School of Dentistry, Aristotle University of Thessaloniki, Greeceand the Laboratory of Polymer and Color Chemistry and Technology, Department of Chemistry, Aristotle University Thessaloniki, Greece.

Conflicts of Interest: The authors declare no conflict of interest.

\section{References}

1. Bowen, R.L. Composite and sealant resins-past, present, and future. Pediatr. Dent. 1982, 4, 10-15.

2. McCabe, J.; Walls, A. Applied Dental Materials, 9th ed.; Blackwell Publishing Ltd.: Oxford, UK, 2008; pp. 188-189, 206-207.

3. Mai, Y.W.; Yu, Z.Z. Polymer Nanocomposites, 1st ed.; Woodhead Publishing Ltd.: Cambridge, UK, 2006; pp. 188-190, 206, 307.

4. Chen, M.-H. Update on Dental Nanocomposites. J. Dent. 2010, 89, 549-560. [CrossRef]

5. Van Noort, R. Introduction to Dental Materials, 4th ed.; Mosby Elsevier Ltd.: Edinburgh, UK, 2013; pp. 73-80.

6. Cramer, N.; Stansbury, J.; Bowman, C. Recent advances and developments in composite dental restorative materials. J. Dent. Res. 2011, 90, 401-416. [CrossRef] [PubMed] 
7. Ilie, N.; Hickel, R. Resin composite restorative materials. Aust. Dent. J. 2011, 56, 59-66. [CrossRef]

8. Saharudin, K.A.; Sreekantan, S.; Basiron, N.; Khor, Y.L.; Harun, N.H.; Mydin, R.B.S.M.N.; Akil, H.M.; Seeni, A.; Vignesh, K.; Mydin, R.S.M.N. Bacteriostatic Activity of LLDPE Nanocomposite Embedded with Sol-Gel Synthesized TiO2/ZnO Coupled Oxides at Various Ratios. Polymers 2018, 10, 878. [CrossRef]

9. Wang, H.-T.; Chiang, P.-C.; Tzeng, J.-J.; Wu, T.-L.; Pan, Y.-H.; Chang, W.-J.; Huang, H.-M.; Choi, I.S.; Mano, J.F. In Vitro Biocompatibility, Radiopacity, and Physical Property Tests of Nano-Fe3O4 Incorporated Poly-l-lactide Bone Screws. Polymers 2017, 9, 191. [CrossRef]

10. Zagho, M.M.; Hussein, E.A.; Elzatahry, A.A. Recent Overviews in Functional Polymer Composites for Biomedical Applications. Polymers 2018, 10, 739. [CrossRef] [PubMed]

11. Liu, Y.; Wu, X.; Sun, Y.; Xie, W. POSS Dental Nanocomposite Resin: Synthesis, Shrinkage, Double Bond Conversion, Hardness, and Resistance Properties. Polymers 2018, 10, 369. [CrossRef] [PubMed]

12. Gilman, J.W.; Morgan, A.B.; Harris, R., Jr.; Manias, E.; Giannelis, E.P.; Wuthenow, M. Polymer layered-silicate nanocomposites: polyamide-6, polypropylene and polystyrene. In Proceedings of the New Advances in Flame Retardant Technology Conference, Tucson, AZ, USA, 27-27 October 1999. Fire Retardant Chemical Association.

13. LeBaron, P. Polymer-layered silicate nanocomposites: an overview. Appl. Clay Sci. 1999, 15, 11-29. [CrossRef]

14. Alexandre, M.; Dubois, P. Polymer-layered silicate nanocomposites: preparation, properties and uses of a new class of materials. Mater. Sci. Eng. R: Rep. 2000, 28, 1-63. [CrossRef]

15. Zanetti, M.; Lomakin, S.; Camino, G. Polymer layered silicate nanocomposites. Macromol. Mater. Eng. 2000, 279, 1-9. [CrossRef]

16. Ahmadi, S.J.; Huang, Y.D.; Li, W. Synthetic routes, properties and future applications of polymer-layered silicate nanocomposites. J. Mater. Sci. 2004, 39, 1919-1925. [CrossRef]

17. Zeng, Q.H.; Yu, A.B.; Lu, G.Q.; Paul, D.R.; Zeng, Q. Clay-Based Polymer Nanocomposites: Research and Commercial Development. J. Nanosci. Nanotechnol. 2005, 5, 1574-1592. [CrossRef]

18. Pavlidou, S.; Papaspyrides, C. A review on polymer-layered silicate nanocomposites. Prog. Sci. 2008, 33, 1119-1198. [CrossRef]

19. Ryu, J.G.; Lee, J.W.; Kim, H. Development of poly(methyl methacrylate)-clay nanocomposites by using power ultrasonic wave. Macromol. Res. 2002, 10, 187-193. [CrossRef]

20. Diaconu, G.; Paulis, M.; Leiza, J.R. High Solids Content Waterborne Acrylic/Montmorillonite Nanocomposites by Miniemulsion Polymerization. Macromol. Eng. 2008, 2, 80-89. [CrossRef]

21. Lakshmi, M.S.; Narmadha, B.; Reddy, B. Enhanced thermal stability and structural characteristics of different MMT-Clay/epoxy-nanocomposite materials. Degrad. Stab. 2008, 93, 201-213. [CrossRef]

22. Dhibar, A.K.; Mallick, S.; Rath, T.; Khatua, B.B. Effect of clay platelet dispersion as affected by the manufacturing techniques on thermal and mechanical properties of PMMA-clay nanocomposites. J. Appl. Polym. Sci. 2009, 113, 3012-3018. [CrossRef]

23. Chen, G.; Chen, X.; Lin, Z.; Ye, W.; Yao, K. Preparation and properties of PMMA/clay nanocomposite. J. Mater. Sci. Lett. 1999, 18, 1761-1763. [CrossRef]

24. Okamoto, M.; Morita, S.; Taguchi, H.; Kim, Y.H.; Kotaka, T.; Tateyama, H. Synthesis and structure of smectic clay/poly(methyl methacrylate) and clay/polystyrene nanocomposites via in situ intercalative polymerization. Polymer 2000, 41, 3887-3890. [CrossRef]

25. Li, Y.; Zhao, B.; Xie, S.; Zhang, S. Synthesis and properties of poly(methyl methacrylate)/montmorillonite (PMMA/MMT) nanocomposites. Polym. Int. 2003, 52, 892-898. [CrossRef]

26. Liu, G.-D.; Zhang, L.-C.; Qu, X.-W.; Wang, B.-T.; Zhang, Y. Tentative study on kinetics of bulk polymerization of methyl methacrylate in presence of montmorillonite. J. Appl. Sci. 2003, 90, 3690-3695. [CrossRef]

27. Ray, S.S.; Okamoto, M. Polymer/layered silicate nanocomposites: A review from preparation to processing. Prog. Sci. 2003, 28, 1539-1641.

28. Essawy, H.; Badran, A.; Youssef, A.; El-Hakim, A.E.-F.A. Synthesis of poly(methylmethacrylate)/ montmorillonite nanocomposites via in situ intercalative suspension and emulsion polymerization. Polym. Bull. 2004, 53, 9-17. [CrossRef]

29. Su, S.; Jiang, D.D.; Wilkie, C.A. Methacrylate modified clays and their polystyrene and poly(methyl methacrylate) nanocomposites. Polym. Adv. Technol. 2004, 15, 225-231. [CrossRef]

30. Huskić, M.; Žigon, M. PMMA/MMT nanocomposites prepared by one-step in situ intercalative solution polymerization. Eur. J. 2007, 43, 4891-4897. [CrossRef] 
31. Cui, L.; Tarte, N.H.; Woo, S.I. Synthesis and properties of poly(methyl methacrylate)/clay nanocomposites prepared via in situ polymerization with $\mathrm{Ni}$ (acac)2catalyst. J. Appl. Sci. 2008, 110, 784-790. [CrossRef]

32. Tiwari, R.R.; Khilar, K.C.; Natarajan, U. Synthesis and characterization of novel organo-montmorillonites. Appl. Clay Sci. 2004, 38, 203-208. [CrossRef]

33. Karger-Kocsis, J.; Wu, C.-M.; Karger-Kocsis, J. Thermoset rubber/layered silicate nanocomposites. Status and future trends. Eng. Sci. 2004, 44, 1083-1093. [CrossRef]

34. Kojima, Y.; Usuki, A.; Kawasumi, M.; Okada, A.; Kurauchi, T.; Kamigaito, O. One-pot synthesis of nylon 6-clay hybrid. J. Polym. Sci. Part A: Polym. Chem. 1993, 31, 1755-1758. [CrossRef]

35. Burnside, S.D.; Giannelis, E.P. Synthesis and properties of new poly(dimethylsiloxane) nanocomposites. Chem. Mater. 1995, 7, 1597-1600. [CrossRef]

36. Wang, Z.; Pinnavaia, T.J. Hybrid Organic-Inorganic Nanocomposites: Exfoliation of Magadiite Nanolayers in an Elastomeric Epoxy Polymer. Chem. Mater. 1998, 10, 1820-1826. [CrossRef]

37. Sciascia, L.; Casella, S.; Cavallaro, G.; Lazzara, G.; Milioto, S.; Princivalle, F.; Parisi, F. Olive mill wastewaters decontamination based on organo-nano-clay composites. Ceram. Int. 2019, 45, 2751-2759. [CrossRef]

38. Calabrese, I.; Cavallaro, G.; Scialabba, C.; Licciardi, M.; Merli, M.; Sciascia, L.; Liveri, M.L.T. Montmorillonite nanodevices for the colon metronidazole delivery. Int. J. Pharm. 2013, 457, 224-236. [CrossRef]

39. Sciascia, L.; Liveri, M.L.T.; Merli, M. Kinetic and equilibrium studies for the adsorption of acid nucleic bases onto K10 montmorillonite. Appl. Clay Sci. 2011, 53, 657-668. [CrossRef]

40. Mahmoodian, M.; Pourabbas, B.; Arya, A. Preparation and characterization of Bis-GMA/TEGDMA/clay nanocomposites at low filler content regimes. J. Compos. Mater. 2010, 44, 1379-1395. [CrossRef]

41. Mucci, V.; Pérez, J.; Vallo, C. Preparation and characterization of light-cured methacrylate/montmorillonite nanocomposites. Polym. Int. 2011, 60, 247-254. [CrossRef]

42. Terrin, M.M.; Poli, A.L.; Horn, M.A., Jr.; Neumann, M.G.; Cavalheiro, E.T.G.; Correa, I.C.; Schmitt, C.C. Effect of the loading of organomodified clays on the thermal and mechanical properties of a model dental resin. Mater. Res. 2016, 19, 40-44. [CrossRef]

43. Campos, L.M.D.P.; Boaro, L.C.; Ferreira, H.P.; Dos Santos, L.K.G.; Dos Santos, T.R.; Parra, D.F. Evaluation of polymerization shrinkage in dental restorative experimental composites based: BisGMA/TEGDMA, filled with MMT. J. Appl. Sci. 2016, 133, 43543. [CrossRef]

44. De Paiva Campos, L.M.; Lugão, A.B.; Vasconcelos, M.R.; Parra, D.F. Polymerization shrinkage evaluation on nanoscale-layered silicates: Bis-GMA/TEGMA nanocomposites, in photo-activated polymeric matrices. J. Appl. Polym. Sci. 2014, 131, 40010. [CrossRef]

45. Campos, L.M.P.; Boaro, L.C.; Santos, T.M.R.; Marques, P.A.; Almeida, S.R.Y.; Braga, R.R.; Parra, D.F. Evaluation of flexural modulus, flexural strength and degree of conversion in BISGMA/TEGDMA resin filled with montmorillonite nanoparticles. J. Compos. Mater. 2017, 51, 927-937. [CrossRef]

46. Campos, L.M.P.; Zaharescu, T.; Boaro, L.C.; Santos, L.K.G.; Santos, T.M.R.; Ferreira, H.P.; Parra, D.F. Thermal and mechanical behavior evaluation of dental composites filled with irradiated montmorillonite. J. Appl. Sci. 2017, 134, 45063. [CrossRef]

47. Munhoz, T.; Fredholm, Y.; Rivory, P.; Balvay, S.; Hartmann, D.; Da Silva, P.; Chenal, J.-M. Effect of nanoclay addition on physical, chemical, optical and biological properties of experimental dental resin composites. Dent. Mater. 2017, 33, 271-279. [CrossRef]

48. Menezes, L.R.; da Silva, E.O.; da Silva Rocha, A.C.; de Oliveira, D.C.R.S.; de Barros Campos, P.R. The applicability of organomodified nanoclays as new fillers for mechanical reinforcement of dental composites. J. Compos. Mater. 2018, 52, 963-970. [CrossRef]

49. Nikolaidis, A.K.; Achilias, D.S.; Karayannidis, G.P. Effect of the type of organic modifier on the polymerization kinetics and the properties of poly(methyl methacrylate)/organomodified montmorillonite nanocomposites. Eur. J. 2012, 48, 240-251. [CrossRef]

50. Nikolaidis, A.K.; Koulaouzidou, E.A.; Achilias, D.S. Synthesis and characterization of novel organomodified nanoclays for application in dental materials. Curr. Nanosci. 2018, 14, 1-12. [CrossRef]

51. Watts, D.; Marouf, A. Optimal specimen geometry in bonded-disk shrinkage-strain measurements on light-cured biomaterials. Dent. Mater. 2000, 16, 447-451. [CrossRef]

52. Watts, D.; Marouf, A.; Al-Hindi, A. Photo-polymerization shrinkage-stress kinetics in resin-composites: methods development. Dent. Mater. 2003, 19, 1-11. [CrossRef] 
53. Al Sunbul, H.; Silikas, N.; Watts, D.C. Polymerization shrinkage kinetics and shrinkage-stress in dental resin-composites. Dent. Mater. 2016, 32, 998-1006. [CrossRef]

54. Rueggeberg, F.; Hashinger, D.; Fairhurst, C. Calibration of FTIR conversion analysis of contemporary dental resin composites. Dent. Mater. 1990, 6, 241-249. [CrossRef]

55. Jlassi, K.; Chandran, S.; Mičušík, M.; Benna-Zayani, M.; Yagci, Y.; Thomas, S.; Chehimi, M.M. Poly(glycidyl methacrylate)-grafted clay nanofiller for highly transparent and mechanically robust epoxy composites. Eur. J. 2015, 72, 89-101. [CrossRef]

56. Jlassi, K.; Kurup, S.C.C.; Poothanari, M.A.; Benna-Zayani, M.; Thomas, S.; Chehimi, M.M. Clay/polyaniline hybrid through diazonium chemistry: conductive nanofiller with unusual effects on interfacial properties of epoxy nanocomposites. Langmuir 2016, 32, 3514-3524. [CrossRef] [PubMed]

57. Campos, L.M.P.; Boaro, L.C.; Santos, T.M.R.; Santos, V.J.; Grecco-Romano, R.; Santos, M.J.; Parra, D.F. Polymerization shrinkage evaluation in experimental dental composite filled with montmorillonite nanoparticles. J. Therm. Anal. Calorim. 2018, 131, 771-774. [CrossRef]

58. Halvorson, R.H.; Erickson, R.L.; Davidson, C.L. The effect of filler and silane content on conversion of resin-based composite. Dent. Mater. 2003, 19, 327-333. [CrossRef]

59. Wilson, K.S.; Zhang, K.; Antonucci, J.M. Systematic variation of interfacial phase reactivity in dental nanocomposites. Biomaterials 2005, 26, 5095-5103. [CrossRef] [PubMed]

60. Wilson, K.S.; Antonucci, J.M. Interphase structure-property relationships in thermoset dimethacrylate nanocomposites. Dent. Mater. 2006, 22, 995-1001. [CrossRef]

61. Gonçalves, F.; Kawano, Y.; Pfeifer, C.; Stansbury, J.W.; Braga, R.R. Influence of BisGMA, TEGDMA, and BisEMA contents on viscosity, conversion, and flexural strength of experimental resins and composites. Eur. J. Oral Sci. 2009, 117, 442-446. [CrossRef]

62. Sideridou, I.D.; Karabela, M.M. Effect of the structure of silane-coupling agent on dynamic mechanical properties of dental resin-nanocomposites. J. Appl. Polym. Sci. 2008, 110, 507-516. [CrossRef]

63. Discacciati, J.A.C.; Oréfice, R.L. Structural analysis on photopolymerized dental resins containing nanocomponents. J. Mater. Sci. 2007, 42, 3883-3893. [CrossRef]

64. Cuevas-Suárez, C.E.; Pimentel-García, B.; Rivera-Gonzaga, A.; Álvarez-Gayosso, C.; Ancona-Meza, A.L.; Grazioli, G.; Zamarripa-Calderón, E. Examining the Effect of Radiant Exposure on Commercial Photopolimerizable Dental Resin Composites. Dent. J. 2018, 6, 55. [CrossRef]

65. Leprince, J.G.; Palin, W.M.; Vanacker, J.; Sabbagh, J.; Devaux, J.; Leloup, G. Physico-mechanical characteristics of commercially available bulk-fill composites. J. Dent. 2014, 42, 993-1000. [CrossRef]

66. Achilias, D.S. A Review of Modeling of Diffusion Controlled Polymerization Reactions. Macromol. Theory Simul. 2007, 16, 319-347. [CrossRef]

67. Achilias, D.S.; Verros, G.D. Modeling of diffusion-controlled reactions in free radical solution and bulk polymerization: Model validation by DSC experiments. J. Appl. Sci. 2010, 116, 1842-1856. [CrossRef]

68. Verros, G.; Latsos, T.; Achilias, D.; Achilias, D. Development of a unified framework for calculating molecular weight distribution in diffusion controlled free radical bulk homo-polymerization. Polymer 2005, 46, 539-552. [CrossRef]

69. Verros, G.D.; Achilias, D.S. Modeling gel effect in branched polymer systems: Free-radical solution homopolymerization of vinyl acetate. J. Appl. Sci. 2009, 111, 2171-2185. [CrossRef]

70. Menezes, L.R.; Silva, E.O. The Use of montmorillonite clays as reinforcing fillers for dental adhesives. Mater. Res. 2016, 19, 236-242. [CrossRef]

71. Ratanajanchai, M.; Tanwilai, D.; Sunintaboon, P. Visible light-induced surfactant-free emulsion polymerization using camphorquinone/tertiary amine as the initiating system for the synthesis of amine-functionalized colloidal nanoparticles. J. Colloid Interface Sci. 2013, 409, 25-31. [CrossRef]

72. Tsagkalias, I.S.; Manios, T.K.; Achilias, D.S. Effect of Graphene Oxide on the Reaction Kinetics of Methyl Methacrylate In Situ Radical Polymerization via the Bulk or Solution Technique. Polymers 2017, 9, 432. [CrossRef] [PubMed]

73. Ilie, N.; Durner, J. Polymerization kinetic calculations in dental composites: a method comparison analysis. Clin. Oral Investig. 2014, 18, 1587-1596. [CrossRef] [PubMed]

74. Kelly, P.; Akelah, A.; Qutubuddin, S.; Moet, A. Reduction of residual stress in montmorillonite/epoxy compounds. J. Mater. Sci. 1994, 29, 2274-2280. [CrossRef] 
75. Salahuddin, N.; Shehata, M. Polymethylmethacrylate-montmorillonite composites: preparation, characterization and properties. Polymer 2001, 42, 8379-8385. [CrossRef]

76. Hussain, F.; Chen, J.; Hojjati, M. Epoxy-silicate nanocomposites: Cure monitoring and characterization. Mater. Sci. Eng. A 2007, 445, 467-476. [CrossRef]

77. Qu, X.; Guan, T.; Liu, G.; She, Q.; Zhang, L. Preparation, structural characterization, and properties of poly(methyl methacrylate)/montmorillonite nanocomposites by bulk polymerization. J. Appl. Sci. 2005, 97, 348-357. [CrossRef]

78. Noh, M.W.; Lee, D.C. Synthesis and characterization of PS-clay nanocomposite by emulsion polymerization. Polym. Bull. 1999, 42, 619-626. [CrossRef]

79. Atai, M.; Pahlavan, A.; Moin, N. Nano-porous thermally sintered nano silica as novel fillers for dental composites. Dent. Mater. 2012, 28, 133-145. [CrossRef]

80. Bee, S.-T.; Hassan, A.; Ratnam, C.T.; Tee, T.-T.; Sin, L.T.; Hui, D. Dispersion and roles of montmorillonite on structural, flammability, thermal and mechanical behaviours of electron beam irradiated flame retarded nanocomposite. Compos. Part B: Eng. 2014, 61, 41-48. [CrossRef]

(C) 2019 by the authors. Licensee MDPI, Basel, Switzerland. This article is an open access article distributed under the terms and conditions of the Creative Commons Attribution (CC BY) license (http://creativecommons.org/licenses/by/4.0/). 
Article

\title{
Polyamine-Based Organo-Clays for Polluted Water Treatment: Effect of Polyamine Structure and Content
}

\author{
Cinzia Cristiani ${ }^{1, *}$, Elena Maria Iannicelli-Zubiani ${ }^{1}$, Giovanni Dotelli ${ }^{1}$, Elisabetta Finocchio ${ }^{2, *}$, \\ Paola Gallo Stampino ${ }^{1}$ and Maurizio Licchelli ${ }^{3}$ \\ 1 Politecnico di Milano, Dipartimento di Chimica, Materiali e Ingegneria Chimica "Giulio Natta," Piazza \\ Leonardo Da Vinci 32, 20133 Milano, Italy; elenamaria.iannicelli@polimi.it (E.M.I.-Z.); \\ giovanni.dotelli@polimi.it (G.D.); paola.gallo@polimi.it (P.G.S.) \\ 2 Università di Genova, Dipartimento di Ingegneria Civile, Chimica e Ambientale, Via all'Opera Pia 15, \\ 16145 Genova, Italy \\ 3 Università degli Studi di Pavia, Dipartimento di Chimica, Via Taramelli 12, 27100 Pavia, Italy; \\ maurizio.licchelli@unipv.it \\ * Correspondence: cinzia.cristiani@polimi.it (C.C.); elisabetta.finocchio@unige.it (E.F.); \\ Tel.: +39-02-23993248 (C.C.); +39-010-3352919 (E.F.)
}

Received: 27 March 2019; Accepted: 13 May 2019; Published: 16 May 2019

\begin{abstract}
Hybrid materials based on clays and polyamines are supposed to be efficient heavy metals sorbents due to the well-known adsorption behaviour of the clay matrix and to the coordination properties of un-protonated amino groups. For this purpose, a montmorillonite clay was modified with three different aliphatic polyamines: L6 and L10 have a linear structure with six and ten amino groups, respectively, while B14 is a branched polyamine with fourteen amino groups. Initial amine concentration was the main parameter investigated and data were fitted with Langmuir and Freundlich models. Interaction mechanisms between clay and amines were deeply investigated by different experimental techniques such as X-ray powder diffraction (XRD), thermal analysis measurements (DTG), Fourier Transform Infrared Spectroscopy (FT-IR) and diffuse reflectance (NIR) spectroscopy. Experimental results showed that the amount of amines efficiently immobilized in the solid phase can be increased by increasing the initial concentration of polyamines in the clay modification process. These data were best fitted by Freundlich model, indicating a presence of surface sites of different nature. In the resulting hybrid materials, neither the accessibility of the $\mathrm{NH} / \mathrm{NH}_{2}$ groups of the amines, nor the accessibility of the structural $\mathrm{OH}$ of the clay was hindered. Several preliminary tests in La ions' uptake and release from aqueous solution were also carried out. In the conditions used for this study, total metal ion removal was achieved at sufficiently low linear amine loadings (i.e., $0.45 \mathrm{mmol} / \mathrm{g}_{\text {clay }}$ for the small $\mathrm{L} 6$ amine), suggesting that these hybrid materials are promising for the proposed application in environmental remediation.
\end{abstract}

Keywords: organo-clays; polyamines; clay-amine interaction mechanisms; structure effects; la uptake and release

\section{Introduction}

In recent years, water issues have progressively gained prominence in view of water relationship with the three pillars of a sustainable development: that is, economic, social and environmental [1]. It is an undeniable fact that unsustainable development pathways have generated immense pressures on water resources, affecting its quality and availability and the Earth's capacity to sustain the growing demands for freshwater is being challenged [2,3]. Water, indeed, is also a key resource for industrial and manufacturing processes (e.g., heating, cooling, cleaning, rinsing, etc.) but wastewater that is generated can cause environmental damage when it is discharged without any treatment. Industrial 
pollutants in water generally include heavy metals and /or huge amounts of organic pollutants whose removal is mandatory indeed but requires the application of complex and expensive processes [4]. The complexity and the cost of the treatments are much more pressing in case of very strict disposal limits, low concentration of the contaminants and/or contaminants hard to be removed.

Conventional techniques proposed for this purpose, such as ion exchange, precipitation, electrodialysis, reverse osmosis, ultrafiltration, flocculation, biosorption, adsorption and so forth $[5,6]$ in some cases are not effective to treat complex and complicated polluted wastewaters or they are too expensive. For instance, conventional water treatment processes are not able to adequately address the removal of a wide spectrum of heavy metals (e.g., $\mathrm{Cd}, \mathrm{Cr}, \mathrm{Cu}, \mathrm{Ni}, \mathrm{As}, \mathrm{Pb}$ and $\mathrm{Zn}$ ), in particular, when they are present in complex mixtures [7].

Numerous approaches have been explored to develop cheaper and more effective technologies. Among these purification methods, the adsorption process, using adequate sorbents, is considered one of the most efficient and economical techniques in view of its simple design and facile handling [8] and the search for low-cost adsorbents with strong metal-binding capacities has been pursued. Consequently, sorption, particularly Solid Phase Extraction (SPE), has become one of the most promising alternative treatments technologies, being simple and potentially low cost. Many different solid adsorbents, such as materials of mineral, organic or biological origin, zeolites, industrial by-products, agricultural wastes, biomass and polymeric materials have been proposed and studied in the literature [9].

In this respect, the use of natural materials as sorbents has gained significant interest in recent years, mainly due to their high selectivity for certain heavy metal ions, their low cost and potential to be more environmentally friendly [10-12]. "Green adsorbents," however, are expected to possess lower adsorption capacity than the super-adsorbents reviewed in the literature, thus a strong improvement of their properties is required to make them competitive. In the above "scenario," clays have been suggested as a "green" alternative being characterized by low cost, high mechanical intensity, good tolerance towards harsh chemical environment, convenient solid-liquid separation and excellent reusability $[13,14]$. More recently, the use of natural clays in treating rare earths-containing wastewaters has been reported [15]. Montmorillonites were selected as sorbent in view of their excellent properties, including high cation exchange capacity, swelling behaviour, adsorption properties and large surface areas $[15,16]$. These clays, however, cannot efficiently remove huge amounts of pollutants and show a negligible selectivity towards the different metal ions. Therefore, layered inorganic-organic hybrid materials (organo-clays) have been proposed in various applications [17-19]. Indeed, montmorillonites can be easily modified by some intercalating agents, which can be allocated in the clay interlayer via a combination of ionic and weaker forces [20,21]. Some Authors, [22], with the final target of Rare Earths (RE) recovery, demonstrated that the use of organo-clays has positive effects on the capture of La ions. They investigated the intercalation of two different montmorillonites with $N$-(methoxy-polyethylene glycol) ethylene diamine. Such a polymer was selected for being characterized by a polyethylene-glycol chain, able to intercalate in the clay interlayer [23] and by two amino groups, already reported in the literature, able to interact and remove heavy metal ions [24,25]. Preparation procedure was deeply investigated at different polyamine concentrations and operating conditions [26]. It was found that the intercalation mechanism was mainly dependent on the $\mathrm{pH}$ of the preparation procedure, which affects the protonation of the amino groups. At alkaline $\mathrm{pH}$, the interaction was mainly based on weak bonds between the free amino groups of the organic moiety and the clay matrix and the obtained organo-clays were much more effective in La uptake than pristine clay. So, as general remark, the clay-polymer system can be considered promising for the here proposed application.

Starting from these data, in the present paper new organo-clay systems are assessed where montmorillonite is modified with polyamines characterized by longer chain length (i.e., larger number of amino groups) or more complex structures (i.e., more steric hindrance). By the use of multi-technique characterization approach (i.e., X-ray powder diffraction, $\mathrm{XRD}$, thermal analyses measurements, TGA-DTG, Fourier Transform Infrared Spectroscopy, FT-IR and Diffuse Reflectance Near Infrared Spectroscopy, DR-NIR) the interaction mechanisms occurring between the clay matrix 
and the polyamines are proposed. The final goal is to identify the best operating conditions, with respect to the chemical nature of the polyamine, to prepare organo-clay system characterized by high metal capture capability and selectivity in wastewaters treatment. Finally, the prepared materials were tested in La ions uptake to assess their usability in the field of environmental water remediation.

\section{Materials and Methods}

\subsection{Materials}

A natural smectite clay was used as sorbent, namely a Ca-montmorillonite (STx-1b, The Clay Minerals Society, STx hereafter). From the supplier datasheet, the chemical composition of the clay is ${ }^{\mathrm{IV}} \mathrm{Si}_{4.0}{ }^{\mathrm{VI}}\left(\mathrm{Al}_{1.21} \mathrm{Fe}^{3+}{ }_{0.05} \mathrm{Mg}^{2+}{ }_{0.36} \mathrm{Ti}_{0.02}\right)^{\mathrm{XII}}\left(\mathrm{Ca}_{0.14} \mathrm{Na}_{0.02} \mathrm{~K}_{0.01}\right) \mathrm{O}_{10}(\mathrm{OH})_{2}$ and the measured Cation Exchange Capacity (CEC) is $89 \mathrm{meq} / 100 \mathrm{~g}$.

Three different polyamines were used as clay modifiers. Polyamines are characterized by several amino group numbers and/or more hindered structures. In particular, two linear ethylene-based amines with different chain length (L6 and L10) and a branched amine (B14) were considered (Table 1, where chemical structures and labels used within the text are summarized).

Table 1. Structures, number of amino groups (n) and labels of the different amines.

\begin{tabular}{|c|c|c|}
\hline Structure & Number of Amino Groups & Label \\
\hline $\mathrm{H}_{2} \mathrm{~N}^{\prime}$ & 6 & L6 \\
\hline $\mathrm{H}_{2} \mathrm{~N}^{\top}$ & 10 & L10 \\
\hline
\end{tabular}

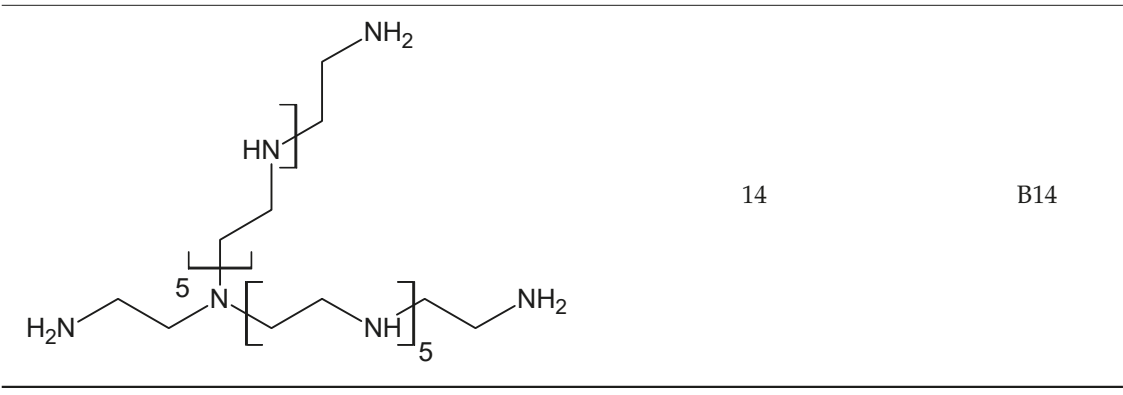

Polyamine L6 technical grade was supplied by Sigma Aldrich; L10 and B14 were prepared according to literature procedure [27].

\subsection{Organo-Clays Preparation}

The preparation of the organo-clays was performed according to a procedure developed elsewhere [28], which implied the mixing of the clay with the aqueous polyamine solution, in a jacketed reactor under vigorous stirring ( $500 \mathrm{rpm}$ ), for $90 \mathrm{~min}$ at controlled temperature of $30^{\circ} \mathrm{C}$. This temperature and time were demonstrated to be the proper ones for the effective amine immobilization, while amine concentration was the main parameter to be considered to affect the intercalation process [23].

The $\mathrm{pH}$ of the solutions was measured before and after the reaction using a Mettler Toledo FE20/EL20 digital pH-meter (Mettler Toledo, Italy). All the polyamine solutions were highly basic, presenting a natural $\mathrm{pH}$ of about 11 and no variation of this parameter was detected during the mixing reaction. 
At the end of the reaction, the solid and liquid phases were separated by centrifugation (3500 rpm for $15 \mathrm{~min}$, RotoFix 32 centrifuge HETTICH, Tuttlingen, Germany). The dried solid (one day at r.t.), was ground in a mortar and fully characterized.

These measurements could be affected by underestimation due to the entrapment in the interlayer of polymers combustion residues.

According to the literature, quantitative evaluation of the amine contents was performed by Chemical Oxygen Demand (COD) $[23,28]$ using a Spectrodirect Lovibond instrument (Tintometer Gmbh, Dortmund, Germany). Measurements were performed according to Reference [29]. Amine content in the solid phase $\left(C_{\mathrm{s}}\right)$ was calculated by difference between the initial $\left(C_{0} \mathrm{mmol} / \mathrm{g}\right)$ and the residual polyamine amount in the liquid phase $\left(C_{\text {res }} \mathrm{mmol} / \mathrm{g}\right)$ according to Equation (1):

$$
C_{\mathrm{s}}=C_{0}-C_{\text {res }}
$$

After the interlayer reaction, also the presence of $\mathrm{Ca}$, that is, the clay interlayer cation and $\mathrm{Mg}$, leached from clay layers or present as impurity, was monitored in the solution. $\mathrm{Ca}$ and $\mathrm{Mg}$ were measured by Inductively Coupled Plasma-Optical Emission Spectroscopy (ICP-OES) analyses using a Perkin Elmer Optima 2000DV spectrometer, estimated error within 1\%).

Throughout the text, all the results of chemical analysis will be referred to $1 \mathrm{~g}$ of clay.

In Table 2, labels of the samples, initial amine solution concentration $(\mathrm{mM})$ and $C_{0}$-initial mmoles of polyamine per $\mathrm{g}$ of clay-are summarized.

Table 2. Sample labels and initial contacted amine concentration used in the clay modification experiments.

\begin{tabular}{ccc}
\hline Sample (Label) & $\begin{array}{c}\text { Amine Solution Initial } \\
\text { Concentration }(\mathbf{m M})\end{array}$ & $\begin{array}{c}\text { Initial Contacted Amine } C_{0} \\
\left(\mathbf{m m o l} / \mathrm{g}_{\text {clay }}\right)\end{array}$ \\
\hline L6-25 & 25 & 0.5 \\
L6-70 & 70 & 1.5 \\
L6-135 & 135 & 3.1 \\
L6-175 & 175 & 4.0 \\
\hline L10-15 & 15 & 0.3 \\
L10-25 & 25 & 0.6 \\
L10-35 & 35 & 0.8 \\
L10-60 & 60 & 1.4 \\
L10-90 & 90 & 2.1 \\
\hline B14-15 & 15 & 0.3 \\
B14-25 & 25 & 0.6 \\
B14-40 & 40 & 0.9 \\
B14-90 & 90 & 2.0 \\
\hline
\end{tabular}

\subsection{Organo-Clays Characterizations}

X-ray powder diffraction (XRD) patterns were recorded with a Bruker D8 Advance diffractometer using a graphite-monochromated $\mathrm{Cu} \mathrm{K} \alpha$ radiation; the scan step was $0.02^{\circ} 2 \theta$ and the measurement time was $1 \mathrm{~s}$ per step. The XRD line profile analysis was performed with TOPAS P 2.1 software (Bruker AXS, Karlsruhe, Germany) using a Pearson VII profile function, after background subtraction. The calculated profiles were used for the determination of basal spacing $\left(\mathrm{d}_{001}\right)$ of the organo-clays.

Thermal analyses measurements (TG-DTG) were performed with a DTA-TG SEIKO 6300 thermal analyser. The experiments were carried out in flowing air in the temperature range of $25-1000{ }^{\circ} \mathrm{C}$, with heating rate of $10^{\circ} \mathrm{C} / \mathrm{min}$.

FT-IR spectra were recorded with a Jasco mod. 615 spectrometer and with a FT-IR Thermo Nicolet 380 Avatar (Thermo Electron Corporation, Madison, WI, USA) using the KBr pressed disk technique (mid-IR region). 
Diffuse reflectance NIR spectra of pure powders have been obtained in air by a Jasco V570 UV-vis-NIR instrument (Jasco-Europe SRL, Jasco ${ }^{\text {TM }}$ Software) and reported in Kubelka-Munk units in the range $9000-4000 \mathrm{~cm}^{-1}$.

\subsection{Metal Ions Uptake and Release Tests}

$\mathrm{La}\left(\mathrm{NO}_{3}\right)_{3}$ solutions were selected as model systems. La ions uptake and release experiments were performed according to literature $[15,26,28]$. The use of concentrated solutions was considered in order to demonstrate the feasibility of the method in industrial applications.

Briefly, weighted amounts of organo-clays were contacted under stirring with model solutions $19 \mathrm{mM}$ concentration for $90 \mathrm{~min}$, solid/liquid ratio $=0.04 \mathrm{~g} / \mathrm{mL}$. $\mathrm{pH}$ of the contacting solution was in the range $5-6$, that is, $\mathrm{pH}$ of the starting La solution before contacting. Solid and liquid were separated by centrifugation (HETTICH 32 RotoFix).

The flowchart of the uptake/release procedure is reported in Figure 1.

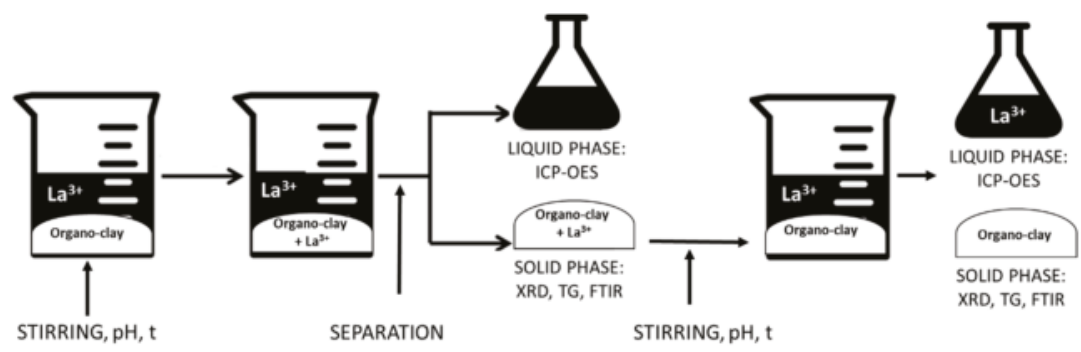

Figure 1. Flowchart of the UPTAKE/RELEASE process.

La ions content in the solutions, (mother, after uptake and after release), was determined by ICP-OES (Perkin Elmer Optima 2000DV spectrometer).

Captured ions were determined according to Equation (2):

$$
q_{t}=\frac{\left(C_{0}-C_{t}\right) V}{W}
$$

where $q_{t}(\mathrm{mmol} / \mathrm{g})$ is the adsorbed metal ion at time $t, C_{0}$ and $C_{t}$ are the initial and the residual metal concentrations in solution $(\mathrm{mmol} / \mathrm{L})$ respectively, $V$ is the volume of solution $(\mathrm{L})$ and $W$ is the organo-clay mass (g).

Release tests were performed by contacting the La-containing organo-clays with $\mathrm{HNO}_{3}$ solution at $\mathrm{pH} 1$ under continuous stirring at room temperature, for $1.5 \mathrm{~h}$, with a solid/liquid ratio of $0.026 \mathrm{~g} / \mathrm{mL}$ [30].

Released ions $q_{w}$ (mmol/g) were calculated according to Equation (3), where $C_{w}$ is the metal concentration in solution $(\mathrm{mmol} / \mathrm{L})$ after release (determined by ICP-OES).

$$
q_{w}=\frac{\left(C_{w}\right) V}{W}
$$

$V$ is the volume of the solution (L) and $W$ is the organo-clay mass (g).

\section{Results and Discussion}

\subsection{Characterization of Modified Clays}

In Figure 2 polyamine contents $\left(C_{\mathrm{s}}\right)$ obtained at different initial amine concentrations are reported. The extent of the intercalation reaction is clearly irrespective of amine structure and molecular weight. The asymptotic behaviour of Figure 2 well fits all the data. The plateau value is reached, by all the 
systems, for $C_{0}$ higher than $0.3 \mathrm{mmol} / \mathrm{g}_{\text {anhydrous clay }}$ and this value represents the maximum loadable polyamine in STx clay in these conditions.

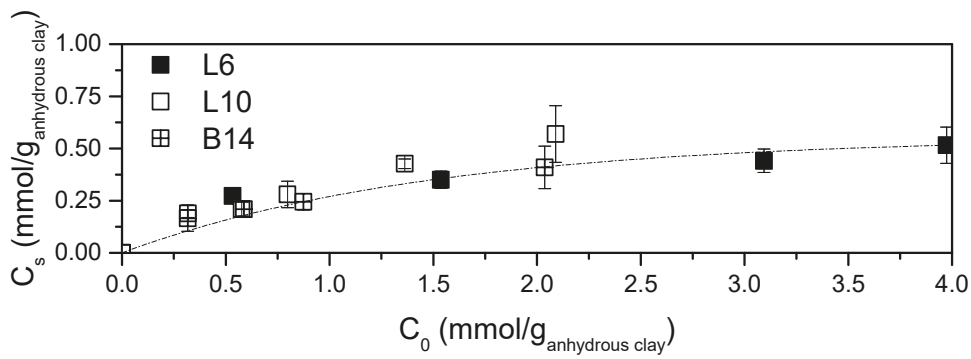

Figure 2. Amine content in the solids as a function of the initial contacted amine.

Considering that the intercalation reaction was performed at $\mathrm{pH}=11$, that is, $\mathrm{pH}$ of the polymer solutions, it can be assumed that polyamines interact with the clay in their neutral form and no quaternary ammonium ions should be present in the organo-clays.

Modified-clays were fully characterized to get information on the polymer-clay interaction.

X-Ray diffractograms of the samples are reported in Figure 3a, where pristine clay (STx) is also reported for comparison.

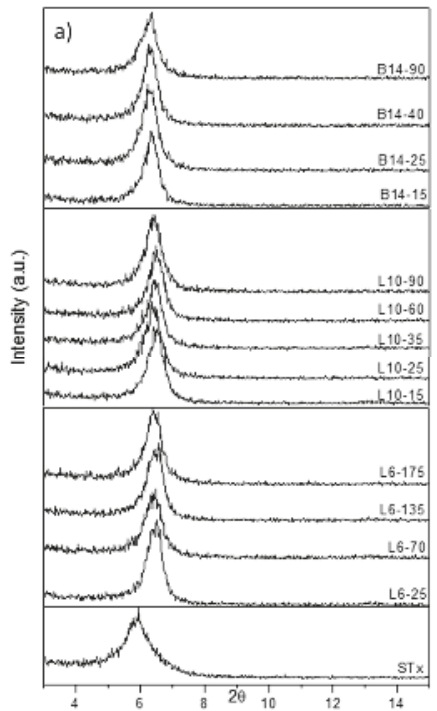

b)

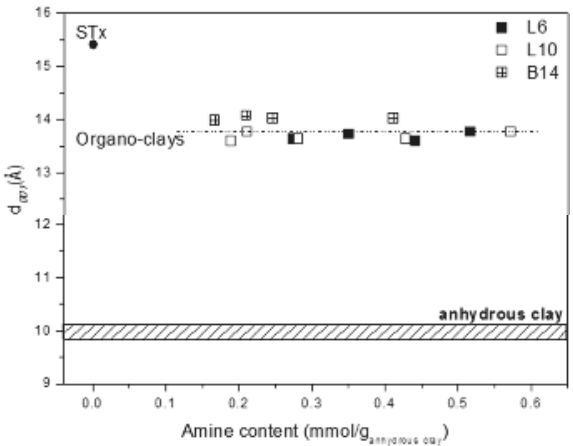

Figure 3. (a) X-ray diffraction (XRD) analysis of pristine and modified clays prepared at different initial amine concentrations and $(\mathbf{b})$ Basal spacing $\left(\mathrm{d}_{001}\right)$ as a function of the amines content of modified clays, (for sake of comparison, $\mathrm{d}_{001}$ of fully hydrated and dehydrated STx are reported).

A displacement of the basal reflection from the original STx towards higher angles is evident, which corresponds to a contraction of the interlayer spacing $\mathrm{d}_{001}$ from 15.4 to $13.5-14 \AA$ for all the organo-clays, regardless the amines structure and content.

To better clarify this point, in Figure $3 \mathrm{~b} \mathrm{~d}_{001}$ are plotted as a function of the corresponding amine content. For sake of comparison, also pristine STx and pristine STx fully dehydrated are also reported. 
It is evident that all the $\mathrm{d}_{001}$ of the modified-clays are very close and their values are intermediate between pristine-fully hydrated and pristine-fully dehydrated clays.

The observed interlayer shrinkage, calculated from XRD, is an indication of the presence of the polyamine interacting with clay interlayer [31]; indeed, no other effect than polymer-clay interaction should be present in this case. However, usually, the accommodation of large organic molecules, such as polyethylenglycols PEG [23,32], inside the clay has been reported to cause interlayer enlargement [33-35]. A contraction of the interlayer spacing has been reported in the literature for the intercalation, via ion exchange mechanism, of a polycationic quaternary amine polymer [31]. Nevertheless, in the considered samples, no cation exchange is expected: intercalation reaction was performed at the amine original $\mathrm{pH}$ of 11 , that is, basic enough to prevent any protonation of the amino-groups or any protonation of the clay interlayers. To verify this point, ICP analyses were performed on the solution after the polyamine-clay contacting reaction: in case of cations exchange the presence of interlayer ions, for example, $\mathrm{Ca}^{2+}$, has to be found. As expected, no $\mathrm{Ca}^{2+}$ ions were found after intercalation, thus confirming that no ions exchange has occurred, and amines are intercalated in their neutral form.

A possible explanation for the observed interlayer shrinkage could be found by considering interlayer water molecules. As reported elsewhere for PEG-based systems, the interaction between the intercalated polymers and the clay involves the formation of weak bonds between the polymer chain and the interlayer water molecules that are in turn coordinated to the interlayer cations [23,31,32]. Similar effects can be claimed also in this case. The interlayer contraction, indeed, could imply a water-amine interaction that, in view of the higher affinity of the amino groups for the interlayer cations could result in water displacement. Moreover, interlayer spacing calculated for the organo-clays, are intermediate between fully hydrated- and fully dehydrated-pristine clay, thus supporting this explanation. Thus, the polyamine chain, which possesses a stronger coordination capability towards the interlayer cations, could displace some interlayer water molecules. The replacement of part of the interlayer water molecules with these new "stronger and shorter" coordination bonds could result in the observed contraction. However, a minimum interlayer water content cannot be overcome to prevent either repulsion phenomena between two faced silico-aluminate layers or the structure collapse. This phenomenon can account for the observed $\left(\mathrm{d}_{001}\right)$ constancy in all the modified-clays.

On the contrary, in the case of PEG intercalation [23,32], in view of the polyoxyethylene chains of the polymer, a weaker H-bond interaction between the etheric oxygen of the polymer chain and the interlayer water molecules occurred. Indeed, water displacement was hardly detected and the hindrance effect of the PEG chain prevailed, resulting in an interlayer enlargement.

To confirm this picture, TG analyses of the solids were performed in air: both polyamine decomposition and the water evolution are detectable by this technique.

DTG curves are shown in Figure 4a. Once more, very similar behaviours were observed for all the different samples.

Three different ranges of temperature can be identified: (1) $100-120^{\circ} \mathrm{C}$ corresponding to water evolution; (2) $150-250{ }^{\circ} \mathrm{C}$ corresponding to amine decomposition; (3) $300-450{ }^{\circ} \mathrm{C}$ corresponding to decomposition of combustion residue and clay collapse.

The above thermal phenomena are discussed below in details. The thermal phenomenon up to $100-120^{\circ} \mathrm{C}$ is present in both pristine and all the modified clays. It accounts for water evolution. However, broader decomposition patterns, accompanied in some case by shoulders, are found in organo-clays with respect to the pristine one. In case of modified-clays, water evolution corresponds to sample water content, mainly interlayer water molecules. Indeed, organo-clays after intercalation were dried at room temperature for one day, conditions that are too mild to modify the interlayer water content that is, therefore, preserved. However, as discussed above, "different" interlayer water molecules are present in the materials, some of them interacting with the clay interlayer cations and some others interacting with the polyamine chain. These last water molecules, in view of their stronger interactions, evolve at temperatures that are slightly different from the others. Therefore, this heterogeneity of bond strength can explain broadness and complexity of the first thermal decomposition. 
Water losses were quantified and plotted as a function of the total intercalated polyamine content (Figure $4 b$ ). Very similar water losses were found for all the samples, irrespective of the polyamine content or structure and definitely lower if compared to the unmodified clay.

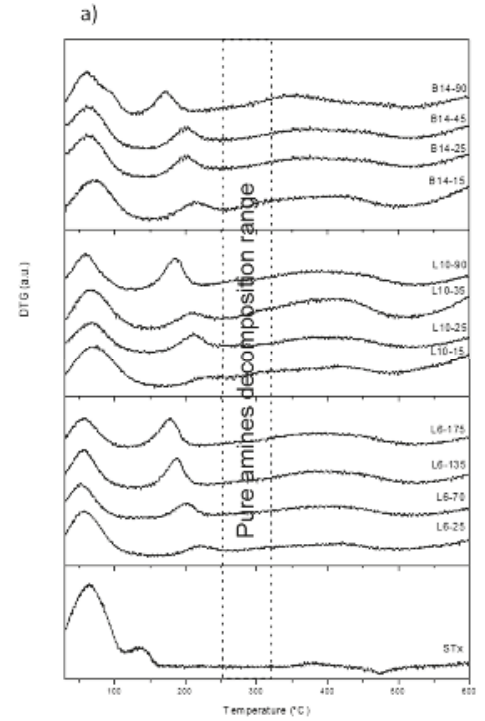

b)

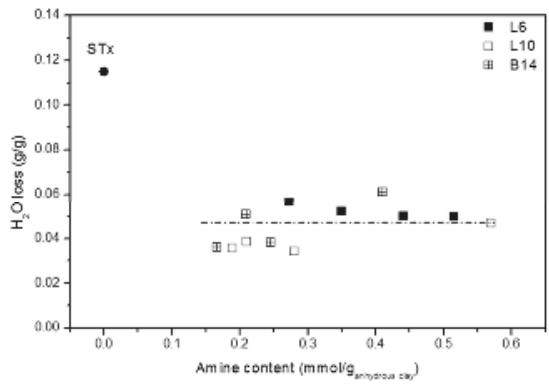

Figure 4. (a) DTG curves of pristine and modified-clays prepared at different polyamine content; and (b) Water loss (calculated from thermal decomposition) as a function of the amine content for pristine and modified clays.

This behaviour is in line with $\mathrm{d}_{001}$ reduction (compared to the unmodified STx) and constancy (for all the modified clays) and confirms that XRD findings were related to interaction of interlayer water and intercalated polyamine. Moreover, it also strongly supports the presence of the threshold amount of water that has to be retained to preserve the clay structure.

Thermal phenomenon at $150-250^{\circ} \mathrm{C}$, not detected in the pristine clay, is attributed to the intercalated polyamines decomposition, which in pure amines are observed at about $300{ }^{\circ} \mathrm{C}$. With respect to the free amines, in the organo-clays the decomposition is anticipated of about $100{ }^{\circ} \mathrm{C}$. In addition, a progressive decrease of the decomposition temperature was observed on increasing amine content in the solid. In line with literature indication, anticipation of polymer decomposition is attributed to the effect of the polymer-clay interaction [23].

Moreover, the last thermal phenomenon, detected between 300 and $450{ }^{\circ} \mathrm{C}$, also in this case is possibly related to decomposition of pitch-like residues of combustion of the polyamine [23]. Such residues trapped inside the clay thus require higher temperature to be decomposed. [23,32].

IR spectra of the L6-70 material as pure powder were also recorded at increasing temperatures from room temperature to $400^{\circ} \mathrm{C}$ in air (Supplementary Material, Figure S1). Pure powder spectra show very strong and noisy bands due to the high concentration of the organic loading. Nevertheless, two broad and ill-defined absorptions are detected, characterizing the amine molecules and corresponding to $\mathrm{CH}$ stretching modes (3000-2800 $\left.\mathrm{cm}^{-1}\right)$ and NH stretching modes $\left(3300-3200 \mathrm{~cm}^{-1}\right)$ as detailed in the following. Their intensity strongly decreases upon heating up to $200{ }^{\circ} \mathrm{C}$ and above, as further confirmation that organic moieties are mainly responsible of the weight loss detected in this temperature range by TG studies.

Therefore, from XRD and DTG combined information, it is possible to state that all the polyamines show quite similar interactions with the clay, partially due to intercalation by means of weak bonds and 
partially due to water interactions, suggesting the presence of a multilayer and heterogeneous situation. Despite a good accordance among XRD and TG experimental data, the presence of some polyamine adsorbed at the surface clay platelets cannot be excluded. Therefore, confirmation of the supposed mechanisms was also assessed by skeletal IR spectra. For this purpose, organo-clay containing L6 was selected. Indeed, the L6 structure is simpler than the other polyamines and, as a consequence, data interpretation is expected to be simpler too. Moreover, it is a commercial product, therefore much more suitable for the production of the organo-clays for industrial application. Organo-clays containing increasing amount of L6 polyamine were hence analysed and resulting spectra are reported in Figure 5. Spectrum of the pristine STx material (dashed line) is reported for sake of comparison.

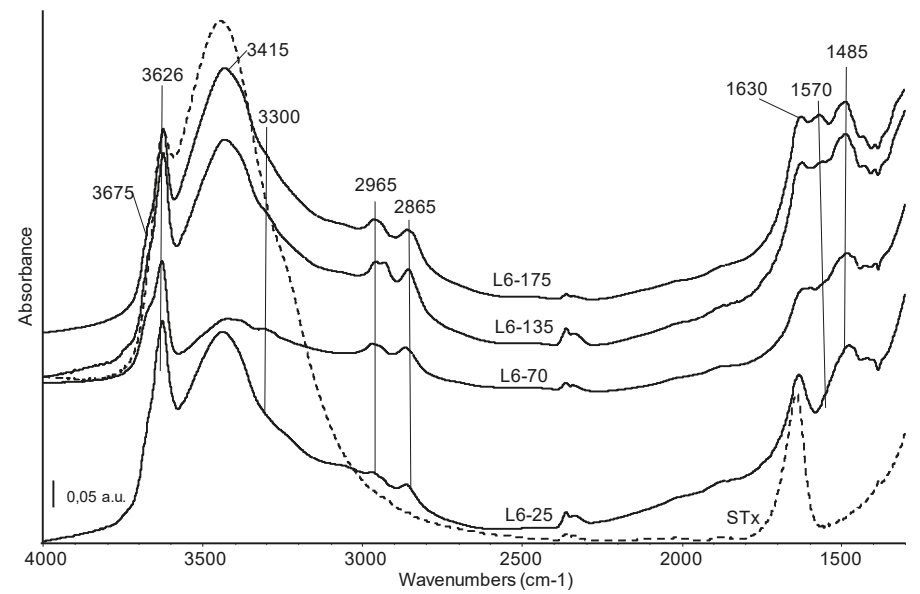

Figure 5. FT-IR skeletal spectra of the organo-clays prepared at different initial L6 amine content (common scale). Broken line: FT IR spectrum of the reference STx material.

Skeletal bands of the pristine montmorillonite material are not affected by the modification with amine and the corresponding spectral region (below $1300 \mathrm{~cm}^{-1}$ ) has not been reported in Figure 5 .

The high frequency region of all the spectra shows bands assigned to $\mathrm{OH}$ stretching modes at $3626 \mathrm{~cm}^{-1}$ (structural $\mathrm{OH}$ groups of montmorillonite, mainly coordinating central $\mathrm{Al}$ atoms) and $3415 \mathrm{~cm}^{-1}$ (water molecules), overlapping the broad band due to water vapour adsorbed on the $\mathrm{KBr}$ matrix [32,36]. The introduction of amines leads to changes in shape and relative intensity of these features. In particular, in this region a decrease in intensity of the band due to water molecule can be detected, especially for the L6-70 sample. The ratio water molecules/structural OH is always slightly lower in the organo-clays than in the pristine STx spectrum and suggests the preferential interaction of amine with water, either adsorbed water either interlayer water, which is partially substituted by the organic molecules. This effect could be considered indirect evidence of some amine intercalation. A weak absorption centred at $3300 \mathrm{~cm}^{-1}$, more evident in the L6-70 spectrum, is assigned to the NH stretching mode of a secondary amino group. Two absorptions, quite complex, at 2965 and $2865 \mathrm{~cm}^{-1}$ correspond to the $\mathrm{CH}$ asymmetric and symmetric stretching modes of the $\mathrm{CH}_{2}$ of the alkyl chain. The complexity of these bands, which are evidently split in the spectrum of the L6-135 sample, is due to the existence of $\mathrm{CH}$ groups having different environment, that is, in the $-\left(\mathrm{CH}_{2}\right)_{2}-$ unit or linked to $-\mathrm{NH}-$ groups. Broadening and splitting of these bands is indeed related to the interaction of the chain with the clay [37]. At lower frequencies, the main bands corresponding to the amine chain are detected at $1570 \mathrm{~cm}^{-1}$, NH deformation mode and at 1480, 1430 and $1390 \mathrm{~cm}^{-1}, \mathrm{CH}$ deformation modes. The relative intensity of the amine band at $1570 \mathrm{~cm}^{-1}$ increases with the increasing amine content in the starting solution, confirming the efficiency of the preparation procedure. Moreover, the detection of this band highlights that amino groups in the organic framework are still "free" after interaction with 
the clay and are therefore available for the uptake of metal ions. As expected, considering the highly basic contacting $\mathrm{pH}$, no amine protonation was detected.

In sum, the immobilization of the organic chain in solid matrix should not hinder the accessibility of the $\mathrm{NH} / \mathrm{NH}_{2}$ groups, which are directly involved in the coordination of metals in environmental remediation applications [24,38].

FT-IR spectra of organo-clays prepared at similar initial amine concentration but with different polyamines are shown in Figure 6 and exploited in the regions of interest.

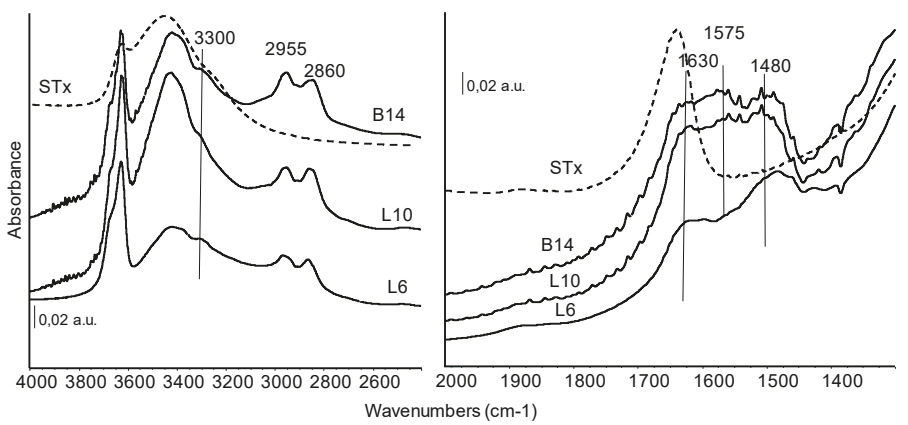

Figure 6. FT-IR skeletal spectra of all the organo-clays samples prepared at similar initial amines molarity. Dashed line: STx reference spectrum.

As discussed in the previous paragraph, in the high frequency region of the spectra, the band centred at $3300 \mathrm{~cm}^{-1} \mathrm{ca}$. is assigned to $\mathrm{NH}$ stretching of the secondary amino groups. The $-\mathrm{NH}_{2}$ vibrational stretching modes fall in the same region, while the corresponding asymmetric stretching absorptions could be masked by the band of H-bound water centred at $3350 \mathrm{~cm}^{-1}$.

Bands at 2955 and $2860 \mathrm{~cm}^{-1}$, are characteristic of the vibrations of $\mathrm{CH}$ bonds in $\mathrm{CH}_{2}$ groups. Correspondingly, the asymmetric $\mathrm{CH}$ deformation mode is detected at about $1480 \mathrm{~cm}^{-1}$ ca quite broad and complex. In this spectral region, also other contributions can actually be identified, although strongly overlapped. The absorptions in the range $1635-1620 \mathrm{~cm}^{-1}$, shifted in wavenumber in comparison to the pristine material, are assigned to deformation modes of water. Another broad absorption growing at $1575 \mathrm{~cm}^{-1}$ is due to $\mathrm{NH}$ deformation mode, slightly stronger in L10 and B14 sample.

The comparison of Figures 5 and 6 showed similar spectral features for the different organo-clays: NH stretching and deformation modes of exposed amino groups, reduced water content of these materials in comparison to the pristine clay, complex $\mathrm{CH}$ stretching and deformation modes slightly perturbed by the interaction with the matrix. As already reported for XRD and TG the presence of only very few and small differences, suggest that similar immobilization mechanisms occurs, in spite of different structure or molecular weight of the considered polyamines.

Diffuse Reflectance-NIR spectra of pristine montmorillonite and of sample L6-135 are reported in Figure 7.

In the pristine clay spectrum, the complex band at $7085 \mathrm{~cm}^{-1}$, with a shoulder at $6865 \mathrm{~cm}^{-1}$, corresponds to the overlapping of the first overtone of vibrational modes of structural $\mathrm{OH}$ stretching and water molecules and to the first overtone of vibrational modes of water molecules strongly bonded through H-bonds. The sharp band centred at $5245 \mathrm{~cm}^{-1}$ and tailing towards lower frequencies has been previously assigned to a $(v+\delta)$ combination mode of water strongly interacting with interlayer cations and with the clay surface [39]. The NIR spectrum of the organo-clay shows a significant decrease in the relative intensity of this band which is also shifted at higher frequencies $\left(5252 \mathrm{~cm}^{-1}\right)$. The modification of this feature suggests that in the studied organoclays the immobilization process affects interlayer water, as also reported by some of us for PEG intercalation in a previous paper [40]. 


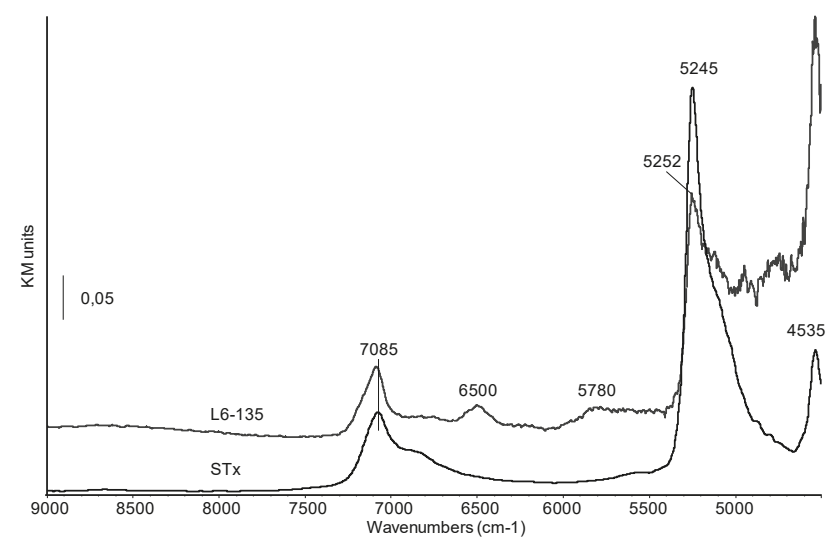

Figure 7. Diffuse Reflectance NIR spectra of STx and organo-clay L6-135.

On the other side, the shift in wavenumbers suggests that the strength of the $\mathrm{H}$-bonds between residual water molecules is also changed, possibly increased [37,41]. The band at $4535 \mathrm{~cm}^{-1}$ is assigned to overtones of structural $\mathrm{OH}$ groups which seem to be only marginally involved in the amine immobilization. Finally, new features appear at 6500 and $5780 \mathrm{~cm}^{-1}$, corresponding to overtones and combination vibrational modes of $-\mathrm{NH}-$ and $-\mathrm{CH}_{2}-$ groups, respectively, thus characterizing the organic chain.

To model the polyamine-clay interaction and trying to support the hypothesis of a complex and heterogeneous situation, the experimental results concerning the polyamines adsorption were interpreted according to Langmuir and Freundlich models [42,43]. It is well known that Langmuir model describes a more homogeneous situation where equal active sites can be occupied only once. On the contrary, Freundlich model is able to describe complex and heterogeneous (multilayer) surfaces.

Langmuir model parameters were calculated according to Equation (4):

$$
\frac{c_{\mathrm{e}}}{q_{\mathrm{e}}}=\frac{c_{\mathrm{e}}}{q_{\max }}+\frac{1}{q_{\max } K_{\mathrm{L}}}
$$

where $q_{\mathrm{e}}(\mathrm{mg} / \mathrm{g})$ is the adsorbed amount at equilibrium, $C_{\mathrm{e}}(\mathrm{mg} / \mathrm{L})$ is the equilibrium concentration, $q_{\max }$ is the maximum monolayer adsorption capacity of the adsorbent and $K_{\mathrm{L}}$ is the Langmuir constant related to the free energy of adsorption.

Freundlich model parameters were obtained according to Equation (5):

$$
\log _{10} q_{\mathrm{e}}=\log _{10} K_{F}+\frac{1}{n} \cdot \log _{10} C_{\mathrm{e}}
$$

where $q_{\mathrm{e}}$ and $C_{\mathrm{e}}$ have the same meaning of Equation (4), while $K_{F}\left(\left(\mathrm{mmol} /\left(\mathrm{g} \cdot(\mathrm{mmol} / \mathrm{L})^{1 / n}\right)\right)\right.$ and $1 / n$ are Freundlich constants related to the adsorption capacity and the adsorption intensity, respectively.

Calculated values of Langmuir and Freundlich constants are compared in Table 3.

Table 3. Langmuir and Freundlich constants calculated from amines adsorption data on STx.

\begin{tabular}{cccc}
\hline Model & $q_{\max }(\mathrm{mmol} / \mathrm{g})$ & $\mathrm{K}_{\mathrm{L}}(\mathrm{L} / \mathrm{mmol})$ & $R^{2}$ \\
\hline Langmuir & 0.47 & 0.08 & 0.71 \\
\hline & $\mathrm{n}$ & $\mathrm{K}_{\mathrm{F}}\left(\mathrm{mmol} /\left(\mathrm{g} \cdot(\mathrm{mmol} / \mathrm{L})^{1 / \mathrm{n}}\right)\right.$ & $\boldsymbol{R}^{2}$ \\
\hline Freundlich & 3.03 & 0.10 & 0.81 \\
\hline
\end{tabular}


Plots of the linearized isotherms evaluated using both models are reported in Figure 8.
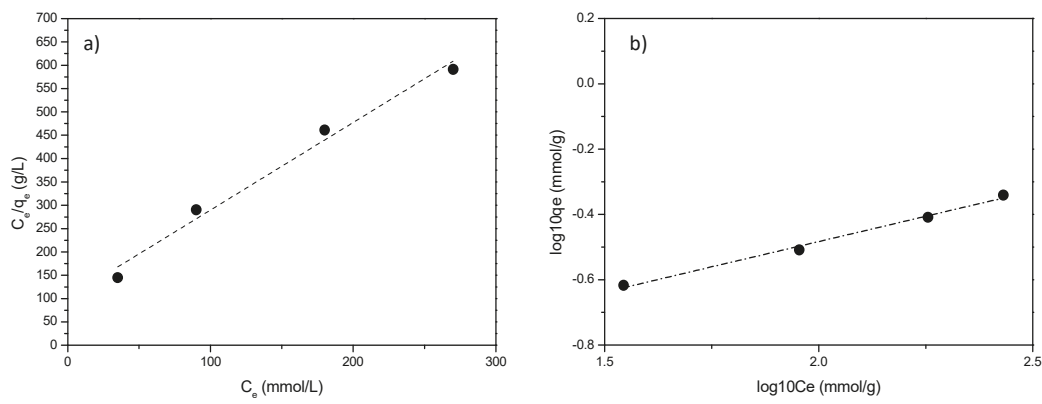

Figure 8. Adsorption isotherms for L6-STx: (a) Langmuir and (b) Freundlich model.

Only small differences were found (Figure 8a,b), however, despite data are only slightly better fitted, Freundlich model is selected as the most proper one to account for the adsorption. This is not surprising, considering that Freundlich empirical model can be applied to multilayer adsorption, with non-uniform distribution of adsorption heat and affinities over sites of different nature, as is the case of the clay here discussed [44]. Freundlich slope, ranging between 0 and 1, is a measure of adsorption intensity or surface heterogeneity: a larger heterogeneity of surface sites is present as the slope value is near to zero. Values of $n>1$ indicate either penetration of a sorbate into a sorbent structure or lateral sorbate-sorbate interactions. In the case here reported, for all the considered polyamines, $n>1$ indicates most probably lateral interactions between polyamine molecules in an adsorbed state [45].

\subsection{Metal Uptake and Release}

Finally, the effect of the nature and the concentration of polyamine on metal capture reaction was performed. A parallel study was performed on the 3 systems, that is, L6, L10 and B14. Uptake reaction at initial La concentration $19 \mathrm{mM}$ (90 min contact time and $\mathrm{pH}$ about 6) was performed using different organo-clays prepared at different polyamine initial concentration (10-90 mM) Results are reported in Figure 9a.
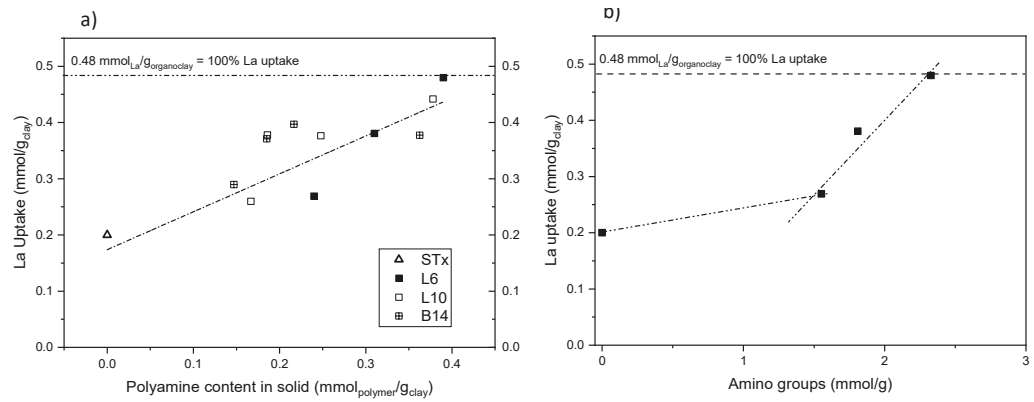

Figure 9. - La uptake as a function of (a) the polyamine content for the different organo-clays and (b) number of amino-groups (STx-L6 and STx-L10). Dashed lines are drawn as guide for the eyes.

All the capture data can be accounted for a linear La uptake up to polyamine content of $0.45 \mathrm{mmol} / \mathrm{g}_{\text {clay }}$. At that value, $100 \%$ of La ions' uptake, that is, total metal ions removal from the solution, was achieved. In particular, the organo-clay functionalized with the smallest amine chain L6 shows good adsorption capacity, that is, $65 \mathrm{mg} / \mathrm{g}$ sorbent, fitting indeed within the adsorption results reported in the open literature for Lanthanum uptake, as very recently reviewed by Iftekhar et al. [46]. 
On average, the uptake reaction is apparently insensitive to the polyamine structure that is, linear or branched and high or low molecular weight, showing a similar linear La uptake. In case of B14-based organo-clay, the lower uptake at increasing amine content is probably due either to lower polyamine content inside the clay or lower availability of the metal coordination sites. Indeed, in view of the highly branched structure of B14, lower polyamine content can be allocated inside the clay and/or the branched chains could interact each other, preventing some coordinating sites. Therefore, this suggests that the capture mechanism could be driven by the number of coordination centres, that is, the number of amino groups in the functionalized clays.

This effect becomes evident when uptake data obtained for L6- and L10-based materials are plotted as a function of the overall number of amino groups, (calculated as the product of the moles of polyamine present in the organo-clay and the number of amino groups per moles of polymer) (Figure 9b). Behaviour of L6- and L10-based materials is so overlapped that the organo-clays becomes indistinguishable, despite of the marked differences between the polyamine structures.

This suggests that the same total amount of amino groups, although included in molecules having different chain length, either linear or branched, has the same metal uptake behaviour. Therefore, shorter and simpler polyamines, easier to handle—for instance, more soluble—can be used without lacking effectiveness in the uptake reaction.

Also release experiments were performed. Knowledge on release mechanism will be useful in case of recovery of the captured metals with their revalorization and re-use as final target. In addition, desorption data could provide additional information to better understand capture mechanism and its relationship with polyamine loading, structure and composition.

The La-enriched organo-clays were thus contacted with solutions at $\mathrm{pH} 1$, value that was proved [47] to be the best to perform the desorption reaction.

Data obtained from release tests are reported in Figure 10, where La release is plotted as a function of La uptake. Also, in the case of release, very close behaviours were found for the different organo-clays. Once more, regardless of polyamine nature, release results can be fitted by a linear plot, where the different systems are indistinguishable.

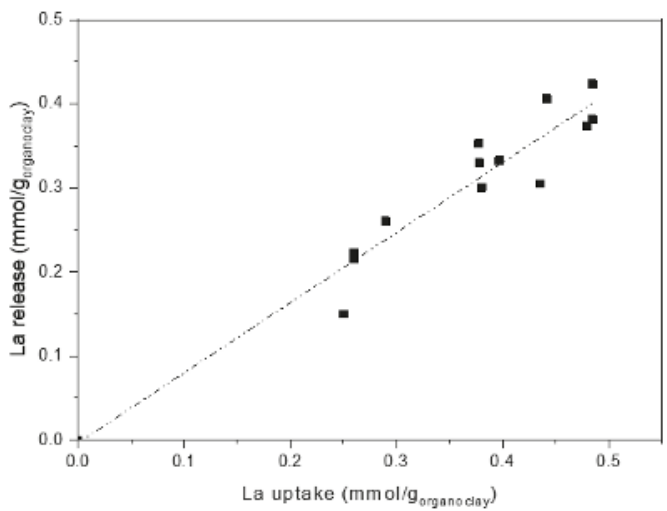

Figure 10. Lanthanum release as a function of lanthanum uptake (Dashed-dotted line: linear fitting).

This behaviour confirms that metal ions uptake is amino-groups dependent only. Indeed, considering release conditions, that is, $\mathrm{pH}=1$, La ions are recovered by replacing them with protons via acidification of the amino groups of the polyamines, whatever their structure. Amino-groups mainly interact with the ions (and not with the clay), therefore are free and easily protonable. The complete conversion of amine to ammonium results in an almost quantitative metal release (90-95\%) that accounts for the linear plot (fitting line near to bisect) observed in Figure 10. 
COD analysis of the solution after both uptake and release tests, evidenced the presence of very limited amount of polyamine, lower than $1 \%$ of the total content. Therefore, data reported on capture and release fit well with the hypothesis of significant polyamine immobilization in the interlayer.

Indeed, a conclusive picture of the clay-polymer interaction cannot be drawn on these bases and possibly a deeper and dedicated investigation by means of other techniques such as TEM and XPS is still required. Evidences from different characterization techniques, such as: a) a slight modification of the $\mathrm{d}_{001}$ observed by XRD; b) the anticipation of the polymer decomposition temperature, due to polymer-clay interaction as already observed by TG for similar systems; c) a decrease of water content due to interlayer water displacement detected both by TG and spectroscopic techniques; e) a negligible amount of polymer dissolved in harsh conditions (i.e., soaking and stirring at $\mathrm{pH} 6$ and 1 for uptake and release test, respectively), accounted for polyamines molecules strongly interacting with the clay matrix. However, it cannot definitely discriminate between intercalated polyamine and/or polyamine strongly bonded to the platelets surface and edges.

\section{Conclusions}

The preparation of organo-clays using polyamines as modifying agents was carried out and the main following conclusions can be drawn:

1. amine content in the solid phase can be increased, increasing the initial amine concentration in solution up to a threshold value;

2. Freundlich isotherm model was found to be the one best fitting the data, meaning the presence of surface sites of different nature. This fact is not surprising because Freundlich isotherm is widely applied in heterogeneous systems especially for organic compounds [45].

3. Common mechanisms occur, in spite of different structures or molecular weights: at $\mathrm{pH} 11$ polyamines are likely intercalated in the clay interlayer, although surface adsorption cannot be ruled out. Both interactions involve preferential interaction of amines with water, either interlayer water either adsorbed water (the first being at least partially substituted by the organic chains).

4. At lower amine content the accessibility of the amino groups of the polyamines is not prevented. Increased amine content leads to a significant interaction between the chains, somehow hindering the availability of the $\mathrm{NH}$ groups, namely for the branched chain. On the other side, amine linear structure does not seem to affect uptake activity. So, shorter and linear polyamines, more easily handled, can be used preferentially

5. In our conditions ( $19 \mathrm{mM}$ initial La concentration, $90 \mathrm{~min}$ contact time and $\mathrm{pH}$ about 6), total $\mathrm{La}^{3+}$ removal was achieved already using the L6 hybrid material at quite low amine loading, that is, $0.45 \mathrm{mmol} / \mathrm{g}_{\text {clay }}$. An almost quantitative ions release was obtained by lowering $\mathrm{pH}$ of the aqueous medium. Thus, uptake and release results suggest that polyamine-based organo-clays are promising materials for the proposed application.

Supplementary Materials: The following are available online at http://www.mdpi.com/2073-4360/11/5/897/s1, Figure S1. FT IR spectra of organo-clay L6-70 pure powder at increasing temperatures. Broken line: pure STx reference spectrum recorded at $100^{\circ} \mathrm{C}$. Arrows: bands assigned to L6 amine.

Author Contributions: Conceptualization and methodology, C.C., E.M.I.-Z., G.D., E.F., P.G.S. and M.L.; investigation, E.M.I.-Z., E.F., P.G.S. and M.L; project administration, C.C.; writing-original draft preparation, C.C., E.M.I.-Z., G.D., E.F., P.G.S. and M.L.

Funding: The research was partially founded by PROGETTO "BLOW UP" ID 16320 EIT RAW MATERIALS

Acknowledgments: Thanks are due to Giuseppina Gasti (Politecnico di Milano) for her help with the experimental work. Reviewers are also acknowledged to help the Authors on paper improvement.

Conflicts of Interest: The authors declare no conflict of interest 


\section{References}

1. Conor, R. Water for a Sustainable World - The United Nations World Water Development Report; Unesco Publishing: Paris, France, 2015.

2. Sis, H.; Uysal, T. Removal of heavy metal ions from aqueous medium using kuluncak (malatya) vermiculites and effect of precipitation on removal. Appl. Clay Sci. 2014, 95, 1-8. [CrossRef]

3. Liu, X.; Hicher, P.; Muresan, B.; Saiyouri, N.; Hicher, P.-Y. Heavy metal retention properties of kaolin and bentonite in a wide range of concentration and different ph conditions. Appl. Clay Sci. 2016, 119, 365-374. [CrossRef]

4. Shen, C.; Chen, C.; Wen, T.; Zhao, Z.; Wang, X.; Xu, A. Superior adsorption capacity of g-C3N4 for heavy metal ions from aqueous solutions. J. Colloid Interface Sci. 2015, 456, 7-14. [CrossRef]

5. Li, X.; Zhou, H.; Wu, W.; Wei, S.; Xu, Y.; Kuang, Y. Studies of heavy metal ion adsorption on chitosan/sulfydryl-functionalized graphene oxide composites. J. Colloid Interface Sci. 2015, 448, 389-397. [CrossRef]

6. Matusik, J.; Wścisło, A. Enhanced heavy metal adsorption on functionalized nanotubular halloysite interlayer grafted with aminoalcohols. Appl. Clay Sci. 2014, 100, 50-59. [CrossRef]

7. Amin, M.T.; Alazba, A.A.; Manzoor, U. A review of removal of pollutants from water/wastewater using different types of nanomaterials. Adv. Mater. Sci. Eng. 2014, 2014, 1-24. [CrossRef]

8. Song, J.; Kong, H.; Jang, J. Adsorption of heavy metal ions from aqueous solution by polyrhodanineencapsulated magnetic nanoparticles. J. Colloid Interface Sci. 2011, 359, 505-511. [CrossRef] [PubMed]

9. Barakat, M.A. New trends in removing heavy metals from industrial wastewater. Arab. J. Chem. 2011, 4, 361-377. [CrossRef]

10. Bedelean, H.; Măicăneanu, A.; Burcă, S.; Stanca, M. Removal of heavy metal ions from wastewaters using natural clays. Clay Miner. 2010, 44, 487-495. [CrossRef]

11. Bourliva, A.; Michailidis, K.; Sikalidis, C.; Filippidis, A.; Betsiou, M. Lead removal from aqueous solutions by natural greek bentonites. Clay Miner. 2013, 48, 771-787. [CrossRef]

12. Kyzas, G.Z.; Azizian, S.; Kostoglou, M. Novel approaches in designing natural/synthetic materials for environmental applications. Adv. Mater. Sci. Eng. 2015, 2015, 1. [CrossRef]

13. Triantafyllou, S.; Christodoulou, E.; Neou-Syngouna, P. Removal of nickel and cobalt from aqueous solutions by Na-activated bentonite. Clays Clay Miner. 1999, 47, 567-572. [CrossRef]

14. Okada, T.; Ehara, Y.; Ogawa, M. Adsorption of Eu3+ to smectites and fluoro-tetrasilicic mica. Clays Clay Miner. 2007, 55, 348-353. [CrossRef]

15. Iannicelli-Zubiani, E.M.; Cristiani, C.; Dotelli, G.; Gallo Stampino, P.; Pelosato, R.; Mesto, E.; Schingaro, E.; Lacalamita, M. Use of natural clays as sorbent materials for rare earth ions: Materials characterization and set up of the operative parameters. Waste Manag. 2015, 46, 546-556. [CrossRef]

16. Rathnayake, S.I.; Xi, Y.; Frost, R.L.; Ayoko, G.A. Structural and thermal properties of inorganic-organic montmorillonite: Implications for their potential environmental applications. J. Colloid Interface Sci. 2015, 459, 17-28. [CrossRef] [PubMed]

17. Yariv, S.; Cross, H. Organo-Clay Complexes and Interactions; Taylor \& Francis: Milton Park, UK, 2001.

18. Dick, S.; Weiss, A. Reactions of smectites with binuclear oxobridged iron complexes of n-alkyl-n,nbis (2-pyridylmethyl)amines. Clay Miner. 1998, 33, 35-42. [CrossRef]

19. Zhang, S.; Qiu, Z.; Huang, W. Water-adsorption properties of xiazijie montmorillonite intercalated with polyamine. Clay Miner. 2015, 50, 537-548. [CrossRef]

20. Malakul, P.; Srinivasan, K.R.; Wang, H.Y. Metal adsorption and desorption characteristics of surfactantmodified clay complexes. Ind. Eng. Chem. Res. 1998, 37, 4296-4301. [CrossRef]

21. Deng, Y.; Dixon, J.B.; White, G.N. Intercalation and surface modification of smectite by two non-ionic surfactants. Clays Clay Miner. 2003, 51, 150-161. [CrossRef]

22. Iannicelli Zubiani, E.M.; Cristiani, C.; Dotelli, G.; Gallo Stampino, P.; Pelosato, R.; Bengo, I.; Masi, M. Polymers modified clays for separating rare earths from WEEE. Environ. Eng. Manag. J. 2013, 12 (Suppl. 11), $23-26$.

23. Zampori, L.; Stampino, P.G.; Cristiani, C.; Dotelli, G.; Cazzola, P. Synthesis of organoclays using non-ionic surfactants: Effect of time, temperature and concentration. Appl. Clay Sci. 2010, 48, 97-102. [CrossRef]

24. Khan, M.A.; Ahmad, A.; Umar, K.; Nabi, S.A. Synthesis, characterization and biological applications of nanocomposites for the removal of heavy metals and dyes. Ind. Eng. Chem. Res. 2015, 54, 76-82. [CrossRef] 
25. Anirudhan, T.S.; Jalajamony, S.; Sreekumari, S.S. Adsorption of heavy metal ions from aqueous solutions by amine and carboxylate functionalised bentonites. Appl. Clay Sci. 2012, 65-66, 67-71. [CrossRef]

26. Iannicelli-Zubiani, E.M.; Cristiani, C.; Dotelli, G.; Gallo Stampino, P. Recovery of valuable metals from electronic scraps by clays and organo-clays: Study on bi-ionic model solutions. Waste Manag. 2017, 60, 582-590. [CrossRef] [PubMed]

27. Basolo, F. Inorganic Syntheses; McGraw-Hill: New York, NY, USA, 1976.

28. Iannicelli-Zubiani, E.M.; Cristiani, C.; Dotelli, G.; Stampino, P.G.; Pelosato, R. Recovery of rare earths and precious metals from waste electrical and electronic equipment by acid leaching and immobilized chelating agents. Chem. Eng. Trans. 2015, 43, 2413-2418. [CrossRef]

29. ASTM. Standard Test Methods for Chemical Oxygen Demand (Dichromate Oxygen Demand) of Water; ASTM: West Conshohocken, PA, USA, 2006; Volume D1252-06.

30. Iannicelli-Zubiani, E.M.; Gallo Stampino, P.; Cristiani, C.; Dotelli, G. Enhanced lanthanum adsorption by amine modified activated carbon. Chem. Eng. J. 2018, 341, 75-82. [CrossRef]

31. Blachier, C.; Michot, L.; Bihannic, I.; Barres, O.; Jacquet, A.; Mosquet, M. Adsorption of polyamine on clay minerals. J. Colloid Interface Sci. 2009, 336, 599-606. [CrossRef]

32. Finocchio, E.; Cristiani, C.; Dotelli, G.; Stampino, P.G.; Zampori, L. Thermal evolution of peg-based and brij-based hybrid organo-inorganic materials. FT-IR studies. Vib. Spectrosc. 2014, 71, 47-56. [CrossRef]

33. Deng, Y.; Dixon, J.; White, G.N. Bonding mechanisms and conformation of poly(ethylene oxide)-based surfactants in interlayer of smectite. Colloid Polym. Sci. 2006, 284, 347-356. [CrossRef]

34. Chiu, C.-W.; Huang, T.-K.; Wang, Y.-C.; Alamani, B.G.; Lin, J.-J. Intercalation strategies in clay/polymer hybrids. Prog. Polym. Sci. 2013, 39, 443-485. [CrossRef]

35. Wang, Y.; Wang, X.; Duan, Y.; Liu, Y.; Du, S. Modification of montmorillonite with poly(oxypropylene) amine hydrochlorides: Basal spacing, amount intercalated and thermal stability. Clays Clay Miner. 2011, 59, 507-517. [CrossRef]

36. Madejová, J.; Komadel, P. Baseline studies of the clay minerals society source clays: Infrared methods. Clays Clay Miner. 2001, 49, 410-432. [CrossRef]

37. Scholtzová, E.; Madejová, J.; Tunega, D. Structural properties of montmorillonite intercalated with tetraalkylammonium cations-Computational and experimental study. Vib. Spectrosc. 2014, 74, 120-126. [CrossRef]

38. Liu, C.; Bai, R.; Hong, L.; Liu, T. Functionalization of adsorbent with different aliphatic polyamines for heavy metal ion removal: Characteristics and performance. J. Colloid Interface Sci. 2010, 345, 454-460. [CrossRef]

39. Pentrák, M.; Bizovská, V.; Madejová, J. Near-IR study of water adsorption on acid-treated montmorillonite. Vib. Spectrosc. 2012, 63, 360-366. [CrossRef]

40. Finocchio, E.; Baccini, I.; Cristiani, C.; Dotelli, G.; Gallo Stampino, P.; Zampori, L. Hybrid organo-inorganic clay with nonionic interlayers. Mid- and Near-IR spectroscopic studies. J. Phys. Chem. A 2011, 115, 7484-7493. [CrossRef]

41. Madejová, J. FTIR techniques in clay mineral studies. Vib. Spectrosc. 2003, 31, 1-10. [CrossRef]

42. Ko, C.H.; Fan, C.; Chiang, P.N.; Wang, M.K.; Lin, K.C. P-nitrophenol, phenol and aniline sorption by organo-clays. J. Hazard. Mater. 2007, 149, 275-282. [CrossRef]

43. Laaz, I.; Stébé, M.-J.; Benhamou, A.; Zoubir, D.; Blin, J.-L. Influence of porosity and surface modification on the adsorption of both cationic and anionic dyes. Colloids Surf. A Physicochem. Eng. Asp. 2016, 490, 30-40. [CrossRef]

44. Foo, K.Y.; Hameed, B.H. Insights into the modeling of adsorption isotherm systems. Chem. Eng. J. 2010, 156, 2-10. [CrossRef]

45. Okeola, F.; Odebunmi, E. Freundlich and Langmuir isotherms parameters for adsorption of methylene blue by activated carbon derived from agrowastes. Adv. Nat. Appl. Sci. 2010, 4, 281-288.

46. Iftekhar, S.; Ramasamy, D.L.; Srivastava, V.; Asif, M.B.; Sillanpää, M. Understanding the factors affecting the adsorption of lanthanum using different adsorbents: A critical review. Chemosphere 2018, 204, 413-430. [CrossRef]

47. Iannicelli-Zubiani, E.M.; Cristiani, C.; Dotelli, G.; Gallo Stampino, P.; Pelosato, R.; Finocchio, E. Effect of pH in the synthesis of organo-clays for rare earths removal. Environ. Eng. Manag. J. 2017, 16, 1719-1727. [CrossRef]

(C) 2019 by the authors. Licensee MDPI, Basel, Switzerland. This article is an open access article distributed under the terms and conditions of the Creative Commons Attribution (CC BY) license (http://creativecommons.org/licenses/by/4.0/). 
Article

\title{
The Stability of Intercalated Sericite by Cetyl Trimethylammonium Ion under Different Conditions and the Preparation of Sericite/Polymer Nanocomposites
}

\author{
Yu Liang ${ }^{1,2}$, Dexin Yang ${ }^{3}$, Tao Yang ${ }^{3}$, Ning Liang ${ }^{2}$ and Hao Ding ${ }^{2, *}$ \\ 1 School of Materials Science and Technology, Shenyang University of Chemical Technology, Shenyang 110142, \\ China; liangyuaadd@126.com \\ 2 Beijing Key Laboratory of Materials Utilization of Nonmetallic Minerals and Solid Wastes, \\ National Laboratory of Mineral Materials, School of Materials Science and Technology, \\ China University of Geosciences (Beijing), Beijing 100083, China; liangning628@126.com \\ 3 College of Materials and Environmental Engineering, Hangzhou Dianzi University, Hangzhou 310018, \\ China; dy263@hdu.edu.cn (D.Y.); yangtao@hdu.edu.cn (T.Y.) \\ * Correspondence: dinghao@cugb.edu.cn or dinghao113@126.com
}

Received: 27 April 2019; Accepted: 12 May 2019; Published: 17 May 2019

\begin{abstract}
Layered silicates are suitable for use as fillers in nanocomposites based on their particular features, such as large aspect ratio, easy availability, and chemical resistance. Among them, sericite is distinguished for its higher aspect ratio, higher resilience, and ultraviolet shielding and absorption. Previously, sericite was structure-modified and intercalated by CTAB to expand its interlayer space. The intercalated sericite seems promising for use in the fabrication of polymer/sericite composites or pillared sericite. However, special attention should be paid to the stability of the intercalated sericite because the CTAB inside the layer may be de-intercalated, which would affect the interlayer spacing and its surface properties. In this article, the stability of the sericite intercalated by CTAB was tested by changing different variables, such as different washing solvents, different temperatures, ultrasonic cleaning, and different solution conditions. Finally, sericite/polymer nanocomposites were produced with the stable intercalated sericite, and showed excellent properties compared with pure epoxy resin.
\end{abstract}

Keywords: layered silicate; sericite; CTAB; intercalation stability; nanocomposites

\section{Introduction}

Recently, nanocomposites have attracted great attention due to their extraordinary properties. A wide variety of fillers, either natural ones or synthetic ones, can be used to intercalate into the polymer [1,2]. The conventional fillers for polymers are inorganic materials of mainly three categories: particles (e.g., calcium carbonate particles), fibers (e.g., glass fibers and carbon fibers), and plate-shaped particles (e.g., montmorillonite and vermiculite) [3-8]. Among them, layered silicates attract the most attention because of their easy availability, large aspect ratios, and chemical resistance [9-12]. However, in most cases, these layered silicates, which are hydrophilic by nature, need to be organically modified to produce polymer-compatible clay, to increase the compatibility between the layered silicate and the polymer matrix, and to lower the surface energy of the layered silicate. Until now, many methods have been developed to modify layered silicate. For example, the replacement of the inorganic exchange cations with alkylammonium surfactants can make clay compatible to the polymer matrix [13-15].

Many layered silicates can be organically modified for the fabrication of polymer/layered silicate nanocomposites. Some commonly used substrates for intercalation are swelling clays, such as kaolinite [16-18], vermiculite [19-22], and montmorillonite [23-25]. A few articles use sericite as a 
raw material for the fabrication of polymer/layered silicate nanocomposites. Sericite is a kind of 2:1 phyllosilicate similar to muscovite with a series of unique properties. It has a higher aspect ratio (more than 1000) [26-28], and higher resilience than other fillers, and can shield and absorb ultraviolet radiation [29]. Unlike other 2:1 phyllosilicates such as montmorillonite and vermiculite, sericite cannot swell in water, and is non-expandable due to the substitution of $\mathrm{Al}^{3+}$ for $\mathrm{Si}^{4+}$ in tetrahedral sheets, which results in a net negative surface charge balanced by $\mathrm{K}^{+}$, filling in the interlayer tightly. Therefore, sericite needs to be modified first in order to allow water and other molecules to enter the interlayer, and for cation exchanges. Previously, our team has successfully modified sericite by thermal modification, acid activation, and sodium modification [30]. The cation exchange capacity of the modified sericite reached $56.37 \mathrm{mmol} / 100 \mathrm{~g}$, making it a suitable material for organic modification. The modified sericite was then intercalated by CTAB to expand its interlayer space from $1.00 \mathrm{~nm}$ to $5.07 \mathrm{~nm}$. Therefore, sericite seems promising for use in the next step-for the fabrication of polymer/sericite composites or pillared sericite (e.g., Al-pillared sericite) in order to obtain better performances.

As for the intercalation of layered silicate, a lot of attention is given to methods that can modify layered silicate from hydrophilic to hydrophobic, but not much attention has been given to the stability of the modified layered silicate (or intercalated layered silicate). However, it should be noted that the stability of the intercalated layered silicate is very important, since the intercalated silicate needs to be used in the next step-for the fabrication of clay-polymer nanocomposites, or for the fabrication of pillared silicates (such as $\mathrm{TiO}_{2}$ pillared montmorillonite). If the intercalated layered silicate is not very stable, the interlayer spacing of the layered silicate will be changed, and its surface properties will be affected. Gu et al. [31] found that part of the intercalated CTAB in montmorillonite was de-intercalated when the colloidal particles produced by the hydrolysis of tetrabutyl titanate were in the suspension. The content of $\mathrm{CTA}^{+}$in the interlayer decreased, and the distribution and inclination angle of CTA ${ }^{+}$ in the interlayer changed accordingly. Therefore, there is a great necessity to study the stability of intercalated layered silicate.

In this article, the stability of intercalated sericite was researched. Raw sericite (S0) was first modified to gain cation exchange capacity by thermal modification, acid activation, and sodium modification. Then, the modified sericite was intercalated by CTAB to increase d-space and change its surface wettability. Different conditions (e.g., washing solvents with different temperatures) were used to test the stability of the intercalated sericite. Finally, the stable intercalated sericite was added into epoxy resin to produce sericite/polymer nanocomposites, and these showed excellent properties. This article aims to provide some guidance to those who use intercalated layered silicate for the fabrication of polymer/layered silicate nanocomposites or pillared silicate.

\section{Materials and Methods}

\subsection{Materials}

Following gravity purification, drying, and grinding, raw sericite materials (S0) were obtained from the natural sericite produced in Anhui, China. S1 was produced by heating raw sericite materials at $800{ }^{\circ} \mathrm{C}$ for $1 \mathrm{~h}$, and cooling them down to room temperature. S2 was produced by mixing $6 \mathrm{~g}$ of the thermally treated sericite with $200 \mathrm{~g} 5 \mathrm{M}$ nitric acid at $95^{\circ} \mathrm{C}$ for $4 \mathrm{~h}$ in a water bath. Stirring at a speed of $150 \mathrm{rpm}$ and drying at ambient temperature was required for the next modification step. $\mathrm{S} 3$ was obtained by mixing $6 \mathrm{~g}$ of $\mathrm{S} 2$ with $200 \mathrm{~g}$ of saturated $\mathrm{NaCl}$ solution, and by stirring at the same rotational speed as before at $95{ }^{\circ} \mathrm{C}$ for $3 \mathrm{~h}$. Then, $\mathrm{S} 3$ was washed with $\mathrm{DI}_{2} \mathrm{O}$ and dried at room temperature. The cation exchange capacity (CEC) value of S3 reached $56 \mathrm{mmol} / 100 \mathrm{~g}$, which is a great improvement compared to raw sericite $(7.5 \mathrm{mmol} / 100 \mathrm{~g})$. The XRD patterns and SEM images of $\mathrm{S} 0$ and S3 are shown in Figures 1 and 2, respectively. All chemicals were of analytical grade, and were used without further purification. 


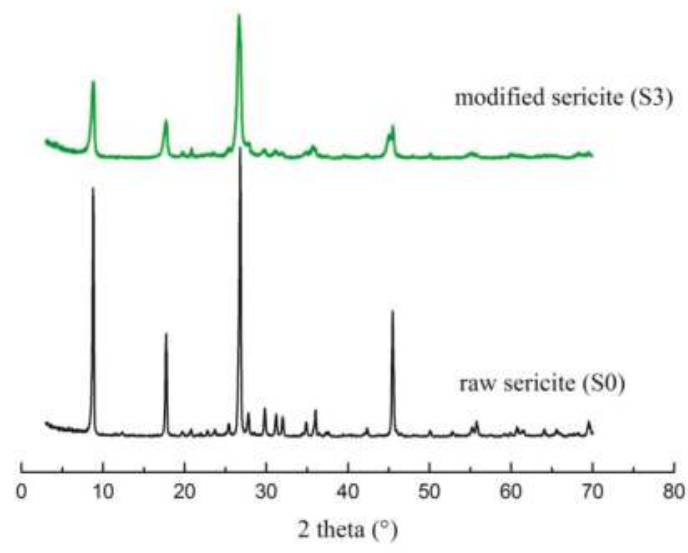

Figure 1. XRD patterns of $\mathrm{S} 0$ and $\mathrm{S} 3$.

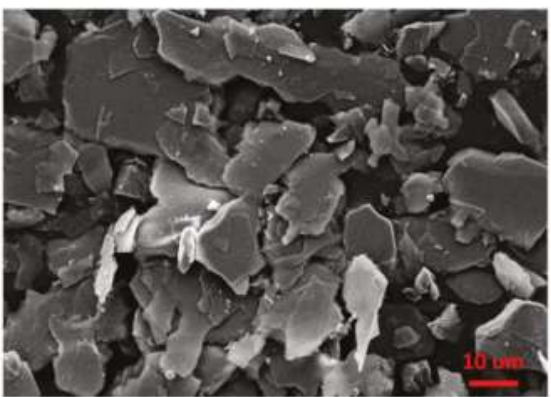

(a)

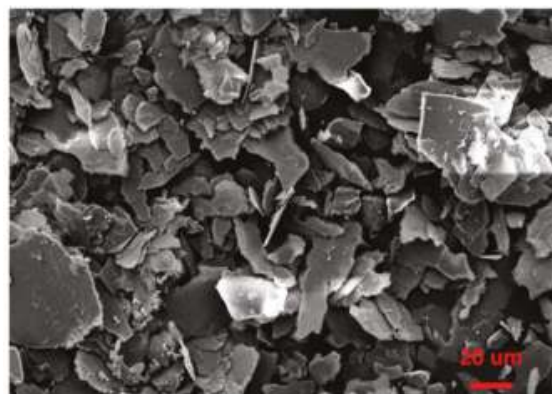

(b)

Figure 2. SEM images of (a) S0 and (b) S3.

\subsection{The Intercalation Process and the Stability Tests}

In order to test the stability of the intercalated product under different washing conditions (different solvents and temperatures), S3 was intercalated with CTAB (the amount of CTAB was 15 times the CEC of S3) at $80^{\circ} \mathrm{C}$ for $24 \mathrm{~h}$ in a water bath. Then the product was washed with hot $\mathrm{DI}_{2} \mathrm{O}$ $\left(50^{\circ} \mathrm{C}\right)$, cold $\mathrm{DI} \mathrm{H}_{2} \mathrm{O}\left(25^{\circ} \mathrm{C}\right)$, and ethanol $\left(25^{\circ} \mathrm{C}\right)$ separately, then centrifuged and dried. Ultrasonic cleaning was used in the procedure of washing to see if it had a negative effect on the stability of the intercalated product. The stability of intercalated product in the solution was also tested by putting the intercalated product in DMSO, and in a solution of CTAB (DMSO was used as solvent, and the amount of CTAB was 15 times theCEC of S3) at $80^{\circ} \mathrm{C}$ for $24 \mathrm{~h}$ in a water bath, separately. Finally, different substrates were used in the intercalation process to see which one was better for future use.

\subsection{The Preparation Process of Sericite/Polymer Nanocomposites}

The epoxy-based matrix consists of a two functional diglycidylether of bisphenol A (DGEBA, Hubei Jusheng Technology, Wuhan, China) which is cured stoichiometrically with 4,4-diaminodiphenyl methane (DDM, Changsha Jiazhen biological Company, Changsha, China). As an accelerator, 3 wt.\% tris(dimethylaminomethyl)phenol (DMP-30, Changzhou Shanfeng Chemical, Changzhou, China) was added to the reactive mixture. All nanocomposites were then cured at $80^{\circ} \mathrm{C}$ for $2 \mathrm{~h}$, and then at $150{ }^{\circ} \mathrm{C}$ for $2 \mathrm{~h}$ to achieve a fully cured polymer matrix. The chemical structures of the reactive components are presented in Figure 3. 

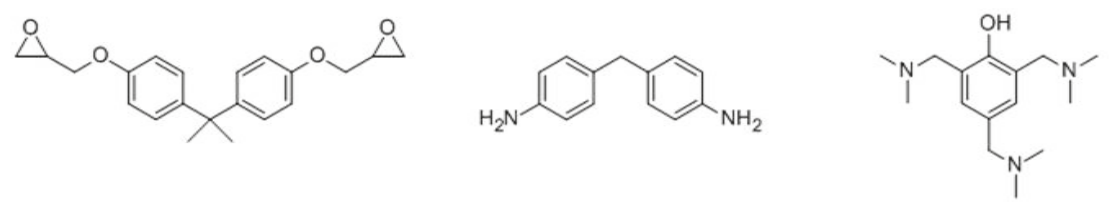

Figure 3. The chemical structure of diglycidylether of bisphenol A (DGEBA) resin (left), 4,4-diaminodiphenyl methane (DDM) hardener (middle), and tris(dimethylaminomethyl)phenol (DMP-30) accelerator (right).

\subsection{Characterization}

The changes in the $\mathrm{d}_{001}$ value of the sericite were determined by X-ray diffraction (XRD) analyses. The percentage of the intercalated layers to the total layers, defined as the intercalation rate, was used to judge the degree of intercalation. The XRD analyses were conducted with a Rigaku D/max-rA $(12 \mathrm{~kW}) \mathrm{X}$-ray powder diffractometer (Rigaku, Tokyo, Japan), operated with $\mathrm{Cu} \mathrm{K} \alpha$ radiation at $40 \mathrm{kV}$ and $100 \mathrm{~mA}$, and with a scanning speed of $0.5^{\circ}(2 \theta) / \mathrm{min}$. The microstructure and morphologies were investigated by a Hitachi S-3500 SEM (Hitachi, Tokyo, Japan). The morphologies of the sericite/epoxy nanocomposites were characterized using a Tecnai F-20 transmission electron microscope (FEI, Hillsboro, OR, USA), applying a $200 \mathrm{kV}$ accelerating voltage. The mechanical properties of the sericite/epoxy nanocomposites were tested by an electronic universal testing machine (WDW-300, Shimadzu, Kyoto, Japan).

\section{Results and Discussion}

\subsection{The Effect of Different Washing Solvents and Temperatures on the Stability of the Intercalated Product}

Figure 4 shows the XRD patterns of the intercalated products washed with different solvents at different temperatures. Obviously, cold $\mathrm{DI}_{2} \mathrm{O}$ had the best effect of keeping the intercalation result. After being washed with cold DI $\mathrm{H}_{2} \mathrm{O}$, the d-value of the newly emerged diffraction peak was $4.96 \mathrm{~nm}$, which was more than five times the d-value of the modified sericite (S3). The peak pattern was sharp as well as symmetric, which means CTAB had ordered arrays in the interlayer of sericite. While the effects of hot DI $\mathrm{H}_{2} \mathrm{O}$ and cold ethanol were much worse than cold $\mathrm{DI}-\mathrm{H}_{2} \mathrm{O}$, no obvious diffraction peaks could be seen in the two XRD patterns. This means that washing with hot $\mathrm{DI}_{2} \mathrm{O}$ and ethanol can make the CTAB in the interlayer de-intercalate and arrange in a disorderly manner. In spite of the de-intercalated effect, the final product still had some intercalated effect compared to S3.

CTAB has a much higher solubility in hot water and in ethanol than in cold water, which caused the de-intercalation of CTAB in the interlayer of sericite. Therefore, cold $\mathrm{DI}_{2} \mathrm{O}$ should be used during the washing procedure in order to guarantee the modification effect. 


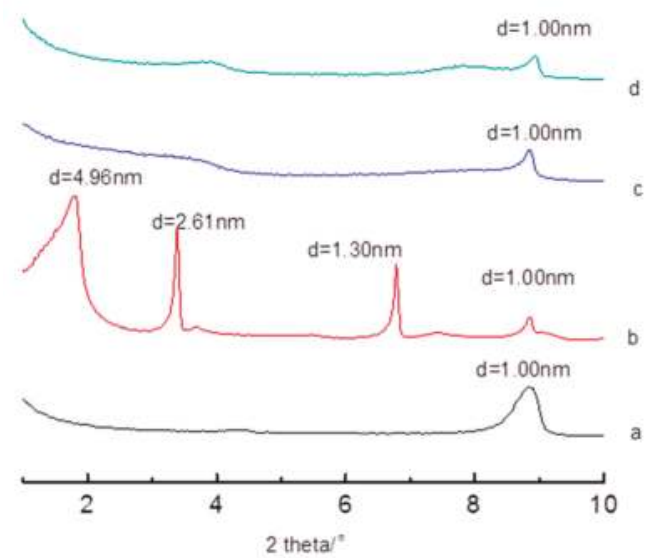

Figure 4. XRD patterns of the intercalated products washed with different solvents at different temperatures. a. S3; b. the intercalated product washed with cold $\mathrm{DI} \mathrm{H}_{2} \mathrm{O}\left(25^{\circ} \mathrm{C}\right)$; c. the intercalated product washed with hot $\mathrm{DI} \mathrm{H}_{2} \mathrm{O}\left(50^{\circ} \mathrm{C}\right)$; $d$. the intercalated product washed with cold ethanol $\left(25^{\circ} \mathrm{C}\right)$.

\subsection{The Effect of Ultrasonic Cleaning on the Stability of the Intercalated Product}

Figure 5 shows the XRD patterns of the intercalated products after being treated with and without ultrasonic waves during the washing procedure. The solvent used in the washing procedure was cold DI $\mathrm{H}_{2} \mathrm{O}$. It can be seen that ultrasonic cleaning had no obvious effect on the intercalation result.

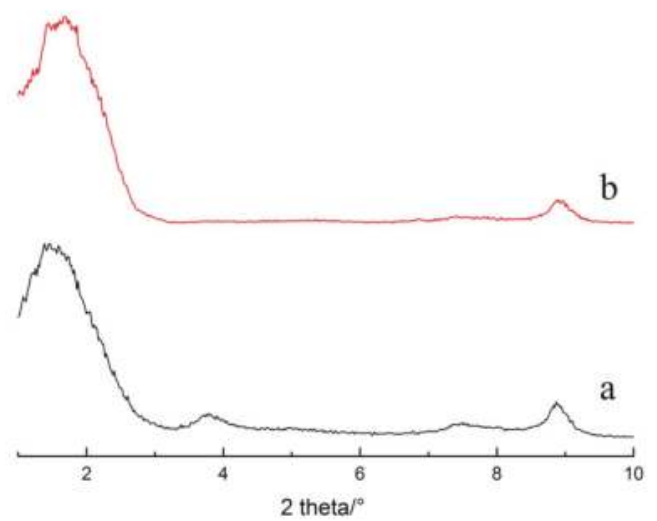

Figure 5. XRD patterns of the intercalated products: $\mathbf{a}$. washed with ultrasonic cleaning; $\mathbf{b}$. washed without ultrasonic cleaning.

\subsection{The Stability of the Intercalated Product in the Solution}

Intercalation is not the final procedure in the fabrication of polymer/clay nanocomposites or pillared composites. The intercalated products need to be used as raw material for the next step. Therefore, it is necessary to keep the intercalated product stable in the solution. In this part, the stability of intercalated product in the solution was tested by putting the intercalated product in DMSO solvent, and in a solution of CTAB (DMSO was used as the solvent and the amount of CTAB was 15 times the CEC of S3) at $80^{\circ} \mathrm{C}$ for $24 \mathrm{~h}$ in a water bath, separately. Figure 6 shows the XRD patterns of the final products. The d-space of the intercalated sericite in the mixed solution of CTAB and DMSO 
was $5.79 \mathrm{~nm}$, and the peak patterns were sharp, which means good crystallinity of the final product. The d-space of the intercalated sericite in the DMSO only reached $3.03 \mathrm{~nm}$, and the diffraction peaks were much flatter. Therefore, it is clear that the stability of the intercalated product in the mixed solution of CTAB and DMSO was much better than in DMSO.

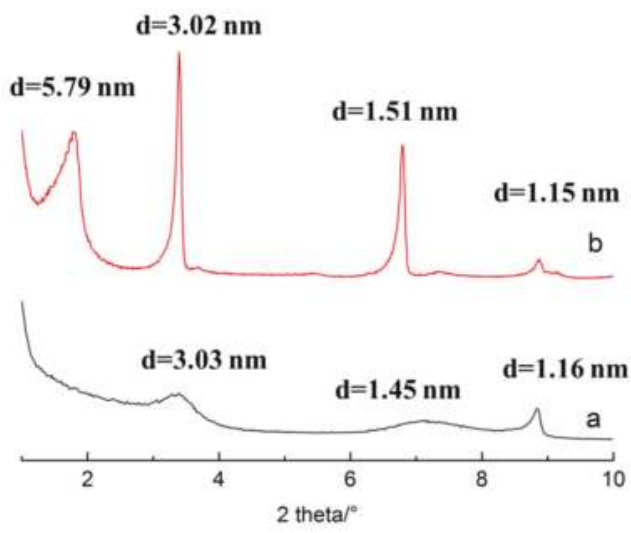

Figure 6. Stability test of the intercalated products in the solution: a. the intercalated sericite was used as raw material and DMSO was used as a solvent, at $80^{\circ} \mathrm{C}$ for $24 \mathrm{~h}$; $\mathbf{b}$. the intercalated sericite was used as raw material, CTAB (the amount of CTAB was 15 times the cation exchange capacity (CEC) of S3) and DMSO were used in the solution at $80^{\circ} \mathrm{C}$ for $24 \mathrm{~h}$.

A hot solvent such as DMSO can dissolve $\mathrm{CTAB}$ and move CTAB out from the interlayer, causing the disorder of CTAB in the interlayer. However, the mixed solution of CTAB and DMSO can ensure a saturated solution of CTAB (the amount of CTAB was 15 times the CEC of S3, much higher than the solubility of CTAB in this temperature), and maintain the stability of CTAB in the interlayer with the help of osmotic pressure. Therefore, if the intercalated sericite needs to be used in a subsequent step, sufficient $\mathrm{CTAB}$ should be put into the solution in order to keep the intercalated CTAB stable inside the interlayer.

\subsection{The Choice of Raw Material for the Intercalation Based on Stability, Intercalation Rate, and Other Experimental Conditions}

Figure 7 shows the XRD patterns of different raw materials used in the intercalation. When S3 was used as a raw material, CTAB was used as an intercalation agent in DMSO solvent at $80^{\circ} \mathrm{C}$ for $24 \mathrm{~h}$. When the intercalated sericite was used as a raw material, CTAB was used as an intercalation agent in the DMSO at $80^{\circ} \mathrm{C}$ for $3 \mathrm{~h}$. It can be seen that it did not matter what the raw material was, the intercalation result was almost the same. The d-space was maintained between 4.88 and $4.99 \mathrm{~nm}$, and both peak patterns were sharp and symmetrical. 


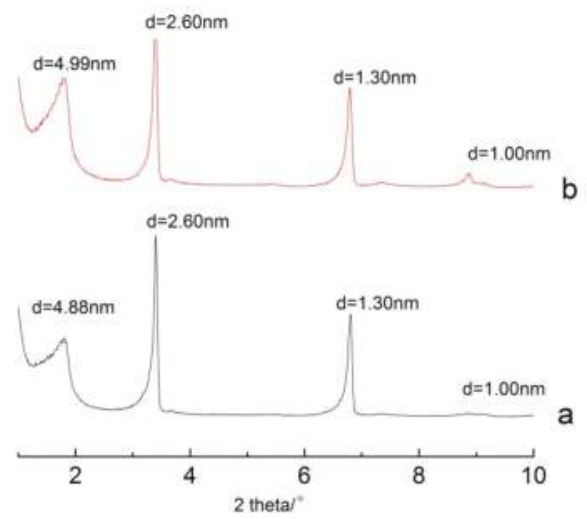

Figure 7. XRD patterns of different raw materials used for the intercalation: a. raw material was S3, $\mathrm{CTAB}$ was used as intercalation agent in DMSO solvent; $\mathbf{b}$. raw material was intercalated S3, CTAB was used as intercalation agent in DMSO solvent.

The intercalation rates were calculated, and are shown in Table 1. From Table 1, it can be seen that both the intercalation rates were relatively high, ranging from $94.83 \%$ to $98.20 \%$.

Table 1. The intercalation rate using different raw materials.

\begin{tabular}{ccc}
\hline & S3 & Intercalated S3 \\
\hline Intercalation rate (\%) & 98.20 & 94.83 \\
\hline
\end{tabular}

The usage of reagents and the experiment time are listed in Table 2. Obviously, the intercalated S3 consumed more reagent and had a longer experiment time. Based on these factors, S3 should be chosen for the intercalation. The intercalation did not improve by repeating it. The intercalated product made from S3 was stable, consumed less reagent, took less time, and had a higher intercalation rate.

Table 2. The usage of reagents and experiment time with different raw materials for the intercalation.

\begin{tabular}{ccc}
\hline & S3 & Intercalated S3 \\
\hline The amount of CTAB & 15 times the CEC of S3 & 30 times the CEC of S3 \\
Experiment time & $24 \mathrm{~h}$ & $24 \mathrm{~h}+$ drying time $+3 \mathrm{~h}$ \\
\hline
\end{tabular}

\subsection{Properties of the Sericite/Polymer Nanocomposites}

Different amounts of stable intercalated sericite, using the optical conditions mentioned above $(0.1 \mathrm{wt} \%, 0.5 \mathrm{wt} \%, 1 \mathrm{wt} \%, 1.5 \mathrm{wt} \%$, and $2 \mathrm{wt} \%)$, were added into epoxy resin to produce sericite/epoxy nanocomposites. In Figure 8, it can be seen that when the amount of sericite was less than $1 \mathrm{wt} \%$, mainly exfoliated nanocomposites were formed. The interlayer spacing could be expanded to more than $8.8 \mathrm{~nm}$, with the sheets of sericite distributed uniformly in the matrix. As the amount of sericite increased to $1.5 \mathrm{wt} \%$, diffraction peaks emerged at $2 \theta<2^{\circ}$, which means that part of the sericite was not exfoliated in the epoxy resin matrix, while the interlayer spacing still increased substantially. The increased amount of sericite increased the background of the previous d002 value of sericite, which means the exfoliation is not that completed, with part of sericite lamellar still in order degree. 


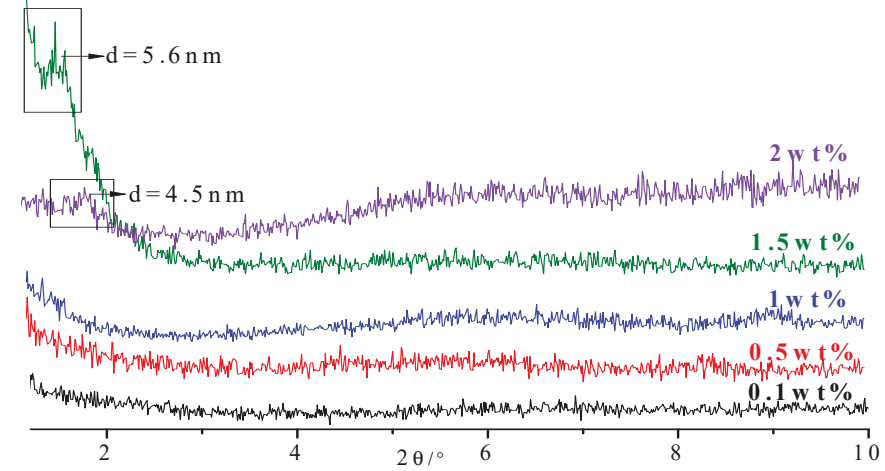

Figure 8. XRD patterns of the sericite/epoxy resin with different amounts of intercalated sericite added.

The tensile strength, flexural strength, and impact strength of the sericite/epoxy nanocomposites with different amounts of sericite added were also tested and compared with pure epoxy resin with no sericite added. In Figure 9, it can be seen that the mechanical strength of the nanocomposites increased substantially compared with pure epoxy resin. The mechanical strength was best when the amount of sericite was $1 \%$. The tensile strength (105 MPa) increased by $110 \%$ compared with pure epoxy resin (50 MPa), the flexural strength $(110 \mathrm{MPa})$ increased by $41 \%$ compared with pure epoxy resin $(78 \mathrm{MPa})$, and the impact strength $\left(35 \mathrm{~kJ} / \mathrm{m}^{2}\right)$ increased by $94 \%$ compared with pure epoxy resin $\left(18 \mathrm{~kJ} / \mathrm{m}^{2}\right)$.

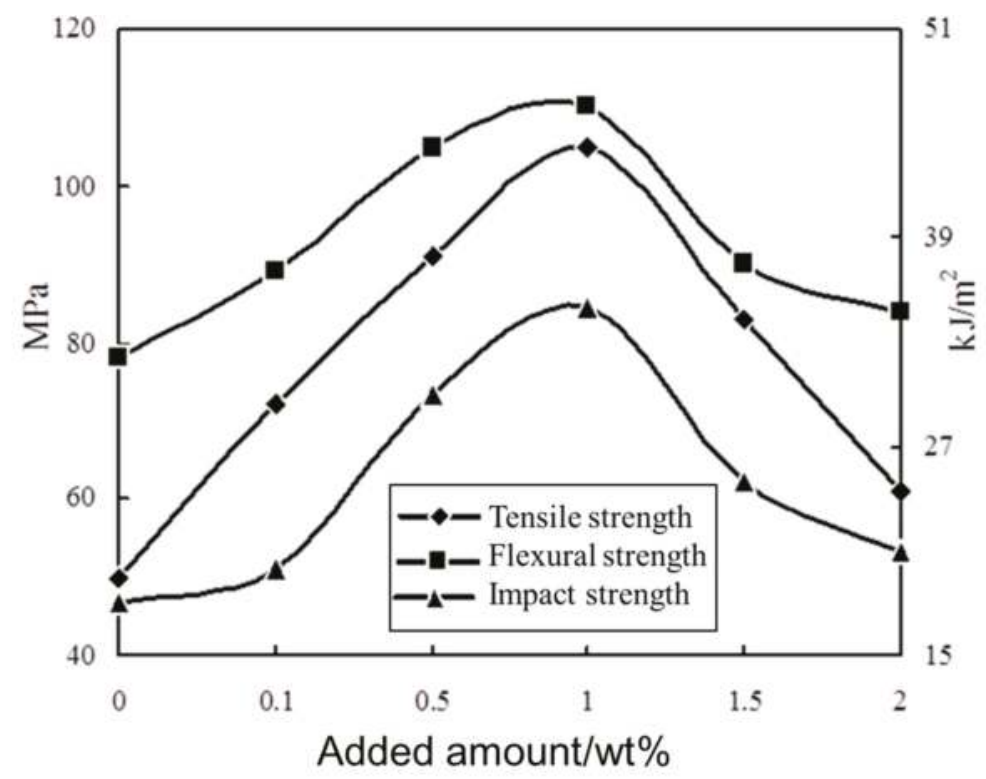

Figure 9. The effect of the added amount of intercalated sericite on the mechanical properties of the composites.

Figure 10 shows TEM images of the sericite/epoxy nanocomposites (the amount of sericite was $1 \mathrm{wt} \%$ ). The dark textures are sericite layers, while the light parts are epoxy resin. The sericite interlayers were still parallel while most of them were exfoliated to a relatively large extent with 
the distance at $20 \mathrm{~nm}$ or more. Together with the XRD patterns, this result proves the formation of exfoliated sericite/epoxy nanocomposites.

(a)

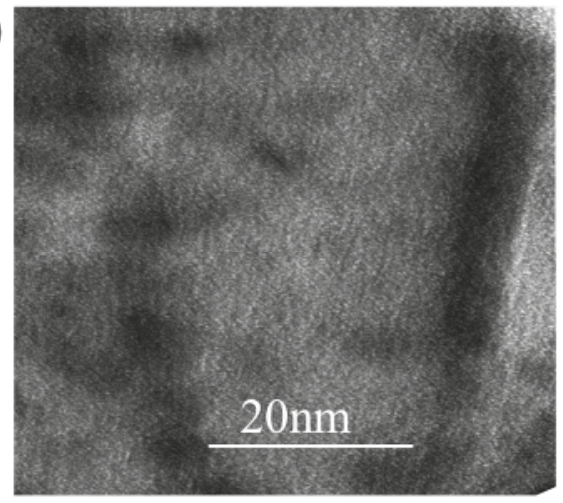

(b)

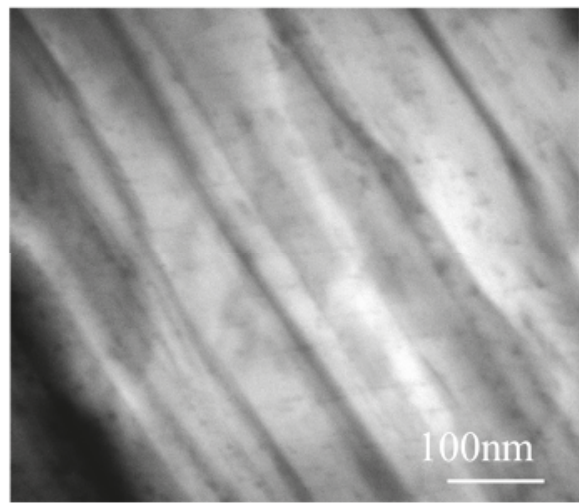

Figure 10. TEM images of the sericite/epoxy nanocomposites (the amount of sericite was $1 \mathrm{wt} \%$ ). (a) scale bar $20 \mathrm{~nm}$; (b) scale bar $100 \mathrm{~nm}$.

When compared with virgin epoxy resin, the sericite/epoxy resin nanocomposites had better mechanical properties. The results above also suggest that there is an optimum clay concentration for the nanocomposite, which was $1 \%$ in our case. Compared with epoxy resin, sericite is more naturally resistant to straining due to its high modulus. Moreover, when the added amount of sericite was less than (or equal to) $1 \%$, mainly exfoliated sericite/epoxy nanocomposites could be formed, with most of the sericite lamella distributed uniformly in the epoxy resin. Therefore, the mechanical properties of sericite/epoxy nanocomposites improved dramatically when compared with pure epoxy resin. As the amount of sericite increased, the relative amount of intercalation/exfoliation of the sericite gradually increased with an increase in the sericite content, which means that the dispersion of sericite in epoxy resin was not as good as before, leading to decreased mechanical properties. The modifier of sericite-CTAB in our case-helped change the surfaces of sericite from hydrophilic to hydrophobic, leading to good adhesion between sericite and epoxy resin, which was the precondition to the improvement of the nanocomposite mechanical properties.

\section{Conclusions}

In summary, the intercalated sericite by cetyl trimethylammonium ion was stable during the washing procedure with cold (room-temperature) $\mathrm{DI} \mathrm{H}_{2} \mathrm{O}$. Hot $\mathrm{DI} \mathrm{H}_{2} \mathrm{O}$ and ethanol could de-intercalate CTAB in the interlayer. Ultrasonic cleaning had no effect on the stability of the intercalated product. If the intercalated product needs to be used in a subsequent reaction step, a sufficient amount (larger than the solubility of CTAB) of CTAB should be put into the solution in order to prevent the de-intercalation of CTAB from the interlayer. The intercalated product made from S3 was stable, consumed less reagents, and had a higher intercalation rate compared with twice-intercalated products. The sericite/epoxy nanocomposites produced with stable intercalated sericite had better mechanical properties than pure epoxy resin. Mainly exfoliated sericite/epoxy nanocomposites with the layers of sericite dispersed very well in the epoxy resin were formed when the amount of sericite was $1 \mathrm{wt} \%$ or less based on the XRD and TEM results. Stable intercalated sericite is the precondition of a good adhesion between sericite and epoxy resin, which leads to good nanocomposite mechanical properties.

Author Contributions: Data curation, Y.L., D.Y., and N.L.; Methodology, Y.L. and T.Y.; writing—original draft preparation, Y.L.; supervision, H.D.

Funding: This research received no external funding. 
Conflicts of Interest: The authors declare no conflict of interest.

\section{References}

1. Ha, C.S. Polymer Based Hybrid Nanocomposites; A Progress Toward Enhancing Interfacial Interaction and Tailoring Advanced Applications. Chem. Rec. 2017, 18, 759-775. [CrossRef] [PubMed]

2. Alexandre, M.; Dubois, P. Polymer-layered silicate nanocomposites: Preparation, properties and uses of a new class of materials. Mater. Sci. Eng. R Rep. 2000, 28, 1-63. [CrossRef]

3. Chen, C.; Xi, J.; Han, Y.; Peng, L.; Gao, W.; Xu, Z.; Gao, C. Ultralight graphene micro-popcorns for multifunctional composite applications. Carbon 2018, 139, 545-555. [CrossRef]

4. Ahn, C.; Kin, S.-M.; Jung, J.-W.; Park, J.; Kim, T.; Lee, S.; Jang, D.; Hong, J.-W.; Han, S.M.; Jeon, S. Multifunctional polymer nanocomposites reinforced by 3D continuous ceramic nanofillers. ACS Nano 2018, 12, 9126-9133. [CrossRef] [PubMed]

5. Hrachova, J.; Komadel, P.; Chodak, I. Natural rubber nanocomposites with organo-modified bentonite. Clays Clay Miner. 2009, 57, 444-451. [CrossRef]

6. Tan, X.; Xu, Y.; Cai, N.; Jia, G. Polypropylene/silica nanocomposites prepared by in-situ melt ultrasonication. Polym. Compos. 2009, 30, 835-840. [CrossRef]

7. Carvalho, D.; Carvalho, J.; Oliveira, S.; Rosa, D. A new approach for flexible PBAT/PLA/CaCO3 film into agriculture. J. Appl. Polym. Sci. 2018, 135, 46660.

8. Mao, Y.; Shao, C.; Shang, P.; Li, Q.; He, X.; Wu, C. Preparation of high strength PET/PE composites reinforced with continued long glass fibers. Mater. Res. Express 2019, 6, 045303. [CrossRef]

9. Chiu, C.-W.; Huang, T.-K.; Wang, Y.-C.; Alamani, B.G.; Lin, J.-J. Intercalation strategies in clay/polymer hybrids. Prog. Polym. Sci. 2014, 39, 443-485. [CrossRef]

10. Ogawa, M.; Kuroda, K. Preparation of Inorganic-Organic Nanocomposites through Intercalation of Organoammonium Ions into Layered Silicates. Bull. Chem. Soc. Jpn. 1997, 70, 2593-2618. [CrossRef]

11. Pavlidou, S.; Papaspyrides, C.D. A review on polymer-layered silicate nanocomposites. Prog. Polym. Sci. 2008, 33, 1119-1198. [CrossRef]

12. Alateyah, A.I.; Dhakal, H.N.; Zhang, Z.Y. Processing, Properties, and Applications of Polymer Nanocomposites Based on Layer Silicates: A Review. Adv. Polym. Technol. 2013, 32, 21368. [CrossRef]

13. Jian, X.; Xuebing, W.; Bingyao, D.; Qingsheng, L. Modification of montmorillonite by different surfactants and its use for the preparation of polyphenylene sulfide nanocomposites. High Perform. Polym. 2016, 28, 618-629. [CrossRef]

14. Xiong, J.; Liu, Y.; Yang, X.; Wang, X. Thermal and mechanical properties of polyurethane/montmorillonite nanocomposites based on a novel reactive modifier. Polym. Degrad. Stab. 2004, 86, 549-555. [CrossRef]

15. Zhang, H.; Jia, X.; Yu, J.; Xue, L. Effect of expanded vermiculite on microstructures and aging properties of styrene-butadiene-styrene copolymer modified bitumen. Constr. Build. Mater. 2013, 40, 224-230. [CrossRef]

16. Liang, S.; Li, C.; Dai, L.; Tang, Q.; Cai, X.; Zhen, B.; Xie, X.; Wang, L. Selective modification of kaolinite with vinyltrimethoxysilane for stabilization of Pickering emulsions. Appl. Clay Sci. 2018, 161, 282-289. [CrossRef]

17. Frost, R.L.; Kristof, J.; Horvath, E.; Kloprogge, J.T. Deintercalation of dimethylsulphoxide intercalated kaolinites: A DTA/TGA and Raman spectroscopic study. Thermochim. Acta 1999, 327, 155-166. [CrossRef]

18. Tchoumene, R.; Dedzo, G.K.; Ngameni, E. Preparation of Methyl Viologen-Kaolinite Intercalation Compound: Controlled Release and Electrochemical Applications. ACS Appl. Mater. Interfaces 2018, 10, 34534-34542. [CrossRef]

19. Isci, S.; Isci, Y. Characterization and comparison of thermal \& mechanical properties of vermiculite polyvinylbutyral nanocomposites synthesized by solution casting method. Appl. Clay Sci. 2018, 151, 189-193.

20. Isci, S. Intercalation of vermiculite in presence of surfactants. Appl. Clay Sci. 2017, 146, 7-13. [CrossRef]

21. Su, X.; Ma, L.; Wei, J.; Zhu, R. Structure and thermal stability of organo-vermiculite. Appl. Clay Sci. 2016, 132, 261-266. [CrossRef]

22. $\mathrm{Wu}, \mathrm{N}$.; Wu, L.; Liao, L.; Lv, G. Organic intercalation of structure modified vermiculite. J. Colloid Interface Sci. 2015, 457, 264-271. [CrossRef]

23. Hattab, Y.; Benharrats, N. Thermal stability and structural characteristics of PTHF-Mmt organophile nanocomposite. Arab. J. Chem. 2015, 8, 285-292. [CrossRef] 
24. Belhouchat, N.; Zaghouaneboudiaf, H.; Viseras, C. Removal of anionic and cationic dyes from aqueous solution with activated organo-bentonite/sodium alginate encapsulated beads. Appl. Clay Sci. 2017, 135, 9-15. [CrossRef]

25. Jin, J.; Tan, Y.; Liu, R.; Zheng, J.; Zhang, J. Synergy Effect of Attapulgite, Rubber, and Diatomite on Organic Montmorillonite-Modified Asphalt. J. Mater. Civ. Eng. 2019, 31, 04018388. [CrossRef]

26. Solomon, M.J.; Almusallam, A.S.; Seefeldt, K.F.; Somwangthanaroj, A.; Varadan, P. Rheology of polypropylene/clay hybrid materials. Macromolecules 2001, 34, 1864-1872. [CrossRef]

27. Mcnally, T.; Murphy, W.R.; Lew, C.Y.; Turner, R.J.; Brennan, G. Polyamide-12 layered silicate nanocomposites by melt blending. Polymer 2003, 44, 2761-2772. [CrossRef]

28. Uno, H.; Tamura, K.; Yamada, H.; Umeyama, K.; Hatta, T.; Moriyoshi, Y. Preparation and mechanical properties of exfoliated mica-polyamide 6 nanocomposites using sericite mica. Appl. Clay Sci. 2009, 46, 81-87. [CrossRef]

29. Liang, Y.; Ding, H.; Wang, Y.; Liang, N.; Wang, G. Intercalation of cetyl trimethylammonium ion into sericite in the solvent of dimethyl sulfoxide. Appl. Clay Sci. 2013, 74, 109-114. [CrossRef]

30. Liang, Y.; Ding, H.; Sun, S.; Chen, Y. Microstructural Modification and Characterization of Sericite. Materials 2017, 10, 1182. [CrossRef]

31. Gu, C.; Peng, T.; Sun, H.; Lv, X.; Luo, L. Assembled Structure and Characterization of $\mathrm{TiO}_{2} / \mathrm{Montmorillonite}^{2}$ nanocomposites. J. Synth. Cryst. 2012, 41, 771-778.

(C) 2019 by the authors. Licensee MDPI, Basel, Switzerland. This article is an open access article distributed under the terms and conditions of the Creative Commons Attribution (CC BY) license (http://creativecommons.org/licenses/by/4.0/). 
Article

\title{
High Performance Attapulgite/Polypyrrole Nanocomposite Reinforced Polystyrene (PS) Foam Based on Supercritical $\mathrm{CO}_{2}$ Foaming
}

\author{
Yidong Liu ${ }^{1,2, \dagger}{ }^{\dagger}$ Lingfeng Jian ${ }^{1, \dagger}{ }^{+}$, Tianhua Xiao ${ }^{1}$, Rongtao Liu ${ }^{1}$, Shun $\mathrm{Yi}^{1}{ }^{1}$, Shiyang Zhang ${ }^{1}$, \\ Lingzhi Wang ${ }^{1}$, Ruibin Wang ${ }^{1, *}$ and Yonggang Min ${ }^{1,2, *}$ \\ 1 School of Materials and Energy, Center of Emerging Material and Technology, Guangdong University of \\ Technology, Guangzhou 510006, Guangdong, China; ydliu@gdut.edu.cn (Y.L.); lif23216@163.com (L.J.); \\ xth1011@126.com (T.X.); liu44101@163.com (R.L.); 13290065303@163.com (S.Y.); syz131139@163.com (S.Z.); \\ lingzhiwanglz@126.com (L.W.) \\ 2 Dongguan South China Design Innovation Institute, Dongguan 523808, Guangdong, China \\ * Correspondence: wang.rb@gdut.edu.cn (R.W.); ygmin@gdut.edu.cn (Y.M.); \\ Tel.: +86-020-3932-2570 (R.W. \& Y.M.) \\ + These authors contributed equally to this work.
}

Received: 21 March 2019; Accepted: 7 May 2019; Published: 4 June 2019

\begin{abstract}
CO}_{2}$ has been regarded as one of the most promising blowing agents for polystyrene (PS) foam due to its non-flammability, low price, nontoxicity, and eco-friendliness. However, the low solubility and fast diffusivity of $\mathrm{CO}_{2}$ in PS hinder its potential applications. In this study, an attapulgite (ATP)/polypyrrole (PPy) nanocomposite was developed using the in situ polymerization method to generate the hierarchical cell texture for the PS foam based on the supercritical $\mathrm{CO}_{2}$ foaming. The results demonstrated that the nanocomposite could act as an efficient $\mathrm{CO}_{2}$ capturer enabling the random release of it during the foaming process. In contrast to the pure PS foam, the ATP/PPy nanocomposite reinforced PS foam is endowed with high cell density (up to $1.9 \times 10^{6}$ ) and similar thermal conductivity as the neat PS foam, as well as high compression modulus. Therefore, the in situ polymerized ATP/PPy nanocomposite makes supercritical $\mathrm{CO}_{2}$ foaming desired candidate to replace the widely used fluorocarbons and chlorofluorocarbons as PS blowing agents.
\end{abstract}

Keywords: supercritical $\mathrm{CO}_{2}$; polystyrene foam; blowing agent; in situ polymerization; attapulgite/ polypyrrole nanocomposite

\section{Introduction}

Polystyrene (PS) foam is one of the most popular and low-cost polymeric foams that is widely used in many applications such as household materials, food containers, lightweight composites, toy models, and packaging [1-6]. As is well known, the blowing agent applied during the foaming process is the key factor for achieving PS foam of good quality. Recently, with the rising environmental concerns from currently used blowing agents such as fluorocarbons (FCs) and chlorofluorocarbons (CFCs), carbon dioxide $\left(\mathrm{CO}_{2}\right)$ has attracted tremendous interests from the scientific and industrial communities. Compared with FCs and CFCs, $\mathrm{CO}_{2}$ at its supercritical state $\left(T=31{ }^{\circ} \mathrm{C}\right.$ and $\left.P=73.8 \mathrm{bar} / 1074 \mathrm{psi}\right)$ can be competitive because it is inexpensive, non-flammable, nontoxic, environmentally friendly, and chemically inert [7-10]. However, $\mathrm{CO}_{2}$ also has some drawbacks, in that it is usually processed at higher pressures and easily escapes from the polymer matrix, which induces processing instability and shaping contraction. These will result in an uncontrollable foam density and cell morphology of PS foam, thus leading to poor thermal and mechanical properties [11-13]. In this case, it is requisite to develop an efficient strategy to manage $\mathrm{CO}_{2}$ for PS foaming. 
In a traditional process based on supercritical $\mathrm{CO}_{2}$ foaming, a large quantity of $\mathrm{CO}_{2}$ is dissolved in PS that can modify the rheological properties of PS in the barrel of the extruder, then resulting in extensive expansion during the relaxation at the die. Consequently, the reduction of viscosity decreases the mechanical constraints and the operating temperature within the extruder [14-16]. It was found that $\mathrm{CO}_{2}$ can decrease the viscosity of PS without otherwise changing their pseudoplastic behavior. The viscosity data for the PS based on supercritical $\mathrm{CO}_{2}$ foaming follow the ideal viscoelastic scaling, whereby the set of viscosity curve can be scaled to a master curve of reduced viscosity vs. reduced shear rate identical to the viscosity curve for the pure PS [17]. It was also reported that $\mathrm{CO}_{2}$ could blow the PS-based composites. Pang et al. studied the effect of $\mathrm{CO}_{2}$ as the processing medium to improve the in situ compatibilization of polypropylene/PS blends via reactive extrusion. $\mathrm{CO}_{2}$ had been proved to enable an improved phase dispersion and thus the mechanical properties of the polypropylene/PS through two functions: $\mathrm{CO}_{2}$-assisted phase dispersion and $\mathrm{CO}_{2}$-promoted in situ compatibilization [18,19]. In addition, the PS/carbon-based nanoparticle composites with different shapes or dimensions were prepared using supercritical $\mathrm{CO}_{2}$ as the foaming agent, of which the nucleation mechanism was well analyzed by the classical nucleation theory [20].

In this study, attapulgite (ATP) and polypyrrole (PPy) have been in situ polymerized to give a nanocomposite to assist $\mathrm{CO}_{2}$ for PS foaming, where the ATP/PPy nanocomposite is expected to act as a " $\mathrm{CO}_{2}$ capturer" to control the $\mathrm{CO}_{2}$ releasing during foaming [21]. The ATP-PS foams possessed cells of the similar three-petal shape as the neat PS foam, while the ATP/PPy nanocomposites reinforced PS foams displayed significantly different cellular structure. As demonstrated in the SEM images, it was found that the ATP/PPy nanocomposites reinforced PS foams were composed of multi-petal $(\geq 3)$ and hierarchical petal-in-petal textures. The foam density and cell size features of the ATP/PPy nanocomposites reinforced PS foams were significantly different from the pure PS foam. This could be understood from the fact that the uniquely fibrous ATP with PPy could form the separated pathways for $\mathrm{CO}_{2}$ during the foaming process, interfering with its spontaneous release. As a result, the ATP/PPy nanocomposites reinforced PS foams were endowed with similar thermal conductivity, as well as up to $8.06{ }^{\circ} \mathrm{C}$ higher thermal decomposition temperature (TDT) and $181 \%$ higher compression modulus, compared to $415.78^{\circ} \mathrm{C}$ and $1.09 \mathrm{MPa}$ of the neat PS foam. The ATP/PPy-PS foam with these excellent performances opens enormous opportunities for them to be used in the lightweight composite, microwave absorption, energy, and catalytic applications.

\section{Materials and Methods}

\subsection{Materials}

Pyrrole, Ammonium persulfate (APS), Sodium hexametaphosphate (SHMP), (3-Aminopropyl) triethoxysilane (KH-550), and other chemicals were purchased from Aladdin Chemistry Co., Ltd. (Shanghai, China). All chemicals were of analytical reagent grade used without further purification. PS (Nova 1600) was provided by NOVA Chemical, Inc. (Painesville, OH, USA). Deionized (DI) water was used throughout this study.

ATP was purified from the crude mineral ores (Gansu, China) as follows: First, the crude mineral ores were put into the planetary ball mill (QM3SP4L, Shanghai Xinnuo Instrument Equipment Co., LTD, Shanghai, China) and ball milled at $400 \mathrm{rpm}$ for $8 \mathrm{~h}$. The resulting solid was further ground in an agate mortar for $10 \mathrm{~min}$, followed by sifting fine ATP ( $400 \mathrm{mesh}$ ) from the ores. After that, the fine ATP was dispersed in DI water then ultra-sonicated for $30 \mathrm{~min}$ to obtain the uniform dispersion. Subsequently, the ATP dispersion was centrifuged at $5000 \mathrm{rpm}$ for $30 \mathrm{~min}$. Finally, further air-drying was conducted at $105^{\circ} \mathrm{C}$ overnight to complete the purification (Figure 1). 

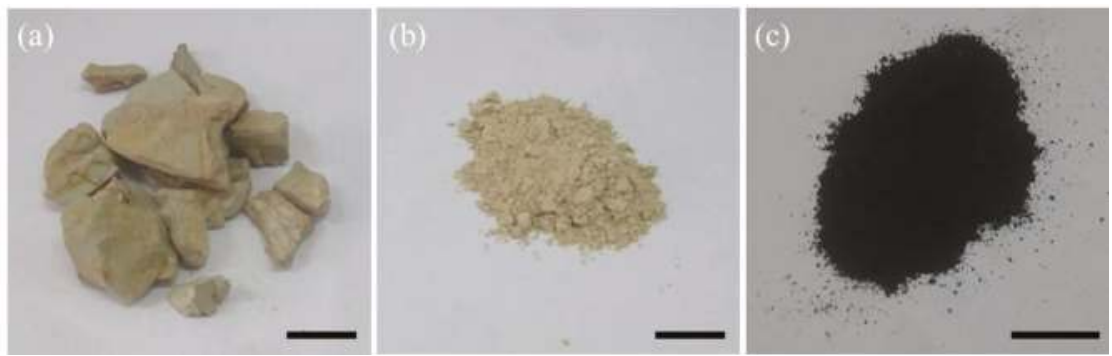

Figure 1. Digital camera images of (a) the crude mineral ore of attapulgite (ATP), (b) purified ATP and (c) the ATP/polypyrrole (PPy) nanocomposite. Scale bar: $1 \mathrm{~cm}$.

\subsection{Foam Extrusion of ATP/PPy Nanocomposite Reinforced PS}

At first, the ATP/PPy nanocomposites were prepared according to the chemical oxidation for the preparation of PPy. Specifically, $0.9 \mathrm{~g}$ of purified ATP was dispersed in $500 \mathrm{~mL}$ of DI water under magnetic stirring overnight at ambient temperature. Pyrrole monomers of desired amount and $500 \mathrm{~mL}$ of $\mathrm{HCl}(2 \mathrm{M})$ were subsequently introduced into the previous solution, under vigorous magnetic stirring for another $24 \mathrm{~h}$ at $5{ }^{\circ} \mathrm{C}$. Then a mixture of APS (13.02 g) and $100 \mathrm{~mL}$ of DI water was added in to initiate the polymerization for about $16 \mathrm{~h}$, during which the temperature of which was kept at $5{ }^{\circ} \mathrm{C}$. The obtained suspension was washed with deionized water three times and centrifuged to obtain the sediment. Finally, the sediment was collected and freeze-dried to give the powdery ATP/PPy nanocomposite (Figure 1c).

The ATP/PPy nanocomposite reinforced PS foams were prepared by a modified method as originally presented by Castro and co-workers [10]. Foaming was done using a twin screw extruder equipped with a pelletizer die as shown in Figure 2 (Leistritz ZSE-27; $D=27 \mathrm{~mm} ; L / D=40$ ). The temperature zones of the extruder barrel were kept at $160^{\circ} \mathrm{C}$ in the feed zone and $180^{\circ} \mathrm{C}$ at the die tip. The extruder was typically run at $100 \mathrm{rpm}$. The 2.0, 2.8, and $3.3 \mathrm{wt} \%$ of ATP/PPy nanocomposite were selected to reinforce the PS foams, thus being labeled as ATP/PPy-PS 1, 2, and 3, respectively. For a comparison purpose, pristine PS and PS reinforced by the purified ATP alone were also prepared, denoting as PS foam and ATP-PS 1, 2, 3 (with respectively 2.0, 2.8, and $3.3 \mathrm{wt} \%$ of purified ATP included). The visual observations of neat PS, ATP-PS 3, and ATP/PPy-PS are presented in Figure 3 (optical camera images).

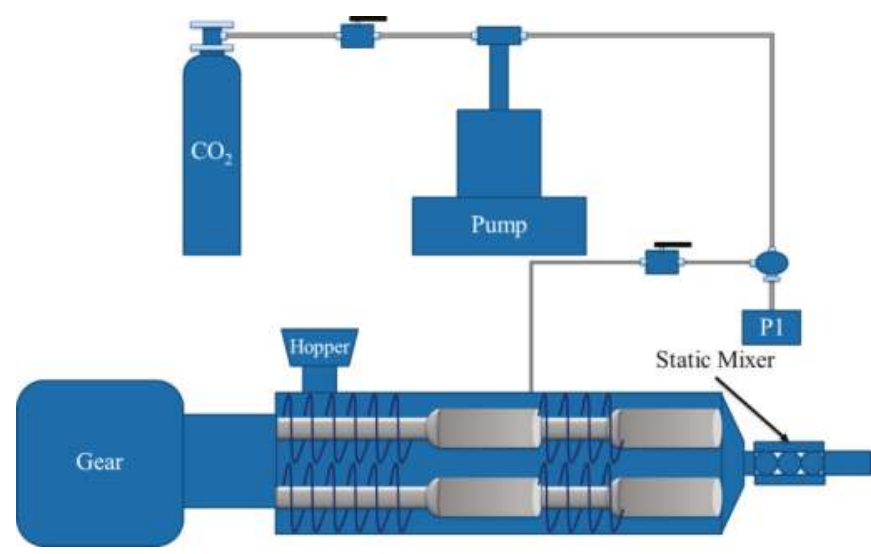

Figure 2. The continuous foaming extrusion process for the fabrication of the polystyrene (PS) foam in this study. 

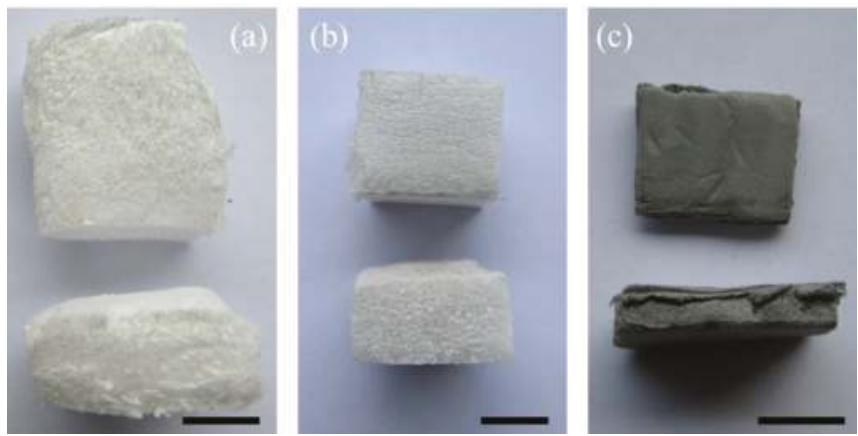

Figure 3. Optical camera images (upper: top view, lower: cross section view) of (a) neat PS foam, (b) ATP-PS 3, (c) ATP/PPy-PS 3. Scale bar: $1 \mathrm{~cm}$.

The cell size and cell density of all PS foams (Figure 4, scanning electron microscopy, SEM images) were determined by using both the inverted microscope (equipped with a $4 \times$ objective lens, ECLIPSE Ti2-U, Nikon Corporation, Tokyo, Japan) and SEM (S3400N, Hitachi Co., Ltd., Tokyo, Japan). At least 70 cells were taken into account to determine the average cell size and cell density. The obtained morphological properties and densities of the neat PS foam, ATP-PS foams, and ATP/PPy-PS foams are summarized in Table 1. Specifically, the foam density of each sample was calculated according to the following equation from the ASTM D792 standard [22]:

$$
\text { FoamDensity }=\frac{M_{\text {air }} \times \rho_{\text {water }}}{M_{\text {air }}-M_{\text {water }}}
$$

where $M_{\text {air }}$ is the apparent mass of the sample in air, $M_{\text {water }}$ is the apparent mass of the sample in water, and $\rho_{\text {water }}$ is the density of water.
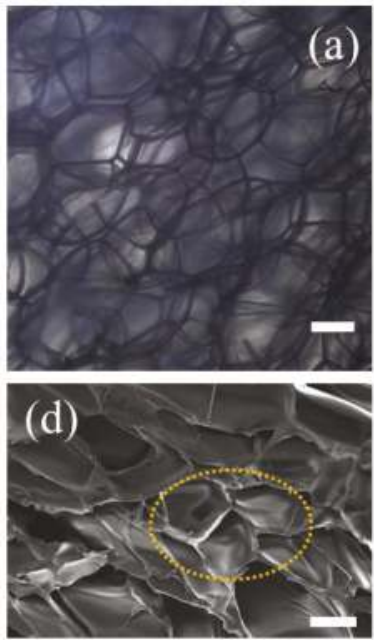
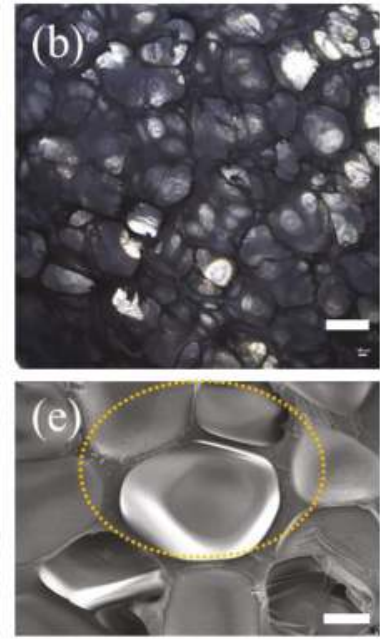

Figure 4. Cont.
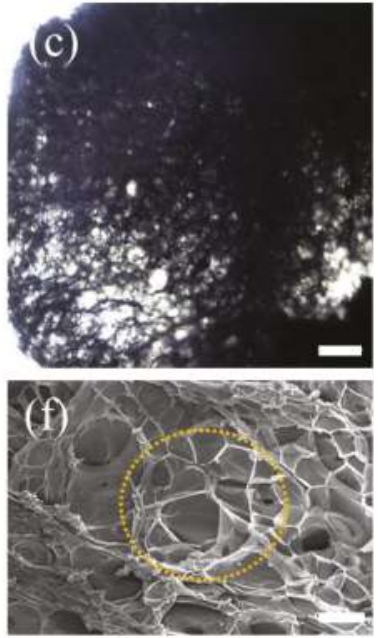


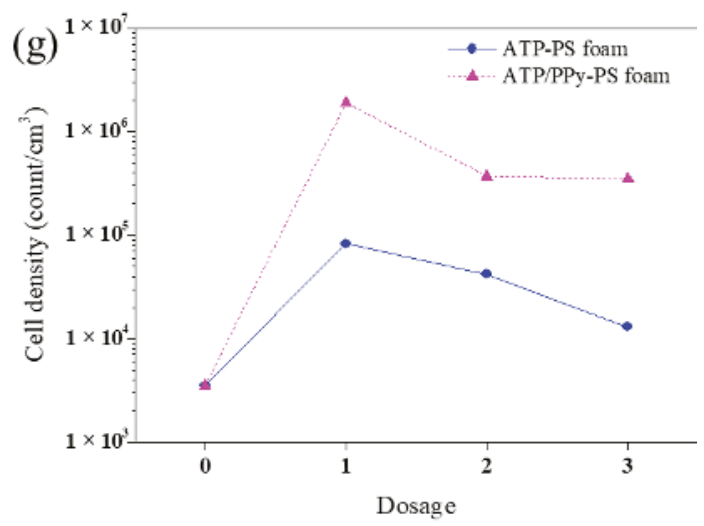

Figure 4. The optical microscopy images showing the cell size and cell density of (a) neat PS foam, (b) ATP-PS 3 and (c) ATP/PPy-PS 3, scale bar: $500 \mu \mathrm{m}$. Top view scanning electron microscopy (SEM) micrographs of (d) neat PS foam, (e) ATP-PS 3 and (f) ATP/PPy-PS 3, scale bar: $2 \mu \mathrm{m}$. (g) Cell density test of ATP-PS foam and ATP/PPy-PS foams.

Table 1. Density and morphological properties of neat PS foam, ATP-PS foam, and ATP/PPy-PS foam.

\begin{tabular}{cccc}
\hline Sample & Foam Density $\left(\mathrm{g} / \mathrm{cm}^{3}\right)$ & Cell Size $(\mathrm{mm})$ & Cell Density $\left(\mathrm{cells} / \mathrm{cm}^{3}\right)$ \\
\hline neat PS & $0.070 \pm 0.004$ & $0.52 \pm 0.06$ & $(3.5 \pm 0.2) \times 10^{3}$ \\
ATP-PS 1 & $0.188 \pm 0.015$ & $0.40 \pm 0.03$ & $(8.3 \pm 0.2) \times 10^{4}$ \\
ATP-PS 2 & $0.132 \pm 0.008$ & $0.53 \pm 0.02$ & $(4.2 \pm 0.2) \times 10^{4}$ \\
ATP-PS 3 & $0.159 \pm 0.016$ & $0.56 \pm 0.04$ & $(1.3 \pm 0.1) \times 10^{4}$ \\
ATP/PPy-PS 1 & $0.052 \pm 0.004$ & $0.19 \pm 0.01$ & $(1.9 \pm 0.1) \times 10^{6}$ \\
ATP/PPy-PS 2 & $0.058 \pm 0.007$ & $0.27 \pm 0.02$ & $(3.7 \pm 0.2) \times 10^{5}$ \\
ATP/PPy-PS 3 & $0.055 \pm 0.003$ & $0.22 \pm 0.01$ & $(3.5 \pm 0.1) \times 10^{5}$ \\
\hline
\end{tabular}

\subsection{Thermal and Mechanical Properties}

Thermogravimetric analysis (TGA) was carried out using a Simultaneous TGA/differential scanning calorimetry (DSC) analyzer (TGA/DSC 3+, Mettler Toledo, Greifensee, Switzerland) with temperature scanning from ambient to $800^{\circ} \mathrm{C}$ at a heating ramp of $10^{\circ} \mathrm{C} / \mathrm{min}$. Besides this, TDT for all samples was defined as the temperature where $50 \%$ of weight loss was viewed. Also, the thermal stability of samples was evaluated by differential scanning calorimetry (DSC, TGA/DSC 3+, Mettler Toledo, Switzerland) while flowing $\mathrm{N}_{2}$ over a range of $25-800^{\circ} \mathrm{C}$ and with a heating rate of $10^{\circ} \mathrm{C} / \mathrm{min}$. In addition, the thermal conductivity was evaluated by using Thermal Constants Analyzer (TPS-500s, Hot Disk AB Co., Gothenburg, Sweden) at room temperature under $20 \mathrm{~mW}$ within $10 \mathrm{~s}$.

The compressive properties measurements were carried out using an electronic universal testing machine (Inspekt table blue $5 \mathrm{kN}$, Hegewald \& Peschke, Nossen, Germany) at a testing speed of $1 \mathrm{~mm} / \mathrm{min}$ for all samples according to the ASTM D1621-16 standard. The sample size is $40 \mathrm{~mm} \times$ $20 \mathrm{~mm} \times 16 \mathrm{~mm}$.

All the above tests were performed on at least five samples from each set of measurements.

\section{Results and Discussion}

\subsection{Foam Morphology}

The morphology of the foam samples was examined to investigate the effect of the ATP/PPy nanocomposites on $\mathrm{CO}_{2}$ for foaming PS. The optical images compare the cell size and cell density of PS foam with others, which illustrate the ATP-PS foam is made up of many three-petal flowers, which is 
similar to that viewed for neat PS foam, whereas the ATP/PPy-PS foam exhibits significantly different multi-petal and hierarchical-petal flower textures (Figure $4 a-c)$. The unique texture of ATP/PPy-PS foam was further determined by SEM (Figure 4d-f). With the addition of ATP/PPy nanocomposite, the obtained foams displaying increased cell density than both the PS foam and the ATP-PS foam as graphed in Figure $4 \mathrm{~g}$. In contrast to $3.5 \times 10^{3}$ of the PS foam, the cell density of the ATP/PPy-PS foams increased 500-fold to within $3.5 \times 10^{5}-1.9 \times 10^{6}$. Meanwhile, as listed in Table 1, both the foam density and average cell size for the ATP/PPy-PS foams were decreased to $0.052-0.058 \mathrm{~g} / \mathrm{cm}^{3}$ and $0.19-0.27 \mathrm{~mm}$, respectively, from $0.132-0.188 \mathrm{~g} / \mathrm{cm}^{3}$ and $0.40-0.56 \mathrm{~mm}$ for the ATP-PS foam. To our knowledge, this may be attributed to that the positive effect brought by overdosage of ATP or ATP/PPy can cause many drawbacks such as low diffusion and long contact time on the supercritical $\mathrm{CO}_{2}$ foaming [23].

On the basis of these results, we propose that the reinforcement of ATP/PPy nanocomposites may be attributed to the physical confinement of them. On one hand, after the inclusion of the nanocomposites, the initial geometry of PS during foaming was changed as the nanocomposites would occasionally stand in the way where the PS cells growing. As a result, it is possible for $\mathrm{CO}_{2}$ to get split or disorder, correspondingly generating the cells of multi-petal and hierarchical-petal flower textures. This constraint effect of the nanocomposites can be understood by regarding them as the $\mathrm{CO}_{2}$ capturer to manage its releasing other than the usual manner [24-26]. On the other hand, both the rod-like ATP and fibrous PPy may mislead $\mathrm{CO}_{2}$ to flow on more directions. Herein, ATP functions as a good substrate for other polymers, such as PPy in this study or polyaniline in our previous papers, to get improved performances $[27,28]$.

\subsection{Thermal and Mechanical Properties}

The thermal properties are crucial for PS-based materials. As addressed above, the incorporation of ATP or ATP/PPy nanocomposites in PS can facilitate the random releasing of $\mathrm{CO}_{2}$, however, the increased thermal conductivity was simultaneously observed (Figure 5a). The highest thermal conductivity was of $0.054 \mathrm{~W} /(\mathrm{mK})$ for ATP-PS $1,38 \%$ higher than $0.039 \mathrm{~W} /(\mathrm{mK})$ of the neat PS foam, which hinders the potential application of ATP-PS, though further increasing the content of ATP in the ATP-PS foams exhibits a decreased trend. This may be due to that, although ATP is a thermally conductive clay $(\sim 0.68 \mathrm{~W} /(\mathrm{mK}))$, it is also easy to get agglomerated to embody decreased heat transfer. In other words, the obtained ATP-PS foams with higher ATP content sacrificed their thermal conductivity to gain increased applicable potential. Note that the thermal conductivity curve of the ATP/PPy-PS foams is much different from that of the ATP-PS foams, which linearly grows along with the content of the ATP/PPy nanocomposite. On one hand, the inclusion of comparably thermally non-conductive PPy in the foam can compromise ATP. On the other hand, the growth of fibrous PPy on ATP in the nanocomposites avoids ATP to agglomerate $[29,30]$. Besides this, TGA is applied to estimate the thermal stability of these foams according to their thermogravimetric behavior (Figure 5b). As shown in Figure 5c, it can be clearly observed that all foams shared similar TGA curve patterns, while ATP/PPy-PS 1 exhibited the highest TDT, which is $\sim 8^{\circ} \mathrm{C}$ higher than that of the PS foam. Encouragingly, all ATP/PPy-PS foams outperformed the ATP-PS foams with respect to TDT. This manifests that ATP has a limited effect on PS here, unless nanocomposited with PPy to form the thermostable interactions between $\mathrm{Fe}_{2} \mathrm{O}_{3}(7.53 \mathrm{wt} \%)$ in ATP and PPy chains [31]. Increasing the dosage of ATP shows a similar trend as seen in Figure 5a, suggesting that the inclusion of highly thermally conductive ATP in the foams facilitates their decomposition under heating. Note that the TDT curve of the ATP/PPy-PS foams is similar to that of ATP-PS foams, though significantly different from its thermal conductivity data. These effects most likely due to the relatively thermal stable dopant anion of PPy, which can increase the TDT of the ATP/PPy-PS foams [32]. In addition, the thermal behavior of all foams was also evaluated by DSC. As elucidated in Figure 5d, the DSC profiles of the PS foam, the ATP-PS foams, and the ATP/PPy-PS foams are alike, indicating that they were based on the same degradation mechanism as the decompositions of neat PS [33]. 

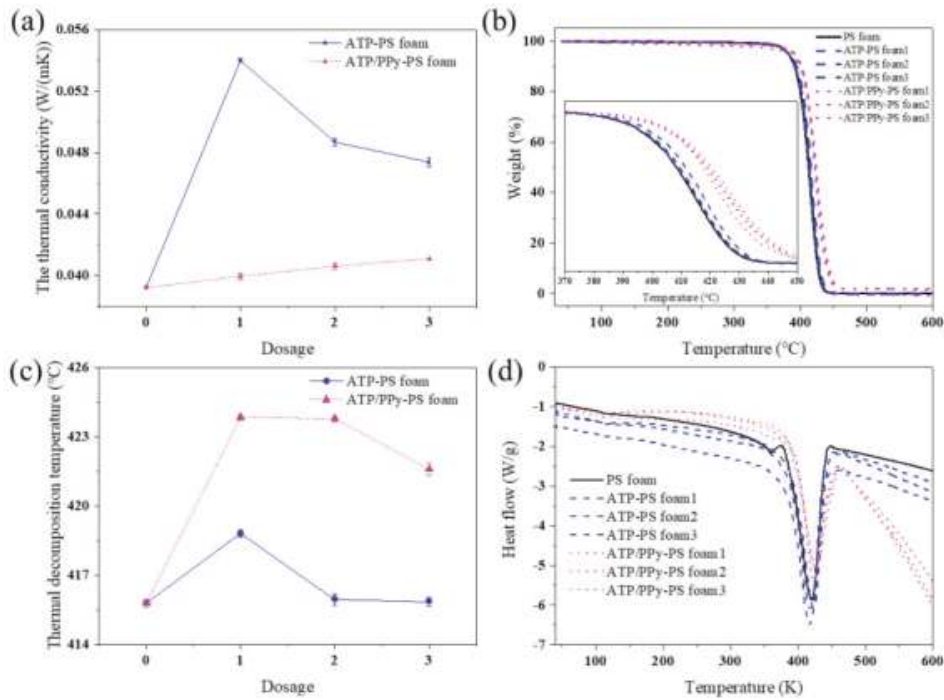

Figure 5. (a) Thermal conductivity, (b) thermogravimetric analysis (TGA, the partial curves within the temperature range of $370-450{ }^{\circ} \mathrm{C}$ were zoomed in as shown in the inset), (c) thermal decomposition temperature (TDT) and (d) differential scanning calorimetry (DSC) analyses of neat PS foam, ATP-PS foams, and ATP/PPy-PS foams, respectively.

As a foam material, mechanical properties are also highly desired. Figure 6 a shows the compression modulus of all foams; the ATP-PS foams and the ATP/PPy-PS foams present up to 4.18 (383\%) and 3.06 $\mathrm{MPa}(281 \%)$, respectively, much higher than $1.09 \mathrm{MPa}(100 \%)$ of the neat PS foam. The compression modulus data of the ATP-PS foams and the ATP/PPy-PS foams are inconsistent with the cell density value of them as listed in Table 1. This may be due to that the calculated cell density calculation concerns all cells in three dimensions, whereas the compression modulus is only directly related to the vertical dimension. Furthermore, the open-cell percentage of the ATP/PPy-PS foams varies a lot from the others, $63.71-78.96 \%$ vs. $33.95 \%$ and $31.00-40.07 \%$ (Figure $6 \mathrm{~b}$ ). This difference can be clearly told in the respective top view SEM images as demonstrated in Figure 6c-i. As reported earlier, materials with open cellular structures are known to exhibit low modulus [34]. Therefore, we believe that when the close-cell statue is maintained, the compression modulus of the ATP-PS foams can be significantly increased from that of the neat PS foam, in agreement with the changing trend of their cell density. The introduction of aromatic tertiary amine groups on the surface of ATP leads to the parallel alignment of aromatic rings in the PS chains that conduces to the effective load transfer between the ATP surface and PS matrix [35]. On the other hand, once it is reversed into the open-cell statue, as happened on the ATP/PPy-PS foams, the contribution of the significantly changed cell density to the compression modulus may be compromised to some extent. Nonetheless, the highly open-cell ATP/PPy-PS foams still outperform their nonporous or poorly porous counterparts in regard to the application of microwave absorption as well as the accessibility of the active surface of the materials [36-40]. 

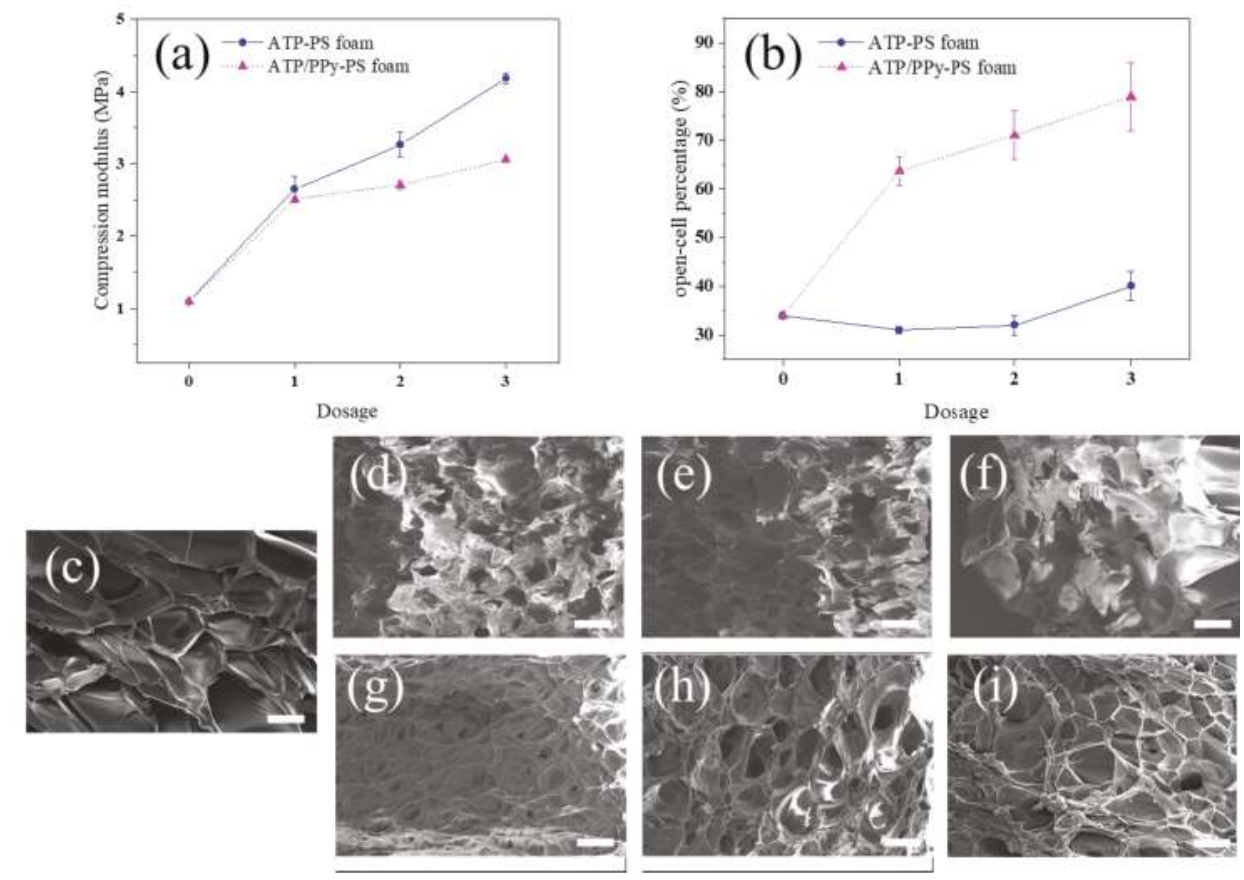

Figure 6. (a) The compression modulus measurements of neat PS foam, ATP-PS foams, and ATP/PPy-PS foams, according to the ASTM D1621-16 standard. (b) Corresponding open-cell percentage of all foams, with respective top view SEM images of (c) neat PS foam, (d-f) ATP-PS 1, 2, 3 and (g-i) ATP/PPy-PS 1 , 2,3 , scale bar: $2 \mu \mathrm{m}$.

\section{Conclusions}

In summary, the in situ polymerized attapulgite/polypyrrole nanocomposite has been successfully introduced into the supercritical $\mathrm{CO}_{2}$-based PS foam. The optical graphs and SEM images of all foams demonstrated that even though the typical foam structure of PS was formed throughout the others, of which the foam density and cell size features were significantly different. By combining the nanocomposites, the thus-obtained foams exhibited the cells of significantly different multi-petal and hierarchical-petal flower textures, along with more than 500 times higher $\left(1.9 \times 10^{6}\right)$ cell density than the pure PS foam. This may be attributed to that the nanocomposite could act as an efficient $\mathrm{CO}_{2}$ capturer so enables the random release of it during the foaming process. Also, the thermal behavior and mechanical properties of the ATP/PPy-PS foams were investigated, which were endowed with up to $8.06^{\circ} \mathrm{C}$ higher TDT and $281 \%$ higher compression modulus than those of the neat PS foam. Overall, the ATP/PPy nanocomposite reinforced PS foams fabricated in this study will open up numerous opportunities for a range of applications based on the supercritical $\mathrm{CO}_{2}$ foaming.

Author Contributions: Conceptualization, Y.L., L.J., T.X., R.L., S.Y., S.Z., and L.W.; methodology, Y.L. and L.J.; software, L.J. and R.W.; validation, Y.L. and Y.M.; formal analysis, Y.L. and L.J.; investigation, T.X.; resources, R.W. and Y.M.; data curation, L.J.; writing—original draft preparation, L.J.; writing-review and editing, Y.L. and R.W; visualization, L.W.; supervision, Y.L.; project administration, Y.M.; funding acquisition, Y.M.

Funding: This research was funded by the Program for Guangdong Introducing Innovative and Entrepreneurial Team (2016ZT06C412) and the Hundred Talent Program of Guangdong University of Technology (220418095).

Conflicts of Interest: The authors declare no conflict of interest. 


\section{References}

1. Dong, M.; Gu, X.; Zhang, S.; Li, H.; Jiang, P. Effects of Acidic Sites in HA Zeolite on the fire performance of polystyrene composite. Ind. Eng. Chem. Res. 2013, 52, 9145-9154. [CrossRef]

2. Demirel, B. Optimization of the composite brick composed of expanded polystyrene and pumice blocks. Constr. Build. Mater. 2013, 40, 306-313. [CrossRef]

3. Chen, W.; Hao, H.; Hughes, D.; Shi, Y.; Cui, J.; Li, Z.-X. Static and dynamic mechanical properties of expanded polystyrene. Mater. Des. 2015, 69, 170-180. [CrossRef]

4. Min, Z.; Yang, H.; Chen, F.; Kuang, T. Scale-up production of lightweight high-strength polystyrene/ carbonaceous filler composite foams with high-performance electromagnetic interference shielding. Mater. Lett. 2018, 230, 157-160. [CrossRef]

5. Jing, X.; Peng, X.-F.; Mi, H.-Y.; Wang, Y.-S.; Zhang, S.; Chen, B.-Y.; Zhou, H.-M.; Mou, W.-J. Cell evolution and compressive properties of styrene-butadiene-styrene toughened and calcium carbonate reinforced polystyrene extrusion foams with supercritical carbon dioxide. J. Appl. Polym. Sci. 2016. [CrossRef]

6. Ji, W.-Y.; Feng, L.-F.; Zhang, C.-L.; Hoppe, S.; Hu, G.-H.; Dumas, D. A concept of reactive compatibilizer-tracer for studying reactive polymer blending processes. AIChE J. 2016, 62, 359-366. [CrossRef]

7. Zeng, C.; Han, X.; Lee, L.J.; Koelling, K.W.; Tomasko, D.L. Polymer-clay nanocomposite foams prepared using carbon dioxide. Adv. Mater. 2003, 15, 1743-1747. [CrossRef]

8. Han, X.; Zeng, C.; Lee, L.J.; Koelling, K.W.; Tomasko, D.L. Extrusion of polystyrene nanocomposite foams with supercritical $\mathrm{CO}_{2}$. Polym. Eng. Sci. 2003, 43, 1261-1275. [CrossRef]

9. Yu, P.; Liu, G.; Li, K.; Huang, A.; Chen, B.; Mi, H.; Zhang, S.; Peng, X. Fabrication of polystyrene/nano-CaCO 3 foams with unimodal or bimodal cell structure from extrusion foaming using supercritical carbon dioxide. Polym. Compos. 2016, 37, 1864-1873. [CrossRef]

10. Cabrera, E.D.; Mulyana, R.; Castro, J.M.; Lee, L.J.; Min, Y. Pressurized water pellets and supercritical nitrogen in injection molding. J. Appl. Polym. Sci. 2013, 127, 3760-3767. [CrossRef]

11. Han, X.; Koelling, K.W.; Tomasko, D.L.; Lee, L.J. Continuous microcellular polystyrene foam extrusion with supercritical $\mathrm{CO}_{2}$. Polym. Eng. Sci. 2002, 42, 2094-2106. [CrossRef]

12. Xu, L.-Q.; Huang, H.-X. Formation mechanism and tuning for bi-modal cell structure in polystyrene foams by synergistic effect of temperature rising and depressurization with supercritical $\mathrm{CO}_{2}$. J. Supercrit. Fluids 2016, 109, 177-185. [CrossRef]

13. Sovova, H.; Nistor, A.; Topiar, M.; Kosek, J. Vitrification conditions and porosity prediction of $\mathrm{CO}_{2}$ blown polystyrene foams. J. Supercrit. Fluids 2017, 127, 1-8. [CrossRef]

14. Sauceau, M.; Nikitine, C.; Rodier, E.; Fages, J. Effect of supercritical carbon dioxide on polystyrene extrusion. J. Supercrit. Fluids 2007, 43, 367-373. [CrossRef]

15. Wang, L.; Feng, L.-F.; Gu, X.-P.; Zhang, C.-L. Influences of the viscosity ratio and processing conditions on the formation of highly oriented ribbons in polymer blends by tape extrusion. Ind. Eng. Chem. Res. 2015, 54, 11080-11086. [CrossRef]

16. Ji, W.-Y.; Feng, L.-F.; Zhang, C.-L.; Hoppe, S.; Hu, G.-H. Development of a reactive compatibilizer-tracer for studying reactive polymer blends in a twin-screw extruder. Ind. Eng. Chem. Res. 2015, 54, 10698-10706. [CrossRef]

17. Kwag, C.; Manke, C.W.; Gulari, E. Rheology of molten polystyrene with dissolved supercritical and near-critical gases. J. Polym. Sci. Part B 1999, 37, 2771-2781. [CrossRef]

18. Wang, K.; Pang, Y.; Liu, W.; Wu, F.; Zheng, W. A new approach designed for improving in situ compatibilization of polypropylene/polystyrene blends via reactive extrusion with supercritical $\mathrm{CO}_{2}$ as the processing medium. J. Supercrit. Fluids 2016, 118, 203-209. [CrossRef]

19. Han, X.; Shen, J.; Huang, H.; Tomasko, D.L.; Lee, L.J. $\mathrm{CO}_{2}$ foaming based on polystyrene/poly(methyl methacrylate) blend and nanoclay. Polym. Eng. Sci. 2007, 47, 103-111. [CrossRef]

20. Xiao, W.; Liao, X.; Li, S.; Xiong, J.; Yang, Q.; Li, G. The distinctive nucleation of polystyrene composites with differently shaped carbon-based nanoparticles as nucleating agent in the supercritical $\mathrm{CO}_{2}$ foaming process. Polym. Int. 2018, 67, 1488-1501. [CrossRef]

21. Wan, H.; Que, Y.; Chen, C.; Wu, Z.; Gu, Z.; Meng, J.; Wang, L.; Guan, G. Preparation of metal-organic framework/attapulgite hybrid material for $\mathrm{CO}_{2}$ capture. Mater. Lett. 2017, 194, 107-109. [CrossRef] 
22. ASTM Committee D-20 on Plastics. Standard Test Methods for Density and Specific Gravity (Relative Density) of Plastics by Displacement; ASTM International: West Conshohocken, PA, USA, 2008.

23. Weidner, E. Impregnation via supercritical $\mathrm{CO}_{2}-$ What we know and what we need to know. J. Supercrit. Fluids 2018, 134, 220-227. [CrossRef]

24. Yang, Y.; Gupta, M.C.; Dudley, K.L.; Lawrence, R.W. Novel carbon nanotube-polystyrene foam composites for electromagnetic interference shielding. Nano Lett. 2005, 5, 2131-2134. [CrossRef] [PubMed]

25. Krause, B.; Diekmann, K.; van der Vegt, N.F.A.; Wessling, M. Open nanoporous morphologies from polymeric blends by carbon dioxide foaming. Macromolecules 2002, 35, 1738-1745. [CrossRef]

26. Reverchon, E.; Cardea, S. Production of controlled polymeric foams by supercritical $\mathrm{CO}_{2}$. J. Supercrit. Fluids 2007, 40, 144-152. [CrossRef]

27. Zhang, S.; Gao, L.; Shan, L.; Wang, R.; Min, Y. Comparative study on the adsorption of $\mathrm{NO}_{2}$ using different clay/polyaniline composites. Ind. Eng. Chem. Res. 2018, 57, 6897-6903. [CrossRef]

28. Huang, J.; Li, H.; Shan, L.; Gao, L.; Yu, Y.; Min, Y. Adsorption of sulfur dioxide in flue gas by polyamine based composite absorbent. Chin. J. Environ. Eng. 2017, 11, 3587-3593.

29. Gu, J.; Li, N.; Tian, L.; Lv, Z.; Zhang, Q. High thermal conductivity graphite nanoplatelet/UHMWPE nanocomposites. RSC Adv. 2015, 5, 36334-36339. [CrossRef]

30. Liu, Y.; Liu, P.; Su, Z. Thermal stabilities of attapulgite/polystyrene (ATP/PS) nanocomposites via microwave-assisted bulk polymerization. J. Dispers. Sci. Technol. 2008, 29, 478-481. [CrossRef]

31. Chen, W.; Li, X.; Xue, G.; Wang, Z.; Zou, W. Magnetic and conducting particles: preparation of polypyrrole layer on $\mathrm{Fe}_{3} \mathrm{O}_{4}$ nanospheres. Appl. Surf. Sci. 2003, 218, 216-222. [CrossRef]

32. Oh, E.J.; Jang, K.S.; MacDiarmid, A.G. High molecular weight soluble polypyrrole. Synth. Met. 2001, 125, 267-272. [CrossRef]

33. Eskander, S.B.; Tawfik, M.E.; Tawfic, M.L. Mechanical, flammability and thermal degradation characteristics of rice straw fiber-recycled polystyrene foam hard wood composites incorporating fire retardants. J. Therm. Anal. Calorim. 2018, 132, 1115-1124. [CrossRef]

34. Ryan, G.; Pandit, A.; Apatsidis, D.P. Fabrication methods of porous metals for use in orthopaedic applications. Biomaterials 2006, 27, 2651-2670. [CrossRef] [PubMed]

35. Yang, H.; Zhang, L.; Ma, W.; Pu, H.; Gong, F. Polystyrene/attapulgite nanocomposites prepared via in situ suspension polymerization with redox initiation system. J. Appl. Polym. Sci. 2015. [CrossRef]

36. Minas, C.; Carnelli, D.; Tervoort, E.; Studart, A.R. 3D printing of emulsions and foams into hierarchical porous ceramics. Adv. Mater. 2016, 28, 9993-9999. [CrossRef] [PubMed]

37. Yan, D.-X.; Ren, P.-G.; Pang, H.; Fu, Q.; Yang, M.-B.; Li, Z.-M. Efficient electromagnetic interference shielding of lightweight graphene/polystyrene composite. J. Mater. Chem. 2012, 22, 18772-18774. [CrossRef]

38. Li, W.; Qiu, T.; Wang, L.; Ren, S.; Zhang, J.; He, L.; Li, X. Preparation and electromagnetic properties of core/shell polystyrene@polypyrrole@nickel composite microspheres. ACS Appl. Mater. Interfaces 2013, 5, 883-891. [CrossRef]

39. Liao, G.; Chen, J.; Zeng, W.; Yu, C.; Yi, C.; Xu, Z. Facile preparation of uniform nanocomposite spheres with loading silver nanoparticles on polystyrene-methyl acrylic acid spheres for catalytic reduction of 4-nitrophenol. J. Phys. Chem. C 2016, 120, 25935-25944. [CrossRef]

40. Zhang, L.; Gu, J.; Song, L.; Chen, L.; Huang, Y.; Zhang, J.; Chen, T. Underwater superoleophobic carbon nanotubes/core-shell polystyrene@Au nanoparticles composite membrane for flow-through catalytic decomposition and oil/water separation. J. Mater. Chem. A 2016, 4, 10810-10815. [CrossRef]

(C) 2019 by the authors. Licensee MDPI, Basel, Switzerland. This article is an open access article distributed under the terms and conditions of the Creative Commons Attribution (CC BY) license (http://creativecommons.org/licenses/by/4.0/). 
Article

\title{
Dispersion and Performance of a Nanoclay/Whey Protein Isolate Coating upon its Upscaling as a Novel Ready-to-Use Formulation for Packaging Converters
}

\author{
Elodie Bugnicourt ${ }^{1, *,+}$, Nicola Brzoska ${ }^{2,+}$, Esra Kucukpinar ${ }^{2}$, Severine Philippe ${ }^{3}$, \\ Enrico Forlin ${ }^{4}$, Alvise Bianchin ${ }^{4}$ and Markus Schmid ${ }^{5}$ \\ 1 IRIS Technology Solutions S.L., Parc Mediterrani de la Tecnologia, 08860 Barcelona, Spain \\ 2 Fraunhofer-Institute for Process Engineering and Packaging IVV, 85354 Freising, Germany \\ 3 Visum, NexusUCD, University College Dublin, D04 V1W8 Dublin, Ireland \\ 4 MBN Nanomaterialia S.p.A., 31050 Vascon di Carbonera (TV), Italy \\ 5 Faculty of Life Sciences, Albstadt-Sigmaringen University, 72488 Sigmaringen, Germany \\ * Correspondence: ebugnicourt@iris.cat; Tel.: +34-93-554-25-00 \\ + These authors contributed equally to this work.
}

Received: 24 July 2019; Accepted: 23 August 2019; Published: 28 August 2019

\begin{abstract}
Studies on composition optimisation showed that the mixing of nanoclays to whey protein-isolate (WPI)-based coating formulations offers an effective strategy to reduce the oxygen permeability of coated polymer films. The scaling up of the various processing stages of these formulations was undertaken to prove their industrial feasibility. The aim was to investigate the effect of various preparation methods at different production scales (pilot- and semi-industrial scale) on the barrier performance and morphological properties of the applied nanocomposites. A nano-enhanced composition was converted into a so-called "ready-to-use" formulation by means of a solid-state pre-dispersion process using ball-milling. The process yielded a nearly dust-free, free-flowing powder containing agglomerated particles, which can easily be mixed with water. The preparation of a coating formulation using the ready-to-use granules and its upscaling for roll-to-roll converting at pilot- and semi-industrial scale was also successfully implemented. The effects of both the production at various scales and ultrasound treatment on the morphology and barrier performance of the nanocomposites were characterized by transmission electron microscopy, scanning electron microscopy, as well as oxygen permeability measurements. Results have shown that the addition of nanoclays to WPI-based coating formulations ultimately led to significantly reduced oxygen permeabilities to $0.59 \mathrm{~cm}^{3}$, $100 \mu \mathrm{m} \cdot \mathrm{m}^{-2} \cdot \mathrm{d}^{-1} \cdot \mathrm{bar}^{-1}$ (barrier improvement factor, BIF of 5.4) and $0.62 \mathrm{~cm}^{3}, 100 \mu \mathrm{m} \cdot \mathrm{m}^{-2} \cdot \mathrm{d}^{-1} \cdot \mathrm{bar}^{-1}$ (BIF of 5.1) in cases of pilot- and semi-industrial-processed coatings, respectively, compared to a reference without nanoclay. In both cases, a similar degree of nanoparticle orientation was achieved. It was concluded that the solid state pre-dispersion of the nanoplatelets during the production of the ready-to-use formulation is the predominant process determining the ultimate degree of nanoparticle orientation and dispersion state.
\end{abstract}

Keywords: dispersion; coatings; whey protein isolate; nanocomposites; nanoclay, barrier; morphology

\section{Introduction}

The ability of protein-based films and coatings to act as a superior barrier against the permeation of oxygen in packaging materials has attracted a great deal of attention in recent years, as extensive research has demonstrated [1-11]. Research has shown that the application of coating formulations based on native whey proteins followed by in-line denaturation, in contrast to the application of preliminary denatured whey proteins formulations, allows the processing of formulations with much higher solid contents while still providing an optimum barrier against oxygen [12,13]. 
While currently being currently routed to commercialisation, whey protein-based coatings offer several advantages compared to conventional petrochemically-sourced oxygen barrier materials, such as poly (ethylene-co-vinyl alcohol) (EVOH), as they are extracted from renewable resources and, despite usually being biodegradable, also offer the opportunity to be recycled [14]. Indeed, upon the enzymatic removal of whey protein-based coating layers from multilayer films and laminate, these coatings can contribute to end-of-life product management as a sub sequential film, and laminate delamination allows for the separate recycling of the materials [15]. Moreover, when applied on biodegradable polymeric substrates, e.g., poly (lactic acid) (PLA), the design and production of compostable packaging concepts is possible [16]. These aspects enable converters in the plastics packaging sector to cope with the EU action plan for a circular economy, which was adopted by the European commission in 2015 and supports the goal that all plastic packaging is recyclable by 2030 [17].

However, protein-based films still have drawbacks that need to be addressed in order to allow them to be competitive with conventional established materials in the market place. This particularly includes the excellent but still higher oxygen permeability of neat whey protein isolate (WPI) in comparison to petroleum-based high barrier materials, such as poly (ethylene-co-vinyl alcohol) (EVOH) or copolymers of poly (vinylidene chloride) [18].

One effective strategy to address this drawback is the application of nanocomposites [19-22]. Among other biopolymers, nanocomposites based on whey proteins have become a promising field of research [23-28]. Amongst other researchers, we have studied nanocomposite coatings for their ability to improve the barrier performance of these coatings by extending the pathway and time and reducing the effective volume fraction for diffusing molecules, which is generally referred to as the tortuous-path effect [29]. In order to fully take advantage of these effects, the uniform and nanoscale dispersion of the nanoparticles is required [30]. In the case of layered silicates, the formation of a nanocomposite depends on several factors, such as the nature and type of the matrix, their compatibility, as well as the dispersion process [31]. True nanocomposites with enhanced properties comprise an intercalated or exfoliated structure, in contrast to phase-separated micro composites, which do not achieve enhanced barrier properties [31,32]. Previously published studies have compared different coating formulations and been dedicated to selecting a suitable plasticiser and nanoparticle type and concentration necessary to obtain optimized oxygen barrier performance with an emphasis on the processability of the nano-enhanced formulations [33,34].

The present study goes one step further than the prior art in the upscaling of nano-enhanced whey protein-based coating formulations and focuses on the influence of the dispersion process of a ready-to-use coating formulation on the final oxygen barrier performance and morphology of nanocomposite coatings processed at different scales.

\section{Materials and Methods}

\subsection{Materials}

WPI was obtained from Agropur Ingredients (Saint-Hubert, Laval, QC, Canada; previously Davisco Food International Inc.). Aqueous nanoclay dispersions were provided by ITENE, Valencia, Spain, as described in our previously published results [34]. The plasticising agent, as selected in a prior published study that focused on formulation optimization [34], was purchased from Panreac S.A., Barcelona, Spain to be used as a plasticiser for the WPI-based film-forming formulations. As a substrate for the coatings, chemically pre-treated poly (ethylene terephthalate) (PET) with thicknesses of 23 and $100 \mu \mathrm{m}$, respectively (PLASTIKA-ANDREJ MESOJEDEC S.P., Polje, Slovenia), were respectively used for the application of coating formulations using a pilot-scale and a semi-industrial-scale processing environment. 


\subsubsection{Preparation of WPI-Based Ready-to-Use Formulations by High-Energy Ball-Milling}

WPI was mixed, using a high-energy ball-milling approach, with plasticizer and nanoclay according to a coating formulation that was selected based on previously published results [33,34]. Dry ball-milling of the formulation constituents in form of a fine powder (WPI, plasticizer and nanoclay) was performed by a proprietary process of MBN, specifically adapted for the manufacturing of ready-to-use formulations for biopolymer nanocomposites. The homogeneous dispersion of formulation constituents as well as an exfoliation effect of the utilized nanoclay was achieved in dry conditions, without the addition of any solvent. The fine and light-weighted powder obtained was then agglomerated in form of granules by using a minimum amount of water, which highly reduced the dustiness of the mixture. The ready-to-use formulation, which is in form of granules, just needs to be mixed with water, as described below, for obtaining the feedstock dispersion for coating processes. In order to be able to interpret the results achieved with the nano-enhanced WPI-based coating formulation, a pristine ready-to-use formulation was also milled and agglomerated without the addition of nanoclay.

\subsubsection{Coating Preparation, Application, and Drying}

Coating formulations for pilot- and semi-industrial-scale processing with and without nanoparticles were obtained by mixing agglomerated ready-to-use formulations with deionized water. In contrast to pilot- and semi-industrial-scale, coating formulations based on pre-dispersed ready-to-use granules were not prepared for lab-scale coatings. Instead, the formulation constituents were mixed and the aqueous nanodispersions were dispersed altogether. The solid content was kept at about $18.2 \%$ ( $w / w$, referred to the total weight of the aqueous formulation) for all coating formulations. The nanoparticle concentration was at about $15 \%(w / w$, relative to the WPI content of the ready-to-use formulation [34]). This corresponds to a final nanoparticle concentration of approximately $9 \%(w / w$, relative to the dry coating weight of the WPI/plasticiser solids).

\section{Lab-Scale}

For lab-scale processing, aqueous protein-plasticizers formulations with (in that case using the above mentioned clay dispersions from ITENE) and without nanoparticles were mixed at $23^{\circ} \mathrm{C}$ and $130 \mathrm{rpm}$ in a magnetic stirrer for $24 \mathrm{~h}$.

In order to understand the effect of an ultrasonication treatment on the intercalation/exfoliation state of the used nanoclay, some of the coating formulations were treated with an ultrasound horn for up to $240 \mathrm{~min}$ at $20 \mu \mathrm{m}$ of amplitude. Treatment by ultrasound was realized by recirculation of the prepared sample. While the treatment with ultrasound was taking place, the sample was agitated with a magnetic stirrer at $100 \mathrm{rpm}$. The ultrasonic system was from ISM-Industrial Sonomechanics (Miami, FL, USA) and included a $1200 \mathrm{~W}$ ultrasonic generator, piezoelectric transducer, a full-wave Barbell Horn, and an $80 \mathrm{~mL}$ reactor chamber with cooling jacket. Subsequently, about $5 \mathrm{~mL}$ of these still native coating formulations were then applied on a $100 \mu \mathrm{m}$ chemically pre-treated PET film using a wired-rod (Lumaquin, Barcelona, Spain, $100 \mu \mathrm{m}$ wet-film thickness).

In order to understand the effect of an ultrasonication treatment on the intercalation/exfoliation state of the used nanoclay, samples coated with and without ultrasound-treated formulations have been compared in terms of their dispersion quality and gas barrier performance. For this purpose, a nanodispersion provided by ITENE (Valencia, Spain) was mixed with a WPI-based aqueous formulation and treated with an ultrasound horn for variable times of up to $240 \mathrm{~min}$ at $20 \mu \mathrm{m}$ of amplitude. Samples were taken at different time-intervals. Treatment by ultrasound was realized by recirculation of the prepared sample. While the treatment with ultrasound was taking place, the sample was agitated with a magnetic stirrer at $100 \mathrm{rpm}$.

The coatings were dried in a stove at $(50 \pm 2)^{\circ} \mathrm{C}$ for $5 \mathrm{~min}$ and then kept at ambient conditions $\left(57 \% \mathrm{RH}\right.$ and $\left.20.7^{\circ} \mathrm{C}\right)$ for at least $15 \mathrm{~h}$. Each coating was carried out in four replicas. Although the 
drying procedure is far from the optimal that induces in-process denaturation to maximise oxygen barrier properties, it allows one to obtain comparative results to show the effect of nanoparticles.

Pilot-Scale

For pilot-line processing, the aqueous coating formulations obtained using the ready-to-use granules were mixed at $23^{\circ} \mathrm{C}$ and $200 \mathrm{rpm}$ in an electrically heatable stirrer (Thermomix 31-1, Vorwerk Deutschland Stiftung \& Co. KG, Berlin, Germany) for $30 \mathrm{~min}$, and then at $90^{\circ} \mathrm{C}$ and $400 \mathrm{rpm}$ for another $30 \mathrm{~min}$. The heat-treatment induces the denaturation of the native whey proteins. Subsequently, both formulations, with and without nanoparticles, were transferred into a $2.5 \mathrm{~L}$ glass bottle and cooled down to room temperature using a water bath and an electrical stirrer at $200 \mathrm{rpm}$. Both formulations were degassed in an ultrasonic bath (DT $514 \mathrm{H}$, Ultrasonic peak output: $860 \mathrm{~W}$, bandelin electronic $\mathrm{GmbH} \& \mathrm{Co}$. KG, Berlin, Germany) at $25^{\circ} \mathrm{C}$ and $37 \mathrm{kHz}$ for $30 \mathrm{~min}$ to allow incorporated air to escape.

Pre-denatured WPI-based coating formulations were applied on a $23 \mu \mathrm{m}$ chemically pre-treated PET film using a reverse-gravure coating system by a pilot-scale lacquering and lamination line (available at Fraunhofer IVV, Freising, Germany) at width of $360 \mathrm{~mm}$. The ceramic-coated anilox roller had a helically-engraved (Hachure) surface with 14 lines per centimeter and an angle of $45^{\circ}$ diagonally to the main axis, resulting in a theoretical wet-film coating volume of approximately $90 \mathrm{~mL} \cdot \mathrm{m}^{-2}$. The web-speed during the coating application was $5 \mathrm{~m} \cdot \mathrm{min}^{-1}$, whereas the rotational speed of the gravure-roll was $6 \mathrm{~m} \cdot \mathrm{min}^{-1}$.

The coatings were dried convectively using hot air at a temperature of $120{ }^{\circ} \mathrm{C}$ in an in-line drying-tunnel with a dwell-time of approximately $90 \mathrm{~s}$. For both coating formulations, with and without nanoparticles, single-, double-, and triple-layer coatings were performed at dry layer thicknesses of approximately 10,17 , and $30 \mu \mathrm{m}$, respectively. This leads to a multiple drying of the first and the second-layer coatings.

Semi-Industrial-Scale

For the semi-industrial processing-line, the aqueous coating formulations obtained using the ready-to-use granules were mixed at $23^{\circ} \mathrm{C}$ and $2 \mathrm{rpm}$ for $30 \mathrm{~min}$ with a three-bladed propeller stirrer (diameter $10 \mathrm{~cm}$ ). The still native WPI-based coating dispersions were then put to rest over night to allow incorporated air to escape. Prior to coating application, a quantity of the aqueous nano-enhanced formulation was exposed to an ultrasonication-treatment ( $1200 \mathrm{~W}, 20 \mu \mathrm{m}$ amplitude) similar to the lab-scale trials with the aim to investigate a possible effect on the barrier performance and layer morphology of the resulting coatings. However, in order to avoid possible re-agglomeration of the particles that were already exfoliated during solid-state pre-dispersion of the ready-to-use formulation, its exposure was limited to a 1 min time-interval.

Native WPI-based coating formulations were applied on a $100 \mu \mathrm{m}$ chemically pre-treated PET film using an engraved application roller on a semi-industrial coating machine (available at the company Lajovic Tuba d.o.o., home built with support from IRIS Technology Solutions, Barcelona, Spain).

The coatings were dried using a specifically built convective and infrared drying section, which provides a finely adjustable temperature profile, following previously published research results [12] and patented conditions [13]. These conditions ensure an optimal in-process denaturation of the native whey protein fraction of the coating formulation. Further visual understanding of the in-line drying process is available in the supplementary materials section.

\subsection{Methods}

\subsubsection{Transmission Electron Microscopy}

To ascertain the need for a sonication of the liquid coating formulations, transmission electron microscopy (TEM) was performed on selected samples coated in lab scale at the UCD Conway Institute of Biomolecular and Biomedical Research (University College Dublin, Dublin, Ireland), using protocols 
developed previously for delicate biological samples to avoid degrading the biopolymer coatings, whereby fixation is performed in two steps, first by glutaraldehyde fumes, then by Osmium fumes. The sample is then plunged into acetone, followed by a multi-step substitution by an epoxy resin, and then a final polymerization step is carried out.

\subsubsection{Scanning Electron Microscopy}

To determine the layer thicknesses of the coated samples and the intercalation/exfoliation state of the nanoparticles within the WPI-based matrix, specimens taken from pilot and semi-industrial scale produced coatings were prepared for scanning electron microscopy (SEM) investigations. Specimens were cut into small pieces and adhered between two silicon-wafers using a conductive two-component-adhesive and were then cured in a vise to maintain a minimal pressure for at least $30 \mathrm{~min}$ at ambient conditions. The cross-sectional interfaces were prepared by an $\mathrm{Ar}^{+}$-ion beam, using a cross-section polisher IB-19530CP (JOEL Ltd., Akishima, Japan). Samples were coated with a thin gold layer to reduce electrical charging of the non-conducting polymeric layers. SEM images were taken using a SEM JSM-7200F (JOEL Ltd., Akishima, Japan) at various accelerating voltages and magnifications. Layer thicknesses of the investigated samples could be determined using a software package (JOEL Ltd., Akishima, Japan).

\subsubsection{Oxygen permeability}

The coulometric oxygen permeability (OP) measurements were performed at $23^{\circ} \mathrm{C}$ and $50 \% \mathrm{RH}$ using an Ox-Tran ${ }^{\circledR} 2 / 20$ measurement device from Mocon, Brooklyn Park, MN, USA according to DIN 53380-3. A two-fold determination was performed in all cases. The oxygen permeability values, $Q_{\text {coating, }}$ for the applied coatings were calculated according to

$$
\frac{1}{Q_{\text {coating }}}=\frac{1}{Q_{\text {total }}}-\frac{1}{Q_{\mathrm{PET}}}
$$

where $Q_{\text {total }}$ is the oxygen permeability measured for the coated PET substrate. $Q_{\mathrm{PET}}$ is the oxygen permeability of the PET, and it is measured as 48.7 and $12.5 \mathrm{~m}^{3} \cdot \mathrm{m}^{-2} \cdot \mathrm{d}^{-1} \cdot \mathrm{bar}^{-1}$ at $23^{\circ} \mathrm{C}$ and $50 \% \mathrm{RH}$ for the PET layer thicknesses of 23 and $100 \mu \mathrm{m}$, respectively. In order to allow direct comparisons between the different coatings independent of the coating layer thicknesses, the oxygen permeability, $Q_{\text {coating, }}$ was calculated for a layer thickness of $100 \mu \mathrm{m}\left(Q_{100}\right)$ by

$$
Q_{100}=Q \times \frac{d}{100}
$$

where $d$ is the dry coating layer thickness.

\section{Results}

\subsection{Effect of Ultrasound-Treatment on Lab-Scale Coating Quality Prepared from Liquid Nanoparticles Suspensions}

At lab scale, nanocomposite coatings of an initially native nano-enhanced formulation were produced with the aim to determine the effect of an additional ultrasound-treatment on the dispersion quality of the aqueous WPI-based coating formulation. As a reference, a formulation produced with the exact same coating process parameters, but without nanoparticles, was prepared as previously described (Lab-Scale).

Transmission electron microscopy (TEM) was used to analyse the layer morphology of untreated coatings and coatings, which were treated with ultrasonication for $240 \mathrm{~min}$ (Figure 1). For coatings that were treated by ultrasonication, it seems that fewer nanoparticle agglomerates were present in the dry coatings, and more individual nanoplatelets, instead of nanoplatelet-stacks, achieved an intercalated or exfoliated state (Figure 1b). 


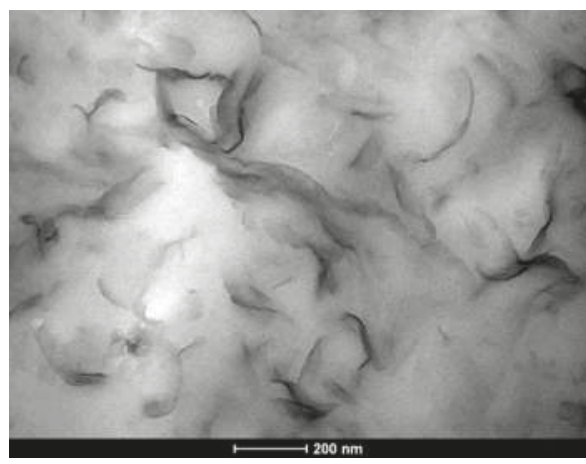

(a)

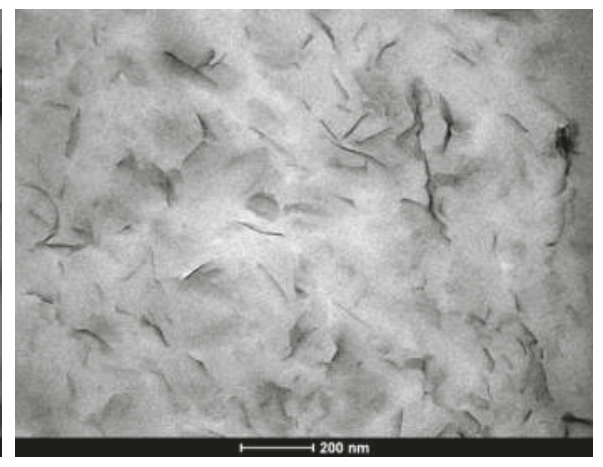

(b)

Figure 1. Transmission electron microscopy (TEM) image of coated samples produced at lab scale (6000× magnification) (a) without ultrasonication-treatment and (b) after $240 \mathrm{~min}$ of ultrasonication-treatment at $20 \mu \mathrm{m}$ amplitude.

Nevertheless, as TEM micrographs cannot lead to a quantitative conclusion, the study was complemented by X-ray diffraction and oxygen permeability measurements. X-ray diffraction measurements on cast-film samples obtained from the same coating formulations did not reveal any relevant diffraction peak, confirming the absence of short distance organisation also observed by the TEM micrographs. There is no difference in the oxygen permeability values measured for the coated specimens produced with and without using ultrasonication treatment, despite a more homogeneous distribution of the nanoparticles (Figure 1b).

\subsection{Oxygen Barrier Performance of Coatings Based on Ready-to-Use Formulations}

Oxygen barrier performance measurements were performed for WPI-based nanocomposite coatings processed at pilot- and semi-industrial-scale at Fraunhofer-IVV and TUBA, respectively. Figure 2 shows the oxygen permeability $\left(Q_{100}\right)$ of the coating layer at $23{ }^{\circ} \mathrm{C}$ and $50 \% \mathrm{RH}$. The permeability values were calculated for a coating layer thickness of $100 \mu \mathrm{m}$ according to Equations (1) and (2) in order to be able to compare the barrier performance of the various coatings with each other.

Barrier performance measurements for the samples produced at semi-industrial scale have shown that for these coatings, an average oxygen barrier performance improvement of a factor of about $5.1\left((0.62 \pm 0.03) \mathrm{cm}^{3} \cdot \mathrm{m}^{-2} \cdot \mathrm{d}^{-1} \cdot \mathrm{bar}^{-1}\right.$ at $23{ }^{\circ} \mathrm{C}$ and $\left.50 \% \mathrm{RH}\right)$ could be achieved due to the addition of nanoclay, in comparison to pristine coatings. On the other hand, no significant difference in the oxygen barrier performance was observed for the coatings pre-treated by ultrasonication. The absence of a significant effect on the barrier performance due to ultrasound treatment might be explained by the efficiency of the solid-state pre-dispersion process. During the high-energy ball-milling process, resulting high shear-rates likely lead to the intercalation of polymeric protein chains between exfoliated nanoplatelets. Good compatibility between the surface of the studied nanoplatelets, owing to their partially polar surface, and the highly polar WPI-based matrix [33], as well as the ability to form interfacial interactions between nanofiller and WPI via hydrogen bonding [35], favor the dispersion of the particles and probably contribute to maintaining their dispersed state. It seems likely that upon reconstitution of the ready-to-use mix with water, the pre-dispersed nanoplatelets remain homogeneously dispersed within the WPI matrix, as they are stabilised by intermolecular interactions with protein side chains. 


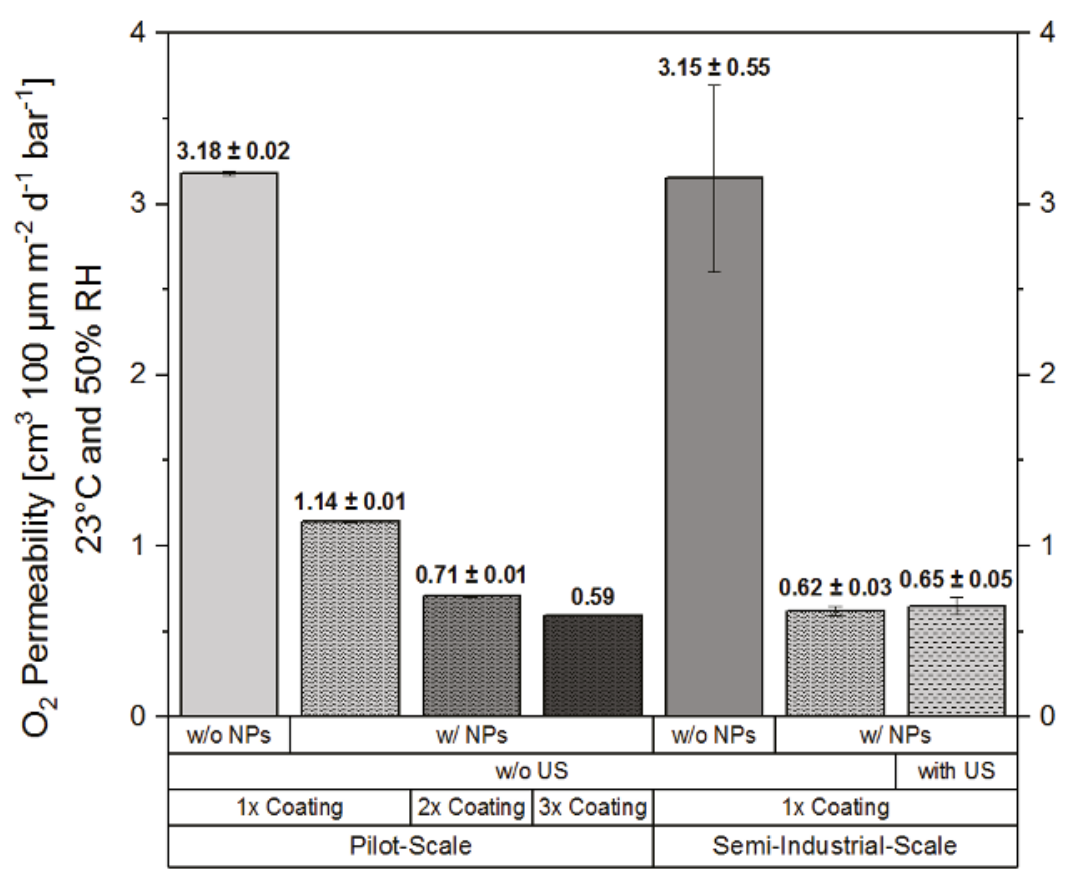

Figure 2. Oxygen permeability $\left(Q_{100}\right.$ at $23^{\circ} \mathrm{C}$ and $50 \% \mathrm{RH}$ of WPI-based coatings calculated for a coating layer thickness of $100 \mu \mathrm{m}$. Effects of nanoparticle (NP) addition, number of coating/drying steps $(1 \times, 2 \times$, and $3 \times$ coating), ultrasound treatment (US, with and without (w/o)), and manufacturing environment (pilot-, semi-industrial-scale) on the WPI-based coating formulations prepared using novel ready-to-use agglomerates are shown. The data shown represent mean values of a two-fold measurement \pm minimum/maximum deviation (range of variation).

At pilot-scale, coating trials were performed using the same nano-enhanced/pristine ready-to-use formulations, except that they were heat-treated prior to their coating on PET, as described in Section 3.2. The aim was to determine the effect of the number of coating/drying runs (single, double, or triple coating) on the oxygen permeability of the produced coatings. As expected, these coatings ultimately showed a very similar $Q_{100}$ value of about $0.59 \mathrm{~cm}^{3} \cdot \mathrm{m}^{-2} \cdot \mathrm{d}^{-1} \cdot \mathrm{bar}^{-1}$ at $23^{\circ} \mathrm{C}$ and $50 \% \mathrm{RH}$, compared to the coatings produced at semi-industrial scale. However, it has to be noted here that the extent of barrier improvement in the case of pilot-scale processed coatings highly depended on the number of coating/drying runs.

After application of a single coating layer of the nano-enhanced formulation, the oxygen permeability was reduced by a factor of about 2.8 , in comparison to pristine WPI coatings, which had a $Q_{100}$ value of about $3.2 \mathrm{~cm}^{3} \cdot \mathrm{m}^{-2} \cdot \mathrm{d}^{-1} \cdot \mathrm{bar}^{-1}$ (Figure 2). By the application of the second coating layer, the $Q_{100}$ value was reduced down to $0.7 \mathrm{~cm}^{3} \cdot \mathrm{m}^{-2} \cdot \mathrm{d}^{-1} \cdot \mathrm{bar}^{-1}$, resulting in a barrier improvement factor of about 4.5. After the application of a third coating layer, the $Q_{100}$ value was reduced to a value of about $0.59 \mathrm{~cm}^{3} \cdot \mathrm{m}^{-2} \cdot \mathrm{d}^{-1} \cdot \mathrm{bar}^{-1}$, which corresponds to a barrier improvement factor of about 5.4 in comparison to the permeability $\left(Q_{100}\right)$ of pristine WPI (Figure 2$)$. 
The improvement of the barrier performance against oxygen permeation, which, in case of pilot-scale coatings, was only achieved after a second and third coating/drying step, can likely be attributed to a lower residual moisture content of the coatings due to longer corresponding drying times. High moisture contents lead to a swollen biopolymer matrix, as residual water molecules cluster between highly polar side chains of the hydrophilic WPI and plasticiser molecules, consequently increasing the free volume within the WPI matrix $[34,36]$. The significance of the residual water content with regard to the possible barrier improvement against oxygen permeation is also underlined by the fact that coatings produced at semi-industrial-scale, in contrast to pilot-scale, already result in low oxygen permeabilities after the first coating and drying step, which might be explained by a better drying performance of the combination or infra-red and convective air dryers at semi-industrial-scale, resulting in optimal in-line process denaturation conditions for the whey proteins $[12,13]$.

Two other aspects need to be discussed regarding the oxygen barrier performance of the studied nanocomposite coating formulations. Firstly, the reduction of the effective volume available for the diffusing gas molecules due to the volume fraction that is occupied by the nanofiller and, secondly, an increase in the effective path length that the diffusing gas molecules, have to be covered due to the incorporated filler material [29].

The effectiveness of the nanoparticles to significantly increase the diffusion path, the so-called tortuosity-effect, largely depends on the shape of the particles, where in an optimum case for barrier improvement platelet-shaped particles are oriented parallel to the plane of the film, and on their uniform distribution within the polymeric matrix. To prove the hypothesis of a lowered volume that is available for diffusion processes and a prolonged diffusion path due to nano-filler addition, the microstructure of the studied coatings has been examined by scanning electron microscopy (Figure 3). In Figure 3, Images $1 \mathrm{a}$ and $1 \mathrm{~b}$ as well as $2 \mathrm{a}$ and $2 \mathrm{~b}$ show specimen of coatings produced by double or triple coating at pilot-scale (IVV), respectively, whereas the images $3 a$ and $3 b$ show specimen of coatings produced at semi-industrial-scale (TUBA). Inorganic materials are perceived as lighter shades, whereas the organic polymer matrix as well as cavities are depicted in darker shades.

Although few nanoparticle agglomerates are visible in all studied specimen, SEM images generally show a homogenous distribution of the added nano-filler within the WPI-based matrix. Based on these images, it can be concluded that in fact volume fraction available for the diffusing gas molecules was effectively reduced due to the volume fraction that is occupied by the nanofiller, and that the effective diffusion path for permeating gas molecules was significantly increased, which led to a reduction in oxygen permeability compared to pristine coatings without any nanofiller. However, despite their homogenous dispersion, no apparent differences regarding the orientation or intercalation/exfoliation state of the nanoparticles could be identified for specimens produced at semi-industrial and pilot-scale, either at a magnification of 20,000× (Images 1a, 2a, and 3a.) or at a higher magnification $(50,000 \times)$ (Images 1b, 2b, and 3b).

This indicates that despite the differences in the preparation method of the aqueous coating formulations at the different production scales, for the most part viscosity differences due to the sequence of the processing steps (since the viscosity of the WPI-based dispersions increases after heat-treatment due to denaturation-associated unfolding of proteins) as well as differences regarding the applied shear-rates (the ultimate degree of particle orientation and the intercalation/exfoliation state of the particles) are mainly affected by the solid state pre-dispersion of the nanoplatelets by means of high-energy ball-milling during the production of the ready-to-use formulation. 


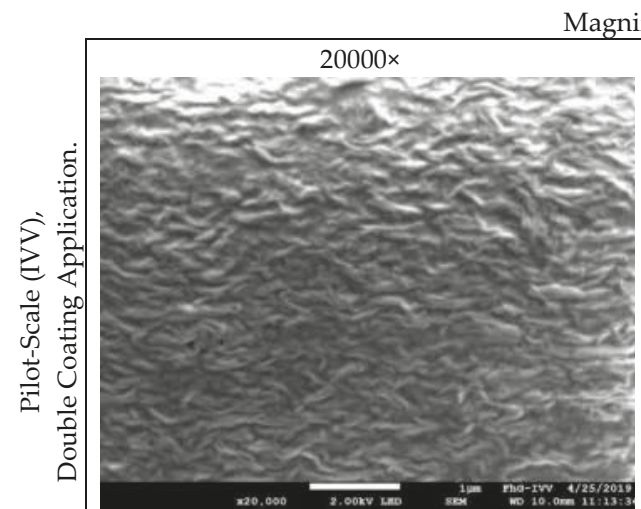

(1a)

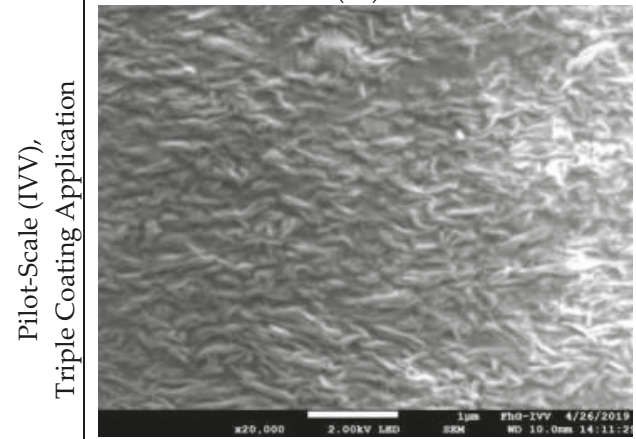

(2a)

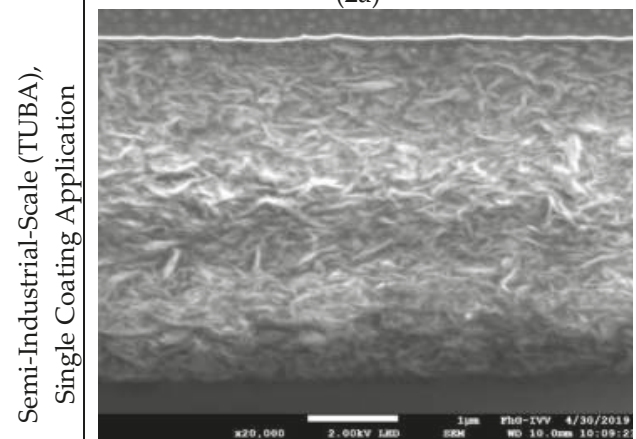

(3a)

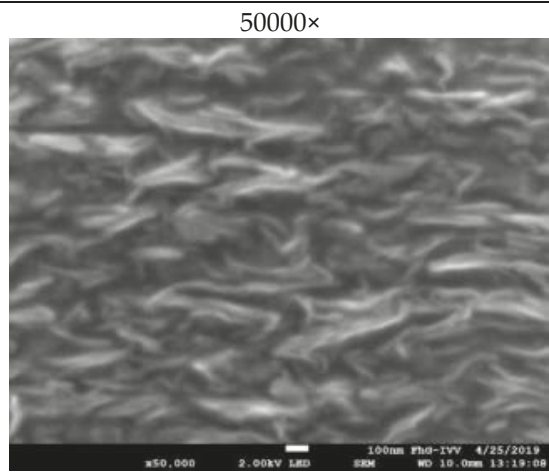

(1b)

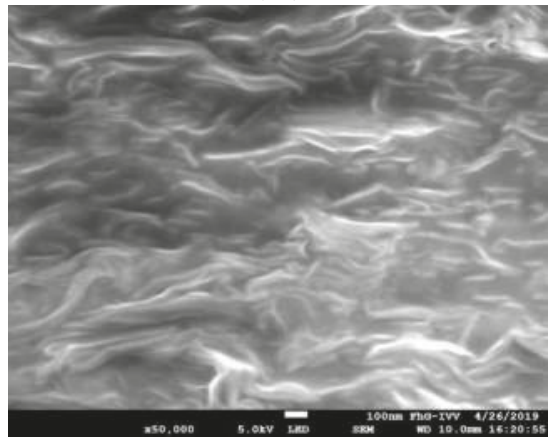

(2b)

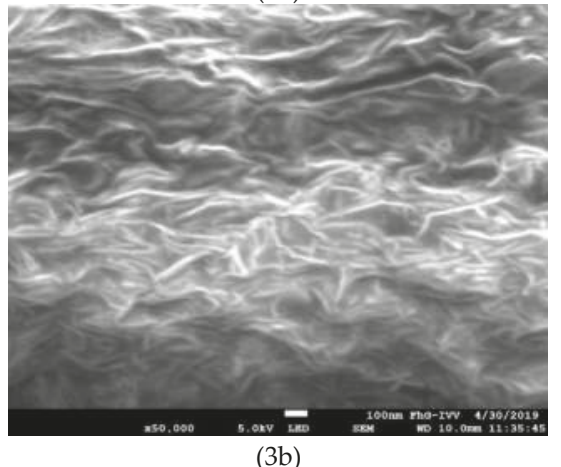

(3b)

Figure 3. Scanning electron microscopy (SEM) images, at different magnifications, (a) at a magnification of 20,000 and (b) at a magnification of 50,000, of whey protein-isolate (WPI)-based nanocomposite coatings produced at both $(1,2)$ pilot- and $(3)$ semi-industrial-scale.

\section{Discussion}

The present study highlights the potential of the studied ready-to-use coating formulations to be utilized at an industrial scale. Homogeneous and nano-scale dispersion of nanoparticles within the continuous (bio)polymer matrix is an essential feature in the formation of (bio-)nanocomposite coatings [30]. More precisely, in the case of layered silicates, to really take advantage of the addition of layered-silicate nano-fillers into a biopolymer matrix, an intercalated or exfoliated structure, which refers to an intermingling of the two phases, has to arise in order to significantly improve the properties 
of the resulting nanocomposites compared to traditional microcomposites, in which the two phases remain separated [20,31,32].

The successful implementation of the nano-enhanced, ready-to-use formulations prepared within this work demonstrates their applicability at different scales and processing conditions. However, it should be noted here that the application of a single coating in contrast to the necessity of multiple coating and drying steps to achieve the desired barrier performance and the in-process partial denaturation of the WPI, compared to time-consuming and costly pre-denaturation processes, is industrially far more feasible.

A significantly reduced oxygen permeability of about $0.6 \mathrm{~cm}^{3}, 100 \mu \mathrm{m} \cdot \mathrm{m}^{-2} \cdot \mathrm{d}^{-1} \cdot \mathrm{bar}^{-1}$ in case of pilot- and semi-industrial-processed coatings represents an improvement factor against the permeation of oxygen of about 5.4 and 5.1, respectively, in comparison to the corresponding pristine reference coatings. The barrier performance against oxygen permeation of nano-enhanced coatings produced at pilot- or semi-industrial-scale seems to be independent of the shear-rates applied during the coating application with different coating units.

A previous publication that studied the addition of layered-silicates into a WPI-based matrix reported a higher oxygen barrier improvement factor of up to 7.1 [34] - for coatings using the same nanofiller concentration as well as plasticiser type and concentration as in the present study, with an absolute oxygen permeability ( $Q_{100}$ coating monolayer) of about $0.6 \mathrm{~cm}^{3}, 100 \mu \mathrm{m} \cdot \mathrm{m}^{-2} \cdot \mathrm{d}^{-1} \cdot \mathrm{bar}^{-1}$. Whereas the absolute permeability value that was achieved by the addition of the nanofiller was the same as previously reported, the relative improvement factor was lower in the present study (factors 5.4 to 5.1), as the pristine reference coating (without any nanofiller-addition) already had a lower oxygen permeability due to the processing method (lab-scale [34] vs. pilot-scale (this study)) and drying procedure, as previously described (Lab-Scale and Pilot-Scale).

Moreover, it should be mentioned here that the used nano-filler material had an aspect ratio of about 70, as previously published by another study [37]. In the case of rectangular, platelet-shaped particles, the aspect ratio was between their diameter and thickness. Further optimization potential lies in the usage of nanoclays with higher aspect ratios, which will likely result in even more pronounced tortuosity effects at even lower nanofiller-loadings than already achieved within this work, as demonstrated in another study [33] with a different layered-silicate.

In terms of their oxygen barrier performance, WPI-based nanocomposite coatings could therefore have the potential to eventually substitute commercially available petroleum-based oxygen barrier materials, such as certain grades of poly (ethylene-co-vinyl alcohol) with high ethylene contents (EVOH $44 \%$ ) or certain halogen-containing poly (vinylidene chloride) copolymers, in multi-layer packaging applications. These high-performance materials exhibit oxygen permeabilities in the magnitude of about 0.24 to $0.11 \mathrm{~cm}^{3}, 100 \mu \mathrm{m} \cdot \mathrm{m}^{-2} \cdot \mathrm{d}^{-1} \cdot \mathrm{bar}^{-1}$, respectively.

The process yielded a nearly dust-free, free-flowing powder containing agglomerated particles that was easily reconstituted with water. The preparation of a coating formulation using the ready-to-use granules and its upscaling for roll-to-roll converting at pilot- and semi-industrial scale was successfully implemented. The effects of production at various scales and of ultrasound treatment on morphology and barrier performance of the WPI-based nanocomposites were characterized by transmission electron microscopy, scanning electron microscopy, as well as oxygen permeability measurements. In both cases, a similar degree of nanoparticle orientation could be achieved. It was concluded that the solid state pre-dispersion of the nanoplatelets during production of the ready-to-use formulation is the predominant factor affecting the ultimate degree of nanoparticle orientation and dispersion state. Thereby, the most challenging process step for the upscaling of nanoclay/whey protein isolate coatings is the fabrication of the pre-dispersed "ready-to-use" granules. This process step facilitated a homogeneous distribution of nanoparticles within the final dry WPI matrix without the need of applying high shear-rates after mixing the "ready-to-use" granules with water prior to its coating. Several other factors, such as type of the coating application system, coating speed, or drying conditions for 
maintaining protein denaturation, as well as the rheology of the nano-enhanced formulations, still need to be considered during the different upscaling stages, as shown in previous publications [12,18,34].

However, one has to bear in mind that images obtained by SEM offer only very limited information due to the small quantity of material investigated and possible heterogeneities of the material. Additionally, it has to be noted that residual moisture within highly hygroscopic biopolymer matrices, at relevant pressures during the imaging process, evaporates, and can influence the microstructure of the material that is investigated.

\section{Conclusions}

This study showed that the utilization of ready-to-use coating formulations in form of pre-dispersed agglomerates offers several advantages for flexible film converters in regard to the processability of nano-enhanced WPI-based coating formulations.

Barrier performance measurements revealed that due to the presence of nanoclay within the biopolymer matrix, the oxygen permeability $\left(Q_{100}\right)$ of WPI-based nanocomposite coatings could be decreased significantly, in the case of coating formulations processed at both pilot- and semi-industrial scales. However, for coatings produced at pilot scale without a specifically adjusted drying process, the ultimate oxygen barrier performance highly depended on effective drying times, as shown by an increasing barrier performance after the second and third drying step.

The utilization of prolonged ultrasound treatment of the aqueous nano-enhanced coating formulations produced at lab scale demonstrated that it had an effect on the homogenous dispersion of larger nanoparticle agglomerates; however, no significant differences regarding the oxygen barrier performance were observed. This approach was shown to be less efficient than the use of a solid-state pre-dispersion, as this process is more feasible at an industrial scale and also does not require a further in-line ultrasonication process.

Images obtained by SEM indicated that the nanoparticle orientation and its state of intercalation/exfoliation in the case of both coatings produced at pilot- or semi-industrial-scale were quite similar. The obtained cross-sectional images of the nanocomposite coatings suggest that the effective diffusion path for permeating gas molecules was significantly increased and the available volume for diffusion processes was significantly reduced by the addition of the nano-filler into the polymeric matrix.

This leads to the conclusion that the ultimate degree of nanoparticle orientation and its intercalation/exfoliation state is mainly affected by the effectiveness of the solid state pre-dispersion of the nanoplatelets by means of ball-milling during the production of the ready-to-use formulation. The coating application and drying methods are additional factors, with a less important contribution allowing the achievement of similar oxygen permeabilities $\left(Q_{100}\right)$ after a single native protein coating in semi-industrial-scale vs. a triple pre-denatured coating at pilot scale.

In addition to the easy handling during processing of the studied ready-to-use formulations, their usage offers various additional advantages for converters, i.e., the possibility to use native, low viscosity WPI-based dispersions, which enable the handling of high solid content formulations (up to $\sim 25 \%$ (w/w) ready-to-use formulation); their effective drying using patented process conditions; as well as a reduced dustiness of the ready-to-use formulations, leading to a greater nano-safety for operators by allowing the handling of agglomerated nanocomposite powder instead of free nanoparticles [38].

Author Contributions: E.B.: fund acquisition for the overall project, coordination of the tasks involved, conceptualisation of the article, the initial drafting thereof, overall critical revision, editing, and contributions; N.B.: preparation of the coating formulations for pilot-scale coating trials, performing of the experimental investigations, editing of the initially drafted parts of the manuscript, interpretation of the results, and writing of the manuscript; E.K.: technical management of the project, supervision of the pilot-scale trials, organization of the experiments, interpretation of the results and writing of the conclusions, overall critical revision, editing, and contributions; S.P.: supervision of the sonication trials as well as of the TEM characterisation and drafting of the related parts of the article; E.F.: investigation and validation of the process for the preparation of WPI-based, ready-to-use formulations by high-energy ball-milling at pilot scale and pre-industrial scale; A.B.: conceptualization of 
ready-to-use formulation using the high-energy ball-milling approach; M.S.: technical management of the project, reviewing and editing the manuscript via critical revision of the texts.

Funding: This research was funded by the European Union's Horizon 2020 research and innovation program under grand agreement number 686116.

Acknowledgments: Further acknowledgements go to project partners who provided materials and production facilities-ITENE and Lajovic TUBA d.o.o, respectively. Authors also would like to acknowledge Dieter Bauer for the SEM investigations, the preparation of test specimens, and images thereof. Marius Jesdinszki is also acknowledged for the permeability and layer thickness measurements at the accredited laboratories of Fraunhofer IVV. The authors sincerely thank the Institute of Occupational Medicine for its contribution to the nanosafety assessment related to the new materials. Finally, we would like to acknowledge Dimitri Scholtz and Tiina O'Neill from the Conway Institute, University College Dublin, for the development of the embedding protocol for the TEM investigation.

Conflicts of Interest: The authors declare no conflict of interest.

\section{References}

1. Zink, J.; Wyrobnik, T.; Prinz, T.; Schmid, M. Physical, chemical and biochemical modifications of protein-based films and coatings: An extensive review. Inter. J. Mol. Sci. 2016, 17, 1376. [CrossRef] [PubMed]

2. Perez-gago, M.B.; Krochta, J.M. Denaturation time and temperature effects on solubility, tensile properties, and oxygen permeability of whey protein edible films. J. Food Sci. 2001, 66, 705-710. [CrossRef]

3. Tunc, S.; Angellier, H.; Cahyana, Y.; Chalier, P.; Gontard, N.; Gastaldi, E. Functional properties of wheat gluten/montmorillonite nanocomposite films processed by casting. J. Mem. Sci. 2007, 289, 159-168. [CrossRef]

4. Were, L.; Hettiarachchy, N.S.; Coleman, M. Properties of cysteine-added soy protein-wheat gluten films. J. Food Sci. 1999, 64, 514-518. [CrossRef]

5. Min, S.; Harris, L.J.; Krochta, J.M. Listeria monocytogenes inhibition by whey protein films and coatings incorporating the lactoperoxidase system. J. Food Sci. 2005, 70, m317-m324. [CrossRef]

6. Janjarasskul, T.; Rauch, D.J.; McCarthy, K.L.; Krochta, J.M. Barrier and tensile properties of whey protein-candelilla wax film/sheet. LWT Food Sci. Technol. 2014, 56, 377-382. [CrossRef]

7. Galus, S.; Kadzińska, J. Whey protein edible films modified with almond and walnut oils. Food Hydrocolloids 2016, 52, 78-86. [CrossRef]

8. Schmid, M.; Sängerlaub, S.; Wege, L.; Stäbler, A. Properties of transglutaminase crosslinked whey protein isolate coatings and cast films. Packag. Technol. Sci. 2014, 27, 799-817. [CrossRef]

9. Di Pierro, P.; Chico, B.; Villalonga, R.; Mariniello, L.; Damiao, A.E.; Masi, P.; Porta, R. Chitosan-whey protein edible films produced in the absence or presence of transglutaminase: Analysis of their mechanical and barrier properties. Biomacromolecules 2006, 7, 744-749. [CrossRef]

10. Gennadios, A.; Brandenburg, A.H.; Weller, C.L.; Testin, R.F. Effect of ph on properties of wheat gluten and soy protein isolate films. J. Agric. Food Chem. 1993, 41, 1835-1839. [CrossRef]

11. Sothornvit, R.; Krochta, J.M. Water vapor permeability and solubility of films from hydrolyzed whey protein. J. Food Sci. 2000, 65, 700-703. [CrossRef]

12. Bugnicourt, E.; Schmid, M.; Kainz, D.M.; Lafortune, P.; Rodriguez-Turienzo, L.; Cinelli, P. Simulation and experimental validation of the denaturation of a whey protein-based coating during convection and/or infrared drying on a plastic film and influence on its oxygen barrier properties. Poly. Plast. Technol. Eng. 2016, 55, 1503-1511. [CrossRef]

13. Schmid, M.; Noller, K.; Wild, F.; Bugnicourt, E. Whey Protein Coated Films. Patent EP2734575A1, 22 July 2011.

14. Hong, S.-I.; Krochta, J.M. Oxygen barrier properties of whey protein isolate coatings on polypropylene films. J. Food Sci. 2003, 68, 224-228. [CrossRef]

15. Cinelli, P.; Schmid, M.; Bugnicourt, E.; Coltelli, M.B.; Lazzeri, A. Recyclability of pet/wpi/pe multilayer films by removal of whey protein isolate-based coatings with enzymatic detergents. Materials (Basel) 2016, 9, 473. [CrossRef] [PubMed]

16. Cinelli, P.; Schmid, M.; Bugnicourt, E.; Wildner, J.; Bazzichi, A.; Anguillesi, I.; Lazzeri, A. Whey protein layer applied on biodegradable packaging film to improve barrier properties while maintaining biodegradability. Polym. Degrad. Stab. 2014, 108, 151-157. [CrossRef]

17. Commission. A European Strategy for Plastics in a Circular Economy; European Commission: Brussels, Belgium, 2018. 
18. Bugnicourt, E.; Schmid, M.; Nerney, O.M.; Wildner, J.; Smykala, L.; Lazzeri, A.; Cinelli, P. Processing and validation of whey-protein-coated films and laminates at semi-industrial scale as novel recyclable food packaging materials with excellent barrier properties. Adv. Mater. Sci. Eng. 2013, 2013, 10. [CrossRef]

19. Ray, S.; Quek Siew, Y.; Easteal, A.; Chen Xiao, D. The potential use of polymer-clay nanocomposites in food packaging. Inter. J. Food Eng. 2006, 2, 1556-3758. [CrossRef]

20. Müller, K.; Bugnicourt, E.; Latorre, M.; Jorda, M.; Echegoyen Sanz, Y.; Lagaron, J.M.; Miesbauer, O.; Bianchin, A.; Hankin, S.; Bölz, U.; et al. Review on the processing and properties of polymer nanocomposites and nanocoatings and their applications in the packaging, automotive and solar energy fields. Nanomaterials 2017, 7, 74 .

21. Giannelis, E.P. Polymer layered silicate nanocomposites. Adv. Mater. 1996, 8, 29-35. [CrossRef]

22. Sinha Ray, S.; Okamoto, M. Polymer/layered silicate nanocomposites: A review from preparation to processing. Prog. Polym. Sci. 2003, 28, 1539-1641. [CrossRef]

23. Hedenqvist, M.S.; Backman, A.; Gällstedt, M.; Boyd, R.H.; Gedde, U.W. Morphology and diffusion properties of whey/montmorillonite nanocomposites. Comp. Sci. Technol. 2006, 66, 2350-2359. [CrossRef]

24. Zhou, J.J.; Wang, S.Y.; Gunasekaran, S. Preparation and characterization of whey protein film incorporated with tio2 nanoparticles. J. Food Sci. 2009, 74, N50-N56. [CrossRef] [PubMed]

25. Sothornvit, R.; Hong, S.-I.; An, D.J.; Rhim, J.-W. Effect of clay content on the physical and antimicrobial properties of whey protein isolate/organo-clay composite films. LWT Food Sci. Technol. 2010, 43, 279-284. [CrossRef]

26. Zolfi, M.; Khodaiyan, F.; Mousavi, M.; Hashemi, M. The improvement of characteristics of biodegradable films made from kefiran-whey protein by nanoparticle incorporation. Carbohydr. Polym. 2014, 109, 118-125. [CrossRef] [PubMed]

27. Azevedo, V.M.; Dias, M.V.; Borges, S.V.; Costa, A.L.R.; Silva, E.K.; Medeiros, É.A.A.; Soares, N.d.F.F. Development of whey protein isolate bio-nanocomposites: Effect of montmorillonite and citric acid on structural, thermal, morphological and mechanical properties. Food Hydrocolloids 2015, 48, 179-188. [CrossRef]

28. Hassannia-Kolaee, M.; Khodaiyan, F.; Pourahmad, R.; Shahabi-Ghahfarrokhi, I. Development of ecofriendly bionanocomposite: Whey protein isolate/pullulan films with nano-sio2. Inter. J. Biol. Macromol. 2016, 86, 139-144. [CrossRef]

29. Langowski, H.C. Permeation of gases and condensable substances through monolayer and multilayer structures. In Plastic Packaging; Piringer, O.G., Baner, A.L., Eds.; Wiley-VCH: Weinheim, Germany, 2008; p. 297.

30. Yang, Y.; Zhu, Z.K.; Yin, J.; Wang, X.Y.; Qi, Z.E. Preparation and properties of hybrids of organo-soluble polyimide and montmorillonite with various chemical surface modification methods. Polymer 1999, 40, 4407-4414. [CrossRef]

31. Paul, D.R.; Robeson, L.M. Polymer nanotechnology: Nanocomposites. Polymer 2008, 49, 3187-3204. [CrossRef]

32. Alexandre, M.; Dubois, P. Polymer-layered silicate nanocomposites: Preparation, properties and uses of a new class of materials. Mater. Sci. Eng. 2000, 28, 1-63. [CrossRef]

33. Müller, K.; Jesdinszki, M.; Schmid, M. Modification of functional properties of whey protein isolate nanocomposite films and coatings with nanoclays. J. Nanomater. 2017, 2017, 10. [CrossRef]

34. Schmid, M.; Merzbacher, S.; Brzoska, N.; Müller, K.; Jesdinszki, M. Improvement of food packaging-related properties of whey protein isolate-based nanocomposite films and coatings by addition of montmorillonite nanoplatelets. Front. Mater. 2017, 4. [CrossRef]

35. Almasi, H.; Ghanbarzadeh, B.; Entezami, A.A. Physicochemical properties of starch-cmc-nanoclay biodegradable films. Int. J. Biol. Macromol. 2010, 46, 1-5. [CrossRef] [PubMed]

36. Lieberman, E.R.; Gilbert, S.G. Gas permeation of collagen films as affected by cross-linkage, moisture, and plasticizer content. J. Polym. Sci. 1973, 41, 33-43. [CrossRef] 
37. Wakai, M.; Almenar, E. Effect of the presence of montmorillonite on the solubility of whey protein isolate films in food model systems with different compositions and pH. Food Hydrocolloids 2015, 43, 612-621. [CrossRef]

38. Cooper, P.; Loughlin, A.; Carnaghan, E.; Read, S. Processing and Control of Novel Nanomaterials in Packaging, Automotive and Solar Panel Processing Lines-optinanopro, Deliverable 9.10, Nanosafety Assessment Report; OptiNanoProGrant Agreement No: 686116; European Commission: Brussels, Belgium, 2018.

(C) 2019 by the authors. Licensee MDPI, Basel, Switzerland. This article is an open access article distributed under the terms and conditions of the Creative Commons Attribution (CC BY) license (http://creativecommons.org/licenses/by/4.0/). 

MDPI

St. Alban-Anlage 66

4052 Basel

Switzerland

Tel. +41616837734

Fax +41 613028918

www.mdpi.com

Polymers Editorial Office

E-mail: polymers@mdpi.com

www.mdpi.com/journal/polymers

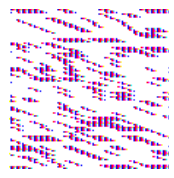



MDPI

St. Alban-Anlage 66

4052 Basel

Switzerland

Tel: +41 616837734

Fax: +41 613028918

www.mdpi.com 